Golden Gate University School of Law GGU Law Digital Commons

1906

Report of progress of stream measurements for the calendar year 1905. Part XIII, Great Basin and Pacific Ocean drainages in California, and Colorado River drainage below Gila River

William Billings Clapp 
DEPARTMENT OF THE INTERIOR

UNITED STATES GEOLOGICAL SURVEY

CHARLES D. WALCOTT, DIRECTOR

\section{REPORT}

$\mathrm{OF}$

\section{PROGRESS OF STREAII MEASUREMENTS}

FOR

\section{THE CALENDAR YEAR 1905}

PREPARED UNDER THE DIRECTION OF F. H. NEWELL

PART XIII.-Great Basin and Pacific Ocean Drainages in California, and Colorado River Drainage below Gila River

BY

W. B. CLAPP and J. C. HOYT

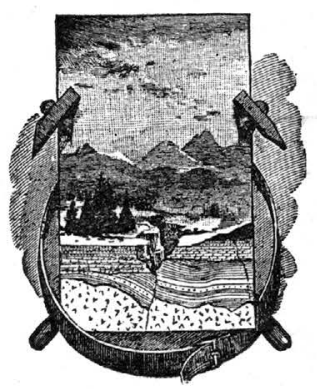

W A SHIN GTON

GOVERNMENT PRINTING OFFICE

1906 



\section{0 N T E T T.}

Introduction Page.

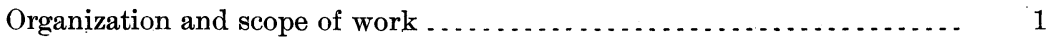

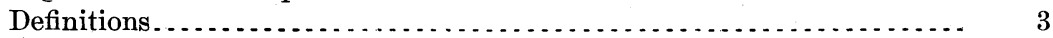

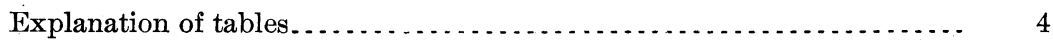

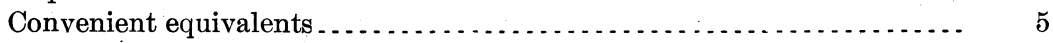

Field methods of measuring stream flow ....................... 6

Office methods of computing run-off . . . . . . . . . . . . . 10

Cooperation and acknowledgments .............................. 12

Colorado River drainage basin below Gila River... . . . . . . . . . . . . . . . . 13

Colorado River at Yuma, Ariz .................................. 13

Colorado River below heading No. 3 of Imperial canal ............... 17

Canals below Yuma, Ariz .................................... 18

Colorado Valley Pumping and Irrigation Company's canal at Yuma,

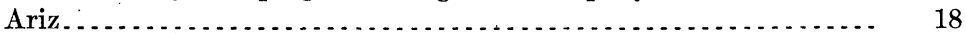

Farmers' canal near. Yuma, Ariz . . . . . . . . . . . . 19

Ludy canal near Yuma, Ariz . . . . . . . . . . . . . . . . . . . . . . .

Imperial canal at headings in United States and Mexico ........... 20

Imperial Valley canals ....................................... 25

Lands irrigated in the Imperial Valley . . . . . . . . . . . . . . . . . 40

Duty of water in the Imperial Valley ....................... 40

McKim flume..................................... 40

California-Mexico Land and Cattle Company's flume .......... 41

Miscellaneous measurements ...................................... 42

The Great Basin drainage . . . . .

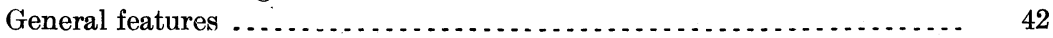

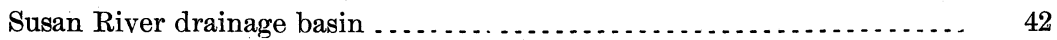

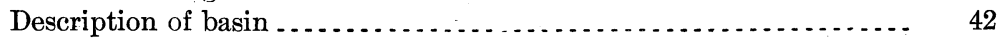

Susan River near Susanville, Cal ......................... 42

Willow Creek at Merrillville, Cal........................... 45

Willow Creek neàr Standish, Cal ........................... 47

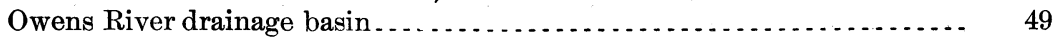

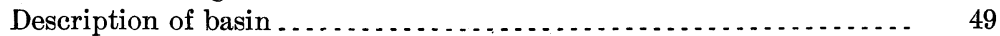

Owens River near Round Valley, Cal.................... 50

Rock Creek near Round Valley, Cal........................ 52

Pine Creek near Round Valley, Cal........................ 55

Owens River canal near Bishop, Cal ....................... 57

Bishop Creek canal near Bishop, Cal . . . . . . . . . . 58

Farmers' canal near Bishop, Cal................................ 59

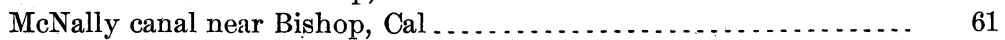

George Collins canal near Bishop, Cal ...................... 61

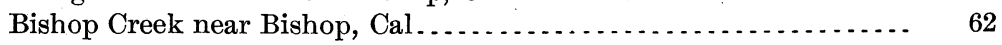

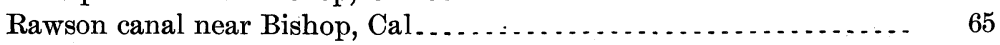

A. O. Collins canal near Bishop, Cal ....................... 66 
The Great Basin drainage-Continued.

Owens River drainage basin-Continued.

Dell canal near Bishop, Cal ................................ 68

Big Pine and Owens River canal near Bishop, Cal .............. 69

Sanger canal at Alvord, Cal .............................. $\quad 70$

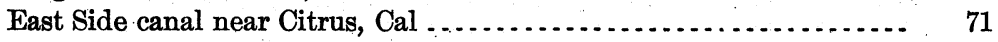

Stevens canal near Citrus, Cal ............................. 73

Owens River near Citrus, Cal ............................. $\quad 74$

Powers canal near Bishop, Cal .......................... 76

South Hillside canal near Bishop, Cal...................... 77

North Hillside canal near Bishop, Cal ..................... 78

Big Pine Creek near Big Pine, Cal................... 78

Birch Creek near Tinemaha, Cal ........................... 80

Independence Creek near Independence, Cal................. 81

Oak Creek near Independence, Cal ......................... 83

Miscellaneous measurements ............................... 84

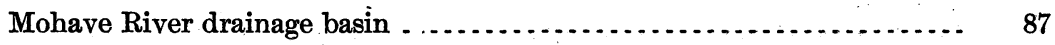

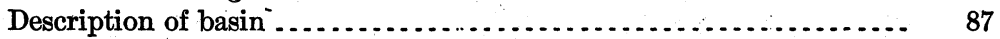

Mohave River at Victorville, Cal .......................... 87

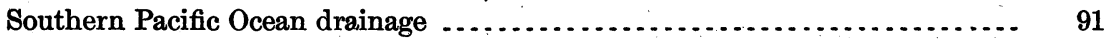

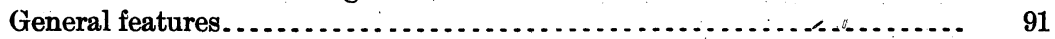

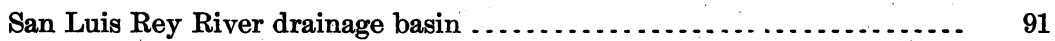

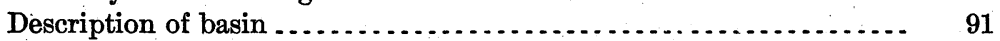

San Luis Rey River near Pala, Cal........................... 91

Santa Ana River drainage basin ............................... 94

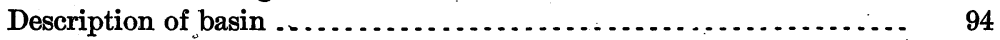

Santa Ana River near Mentone, Cal ......................... 94

Seepage measurements.................................... 99

Miscellaneous measurements .............................. 102

San Gabriel River drainage basin . . . . . . . . . . . . . . . . . . . . . . . 104

Description of basin . ...................................... 104

San Gabriel River and canals near Azusa, Cal .................. 104

Miscellaneous measurements ............................. 108

Los Angeles River drainage basin . .............................. 109

Description of basin ...................................... 109

Miscellaneous measurements ............................... 109

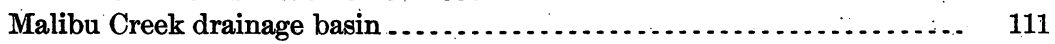

Description of basin ...................................... 111

Malibu Creek near Calabasas, Cal ........................... 111

Triunfo Creek near Calabasas, Cal ............................ 113

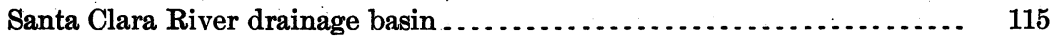

Description of basin .................................... 115

Miscellaneous measurements .............................. 115

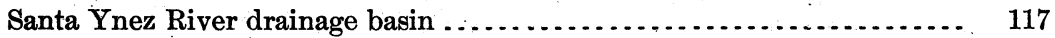

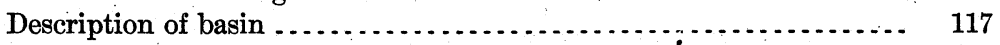

Santa Ynez River near Santa Barbara, Cal . . . . . . . . . . . . . . . ... 117

Santa Maria River drainage basin . . . . . . . . . . . . . . . . 120

Description of basin ......................................... 120

Santa Maria River near Santa Maria, Cal...................... 120

Salinas River drainage basin .............................. 123

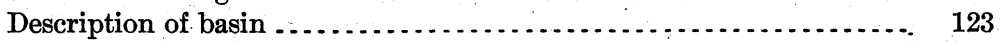

Arroyo Seco near Soledad, Cal . ............................. 123

Miscellaneous measurements in southern California................ 126 
San Francisco Bay drainage basin . . . . . . . . . . . . . . . . . . . . . . . . . . . . . 127

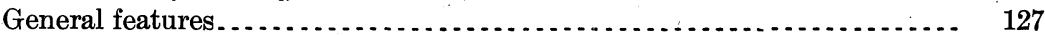

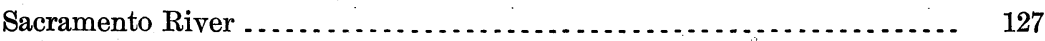

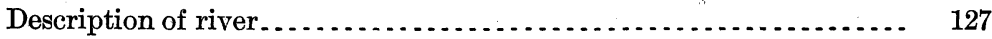

Sacramento River near Red Bluff, Cal . . . . . . . . . . . . . . . . 128

Sacramento River at Sacramento, Cal. . . . . . . . . . . . . . . . . 131

Miscellaneous measurements............................... 131

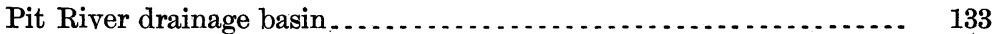

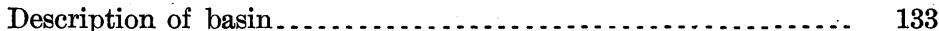

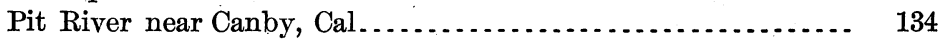

Pit River near Bieber, Cal ............................... 136

South Fork of Pit River near Ivy, Cal. . . . . . . . . . . . . . . . . . . 139

West Valley Creek near Likely, Cal. ........................ 140

Ash Creek at Adin, Cal................................. 144

McCloud River near Gregory, Cal........................ 147

Miscellaneous measurements. ............................ 151

Stony Creek drainage basin . . . . . . . . . . . . . . . . . . . . . . . 152

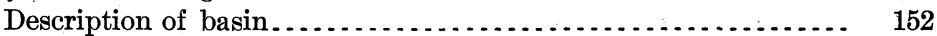

Stony Creek near Fruto, Cal............................... 153

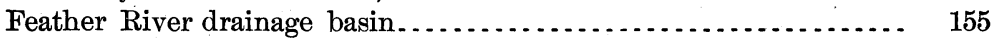

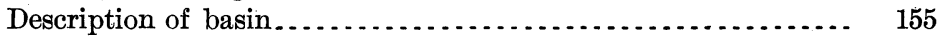

Feather River at Oroville, Cal . . . . . . . . . . . . . . . . 155

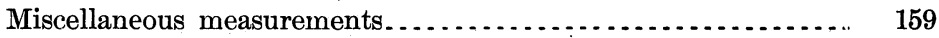

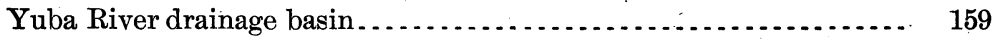

Description of basin.....

Yuba River near Smartsville, Cal _ . . . . . . . . . . . . . . . . 160

Miscellaneous measurements ............................ 164

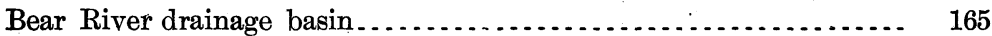

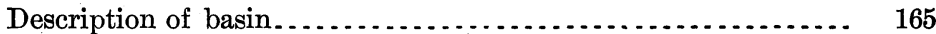

Bear River above Wheatland, Cal ..................... 165

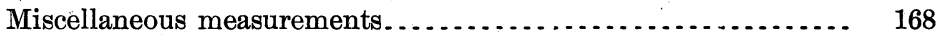

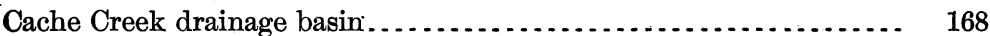

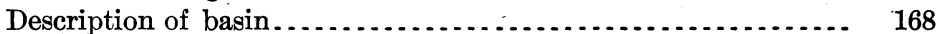

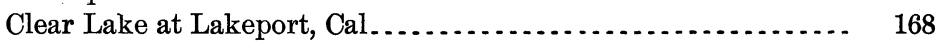

Cache Creek at Lower Lake, Cal......................... 169

Cache Creek near Yolo, Cal............................... 172

Miscellaneous measurements. ............................... 175

American River drainage basin. . . . . . . . . . . . . . . . . . . . . . . 175

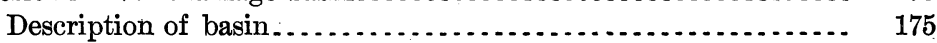

American River near Fairoaks, Cal....................... 176

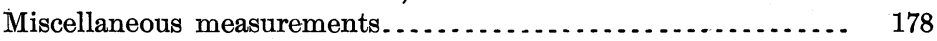

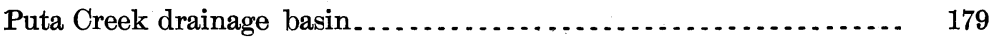

Description of basin.................................. 179

Puta Creek near Guenoc, Cal............................ 180

Puta Creek at Winters, Cal............................. 182

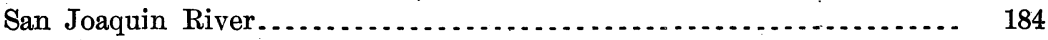

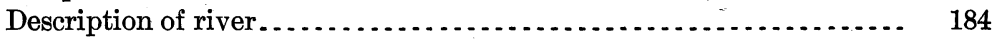

San Joaquin River at Herndon, Cal ... . . . . . . . . . . . . . . . . . 184

Miscellaneous measurements. . . . . . . . . . . . . . . . . . . . . . . . . 185

Kern River drainage basin . . . . . . . . . . . . . . . . . . . . . . . . . . . . 187

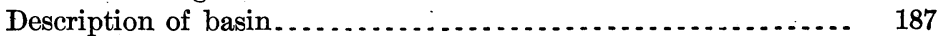

Kern River near Bakersfield, Cal......................... 187 
San Francisco Bay drainage basin-Continued.

San Joaquin River-Continued.

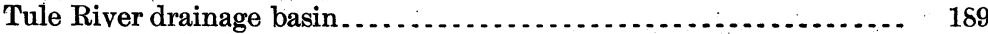

Description of basin .................................. 189

Tule River near Portersville, Cal ........................... " 189

Miscellaneous measurements .............................. 191

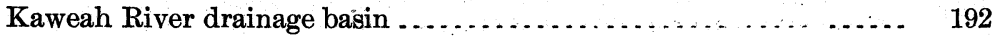

Description of basin . .................................... 192

Kaweah River below Three Rivers, Cal................... 192

Miscellaneous measurements ............................... 194

Kings River drainage basin ................................ 195

Description of basin ..........

Kings River near Sanger, Cal. . . . . . . . . . . . . . . . . . . . . 196

Miscellaneous measurements .............................. 198

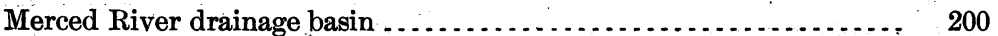

Description of basin ..................................... 200

Merced River in Yosemite Valley, Cal...................... 201

Merced River above Merced Falls, Cal..................... 203

Yosemite Creek in Yosemite Valley, Cal.................... 205

Tenaya Creek in Yosemite Valley, Cal...................... 207

Miscellaneous measurements .............................. 209

Tuolumne River drainage basin . . . . . . . . . . . . . . . . . . . . . 209

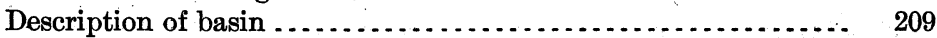

Tuolumne River at Lagrange, Cal . . . . . . . . . . . . . . . . . . . . 209

Modesto canal at Lagrange, Cal . . . . . . . . . . . . . . . . . . . 212

Turlock canal at Lagrange, Cal . . . . . . . . . . . . .

Miscellaneous measurements . . . . . ..................... 216

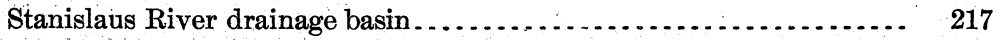

Description of basin ................................... 217

Stanislaus River at Knights Ferry, Cal ................... 217

Stanislaus Water Company's ditch at Knights Ferry, Cal........ 220

Miscellaneous measurements .............................. 222

Mokelumne River drainage basin ............................. 223

Description of basin .................................. 223

Mokelumne River near Clements, Cal..................... 223

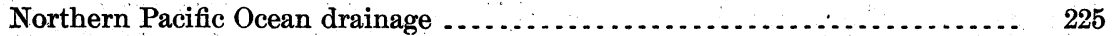

Russian River drainage basin. . . . . . . . . . . . . . . 225

Miscellaneous measurements ................................ 225

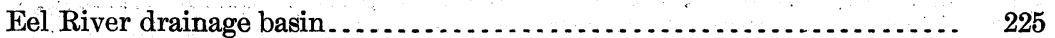

Miscellaneous measurements . . . . . . . . . . . . .

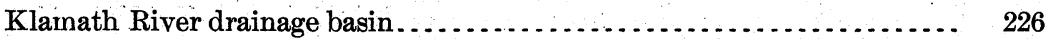

Link River at Klamath Falls, Oreg ....................... 226

Klamath River at Keno, Oreg............................. 229

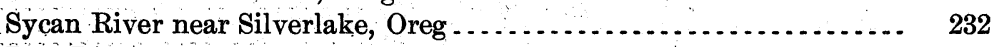

Lost River near Clear Lake, Cal . ............................. 233

Lost River near Merrill, Oreg............................. 236

Tule Lake near Merrill, Oreg. . . . . . . . . . . . . . . . . . . . . . . 238

Miller Creek near Lorella, Oreg ........................... 239

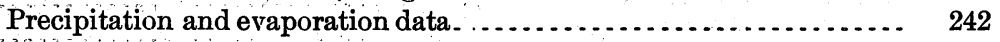

Miscellaneous measurements ..................................... 242

Rogue River drainage basin . . . . . . . . 249

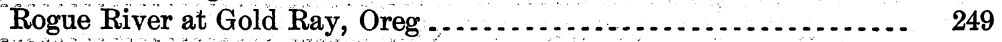


Northern Pacific Ocean drainage-Continued.

Umpqua River drainage basin . ................................. 250

South Fork of Umpqua River near Brockway, Oreg............... 250

North Fork of Umpqua River near Oakcreek, Oreg ............... 251

Siletz River drainage basin ...................................... 252

Siletz River at Siletz, Oreg ................................. 252

Index

\section{IL L USTRATIONS.}

Plate I. Map of the United States, showing location of principal gaging sta-

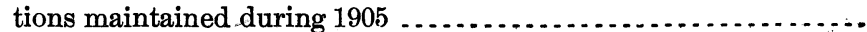

Frg. 1. Cable station, showing section of river, car, gage, etc ...................

2. Dischargє, mean-velocity, and area curves for South Fork of Skykomish

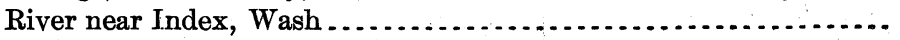

Page. 



\title{
PROGRESS REPORT OF STREAM MEASUREMENTS FOR THE CALENDAR YEAR 1905.
}

\author{
PART XIII.
}

By W. B. Clapp and J. C. HoYt.

INTRODUCTION.

ORGANIZATION AND SCOPE OF WORK.

The hydrographic work of the United States Geological Survey includes the collection of facts concerning and the study of conditions affecting the behavior of water from the time it reaches the earth as rain or snow until it joins the oceans or great navigable rivers. These investigations became a distinct feature of the work of the Survey in the fall of 1888, when an instruction camp was established at Embudo, N. Mex. The first specific appropriation for gaging streams was made by the act of August 18, 1894, which contained an item of $\$ 12,500$ "for gauging the streams and determining the water supply of the United States, including the investigation of underground currents and artesian wells in the arid and semiarid sections." (28 Stat. L., p. 398.)

Since that time the appropriations have been gradually. increased, as shown by the following table:

Annual appropriation for hydrographic surveys, fiscal years ending June 30, 1895 to 1906.

\begin{tabular}{|c|c|}
\hline $1895 \ldots . . .$. & .. $\$ 100,000$ \\
\hline$\ldots \quad 20,000$ & 100,000 \\
\hline$\ldots \ldots \ldots \ldots$ & $\ldots 200,000$ \\
\hline (n) & 200,000 \\
\hline 1899 & $1905 \ldots 000$ \\
\hline 1900 & 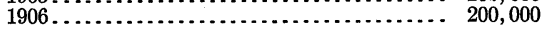 \\
\hline
\end{tabular}

As a result of the increased appropriations the work has been greatly extended, and at the same time it has been more thoroughly systematized by the adoption of standard methods and by grouping the States into districts, in each of which a district hydrographer and a corps of assistants carry on a comprehensive study of the hydrographic resources.

The chief features of the hydrographic work are the collection of data relating to the flow of the surface waters and the study of the conditions affecting this flow. Information is also collected concerning river profiles, duration and magnitude of floods, water power, etc., which may be of use in hydrographic studies. This work includes the study of the hydrography of every important river basin in the United States and is of direct value in the commercial and agricultural development of the country.

In order to collect the material from which estimates of daily flow are made, gaging stations are established. The selection of a site for a gaging station and the length of time it is maintained depend largely on the physical features and the needs of each locality. If the water is to be used for power, special effort is made to obtain information concerning 
the minimum flow; if water is to be stored, the maximum flow receives special attention. In all sections of the country permanent gaging stations are maintained for general statistical purposes, to show the conditions existing through long periods. They are also used as primary stations, and their records, in connection with short series of measurements, serve as bases for estimating the flow at other points in the drainage basin.

During the calendar year 1905 the division of hydrography has continued measuring the flow of streams on the same general lines as in previous years. Many new and improved methods have been introduced, by which the accuracy and value of the results have been increased. Approximately 800 regular gaging stations were maintained during the year, and an exceptionally large number of miscellaneous measurements and special investigations were made. The "Report of Progress of Stream Measurements," which contains the results of this work, is published in a series of fourteen Water-Supply and Irrigation Papers, Nos. 165-178, as follows:

No. 165. Atlantic coast of New England drainage.

No. 166. Hudson, Passaic, Raritan, and Delaware river drainages.

No. 167. Susquehanna, Gunpowder, Patapsco, Potomac, James, Roanoke, and Yadkin river drainages.

No. 168. Santee, Savannah, Ogeechee, and Altamaha rivers and eastern Gulf of Mexico drainages.

No. 169. Ohio and lower eastern Mississippi River drainages.

No. 170. Great Lakes and St. Lawrence River drainages.

No. 171. Hudson Bay and upper eastern and western Mississippi River drainages.

No. 172. Missouri River drainage.

No. 173. Meramec, Arkansas, Red, and lower western Mississippi river drainages.

No. 174. Western Gulf of Mexico and Rio Grande drainages.

No. 175. Colorado River drainage.

No. 176. The Great Basin drainage.

No. 177. The Great Basin and Pacific Ocean drainages in California.

No. 178. Columbia River and Puget Sound drainages.

These papers embody the data collected at the regular gaging stations, the results of the computations based on the observations, and such other information as may have a direct bearing on the study of the subject, and include, as far as practicable, descriptions of the basins and the streams draining them.

For the purpose of introducing uniformity into the reports for the various years the drainages of the United States have been divided into eleven grand divisions, which have been again divided into secondary divisions, as shown in the following list. The Progress Report has been made to conform to this arrangement, each part containing the data for one or more of the secondary divisions. The secondary divisions have in most cases been redivided, and the facts have been arranged, as far as practicable, geographically.

\section{List of drainage basins in the United States.}

NORTHÉRN ATLANTIC DRAINAGE BASINS.

St. John.

St. Croix.

Penobscot.

Kennebec.

Androscoggin.

Presumpscot.

Saco.

Merrimac.

Connecticut.

Blackstone.

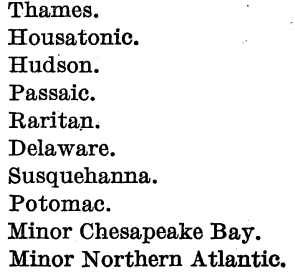

SOUTHERN ATLANTIC DRAINAGE BASINS.

James.

Chowan.

Roanoke.

Tar.

Neuse.

Cape Fear.

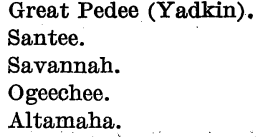

Minor Southern Atlantic. 


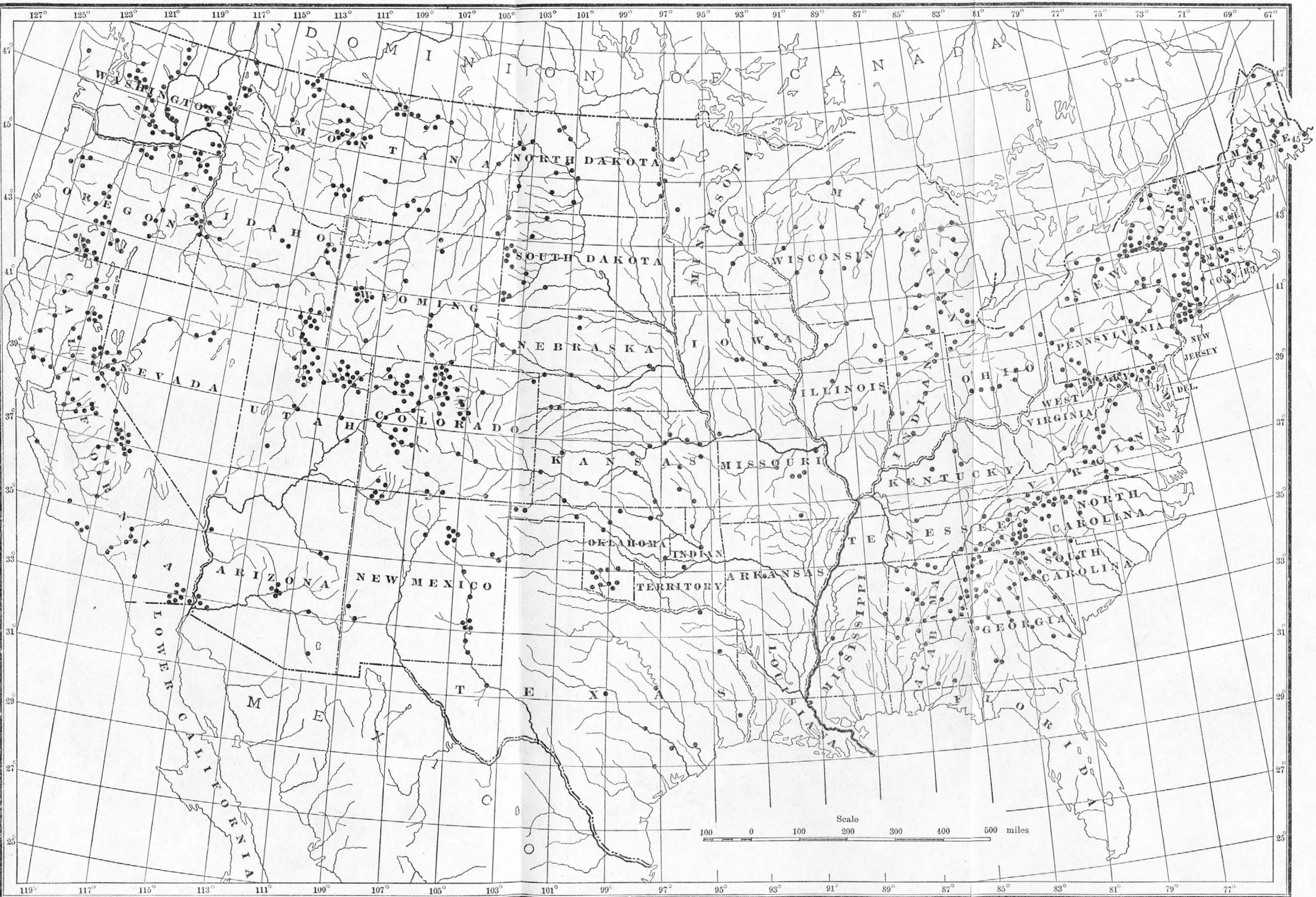

MAP OF THE UNITED STATES, SHOWING LOCATION OF PRINCIPAL RIVER STATIONS MAINTAINED DURING 1905 
EASTERN GULF OF MEXICO DRAINAGE BASINS.

Suwanee.

Apalachicola.

Mobile.
Pearl.

Minor Eastern Gulf of Mexico.

EASTERN MISSISSIPPI RIVER DRAINAGE BASINS.

Lower eastern Mississippi. Ohio.

Upper eastern Mississippi.

ST. LAWRENCE RIVER DRAINAGE BASINS.

Lake Superior.

Lake Michigan.

Lake Huron.

Lake St. Clair.

Lake Erie.

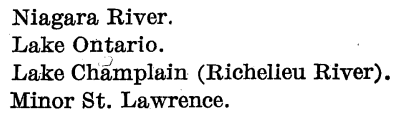

WESTERN MISSISSIPPI RIVER DRAINAGE BASINS.

Upper western Mississippi.

Missouri.

Meramec.

Sabine.

Neches.

Trinity.

Brazos.

Colorado (of Texas).

Wasatch Mountains.

Humboldt.

Southern Pacific.

San Francisco Bay.

Northern Pacific.

Lower western Mississippi.

Arkansas.

Red.

WESTERN GULF OF MEXICO DRAINAGE BASINS.

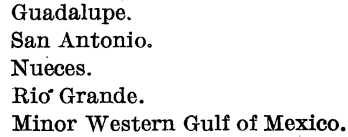

COLORADO RIVER DRAINAGE BASIN.

THE GREAT BASIN.

Sierra Nevada.

Minor streams in Great Basin.

PACIFIC COAST DRAINAGE BASINS.

Columbia.

Puget Sound.

HUDSON BAY DRAINAGE BASINS.

\section{DEFINITIONS.}

The volume of water flowing in a stream, the "run-off" or "discharges," is expressed in various terms, each of which has become associated with a certain class of work. These terms may be divided into two groups-(1) those which represent a rate of flow, as secondfeet, gallons per minute, miner's inch, and run-off in second-feet per square mile; and (2) those which represent the actual quantity of water, as run-off in depth in inches and acrefeet. They may be defined as follows:

"Second-foot" is an abbreviation for cubic foot per second, and is the quantity of water flowing in a stream 1 foot wide, 1 foot deep, at a rate of 1 foot per second. It is generally used as a fundamental unit from which others are computed.

"Gallons per minute" is generally used in connection with pumping and city water supply.

The "miner's inch" is the quantity of water that passes through an orifice 1 inch square under a head which varies locally. It has been commonly used by miners and irrigators throughout the West, and is defined by statute in each State in which it is used. In most States the California miner's inch is used, which is the fiftieth part of a second-foot. 
"Second-feet per square mile" is the average number of cubic feet of water flowing per second from each square mile of area drained, on the assumption that the run-off is distributed uniformly, both as regards time and area.

"Run-off in inches" is the depth to which the drainage area would be covered if all the water flowing from it in a given period were conserved and uniformly distributed on the surface. It is used for comparing run-off with rainfall, which is usually expressed in depth in inches.

"Acre-foot" is equivalent to 43,560 cubic feet, and is the quantity required to cover an acre to the depth of 1 foot. It is commonly used in connection with storage for irrigation work. There is a convenient relation between the second-foot and the acre-foot. One second-foot flowing for twenty-four hours will deliver 86,400 cubic feet, or approximately 2 acre-feet.

\section{EXPLANATION OF TABLES.}

For each regular gaging station are given, as far as available, the following data:

- 1. Description of station.

2. List of discharge measurements.

3. Gage-height table.

4. Rating table.

5. Table of estimated monthly and yearly discharges and run-off, based on all the facts obtained to date.

The descriptions of stations give such general information about the locality and equipment as would enable the reader to find and use the station. They also give, as far as possible, a complete history of all the changes since the establishment of the station that would be factors in using the data collected.

The discharge-measurement table gives the results of the discharge measurements made during the year, including the date, the name of the hydrographer, the area of cross section, the mean velocity, the gage height, and the discharge in second-feet.

The table of daily gage heights gives the daily fluctuations of the surface of the river as found from the mean of the gage readings taken each day. The gage height given in the table represents the elevation of the surface of the water above the zero of the gage. At most stations the gage is read in the morning and in the evening.

The rating table gives discharges in second-feet corresponding to each stage of the river as given by the gage heights.

In the table of estimated monthly discharge, the column headed "Maximum" gives the mean flow for the day when the mean gage height was highest, and it is the flow as given in the rating table for that mean gage height. As the gage height is the mean for the day, there might have been short periods when the water was higher and the corresponding discharge larger than given in this column. Likewise in the column of "Minimum" the quantity given is the mean flow for the day when the mean gage height was lowest. The column headed "Mean" is the average flow for each second during the month. On this are based the computations for the three remaining columns, which are defined above.

In the computations for the tables of this report the following general and special rules have been used:

\section{Fundamental rules for computation.}

1. The highest degree of precision consistent with the rational use of time and money is imperative.

2. All items of computation should be expressed by at least two and not more than four significant figures.

3. Any measurement in a'vertical velocity, mean velocity, or discharge curve whose per cent of error is five times the average per cent of error of all the other measurements should be rejected.

4. In reducing the number of significant figures, or the number of decimal places, by dropping the last figure, the following rules apply:

(a) When the figure in the place to be rejected is less than 5, drop it without changing the preceding figure. Example: 1,827.4 becomes 1,827.

(b) When the figure in the place to be rejected is greater than 5, drop it and increase the preceding figure by 1. Example: $1,827.6$ becomes 1,828 . 
(c) When the figure in the place to be rejected is 5 and it is preceded by an even figure, drop the 5 . Example: $1,828.5$ becomes 1,828 .

(d) When the figure in the place to be rejected is 5 and it is preceded by an odd figure, drop the 5 and increase the preceding figure by 1 . Example: $1,827.5$ becomes 1,828 .

\section{Special rules for computation.}

1. Rating tables are to be constructed as closely as the data on which they are based will warrant. No decimals are to be used when the discharge is over 50 second-feet.

2. Daily discharges shall be applied directly to the gage heights as they are tabulated.

3. Monthly means are to be carried out to one decimal place when the quantities are below 100 secondfeet. Between 100 and 10,000 second-feet, the last figure in the monthly mean shall be a significant figure. This also applies to the yearly mean.

4. Second-feet per square mile and depth in inches for the individual months shall be carried out to at least three significant figures, except in the case of decimals where the first significant figure is preceded by one or more naughts $(0)$, when the quantity shall be carried out to two significant figures. Example: $1.25 ; .125 ; .012 ; .0012$. The yearly means for these quantities are always to be expressed in three significant figures and at least two decimal places.

\section{CONVENIENT EQUTVALENTS.}

1 second-foot equals 50 California miner's inches.

1 second-foot equals 38.4 Colorado miner's inches.

1 second-foot equals 40 Arizona miner's inches.

1 second-foot equals 7.48 United States gallons per second; equals 448.8 gallons per minute; equals 646,272 gallons for one day.

1 second-foot equals 6.23 British imperial gallons per second.

1 second-foot for one year covers 1 square mile 1.131 feet deep, 13.572 inches deep.

1 second-foot for one year equals 0.000214 cubic mile; equals $31,536,000$ cubic feet.

1 second-foot equals about 1 acre-inch per hour.

1 second-foot falling 10 feet equals 1.136 horsepower.

100 California miner's inches equal 15 United States gallons per second.

100 California miner's inches equal 77 Colorado miner's inches.

100 California miner's inches for one day equal 4 acre-feet.

100 Colorado miner's inches equal 2.60 second-feet.

100 Colorado miner's inches equal 19.5 United States gallons per second.

100 Colorado miner's inches equal 130 California miner's inches.

100 Colorado miner's inches for one day equal 5.2 acre-feet.

100 United States gallons per minute equal 0.223 second-foot.

100 United States gallons per minute for one day equal 0.44 acre-foot.

$1,000,000$ United States gallons per day equal 1.55 second-feet.

$1,000,000$ United Sta.tes gallons equal 3.07 acre-feet.

$1,000,000$ cubic feet equal 22.95 acre-feet.

1 acre-foot equals 325,850 gallons.

1 inch deep on 1 square mile equals $2,323,200$ cubic feet.

1 inch deep on 1 square mile equals 0.0737 second-foot per year.

1 inch equals 2.54 centimeters.

1 foot equals 0.3048 meter.

1 yard equals 0.9144 meter.

1 mile equals 1.60935 kilometers.

1 mile equals 1,760 yards; equals 5,280 feet; equals 63,360 inches.

1 square yard equals 0.836 meter.

1 acre equals 0.4047 hectare.

1 acre equals 43,560 square feet; equals 4,840 square yards.

1 acre equals 209 feet square, nearly.

1 square mile equals 259 hectares.

1 square mile equals 2.59 square kilometers.

1 cubic foot equals 0.0283 cubic meter.

1 cubic foot equals 7.48 gallons; equals 0.804 bushel.

1 cubic foot of water weighs 62.5 pounds.

1 cubic yard equals 0.7646 cubic meter.

1 cubic mile equals $147,198,000,000$ cubic feet.

1 cubic mile equals 4,667 second-feet for one year.

1 gallon equals 3.7854 liters.

1 gallon equals 8.36 pounds of water.

1 gallon equals 231 cubic inches (liquid measure).

1 pound equals 0.4536 kilogram.

1 avoirdupois pound equals 7,000 grains. 
1 troy pound equals 5,760 grams.

1 meter equals 39.37 inches. Log. 1.5951654 .

1 meter equals 3.280833 feet. Log. 0.5159842 .

1 meter equals 1.093611 yards. Log. 0.0388629 .

1 kilometer equals 3,281 feet; equals five-eighths mile, nearly.

1 square meter equals 10,764 square feet; equals 1,196 square yards.

1 hectare equals 2.471 acres.

1 cubic meter equals 35.314 cubic feet; equals 1.308 cubic yards.

1 liter equals 1.0567 quarts.

1 gram equals 15.43 grains.

1 kilogram equals 2.2046 pounds.

1 tonneau equals $2,204.6$ pounds.

1 foot per second equals 1.097 kilometers per hour.

1 foot per second equals 0.68 mile per hour.

1 cubic meter per minute equals 0.5886 second-foot.

1 atmosphere equals 15 pounds per square inch; equals 1 ton per square foot; equals 1 kilogram per square centimeter.

Acceleration of gravity equals 32.16 feet per second every second.

1 horsepower equals 550 foot-pounds per second.

1 horsepower equals 76 kilogram-meters per second.

1 horsepower equals 746 watts.

- 1 horsepower equals 1 second-foot falling 8.8 feet.

$1 \frac{1}{3}$ horsepowers equal about 1 kilowatt.

To calculate water power quickly: $\frac{\text { Sec.-ft. } X \text { fall in feet }}{11}=$ Net horsepower on water wheel, realizing 80 per cent of the theoretical power.

Quick formula for computing discharge over weirs: Cubic feet per minute equals $0.4025 l V \overline{h^{3}}$; $l=$ length of weir in inches; $h=$ head in inches flowing over weir, measured from surface of still water.

To change miles to inches on map:

Scale $1: 125000,1$ mile $=0.50688$ inch.

Scale $1: 90000,1$ mile $=0.70400$ inch.

Scale $1: 62500,1$ mile $=1.01376$ inches.

Scale $1: 45000,1$ mile $=1.40800$ inches.

\section{FIELD METHODS OF MEASURING STREAM FLOW.}

The methods used in collecting these data and in preparing them for publication are given in detail in Water-Supply. Papers No. 94 (Hydrographic Manual, U. S. Geol. Survey) and No. 95 (Accuracy of Stream Measurements). In order that those who use this report may readily become acquainted with the general methods employed, the following brief description is given:

Streams may be divided, with respect to their physical conditions, into three classes(1) those with permanent beds; (2) those with beds which change only during extreme low or high water; (3) those with constantly shifting beds. In estimating the daily flow special methods are necessary for each class. The data on which these estimates are based and the methods of collecting them are, however, in general the same.

There are three distinct methods of determining the flow of open-channel streams-(1) by measurements of slope and cross section and the use of Chezy's and Kutter's formulas; (2) by means of a weir; (3) by measurements of the velocity of the current and of the area of the cross section. The method chosen for any case depends on the local physical conditions, the degree of accuracy desired, the funds available, and the length of time that the record is to be continued.

Slope method.-Much information has been collected relative to the coefficients to be used in the Chezy formula, $v=c \sqrt{R s}$. This has been utilized by Kutter, both in developing his formula for $c$ and in determining the values of the coefficient $n$ which appears therein. The results obtained by the slope method are in general only roughly approximate, owing to the difficulty in obtaining accurate data and the uncertainty of the value for $n$ to be used in Kutter's formula. The most common use of this method is in estimating the flood discharge of a stream when the only data available are the cross section, the slope as shown by marks along the bank, and a knowledge of the general conditions. 
Weir method.-When funds are available and the conditions are such that sharp-crested weirs can be erected, these offer the best facilities for determining flow. If dams are suitably situated and constructed, they may be utilized for obtaining reliable estimates of flow. The conditions necessary to insure good results may be divided into two classes-(1) those relat$i_{n g}$ to the physical characteristics of the dam itself, and (2) those relating to the diversion and use of water around and through the dam.

The physical requirements are as follows: $(a)$ Sufficient height of dam, so that backwater will not interfere with free fall over it; $(b)$ absence of leaks of appreciable magnitude; $(c)$ topography or abutments which confine the flow over the dam at high stages; $(d)$ level crests, which are kept free from obstructions caused by floating logs or ice; $(e)$ crests of a type for which the coefficients to be used in $Q=c b h^{\frac{3}{2}}$, or some similar standard weir formula, are known (see Water-Supply Paper No. 150); $(f)$ either no flash-boards or exceptional care in reducing leaking through them and in recording their condition.

Preferably there should be no diversion of water through or around the dam. Generally, however, the dam is built for purposes of power or navigation, and part or all of the "water flowing past it is diverted for such uses. This water is measured and added to that passing over the dam. To insure accuracy in such estimates the amount of water diverted should be reasonably constant. Furthermore, it should be so diverted that it can be measured, either by a weir, a current meter, or a simple system of water wheels which are of standard make, or which have been rated as meters under working conditions and so installed that the gate openings the heads under which they work, and their angular velocities may be accurately abserved.

The combination of physical conditions and uses of the water should be such that the estimates of flow will not involve, for a critical stage of considerable duration, the use of a head on a broad-crested dam, of less than 6 inches. Moreover, when all other conditions are good, the cooperation of the owners or operators of the plant is still essential if reliable results are to be obtained.

A gaging station at a weir or dam has the general advantage of continuity of record through the periods of ice and floods, and the disadvantages of uncertainty of coefficient be used in the weir formula, and of complications in the diversion and use of the water.

$V$ elocity method.-The determination of the quantity of water flowing past a certain section of a stream at a given time is termed a discharge measurement. This quantity is the product of two factors-the mean velocity and the area of the cross section. The mean velocity is a function of surface slope, wetted perimeter, roughness of bed, and the channel conditions at, above, and below the gaging section. The area depends on the contour of the bed and the fluctuations of the surface. The two principal ways of measuring the velocity of a stream are by floats and current meters.

Great care is taken in the selection and equipment of gaging stations for determining discharge by velocity measurements in order that the data may have the required degree of accuracy. Their essential requirements are practicaliy the same whether the velocity is determined by meters or floats. They are located as far as possible where the channel is straight both above and below the gaging section; where there are no cross currents, backwater, or boils; where the bed of the stream is reasonably free from large projections of a permanent character; and where the banks are high and subject to overflow only at flood stages. The station must be so far removed from the effects of tributary streams and dams or other artificial obstructions that the gage height shall be an index of the discharge.

Certain permanent or semi-permanent structures, usually referred to as "equipment," are generally pertinent to a gaging station. These are a gage for determining the fluctuations of the water surface, bench marks to which the datum of the gage is referred, permanent marks on a bridge or a tagged line indicating the points of measurement, and where the current is swift, some appliance (generally a secondary cable) to hold the meter in position in the water. As a rule, the stations are located at bridges if the channel conditions are satisfactory, as from them the observations can more readily be made and the cost of the equipment is small. 
The floats in common use are the surface, subsurface, and tube or rod floats. A corked bottle with a flag in the top and weighted at the bottom makes one of the most satisfactory surface floats, as it is affected but little by wind. In case of flood measurements, good results can be obtained by observing the velocity of floating cakes of ice or débris. In case of all surface-float measurements coefficients must be used to reduce the observed velocity to the mean velocity. The subsurface and tube or rod floats are intended to give directly the mean velocity in the vertical. Tubes give excellent results when the channel conditions are good, as in canals.

In measuring velocity by a float, observation it made of the time taken by the float to pass over the "run," a selected stretch of river from 50 to 200 feet long. In each discharge measurement a large number of velocity determinations are made at different points across the stream, and from these observations the mean velocity for the whole section is determined. This may be done by plotting the mean positions of the floats as indicated by the distances from the bank as ordinates, and the corresponding times as abscissas. A curve through these points shows the mean time of run at any point across the stream, and the mean time for the whole stream is obtained by dividing the area bounded by this curve and its axis by the width. The length of the run divided by the mean time gives the mean velocity.

The area used in float measurements is the mean of the areas at the two ends of the run and at several intermediate sections.

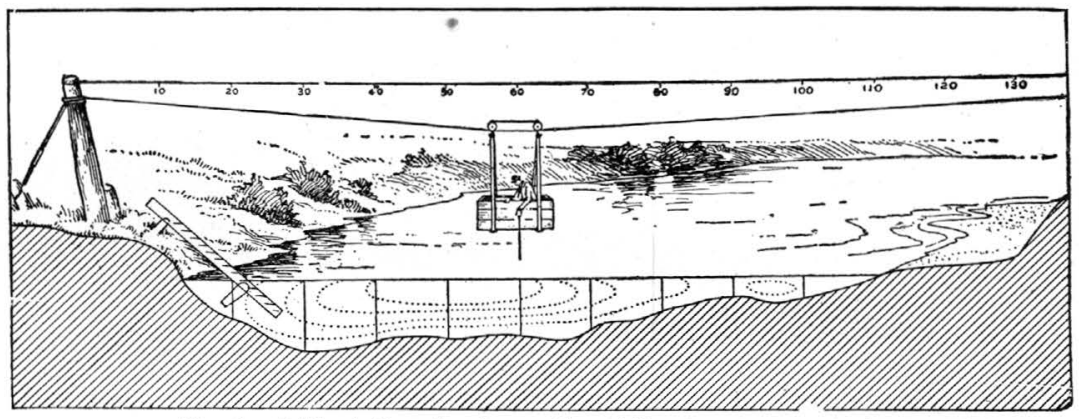

FIG. 1.-Cable station showing section of the river, car, gage, etc.

The essential parts of the current meters in use are a wheel of some type, so constructed that the impact of flowing water causes it to revolve, and a device for recording or indicating the number of revolutions. The relation between the velocity of the moving water and the revolutions of the wheel is determined for each meter. This rating is done by drawing the meter through still water for a given distance at different speeds, and noting the number of revolutions for each run. From these data a rating table is prepared which gives the velocity per second for any number of revolutions.

Many kinds of current meters have been constructed. They may, however, be classed in two general types-those in which the wheel is made up of a series of cups, as the Price, and those having a screw-propeller wheel, as the Haskell. Each meter has been developed for use under some special condition. In the case of the small Price meter, which has been largely developed and extensively used by the United States Geological Survey, an attempt has been made to get an instrument which could be used under practically all conditions.

Current-meter measurements may be made from a bridge, cable, boat, or by wading, and gaging stations may be classified in accordance with such use. Fig. 1 shows a typical cable station.

In making the measurement an arbitrary number of points are laid off on a line perpendicular to the thread of the stream. The points at which the velocity and depth are observed are known as measuring points and are usually fixed at regular intervals, varying from 2 to 20 feet, depending on the size and conditions of the stream. Perpendiculars 
dropped from the measuring points divide the gaging section into strips. For each strip or pair of strips the mean velocity, area, and discharge are determined independently, so that conditions existing in one part of the stream may not be extended to parts where they do not apply.

Three classes of methods of measuring velocity with current meters are in general usemultiple point, single point, and integration.

The three principal multiple-point methods in general use are the vertical velocity-curve; 0.2 and 0.8 depth; and top, bottom, and mid-depth.

In the vertical velocity-curve method a series of velocity determinations are made in each vertical at regular intervals, usually from 0.5 to 1 foot apart. By plotting these velocities as abscissas and their depths as ordinates, and drawing a smooth curye among the resulting points, the vertical velocity-curve is developed. This curve shows graphically the magnitude and changes in velocity from the surface to the bottom of the stream. The mean velocity in the vertical is then obtained by dividing the area bounded by this velocity-curve and its axis by the depth. On account of the length of time required to make a complete measurement by this method, its use is limited to the determination of coefficients for purposes of comparison, and to measurements under ice.

In the second multiple-point method the meter is held successively at 0.2 and 0.8 of the depth, and the mean of the velocities at these two points is taken as the mean velocity for that vertical. Assuming that the vertical velocity-curve is a common parabola with horizontal axis, the mean of the velocities at 0.22 and 0.79 of the depth will give (closely) the mean velocity in the vertical. Actual observations under a wide range of conditions show that this second multiple-point method gives the mean velocity very closely for openwater conditions where the depth is over 5 feet and the bed comparatively smooth, and moreover the indications are that it will hold nearly as well for ice-covered rivers.

In the third multiple-point method the meter is held at mid-depth, at 0.5 foot below the surface, and at 0.5 foot above the bottom, and the mean velocity is determined by dividing by 6 the sum of the top velocity, four times the mid-depth velocity, and the bottom velocity. This method may be modified by observing at $0.2,0.6$, and 0.8 depth.

The single-point method consists in holding the meter either at the depth of the thread of mean velocity, or at an arbitrary depth for which the coefficient for reducing to mean velocity has been determined.

Extensive experiments by vertical velocity-curves show that the thread of mean velocity generally occurs at from 0.5 to 0.7 of the total depth. In general practice the thread of mean velocity is considered to be at $0.6 \mathrm{depth}$, at which point the meter is held in a majority of the measurements. A large number of vertical velocity-curve measurements taken on many streams and under varying conditions show that the average coefficient for reducing the velocity obtained at 0.6 depth to mean velocity is practically unity.

In the other principal single-point method the meter is held near the surface, usually 1 foot below, or low enough to be out of the effect of the wind or other disturbing influences. This is known as the subsurface method. The coefficient for reducing the velocity taken at the subsurface to the mean has been found to be from 0.85 to 0.95 , depending on the stage, velocity, and channel conditions. The higher the stage the larger the coefficient. This method is specially adapted for flood measurements or when the velocity is so great that the meter can not be kept at 0.6 depth.

The vertical-integration method consists in moving the meter at a slow uniform speed from the surface to the bottom and back again to the surface, and noting the number of revolutions and the time taken in the operation. This method has the advantage that the velocity at each point of the vertical is measured twice. It is well adapted for measurements under ice and as a check on the point methods.

The area, which is the other factor in the velocity method of determining the discharge of a stream, depends on the stage of the river, which is observed on the gage, and on the general contour of the bed of the stream, which is determined by soundings. The soundings are usually taken at each measuring point at the time of the discharge measurement, either IRR $177-06-2$ 
by using the meter and cable, or by a special sounding line or rod. For streams with permanent beds standard cross sections are usually taken during low water. These sections serve to check the soundings which are taken at the time of the measurements, and from them any change which may have taken place in the bed of the stream can be detected. They are also of value in obtaining the area for use in computations of high-water measurements, as accurate soundings are hard to obtain at high stages.

In computing the discharge measurements from the observed velocities and depths at various points of measurement, the measuring section is divided into elementary strips, as shown in fig. 1 , and the mean velocity, area, and discharge are determined separately for either a single or a double strip. The total discharge and the area are the sums of those for the various strips, and the mean velocity is obtained by dividing the total discharge by the total area.

The determination of the flow of an ice-covered stream is difficult, owing to diversity and instability of conditions during the winter period, and also to lack of definite information in regard to the laws of flow of water under ice. The method now employed is to make frequunt discharge measurements during the frozen periods by the vertical velocity-curve method, and to keep an accurate record of the conditions, such as the gage height to the surface of the water as it rises in a hole cut in the ice, the thickness and character of the ice, etc. From these data an approximate estimate of the daily flow can be made by constructing a rating curve (really a series of curves) similar to that used for open channels, but considering in addition to gage heights and discharge, the varying thickness of ice. Such data as are available in regard to this subject are published in Water-Supply Paper No. 146, pages 141-148.

\section{OFFICE METHODS OF COMPUTING RUN-OFF.}

There are two principal methods of estimating run-off, depending on whether or not the bed of the stream is permanent.

For stations on streams with permanent beds the first step in computing the run-off is the construction of a rating table, which shows the discharge corresponding to any stage of the stream. This rating table is applied to the record of stage to determine the amount of water flowing. The construction of the rating table depends on the method used in measuring flow.

For a station at a weir or dam, the basis for the rating table is some standard weir formula. The coefficients to be used in its application depend on the type of dam and other conditions near its crest. After inserting in the weir formula the measured length of crest and the assumed coefficient, the discharge is computed for various heads and the rating table constructed.

The data necessary for the construction of a rating table for a velocity-area station are the results of the discharge measurements, which include the record of stage of the river at the time of measurement, the area of the cross section, the mean velocity of the current, and the quantity of water flowing. A thorough knowledge of the conditions at and in the vicinity of the station is also necessary.

The construction of the rating table depends on the following laws of flow for open permanent channels: (1) The discharge will remain constant so long as conditions at or near the gaging station remain constant. (2) The discharge will be the same whenever the stream is at a given stage if the change of slope due to the rise and fall of the stream be neglected. (3) The discharge is a function of and increases gradually with the stage.

. The plotting of results of the various discharge measurements, using gage heights as ordinates; and discharge, mean velocity, and area as abscissas will define curves which show the discharge, mean velocity, and area corresponding to any gage height. For the development of these curves there should be, therefore, a sufficient number of discharge measurements to cover the range of the stage of the stream. Fig. 2 shows a typical rating curve with its corresponding mean-velocity and area curves. 
As the discharge is the product of two factors, the area and the mean velocity, any change in either factor will produce a corresponding change in the discharge. Their curves are therefore constructed in order to study each independently of the other.

The area curve can be definitely determined from accurate soundings extending to the limits of high water. It is always concave toward the hortzontal axis or on a straight line, unless the banks of the stream are overhanging.

The form of the mean-velocity curve depends chiefly on the surface slope, the roughness of the bed, and the cross section of the stream. Of these, the slope is the principal factor. In accordance with the relative changes of these factors the curve may be either a straight line, convex, or concave toward either axis, or a combination of the three. From a careful study of the conditions at any gaging station the form which the vertical velocity-curve will take can be predicted, and it may be extended with reasonable certainty to stages beyond the limits of actual measurements. Its principal use is in connection with the area curve in locating errors in discharge measurements and in constructing the rating table.

The discharge curve is defined primarily by the measurements of discharge, which are studied and weighted in accordance with the local conditions existing at the time of each

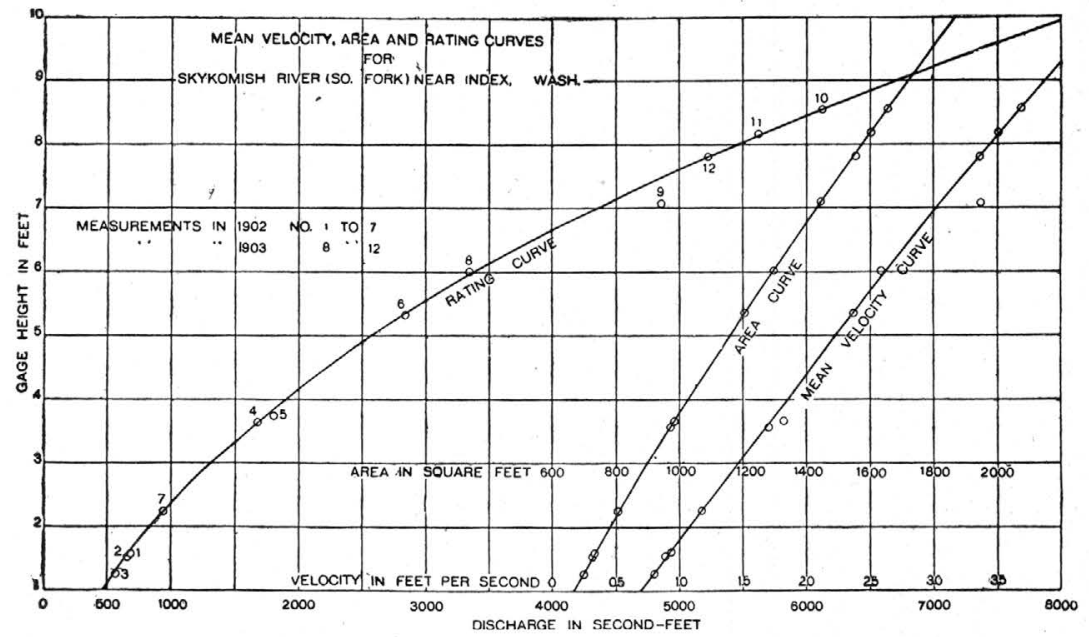

FIG. 2.-Discharge mean-velocity and area curves for South Fork of Skykomish River near Index.

measurement. The curve may, however, best be located between and beyond the measurements by means of curves of area and mean velocity. The discharge curve under normal conditions is concave toward the horizontal axis and is generally parabolic in form.

In the preparation of the rating table the discharge for each tenth or half tenth on the gage is taken from the curve. The differences between successive discharges are then taken and adjusted according to the law that they shall be either constant or increasing.

The determination of daily discharge of streams with changeable beds is a difficult problem In case there is a weir or dam available, a condition which seldom exists on streams of this class, estimates can be obtained by its use. In case of velocity-area stations frequent discharge measurements must be made if the estimates are to be other than rough approximations. For stations with beds which shift slowly, or are materially changed only during floods, rating tables"can be prepared for periods between such changes and satisfactory results obtained with a limited number of measurements, provided that some of them are taken soon after the change occurs. For streams with continually shifting beds, such as the Colorado and Rio Grande, discharge measurements should be made every two or three days and the discharges for intervening days obtained either by interpolation modified by 
gage height or by Professor Stout's method, which has been described in full in the Nineteenth Annual Report of the United States Geological Survey, Part IV, page 323, and in the Engineering News of April 21,1904. This method, or a graphical application of it, is also much used in estimating flow at stations where the bed shifts but slowly.

\section{COOPERATION AND ACKNOWLEDGMENTS.}

Most of the measurements presented in this paper have been obtained through local hydrographers. Acknowledgment is due to each of these persons, and thanks are extended to other persons and corporations who have assisted local hydrographers or have cooperated in any way, either by furnishing records of the height of water or by assisting in transportation.

The hydrographic work of the United States Geological Survey in California is being carried on in cooperation with the State, in accordance with acts of the State legislature approved March 16, 1903, and March 20, 1905.

The act of March 16, 1903, which covered the period from July 1, 1903, to June 30, 1905, is in substance as follows:

The State board of examiners are hereby empowered to enter into contracts with the Director of the United States Geological Survey for the purpose of making topographic maps to the extent of twenty thousand dollars; also for the purpose of gaging streams, surveying reservoir sites and canal locations, for the conservation and utilization of the flood or storm waters of the State, to the extent of fifteen thousand dollars, [etc.]

The act of March 20,1905, is in substance the same as previous act, the appropriations being increased to $\$ 30,000$ for topography and $\$ 20,000$ for hydrography, and covering the two fiscal years July 1, 1905, to June 30, 1907.

The State board of examiners is composed of the following members: George C. Pardee, governor; C. F. Curry, secretary of state; U. S. Webb, attorney-general.

Although the portions of these bills referring to hydrographic work provide for making surveys of reservoir sites and canal locations, none of the State money has been expended for this purpose. The Geological Survey has made these investigations on Puta Creek, Cache Creek, Sacramento River, Pit River and tributaries; Feather River and tributaries, Owens River, and Colorado River, and has paid the entire expense from its own funds.

The State appropriation of $\$ 15,000$, which was available between July 1, 1903, and June 30,1905 , was used exclusively for gathering general stream-flow data. The Survey also apportioned $\$ 20,000$ for the same purpose during the same period. The data being accumulated have a specific value in connection with the future development of the resources of the State. The information will be invaluable in designing and making estimates of cost for storage, irrigation, power and drainage works, and for use in litigation.

The work in California is under the direction of Supervising Engineer J. B. Lippincott, $a$ assisted by Engineer W. B. Clapp, who has immediate charge of the accumulation of hydrographic data. Acknowledgments are also due to the following individuals and corporations for assistance rendered and data furnished: To J. C. Pierson, city surveyor of Sacramento, Cal., for river-stage and turbidity records of Sacramento River at Sacramento; to the Kern County Land Company, through A. K. Warren, engineer in charge of water measurements, for the record of Kern River; to the city of Santa Barbara for cooperation in gaging Santa Ynez River; to the Bay Cities Water Company, through Edwin Duryea, jr., chief engineer, for precipitation and run-ofl data; to the Southern Pacific Company, through its chief engineer, William Hood, for river-stage records of San Joaquin River at Herndon, Cal., and for transportation furnished the supervising engineer and assistants; and to the officials of the Santa Fe Railway for transportation furnished to the supervising engineer and assistants.

The work in the extreme eastern portion of California was carried on under the direction of Henry Thurtell, b State engineer of Nevada, assisted by W. A. Wolf, J. T. Shaw, R. A. Craig, O. F. Heizer, and L. A. Wooley. Acknowledgment is due to the Southern Pacific Company for transportation furnished the district hydrographers and assistants. 


\section{COLORADO RIVER DRAINAGE BASIN BELOW GILA RIVER.}

\section{COLORADO RIVER AT YUMA, ARIZ.}

This station is located in the town of Yuma, Ariz., $1 \frac{1}{2}$ miles below the mouth of Gila River and 10 miles, by river, above the Mexican boundary. Records of river height have been kept by the Southern Pacific Company since April 1, 1878, on the gage which was established by Arthur Brown, superintendent of the company's bridge and building department, during the summer of 1876 .

The channel of the main river is straight for 600 feet above and 5,000 feet below the station. The right bank is low, wooded, and liable to overflow; the left bank is not subject to overflow. The bed of the stream is composed of silt and sand and is very unstable. At low water the channel has a width of 300 feet; at flood stages a large part of the water flows through an old channel and does not pass under the cable. The current is swift and the gaging section regular. At all stages of the river the depth and the velocity are uniform and there are no large eddies.

Prior to May 31, 1903, discharge measurements were made from the railroad bridge. On that date a cable station was established at a point 600 feet below the bridge, and all measurements are now made from a car, except during highest floods, when a boat is used. The cable is supported on masts and is equipped with a winch, by which it can be lowered for the passage of boats. The cable has a span of 650 feet. The initial point for soundings is the cable support on the south or left bank about 20 feet from the water's edge at high water. The water that flows in the old channel at flood stages is measured at the point where it passes under the railway trestle, one-third mile north of the main channel. During the flood of May and June, 1905, there were two breaks in the railroad grade at Araz, Cal., 4 miles from Yuma, and the water passing through these is included in each discharge measurement of the Colorado.

The gage, which was read twice each day during 1905 by W. D. Smith, the resident hydrographer, is in two sections. The lower section of the rod, reading from 15 to 25 feet, is nailed to the pile protection on the south bank of the river 50 feet below the Southern Pacific Railroad bridge. The upper section, reading above 24 feet, is a long pile on the north bank of the river 100 feet above the railway bridge, and is the original gage established in 1876. The bench mark is a standard United States Geological Survey bronze cap located at the railway bridge on the first pier from the south bank; elevation, 35.31 feet above the zero of the gage and 137.4 feet above sea level.

Information in regard to this station is contained in the following publications of the United States Geological Survey (Ann=Annual Report; Bull=Bulletin; WS=Water Supply Paper):

Description: Ann 18, iv, pp 298-299; Bull 131, p 51; 140, pp 207-208; Ws 16, p 151; 28, p 133; 38, p 324; 50 , p 387; 66, p 104; 85, p 17; 100, pp 19-20; 133, pp 25-26.

Discharge: Ann 18, iv, p 299; Bull 131, p 51; 140, p 208; 66, p 104; 85, p 18; 100, pp 20-24; 133, pp $26-29$.

Discharge, mean daily: WS $133, \mathrm{p} 31$.

Discharge, monthly: WS $85, \mathrm{p} 20 ; 100, \mathrm{p} 25 ; 133, \mathrm{p} 32$.

Evaporation record: WS 133, p 32 .

Gage heights: Bull 131, p 52; 140, pp 208-210; WS 11, p 73; 16, p 151; 28, p 141; 38, p 325; 50, p 387; 66, p. $104 ; 85, \mathrm{p} 19 ; 100, \mathrm{pp} 24-25 ; 133, \mathrm{p} 30$.

Hydrograph: Ann 12, ii, p 290.

Rating table: WS $85, \mathrm{p} 19$. 
Discharge measurements of Colorado River at Yuma, Ariz., in 1905.

\begin{tabular}{|c|c|c|c|c|c|c|c|}
\hline Date. & Hydrog & $\begin{array}{c}\text { Gage } \\
\text { height. }\end{array}$ & $\begin{array}{c}\text { Dis- } \\
\text { charge. }\end{array}$ & Da & Hyd & $\begin{array}{c}\text { Gage } \\
\text { height. }\end{array}$ & $\begin{array}{c}\text { Dis- } \\
\text { charge. }\end{array}$ \\
\hline anuary & W. D. Smith. & $\begin{array}{l}\text { Feet. } \\
18.60\end{array}$ & $\begin{array}{r}\text { Sec.-ft. } \\
3,985\end{array}$ & April 2 & W. D & $\begin{array}{l}\text { Feet. } \\
23.40\end{array}$ & $\begin{array}{r}\text { Sec.-ft. } \\
37,160\end{array}$ \\
\hline Tanuary 6 & ....do. & 18.95 & 4,573 & April 27. & A. N. & 23.95 & 41,630 \\
\hline anuary 9. & ...do. & 18.65 & 4,170 & April 29. & W. D. & 23.95 & 34,990 \\
\hline anuary 10. & ...do. & 21.10 & 16,090 & May 1. & .....do. & 25.00 & 41,520 \\
\hline January 11. & .....do. & 19.20 & 6,401 & May 4. & ....do. & 24.25 & 37,280 \\
\hline January & .....do & 19.25 & 6,348 & May 6.. & $\ldots . \mathrm{d}$ & 24.55 & 37,410 \\
\hline January 17. & J.S. Ev & 22.30 & 20,420 & $\mathrm{Mr}$ & d & 25.10 & 40,050 \\
\hline January 18 & .....do. & 23.95 & 27,450 & $\mathrm{Ma}$ & $\ldots d$ & 26.10 & 49,200 \\
\hline January & ....do & 21.00 & 12,170 & Mas & $\ldots . d$ & 25.40 & 38,840 \\
\hline anuary & W. D. S & 20.20 & 7,863 & May 16. & ...do. & 25.00 & 37,320 \\
\hline Januar & J.S. Ev & 19.80 & 7,055 & May 18. & $\ldots . \mathrm{dc}$ & 24.70 & 33,910 \\
\hline Januar & ...do. & 19.55 & 5,727 & $\mathrm{Ma}$ & ...do. & 24.30 & 34,580 \\
\hline nua & & 19.30 & 5,278 & & $\ldots . d$ & 24.90 & \\
\hline $\mathrm{Fe}$ & $\ldots . d c$ & 19.60 & 6,054 & & $\ldots d$ & 25.80 & \\
\hline Febru & $\ldots d$ & 19.70 & 6,632 & $\mathrm{Ma}$ & $\ldots$. & 26.80 & \\
\hline Februa & d & 21.40 & 16,600 & $\mathrm{Ma}$ & $\cdots$ & 27.40 & \\
\hline Februar & $\ldots d$ & 28.00 & 67,730 & Jur & $\ldots d$ & 28.00 & 68 \\
\hline oru & $\ldots d$ & 28.75 & 82,820 & $\mathrm{Ju}$ & $\ldots$ & 28.45 & \\
\hline Februar & $\ldots d$ & 26.20 & 39,580 & Jun & $\cdots$ & -28.30 & 72 \\
\hline ruar & $\ldots c$ & 24.10 & 32,120 & ur & $\ldots$ & 28.25 . & \\
\hline Februar & $\ldots d$ & 21.50 & 18,610 & Jun & $\cdots$ & 28.70 & 82,020 \\
\hline 12 & W. I & 21.40 & 16,490 & & $\ldots$ & 29.15 & \\
\hline Febru & $\ldots d$ & 25.10 & 47,000 & & 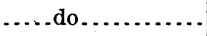 & 29.10 & \\
\hline ua & $\ldots d$ & 25.80 & 54,730 & & $\ldots$ & 28.55 & \\
\hline $\mathrm{Fe}$ & $\ldots d$ & 23.70 & 990 & & $\ldots c$ & .60 & \\
\hline & $\ldots d$ & 22.05 & 21,990 & & $\ldots d$ & 25.90 & \\
\hline & $\ldots$ & 21.50 & & & & .45 & \\
\hline Februar & $\ldots$ & 23.45 & 27,730 & July 1 & $\ldots$ & 23.35 & \\
\hline & $\ldots . d$ & 23.95 & 29,070 & 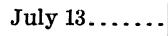 & F.R. & 22.05 & 30,870 \\
\hline & $\cdots c$ & 24.90 & 39,260 & & $\ldots . d$ & 21.90 & \\
\hline & $\ldots . c$ & 26.70 & 70,170 & 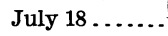 & -0 & 21.75 & 25,300 \\
\hline & $\cdots$ & 24.80 & 310 & & . & 21.30 & \\
\hline 9 & g & 24.10 & 36,400 & 21 & s & 20.90 & 22,000 \\
\hline & $\ldots . \alpha$ & 24.20 & 38,625 & & $\cdots$ & 20.50 & \\
\hline & $\cdots . c$ & 24.45 & 38,870 & & $\ldots$ & 0.35 & \\
\hline & $\ldots$ & 24.00 & 36,720 & & . & .05 & \\
\hline & & 26.10 & 60,640 & & $\ldots$ & 95 & \\
\hline & & 27.35 & 820 & & & .90 & \\
\hline & $\cdots$ & 28.00 & & & $\ldots c$ & 9.80 & \\
\hline & 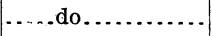 & 30.25 & 110,800 & Alpoust 7 & . & 0.20 & 15 \\
\hline & $\cdots$ & 8.90 & & & $\cdots$ & 0.30 & \\
\hline & 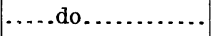 & 27.75 & 930 & & & 0.40 & 16 , \\
\hline & $\ldots d$ & 5.60 & 58,600 . & & & 20.10 & 60 \\
\hline & & 4. 10 & 43,050 & & & 9.60 & 13,800 \\
\hline & $\ldots c$ & 23.10 & 31,020 & & W. I & 19.10 & 9,757 \\
\hline & & 2.00 & & & $\ldots . . d$ & 18.85 & 8,690 \\
\hline & 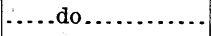 & 21.50 & 20,690 & & & 8.75 & \\
\hline & $\cdots$ & 1.30 & 19,480 & & $\ldots .$. & 8.65 & 7,241 \\
\hline & & 21.60 & 21,000 & & & 18.70 & 7,013 \\
\hline & $\ldots . d$ & 22.50 & & & $\ldots c$ & 19.60 & 10,290 \\
\hline & $\ldots . . d$ & 25.30 & & & & 18.50 & 6,440 \\
\hline & on.. & 29.40 & & & 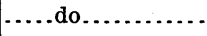 & 18.45 & 6,464 \\
\hline & W. D. Smith. & 24.90 & 45,050 & September 6. & $\ldots c$ & 18.30 & 5,644 \\
\hline & .....do. & 23.30 & & & & 18.45 & 6,051 \\
\hline April 24... & $\ldots$. do & 22.30 & 31,690 & September 11. & ...do & 18.95 & 7,631 \\
\hline
\end{tabular}


Discharge measurements of Colorado River at Yuma, Ariz., in 1905-Continued.

\begin{tabular}{|c|c|c|c|c|c|c|c|}
\hline Date. & Hydrographer. & $\begin{array}{c}\text { Gage } \\
\text { height. }\end{array}$ & $\begin{array}{c}\text { Dis- } \\
\text { charge. }\end{array}$ & Date. & Hydrographer. & $\begin{array}{c}\text { Gage } \\
\text { height. }\end{array}$ & $\begin{array}{c}\text { Dis- } \\
\text { charge. }\end{array}$ \\
\hline September 13. & W. D. Smith. & $\begin{array}{l}\text { Feet. } \\
18.85\end{array}$ & $\begin{array}{r}\text { Sec.-ft. } \\
7,706\end{array}$ & November 3 . & W. D. Smitl & $\begin{array}{l}\text { Feet. } \\
18.50\end{array}$ & $\begin{array}{r}\text { Sec.-ft. } \\
5,812\end{array}$ \\
\hline September 14 & ...do.. & 19.40 & 9,667 & November 6 . & ....do... & 18.35 & 5,709 \\
\hline September 16. & ..do. & 18.80 & 7,700 & November 9 . & ..do. & 18.75 & 6,632 \\
\hline September 18. & ..do. & 18.60 & 6,743 & November 13 & .do & 18.75 & 6,308 \\
\hline September 20 . & ....do. & 18.70 & 7,131 & November 16 & ..do & 18.85 & 6,787 \\
\hline September 23. & ...do. & 18.45 & 6,063 & November 23 & ..do. & 18.80 & 6,495 \\
\hline September 26. & ...do. & 18.20 & 5,260 & November 27 & ...do & 19.00 & 6,652 \\
\hline September 29 . & ....do & 18.00 & 5,287 & Noviember 30 & . .do. & 31.30 & 102,700 \\
\hline October $2 \ldots$ & ...do. & 18.00 & 5,222 & December $1 .$. & 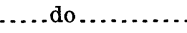 & 28.78 & 77,360 \\
\hline October 4.. & $\ldots$..do & 18.65 & 7,172 & December 2.. & ..d & 23.50 & 37,160 \\
\hline October 6 ... & $\ldots$...do & 18.80 & 7,295 & December $5 .$. &.. $\mathrm{d}$ & 21.30 & 28,650 \\
\hline October 7. . & ...do. & 20.10 & 13,080 & December $8 .$. & ..do & 19.50 & 16,970 \\
\hline October 10.. & ....do. & 20.30 & 14,060 & December 11 . & ..do. & 18.75 & 12,620 \\
\hline October 13.. & ...do. & 19.70 & 10,660 & December 14 . & .. do & 18.30 & 9,613 \\
\hline October $16 .$. & ....do. & 19.10 & 8,290 & December 16 . & ..do & 18.20 & 8,795 \\
\hline October 19. & ....do. & 18.70 & $\cdot 6,779$ & December 18 . & .do & 17.95 & 7,807 \\
\hline October 23. & ...do & 18.60 & 6,025 & December 21 . &..$d$ & 17.85 & 7,670 \\
\hline October 27. . & .....do & 18.40 & 5,507 & December 26 . &.$d$ & 17.95 & 7,502 \\
\hline October 31 . & ....do & 18.40 & 5,579 & December 30 . & ..do. & 17.80 & 5,981 \\
\hline
\end{tabular}

Daily gage height, in feet, of Colorado River at Yuma, Ariz., for 1905.

\begin{tabular}{|c|c|c|c|c|c|c|c|c|c|c|c|c|}
\hline Day. & Jan. & Feb. & Mar. & Apr. & May. & June. & July. & Aug. & Sept. & Oct. & Nov. & Dec \\
\hline & .5 & 19.45 & 3.9 & 21.5 & 25.0 & 27.55 & 26.8 & 19.95 & 18.5 & 18.0 & 18.4 & 28.05 \\
\hline 2 & .5 & 19.6 & 5.05 & 21.4 & 24.7 & 27.8 & 3.5 & 19.9 & 18.4 & 18.0 & 8.45 & 24. \\
\hline 3. & 18.5 & 19.7 & 6.7 & 21.3 & 24.7 & 28.0 & 5.9 & 19.85 & 18.4 & 18.3 & 18.5 & 23.95 \\
\hline 4. & 18.6 & 19.7 & 6.7 & 21.3 & 24.25 & 28.2 & 25.45 & 19.8 & 18.45 & 18.8 & 18.4 & 22.9 \\
\hline 5. & 18.75 & 20.3 & 25.3 & 21.65 & 24.25 & 28.35 & 25.45 & 19.8 & 18.4 & 19.0 & 18.35 & 21.3 \\
\hline 6.. & 18.95 & 21.2 & 24.8 & 22.6 & 24.55 & 28.45 & 25.0 & 19.9 & 18.3 & 18.95 & 18.35 & 20.5 \\
\hline 7. & 19.0 & 21.05 & 24.8 & 22.5 & 24.7 & 28.55 & 24.6 & 20.3 & 8.2 & 20.05 & 18.4 & 20.0 \\
\hline 3. & .9 & 27.2 & 24.7 & 22.1 & 25.1 & 28.3 & 24.1 & 20.5 & 18.3 & 20.2 & 8.65 & 19.5 \\
\hline 9. & 18.65 & 28.75 & 24.1 & 22.0 & 25.75 & 28.2 & 3.8. & 20.3 & 18.45 & 20.6 & 8.75 & 19.2 \\
\hline 0. & 21.0 & 26,6 & 23.9 & 22.0 & 26.1 & 28.15 & 23.35 & 20.2 & 18.95 & 20.3 & 8.8 & 18.95 \\
\hline 1. & 19.25 & 24.1 & 24.1 & 21.85 & 26.3 & 28.2 & 22.8 & 20.45 & 18.7 & 20.1 & 18.8 & 18.75 \\
\hline . & 19.2 & 22.95 & 24.75 & 22.10 & 26.1 & 28.25 & 22.6 & 20.4 & 18.6 & 19.95 & 19.0 & 18.45 \\
\hline .. & 19.25 & 22.4 & 24.35 & 25.45 & 25.5 & 28.4 & 22.1 & 20.15 & 18.85 & 19.7 & 8.75 & 18.4 \\
\hline 4.. & 19.45 & 22.2 & 3.7 & 29.25 & 25.1 & 28.7 & 2.0 & .1 & & 19.3 & .65 & 18.3 \\
\hline 5. & 65 & 22.25 & 24.35 & 29.65 & 25.0 & 28.7 & 1.9 & 19.75 & 19.2 & 19.2 & 3.75 & 18.2 \\
\hline $6 .$. & 19.75 & 21.6 & 26.35 & 27.2 & 25.05 & 28.75 & 21.95 & 19.6 & 18.8 & 19.1 & 8.85 & 18.2 \\
\hline 7. & 22.2 & 21.1 & 27.35 & 24.9 & 24.95 & 28.85 & 22.25 & 19.35 & 18.65 & 18.9 & 18.85 & 18.0 \\
\hline 18. & 23.8 & 21.4 & 26.55 & 24.55 & 24.7 & 28.95 & 21.7 & 19.15 & 18.6 & 18.75 & 18.8 & 17.95 \\
\hline 19.. & 22.2 & 22.65 & 28.1 & 24.9 & 24.5 & 29.15 & 21.3 & 19.05 & 18.6 & 18.7 & 18.95 & 17.9 \\
\hline 20. & 21.0 & 24.9 & 30.3 & 24.4 & 24.3 & 29.05 & 21.25 & 19.0 & 18.7 & 18.7 & 8.9 & 17.95 \\
\hline 1. & 45 & 25.75 & 29.75 & 23.3 & 24.45 & 29.10 & 0.95 & 18.85 & 18.6 & 3.65 & 3.8 & 17.8 \\
\hline & 20.55 & 25. & 28.9 & 22.8 & 24.65 & 29.1 & 0.7 & 18 & 18 & 18 & .8 & 17.85 \\
\hline -. & 20.3 & 23.55 & 27.75 & 22.6 & 24.85 & 29.0 & 0.5 & 18.75 & 18.45 & 18.6 & 8.8 & 17.85 \\
\hline 4. & 20.0 & 22.0 & 25.6 & 22.35 & 25.25 & 28.85 & 0.5 & 18.7 & 18 & 18.6 & 18.7 & 17.9 \\
\hline 5. & 19.8 & 21.45 & 24.1 & 22.6 & 25.55 & 28.75 & 20.45 & 18.65 & 18.35 & 18.6 & 8.75 & 17.85 \\
\hline 26. & 19.75 & 23.65 & 23.4 & 23.45 & 25.75 & 28.55 & 20.35 & 18.65 & 18.2 & 18.5 & 18.8 & 17.95 \\
\hline 27. & 19.65 & 23.5 & 23.1 & 23.95 & 26.0 & 28.25 & 20.2 & 18.7 & 18.0 & 18.4 & 9.05 & 17.9 \\
\hline & 18.55 & 23.5 & 22.85 & 24.15 & 26.4 & 27.9 & 0.05 & 18.7 & 18.0 & 8.35 & 21.3 & 17.8 \\
\hline & 19.4 & & 22.4 & 23.95 & 26.8 & 27.6 & 19.95 & 18.65 & 18.0 & 3.4 & 6.2 & 17.8 \\
\hline U. & 19.35 & & 22.0 & 24.55 & 27.0 & 27.25 & 2 & 19.5 & 18.0 & 18.45 & 31.3 & 17.8 \\
\hline & 19.3 & & 21.9 & $\cdots$ & 27.4 & & 19.95 & 18.8 & & 18.4 & & 17.8 \\
\hline
\end{tabular}


Daily discharge, in second-feet, of Colorado River at Yuma, Ariz., for 1905.

\begin{tabular}{|c|c|c|c|c|c|c|c|c|c|c|c|c|}
\hline & n. & Feb. & Mar. & Apr. & May. & June. & July. & Aug. & Sept. & Oct. & Nov. & Dec. \\
\hline & & 0 & 2 & 20 & 20 & 500 & 00 & 16,400 & 440 & 240 & 620 & , \\
\hline & & 44 & 60 & 20,100 & ,700 &, 300 & 55,500 & 15,790 & 280 & 20 & 720 & 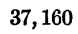 \\
\hline & & 0 & 170 & 19,480 & , 700 & 160 & ,640 & 14,700 & 250 & 40 & & \\
\hline & & 32 & & 19,450 & 280 & 0 & 000 & 13 & 64 & & & \\
\hline & 300 & 9,800 & & 21,000 & 100 & 600 & 950 & 12,900 & 160 & 250 & 00 & \\
\hline & 570 & 16,590 & 310 & 30,100 &, 410 & 67,600 & 400 & 14,200 & 644 & 300 & 10 & \\
\hline & 700 & 9,400 & 44,100 & 29,840 & 000 & 69,500 & 100 & 15,940 & 5,060 & 13,080 & 360 & \\
\hline & 500 & 62,080 & 43,100 & 25,800 & 050 & 30 & ,200 & 17,450 & 560 & 13,560 & 360 & \\
\hline & 170 & 82,820 & 36,400 & 24,800 & 46,000 & 100 & ,500 & 16,770 &, 051 & 15,500 & 630 & \\
\hline & 090 & 52,580 & 34,400 & 24,900 & , 200 & 0 & 980 & 16,200 & 000 & 14,060 & 30 & \\
\hline & 0 & 37,320 & 38,620 & 23,000 & 00 & 00 & 100 & 1 & 31 & 1 & 50 & \\
\hline & 300 & 29,700 & 42,000 & 26,100 &, 000 & 7 & 720 & 16 & 300 & 1 & 950 & \\
\hline & 350 & 22,800 & $|38,870|$ & 45,800 & 38,840 & 76,000 & 870 & 16,150 & 706 & 10 & 10 & 1 \\
\hline & 000 & 21,900 & 32,000 & 93,800 & 37,800 & 20 & 500 & 15,960 & 667 & 100 & & \\
\hline & 370 & 22,500 & $|36,720|$ & 97,500 & 300 & & 710 & 00 & 00 & & & \\
\hline & 600 & 18,610 & 60,640 & 70,100 & 37,320 & 000 & 28,300 & 13,800 & 700 & 290 & 90 & 00 \\
\hline & 100 & 14,600 & 65,820 & $.45,000$ & 37,000 & 00 & 31,100 & 11,900 & 000 & 500 & 640 & \\
\hline & 0 & 16 , & 62,400 & 43,600 & 33 & & 25,300 & 10,300 & 43 & 50. & & \\
\hline & 300 & 31, & 73,440 & 45,050 & 34 & & 320 & 9,757 & 720 & 780 & & 7 \\
\hline & 120 & 47,000 & 11 & 43,400 & 34,580 & 91,500 & 250 & 350 & 31 & 00 & 240 & 7 \\
\hline & 0 & 54,200 & 10 & 39,500 & 700 & 00 &, 000 & 690 & 00 & 400 & 320 & 6 \\
\hline & 170 & 54,730 & 200 & 35,900 & 37,000 & 390 & 21,500 & 100 & 080 & 00 & 00 & \\
\hline & 7,863 & 32,990 & 76,930 & 33,900 & 38,390 & 89,800 & 20,900 & 7,796 & 063 & 020 & 500 & 6 \\
\hline & 900 & 21,990 & 58,600 & 31,690 & 41,500 & $84,800 \mid$ & 20,800 & 7,550 & 890 & 000 & 520 & 5 \\
\hline & & 18,850 & & 33,000 & 43,700 & 82,000 & 20,650 & 7,380 & 40 & & & \\
\hline & & 30 & & & & & 20,460 & 7,241 & 260 & & & \\
\hline & & & & & & & 19,700 & 7,240 & 80 & 510 & & \\
\hline & & 00 & & & & & & 150 & 280 & \pm 00 & & \\
\hline & 5,400 & & & & & & & 850 & 287 & 540 & & \\
\hline & 5,070 & & 24,390 & 38,700 & 56,300 & 61,500 & 17,500 & 10,290 & 5,260 & 5,670 & 102,700 &, 98 \\
\hline & & & 23,50 & & 00 & & 16,750 & 7,500 & & 5,580 & & 5,90 \\
\hline
\end{tabular}

Note.-Daily discharge obtained by indirect method.

Estimated monthly discharge of Colorado River at Yuma, Ariz., for 1905.

[Drainage area, 225,000 square miles.]

\begin{tabular}{|c|c|c|c|c|c|c|}
\hline \multirow[b]{2}{*}{ Month. } & \multicolumn{3}{|c|}{ Discharge in second-feet. } & \multirow[b]{2}{*}{$\begin{array}{l}\text { Total in } \\
\text { acre-feet. }\end{array}$} & \multicolumn{2}{|c|}{ Run-off. } \\
\hline & Maximum. & Minimum. & Mean. & & $\begin{array}{l}\text { Second-feet } \\
\text { per square } \\
\text { mile. }\end{array}$ & $\begin{array}{l}\text { Depth in } \\
\text { inches. }\end{array}$ \\
\hline January.. & 27,500 & 3,750 & 8,130 & 499,900 & 0.036 & 0.042 \\
\hline February.. & 82,820 & 5,800 & 28,100 & $1,561,000$ & .125 & $\cdot .130$ \\
\hline March..... & 110,800 & 23,500 & 50,540 & $3,108,000$ & .225 & .259 \\
\hline April.... & 97,500 & 19,450 & 37,830 & $2,251,000$ & .168 & .187 \\
\hline May.... & 59,020 & 33,910 & $42,170^{\circ}$ & $2,593,000$ & .187 & .216 \\
\hline June... & 94,320 & 61,500 & 76,470 & $4,550,000$ & .340 & .379 \\
\hline July.... & 57,800 & 16,750 & 30,310 & $1,864,000$ & .135 & .156 \\
\hline August.. & 17,450 & 6,850 & 12,100 & 744,000 & .054 & .062 \\
\hline September. & 9,667 & 5,060 & 6,495 & 386,500 & .029 & .032 \\
\hline October.. & 15,500 & 5,220 & 8,037 & 494,200 & .036 & .042 \\
\hline November..... & 102,700 & 5,620 & 12,000 & 714,000 & .053 & .059 \\
\hline December. & 77,360 & 5,900 & 15,400 & 946,900 & .068 & .078 \\
\hline The year.. & 110,800 & 3,750 & 27,300 & $19,710,000$ & .121 & 1.64 \\
\hline
\end{tabular}


Yearly maximum and minimum gage heights, in feet, of Colorado River at Yuma, Ariz., 1878 to 1905 .

[Compiled from the records of Capt. Isaac Polhamus, the Southern Pacific Railroad, and the United States Geological Survey.]

\begin{tabular}{|c|c|c|c|c|c|c|c|c|c|}
\hline \multirow{2}{*}{ Year. } & \multicolumn{2}{|c|}{ Maximum. } & \multicolumn{2}{|c|}{ Minimum. } & \multirow{2}{*}{ ear. } & \multicolumn{2}{|c|}{ Maximum. } & \multicolumn{2}{|c|}{ Minimum. } \\
\hline & Height. & Date. & Ieight. & Date. & & Height. & Date. & Height. & Date. \\
\hline 378 & 23.0 & June 24 & 14.7 & Dec. 31 & 1892 & 25.5 & July 3 & 15.5 & Dec. 31 \\
\hline 1879. & 20.0 & May 12 & 13.2 & Oct. 14 & 1893 & 25.2 & May 28 & 15.5 & Jan. 2 \\
\hline 1880. & 24.0 & May 31 & 14.9 & Dec. 8 & 1894 & 23.7 & June 14 & 15.9 & Jan. 23 \\
\hline 1881. & 23.5 & June 14 & 15.0 & Jan. 25 & 1895. & 28.2 & Jan. 20 & 16.8 & Feb. 13 \\
\hline 1882. & 22.6 & June 18 & 15.5 & Dec. 20 & 1896. & 24.5 & Sept. 30 & 17.4 & Dec. 17 \\
\hline 1883. & 24.5 & July 3 & 14.0 & Dec. 14 & 1897. & 26.1 & June 9 & 17.9 & Dec. 21 \\
\hline 1884. & $a 28.5$ & June 27 & 14.2 & Dec. 5 & 189 & 23.6 & June 27 & 17.5 & Jan. \\
\hline 1885. & 24.7 & June 13 & 13.7 & Feb. 8 & 1899 & 27.0 & July 1 & 17.0 & Oct. 17 \\
\hline 1886. & 26.8 & June 6 & 14.4 & Jan. 19 & 1900. & 26.0 & June 10 & 16.4 & Sept. 10 \\
\hline 1887. & 23.5 & June 10 & 14.9 & Jan. 26 & 1901. & 27.2 & May 31 & 16.2 & Jan. .14 \\
\hline 1888. & 21.8 & June 25 & 14.8 & Jan. 4 & 1902. & 24.5 & May 26 & 16.6 & Sept. 28 \\
\hline 1889. & 22.4 & June 7 & 15.4 & Sept. 27 & 1903. & 27.7 & June 26 & 16.8 & Jan. 13 \\
\hline 1890. & 25.5 & June 5 & 16.4 & Jan. 29 & 1904. & 26.3 & June 5 & 18.3 & Dec. 27 \\
\hline $1891 .$. & $b 33.2$ & Feb. 26 & 16.4 & Sept. 22 & 1905. & 31.3 & Nov. 30 & 17.8 & Dec. 31 \\
\hline
\end{tabular}

$a$ Said to be highest flood for 17 years preceding.

$b$ Highest flood recorded.

COLORADO RIVER BELOW HEADING NO. 3 OF THE IMPERIAL CANAL.

Measurements at this point were begun July 12,1905 , as so much water was at that date going down the canal that it was simpler and more economical to measure the water in the old channel of the Colorado than to continue the measurements on the canal itself.

In the center of the river, opposite heading No. 3, is an island nearly a mile long. The channel on the west side of the island is about 600 feet wide; that on the east side is about 800 feet wide. At first the discharge was measured at a point below the island, but by August 15 a sand bar had formed from the island to the west bank of the river below the heading, and all water passing down on the west of the island entered the canal. August 31 a gage was established above the head of the island, and after that date all discharge measurements were made at the head of the island. Measurements were not made at one certain point, but at any favorable cross section among the shifting bars of mud and quicksand that could be reached and measured by boat or by wading.

The old channel continued to silt up gradually and the new one to scour deeper, and Oetober 20,1905 , all the water was carried by the new channel, i. e., the Imperial canal. The California Development Company made a serious and expensive effort to turn the water back by constructing a dam of piles, brush mats, brush, and sand bags. By the latter part of November this dam had progressed sufficiently to raise the height of the water 2.5 feet, and about 300 second-feet of water flowed down the old channel. Most of the great flood of November 29 at its crest went down the old channel; but by the time it had receded the dam was washed away, the old channel was silted up higher than before, and the new channel was scoured still deeper. 
The gage is a vertical rod nailed to the root of a large willow stump on the east bank of the river. It is located at a point nearly opposite the upper end of the upper row of piling, extending from the head of the island obliquely upstream to the west bank, and is opposite station 425 of the Yuma Valley levee line. The bench mark is a nail in a willow tree 20 feet above the gage and 25 feet from the bank; elevation, 13.05 feet above the zero of the gage. Levels from the levee line show the elevation of the zero of the gage to be 109.19 feet above sea level.

Diseharge measurements of Colorado River, below heading No. 3 of Imperial canal in 1905.

\begin{tabular}{|c|c|c|c|c|c|c|c|}
\hline Date. & Hydrographer. & $\begin{array}{c}\text { Gage } \\
\text { height. }\end{array}$ & $\begin{array}{c}\text { Dis- } \\
\text { charge. }\end{array}$ & Date. & Hydrographer. & $\begin{array}{c}\text { Gage } \\
\text { height. }\end{array}$ & $\begin{array}{c}\text { Dis- } \\
\text { charge. }\end{array}$ \\
\hline July 12 . & W. D. Smith . & $\begin{array}{c}\text { Feet. } \\
\ldots . . . .\end{array}$ & $\begin{array}{c}\text { Sec. }-f t \\
11,610\end{array}$ & October $18 \ldots$ & W. D. Smith. & $\begin{array}{c}\text { Feet. } \\
1.50\end{array}$ & $\begin{array}{r}\text { Sec.-ft. } \\
25\end{array}$ \\
\hline July $30 \ldots$ & L. B. Brainard .. & $\ldots \ldots$ & 2,484 & October $25 \ldots$ & .....do...... & 1.00 & $a_{0}$ \\
\hline August 3 . & F. R. S. Butteme & $\ldots$. & 1,999 & October $30 . .$. & .....do.... & .95 & $a_{0}$ \\
\hline August 10. & .....do....... & $\ldots$ & 2,218 & November $4 .$. & .....do...... & .95 & $a_{0}$ \\
\hline August 18 & W. D. Smith . & $\ldots . .$. & 1,169 & November 11 & .....do. & $\cdots$ & $a_{0}$ \\
\hline August $24 \ldots$. ... & $\ldots$. do...... & ........ & 769 & November 18 & .....do... & 2.10 & 87 \\
\hline August 31 . & .....do..... & 2.90 & 557 & November 25 & ....do..... & 2.45 & 205 \\
\hline September $7 .$. & .....do...... & 1.80 & 90 & November 30 & J. A. Tolin. & & b 70,000 \\
\hline September 14 . & .....do... & 3.80 & 1,232 & December 7 & W. D. Smith . & & 878 \\
\hline September 22 . & .....do.... & 2.10 & 175 & December 13 & .....do. & & $c_{0}$ \\
\hline September 28 . & ......do.... & 1.60 & 37 & December 17 & .....do.... & & $c_{0}$ \\
\hline October $11 .$. & .....do. . & 3.50 & 1,014 & December 23 & .....do . . & & $a_{0}$ \\
\hline
\end{tabular}

$a$ No discharge October 20 to November 11.

$b$ Estimated.

$c$ No discharge December 13-31.

CANAIS BELOW YUMA, ARIZ.

\section{COLORADO VALIEY PUMPING AND IRRIGATION COMPANY'S CANAL AT YUMA, ARIZ.}

This canal diverts water from the Colorado River at Yuma, Ariz., for the purpose of irrigating land in the upper part of Yuma Valley. The water is raised by a large centrifugal pump operated by a steam engine of 125 horsepower. The lift varies from 2 to 12 feet in ordinary seasons. The expense of pumping is about $\$ 0.60$ per acre-foot; not including interest on investment and deterioration of plant. The amount of water used during 1904 was accurately determined by W. D. Smith, and reported in Water-Supply Paper No. 134, page 42, under the head of "Duty of water and seepage investigations." Measurements made in 1903 are reported in Water-Supply Paper No. 100. During 1905 the zanjero used the same "irrigating head" as for the year preceding, his measurements being made by weirs. This was checked by a meter gaging on September 26 , which gave a discharge of 34.1 second-feet. The engineer, H. H. Alexander, kept a record of the number of irrigating heads pumped every hour the pump was in operation during 1905. The following table is based on his record. The rainfall data were furnished by S. H. Hackett, of the United States Weather Bureau. 
Monthly duty of water of the Colorado Valley Pumping and Irrigation Company's canal and rainfall at Y uma, Ariz., for 1905.

\begin{tabular}{|c|c|c|c|c|}
\hline \multirow[t]{2}{*}{. } & \multicolumn{3}{|c|}{ Duty of water (acre-feet). } & \multirow{2}{*}{$\begin{array}{l}\text { Rainfall } \\
\text { (inches). }\end{array}$} \\
\hline & Total. & $\begin{array}{c}\text { Per acre, } \\
\text { gross area. }\end{array}$ & $\begin{array}{l}\text { Per acre, } \\
\text { net area. }\end{array}$ & \\
\hline January ........... & 220 & 0.03 & 0.10 & 1.15 \\
\hline February........ & 250 & .04 & .11 & 3.43 \\
\hline March.................................... & 0 & .00 & .00 & 3.33 \\
\hline April............. & 1,180 & .18 & .51 & .16 \\
\hline 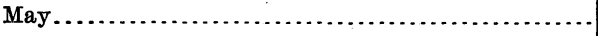 & 1,470 & .23 & .64 & .00 \\
\hline June.......................... & 1,240 & .19 & .54 & .00 \\
\hline July................... & 1,260 & .19 & .55 & .00 \\
\hline August...................... & 1,550 & .24 & .67 & .02 \\
\hline 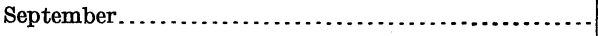 & 1,310 & .20 & .57 & .52 \\
\hline October................................... & 530 & .08 & .23 & .02 \\
\hline November.................... & 350 & .05 & .15 & 2.44 \\
\hline 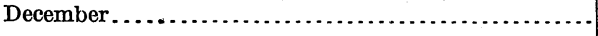 & 300 & .05 & .13 & .32 \\
\hline Total irrigation $\ldots \ldots \ldots \ldots \ldots \ldots \ldots \ldots \ldots \ldots \ldots \ldots \ldots \ldots$ & 9,660 & 1.48 & 4.20 & 11.39 \\
\hline Total, including rainfall..... & & & 5.15 & \\
\hline
\end{tabular}

Mixed crops: Alfalfa, 1,350 acres; grain, 470 acres; corn and sorghum, 250 acres; miscellaneous, 230 acres. Total net area, 2,300 acres; total gross area, 6,500 acres; number of irrigators, 75. Average annual rainfall at Yuma, 3 inches.

The above includes loss by seepage.

\section{FARMERS' CANAI NEAR YUMA, ARIZ.}

This canal, the property of the Yuma Valley Union Land and Water Company, diverts water from Colorado River at a point 1 mile below Yuma, Ariz., for the purpose of irrigating lands in Yuma Valley.

Measurements have been made at the heading immediately at the river since May, 1903, by means of a boat and cable or by wading. As the point of gaging is above the controlling head gate, gage heights would be of no value for the purpose of constructing rating tables and no gage has been established. The height at any date may be obtained approximately by subtracting 1 foot from the gage reading at Yuma.

The canal is heavily silted, and during 1905 has been dry much of the time.

Results of measurements made in previous years are contained in Water-Supply Papers Nos. 100 and 134 of the United States Geological Survey.

Discharge measurements of Farmers' Canal near Yuma, Ariz., for 1905.

\begin{tabular}{|c|c|c|c|c|}
\hline Date. . & Hydrographer. & $\begin{array}{l}\text { Area of } \\
\text { section. }\end{array}$ & $\begin{array}{c}\text { Mean } \\
\text { velocity. }\end{array}$ & $\begin{array}{c}\text { Dis- } \\
\text { charge. }\end{array}$ \\
\hline$\cdot$ & . & sq. feet. & Ft.per sec. & Sec.-ft. \\
\hline January 13 .... & W. D. Smith..... & 31 & 0.71 & 22 \\
\hline March $7 \ldots$ & …do........... & 31 & .58 & 18 \\
\hline May 11... & .....do...... & 67 & 1.91 & 128 \\
\hline June 30. & .....do........ & 37 & 2.27 & 84 \\
\hline
\end{tabular}

LUDY CANAL NEAR YUMA, ARIZ.

This canal is the property of the Irrigation Land and Improvement Company. It diverts water from Colorado River at a point 3 miles below Yuma, Ariz., for the purpose of irrigating lands in Yuma Valley.

Miscellaneous measurements have been made at the heading since March, 1903. June 6, 1905, a gage was established and a nilometer placed on the canal by W. D. Smith, at the 
second gate and bridge, which is 1 mile from the river. No water is taken from the cana above this gate. The gage is nailed to the west wall of the flume below the bridge. The bench mark is a nail driven into the top of the west end of an 8 by 8 inch timber at the lower side of the bridge. Its elevation is $\mathbf{1 5 . 0 0}$ feet above the zero of the gage. The elevation of the top of the floor of the bridge at the southwest corner is 16.10 feet above the zero of the gage. Discharge measurements are made from the lower side of the bridge. The initial point is the east end of the 8 by 8 inch timber referred to above. The canal is straight both above and below the gate. The velocity of the cross section is fairly uniform. The gage height is not affected by any gates farther down the canal.

The canal is badly silted up and is dry much of the time at low water. During the floods of 1905 the banks were overflowed and considerably damaged.

Discharge measurements of Ludy canal near Yuma, Ariz., for 1905.

\begin{tabular}{|c|c|c|c|c|c|}
\hline Date. & Hydrographer. & Area. & $\begin{array}{c}\text { Mean } \\
\text { velocity. }\end{array}$ & $\begin{array}{c}\text { Gage } \\
\text { height. }\end{array}$ & $\begin{array}{c}\text { Dis- } \\
\text { charge. }\end{array}$ \\
\hline March 21. & W. D. Smith. & $\begin{array}{r}S q \cdot f t . \\
405\end{array}$ & $\begin{array}{r}\text { Ft.per sec. } \\
0.90\end{array}$ & Feet. & $\begin{array}{r}\text { Sec.-ft. } \\
\quad 365\end{array}$ \\
\hline April 6. & .....do.. & 128 & .18 & & 23 \\
\hline May $5 . .$. & .....do.. & 104 & .64 & & 67 \\
\hline June $6 .$. & .....do...... & 174 & 1.97 & & 343 \\
\hline June $17 \ldots$ & .....do........ & 799 & 2.42 & 12.60 & 1,932 \\
\hline July $11 .$. & ......do......... & 235 & 1.15 & 10.40 & 271 \\
\hline July $15 .$. & .....do... ............ & 152 & .19 & 9.30 & 29 \\
\hline
\end{tabular}

\section{IMPERIAL CANAL AT HEADINGS IN UNITED STATES AND MEXICO.}

General statement.-The original heading of the Imperial canal, designated in the following tables as heading No. 1, is located about 10 miles by Colorado River below Yuma, Ariz., on the California side, immediately above the international boundary line and opposite Pilot Knob Mountain. The California Development Company is now constructing a permanent gate on bed rock at a spur of this mountain in place of the wooden one now in use. During the summer of 1904 a second heading was made immediately below the boundary line, and in October of the same year an opening was made to the river at a point 4 miles below the boundary. This last opening is designated as heading No. 3. No gage was placed at either heading No. 2 or No. 3. The canal runs parallel to the river for the first 4 miles of its course, so that it was necessary to cut a channel only one-half mile long.

The first 12 miles of the canal was constructed; the remaining 40 miles is an old natural channel, called Alamo River. The whole length of the canal lies in the Colorado River delta, which is composed of loose, sandy soil, covered with a heavy growth of mesquite, willow, and arrowwood. The canal as a whole had a greater fall per mile than the river, but for the first 15 miles the fall was less than that in the river, and for this reason the canal had to be dredged constantly to keep it open. The exceptional floods of January, February, March, and April, 1905, scoured it out to a channel of considerable depth, and during the regular annual flood of May and June, which was unusually high and long, this scouring was continued to such an extent that when the flood began to recede and the river again began to deposit mud, the velocity in the canal was much greater than in the river below heading No. 3. The result was that the old river channel silted up heavily while the new channel, the canal from heading No. 3 down, was still scouring. As the old river channel filled with silt a greater proportion of the water went down the canal, increasing the velocity; and as the canal scoured deeper the amount and velocity of the water going down the old channel were decreased. These reactions were so great that the process of scouring a new channel, which had been going on slowly for several months, began to make rapid progress. The canal was widened in a few days from 100 to 300 or 400 feet. June 30 the total discharge of the river was 61,500 second-feet, 22 per cent of which went down the canal; July 8 the total 
discharge was 37,200 second-feet, 67 per cent of which went down the canal. All efforts to stop the flood proved ineffectual.

Meter measurements have been made weekly during the year to determine the total amount of water diverted by the canal, the amount diverted in the United States, the amount diverted in Mexico, and the amount going down the old river channel.

Besides miscellaneous gagings farther down the canal, measurements were made at five points, as follows: Heading No. 1, heading No. 2, heading No. 3 above its junction with the original canal (giving discharge of heading No. 3 alone), heading No. 3 below the junction (giving the total discharge of the canal), and the old channel of the Colorado below heading No. 3. At all of these stations except the last the conditions are constantly changing. The banks are washing away or being filled in, and the bed is silting up or scouring out and being dredged out. For these reasons it has been necessary to put in new gages often. The frequent passage of barges and dredges has rendered the maintenance of permanent cables impracticable, and a light cable has been stretched for each gaging. Measurements each week have been made at such points as would give the desired results, directly or indirectly, with the least expenditure of time and money.

The total discharge of the canal during 1905 was obtained as follows: From January 1 to March 10 by direct meter measurements at heading No. 3 below the junction; from March 11 to June 30 by adding discharges of headings Nos. 1,2, and 3; from July 1 to December 31 by subtracting the discharge of the old channel below heading No. 3 from the discharge of Colorado River at Yuma. To obtain the discharge of the old channel below heading No. 3 on the dates when it was not measured directly, the total discharge of the canal was subtracted from the discharge of the Colorado at Yuma.

The mean discharge of heading No. 1 for each month was obtained by averaging results of meter measurements. The mean discharge of the whole canal for each month was obtained in the same way. Measurements for the first ten months are so well distributed that they are given equal weight. On account of the flood of November 29 , the estimate for November and December is based on an interpolation of the percentage of the total flow of the river diverted by the canal. As the flood in the old channel lasted only five days, any error from this method of calculation is not great.

The monthly summary of the discharge of headings Nos. 2 and 3, showing total diversion made in Mexico, is obtained by subtracting the discharge of heading No. 1 from the total. The monthly summary of the discharge of the old channel below heading No. 3 is obtained by subtracting the total discharge of the canal from the discharge of Colorado River at Yuma.

No gage observer was obtainable for the three lower stations, but gage-height records were kept at headings Nos. 1 and 2.

June 15, 1904, a waste gage was put in operation at a point 12 miles below heading No. 1, called the Quail River waste gate. Water going through this gate passes through Quail River into Padrones River and thence into Volcano Lake. Volcano Lake has two outletsNew River, which discharges into Salton Basin, and Hardy River, which discharges into the Gulf of California through the old channel of the Colorado. March 20, 1905, the flood silted up the channel leading from the waste gate and no more water has been diverted from the canal at this point.

After the flood of November 29, 1905, a channel was dredged from the canal to Quail River from a point 15 miles below heading No. 1, and a considerable amount of water diverted to Padrones River and Volcano Lake. All the water going down the canal past this point discharges through Alamo and New rivers into Salton Basin, except what is lost by seepage and used for irrigation in the Imperial Valley.

The results of the discharge measurements made at the two waste channels mentioned above will be found in the list of miscellaneous discharge measurements.

Heading No. 1.-This station was established October 24, 1903. It is located half a mile from the river, 600 feet below the wooden head gates, and is 10 miles by river below Yuma, Ariz., on the California side. 
The channel is straight for 600 feet above and 300 feet below the cable and has a width of 70 feet. The right bank is low and is liable to overflow; the left bank has an elevation of 6 feet above high water. The bed of the canal is composed of silt and sand, is free from vegetation, and is very unstable. There is but one channel at all stages, but when the gage at Yuma reads about 26 feet the river overflows into the channel below the gaging section. The current has a moderate velocity.

Discharge measurements are made by means of a boat and cable. The initial point for soundings is a charred post at the southeast corner of the corral about 150 feet west of the right bank.

A vertical gage, which is read twice each day by J. S. Carter, is located just above the international boundary line, on the right bank. The gage is referred to bench marks as follows: (1) A standard United States Geological Survey iron bench-mark post, located near monument 207 of the United States and Mexico boundary line, on a hill about half a mile west of the gaging station; elevation, 52.41 feet above the zero of the gage and 155 feet above sea level. (2) A nail in a tree on the right bank; elevation, 15.20 feet above the zero of the gage. (3) A nail in a post near the ground on the right bank near the corral; elevation, 14.90 feet above the zero of the gage.

The canal at this heading is filled with mud and has been temporarily abandoned, but some flood water passed through it during 1905 and was carefully measured.

Heading No. 2.-This heading diverts water from Colorado River at a point in Mexico 50 feet below the international boundary line, 400 feet below heading No. 1 , and 10 miles by river from Yuma, Ariz. The station was established January 12, 1905, and weekly discharge measurements were made until August 31, 1905.

The channel is straight for 200 feet above and 600 feet below the cable and is about 80 feet wide. The bed and banks are composed of clay and silt and are unstable. There is but one channel at all stages, but when the gage in Yuma reads about 28 feet the river overflows into the canal below the gaging section. As the canal from heading No. 1 joins this canal about 800 feet below, the gage rod, the two gages read about the same.

Discharge measurements are made by means of a boat and cable. The initial point for soundings is a clump of three willow trees growing near the north bank 200 feet below the gage.

A vertical rod gage, which is read daily by $\mathrm{A}$. H. Chance, is driven into the north bank of the canal at a point 50 feet from the river. The bench marks and the elevation of the zero of the gage are the same as for heading No. 1.

Heading No. 3 (above the junction).-This heading or intake is in Mexico, 4 miles below the California-Mexico boundary line and 14 miles by Colorado River below Yuma, Ariz. The station was established March 8, 1905. The point of gaging is about one-half mile from the river and 150 feet above the junction of heading No. 3 with the original canal. Gagings at this point give the discharge of heading No. 3 alone.

The channel is straight for 500 feet above the point of gaging and nearly straight for 500 feet below. In the highest floods the banks are overflowed both above and below the station. The cross section is regular, the bed is unstable, and the current is swift. During July, 1905, the flood widened the channel from 100 to 300 feet and lowered the bed about 10 feet.

Discharge measurements are made by means of a boat and cable. The initial point for soundings, a willow stake on the north bank, was washed away by the flood in July, 1905.

The gage and bench marks are the same as for heading No. 3 below the junction, described below. The gage height is an indication of the total discharge of the canal and not of this heading alone.

Heading No. 3 (below the junction).-This station was established October 7, 1904. The point of gaging is about one-half mile from the river and 300 feet below the junction of heading No. 3 with the original canal. Gagings at this point give the total discharge of the canal. 
The channel is straight for 300 feet above and 500 feet below the cable. The banks are subject to overflow: The cross section is regular. The bed of the canal is composed of silt and sand and is very unstable. During July, 1905, the canal which at this point was about 100 feet wide, was scoured out to a width of 400 feet and the depth increased about 8 feet.

Discharge measurements are made by means of a boat and cable.

The gage is a vertical rod on the south bank and is referred to bench marks as follows: (1) A nail in the central one of three willow trees 20 feet from the bank and 100 feet above the gage; elevation, $\mathbf{1 5 . 8 3}$ feet above the zero of the gage. This beneh mark was originally 2 feet above the surface of the ground but is now 2 feet below, as soil was deposited here to a depth of about 4 feet by the flood of March 20, 1905. (2) A nail on the north side of a large willow stump 100 feet south of the south bank and 500 feet upstream from the gage; elevation, 22.26 feet above the zero of the gage.

Information in regard to this canal is contained in the following Water-Supply Papers of the United States Geological Survey: 85, p. 77; 100, pp. 27-29; 134, pp. 26-39.

Discharge measurements made at the Imperial canal headings during 1905.

\begin{tabular}{|c|c|c|c|c|c|}
\hline \multirow[b]{2}{*}{ Date. } & \multirow{2}{*}{$\begin{array}{l}\text { Heading. } \\
\text { No. } 1 .\end{array}$} & \multirow{2}{*}{$\begin{array}{l}\text { Heading } \\
\text { No. } 2 .\end{array}$} & \multicolumn{2}{|c|}{ Heading No. 3.} & \multirow{2}{*}{$\begin{array}{c}\text { Total } \\
\text { discharge } \\
\text { of canal. }\end{array}$} \\
\hline & & & $\begin{array}{c}\text { Above } \\
\text { junction. }\end{array}$ & $\begin{array}{c}\text { Below } \\
\text { junction. }\end{array}$ & \\
\hline January $12 . . . .$. & & 380 & & 900 & 900 \\
\hline January $26 . . . . .$. & 90 & 350 & & 990 & 990 \\
\hline February $8 \ldots$ & $\cdots$ & & & 6,690 & 6,690 \\
\hline February $14 \ldots$ & 460 & 900 & & 2,480 & 2,480 \\
\hline February 28 & ..... & ...... & $\cdots \cdots$ & 3,100 & 3,100 \\
\hline March $8 . . . . . . .$. & 1,110 & 1,530 & 1,180 & 3,600 & 3,600 \\
\hline March $18 \ldots . . . . .$. & 1,270 & 2,200 & 1,530 & & 5,000 \\
\hline March $21 \ldots$ & 2,590 & 2,240 & 1,920 & *- & 6,750 \\
\hline March $28 \ldots \ldots \ldots \ldots$ & 530 & 1,180 & 1,750 & & 3,460 \\
\hline April $6 \ldots . . . .$. & 450 & 1,190 & 1,650 & $\cdots$ & 3,290 \\
\hline April $20 \ldots \ldots \ldots$ & 760 & 1,190 & 2,610 & …..... & 4,560 \\
\hline April $27 . . . . . .$. & 460 & 1,100 & $\cdots \cdots$ & & ...... \\
\hline May $3 . . . . . . .$. & 180 & 1,470 & 3,360 & & 5,010 \\
\hline May $11 \ldots . . . .$. & & 1,940 & 4,160 & $\cdots$ & 6,100 \\
\hline May $17 . . . . . .$. & & 1,580 & 3,780 & $\ldots$ & 5,360 \\
\hline May $25 . . . . . . . . . . .$. & & 1,790 & 3,560 & 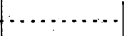 & . 5,350 \\
\hline June $1 . . . . .$. & $\cdots$ & 2,240 & 4,500 & 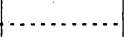 & 6,740 \\
\hline June $7 . . . . . .$. & & 2,660 & 5,830 & & 8,490 \\
\hline June $15 . . .$. & $\cdots$ & 2,700 & 6,130 & 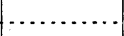 & 8,830 \\
\hline June $23 . . .$. & ... & 3,260 & 6,880 & & 10,140 \\
\hline June $30 \ldots .$. & 390 & 3,140 & 10,430 & ........ & 13,960 \\
\hline July $8 \ldots \ldots$ & & ...... & $\cdots$ & $a 25,000$ & $a 25,000$ \\
\hline July $20 \ldots \ldots$ & & 1,720 & 15,480 & $\cdots \ldots \ldots$ & 17,200 \\
\hline July $25 \ldots . .$. & & ...... & 11,220 & & $\cdots$ \\
\hline August $3 . .$. & & 840 & & & \\
\hline August $10 . . . .$. & & 750 & & & \\
\hline August $18 . . .$. & & 480 & & & \\
\hline August $24 . \ldots \ldots \ldots . .$. & & 440 & & $\cdots$ & \\
\hline August $31 \ldots \ldots \ldots$. & & 470 & & $\ldots: \ldots \ldots$ & \\
\hline
\end{tabular}

NoTE.-Figures in first four columns represent measurements; figures in fifth columen are derived from the preceding. For discharge of canal J uly 25 to December 31 , see following table. 
Portion of Colorado River diverted by Imperial canal during 1905.

\begin{tabular}{|c|c|c|c|c|}
\hline \multirow[b]{2}{*}{ 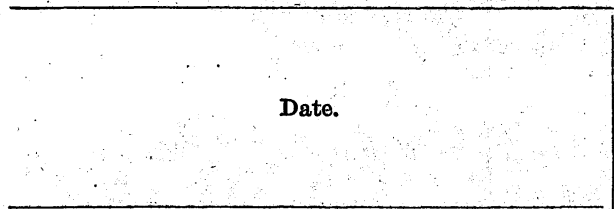 } & \multicolumn{2}{|c|}{$\begin{array}{l}\text { Discharge of Colorado } \\
\text { River in second-feet. }\end{array}$} & \multicolumn{2}{|c|}{$\begin{array}{c}\text { Diversion by Impe- } \\
\text { rial canal. }\end{array}$} \\
\hline & At Yuma. & $\begin{array}{l}\text { Below } \\
\text { heading } \\
\text { No. 3. of } \\
\text { Imperial } \\
\text { canal. }\end{array}$ & $\begin{array}{l}\text { Second- } \\
\text { feet. }\end{array}$ & Per cent. \\
\hline January 12. & 6,300 & 5,400 & 900 & 14 \\
\hline January 26. & 6,770 & 5,780 & 990 & 15 \\
\hline February 8. & 62,080 & 55,390 & 6,690 & 11 \\
\hline February 14.. & 21,900 & 19,420 & 2,480 & 11 \\
\hline February 28. & 25,000 & 21,900 & 3,100 & 12 \\
\hline March 8..... & 43,100 & 39,500 & 3,600 & 8 \\
\hline March 18. & 62,400 & 57,400 & 5,000 & 8 \\
\hline March 21 . & 103,500 & 96,750 & 6,750 & 7 \\
\hline March 28 . & 29,500 & 26,040 & 3,460 . & 12 \\
\hline April $6 . . . . . .$. & 30,100 & 26,810 & 3,290 & 11. \\
\hline April $20 \ldots$ & 43,400 & 38,840 & 4,560 & 11 \\
\hline 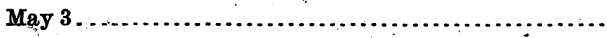 & 39,703 & 34,690 & 5,010 & 13 \\
\hline May $11 . . . \ldots \ldots$ & 52,000 & 45,900 & 6,100 & 12 \\
\hline May $17 . . . .$. & 37,000 & $31 ; 640$ & 5,360 & 14 \\
\hline May 25 ....... & 43,700 & 38,350 & 5,350 & 12 \\
\hline June $1 \ldots$ & $61 ; 500$ & 54,760 & 6,740 & 11 \\
\hline June $7 \ldots . .$. & 69,500 & 61,010 & 8,490 & 12 \\
\hline June 15. & 82,000 & 73,170 & 8,830 & 11 \\
\hline June $23 \ldots$ & 89,800 & 79,660 & 10,140 & 11 \\
\hline June $30 \ldots$ & 61,500 & 47,540 & 13,960 & 23 \\
\hline July $8 \ldots$ & 37,200 & 12,200 & $a 25,000$ & 67 . \\
\hline July $12 \therefore \ldots$ & 31,720 & 11,610 & 20,110 & 63 \\
\hline July $20 \ldots$ & 22,250 & 5,050 & 17,200 & 77 \\
\hline July $25 \ldots . . .$. & 20,650 & $a 8,150$ & a 12,500 & 61 \\
\hline July $30 \ldots$ & 17,500 & 2,480 & 15,020 & 86 \\
\hline August $3 . .$. & 14,700 & 2,000 & 12,700 & 86 \\
\hline August $10 \ldots$ & 16,200 & 2,220 & 13,980 & 86 \\
\hline August 18.... & 10,300 & 1,170 & 9,130 . & 89 \\
\hline August 24 & 7,550 & 770 & 6,780 & 90 \\
\hline August $31, \ldots$ & 7,500 & 560 & 6,940 & 93 \\
\hline September 7 & 5,060 & 90 & 4,970 & 98 \\
\hline September 14 & 9,670 & 1,230 & 8,440 & 87 \\
\hline September 22 & 6,080 & 180 & 5,900 & 97 \\
\hline September 28 . & 5,280 & 40 & 5,240 & 99 \\
\hline October 11.... & 12,950 & 1,010 & 11,940 & 92 \\
\hline October $18 . . .$. & 6,950 & 25 & 6,925 & $99 \frac{2}{3}$ \\
\hline October 25.... & 5,970 & & 5,970 & 100 \\
\hline October $30 . . .$. & 5,670 & & 5,670 & 100 \\
\hline November 4 & 5,750 & & 5,750 & 100 \\
\hline November 11. & 6,550 & $\cdots$ & 6,550 & 100 \\
\hline November 18 & 6,560 & 90 & 6,470 & 99 \\
\hline November $25 \ldots$ & 6,550 & 200 & 6,350 & 97 \\
\hline November $30 \ldots$ & 102,700 & $a 70,000$ & $a 32,700$ & 32 \\
\hline December $7 \ldots$ & 20,100 & 880 & 19,220 & 96 \\
\hline December $13 \ldots$ & 10,200 & & 10,200 & 100 \\
\hline December $17 \ldots$ & 8,100 & & 8,100 & 100 \\
\hline December $23 \ldots$ & 7,600 . & & $7 ; 600$ & 100 \\
\hline December $31 \ldots \ldots$. & 5,900 & & 5,900 & 100 \\
\hline
\end{tabular}


Estimated monthly discharge of Imperial canal during 1905.

\begin{tabular}{|c|c|c|c|c|c|c|}
\hline \multirow{2}{*}{ Month. } & \multicolumn{2}{|c|}{$\begin{array}{l}\text { Heading No.1, includ- } \\
\text { ing total diversion } \\
\text { in United States. }\end{array}$} & \multicolumn{2}{|c|}{$\begin{array}{l}\text { Headings Nos. } 2 \text { and } \\
3 \text {, including total } \\
\text { diversion in Mex- } \\
\text { ico. }\end{array}$} & \multicolumn{2}{|c|}{ Total discharge. } \\
\hline & $\begin{array}{l}\text { Second- } \\
\text { feet. }\end{array}$ & $\begin{array}{l}\text { Total in } \\
\text { acre-feet. }\end{array}$ & $\begin{array}{l}\text { Second- } \\
\text { feet. }\end{array}$ & $\begin{array}{l}\text { Total in } \\
\text { acre-feet. }\end{array}$ & $\begin{array}{l}\text { Second- } \\
\text { fcet. }\end{array}$ & $\begin{array}{l}\text { Total in } \\
\text { acre-feet. }\end{array}$ \\
\hline January .. & 45 & 2,770 & 900 & 55,330 & 945 & 58,100 \\
\hline February .... & 460 & 25,550 & 3,630 & 201,600 & 4,090 & 227,200 \\
\hline March .... & 1,370 & 84,240 & 3,330 & 204,800 & 4,700 & 289,000 \\
\hline$\Lambda$ pril $\ldots \ldots \ldots \ldots \ldots \ldots \ldots \ldots$ & 555 & 33,020 & 3,370 & 200,500 & 3,925 & 233,600 \\
\hline May ........ & 20 & 1,230 & 5,435 & 334,200 & 5,455 & 335,400 \\
\hline June......... & 200 & 11,900 & 9,430 & 561,100 & 9,630 & 573,000 \\
\hline July ......... & 130 & 7,990 & 17,840 & $1,097,000$ & 17,960 & $1,105,600$ \\
\hline Augrust ......... & & $\cdots$ & 9,905 & 609,000 & 9,905 & 609,000 \\
\hline September... & & & 6,140 & 365,400 & 6,140 & 365,400 \\
\hline October.... & $\cdots$ & $\cdots$ & 7,625 & 468,800 & 7,625 & 468,800 \\
\hline November... & . & $\ldots$ & 8,400 & 500,000 & $a 8,400$ & a 500,000 \\
\hline December... & & $\ldots$ & 12,200 & 750,000 & $a 12,200$ & $a 750,000$ \\
\hline The year. & 232 & 166,700 & 7,350 & $5,348,000$ & 7,582 & $5,514,000$ \\
\hline
\end{tabular}

a Approximate.

IMPERIAL VALLEY CANALS.

In July, 1904, stations were established on all canals entering the Imperial Valley, as follows: Folt canal, Hemlock canal, Alamo channel, Alamitos canal, Main canal, and Boundary canal. These stations are located on United States territory, and each is near the California-Mexico boundary line.

Discharge measurements are made from footbridges constructed at each station.

The gages are vertical 4 by 4 inch timbers substantially embedded in the ground. Automatic water-stage registers, from which the daily gage-height record is compiled, were placed at the first five stations mentioned above. These canals enter the valley east of Calexico, Cal.

In October a canal, known as canal No. 6, or Wisteria canal, was completed, which enters the valley west of Calexico, Cal. A station was established on this canal in November and weekly discharge measurements made. After April 11, 1905, the Holt canal supplied a much larger district than originally, covering territory which had been taking its supply from Alamo channel, near Eastside levee. A new canal, known as New Holt or No. 7, was built to replace the Holt, and a station was established in May. A station was also established on the Tamareck canal in June. The latter canal diverted water from New River to a district on the west side of New River below Imperial, which formerly obtained its supply from Main canal. Gages were established at both of these stations, but no automatic registers were placed on either canal.

The station on Alamo channel was inaccessible after the end of February on account of the flooded condition of the country. Water was not taken from the Alamo for irrigation after March. Discharge measurements wore also made at Rockwood, on Alamo River, but no gage heights were kept. They show the amount of water flowing through this channel to Salton Sea.

Measurements were made at Brawley, on New River, for a similar purpose. They were made under such difficulties, however, that they are of little value. Measurements made at Calexico were used to determine the waste into Salton Sea, since the only diversion was through Tamarack canal. A large quantity of water was wasted from Main canal into New River below the gaging station at Calexico; this waste was measured and added to the discharge of New River in making estimate of total discharge of New River into Salton Lake.

IRR $177-06-3$ 
A description of these stations, with gage height and discharge data, is contained in Water-Supply Paper No. 134, United States Geological Survey, pages 29-39.

Discharge measurements of Holt canal, near Calexico, Cal., in 1905.

\begin{tabular}{|c|c|c|c|c|c|c|c|}
\hline Date. & Hydrographer. & $\begin{array}{l}\text { Gage } \\
\text { height }\end{array}$ & $\begin{array}{c}\text { Dis- } \\
\text { charge. }\end{array}$ & Date. & Hydrographer. & $\begin{array}{c}\text { Gage } \\
\text { height. }\end{array}$ & $\begin{array}{c}\text { Dis- } \\
\text { charge. }\end{array}$ \\
\hline January $4 . .$. & W. V. Hardy. & $\begin{array}{r}\text { Feet. } \\
1.30\end{array}$ & $\begin{array}{r}\text { Sec.-ft. } \\
19.5\end{array}$ & June $26 . . . .$. & R. R. McCoslin. & $\begin{array}{l}\text { Feet. } \\
1.64\end{array}$ & $\begin{array}{c}\text { Sec. }-f t . \\
50\end{array}$ \\
\hline January 12. & ....do. & 1.41 & 30 & July 3. . & .....do. & 0.38 & 6.9 \\
\hline January 19. & . .do. & 1.43 & 28 & July $10 \ldots$ & .....do. & .33 & 6.1 \\
\hline January 26 . & ....do. & 1.23 & 19.5 & July 17. . & .....do. & .35 & 5.8 \\
\hline February 2. & ....do & 1.23 & 18.1 & July $24 \ldots$ & .....do... & .36 & 4.8 \\
\hline February $9 \ldots$ & .....do & 1.07 & 13.5 & July $31 . .$. & ......do.. & .36 & 4.2 \\
\hline February 17. & $\ldots$. do & 1.90 & 40 & August $7 . . .$. & .....do.. & 1.45 & 30 \\
\hline February $24 .$. & .....do do & .93 & 11.1 & August 14 . & .....do.. & 1.25 & 38 \\
\hline March 13. & R. R. McCoslin & 1.88 & 51 & August 21.. & .....do. do. & ....... & $85^{-}$ \\
\hline March 20 & .....do.. & 2.06 & 64 & August $21 . . .$. & .....do.. & .039 & 5.1 \\
\hline March 27. & ....do. & 2.84 & 118 & August 28..... & .....do.. & 2.62 & 133 \\
\hline April 3... & ....do. & 2.81 & 131 & September 4 .. & .....do... & 2.67 & 131 \\
\hline April 10. & $\ldots$. do & 1.13 & 21 & October $10 \ldots$ & W. V. Hardy. & 3.08 & 169 \\
\hline April 17. . & ....do & 3.70 & 246 & October $17 \ldots$ & ......do... & .25 & 2 \\
\hline April 24 . & .....do. & 3.70 & 256 & October $23 . .$. & .....do. & 2.65 & 128 \\
\hline May 1. & $\ldots . d c$ & 3.37 & 202 & November $6 .$. & .....do.. & 1.40 & 21 \\
\hline May 8... & ....do & 2.76 & 143 & November 13. & .....do. do. & 1.51 & 35 \\
\hline May 15... & ....do. & 2.91 & 163 & November 20 .. & .....do. & 1.33 & 40 \\
\hline May $22 \ldots$ & ....do. & 2.92 & 169 & November 29 . & ......do....... & 1.31 & 22 \\
\hline May 29. . & ....do. & 2.70 & 128 & October $30 . .$. & .....do. & 2.55 & 124 \\
\hline June $5 . .$. & ....do. & 2.86 & 150 & September 11. & ..... do. & 2.88 & 159 \\
\hline June 12. & .....do.. & 3.02 & 177 & September 25 . & ....do. & 2.75 & 128 \\
\hline June $18 .$. & .....do... & 1.69 & 57 & October $2 \ldots \ldots$ & .....do... & 2.80 & 137 \\
\hline
\end{tabular}


Mean daily gage height, in feet, of Holt canal, near Calexico, Cal., for 1905.

\begin{tabular}{|c|c|c|c|c|c|c|c|c|c|c|c|c|}
\hline Day. & Jan. & Feb. & Mar. & Apr. & May. & June. & July. & Alig. & Sept. & Oct. & Nov. & Dec. \\
\hline & $(1.0)$ & 1.25 & 1.4 & z. & 3.3 & 2.8 & 0.0 & 0.35 . & 3.05 & 2.8 & 2.0 & 1.3 \\
\hline 2. & $(1.0)$ & 1.25 & 1.3 & 2.9 & 3.25 & 2.85 & .0 & .35 & 3.0 & 2.8 & 1.7 & 2.6 \\
\hline $3 .$. & $(1.2)$ & 1.25 & $(1.45)$ & 2.8 & 3.3 & 2.8 & .4 & .35 & 2.75 & 2.8 & 1.7 & 2.8 \\
\hline $4 \ldots$ & $(1.3)$ & 1.25 & $(1.6)$ & 2.5 & 3.2 & 2.8 & .35 & .5 & 2.65 & 2.85 & 1.7 & 3.0 \\
\hline 5. & $(1.3)$ & 1.25 & $(1.4)$ & 2.6 & 2.9 & 2.85 & .35 & 1.15 & 2.7 & 2.85 & 1.5 & 3.0 \\
\hline 6.. & $(1.5)$ & 1.3 & 1.4 & 3.05 & $(2.75)$ & 2.95 & .35 & 1.45 & 2.9 & 2.95 & 1.4 & 2.95 \\
\hline 7.. & $(1.5)$ & 1.15 & 1.55 & (3.3) & $(2.75)$ & 3.0 & .35 & 1.55 & 2.7 & 3.1 & 1.35 & 2.9 \\
\hline 8. & $(1.5)$ & (1.1) & 1.7 & $(3.25)$ & 2.75 & 2.95 & .35 & 1.65 & 2.5 & 3.1 & 1.35 & 2.9 \\
\hline $9 .$. & $(1.5)$ & 1.05 & 1.8 & $(3.25)$ & 2.75 & 3.0 & .35 & 1.65 & 2.75 & 3.15 & 1.35 & 2.85 \\
\hline 10. & $(1.5)$ & 1.05 & 1.65 & 2.15 & 2.75 & 3.05 & .35 & 1.65 & 2.85 & 3.05 & 1.4 & 2.7 \\
\hline $11 .$. & $(1.5)$ & .1 .05 & (1.8) & 3.6 & 2.75 & 3.0 & .35 & 1.55 & 2.9 & 3.15 & 1.45 & 2.7 \\
\hline $12 .$. & 1.4 & 1.0 & $(1.9)$ & 3.65 & 2.8 & 2.95 & .35 & 1.35 & 3.0 & 1.25 & 1.5 & \\
\hline $13 .$. & 1.4 & 1.2 & 1.95 & 3.6 & 2.9 & 2.6 & .35 & 1.3 & 3.1 & .25 & 1.55 & \\
\hline $14 .$. & 1.35 & 1.4 & 2.0 & 3.55 & 2.9 & 2.5 & .35 & 1.25 & 3.05 & .25 & 1.7 & \\
\hline 15. & 1.35 & 1.6 & 1.55 & 3.65 & 2.85 & 2.5 & $.35^{\circ}$ & 1.1 & 3.0 & .25 & 1.1 & \\
\hline 16. & 1.35 & 1.75 & 1.3 & 3.65 & 2.9 & 2.5 & .35 & .95 & 3.1 & .25 & .95 & \\
\hline 17. & 1.35 & 1.8 & 1.5 & 3.7 & 2.85 & $2 . v$ & .35 & .95 & 3.15 & .25 & .9 & \\
\hline $18 .$. & 1.35 & 1.35 & 1.55 & 3.8 & 2.85 & 1.75 & .35 & .95 & 3.1 & .25 & .9 & \\
\hline $19 .$. & 1.4 & 1.4 & 1.95 & 3.75 & 2.9 & 1.7 & .35 & 1.0 & 2.6 & .25 & .85 & \\
\hline 20.. & 1.55 & 1.4 & 1.8 & 3.8 & 2.9 & 1.65 & .35 & 1.0 & 2.3 & 1.2 & 1.05 & \\
\hline $21 \ldots$ & 1.65 & 1.4 . & 1.85 & 3.75 & 2.95 & 1.65 & .35 & 1.05 & 2.75 & 1.3 & .95 & \\
\hline $22 .$. & 1.7 & 1.4 & 2.05 & 3.75 & 2.95 & 1.6 & .35 & 1.5 & 2.95 & 1.5 & .9 & \\
\hline $23 .$. & 1.6 & 1.4 & 2.2 & 3.75 & 2.7 & 1.65 & .35 & 1.95 & 2.9 & 2.65 & .95 & \\
\hline 24.. & $(1.45)$ & .95 & 2.35 & 3.7 & 2.6 & 1.6 & .35 & 2.0 & 2.8 & 2.65 & 1.0 & \\
\hline 25. & 1.3 & 1.1 & 2.2 & 3.5 & 2.5 & 1.65 & .35 & 2.0 & 2.0 & 2.7 & .95 & \\
\hline 26. & $(1.25)$ & 1.4 & $(2.6)$ & 3.35 & 2.45 & 1.65 & .35 & 2.0 & 1.35 & 2.7 & .95 & \\
\hline 27.. & 1.1 & 1.55 & 2.85 & 3.35 & 2.5 & 0 & .35 & 2.0 & 2.25 & 2.7 & 85 & \\
\hline 28. & .95 & 1.5 & 2.8 & 3.35 & 2.55 & 0 & .35 & 2.6 & 2.8 & 2.7 & .8 & \\
\hline $29 .$. & 1.0 & & 2.6 & 3.35 & 2.9 & 0 & .35 & 2.7 & 2.8 & 2.6 & .9 & \\
\hline & $(1.1)$ & & 2.7 & 3.35 & 2.7 & 0 & .35 & 2.75 & 2.8 & 2.4 & 1.25 & \\
\hline $31 .$. & $(1.2)$ & 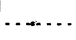 & 2.80 & & 2.65 & & .35 & 2.85 & & 2.3 & . . . . . . & \\
\hline
\end{tabular}

NOTE.-Gage heights in parentheses are estimated.

Estimated monthly discharge of Holt canal near Calexico, Cal., for 1905.

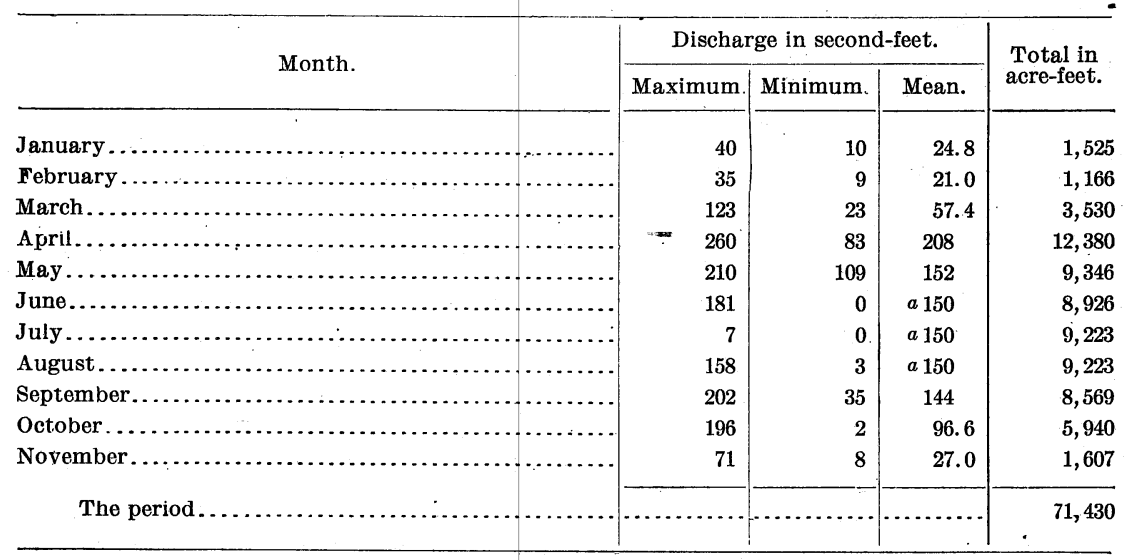

$a$ Estimated. Flood water used for irrigation. Beginning with March 6 Holt heading was used to divert water in No. 5 main canat, but its capacity was not sufficient until April 16. 
Estimated monthly discharge of New Holt or No. 7 canal near Calexico, Cal., for 1905.

\begin{tabular}{|c|c|c|c|c|}
\hline \multirow{2}{*}{ Month. } & \multicolumn{3}{|c|}{ Discharge in second-feet. } & \multirow{2}{*}{$\begin{array}{l}\text { Total in } \\
\text { acre-feet. }\end{array}$} \\
\hline & Maximum. & Minimum. & Mean. & \\
\hline May ....... & & & 18 & 1,107 \\
\hline June.... & & $\ldots \ldots$ & 33 & 1,964 \\
\hline July...... & $\therefore$ & $\ldots \ldots \ldots \ldots$ & 33 & 2,029 \\
\hline August . ...... & & $\cdots$ & 33 & 2,029 \\
\hline September.... & $\cdots$ & $\ldots \ldots \ldots \ldots$ & 33 & 1,964 \\
\hline October............... & a...... & $\ldots \ldots \ldots \ldots$ & 33 & 2,029 \\
\hline November............... & $\cdots$ & $\cdots, \cdots$ & 33 & 1,964 \\
\hline The period. & & & & 13,090 \\
\hline
\end{tabular}

NoTE.-The station record is of no value because most of the water used was obtained from flooded basins to the east. The mean discharge for the entire district covered by the canal was estimated in the field at 33 second-feet.

Discharge measurements of Hemlock canal near Calexico, Cal., in 1905.

\begin{tabular}{|c|c|c|c|c|c|c|c|}
\hline Date. & Hydrogr & $\begin{array}{c}\text { Gage } \\
\text { height. }\end{array}$ & $\begin{array}{l}\text { Dis- } \\
\text { charge. }\end{array}$ & Date. & Hydrogra & $\begin{array}{c}\text { Gage } \\
\text { height }\end{array}$ & $\begin{array}{l}\text { Dis- } \\
\text { charge. }\end{array}$ \\
\hline & & Feet. & Sec.-ft. & & & Feet. & Sec.-ft. \\
\hline January 4 & W. V.H & 0.87 & 2.0 & June 26 . & R. R. & 1.96 & 21.0 \\
\hline January 12 & .... do & 0.88 & 2.2 & July 3 & $\ldots . d$ & 1.23 & 7.2 \\
\hline January 19 & $\ldots$...do. & 1.41 & 9.5 & July 10 . & $\ldots . . \mathrm{dc}$ & 1.73 & 17.9 \\
\hline January & ...do. & 0.84 & 1.9 & July 17 & ....do. & 1.69 & 15.9 \\
\hline February & …do. & 1.25 & 7.4 & July 24 . & $\ldots d$ & 0.75 & 0. \\
\hline Februa & $\ldots \ldots c$ & 1.64 & 16.4 & July $31 \ldots$ & & 3 & 7.8 \\
\hline Februar & R. R.Mc & 1.59 & 12.5 & Augus & $\therefore d$ & 1.43 & 12.7 \\
\hline Februa & .....do & 1.79 & 17.2 & Augus & & 99 & 17.4 \\
\hline Marcl & $\ldots . . \mathrm{dc}$ & 1.20 & 8.7 & Augus & $\ldots$ d & 1.23 & 9. \\
\hline March & $\ldots . . d c$ & 1.45 & 13.2 & Augu & $\ldots . d$ & 1.12 & 7.3 \\
\hline Mar & & 1.73 & 23.0 & 4. & W. & 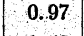 & 4. \\
\hline April 3 & $\ldots . \mathrm{d} c$ & 1.85 & 20.0 & Septer & $\ldots . \mathrm{d}$ & 1.42 & 13.3 \\
\hline April 10: & $\ldots . d$ & 1.42 & 12.7 & Septe & $\cdots$ & 2 & 2. \\
\hline pril & $\ldots$ & 2.45 & 36.0 & oet & $\cdots$ & 1 & 7. \\
\hline pril 24. & $\ldots . . d$ & 0.70 & 1.6 & Octol & $\ldots$. & 1.30 & 8. \\
\hline ay 1 . & $\ldots \mathrm{d}$ & 1.10 & 6.4 & Octob & $\cdots$ & & 8.0 \\
\hline [ay 8. & $\ldots$ & 2.04 & 20.4 & Octol & $\ldots$ & 1.20 & 8.4 \\
\hline ay 15 . & $\ldots$ & 1.94 & 18.6 & Octo & $\ldots$ & 1.04 & 5. \\
\hline May 22. & $\ldots \ldots d$ & 1.75 & 14.5 & November $6 .$. & ...do. & 0.90 & 4.0 \\
\hline May 29 &...$- \mathrm{dc}$ & 1.54 & 12.1 & November 13 . & ... do & 1.00 & 6. \\
\hline June 5 & $\ldots d$ & 1.81 & 16.9 & November 20 . & ....do. & 0.70 & 2.0 \\
\hline June 12. . & ....d & 0.78 & 2.1 & November 29. & ....do. & 0.89 & 3.1 \\
\hline June 18.. & $\ldots . . . d c$ & 1.7 & 13.2 & & 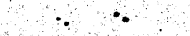 & & \\
\hline
\end{tabular}


Mean daily gage height, in feet, of Hemlock canal near Calexico, Cal., for 1905.

\begin{tabular}{|c|c|c|c|c|c|c|c|c|c|c|c|c|}
\hline Day. & Jan. & Feb. & Mar. & Apr. & May. & June. & July. & Aug. & Sept. & Oct. & Nov. & Dec. \\
\hline $1 .$. & 1.0 & 1.0 & 0.9 & (1.8) & 1.1 & 1.55 & 1.6 & 1.2 & 1.15 & 1.0 & 1.1 & 1.0 \\
\hline $2 .$. & .9 & 1.25 & .9 & 1.9 & 1.05 & 1.55 & 1.5 & 1.2 & 1.1 & 1.1 & 1.15 & 1.0 \\
\hline 3. & .95 & 1.3 & .9 & 1.85 & 1.2 & 1.6 & 1.25 & 1.2 & .9 & 1.1 & 1.25 & 2.0 \\
\hline $4 .$. & 1.0 & 1.3 & .9 & 1.8 & 1.35 & 1.7 & 1.2 & 1.3 & .95 & 1.15 & 1.1 & 1.5 . \\
\hline 5. & 1.05 & 1.1 & 1.05 & 2.0 & 1.7 & 1.8 & 1.2 & 1.3 & 1.2 & 1.2 & .95 & 1.5 \\
\hline 6. & 1.15 & .8 & 1.25 & $(2.0)$ & 1.95 & 1.9 & 1.2 & 1.4 & 1.35 & 1.3 & .9 & 1.7 \\
\hline $7 .$. & 1.3 & .8 & 1.3 & (1.9) & 2.0 & 1.95 & 1.55 & 1.45 . & 1.35 & 1.45 & .9 & 1.65 \\
\hline 8. & 1.4 & 1.0 & 1.3 & (1.6) & 2.1 & 2.05 & 1.75 & 1.4 & 1.35 & 1.45 & .9 & 1.6 \\
\hline 9. & 1.0 & 1.65 & 1.5 & $(1.5)$ & 2.1 & 2.1 & 1.75 & 1.4 & 1.3 .5 & 1.4 & .95 & 1.55 \\
\hline $10 .$. & .75 & 1.65 & 1.25 & (1.4) & 2.1 & 2.15 & 1.75 & 1.4 & 1.4 & 1.35 & 1.0 & 1.6 \\
\hline $11 .$. & .75 & 1.55 & 1.25 & (1.6) & 2.1 & 2.15 & 1.75 & 1.5 & 1.4 & 1.4 & 1.05 & \\
\hline $12 .$. & .75 & 1.35 & 1.3 & $(1.7)$ & 2.1 & .8 & 1.75 & 1.55 & 1.45 & 1.35 & 1.1 & \\
\hline $13 .$. & .75 & 1.1 & 1.2 & 1.9 & 2.1 & .8 & 1.7 & 1.55 & 1.5 & 1.5 & 1.05 & \\
\hline $14 .$. & .75 & 1.15 & 1.05 & 1.8 & 2.1 & .9 & 1.7 & 1.6 & 1.55 & 1.0 & .95 & \\
\hline $15 .$. & .75 & 1.4 & .95 & 2.0 & 1.95 & .9 & 1.7 & 1.4 & 1.55 & 1.15 & .75 & \\
\hline 16. & .75 & 1.8 & .9 & (2.2) & 2.0 & .9 & 1.7 & 1.25 & 1.55 & 1.25 & .8 & \\
\hline 17. & .75 & 1.6 & 1.05 & 2.45 & 1.95 & 1.4 & 1.7 & 1.2 & 1.55 & 1.1 & .8 & \\
\hline 18. & 1.0 & 1.75 & 1.0 & 2.5 & 1.85 & 1.7 & 1.8 & 1.2 & 1.3 & 1.0 & .85 & \\
\hline $19 .$. & 1.4 & 1.75 & 1.05 & 2.2 & 1.85 & 1.65 & 1.85 & 1.2 & .95 & 1.05 & .8 & \\
\hline 20. & 1.55 & 1.65 & 1.45 & 2.45 & 1.8 & 1.6 & 1.45 & 1.2 & 1.0 & 1.1 & .7 & \\
\hline $21 \ldots$ & $(1.4)$ & 1.5 & 1.5 & 2.35 & 1.75 & 1.55 & 1.0 & 1.25 . & 1.05 & 1.0 & .8 & \\
\hline 22. & (1.3) & 1.45 & 1.4 & 2.2 & 1.75 & 1.5 & 1.0 & 1.2 & 1.0 & 1.0 & .9 & \\
\hline $23 .$. & $(1.2)$ & 1.55 & 1.25 & $(2.0)$ & 1.7 & 1.5 & 1.0 & 1.2 & 1.0 & 1.2 & .85 & \\
\hline $24 \ldots$ & $(1.0)$ & 1.8 & 1.3 & 1.45 & 1.75 & 1.75 & .75 & 1.2 & .9 & 1.15 & .75 & \\
\hline $25 .$. & $(.9)$ & 1.65 & 1.55 & 1.45 & $(1.7)$ & 1.95 & .75 & 1.2 & .95 & 1.05 & .75 & \\
\hline $26 .$. & .85 & 1.05 & 1.55 & 1.2 & $(1.65)$ & 1.85 & .75 & 1.2 & 1.0 & 1.05 & .75 & \\
\hline $27 .$. & 1.0 & .9 & 1. 75 & 1.1 & $\cdot(1.6)$ & 1.8 & .75 & 1.2 & 1.0 & 1.1 & .75 & \\
\hline $28 \ldots$ & 1.0 & .9 & 1.55 & 1.1 & $(1.55)$ & 1.8 & 1.05 & 1.1 & .9 & 1.1 & .85 & \\
\hline $29 \ldots$ & .7 & & 1. 15 & 1.1 & 1.5 & 1.75 & 1.25 & 1.1 & .95 & 1.2 & .95 & \\
\hline $30 .$. & .6 & & 1.5 & 1.1 & 1.55 & 1.75 & 1.25 & 1.05 & .95 & .9 & 1.3 & \\
\hline $31 \ldots$ & .55 & 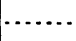 & 1.9 & & 1.6 & 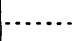 & 1.25 & 1.0 & 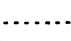 & .85 & & \\
\hline
\end{tabular}

NoTE.-Gage heights in parentheses are estimated.

Estimated monthly discharge of Hemlock canal near Calexico, Cal., for 1905.

\begin{tabular}{|c|c|c|c|c|}
\hline \multirow{2}{*}{ Month. } & \multicolumn{3}{|c|}{ Discharge in second-feet. } & \multirow{2}{*}{$\begin{array}{l}\text { Total in } \\
\text { acre-feet. }\end{array}$} \\
\hline & Maximum. & Minimum. & Mean. & \\
\hline January ............ & 12.0 & 0.4 & 4.3 & 264 \\
\hline February ....................... & 17.4 & 2.0 & 10.0 & 555 \\
\hline 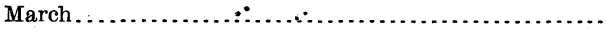 & 23.0 & 3.3 & 10.7 & 658 \\
\hline April.................. & 39.0 & 6.6 & 19.3 & 1,149 \\
\hline May............... & 21.6 & 5.4 & 15.7 & 965 \\
\hline June............... & 25.2 & 1.8 & 14.1 & 839 \\
\hline 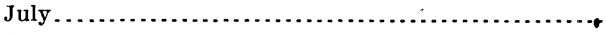 & 18.4 & 0.8 & 10.9 & 670 \\
\hline 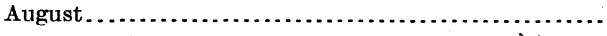 & 17.5 & 5.2 & 10.4 & 639 \\
\hline 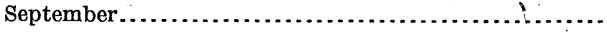 & 14.0 & 3.3 & 8.1 & 482 \\
\hline October............ & 12.6 & 2.4 & 7.5 & 461 \\
\hline 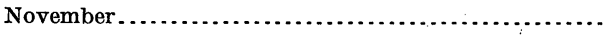 & 9.6 & 1.4 & 4.6 & 274 \\
\hline The period.. & & & $\therefore$ & 6,955 \\
\hline
\end{tabular}


Discharge measurements of New River near Calexico, Cal., in 1905.

\begin{tabular}{|c|c|c|c|c|c|c|c|}
\hline - Date. & Hydrographer. & $\begin{array}{c}\text { Gage } \\
\text { height. }\end{array}$ & $\begin{array}{c}\text { Dis- } \\
\text { charge. }\end{array}$ & Date. & Hydrographer. & $\begin{array}{l}\text { Gage } \\
\text { height. }\end{array}$ & $\begin{array}{c}\text { Dis- } \\
\text { charge. }\end{array}$ \\
\hline January 5 . & W. V. Hardy & $\begin{array}{c}\text { Feet. } \\
2.00\end{array}$ & $\begin{array}{r}\text { Sec.-ft. } \\
12\end{array}$ & May 23.. & R. R. McCoslin .. & $\begin{array}{r}\text { Feet. } \\
9.24\end{array}$ & $\begin{array}{r}\text { Sec.-ft. } \\
3,097\end{array}$ \\
\hline January 13. & .....do. & 3.20 & 82 & May 30. & .....do. & 9.38 & 3,400 \\
\hline January 20 & .....do. & 3.30 & 99 & June 6 . & .....do...... & 9.77 & 3,817 \\
\hline January 27. & .....do. do. & 5.20 & 323 & June 13 & ......do.... & 10.85 & 4,958 \\
\hline February 3 & .....do. do. & 4.00 & 126 & June 20. & .....do.. & 11.81 & 5,949 \\
\hline February 10 & .....do.. & 5.70 & 451 & June 27. & .....do........... & 12.70 & 10,810 \\
\hline February 18 & .....do.. & 6.90 & 986 & July 5. & .....do... & 13.21 & 11,940 \\
\hline February 2 & R. R. MeCoslin.. & 7.14 & 1,063 & July 12 , & ....do......... & 13.34 & 13,640 \\
\hline March $2 .$. & .....do. & 7.48 & 1,461 & July 18 . & and Mc- & & \\
\hline March 9. & .....do. & 7.90 & 1,702 & & & 13.11 & 13,220 \\
\hline March 16. & .....do do & 7.77 & 1,648 & Ju & $\ldots$ & 12.42 & 10,040 \\
\hline March 23. & .....do & 6.74 & 1,159 & August 1. & R. R. McCoslin... & 11.38 & 8,221 \\
\hline March 29 . & .....da. & 8.93 & 2,916 & August 8 & $\begin{array}{l}\text { MeCosland and } \\
\text { Hardy }\end{array}$ & 10.72 & 6,531 \\
\hline April 6 . & ......do & 6.95 & 1,307 & August 15 . & .....do.......... & 10.90 & 6,997 \\
\hline April 12 & ......do & 6.50 & 949 & August 22 . & .....do... & 10.22 & 5,529 \\
\hline April 18. & .....do & 6.80 & 1,149 & August $29 \ldots$ & M. v. Hardy ..... & 9.42 & 4,308 \\
\hline April 25 . & ......do. & 8.71 & 3,052 & September .5.. & Hardy and Mc- & & \\
\hline May 2. & $\ldots . . \mathrm{d}$ & 8.47 & 2,417 & & & 9.31 & 3,830 \\
\hline May 9. & .....do. & 8.80 & 2,890 & September 12 . & .....do... & 7.52 & 1,892 \\
\hline May 16. & .....do...... & 9.19 & 3,237 & & . & . & . \\
\hline
\end{tabular}

Discharge measurements of Alamo Channel near Rockwood, Cal., in 1905.

\begin{tabular}{|c|c|c|c|c|c|c|c|}
\hline Date. & Hydrographer. & $\begin{array}{c}\text { Gage } \\
\text { height. }\end{array} \mid$ & $\begin{array}{l}\text { Dis- } \\
\text { charge. }\end{array}$ & Date. & Hydrographer. & $\begin{array}{c}\text { Gage } \\
\text { height. }\end{array}$ & $\begin{array}{c}\text { Dis- } \\
\text { charge }\end{array}$ \\
\hline & & Feet. & sec.-ft. & & & Feet. & Sec.-ft. \\
\hline January $7 .$. & W. V. Harc & $\ldots$ & 49 & May 17. & R. R. McCoslin & 6.00 & 990 \\
\hline January 14. & ......do. & ... & 205 & May 24. & w. V. Eardy.. & 6.37 & 1,562 \\
\hline January 21. & . .do. & . & 77 & May 31. & .....do. & 5.92 & 1,692 \\
\hline January 28. & .....do. & & 112 & June 7 & .....do. & 6.28 & 2,110 \\
\hline February 4... & .....do. & 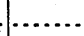 & 151 & June 14 & ....do. do. & 6.85 & 3,644 \\
\hline February $11 .$. & ......do. & & 61 & June $21 \ldots$ & .....do.. & $\ldots \ldots$ & 5,348 \\
\hline February 20. & $\therefore$...do & & 530 & June 28... & ....do. & 9.60 & 3,253 \\
\hline February 25. & ....do & & 94 & July $6 \ldots$ & .....do.. & 9.00 & 4,234 \\
\hline March 3. & .....do. & 7.48 & 387 & July $13 \ldots$ & ....do... & 9.50 & 3,932 \\
\hline March 11 . & $\ldots$ do & 6.80 & 1,088 & July 19 & ....do.. & & 3,501 \\
\hline March 17 . & .....do & 7.15 & 1,379 & July 26 . & 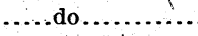 & & 3,104 \\
\hline March 25. & .....do. & 10.00 & 1,806 & August 2. & $\ldots . d c$ & 8.80 & 3,551 \\
\hline March 30. & .....do. & 9.50 & 1,443 & August $9 \ldots$ & ....do.... & 8.60 & 2,815 \\
\hline April 8. . & .....do & 6.20 & 635 & August $16 \ldots$ & ....do & 8.50 & 2,874 \\
\hline April 13... & .....do.. & 6.00 & 692 & August 23... & ....do... & 9.80 & 2,439 \\
\hline April 19...... & R. R. McCoslin.. & 6.00 & 772 & August $30 \ldots$ & $\ldots$...do ...... & 9.93 & 2,509 \\
\hline April 26.. & .....do. & (a) & $a 632$ & September 6 . & Hardy and Mc- & & \\
\hline May 3.. & .....do & ........ & 1,044 & & & 9.95 & 2,115 \\
\hline May 10. & .....do. & ....... & 1770 & Septemberr 13 & ......do. & 10.45 & 3,500 \\
\hline
\end{tabular}


Discharge measurements of Alamitos canal, Calexico, Cal., in 1905.

\begin{tabular}{|c|c|c|c|c|c|c|c|}
\hline Date. & Hydrographer. & $\begin{array}{c}\text { Gage } \\
\text { height. }\end{array}$ & $\begin{array}{c}\text { Dis- } \\
\text { charge. }\end{array}$ & Date. & Hydrographer. & \begin{tabular}{|c|} 
Gage \\
height
\end{tabular} & $\begin{array}{c}\text { Dis- } \\
\text { charge. }\end{array}$ \\
\hline January 3. & W. V. Hardy. & $\begin{array}{r}\text { Feet. } \\
1.48\end{array}$ & $\begin{array}{r}\text { Sec.-ft. } \\
30\end{array}$ & June $12 .$. & R. R. McCoslin.. & $\begin{array}{r}\text { Feet. } \\
1.32\end{array}$ & $\begin{array}{c}\text { Sec.-ft. } \\
37\end{array}$ \\
\hline January 11. & ...do.. & 1.54 & 39 & June 18. & w. V. Hardy.. & 1.30 & 30 \\
\hline January 18. & ....do.. & 1.11 & 15.1 & June 26. & R. R. McCoslin... & 1.35 & 36 \\
\hline January 26 . & ....do.. & 1.05 & 12.9 & July $5 .$. & .....do... & 1.33 & 34 \\
\hline February 2. & ....do... & 1.06 & 13.6 & July $10 .$. & .....do.. & 1.34 & 33 \\
\hline February 9. & ......do.. & 0.52 & 1.0 & July $17 .$. & .....do... & 1.35 & 34 \\
\hline February $17 .$. & R. R. McCoslin.. & 1.05 & 16.8 & July $24 \ldots$ & ......do... & 1.34 & 33 \\
\hline February 24 . & ......do.. & 1.00 & 15.7 & July $31 .$. & ....do... & 1.34 & 33 \\
\hline March $1 .$. & ....do.. & 0.82 & 9.1 & Aug. $7 \ldots$ & ......do.. & 1.12 & 22 \\
\hline March 8.. & ....do.. & . & 0 & August $14 .$. & .....do.... & 1.12 & 22 \\
\hline March 15. & ...do.. & $\ldots$ & 0 & August 21. & ....do... & 1.17 & $24^{\prime}$ \\
\hline March 22. & ....do.. & ...... & 0 & August 28.. & ....do... & 1.27 & 31 \\
\hline March 29. & ....do. & 1.20 & 24 & September 4. & .....do........ & 1.34 & 39 \\
\hline April 5... & ....do.. & 1.53 & 44 & September 11. & W. V. Hardy & 1. 42 & 43 \\
\hline April 11. & ....do. & 1.63 & 50 & September 25 . & ......do.... & 1.23 & 36 \\
\hline April 17.. & …..do. & 1.67 & 57 & October $9 . . .$. & .....do. & 1.30 & 40 \\
\hline April 24. & $\ldots$ do & 1.39 & 40 & October $16 \ldots$. & ....do.. & 1.32 & 45 \\
\hline May 1. & ....do. & 1.10 & 26 & October $23 \ldots$ & .....do.. & 1.27 & 38 \\
\hline May $8 .$. & ....do. & 1.15 & 28 & November $13 .$. & ....do.. & $\therefore \cdots$ & 0 \\
\hline May 15. & .....do & 1.41 & 39 & November 18.. & .... do. & ....... & 0 \\
\hline May $22 .$. & ....do. & 1.34 & 35 & November 30. & ....do.. & 0.42 & 5.4 \\
\hline May 29. & ....do & 1.37 & 36 & October $30 \ldots$ & ....do.... & 0.90 & 18.3 \\
\hline June $5 \ldots$ & ....do & 1.35 & 36 & November $6 \ldots$ & ...do. & & 0 \\
\hline
\end{tabular}


Mean daily height in feet, of Alamitos canal near Calexico, Cal., for 1905.

\begin{tabular}{|c|c|c|c|c|c|c|c|c|c|c|c|c|}
\hline Day. & Jan. & Feb. & Mar. & Apr. & May. & June. & July. & Aug. & Sept. & Oct. & Nov. & Dec. \\
\hline 1.. & 1.5 & 1.2 & 0.8 & 1.35 & 1.1 & 1.35 & 1.3 & 1.05 & 1.4 & 1.1 & 0.45 & 0.2 \\
\hline 2... & 1.5 & 1.1 & .8 & 1.5 & 1.1 & 1.35 & 1.3 & .9 & 1.4 & 1.25 & .45 & .25 \\
\hline 3.. & 1.5 & $1 . \tilde{z}_{b}$ & .6 & 1.6 & 1.1 & 1.35 & 1.35 & 1.05 & 1.35 & 1.15 & .2 & .25 \\
\hline 4.: & 1.45 & 1.1 & .4 & 1.55 & 1.1 & 1.35 & 1.35 & 1.1 & 1.35 & 1.3 & .1 & .2 \\
\hline 5. & 1.45 & .95 & .35 & 1.5 & 1.1 & 1.35 & 1.35 & 1.1 & 1.3 & 1.3 & .0 & .2 \\
\hline 6. & 1.4 & .95 & .35 & 1.55 & 1.1 & 1.35 & 1.35 & 1.1 & 1.35 & 1.3 & .15 & .2 \\
\hline 7.. & 1.45 & .95 & .3 & 1.6 & 1.1 & 1.35 & 1.35 & 1.1 & 1.45 & 1.25 & .25 & .2 \\
\hline 8. & 1.5 & .9 & .1 & 1.6 & 1.15 & 1.35 & 1.35 & 1.1 & 1.45 & 1.25 & .3 & .2 \\
\hline 9.. & 1.6 & .7 & .1 & 1.6 & 1.2 & 1.3 & 1.35 & 1.15 & 1.4 & 1.25 & .3 & .2 \\
\hline $10 .$. & 1.6 & .5 & .1 & 1.65 & 1.25 & 1.35 & 1.35 & 1.15 & 1.4 & 1.25 & .3 & .2 \\
\hline $11 .$. & 1.6 & .5 & .1 & 1.65 & 1.3 & 1.3 & 1.35 & 1.15 & 1.4 & 1.25 & .25 & .2 \\
\hline 12. & 1.6 & .5 & .1 & 1.65 & 1.4 & 1.3 & 1.35 & 1.15 & 1.45 & 1.25 & .3 & .25 \\
\hline 13.. & 1.6 & .5 & .1 & 1.65 & 1.4 & 1.3 & 1.35 & 1.15 & 1.5 & 1.25 & 3 & \\
\hline 14.. & 1.7 & .5 & .1 & 1.65 & 1.4 & 1.:35 & 1.35 & 1.1 & 1.45 & 1.3 & .25 & \\
\hline 15. & 1.45 & .55 & .0 & 1.65 & 1.4 & 1.35 & 1.35 & 1.1 & 1.4 & 1.3 & .3 & \\
\hline 16.. & 1.5 & .65 & .0 & 1.65 & 1.3 & 1.35 & 1.35 & 1.1 & 1.4 & 1.35 & .3 & \\
\hline $17 .$. & 1.15 & 1.05 & .0 & 1.65 & 1.2 & 1.3 & 1.35 & 1.1 & 1.4 & 1. 35 & .25 & \\
\hline 18. & 1.1 & 1.05 & .0 & 1.7 & 1.2 & 1.3 & 1.35 & 1.1 & 1.4 & 1.35 & .2 & \\
\hline 19. & 1.1 & $1.05^{\circ}$ & .0 & 1.75 & 1.2 & 1.3 & 1.35 & 1.15 & 1.35 & 1.25 & .2 & \\
\hline 20. & 1.1 & 1.05 & .0 & 1.35 & 1.25 & 1.3 & 1.35 & 1.2 & 1.4 & 1.2 & .2 & \\
\hline 21. & 1: 05 & 1.05 & .0 & 1.4 & 1.35 & 1.3 & 1.35 & 1.2 & 1.45 & 1.2 & .15 & \\
\hline $22 .$. & 1.1 & 1.05 & .0 & 1.55 & 1.35 & 1.3 & 1.35 & 1.15 & 1.45 & 1.15 & .15 & \\
\hline 23. & 1.1 & 1.0 & .15 & 1.45 & 1.35 & 1.3 & $1.35^{\circ}$ & 1.2 & 1.4 & 1.25 & .15 & \\
\hline 24. & 1.1 & 1.0 & .65 & 1.4 & 1.35 & 1.3 & 1.35 & 1.25 & 1.2 & 1.25 & .2 & \\
\hline 25.: & 1.0 & 1.05 & .7 & 1.4 & 1. 35 & 1.35 & 1.35 & 1.25 & 1.25 & 1.2 & .25 & \\
\hline 26.. & 1.05 & 1.1 & .7 & 1.4 & 1.35 & 1.35 & 1.35 & 1.25 & 1.25 & 1.2 & .25 & \\
\hline $27 .$. & 1.15 & .8 & .85 & 1.4 & 1.35 & 1.35 & 1.35 & 1.3 & 1.2 & 1.15 & .35 & \\
\hline 28. & 1.2 & .75 & .9 & 1.25 & 1. 35 & 1.35 & 1.35 & 1.3 & 1.1 & 1.15 & .4 & \\
\hline 29. & 1.2 & 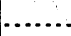 & 1.3 & 1.15 & 1.35 & 1.35 & 1.35 & 1.3 & 1.15 & 1.15 & .35 & \\
\hline $30 .$. & 1.2 & 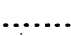 & 1.4 & 1.15 & 1.35 & 1.3 & 1.35 & 1.25 & 1.1 & .9 & .25 & \\
\hline 31. & 1.25 & . & 1.3 & ....... & 1.35 & ..... & 1.35 & 1.3 & 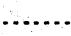 & .8 & & \\
\hline
\end{tabular}

Estimated monthly discharge of Alamitos canal near Calexico, Cal., for 1905.

\begin{tabular}{|c|c|c|c|c|}
\hline \multirow{2}{*}{ Month. } & \multicolumn{3}{|c|}{ Discharge in second-feet. } & \multirow{2}{*}{$\begin{array}{l}\text { Total in } \\
\text { acre-feet. }\end{array}$} \\
\hline & Maximum. & Minimum. & Mean. & \\
\hline January... & 50 & 11 & 26.6 & 1,636 \\
\hline February ........... & 23 & .5 & 11.4 & 633 \\
\hline March..... & 36 & 0 & 4.9 & 301 \\
\hline April........... & 65 & 28 & 45.6 & 2,714 \\
\hline May......... & 40 & 25 & 31.8 & 1,955 \\
\hline June.......... & 38 & 30 & 34.4 & 2,047 \\
\hline July $\ldots \ldots \ldots \ldots \ldots \ldots$ & 35 & 32 & 33.9 & 2,084 \\
\hline August................ & 34 & 11 & 23.9 & 1,470 \\
\hline September.................. & 49 & 28 & 40.8 & 2,428 \\
\hline October...$\ldots \ldots \ldots \ldots \ldots$ & 47 & 18 & 35.8 & 2,201 \\
\hline November.............. & 6 & 0 & 2.7 & 161 \\
\hline The period & & & & 17,630 \\
\hline
\end{tabular}


Discharge measurements of Main canal near Calexico, Cal., in 1905.

\begin{tabular}{|c|c|c|c|c|c|c|c|}
\hline Date. & Hydrographer. & $\mid \begin{array}{c}\text { Gage } \\
\text { height. }\end{array}$ & $\begin{array}{c}\text { Dis- } \\
\text { charge. }\end{array}$ & Date. & Hydrographer. & $\left|\begin{array}{c}\text { Gage } \\
\text { height. }\end{array}\right|$ & $\begin{array}{c}\text { Dis- } \\
\text { charge. }\end{array}$ \\
\hline & & Feet. & Sec.ft. & & & Feet. & Sec.ft. \\
\hline Jantary 3 . & W. V. Hardy. & 3.90 & 439 & June $12 .$. & R. R. McCoslin. & 4.17 & 515 \\
\hline Janvary $11 .$. & .....do. . & 4.20 & 524 & June 18. & ....do. & 4.11 & 540 \\
\hline January $18 \ldots$ & .....do. . & 4.50 & 567 & June 26.. & ....do. & 4.23 & $5 / 1$ \\
\hline January 26 . & .....do.. & 4.20 & 510 & July 3... & .....do & 4.25 & 5.2 \\
\hline February 2... & .....do. . & 4.18 & 507 & July 10. & ....do.. & 4.38 & 567 \\
\hline February 15. & .....do...... & 4.09 & 485 & July $17 .$. & ...do. & 4.36 & 582 \\
\hline February $17 .$. & R. R. McCoslin. & 4.15 & 472 & July $24 \ldots$ & ....do.. & 4.32 & 583 \\
\hline February $23 .$. & .....do... & 3.95 & 447 & July $31 .$. & ...do.. & 4.23 & 580 \\
\hline March $1 .$. & .....do. . & 4.49 & 595 & August 7 . & ...do. & 4.29 & 556 \\
\hline March $8 \ldots$ & .....do. & ....... & 0 & August $14 \ldots$ & , . do. & 4.33 & 562 \\
\hline March 15. & ..... do. & ........ & 0 & August 21 . & ...do. & 4.33 & 540 \\
\hline March 22. & ....do. do. & 3.52 & 410 & August $28 \ldots$ & ....do. & 4.29 & 536 \\
\hline March 29. & .....do. & 4.79 & 651 & September 4 & ....do.. & 4.30 & 558 \\
\hline April 5... & ..... do. . & 4.84 & $675^{\circ}$ & September 11 . & W. V. Hardy. & $\ldots .$. & 0 \\
\hline April 11... & .....do.. & 4.98 & 729 & September 25 . & ....do.. & 4.27 & 551 \\
\hline April 17.. & .....do. & 5.04 & 735 & October $2 \ldots$. & ....do.. & $\ldots .$. & 0 \\
\hline April $24 \ldots$ & .....do. do. & 4.91 & 725 & Qctober $9 . .$. & ...do.... & 4.29 & 559 \\
\hline May $1 .$. & .....do. & 4.47 & 648 & October $16 . .$. & ....do. & 4.30 & 546 \\
\hline May $8 . .$. & .....do. & 4.42 & 623 & November $3 .$. & ....do.. & 2.90 & 298 \\
\hline May $15 .$. & ....do. & 4.34 & 586 & November 6 & ....do. & 3.60 & 380 \\
\hline May $22 .$. & ....do. & 4.24 & 562 & November 13 & ....do.. & 3.60 & 380 \\
\hline May $29, \ldots$ & ....do. & 4.32 & 595 & November 18 & .....do.. & 3.55 & 378 \\
\hline June $5 . .$. & ......do... & 4.19 & 566 & November 30 & .....do..... & 3.50 & 376 \\
\hline
\end{tabular}


Mean daily gage height, in feet, of Main canal near Calexico, Cal., for 1905.

\begin{tabular}{|c|c|c|c|c|c|c|c|c|c|c|c|c|}
\hline Day. & Jan. & Feb. & Mar. & Apr: & May. & June. & July. & Aug. & Sept. & oct. & Nov. & Dec. \\
\hline $1 \ldots$ & 3.9 & 4.05 & 4.5 & 4.7 & 4. 45 & 4.2 & 4.25 & 4.2 & 4.3 & (a) & 2.3 & 3.4 \\
\hline $2 .$. & 3.95 & 4.2 & 4.45 & 4.85 & 4.4 & 4.2 & 4.25 & 4.2 & 4.3 & (a) & 2.9 & 3. 45 \\
\hline 3.. & 3.9 & 4.25 & 4.05 & 4.95 & 4.4 & 4.2 & 4.25 & 4.2 & 4.3 & (a) & 3.22 & 3. 75 \\
\hline $4 .$. & 3.85 & 4.15 & (a) & 4.9 & 4.4 & 4.2 & 4. 25 & 4.25 & 4.3 & (a) & 3.55 & 3.5 \\
\hline 5. & 3.85 & 3.95 & (a) & 4.85 & 4.45 & 4.2 & 4.25 & 4.25 & 4.3 & 3.95 & 3.45 & 3.9 \\
\hline 6.. & 3. 75 & 3.8 & (a) & 4.9 & 4.4 & 4.2 & 4.25 & 4.25 & 4.3 & 4.2 & $(3.55)$ & 3. 95 \\
\hline 7. & 3.7 & 3.8 & (a) & 4.95 & 4.4 & 4.2 & 4.3 & 4.3 & (a) & 4.25 & (3.6) & 3.95 \\
\hline 8: & 3.75 & 3.8 & (a) & 4.95 & 4.45 & 4.2 & 4.35 & 4.3 & (a) & 4.2 & (3.6) & 3.95 \\
\hline $9 \therefore$ & 3.95 & 3.85 & (a) & 5.0 & 4.45 & 4.2 & 4. 35 & 4.3 & (a) & 4.3 & (3.6) & 3.95 \\
\hline 10. & 4.2 & 3.85 & (a) & 5.0 & 4.4 & 4.2 & 4.35 & 4.3 & (a) & 4.3 & $(3.6)$ & 3.6 \\
\hline $11 .$. & 4.2 & 3.85 & (a) & 5. 0 & 4.4 & 4.2 & 4.35 & 4.35 & (a) & 4.3 & 3.6 & .... \\
\hline $12:$ & 4.4 & 3.85 & (a) & 5.0 & 4.45 & 4.2 & 4.35 & 4.35 & .5 & 4.3 & 3.6 & \\
\hline 13.. & 4.55 & 3.8 & (a) & 5.0 & 4.45 & 4.2 & 4. 35 & 4.35 & 2.0 & 4.3 & 3.6 & \\
\hline 14.. & 4.4 & 3.95 & $(a)$ & 5.0 & 4.4 & 4.25 & 4.35 & 4.35 & 2.5 & 4.3 & 3.6 & \\
\hline 15. & 4.3 & 4.1 & (a) & 5.05 & 4. 35 & 4.2 & 4.35 & 4.35 & .2 .95 & 4.3 & (3.6) & \\
\hline 16. & 4.35 & 4. 2 & (a) & 5.05 & 4.3 & 4.2 & 4. 35 & 4.35 & 3.1 & 4.35 & (3.6) & \\
\hline 17. & 4.4 & 4.1 & (a) & 5.05 & 4.3 & 4.25 & 4.35 & 4.35 & 3.15 & 4.35 & (3.6) & \\
\hline 18. & $(4.5)$ & 4.05 & (a) & 5.1 & 3 & 4.2 & 4.35 & 4.35 & 3.15 & 4.35 & 3.6 & \\
\hline 19. & 4.5 & 4.05 & (a) & 5.1 & 4.3 & 4.2 & 4. 35 & 4.35 & 3.5 & $4.3^{\prime}$ & 3.55 & \\
\hline 20. & 4.55 & 4.0 & (1.5) & 5.0 & 4.3 & 4.2 & 4.35 & 4.35 & 3.9 & 4.3 & 3.4 & \\
\hline $21 .$. & 4.5 & 3.9 & 3.35 & 5.0 & 4.3 & 4.2 & 4.35 & 4.35 & 4.05 & 4.3 & 3.4 & \\
\hline 22. & 4.55 & 3.9 & 3.5 & 5.0 & 4. 25 & 4.25 & 4.3 & 4.3 & 4.2 & 4.3 & 3.4 & \\
\hline 23... & 4.5 & 3.95 & 3.8 & 4.9 & 4.3 & 4.3 & 4.3 & 4.3 & 4.35 & (a) & 3.2 & . \\
\hline 24. & 4. 35 & 4.1 & 4. 15 & 4.95 & 4.3 & 4.3 & 4.3 & 4.3 & 4.35 & (a) & 3.05 & $\ldots \ldots$ \\
\hline 25. & 4.25 & 4.3 & (4.5) & 4.9 & 4.3 & 4.25 & 4.35 & 4.3 & 4.3 & (a) & 3.05 & $\cdots$ \\
\hline 26. & 4.2 & 4.4 & (4.8) & 4.9 & 4.3 & 4.25 & 4.3 & 4.3 & 4.25 & (a) & 3.05 & .... \\
\hline 27. & 4.05 & 4.5 & (4.8) & 4.9 & 4.3 & 4.25 & 4.3 & 4.3 & 4.25 & .1 & 3.05 & $\cdots$ \\
\hline 28. & 3.95 & 4.5 & $(4.8)$ & 4.9 & 4.3 & 4.25 & 4.3 & 4,3 & 4.25 & .8 & 3.05 & .... \\
\hline 29. & 3.9 & $\therefore . .$. & $(4.8)$ & 4.8 & 4.3 & 4.25 & 4.3 & 4.3 & 4.25 & .85 & 3.4 & \\
\hline 30. & 3.85 & & 4.8 & 4.6 & 4.2 & 4.25 & $4: 3$ & 4.3 & 3.1 & 1.7 & 3.45 & \\
\hline 31. & 3.85 & ........ & 4. 7 & .... & 4.2 & ........ & 4.25 & 4.3 & : & 1.7 & & \\
\hline
\end{tabular}

NotE.-Gage heights in parentheses are estimated.

Estimated monthly discharge of Main canal near Calexico, Cal., for 1905.

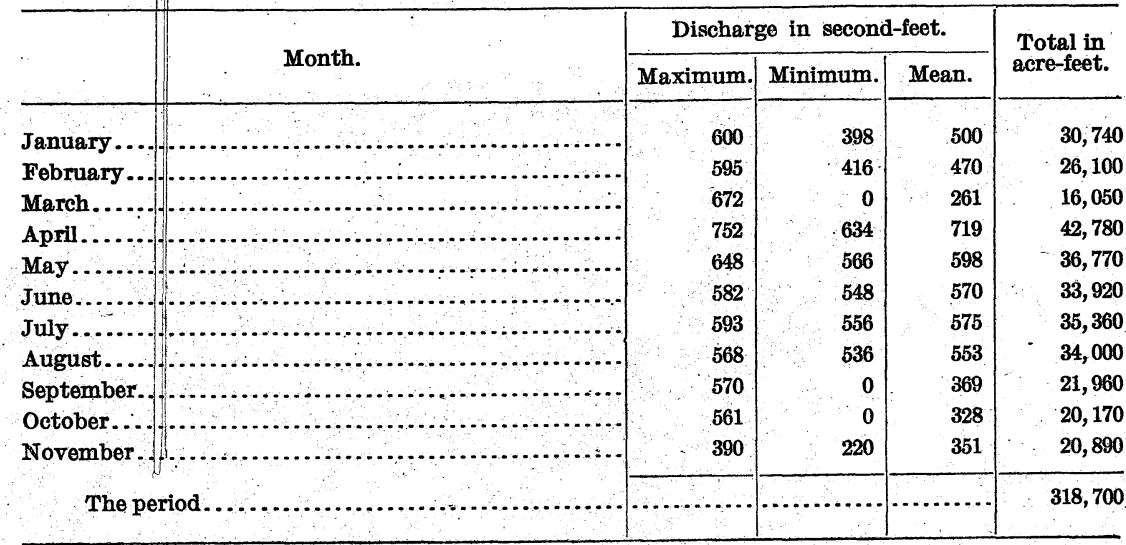


Discharge measurements of Boundary canal near Calexico, Cal., in 1905.

\begin{tabular}{|c|c|c|c|c|c|c|c|}
\hline Date. & Hydrographer. & $\begin{array}{c}\text { Gage } \\
\text { height. }\end{array}$ & $\begin{array}{c}\text { Dis- } \\
\text { charge. }\end{array}$ & Date. & Hydrographer. & $\left|\begin{array}{c}\text { Gage } \\
\text { height. }\end{array}\right|$ & $\begin{array}{c}\text { Dis- } \\
\text { charge. }\end{array}$ \\
\hline & & Feet. & Sec.-ft. & & & Feet. & Sec.-ft. \\
\hline January 3 & W.V.Hardy . & 0.80 & 6.4 & June 12.. & R. R. McCoslin & 0.35 & 0.22 \\
\hline January 11 & ....do. & .98 & 8.6 & June 18 & .....do . & 1.00 & 11.0 \\
\hline January 18 & ..... do . & 1.00 & 8.9 & June $26 .$. & ....do. & .91 & 7.5 \\
\hline January 25 & .....do & $\therefore .$. & 7.8 & July $3 .$. & ....do & .93 & 6.4 \\
\hline February 2. & .....do & .99 & 9.1 & July 10 . & ...do. & .78 & 4.8 \\
\hline February 9 & $\ldots . . d$ & .95 & 8.3 & July $17 \ldots$ & ....do. & .77 & 4.2 \\
\hline -February 17 & R. R. McCoslin & .79 & 5.3 & July $24 \ldots$ & $\therefore$.do & .96 & 6.6 \\
\hline Pebruary 23 & .....do. & .98 & 8.3 & July $31 \ldots$ & ....d & .87 & 5.3 \\
\hline March 1 & ....do & 1.00 & 10.0 & August 7 & $\ldots . . d$ & .83 & 6.2 \\
\hline $\operatorname{March} 5$ & $\ldots . d o$ & $\ldots$ & 0 & Augus & $\ldots . d$ & .78 & 5.7 \\
\hline March 8 & $\ldots . . \mathrm{do}$ & $\ldots$. & 0 & Augus & $\ldots . . d$ & .79 & 5.7 \\
\hline March 22 & $\ldots . . d$ & .88 & 7.8 & August 28 & $\ldots . . d$ & .79 & 4.8 \\
\hline March 29 & $\ldots \ldots c$ & .80 & 6.2 & September 4 . . & & .80 & 3.8 \\
\hline April 5 & $\ldots . . d$ & .85 & 8.5 & September 11 . & $\ldots . . . d$ & ..... & 0 \\
\hline April 11 & $\ldots . . \mathrm{do}$ & .99 & 10.4 & October $2 \ldots$ & W.V.Hardy & $\ldots . .$. & 0 \\
\hline April 17 & $\ldots . d$ & 1.00 & 11.7 & October $6 \ldots .$. & .....do & .90 & 8.4 \\
\hline April 24. & $\ldots . . d$ & 1.08 & 13.0 & Octob & $\ldots .$. & .87 & 5.3 \\
\hline Tay 1. & $\ldots . d$ & .83 & 6.1 & October $16 \ldots$ & $\ldots . . d$ & .80 & 5.0 \\
\hline May 8 ... & $\ldots . . d$ & .83 & 7.5 & October $18 . .$. & $\ldots . . d$ & .25 & 0 \\
\hline May 15 . & $\ldots . . d$ & .74 & 6.1 & October $30 . .$. & $\ldots . . d$ & .85 & 8.0 \\
\hline May 22 . & $\ldots . . d c$ & .71 & 5.1 & November 13 & $\ldots . d$ & .90 & 8.4 \\
\hline May 29 & $\ldots \ldots c$ & .85 & 8.0 & November 18 & $\ldots .$. & .95 & 7.0 \\
\hline June $5 .$. & .....do & .90 & 8.4 & November 30 & ...do & 1.03 & 12.7 \\
\hline
\end{tabular}

Estimated monthly discharge of Boundary canal near Calexico, Cal., for 1905.

\begin{tabular}{|c|c|c|c|c|}
\hline \multirow{2}{*}{ Month. } & \multicolumn{3}{|c|}{ Discharge in second-feet. } & \multirow{2}{*}{$\begin{array}{l}\text { Total in } \\
\text { acre-feet. }\end{array}$} \\
\hline & Maximum. & Minimum. & Mean. & \\
\hline January ............ & 8.9 & 6.0 & 8.1 & 498 \\
\hline February...$\ldots \ldots \ldots$ & 9.8 & 5.1 & 7.8 & 433 \\
\hline March......... & 10.0 & 0 & 3.6 & 221 \\
\hline April................. & 13.0 & 7.0 & 10.2 & 607 \\
\hline May ....................... & 8.0 & 5.1 & 6.5 & 400 \\
\hline June................... & 11.0 & .2 & 7.9 & 471 \\
\hline July ................... & 6.8 & 4.2 & 5.4 & 332 \\
\hline August............. & 6.2 & 4.4 & 5.6 & 344 \\
\hline September............ & 6.0 & $\cdot$ & 3.8 & 227 \\
\hline October.............. & 8.0 & $\mathbf{0}$ & 4.4 & 271 \\
\hline 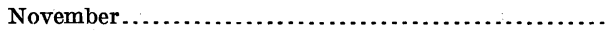 & 12.7 & 7.0 & 9.0 & 536 \\
\hline The period. & & & & 4,340 \\
\hline
\end{tabular}


Discharge measurements of Wisteria canal near Calexico, Cal., in 1905.

\begin{tabular}{|c|c|c|c|c|c|c|c|}
\hline Date. & Hydrographer. & $\begin{array}{c}\text { Gage } \\
\text { height. }\end{array}$ & $\begin{array}{c}\text { Dis- } \\
\text { charge. }\end{array}$ & Date. & Hydrographer. & $\begin{array}{c}\text { Gage } \\
\text { height. }\end{array}$ & $\begin{array}{c}\text { Dis- } \\
\text { charge. }\end{array}$ \\
\hline January 5 .... & W.V.Hardy. & $\begin{array}{r}\text { Freet. } \\
1.12\end{array}$ & $\begin{array}{r}\text { Sec.-ft. } \\
3.0\end{array}$ & June 13... & R. R. McCoslin... & $\mid \begin{array}{r}\text { Feet. } \\
0.35\end{array}$ & $\begin{array}{r}\text { Sec.-ft. } \\
10.6\end{array}$ \\
\hline January 11 . & .....do. & .15 & $\mathbf{0}$ & June 19... & w. V.Hardy... & .57 & 13.5 \\
\hline January 18 ... & .....do. & .52 & 7.7 & June $26 . .$. & R.R.McCoslin . & .38 & 10.6 \\
\hline January 25 . & .....do. & .92 & 22 & July 5. . & .....do. . & .30 & 9.4 \\
\hline February $1 \ldots$ & .....do. & .80 & 16.5 & July 11.... & ....do.. & .96 & 32 \\
\hline February 10 . & .....do. & $\ldots$. & $\mathbf{0}$ & July 18. . & ....do... & .66 & 17.8 \\
\hline February 18 . & .....do. & & 0 & July $25 . . .$. & W.V.Hardy .. & & 0 \\
\hline February $25 .$. & R.R.McCoslin. & .. & $\mathbf{0}$ & August 1 . & ....do....... & .33 & 7.8 \\
\hline March 1. . & .....do. & ... & 0 & August $8 \ldots$. & ....do........ & & 0 \\
\hline March $15 \ldots .$. & ......do. & $\cdots$ & 0 . & August $15 \ldots$ & R.R.McCoslin . & & 0 \\
\hline March 23 ... & .....do. & $\cdots$ & 0 & August $22 \ldots$. & W.V.Hardy .. & & 0 \\
\hline March 29 & .....do. & .90 & 26 & August $29 \ldots$... & R. R.McCoslin ... & & 0 \\
\hline April 6....... & .....do. & 1.65 & 64 & September 5. & W.V.Hardy . & & 0 \\
\hline April 12 ...... & .....do. & 1.29 & 47 & September 12 . & .....do... & & 0 \\
\hline April 18 & .....do. & 1.00 & 33 & September 26 . & ....do. & & $\mathbf{0}$ \\
\hline April 25 & .....do. & .95 & 32 & October 3..... & ....do. & & $\mathbf{0}$ \\
\hline May 2.. & .....do. & .50 & 13.1 & October $11 . .$. & ....do. & & 0 \\
\hline May 9... & .....do. & .85 & 26 & October $18 . .$. & ....do.. & .60 & 12.8 \\
\hline May 16.. & .....do. & .43 & 12.2 & October $31 . .$. & ....do... & .60 & 14 \\
\hline May 23.. & ......do. . & .63 & 17.1 & November 7 .. & ....do..... & .40 & 12 \\
\hline May 30. & .....do... & .42 & 12.1 & November 14 & ....do.. & $\cdots \cdot \cdot$ & 0 \\
\hline June 6... & .....do..... & .38 & 11.9 & November 21 . & ....do......... & .15 & 0 \\
\hline
\end{tabular}

a Estimated. 
Daily gage height, in feet, of Wisteria canal near Calexico, Cal., for 1905.

\begin{tabular}{|c|c|c|c|c|c|c|c|c|c|c|c|}
\hline Day. & Jan. & Feb. & Mar. & Apr. & May. & June. & July. & Aug. & Sept. & Oct. & Nov. \\
\hline 1. & 0.95 & 0.8 & 0.25 & 1.5 & 0.3 & 0.4 & 0.3 & 0.35 & 0 & 0.0 & 0.6 \\
\hline 2. & .9 & .8 & .2 & 1.2 & .4 & .4 & .3 & .05 & 0 & .0 & .6 \\
\hline 3.. & 1.15 & .8 & .2 & 1.7 & .4 & .4 & .3 & .05 & 0 & .0 & .6 \\
\hline $4 .$. & 1.1 & 1.0 & .2 & 1.8 & .4 & .4 & .3 & .05 & 0 & .0 & .6 \\
\hline $5:$ & 1.1 & .55 & .2 & 1.6 & .7 & .4 & .3 & .0 & 0 & .0 & .0 \\
\hline $6 .$. & 1.1 & .3 & .2 & 1.65 & .7 & .4 & .3 & .0 & 0 & .0 & .4 \\
\hline $7 \ldots$ & 1.25 & .0 & .2 & 1.7 & .8 & .4 & .3 & 0 & 0 & .0 & .4 \\
\hline 8. & 1.3 & .0 & .15 & 1.6 & .8 & .4 & .3 & .0 & 0 & .0 & .75 \\
\hline $9 .$. & .95 & .0 & .15 & 1.5 & .8 & .4 & .3 & .0 & 0 & .0 & .75 \\
\hline 10. & .95 & .0 & .15 & 1.45 & .8 & .3 & .3 & .0 & 0 & .0 & .0 \\
\hline $11 .$. & .15 & .0 & .15 & 1.35 & .5 & .3 & .95 & .0 & 0 & .0 & .0 \\
\hline 12. & .1 & .3 & .35 & 1.15 & .45 & .3 & 1.8 & .0 & 0 & .0 & .0 \\
\hline 13. & .1 & .2 & .35 & 1.1 & .45 & .3 & 1.1 & .0 & 0 & 0.6 & .0 \\
\hline $14 .$. & .0 & .25 & .40 & .95 & .45 & .25 & 1.0 & .0 & 0 & .6 & .0 \\
\hline $15 \ldots$ & .0 & .3 & :35 & 1.0 & .45 & .25 & 1.0 & .0 & 0 & .6 & .5 \\
\hline 16. & .0 & .35 & .3 & 1.0 & .45 & .3 & .9 & .0 & 0 & .6 & .0 \\
\hline $17=$ & .6 & .25 & .3 & 1.0 & .45 & .0 & .6 & .0 & 0 & .6 & .0 \\
\hline 18. & .2 & .1 & .3 & 1.0 & .45 & .0 & .65 & .0 & 0 & .6 & .0 \\
\hline 19. & .65 & .0 & .5 & .8 & .75 & .4 & .5 & 0 & 0 & .6 & .0 \\
\hline 20. & .8 & .0 & .5 & . . .7 & .8 & .4 & .5 & .0 & 0 & .6 & .0 \\
\hline $21 \ldots$ & .8 & .0 & .5 & .65 & .8 & .4 & .5 & .0 & 0 & .45 & .15 \\
\hline 22. & .8 & .0 & .5 & 1.0 & .7 & .4 & .45 & .0 & 0 & .45 & .35 \\
\hline $23 .$. & .8 & .0 & .8 & .1 & .6 & .4 & .0 & .0 & 0 & .0 & .35 \\
\hline $24 \ldots$ & .9 & .0 & .8 & .9 & .6 & $\therefore 4$ & .0 & .0 & 0 & .0 & .1 \\
\hline 25. & .9 & .0 & .8 & 1.1 & .7 & .4 & .0 & .0 & 0 & .0 & $\cdot .0$ \\
\hline 26. & 1.0 & 2 & .65 & 1.15 & .7 & .4 & .0 & .0 & 0 & .0 & .0 \\
\hline $27 \ldots \ldots$ & 1.1 & .2 & .6 & .9 & .65 & .4 & .0 & .0 & 0 & .05 & .0 \\
\hline 28 .. & 1.0 & .3 & .8 & .45 & .65 & .3 & .0 & .0 & 0 & .05 & .0 \\
\hline $29 \ldots \ldots$ & 1.0 & ..... & .9 & .4 & .4 & .2 & .0 & .0 & 0 & .6 & .0 \\
\hline $30 \ldots \ldots$ & 1.0 & ....... & 1.0 & .35 & .4 & .3 & .1 & .0 & 0 & .6 & .0 \\
\hline $31 \ldots \ldots \ldots \ldots$ & .8 & ....... & 1.15 & $\ldots$ & .4 & $\ldots .$. & .35 & .0 & 0 & .6 & $\ldots$. \\
\hline
\end{tabular}

Estimated monthly discharge of Wisteria canal near Calexico, Cal., for 1905.

\begin{tabular}{|c|c|c|c|c|}
\hline \multirow{2}{*}{ Month. } & \multicolumn{3}{|c|}{ Discharge in second-feet. } & \multirow{2}{*}{$\begin{array}{l}\text { Total in } \\
\text { acre-feet. }\end{array}$} \\
\hline & Maximum. & Minimum. & Mean. & \\
\hline January ............... & 40 & 0 & 17.9 & 1,100 \\
\hline February $\ldots \ldots \ldots \ldots \ldots \ldots$ & 25 & 0 & 3.8 & 211 \\
\hline March....$\ldots \ldots \ldots \ldots \ldots \ldots \ldots \ldots \ldots \ldots$ & 32 & 0 & 7.1 & 436 \\
\hline April............... & 75 & 0 & 39.5 & 2,325 \\
\hline 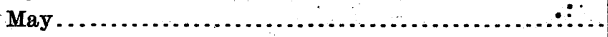 & $=25$ & $\mathbf{0}$ & 16.7 & 1,027 \\
\hline sune................... & 12 & 0 & 9.6 & 572 \\
\hline July $\ldots \ldots \ldots \ldots \ldots \ldots$ & 80 & 0 & 14.2 & 873 \\
\hline August................ & 11 & 0 & .4 & 25 \\
\hline September.............. & 0 & 0 & .0 & 0 \\
\hline October...$\ldots \ldots \ldots \ldots \ldots \ldots \ldots \ldots \ldots \ldots \ldots \ldots$ & 14 & 0 & 5.4 & 332 \\
\hline 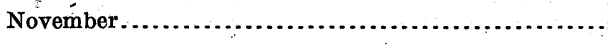 & 25 & 0 & 5.6 & 334 \\
\hline The period. & & & & 7,261 \\
\hline
\end{tabular}


Discharge measurements of Tamarack canal near Imperial, Cal., in 1905.

\begin{tabular}{|c|c|c|c|c|c|c|c|}
\hline Date. & Hydrographer. & $\begin{array}{c}\text { Gage } \\
\text { height. }\end{array}$ & $\begin{array}{c}\text { Dis- } \\
\text { charge. }\end{array}$ & Date. & Hydrographer. & $\begin{array}{c}\text { Gage } \\
\text { height. }\end{array}$ & $\begin{array}{c}\text { Dis- } \\
\text { charge. }\end{array}$ \\
\hline May 25.. & W. V. Hardy ... & $\begin{array}{l}\text { Feet. } \\
\ldots . . . .\end{array}$ & $\begin{array}{r}\text { Sec.-ft. } \\
52\end{array}$ & October $19 \ldots$ & W. V. Hardy & $\begin{array}{c}\text { Feet. } \\
\ldots \ldots\end{array}$ & $\begin{array}{r}\text { Sec. }-f t . \\
a 40\end{array}$ \\
\hline June $1 . .$. & R. R. McCoslin.. & 1.70 & 51 & October $26 \ldots$ & .....do ........ & & a 40 \\
\hline June $8 \ldots$ & W. V. Hardy . & 1.80 & 56 & November 2.. & .....do.... & & a 40 \\
\hline June $14 \ldots$ & .....do......... & 1.64 & 43 & November $9 .$. & ..... do ..... & & a 40 \\
\hline June 22 . & .....do.......... & 14.50 & 14 & November 16. & .....do....... & & a 40 \\
\hline October 4 . & .....do.... & ....... & $a 40$ & November 23 . & .....do ..... & ......... & a 30 \\
\hline October 12 & .....do.... & ....... & $a 40$ & & . & & \\
\hline
\end{tabular}

$a$ Estimated.

Daily gage height, in feet, of Tamarack canal near Imperial, Cal., for 1905.

\begin{tabular}{|c|c|c|c|c|c|c|c|c|c|}
\hline Day. & June. & Day. & June. & Day. & June. & Day. & June. & Day & June. \\
\hline $1 \ldots$ & 1.7 & 7. & 1.8 & 13. & 1.35 & 19. & 1.65 & $25 .$. & 1.2 \\
\hline 2... & 1.68 & $8 \ldots \ldots \ldots$ & 1.8 & 14. & 1.65 & 20. & 1.75 & 26. & $1: 05$ \\
\hline $3 \ldots$ & 1.7 & $9 \ldots$ & 1.8 & 15. & 1.65 & 21. & 1.5 & $27 .$. & 1. 0 . \\
\hline $4 \ldots .$. & 1.55 & $10 \ldots$ & 1.9 & 16. & 1.6 & 22. & 1.45 & $28 \ldots \ldots \ldots \ldots$ & .9 \\
\hline $5 \ldots \ldots \ldots \ldots$ & 1.65 & $11 .$. & 1.95 & 17. & 1.65 & 23. & 1.35 & 29. & .9 \\
\hline $6 \ldots \ldots \ldots$ & 1.75 & $12 \ldots$ & 2.05 & $18 \ldots$. & 1.7 & $24 \ldots$ & 1.2 & $30 \ldots$. & .7 \\
\hline
\end{tabular}

Estimated monthly discharge of Tamarack canal near Imperial, Cal., for 1905.

\begin{tabular}{|c|c|c|c|c|}
\hline \multirow{2}{*}{ Month. } & \multicolumn{3}{|c|}{ Discharge in second-feet. } & \multirow{2}{*}{$\begin{array}{l}\text { Total in } \\
\text { acre-feet. }\end{array}$} \\
\hline & Maximum. & Minimum. & Mean. & \\
\hline June........... & & & 34.3 & 2,041 \\
\hline July .......... & & & $(40)$ & 2,460 \\
\hline August.............. & & & (40) & 2,460 \\
\hline September............ & & & $(40)$ & 2,380 \\
\hline October.... & & & $(40)$ & 2,460 \\
\hline November.............. & (n...... & $\cdots$ & (40) & 2,380 \\
\hline The period... & & & .. & 14,180 \\
\hline
\end{tabular}

Note.-Monthly means in parenthesis estimated in the field. 
Total amount of water (in acre-feet) entering the Imperial Valley through irrigation canals at international boundary, January to November, 1905, inclusive.

\begin{tabular}{|c|c|c|c|c|c|c|}
\hline Canal. & January. & February. & March. & April. & May. & June. \\
\hline Hemlock. . . . . . . . . . & 263 & 555 & 658 & 1,149 & 965 & 839 \\
\hline Boundary... & 249 & 216.5 & 110.5 & 303.5 & 200 & 235.5 \\
\hline Wisteria......... & 1,100 & 211 & 436 & 2,351 & 1,027 & 572 \\
\hline Tamarack... & ......... & .... & $\ldots$ & & ...... & 2,041 \\
\hline New Holt, or No. $7 \ldots$ & .......... & ........ & .......... & ............ & 1,107 & 1,964 \\
\hline 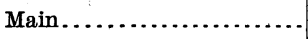 & 30,744 & 26,102 & 16,048 & 42,783 & 36,770 & 33,917 \\
\hline Alamitos............. & 1,636 & 633 & 301 & 2,714 & 1,955 & 2,047 \\
\hline Holt, or No. 5 Main .... & 1,525 & 1,162 & 3,530 & 12,377 & 9,346 & 8,926 \\
\hline Alamo Channel.............. & 5,500 & 8,330 & 9,223 & ........... & & ….... \\
\hline Total...... & 41,017 & 37,209 & 30,306 & 61,677 & 51,370 & 50,541 \\
\hline Canal. & July. & August. & September. & October. & November. & Total. \\
\hline Hemlock. . & 670 & 639 & 482 & 461 & 274 & 6,955 \\
\hline Boundary... & 166 & 172 & 113.5 & 135.5 & 268 & 2,170 \\
\hline Wisteria .... & 873 & 25 & 0 & 332 & 334 & 7,261 \\
\hline Tamarack......... & 2,460 & 2,460 & 2,380 & 2,460 & 2,380 & 14,181 \\
\hline New Holt, or No. $7 .$. & 2,029 & 2,029 & 1,964 & 2,029 & 1,964 & 13,086 \\
\hline Main............ & 35,355 & 34,003 & 21,957 & 20,168 & 20,886 & 318,733 \\
\hline Alamitos...... & 2,084 & . 1,470 & 2,428 & 2,201 & 161 & 17,630 \\
\hline Holt, or No. 5 Main .... & 9,223 & 9,223 & 8,569 & 5,940 & 1,607 & 71,428 \\
\hline Alamo Channel...... & & & & & - & 23,053 \\
\hline Total & $.52,860$ & 50,021 & 37,893 & 33,726 & $27,87 \dot{4}$ & 474,494 \\
\hline
\end{tabular}

Total amount of water (in acre-feet) entering Imperial V alley, including canals, New River, and Alamo Channel; also amount of waste water discharging into Salton Sea and amount used for irrigation, January to November, 1905, inclusive.

\begin{tabular}{|c|c|c|c|c|}
\hline Month. & $\begin{array}{c}\text { Total discharge } \\
\text { Imperial canal } \\
\text { at headings } \\
\text { below Yuma, } \\
\text { Ariz. }\end{array}$ & $\begin{array}{c}\text { Total discharge } \\
\text { into Imperial } \\
\text { Valley: }\end{array}$ & $\begin{array}{c}\text { Total discharge } \\
\text { (waste) into Sal- } \\
\text { ton Sea. }\end{array}$ & $\begin{array}{l}\text { Total amount } \\
\text { of water used } \\
\text { for irrigation. }\end{array}$ \\
\hline January... & $\begin{array}{l}\text { Acre-feet. } \\
58,100\end{array}$ & $\begin{array}{l}\text { Acre-feet. } \\
\qquad 55,590\end{array}$ & $\begin{array}{l}\text { Acre-feet. } \\
35,356\end{array}$ & $\begin{array}{l}\text { Acre-feet. } \\
\qquad 20,234\end{array}$ \\
\hline February....... & 227,150 & 92,913 & 77,919 & 14,994 \\
\hline March.............. & 288,990 & 210,896 & 194,548 & 16,348 \\
\hline April.......... & 233,550 & $223,1.71$ & 182,494 & 40,677 \\
\hline May........... & 335,420 & 321,239 & 287,869 & 33,370 \\
\hline June....................... & 573,020 & 622,911 & 587,370 & 35,541 \\
\hline July................... & $1,104,620$ & $1,037,724$ & 978,244 & 50,480 \\
\hline August........ & 609,030 & 597,751 & 564,946 & 32,805 \\
\hline September.... & 365,360 & $a 353,360$ & 324,869 & 28,491 \\
\hline October...... & 468,840 & $a 456,840$ & 435,227 & 21,613 \\
\hline November..... & $\quad 500,000$ & $a 488,000$ & 477,977 & 10,023 \\
\hline Total. & $4,764,080$ & $4,460,395$ & $4,155,819$ & 304,576 \\
\hline
\end{tabular}

$a$ Estimated.

Total amount of irrigated land (as per examination made in March and April, 1905)...acres... 79,591 Amount of water used per acre (in acre-feet) 
LANDS IRRIGATED IN THE IMPERIAL VALLEY.

In April, 1904, an examination was made to determine the amount of lands irrigated in the valley. The gross area under irrigation at that time was 66,741 acres. A second examination was made in September of the same year by W. V. Hardy, of the Reclamation Service, and showed the total area irrigated at that time to be 31,318 acres. Another examination by Mr. Hardy in March and April, 1905, gave a total irrigated area of 79,591 acres.

The examination of area of irrigated land made in March and April, 1905, is used in computing the amount of water per acre used during 1905. It is probable that this area was greatly reduced during the summer, as areas for the raising of hay and grain were not again used after the crops were harvested until the late fall months, when they were again seeded. Large areas of alfalfa and Kaffir corn were necessarily irrigated throughout the year.

\section{DUTY OF WATER IN THE IMPERIAL VALLEY.}

During the fall of 1904 rating flumes were placed on two canals in Imperial Valley for the study of the duty of water. Two types of soil were selected for these investigations, one known as "hard soil" and classified as "Imperial loam" as shown on soil map of Bureau of Soils, United States Department of Agriculture. The other type is known as "soft soil" and classified as "Imperial sandy loam."

The location of the investigation on "Imperial loam" is at the ranch of W. W. McKim, 7 miles east of Imperial, being the west half of sec. 8, T. 15 S., R. 14 E.

The location of the investigation on "Imperial sandy loam" is on lands of the CaliforniaMexico Land Company, $3 \frac{1}{2}$ miles east of Mexicala in Mexican territory and directly south of the California-Mexico boundary line in T. 17 S., R. 15 E.

Permanent flumes were constructed at the head of each of these canals and a sufficient number of measurements were made throughout the year for rating them. Self-recording registers were placed at each of these stations, and the mean daily gage height was computed from the register records.

During 1905 the Imperial Valley received a very unusual rainfall. The precipitation at Imperial was 10.06 inches for the year. At Calexico the precipitation was 9.33 inches for the year. The mean annual rainfall will probably not exceed 3 inches in normal years.

\section{M'KIM FLUME.}

This station was established on September 23, 1904. The amount of land irrigated under this flume is $\mathbf{3 8 0}$ acres. The soil is classified as "Imperial loam." The crops raised during 1905 consisted of 100 acres in barley, which was sown in October, 1904. The crop was only fair and was used for the pasturage of hogs. The balance of the area, 280 acres, had been previously planted to alfalfa, 100 acres having been sown in October, 1902, and 100 acres in October, 1903. This area in alfalfa was in fair condition at the time the station was established. The crop of 1905 was fair and used only for the pasturage of hogs. The supply of water furnished for this area was not enough for thorough irrigation. Water was taken from Alamo channel until March, 1905, and up to this time the supply was suffcient for thorough irrigation. After March, 1905, water was taken from Alamitos canal on account of flood conditions in the Alamo channel which destroyed the levee at point where the diversion was made. The water supply received from the Alamitos canal was not enough for the thorough irrigation of this area and crops suffered considerably from lack of water after March.

The rainfall for this station is taken from the United States Weather Bureau records kept at Imperial, Cal. 
Monthly duty of water under McKim flume during 1905.

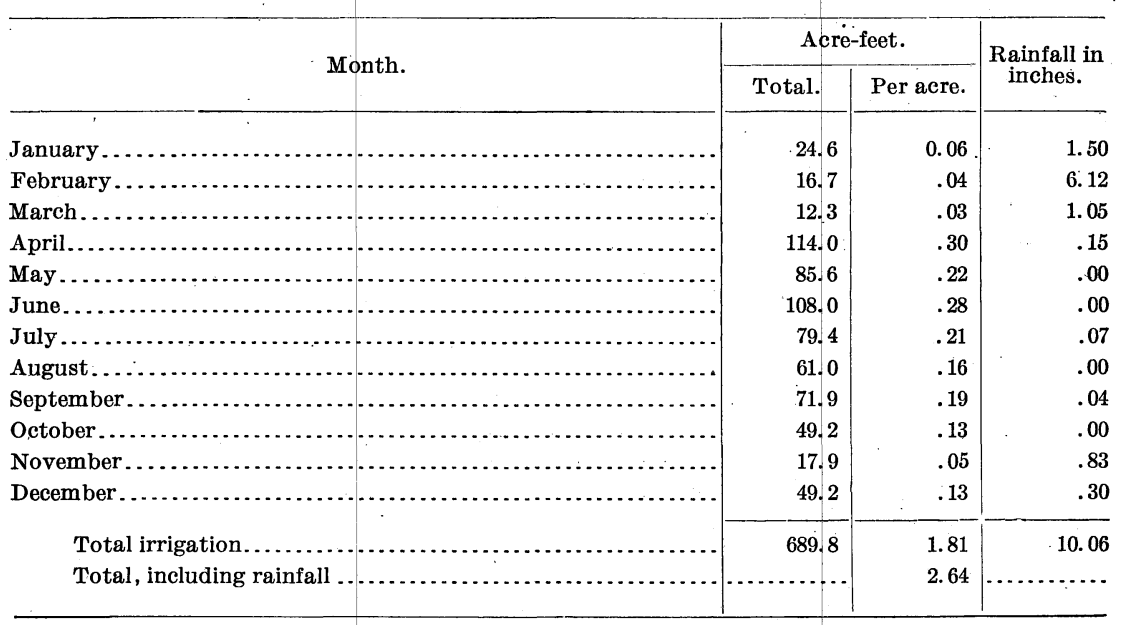

CALIFORNIA-MEXICO LAND AND CATTLE COMPANY'S FLUME.

This station was established November 28, 1904. It is located $3 \frac{1}{2}$ miles east of Mexicala. The total area irrigated under this flume is $\mathbf{5 7 5}$ acres. The soil is classified as "Imperial sandy loam." In December, 1904, the entire area was sown to barley, 75 acres were cut for hay, averaging $1 \frac{1}{4}$ tons to the acre, the balance of the acreage was used for pasturage. The crop was equally as good over the entire area as that on the 75 acres which was cut for hay. In May, 1905, the entire area was sown to Kaffir corn. This crop was poor and scattering and used in the fall for pasturage. This area received an ample supply of water for the crops raised.

The rainfall for this station is taken from the United States Weather Bureau records kept at Calexico, Cal.

Monthly duty of water under California-Mexico Land and Cattle Company's flume during 1905.

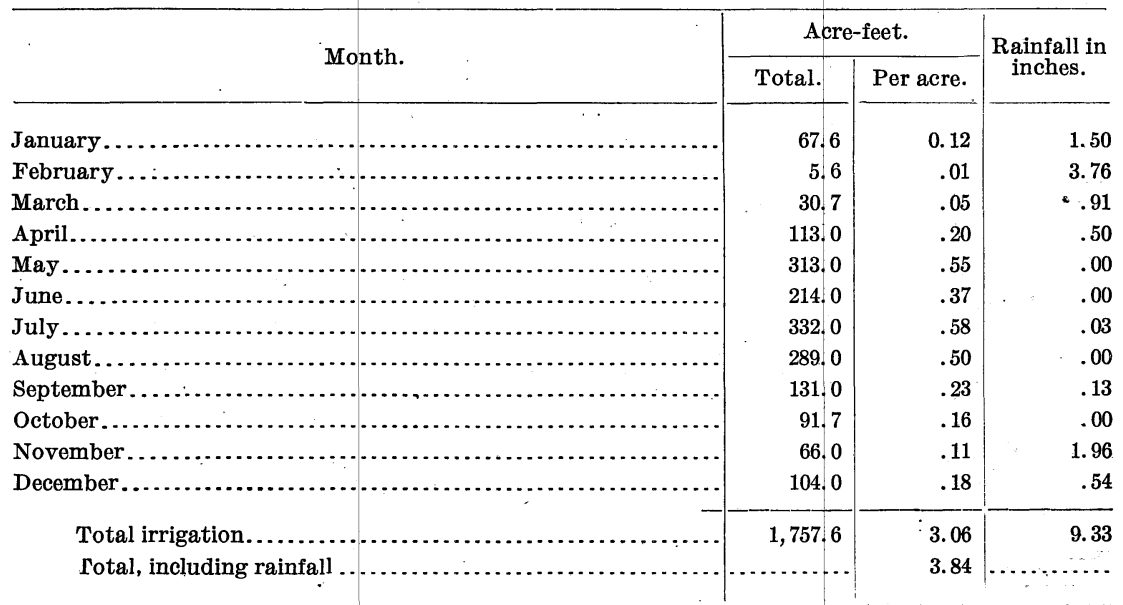




\section{MISCELLANEOUS MEASUREMENTS IN COLORADO RIVER DRAINAGE BASIN,}

The following miscellaneous measurements were made in canals in the Colorado River drainage basin by W. D. Smith during 1905:

Miscellaneous discharge measurements made in Colorado River drainage basin in 1905.

\begin{tabular}{|c|c|c|c|c|c|}
\hline Date. & Locality. & Width. & $\begin{array}{l}\text { Area of } \\
\text { section. }\end{array}$ & $\begin{array}{c}\text { Mean ve- } \\
\text { locity. }\end{array} \mid$ & $\begin{array}{c}\text { Dis- } \\
\text { charge. }\end{array}$ \\
\hline February $15 \ldots$ & $\begin{array}{l}\text { Imperial canal in Mexico immediately below } \\
\text { Quail River waste gate.a }\end{array}$ & Feet. & $S q \cdot f t$. & $\begin{array}{c}\text { Ft. per sec. } \\
3.46\end{array}$ & $\begin{array}{r}\text { Sec.-ft. } \\
1,937\end{array}$ \\
\hline February $28 \ldots$ & 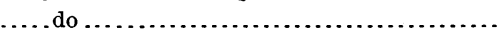 & $\ldots$. & 556 & 3.28 & 1,821 \\
\hline December $17 \ldots$ & $\begin{array}{l}\text { Imperial canal in Mexico immediately below } \\
\text { Quail River cut-off.b }\end{array}$ & 170 & 1,640 & 3.21 & 5,265 \\
\hline December. & $\begin{array}{l}\text { Quail River cut-off in Mexico at point of } \\
\text { diversion from Imperial canal.b }\end{array}$ & 150 & 632 & 2.68 & 1,693 \\
\hline
\end{tabular}

$a$ Quail River waste gate is in Mexico, 12 miles below heading No. 1 of the Imperial canal. Gagings by means of.boat and cable were made at this point February 15 and 28 to determine amount of water being carried to Imperial Valley by the canal.

$b$ For location and significance of this gaging see general description of Imperial canal, page 21. A measurement was made by means of boat and cable.

\section{THE GREAT BASIN DRAINAGE.}

\section{GENERAL FEATURES.}

The Great Basin drainage in California is comprised within the subdrainages, Sierra Nevada and Minor Great Basin. Within the Sierra Nevada drainage is a limited area of arid country lying on the eastern slope of the Sierra Nevadas. This area includes the Susan and Owens River drainage basins. Within the Minor Great Basin drainage lies the Mohave River drainage basin. Having no outlet to the sea, the entire drainage of these basins is lost mainly through evaporation from the lakes and sinks in which the waters of these rivers collect.

\section{SUSAN RIVER DRAINAGE BASIN.}

DESCRIPTION OF BASIN.

Susan River has its source in the Sierra Nevada in northeastern California, and flows eastward, discharging into Honey Lake - one of the landlocked lakes of the Great Basinof which it is the principal feeder. A considerable area of land is irrigated from the waters of the river below the gaging station, and during the last ten or twelve years several projects have been started for irrigating other extensive areas by. the storage of its waters both above and below the town of Susanville.

\section{SUSAN RIVER NEAR SUSANVILLE, CAL.}

This station was established June 3, 1900, by L. H. Taylor. It is located about threefourths of a mile southwest of Susanville, at the electric light plant.

The channel is straight for 150 feet above and 250 feet below the station. The current is swift. There is a riffle immediately above the cable. The right bank is high and is composed of clay covered with vegetation. It is not liable to overflow. The left bank is low, liable to overflow, and covered with a sparse growth of willows. The bed of the stream is composed of gravel and cobblestones and is permanent. A short distance above the station a small irrigating ditch, known as the "Masten ditch," is taken out on the right bank.

High-water measurements are made by means of a cable and car. Measurements at low and ordinary stages are made by wading. The initial point for soundings is a post in the fence in line with the cable, 34.8 feet from the cable support on the left bank. 
On December 20,1903, the station was reestablished, and the datum of the gage was raised 2.00 feet. A gage is also placed in the flume near the head of Masten ditch. During 1905 the gage was read once each day by James Branham. Bench marks were established as follows: (1) A nail in the fence post which is used as the initial point for soundings; elevation, 11.35 feet. (2) A nail in the cable post; elevation, 9.00 feet. (3) A nail in the cottonwood tree to which the cable is attached; elevation, 10.00 feet. Elevations refer to the datum of the gage.

Information in regard to this station is contained in the following Water-Supply Papers of the United States Geological Survey:

Description: 51, p 408; 66, pp 115-116; 85, p 123; 100, pp 204-205; 133, p 197.

Discharge: 51 , p 408; 66, p 116; 100, p 205; 133, p 197 .

Discharge, monthly: 75, p 190; 133, p 198.

Gage heights: 51, p 408; 66, p 116; 85, p 124; 100, p 205; 133, p 198.

Rating table: 566, p 175 .

Discharge measurements of Susan River near Susanville, Cal., in 1905.

\begin{tabular}{|c|c|c|c|c|c|c|}
\hline Date. & Hydrographer. & Width. & $\begin{array}{l}\text { Area of } \\
\text { section. }\end{array}$ & $\underset{\text { velocity. }}{\text { Mean }}$ & $\begin{array}{c}\text { Gage } \\
\text { height. }\end{array}$ & $\begin{array}{l}\text { Dis- } \\
\text { charge. }\end{array}$ \\
\hline March 21...... & J. Branham. . & $\begin{array}{r}\text { Feet. } \\
44\end{array}$ & $\begin{array}{c}S q . f t . \\
92\end{array}$ & $\begin{array}{r}\text { Ft. per sec. } \\
4.79\end{array}$ & $\begin{array}{r}\text { Feet. } \\
6.60\end{array}$ & $\begin{array}{c}\text { Sec.-ft. } \\
441\end{array}$ \\
\hline May $12 \ldots$ & .....do.. & 29 & 48 & 3.27 & 5.40 & 157 \\
\hline June $12 \ldots$ & ....do... & 25 & 31 & 2.32 & 4.70 & 72 \\
\hline June 25. . . & ....do.. & 24 & 18 & 1.66 & 4.20 & 30 \\
\hline July $23 . .$. & ....do. & 18 & 10.8 & 1.19 & 3.85 & 12.8 \\
\hline August $29 \ldots$ & ....do.. & 14. & 8.1 & 0.85 & 3.75 & 6.9 \\
\hline September 26 . & ....do.. & 19 & 13.6 & 1.13 & 4.00 & 15.4 \\
\hline
\end{tabular}


Daily gage height, in feet, of Susan River near Susanville, Cal., for 1905.

\begin{tabular}{|c|c|c|c|c|c|c|c|c|c|c|c|c|}
\hline Day. & Jan. & Feb. & Mar. & Apr. & May. & June. & July. & Avg. & Sept. & Oct. & Nov. & Dec. \\
\hline $1 \ldots$ & 4.95 & 5.2 & 5.25 & 6.15 & 5.95 & 5.15 & 4.1 & 4.1 & 3.8 & 3.8 & 4.0 & 4.0 \\
\hline $2 .$. & 4.8 & 5.2 & 5. 25 & 5.95 & 5.9 & 5.1. & 4. 1 & 4.1 & 3.8 & 3.8 & 4.0 & 4.0 \\
\hline 3. & .4 .75 & 5.2 & 5.25 & 5.95 & 5.8 & 5.05 & 4.45 & 4.1 & 3.8 & 3.9 & 4.0 & 4.0 \\
\hline $4 .$. & 4.7 & 5.2 & 5.25 & 5.9 & 5.8 & 5.05 & 4.8 & 4.1 & 3.8 & 3.95 & 4.0 & 4.0 \\
\hline $5 .$. & 4.65 & 5.15 & 5.25 & 5.9 & 5.65 & 5.0 & 4.6 & 4.1 & 3.85 & 3.95 & 4.0 & 4.0 \\
\hline $6 .$. & 4.6 & 5.05 & 5.25 & 6.0 & 5.55 & 5.0 & 4.4 & 4.1 & 3.85 & 3.95 & 4.0 & 4.0 \\
\hline 7.. & 4.6 & 4.85 & 5. 25 & 6.1 & 5.5 & 5.0 & 4.25 & 4.1 & 3.85 & 4.0 & 4.0 & 4.0 \\
\hline $8 .$. & 4.55 & 4.85 & 5.25 & 6. 1 & 5.5 & 4.9 & 4.1 & 4.0 & 3.85 & 3.95 & 4.0 & 4.0 \\
\hline 9. & $4 . \dot{55}$ & 4.75 & 5.2 & 6.1 & 5.5 & 4.85 & 4.1 & 3.9 & 3.85 & 3.9 & 4.0 & 4.0 \\
\hline $10 .$. & 4.55 & 4.8 & 5.2 & 6.2 & 5.45 & 4.75 & 4.0 & 3.8 & 3.85 & 3.9 & 4.0 & 4.0 \\
\hline 11.. & 4.55 & 4.7 & 5.2 & 6.1 & 5.4 & 4.7 & 4.0 & 3.8 & 3.9 & 3.9 & 4.0 & 4.0 \\
\hline $12 .$. & 4.6 & 4.65 & 5.4 & 6.1 & 5.4 & 4.7 & 4.0 & 3.8 & 3.9 & 3.9 & 4.0 & 4.0 \\
\hline $13 .$. & 4.65 & 4.55 & 5.9 & 6.1 & 5.35 & 4.65 & 3.95 & 3.8 & 3.9 & 3.9 & 4.0 & 4.0 \\
\hline $14 .$. & 4.7 & 4.5 & 5.9 & 6.1 & 5.3 & 4.65 & 4.2 & 3.8 & $3 . \dot{85}$ & 3.9 & 4.0 & 4.0 \\
\hline 15.. & 4.7 & 4.45 & 6.05 & 6.1 & 5.3 & 4.6 & .4 .2 & 3. 75 & 3.85 & 3.9 & 4.0 & 4.0 \\
\hline 16.. & 4.7 & 4.45 & 6.1 & 6.1 & 5.4 & 4.5 & 4.2 & 3. 75 & 3.8 & 3.9 & 4.0 & 4.0 \\
\hline 17. & 4.7 & 4.5 & 6.05 & 6.05 & 5.4 & 4.5 & 4.0 & $3 ; 75$ & 3.8 & 4.0 & 4.0 & 4.0 \\
\hline 18. & 4.65 & 4.55 & 6.0 & 6.05 & 5. 4 & 4.4 & 4.0 & 3.75 & 3.8 & 4.05 & 4.0 & 4.0 \\
\hline $19 .$. & 4.65 & 5.05 & 6.6 & 6.05 & 5.4 & 4.4 & 4.1 & 3.75 & 3.9 & 4.0 & 4.0 & 4.0 \\
\hline 20. & 4. 65 & 5.35 & 6.25 & 5.95 & 5.4 & 4.4 & 4.05 & 3. 75 & 3.9 & 4.0 & 4.0 & 4.0 \\
\hline $21 .$. & 4.65 & 5.35 & 6.45 & 5.8 & 5.4 & 4.3 & 3.9 & 3.8 & 3.95 & 4.0 & 4.0 & 4. 0 \\
\hline $22 \therefore$ & 4.8 & 5.25 & 6.15 & 5.8 & 5.4 & 4.3 & 3.9 & 3.8 & 3.95 & 4.0 & 4.0 & 4.0 \\
\hline $23:$ & 5.5 & 5.1 & 6.1 & 5.8 & 5.35 & 4.25 & 3.85 & 3.8 & 3.95 & 4.0 & 4.05 & 4.0 \\
\hline $24 .$. & 5.4 & 5.1 & 6.05 & 5.85 & 5.35 & 4.2 & 3.85 & 3.75. & 3.95 & 4.0 & 4.05 & 4.0 \\
\hline $25 .$. & 5.2 & 5.2 & 5.85 & 5.9 & 5.3 & 4.2 & 3.85 & 3. 75 & 3.95 & 4.0 & 4.1 & 4.0 \\
\hline $26 .$. & 4.95 & 5.2 & 6.9 & 6.0 & 5.3 & 4.2 & 3.85 & 3.75 & 4.0 & 4.0 & 4.1 & 4.0 \\
\hline $27 \ldots$ & 4.9 & 5.25 & 6.3 & 6.0 & 5.3 & 4.2 & 3.8 & 3.75 & 3.9 & 4.0 & 4.1 & 4.0 \\
\hline $28 \ldots$ & 4.9 & 5.25 & 6.1 & 6.0 & 5.25 & 4.2 & 3.8 & 3. 75 & 4.0 & 4.0 & 4.05 & 4.0 \\
\hline & 4.85 & $\therefore .$. & 6.0 & 6.0 & 5.25 & 4.15 & 3. 75 & 3.8 & 3.95 & 4.0 & 4.05 & 4.0 \\
\hline & 4.8 & & 6.1 & 6.0 & 5.2 & 4.15 & 3.75 & 3.8 & 3.9 & 4.0 & 4.0 & 4.0 \\
\hline 31... & 4.8 & ....... & 6.0 & ....... & 5.2 & -1 & 4.1 & 3.8 & r. & 4.0 & $\ldots$ & 4.0 \\
\hline
\end{tabular}

Station rating table for Susan River near Susanville, Cal., from January 1 to December 31, 1905.

\begin{tabular}{|c|r||r|r||r|r||r|r|}
\hline $\begin{array}{c}\text { Gage } \\
\text { height. }\end{array}$ & Discharge. & $\begin{array}{c}\text { Gage } \\
\text { height. }\end{array}$ & Discharge. & $\begin{array}{c}\text { Gage } \\
\text { height. }\end{array}$ & Discharge. & $\begin{array}{c}\text { Gage } \\
\text { height. }\end{array}$ & Discharge. \\
\hline Feet. & Second-feet. & Feet. & Second-feet. & Feet. & Second-feet. & Feet. & Second-feet. \\
3.75 & 7 & 4.60 & 59 & 5.50 & 168 & 6.40 & 356 \\
3.80 & 9 & 4.70 & 68 & 5.60 & 185 & 6.50 & 382 \\
3.90 & 13 & 4.80 & 77 & 5.70 & 203 & 6.60 & 410 \\
4.00 & 18 & 4.90 & 87 & 5.80 & 222 & 6.70 & 440 \\
4.10 & 24 & 5.00 & 98 & 5.90 & 242 & 6.80 & 470 \\
4.20 & 60 & 5.10 & 110 & 6.00 & 262 & 6.90 & 500 \\
4.30 & 37 & 5.20 & 123 & 6.10 & 284 & & \\
4.40 & 44 & 5.30 & 137 & 6.20 & 307 & & \\
4.50 & 51 & 5.40 & 152 & 6.30 & 331 & & \\
\hline
\end{tabular}

Note.-The above table is based on 11 discharge measurements made during 1904 and 1905 and is well defined. 
Estimated monthly discharge of Susan River near Susanville, Cal., for 1905.

[Drainage area, 256 square miles.]

\begin{tabular}{|c|c|c|c|c|c|c|}
\hline \multirow[b]{2}{*}{ Month. } & \multicolumn{3}{|c|}{ Discharge in second-feet. } & \multirow[b]{2}{*}{$\begin{array}{l}\text { Total in } \\
\text { acre-feet. }\end{array}$} & \multicolumn{2}{|c|}{ Run-off. } \\
\hline & Maximum. & Minimum. & Mean. & & $\begin{array}{c}\text { Second-feet } \\
\text { per square } \\
\text { mile. }\end{array}$ & $\begin{array}{l}\text { Depth } \\
\text { in inches. }\end{array}$ \\
\hline January... & 168 & 55 & 76.9 & 4,728 & 0.300 & 0.346 \\
\hline February .......... & 144 & 48 & 96.9 & 5,382 & .379 & .395 \\
\hline March........... & 500 & 123 & 234 & 14,390 & .914 & 1.05 \\
\hline April....... & 307 & 222 & 264 & 15,710 & 1.03 & 1.15 \\
\hline May......... & 252 & 123 & 160 & 9,838 & .625 & .721 \\
\hline June......... & 116 & 27 & 61.3 & 3,648 & .239 & .267 \\
\hline July ............. & 77 & 7 & 23.4 & 1,439 & .091 & .105 \\
\hline August...... & 24 & 7 & 12.1 & 744 & .047 & .054 \\
\hline September.... & 18 & 9 & 12.3 & 732 & .048 & .054 \\
\hline October.......... & 21 & 9 & 15.7 & 965 & .061 & .070 \\
\hline November........ & 24 & 18 & 19.0 & 1,131 & .074 & .083 \\
\hline December, $\ldots \ldots \ldots \ldots \ldots$ & 18 & 18 & 18.0 & 1,107 & .070 & .081 \\
\hline The year. & 500 & 7 & 82.8 & 59,810 & .323 & - 4.38 \\
\hline
\end{tabular}

WILLOW CREEK AT MERRILLVILLE, CAL,

This station was established June 18, 1904, by S. G. Bennett. It is located at the old bridge, 100 feet above the present wagon bridge at Merrillville.

The channel is straight above and below the bridge for 100 feet. The banks on each side are low, but not subject to overflow, and there is very little fluctuation in the discharge of the creek. The bed of the stream is composed of gravel and is not subject to much change.

Discharge measurements are made from the bridge. The initial point for soundings is on the left bank of the stream.

The gage is a staff fastened vertically to the left end of the bridge. During 1905 the gage was read once each day by $R$. W. Hurlbut.

A description of this station and gage height and discharge data are contained in WaterSupply Paper No. 134, United States Geological Survey, pages 199-200. 
Daily gage hetght, in feet, of Willow Creek at Merrillville, Cal., in 1905.

\begin{tabular}{|c|c|c|c|c|c|c|c|c|c|c|c|c|}
\hline Day. & Jan. & Feb. & Mar. & Apr. & May. & June. & July. & Aug. & Sept. & Oct. & Nov. & Dec. \\
\hline 1.. & 1. 1 & 1.1 & 0.95 & 1.1 & 0.9 & 0.95 & 0.9 & 0.95 & 1.0 & 1.1 & 1.3 & 1. 25 \\
\hline 2. & 1.05 & 1.1 & 1.0 & 1.05 & .9 & .95 & .9 & .95 & 1.0 & 1.1 & 1. 25 & 1.3 \\
\hline 3.. & 1.0 & 1.1 & 1.0 & 1.0 & .95 & .95 & .9 & .95 & 1.0 & 1.1 & 1.2 & 1.3 \\
\hline 4.. & 1.0 & 1.05 & 1.0 & 1.0 & .95 & .95 & .9 & .95 & 1.0 & 1.1 & 1.2 & 1.3 \\
\hline 5. & 1.0 & 1.1 & -.95 & 1.0 & .95 & .95 & .9 & .95 & 1.0 & 1.1 & 1.2 & 1.3 \\
\hline 6.. & 1.0 & 1.05 & .95 & 1.0 & .95 & .95 & .9 & .95 & 1.0 & 1.2 & 1.2 & 1.3 \\
\hline 7.. & 1.0 & 1.0 & 1.0 & 1.0 & .95 & .95 & .9 & $: 95$ & 1.0 & 1.3 & 1.2 & 1.3 \\
\hline 8.. & 1.0 & 1.0 & 1.05 & 1.0 & .95 & 1.0 & .9 & .95 & .1 .0 & 1.2 & 1.2 & 1.3 \\
\hline 9. & 1.0 & 1.0 & 1.0 & 1.0 & .95 & 1.0 & .9 & 1. & 1.0 & 1.2 & 1.2 & 1.3 \\
\hline o. & 1.0 & 1.0 & 1.0 & .95 & 1.0 & .95 & .95 & 1.0 & 1.0 & 1.2 & 1.2 & 1.3 \\
\hline$\ldots$ & 1.0 & 1.0 & 1.0 & .95 & 1.05 & .95 & .95 & 1. & 1.0 & 1.2 & 1.2 & 1.3 \\
\hline a. & 1.0 & 1.0 & 1.0 & .95 & 1.1 & .95 & 1.05 & 1.0 & 1.0 & 1.2 & 1.2 & 1.3 \\
\hline 13.. & 1.0 & 1.0 & 1.05 . & 1.0 & 1.05 & .95 & 1.05 & 1.0 & 1.0 & 1,2 & 1.2 & 1.3. \\
\hline 4.. & 1.05 & 1.0 & .95 & 1.0 & 1.0 & .95 & 1.0 & 1.0 & 1.0 & 1.2 & 1.2 & 1.3 \\
\hline $15 .$. & 1.05 & .95 & 1.05 & 1.0 & .95 & .95 & 1.0 & 1.0 & 1.0 & 1.2 & 1.2 & 1.3. \\
\hline 6. & 1.05 & 1.0 & 1.1 & 1.0 & .9 & .95 & 1.0 & 1.0 & 1.0 & 1.2 & 1.2 & 1.3 \\
\hline 7.. & 1.05 & 1.0 & 1. & 1. & 9 & .95 & .95 & 1.0 & 1.05 & 1.2 & 1.2 & 1.3 \\
\hline $18 .$. & 1.05 & 1.0 & 1.05 & 1.0 & .9 & .95 & .95 & 1.0 & 1.05 & 1.2 & 1,2 & 1.3 \\
\hline 19.. & 1.05 & 1.25 & 1.1 & 1.0 & .9 & .95 & .95 & 1.0 & 1.05 & 1.2 & 1.2 & 1.3 \\
\hline $20 .$. & 1.0 & 1. 15 & 1.05 & 1.0 & .9 & .95 & .95 & 1.0 & 1.05 & 1.2 & 1.2 & 1.3 \\
\hline 21.. & 1.05 & 1.05 & 1.05 & 1.0 . & .9 & .95 & .95 & 1.0 & 1. 05 & 1.2 & 1.2 & 1.3 \\
\hline 22. & 1. 1 & 1.05 & 1.05 & 1.0 & .95 & .95 & .95 & 1.0 & 1.05 & 1. 25 & 1.2 & 1.3. \\
\hline $23 .$. & 1.1 & 1.05 & 1.05 & .9 & .95 & .95 &, .95 & 1.0 & .05 & 1.25 & 1.2 & 1.3 \\
\hline 64. & 1. 1 & 1. & 1. & .9 & 95 & .95 & .95 & 1. & 1.05 & 1.25 & 1.2 & 1.3 \\
\hline 5. & 1.05 & 1.0 & 1. & .95 & .95 & .95 & .95 & 1.0 & 1.05 & 1. 25 & 1.2 & 1.3 \\
\hline $26 .$. & 1.0 & 1.05 & 1.1 & .95 & 1.0 & .95 & .95 & 1.0 & 1.05 & 1.2 & 1.2 & 1.3 \\
\hline $27 .$. & 1.0 & 1.0 & 1.1 & .95 & 1.0 & .95 & .95 & 1.0 & 1.1 & 1.2 & 1.2 & 1.3 \\
\hline 28. & 1.0 & 1.0 & 1.0 & .95 & 1.0 & .95 & .95 & 1.0 & 1.15 & 1.2 & 1.25 & 1.3 \\
\hline 29 . & 1.0 & $\ldots$ & 1.1 & .9 & .95 & .95 & .95 & 1.0 & 1.2 & 1.2 & 1. 25 & 1.3 \\
\hline 30. & 1.0 & $\cdots$ & 1.05 & .9 & .95 & .9 & .95 & 1.0 & 1.1 & 1.2 & 1.25 & 1.3 \\
\hline 31. & 1.0 & & 1.05 & & .95 & & $:^{.95}$ & 1.0 & & 1.25 & & 1.3 \\
\hline
\end{tabular}

Station rating table for Willow Creek at Merrillville, Cal., from July 1, 1904, to December 31, 1905.

\begin{tabular}{|c|c|c|c|c|c|c|c|}
\hline $\begin{array}{l}\text { Gage } \\
\text { height. }\end{array}$ & Discharge. & $\begin{array}{c}\text { Gage } \\
\text { height. }\end{array}$ & Discharge. & $\begin{array}{c}\text { Gage } \\
\text { height. }\end{array}$ & Discharge. & $\begin{array}{l}\text { Gage } \\
\text { height. }\end{array}$ & Discharge. \\
\hline $\begin{array}{r}\text { Feet. } \\
0.90\end{array}$ & $\begin{array}{c}\text { Second-feet. } \\
16\end{array}$ & $\begin{array}{l}\text { Freet. } \\
1.10\end{array}$ & $\begin{array}{c}\text { Second-feet. } \\
20\end{array}$ & $\begin{array}{l}\text { Feet. } \\
1.20\end{array}$ & $\begin{array}{r}\text { Second-feet. } \\
22\end{array}$ & $\begin{array}{c}\text { Feet. } \\
1.30\end{array}$ & $\begin{array}{c}\text { Second-feet } \\
-24\end{array}$ \\
\hline 1.00 & 18 & & & & & & \\
\hline
\end{tabular}

NoTE.-The above table'is based on four discharge measurements made during 1904, and is fairly well defined. 
Estimated monthly discharge of Willow Creek at Merrillville, Cal., for 1905.

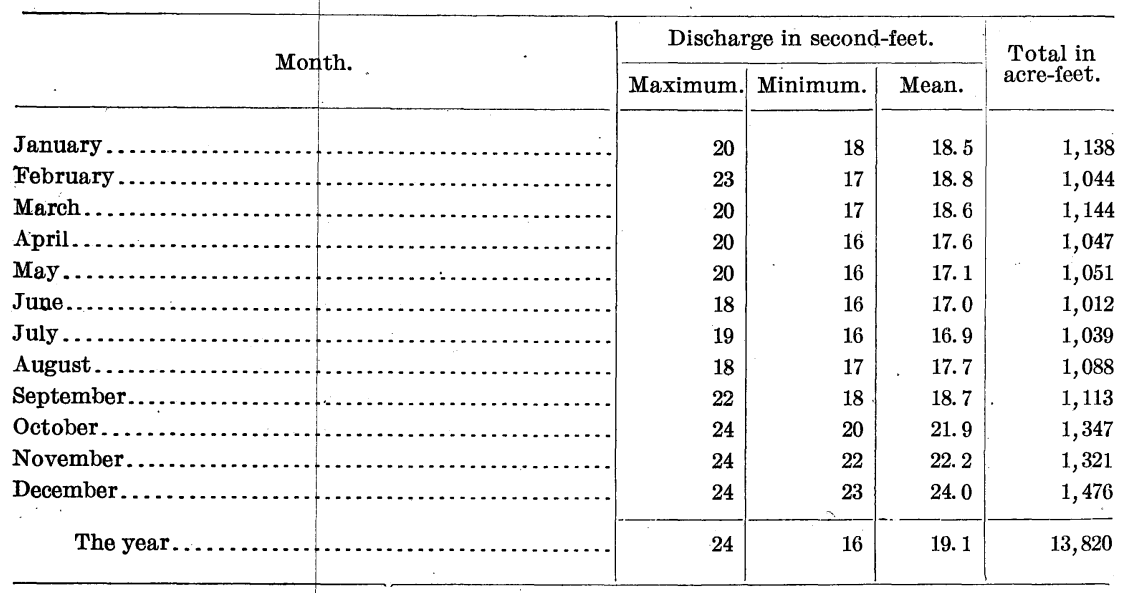

WILLOW CREEK NEAR STANDISH, CAL.

This station was originally established June 4, 1900. It was reestablished January 1, 1905, and discontinued December 31 of the same year. It is located at the bridge on the road from Susanville to Hot Springs and is about $1 \frac{1}{2}$ miles above the junction of the creek with Susan River. It is about 4 miles west of north from Standish.

The channel is straight for 300 feet above and 250 feet below the station. The right bank is rather low and is subject to overflow at extreme high water; "the left bank is high and not liable to overflow. The stream bed is sandy and liable to shift somewhat.

Discharge measurements are made from the bridge. The initial point for soundings is on the end of the bridge on the left bank.

The gage is a staff fastened vertically to the left abutment of the bridge. During 1905 the gage was read once each day by T. E. Ravenscroft

Information in regard to this station is contained in the following Water-Supply Papers of the United States Geological Survey:

Description: 51, p 407; 66, p 115.

Discharge: $51, \mathrm{p} 407$.

Gage heights: 51, p 407; 66, p 115.

Discharge measurements of Witlow Creek near Standish, Cal., in 1905.

\begin{tabular}{|c|c|c|c|c|c|c|}
\hline Date. & Hydrographer. & Width. & $\begin{array}{l}\text { Area of } \\
\text { section. }\end{array}$ & $\begin{array}{c}\text { Mean } \\
\text { velocity. }\end{array}$ & $\begin{array}{c}\text { Gage } \\
\text { height. }\end{array}$ & $\begin{array}{c}\text { Dis- } \\
\text { charge. }\end{array}$ \\
\hline January 24 & J. Branham.......... & $\begin{array}{r}\text { Feet. } \\
57\end{array}$ & Sq. ft. & $\begin{array}{r}\text { Ft. per sec. } \\
0.80\end{array}$ & $\begin{array}{l}\text { Feet. } \\
\quad 6.15\end{array}$ & $\begin{array}{r}S e c .-f t . \\
94\end{array}$ \\
\hline January 29 & .....do... & 57 & 87 & .79 & 5. 35 & 69 \\
\hline February $26 \ldots$ & .....do... & 57 & 105 & .79 & 5.75 & 83 \\
\hline April $23 . . . . .$. & .....do. . & 32 & 21 & .95 & 4: 00 & 20 \\
\hline November 19 . & ....do.. & 57 & 56 & .61 & 4.80 & 34 \\
\hline
\end{tabular}


Daily gage height, in feet, of Willow Creek near Standish, Cal., for 1905.

\begin{tabular}{|c|c|c|c|c|c|c|c|c|c|c|c|c|}
\hline Day. & Jan. & Feb. & Mar. & Apr. & May. & June. & July. & Aug. & Sept. & Oct. & Nov. & Dec. \\
\hline 1.. & .6 & 6.8 & 5.0 & 5.5 & 4.0 & 4.0 & 3.0 & 4.0 & 4.2 & 5.0 & 5.0 & 5.0 \\
\hline $2 \ldots$ & 6.6 & 6.7 & 4.8 & 5.3 & 4.0 & 4.0 & 3.0 & 4.0 & 4.5 & 4.8 & 5.1 & 5.3 \\
\hline 3... & 5.0 & 5.9 & 4.0 & 5.0 & 4.0 & 4.0 & 3.0 & 4.0 & 4.4 & 4.6 & 5.0 & 5.2 \\
\hline 4.. & 5.0 & & 4.0 & 5.0 & 4.0 & 4.0 & 6.0 & 4.0 & 4.4 & 4.3 & 5.0 & 5.0 \\
\hline 5... & 4.6 & 5.8 & 4.0 & 4.8 & 4.0 & 4.0 & 5.8 & 4.0 & 4.4 & 4.0 & 5.0 & 5.1 \\
\hline $6 \ldots$ & 4.6 & 6.0 & 4.4 & 4.5 & 4.0 & 4.0 & 4.8 & 4.0 & 4.4 & 4.0 & 5.1 & 5.0 \\
\hline 7... & 4.6 & 6.0 & 4.4 & 4.0 & 4.0 & 4.0 & 4.0 & 4.0 & 4.4 & 4.0 & 5.2 & 5.2 \\
\hline 8.. & 4.6 & 5.8 & 4.4 & 4.0 & 4.0 & 4.2 & 4.0 & 4.0 & 4.4 & 4.2 & 5.0 & 4:1 \\
\hline $9 .$. & 4.6 & 5.6 & 4.0 & 4.0 & 4.0 & 4.1 & 4.0 & 4.0 & 4.4 & 4.2 & 5.0 & 4.1 \\
\hline ). & 4.6 & 5.0 & 4.0 & 4.0 & 4.0 & 4.0 & 4.0 & 4.0 & 4.0 & 4.1 & 5.2 & 4.4 \\
\hline .. & 4.6 & 5.0 & 4.4 & 4.0 & 4.2 & 4.0 & 4.0 & 4.2 & 4.0 & 4.1 & 5.1 & 4.4 \\
\hline$\ldots$ & 4.7 & 5.0 & 4.0 & 3.8 & 4.2 & 4.0 & 6.0 & 4.0 & 4.0 & 4.0 & $4: 6$ & 4.4 \\
\hline 13.. & 4.7 & 5.0 & 4.0 & 4.0 & 4.0 & 4.0 & 6.0 & 4.0 & 4.0 & 4.0 & 4.4 & 4.3 \\
\hline ... & 4.7 & 5.0 & 4.0 & 4.0 & 4.0 & 4.0 & 5.8 & 4.0 & 6.0 & 4.0 & 4.4 & 4.5 \\
\hline $5 .$. & 5.8 & 4.6 & 4.4 & 4.0 & 4.0 & 4.0 & 5.0 & 4.0 & 5.8 & 4.0 & 4.4 & 4.1 \\
\hline$\ldots$ & 5.8 & 4.7 & 4.4 & 4.0 & 4.0 & 4.0 & 5.8 & 4.0 & 5.0 & 4.1 & 4.6 & 4.1 \\
\hline .. & 6.0 & 4.7 & 4.4 & 4.0 & 4.0 & 4.0 & 5.8 & 4.0 & 5.0 & 4.5 & 4.8 & 4.3 \\
\hline $18 \ldots$ & 6.0 & 4.7 & 4.4 & 4.0 & 4.0 & 4.0 & 5.8 & 4.2 & 5.0 & 4.5 & 4.8 & 4.3 \\
\hline 19.. & 6.4 & 5.1 & 5.0 & 4.0 & 4.0 & 4.0 & 6.0 & 4.2 & 5.0 & 4.5 & 5.0 & 4.3 \\
\hline .. & 6.35 & 6.0 & 5.0 & 4.0 & 4.0 & 4.0 & 6.0 & 4.6 & 5.0 & 4.5 & 5.0 & 4.3 \\
\hline .. & 6.0 & 7.8 & 5.2 & 4.0 & 4.1 & 4.0 & 6.0 & 4.5 & 4.8 & 4.5 & 4.8 & 4.0 \\
\hline $2 .$. & 7.6 & 7.8 & 5.2 & 4.0 & 4.1 & 4.0 & 5.0 & 4.6 & 4.8 & 4.5 & 4.6 & $5 . \theta$ \\
\hline ... & 7.8 & 7.0 & 5.0 & 4.0 & 4.0 & 4.0 & 5.0 & 4.2 & 4.8 & 5.0 & 4.6 & 5.0 \\
\hline 24. & 6.0 & 6.2 & 4.8 & 4.0 & 4.0 & 4.0 & 4.8 & 4.0 & 4.6 & 5.1 & 4.5 & 5.2 \\
\hline $25 \ldots$ & 6.0 & 6.0 . & 4.6 & 4.0 & 4.0 & 4.0 & 4.1 & 4.0 & 4.5 & 5.2 & 4.4 & 5.0 \\
\hline 26. & 5.8 & 6.3 & 5.3 & 4.0 & ، 4.0 & 6.2 & 4.0 & 4,2 & 5.0 & 5.2 & 4.8 & 5.2 \\
\hline 27. & 5.6 & 5.0 & 5.0 & 4.0 & 4.0 & 5.0 & 4.0 & 4.2 & 6.0 & 5.1 & 4.8 & 5.3 \\
\hline 28.. & 5.6 & 5.0 & 5.0 & 4.0 & 3.8 & 4.0 & 4.0 & 4.2 & 5.8 & 5.5 & 5.0 & 5.3 \\
\hline 29. & 5.7 & & 5.0 & 4.0 & 3.8 & 4.0 & 4.0 & 4.2 & 5.8 & 5.2 & 5.0 & 5.3 \\
\hline 30.. & 5.0 & & 5.0 & 4.0 & 3.8 & 3.6 & 4.0 & 4.3 & 5.0 & 5.0 & 5.0 & 5.3 \\
\hline $31 .$. & 5.6 & 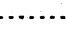 & 5.5 & & .. & & 4.0 & 4.2 & & 5.0 & & 5.3 \\
\hline
\end{tabular}

Station rating table for Willow Creek near Standish, Cal., from January 1 to December 31, 1905.

\begin{tabular}{|r|r||r|r||r|r||r|r|}
\hline $\begin{array}{c}\text { Gage } \\
\text { height. }\end{array}$ & Discharge. & $\begin{array}{c}\text { Gage } \\
\text { height. }\end{array}$ & Discharge. & $\begin{array}{c}\text { Gage } \\
\text { height. }\end{array}$ & Discharge. & $\begin{array}{c}\text { Gage } \\
\text { height. }\end{array}$ & Discharge. \\
\hline Feet. & Second-feet. & Feet. & Second-feet. & Feet. & Second-feet. & Feet.. & Second-feet. \\
3.60 & 10 & 4.60 & 31 & 5.60 & 74 & 6.60 & 141 \\
3.70 & 11 & 4.70 & 34 & 5.70 & 80 & 6.70 & 149 \\
3.80 & 13 & 4.80 & 37 & 5.80 & 86 & 6.80 & 157 \\
3.90 & 15 & 4.90 & 41 & 5.90 & 92 & 6.90 & 165 \\
4.00 & 17 & 5.00 & 45 & 6.00 & 98 & 7.00 & 173 \\
4.10 & 19 & 5.10 & 49 & 6.10 & 105 & 7.20 & 189 \\
4.20 & 21 & 5.20 & 54 & 6.20 & 112 & 7.40 & 205 \\
4.30 & 23 & 5.30 & 59 & 6.30 & 119 & 7.60 & 221 \\
4.40 & 25 & 5.40 & 64 & 6.40 & 126 & 7.80 & 238 \\
4.50 & 28 & 5.50 & 69 & 6.50 & 133 & & \\
\hline
\end{tabular}

Nore.-The above table is based on five discharge measurements made during 1905 and is not well defined. 
Estimated monthly discharge of Willow Creek near Standish, Cal., for 1905.

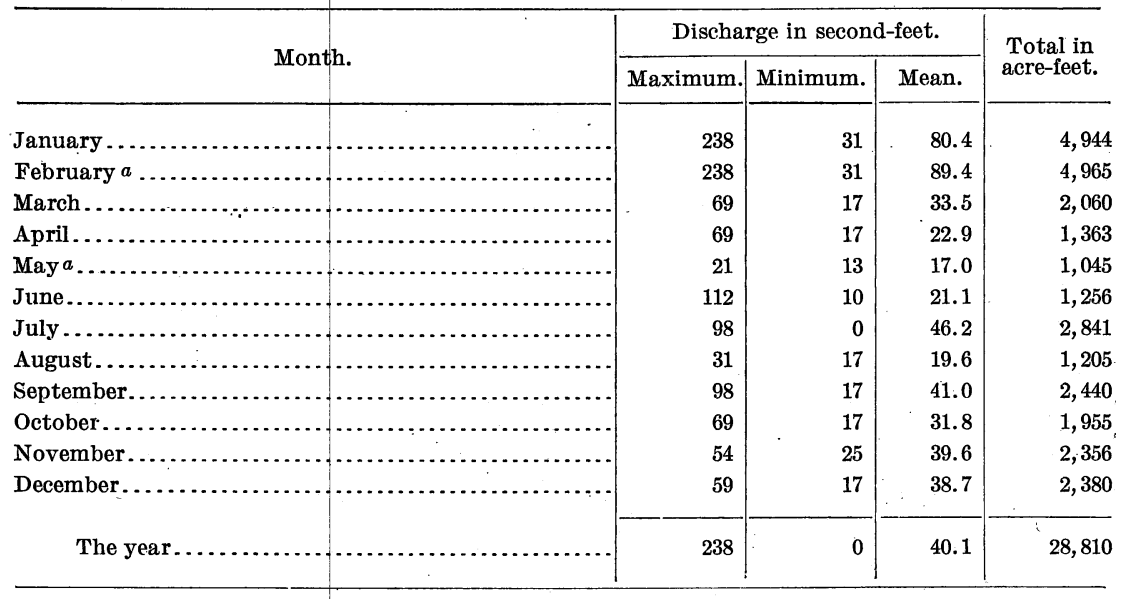

$a$ Discharge interpolated February 4 and May 31.

\section{OWENS RIVER DRAINAGE BASIN.}

\section{DESCRIPTION OF BASIN.}

Owens River has its source in the Sierra Nevadas in eastern California and fows southeast parallel with this range, finally discharging its waters into Owens Lake. This basin has a length from north to south of approximately 150 miles with a width of from 20 to 25 miles. It lies between the Sierra Nevadas on the west and the White Mountains on the east. Practically the entire flow of this river is derived from the Sierra Nevadas, as it drains the entire eastern slope of this range from Mount Lyell on the north to a point some distance below Mount Whitney on the south. The White Mountains furnish no water for this stream except in times of exceptionally heavy rain storms, which seldom nccur on this range. There are numerous tributaries entering Owens River from the west which have their source in the high elevations of the Sierra Nevadas, extending from the northern to the southern limits of this basin. The topography of the portion of the Sierra Nevadas which is drained by this stream is extremely rough and precipitous, the mountains rising abruptly from Owens Valley to elevations of 13,000 to 14,000 feet. The formation is of granite, with very little soil covering and sparse timber growth. Numerous lakes and marshes are found in the upper reaches of this portion of the drainage basin. Owens River, a short distance below its source, enters a flat, swampy country known as Long Valley, where a considerable quantity of its flow is used for the irrigation of meadow lands for stock raising. This water returns to the river channel at the lower end of this valley, at which point the stream enters a deep, narrow gorge with heavy grade. As the river breaks from this canyon it enters Owens Valley; through which it flows for a distance of about 80 miles, finally discharging into Owens Lake. The gaging station at Round Valley is located at the lower end of this canyon. Below this point numerous diversions are made for the irrigation of land in Owens Valley, where the soil is extensively cultivated and large areas are used for the raising of hay and grain. This country is particularly adapted to stock raising, which is carried on extensively throughout the valley. There are numerous opportunities for the construction of storage reservoirs within this basin, both on the main stream and also on the upper reaches of its tributaries, although none have been constructed as yet. The precipitation is extremelly light within the area of this basin with the exception of the high elevations of the Sierra Nevadas, where there is a heavy fall of snow. The 
melting of the snow in the spring and summer months feeds the numerous tributaries of this river, insuring a continued flow throughout the year.

The gaging stations on canals taking water from Owens River may be considered as temporary. The gage rods in most instances are securely fastened to footbridges and are not liable to change. No permanent bench marks were established.

\section{OWENS RIVER NEAR ROUND VALLEY, CAL.}

This station was established August 3, 1903, by J. C. Clausen. It is located at the footbridge, 700 feet above the junction of Owens River and Rock Creek.

The river at this point cuts through a lava deposit about 100 feet thick and forms a gorge, which is about 250 feet wide at the top. The channel is straight for $\mathbf{1 7 5}$ feet above and 250 feet below the station. The current is swift at all stages. Both banks are high and rocky and are not liable to overflow. The bed of the stream is composed of rock and lava bowlders and is not subject to much change.

Discharge measurements are made from the single-span footbridge to which the gage is attached. The bridge is 37 feet long and has a clear span of 35 feet. The initial point for soundings is the anchor bolt of the right abutment.

The gage is a vertical rod, fastened to the concrete bridge abutment on the left bank. During 1905 the gage was read once each day by $\mathrm{T}$. E. Jones. The bench mark is a bolt set in a lava bowlder 97.4 feet north of the right abutment.

Information in regard to this station is contained in the following Water-Supply Papers of the United States Geological Survey:

Description: 100, p 206; 134, p 200.

Discharge: 100, p 206; 134, p 201.

Discharge, monthly: 134, p 203.

Gage heights; 100, p 207; 134, p 202.

Rating table: 134, p 203.

Discharge measurements of Owens River near Round Valley, Cal., in 1905.

\begin{tabular}{|c|c|c|c|c|c|c|}
\hline Date. & Hydrographer. & Width. & $\begin{array}{l}\text { Area of } \\
\text { section. }\end{array}$ & $\begin{array}{l}\text { Mean } \\
\text { velocity. }\end{array}$ & $\begin{array}{c}\text { Gage } \\
\text { height. }\end{array}$ & $\begin{array}{c}\text { Dis- } \\
\text { charge. }\end{array}$ \\
\hline$\cdot \cdot$ & $\therefore \ldots, \cdots$ & Feet: & $s q . f t$ & Ft.per sec. & Feet. & Sec.-ft.' \\
\hline January $16: .$. & R. J. Taylor. & 34 & 73 & 2.52 & 1.97 & 184 \\
\hline February $9 \ldots$ & ....do do... & 34 & 76 & 2. 20 & 1.95 & 175 \\
\hline April 4. . & J. S. Evans ............ & 34 & 72 & 2.49 & 2.00 & 179 \\
\hline May $22 \ldots$ & ....do. & 34 & 91 & 3.36 & 2.39 & 306 \\
\hline June 27... & .....do. & 34 & 102 & 3.74 & 2.58 & 382 \\
\hline July 25. & .....do. . & 34 & 76 & 2.73 & 2.15 & 207 \\
\hline August $15 . .$. & .....do.. & 34 & 72 & 2.12 & 1.90 & 154 \\
\hline September 8. & .....do. & 34 & 76 & 2.33 & 2.00 & 177 \\
\hline September 27 . & ....do. & 34 & 72 & 2.15 & 1.90 & 155 \\
\hline November 2 .. & F. R. S. Buttemer. & 34 & 72 & 2.28 & 1.80 & 164 \\
\hline November 24 . & ....do........ & 34 & 75 & 2.40 & 1.85 & 180 \\
\hline December $10 .$. & .....do... & 34 & 70 & 2.16 & 1.73 & 151 \\
\hline
\end{tabular}


Daily gage height, in feet, of Owens River near Round Valley, Cal., for 1905.

\begin{tabular}{|c|c|c|c|c|c|c|c|c|c|c|c|c|}
\hline Day. & Jan. & Feb. & Mar. & Apr. & May. & June. & July. & Aug. & Sept. & Oct. & Nov. & Dec. \\
\hline $1 .$. & 2.15 & 2.2 & 2.2 & 1.9 & 2.1 & 2.3 & 2.5 & 2.1 & 1.9 & 2.0 & 1.8 & 1.9 \\
\hline $2 .$. & 2.1 & 2.15 & 2.25 & 1.95 & 2.1 & 2.3 & 2.5 & 2.05 & 1.9 & 2.0 & 1.8 & 1.9 \\
\hline 3.. & 2.1 & 2.1 & 2.2 & 2.0 & 2.1 & 2.3 & 2.6 & 2.05 & 1.9 & 2.0 & 1.75 & 1.95 \\
\hline $4 .$. & 2.0 & 2.1 & 2.2 & 2.0 & 2.1 & 2.3 & 2.6 & 2.0 & 1.9 & 1.95 & 1.75 & 1.95 \\
\hline $5 .$. & 2.0 & 2.1 & 2.2 & 2.0 & 2.1 & 2.3 & 2.6 & 2.0 & 1.95 & 1.95 & 1.8 & 1.95 \\
\hline $6 \ldots$ & 2.0 & 2.0 & 2.25 & 2.0 & 2.05 & 2.3 & 2.6 & 1.95 & 2.0 & 1.9 & 1.8 & 2.0 \\
\hline $7 \ldots$ & 2.0 & 2.1 & 2.25 & 2.0 & 2.0 & 2.35 & 2.5 & 1.95 & 2.0 & 1.9 & 1.85 & 2.0 \\
\hline $8 .$. & 2.0 & 2.1 & 2.2 & 2.0 & 2.0 & 2.35 & 2.5 & 2.0 & 2.0 & 1.9 & 1.85 & 2.0 \\
\hline $9 \ldots$ & 2.0 & 2.1 & 2.1 & 2.1 & 2.0 & 2.4 & 2.5 & 2.0 & 2.0 & 1.9 & 1.9 & 1.95 \\
\hline $10 \ldots$ & 2.0 & 2.1 & 2.1 & 2.1 & 2.0 & 2.4 & 2.5 & 2.0 & 2.0 & 1.95 & 1.9 & 1.9 \\
\hline $11 .$. & 2.0 & 2.1 & 2.0 & 2.0 & 2.0 & 2.4 & 2.5 & 1.9 & 2.0 & 1.95 & 1.9 & 1.9 \\
\hline 12. & 1.95 & 2.0 & 2.0 & 2.0 & 2.0 & 2.55 & 2.45 & 1.9 & 2.0 & 2.0 & 1.9 & 1.9 \\
\hline 13. & 1.95 & 2.0 & 2.5 & 2.0 & 2.0 & 2.7 & 2.4 & 1.9 & 2.0 & 2.0 & 1.9 & $1.9^{\circ}$ \\
\hline $14 .$. & 1.9 & 2.0 & 2.15 & 1.95 & 2.0 & 2.75 & 2.4 & 2.0 & 2.0 & 2.0 & 1.95 & 1.85 \\
\hline 15. & 1.9 & 2.0 & 2.15 & 1.9 & 2.0 & 2.75 & 2.4 & 2.0 & 2.0 & 2.0 & 2.0 & 1.85 \\
\hline $16 \ldots$ & 1.9 & 2.0 & 2.15 & 1.9 & 2.0 & 2.75 & 2.3 & 2.0 & 2.0 & 2.0 & 2.0 & 1.85 \\
\hline $17 .$. & 1.95 & 2.0 & 2.1 & 1.95 & 2.2 & 3.0 & 2.2 & 1.9 & 2.0 & 1.95 & 2.0 & 1.8 \\
\hline $18 .$. & 1.95 & 2.1 & 2.1 & 1.95 & 2.35 & 3.0 & 2.2 & 1.9 & 2.0 & 1.95 & 2.0 & 1.8 \\
\hline 19.. & 2.0 & 2.2 & 2.05 & 1.95 & 2.35 & 3.0 & 2.2 & 1.9 & 2.0 & 1.9 & 2.0 & 1.8 \\
\hline $20 .$. & 2.0 & 2.1 & 2.05 & 1.95 & 2.4 & 3.0 & 2.2 & 1.85 & 2.0 & 1.85 & 2.0 & 1.75 \\
\hline $21 .$. & 2.1 & 2.0 & 2.1 & 1.9 & 2.4 & 3.0 & 2.15 & 1.85 & 2.0 & 1.85 & 2.0 & 1.75 \\
\hline 22. & 2.1 & 1.95 & 2.1 & 1.9 & 2.4 & 3.0 & 2.1 & 1.9 & 2.0 & 1.85 & 2.0 & 1.75 \\
\hline $23 .$. & 2.0 & 1.95 & 2.1 & 1.9 & 2.4 & 3.0 & 2.1 & 1.9 & 2.0 & 1.85 & 2.0 & 1.75 \\
\hline $24 .$. & 2.0 & 1.9 & 2.0 & 1.95 & 2.4 & 2.95 & 2.1 & 1.9 & 2.0 & 1.85 & 2.0 & 1.75 \\
\hline $25 .$. & 2.1 & 1.85 & 2.0 & 1.95 & 2.4 & 2.8 & 2.1 & 1.9 & 2.0 & 1.8 & 2.0 & 1.75 \\
\hline 26. & 2.1 & 1.85 & 2.0 & 1.95 & 2.4 & 2.7 & 2.0 & 1.9 & 2.0 & 1.8 & 2.0 & 1.8 \\
\hline $27 .$. & 2.1 & 2.0 & 2.0 & 1.95 & 2.45 & 2.6 & $2.0^{\circ}$ & 1.9 & 2.0 & 1.75 & 2.0 & 1.8 \\
\hline 28. & 2.1 & 2.1 & 1.95 & 2.0 & 2.55 & 2.6 & 2.0 & 1.9 & 2.0 & 1.75 & 1.95 & 1.8 \\
\hline $29 .$. & 2.1 & & 1.9 & 2.05 & 2.5 & 2.55 & 2.0 & 1.9 & 2.0 & 1.75 & 1.9 & 1.8 \\
\hline $30 \ldots$ & 2.2 & & 1.9 & 2.05 & 2.4 & 2.5 & 2.0 & 1.9 & 2.0 & 1.75 & 1.9 & 1.8 \\
\hline $31 \ldots$ & 2.2 & & 1.9 & & 2.3 & $\ldots \ldots$ & 2.0 & 1.9 & $\ldots$ & 1.75 & . & 1.8 \\
\hline
\end{tabular}

Station ratingtable for Owens River near Round V alley, Cal., from January 1 to October 10, 1905.

\begin{tabular}{|c|c||c|c|c|c||c|c|}
\hline $\begin{array}{c}\text { Gage } \\
\text { height. }\end{array}$ & Discharge. & $\begin{array}{c}\text { Gage } \\
\text { height. }\end{array}$ & Discharge. & $\begin{array}{c}\text { Gage } \\
\text { height. }\end{array}$ & Discharge. & $\begin{array}{c}\text { Gage } \\
\text { height. }\end{array}$ & Discharge. \\
\cline { 1 - 6 } Feet. & Second-feet. & Feet. & Second-feet. & Feet. & Second-feet. & Feet. & Second-feet. \\
1.80 & 134 & 2.20 & 240 & 2.60 & 377 & 3.00 & 533 \\
1.90 & 158 & 2.30 & 271 & 2.70 & 415 & & \\
2.00 & 184 & 2.40 & 304 & 2.80 & 453 & & \\
2.10 & 211 & 2.50 & 339 & 2.90 & 493 & & \\
& & & & & & \\
\hline
\end{tabular}

Note. - The above table is based on 12 discharge measurements made during 1904-5. It is fairly well defined between gage heights 1.9 feet and 3.1 feet. 
Station rating lable for Owens River near Round Valley, Cal., from October 11 to December 31, 1905.

\begin{tabular}{|c|c|c|c|c|c|c|c|}
\hline $\begin{array}{c}\text { Gage } \\
\text { height. }\end{array}$ & Discharge. & $\begin{array}{c}\text { Gage } \\
\text { height. }\end{array}$ & Discharge. & $\begin{array}{c}\text { Gage } \\
\text { height. }\end{array}$ & Discharge. & $\begin{array}{c}\text { Gage } \\
\text { height. }\end{array}$ & Discharge. \\
\hline Feet. & Second-feet. & Feet. & Second-feet. & Feèt. & second-feet. & Feet. & Second-feet. \\
\hline 1.70 & 141 & 1.80 & 165 & 1.90 & 191 & 2.00 & 219 \\
\hline
\end{tabular}

NoTE.-The above table is based on three discharge measurements made during the latter part of 1905 and is fairly well defined.

Estimated monthly discharge of Owens River near Round Valley, Cal., for 1905.

\begin{tabular}{|c|c|c|c|c|}
\hline \multirow{2}{*}{ Month. } & \multicolumn{3}{|c|}{ Discharge in second-feet. } & \multirow{2}{*}{$\begin{array}{l}\text { Total in } \\
\text { acre-feet. }\end{array}$} \\
\hline & Maximum. & Minimum. & Mean. & \\
\hline January . . . . . . . . & 240 & 158 & 193 & 11,870 \\
\hline February ........... & 240 & 146 & 196 & 10,880 \\
\hline March............... & 339 & 158 & 213 & 13,100 \\
\hline April.............. & 211 & 158 & 177 & 10,530 \\
\hline May.................... & 358 & 184 & 246 & 15,130 \\
\hline June......... & 533 & 271 & 392 & 23,330 \\
\hline July............ & 377 & 184 & 275 & 16,910 \\
\hline August. ............ & 211 & 146 & 169 & 10,390 \\
\hline September............ & 184 & 158 & 180 & 10,710 \\
\hline October.................. & 219 & 153 & 180 & 11,070 \\
\hline November........ & 219 & 153 & 197 & 11,720 \\
\hline December.................... & 219 & 153 & 179 & 11,010 \\
\hline The year. & 533 & 146 & 216 & 156,600 \\
\hline
\end{tabular}

ROCK CREEK NEAR ROUND VALIEY, CAL.

This station was established August 3, 1903, by J. C. Clausen. It is located at the wagon bridge on the road from Long Valley to Bishop 3,500 feet above the mouth of the creek.

The channel is straight for 50 feet above and 40 feet below the footbridge. The current is swift. Both banks are high and rocky and are not liable to overflow. The bed of the stream is composed of gravel and is not subject to much change.

Discharge measurements are made from a footbridge, which has a span of 18 feet. The initial point for soundings is on right bank of stream.

The gage is a vertical rod fastened to the left end of the bridge. During 1905 the gage was read once each day by T. E. Jones. The bench mark is a point marked on a lava rock 15 feet east of the left end of the footbridge.

Information in regard to this station is contained in the following water-supply papers of the United States Geological Survey:

Description: 100, p 207; 134, p 204.

Discharge: 100, p 207; 134, p 204.

Discharge, monthly: 134, p 207.

Gage heights: 100, p 208; 134, pp 205-206.

- Rating table: 134, p 206. 
Discharge measurements of. Rock Creek near Round Valley, Cal., in 1905.

\begin{tabular}{|c|c|c|c|c|c|c|}
\hline Date: & Hydrographer. & Width. & $\begin{array}{l}\text { Area of } \\
\text { section. }\end{array}$ & $\underset{\text { velocity. }}{\text { Mean }}$ & $\begin{array}{c}\text { Gage } \\
\text { height. }\end{array}$ & $\begin{array}{c}\text { Dis- } \\
\text { charge. }\end{array}$ \\
\hline January $16 \ldots$ & R. J. Taylor. & $\begin{array}{c}\text { Feet. } \\
13\end{array}$ & $\begin{array}{c}S q . f t . \\
16\end{array}$ & $\begin{array}{r}\text { Ft. per sec. } \\
1.76\end{array}$ & $\begin{array}{l}\text { Feet. } \\
\quad 1.52\end{array}$ & $\begin{array}{r}\text { Sec.-ft. } \\
28\end{array}$ \\
\hline February 9. & .....do. & 13 & 12.8 & 1.56 & 1.30 & 20 \\
\hline April $4 . . . . .$. & J. S. Evans... & 13 & 12.8 & 1.55 & 1.40 & 19.9 \\
\hline April $25 . .$. & .....do. & 12 & 11.6 & 1.60 & 1.15 & 18.6 \\
\hline May $24 \ldots . .$. & .....do... & 14 & 19.3 & 2.02 & 1.80 & 39 \\
\hline June $27 . .$. & ......do. . & 14.5 & 23 & 2.22 & 2.00 & 51 \\
\hline July $25 . . .$. & ......do.. & 13 & 12.6 & 1.90 & 1.30 & 24 \\
\hline August $15 \ldots .$. & .....do.. & 12.5 & 11.6 & 1.69 & 1.20 & 19.6 \\
\hline September $8 .$. & ....do.. & 12 & 11.2 & 1.57 & 1.18 & 18.6 \\
\hline September $27 .$. & .....do.... & 12 & 11.6 & 1.57 & 1.20 & 18.2 \\
\hline November $2 \ldots$ & F. R. S. Buttemer. & 13.5 & 14 & 1.50 & 1.35 & 21 \\
\hline November 24 .. & .....do... & 13.5 & 15.3 & 1.50 & 1.46 & 23 \\
\hline December $10 \ldots$ & .....do.. & 13.5 & 16 & 1.69 & 1.60 & 27 \\
\hline
\end{tabular}

Daily gage height, in feet, of Rock Creek near Round Valley, Cal., for 1905.

\begin{tabular}{|c|c|c|c|c|c|c|c|c|c|c|c|c|}
\hline Day. & Jan. & Feb. & Mar. & Apr. & May. & June. & July. & Aug. & Sept. & Oct. & Nov. & Dec. \\
\hline $1 .$. & 1.7 & 2.1 . & 1.4 & 1.4 & 1.3 & 1.7 & 1.8 & 1.35 & 1.2 & 1.2 & 1.3 & 1.6 \\
\hline 2. & 1.6 & 1.6 & 1.4 & 1.4 & 1.4 & 1.65 & 1.8 & 1.35 & 1.2 & 1.2 & 1.3 & 1.6 \\
\hline 3.. & 1.6 & 1.6 & 1.4 & 1.4 & 1.35 & 1.65 & 1.75 & 1.35 & 1.2 & 1.2 & 1.3 & 1.65 \\
\hline $4 .$. & 1.5 & 1.5 & 1.4 & 1.4 & 1.3 & 1.6 & 1.75 & 1.3 & 1.2 & 1.25 & 1.3 & 1.65 \\
\hline 5. & 1.5 & 1.5 & 1.35 & 1.4 & 1.3 & 1.6 & 1.8 & 1.3 & 1.2 & 1.25 & 1.3 & 1.65 \\
\hline $6 .$. & 1.5 & 1.5 & 1.3 & 1.4 & 1.2 & 1.6 & 1.8 & 1.25 & 1.2 & 1.25 & 1.3 & 1.65 \\
\hline 7.. & 1.5 & 1.4 & 1.2 & 1.4 & 1.2 & 1.5 & 1.9 & 1.3 & 1.2 & 1.25 & 1.3 & 1.65 \\
\hline 8.. & 1.5 & 1.35 & 1.2 & $1.4^{\circ}$ & 1.2 & 1.6 & 2.0 & 1.3 & 1.2 & 1.25 & 1.25 & 1.65 \\
\hline 9. & 1.4 & 1.3 & 1.1 & 1.3 & 1.25 & 1.7 & 2.0 & 1.4 & 1.2 & 1.25 & 1.25 & 1.65 \\
\hline 10. & 1.3 & 1.3 & 1.1 & 1.3 & 1.25 & 1.7 & 2.0 & 1.4 & 1.2 & 1.25 & 1.25 & 1.6 \\
\hline $11 .$. & 1.3 & 1.3 & 1.2 & 1.25 & 1.25 & 1.8 & 2.0 & 1.3 & 1.2 & 1.2 & 1.2 & 1.6 \\
\hline 12. & 1.8 & 1.4 & 1.2 & 1.25 & 1.3 & 2.1 & 2.0 & 1.25 & 1.2 & 1.2 & 1.2 & 1.6 \\
\hline 13. & 1.3 & 1.4 & 1.5 & 1.25 & 1.3 & 2.1 & 2.0 & 1.25 & 1.2 & 1.2 & 1.25 & 1.6 \\
\hline $14 .$. & 1.3 & 1.4 & 1.35 & 1.2 & 1.3 & 2.15 & 2.0 & 1.2 & 1.15 & 1.2 & 1.3 & 1.6 \\
\hline 15. & 1.3 & 1.4 & 1.35 & 1.2 & 1.3 & 2.3 & 2.0 & 1.2 & 1.1 & 1.25 & 1.3 & 1.6 \\
\hline 16. & 1.35 & 1.45 & 1.4 & 1.2 & 1.3 & 2.3 & 1.9 & 1.15 & 1.1 & 1.25 & 1.3 & 1.6 \\
\hline 17. & 1.4 & 1.5 & 1.45 & 1.15 & 1.7 & 2.35 & 1.8 & 1.15 & 1.15 & 1.3 & 1.3 & 1.6 \\
\hline 18. & 1.4 & 1.4 & 1.45 & 1.1 & 1.9 & 2.4 & 1.7 & 1.15 & 1.15 & 1.3 & 1.3 & 1.6 \\
\hline 19. & 1.45 & 1.4 & 1.4 & 1.1 . & 1.9 & 2.5 & 1.6 & 1.15 & 1.2 & 1.3 & 1.3 & 1.6 \\
\hline 20. & 1.45 & 1.4 & 1.4 & 1.0 & 1.9 & 2.45 & 1.6 & 1.15 & 1.2 & 1.3 & 1.3 & 1.6 \\
\hline $21 .$. & 1.7 & 1.4 & 1.35 & 1.0 & 1.8 & 2.4 & 1.6 & 1.15 & 1.25 & 1.3 & 1.3 & 1.55 \\
\hline 22. & 1.7 & 1.4 & 1.35 & 1.0 & 1.8 & 2.3 & 1.55 & 1.15 & 1.3 & 1.3 & 1.35 & 1.55 \\
\hline $23 \ldots$ & 1.6 & 1.4 & 1.3 & 1.0 & 1.75 & 2.3 & 1.55 & 1.15 & 1.3 & 1.3 & 1.4 & 1.55 \\
\hline $24 .$. & 1.6 & 1.45 & 1.35 & 1.1 & 1.75 & 2.2 & 1.5 & 1.1 & 1.3 & 1.3 & 1.45 & 1.5 \\
\hline $25 .$. & 1.5 & 1.45 & 1.4 & 1.15 & 1.7 & 2.2 & 1.5 & 1.1 & 1.3 & 1.3 & 1.5 & 1.5 \\
\hline 26.. & 1.4 & 1.45 & 1,4 & 1.15 & 1.7 & 2.1 & 1.4 & 1.1 & 1.25 & 1.3 & 1.5 & 1.55 \\
\hline & 1.4 & 1.4 & 1.4 & 1.15 & 1.75 & 2.0 & 1.4 & 1.1 & 1.2 & 1.25 & 1.55 & 1.6 \\
\hline $28 .$. & 1.4 & 1.4 & 1.4 & 1.2 & 1.9 & 1.9 & 1.3 & 1.1 & 1.2 & 1.25 & 1.6 & 1.6 \\
\hline & 1.4 & & 1.4 & 1.2 & 1.8 & 1.85 & 1.25 & 1.1 & 1.2 & 1.25 & 1.6 & 1.6 \\
\hline $30 \ldots$ & 1.3 & & 1.4 & 1.25 & 1.8 & 1.8 & 1.25 & 1.15 & 1.2 & 1.25 & 1.55 & 1.6 \\
\hline 31... & 1.3 & & 1.4 & 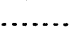 & 1.75 & ...... & 1.4 & 1.15 & $\ldots . .$. & 1.25 & & 1.55 \\
\hline
\end{tabular}


Daily discharge, in second-feet, of Rock Creek near Round Valley, Cal., for 1905.

\begin{tabular}{|c|c|c|c|c|c|c|c|c|c|c|c|c|}
\hline ay. & Jan. & Feb. & Mar. & Apr. & May. & June. & July. & Aug. & Sept. & Oct. & Nov. & Dec. \\
\hline 1. & 34 & 56 . & 23 & 23 & 20 & 34 & 42 & 24 & 19 & 19 & 20 & 27 \\
\hline 2. & 30 & 30 & 23 & 23 & 23 & 32 & 42 & 24 & 19 & 19 & 20 & 27 \\
\hline 3.. & 30 & 30 & 23 & 23 & 21 & 32 & 40 & 24 & 19 & 19 & 20 & 28 \\
\hline 4.: & 26 & 26 & 23 & 23 & 20 & 30 & 40 & 23 & 19 & 19 & 20 & 28 \\
\hline 5. & 26 & 26 & 21 & 23 & 20 & 30 & 42 & 23 & 19 & 19 & 20 & 28 \\
\hline 6. & 26 & 26 & 20 & 23 & 18 & 30 & 42 & 21 & 19 & 19 & 20 & .28 \\
\hline $7 .$. & 26 & 23 & 18 & 23 & 18 & 26 & 46 & 23 & 19 & 19 & 20 & 28 \\
\hline 8. & 26 & 21 & 18 & 23 & 18 & 30 & 51 & 23 & 19 & 19 & 19 & 28 \\
\hline 9. & 23 & 20 & 16 & 20 & 19 & 34 & 51 & 26 & 19 & 19 & 19 & 28 \\
\hline 10. & 20 & 20 & 16 & 20 & 19 & 34 & 51 & 26 & 19 & 19 & 19 & 27 \\
\hline 11. & 20 & 20 & 18 & 19 & 19 & 39 & 51 & 23 & 19 & 19 & 19 & 27 \\
\hline 12. & 20 & 23 & 18 & 19 & 20 & 56 & 51 & 21 & 19 & 19 & 19 & 27 \\
\hline 3.. & 20 & 23 & 26 & 19 & 20 & 56 & 51 & 21 & 19 & 19 & 19 & 27 \\
\hline $14 .$. & 20 & 23 & 21 & 18 & 20 & 59 & 51 & 20 & 18 & 19 & 20 & 27 \\
\hline 15. & 20 & 23 & 21 & 18 & 20 & 68 & 51 & 20 & 18 & 19 & 20 & 27 \\
\hline 16.. & 21 & 24 & 23 & 18 & 20 & 68 & 46 & 18 & 18 & 19 & 20 & 27 \\
\hline $17 .$. & 23 & 26 & 24 & 17. & 34 & 71 & 42 & 18 & 18 & 20 & 20 & 27 \\
\hline 18. & 23 & 23 & 24 & 16 & 44 & 74 & 38 & 18 & 18 & 20 & 20 & 27 \\
\hline 19. & 24 & 23 & 23 & 16 & 44 & 81 & 33 & 18 & 19 & 20 & 20 & 27 \\
\hline 20. & 24 & 23 & 23 & 14 & 44 & 78 & 33 & 18 & 19 & 20 & 20 & 27 \\
\hline $21 \ldots$ & 34 & 23 . & 21 & 14 & 39 & 74 & 33 & 18 & 19 & 20. & 20 & 26 \\
\hline $22 .$. & 34 & 23 & 21 & 14 & 39 & 68 & 31 & 18 & 20 & 20 & 21 & 26 \\
\hline $23 \ldots$ & 30 & 23 . & 20 & 14 & 36 & 68 & 31 & 18 & 20 & 20 & 22 & 26 \\
\hline $24 .:$ & 30 & 24 & 21 & 16 & 36 & 62 & 30 & 17 & 20 & 20 & 23 & 24 \\
\hline $25 \ldots$ & 26 & 24 & 23 & 17 & 34 & 62 & 30 & 17 & 20 & 20 & 24 & 24 \\
\hline $26 \ldots \ldots \ldots$ & 23 & 24 & 23 & 17 & 34 & 56 & 26 & 17 & 19 & 20 & 24 & 26 \\
\hline $27 \ldots .$. & 23 & 23 & 23 & 17 & 36 & 50 & 26 & 17 & 19 & 19 & 26 & 27 \\
\hline $28 \ldots \ldots \ldots$ & 23 & 23 & 23 & 18 & 44 & $a^{46}$ & 23 & 17 & 19 & 19 & 27 & $\cdot 27$ \\
\hline $29 .$. & 23 & & 23 & 18 & 39 & 44 & 21 & 17 & 19 & 19 & 27 & 27 \\
\hline $30 \ldots$ & 20 & & 23 & 19 & 39 & 42 & 21 & 18 & 19 & 19 & 26 & 27 \\
\hline & 20 & & 23 & & 36 & & 26 & 18 & & 19 & & 26 \\
\hline
\end{tabular}

NoTE.-Owing to shifting conditions, the daily discharge has been computed from several curves, each covering a short period of time.

Estimated monthly discharge of Rock Creek near Round Valley, Cal., for 1905.

\begin{tabular}{|c|c|c|c|c|}
\hline \multirow{2}{*}{ Month. } & \multicolumn{3}{|c|}{ Discharge in second-feet. } & \multirow{2}{*}{$\begin{array}{l}\text { Total in } \\
\text { acre-feet. }\end{array}$} \\
\hline & Maximum. & Mịimum. & Mean. & \\
\hline January... & 34 & 20 & 24.8 & 1,525 \\
\hline 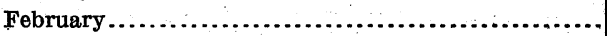 & 56 & 20 & 24.9 & 1,383 \\
\hline March........ & 26 & 16 & 21.5 & 1,322 \\
\hline April.................. & 23 & . 14 & 18.7 & 1,113 \\
\hline May............... & 44 & 18 & 28.8 & 1,771 \\
\hline June................ & 81 & 26 & 51.1 & 3,041 \\
\hline July............... & 51 & 21 & 38.5 & 2,367 \\
\hline Auguist. .............. & 26 & 17 & 20.1 & 1,236 \\
\hline September.............. & 20 & 18 & 19.0 & 1,131 \\
\hline October.... & 20 & 19 . & 19.3 & 1,187 \\
\hline November............. & 27 & 19 & 21.1 & 1,256 \\
\hline December..,,$\ldots \ldots \ldots$ & 28 & 24 & 26.9 & 1,654 \\
\hline The year. & 81 & 14 & 26.2 & 18,990 \\
\hline
\end{tabular}




\section{OWENS RIVER DRAINAGE BASIN. \\ PINE CREEK NEAR ROUND VALIEY, CAL.}

This station was established August 3, 1903, by J. C. Clausen. It is located 150 feet below the wagon bridge on the road from Bishop to Long Valley and 100 feet above the mouth of the creek.

The channel is straight for about 50 feet above and 100 feet below the station. The current has a velocity of about 1 foot per second at ordinary stages. Both banks are high and rocky and are not liable to overflow. The bed of the stream is rocky and permanent.

Discharge measurements are made by wading. The initial point for soundings is a stake on the right bank of the stream.

The gage is a vertical rod fastened in the rocks near the right bank. During 1905 the gage was read once each day by T. E. Jones. The bench mark is the one at the Rock Creek station. It is a point marked on the lava rock 15 feet east of the left end of the footbridge.

Information in regard to this station is contained in the following Water-Supply Papers of the United States Geological Survey:

Description: 100, p 208; 134, p 207:

Discharge: 100, p 209; 134, p 208.

Discharge, monthly: $134, \mathrm{p} 210$.

Gage heights: 100, p 209; 134, pp 208-209.

Rating table: 134, p 210.

Discharge measurements of Pine Creek near Round Valley, Cal., in 1905.

\begin{tabular}{|c|c|c|c|c|c|c|}
\hline Date. & Hydrographer. & Width. & $\begin{array}{l}\text { Area of } \\
\text { section. }\end{array}$ & $\begin{array}{c}\text { Mean } \\
\text { velocity. }\end{array}$ & $\begin{array}{c}\text { Gage } \\
\text { height. }\end{array}$ & $\begin{array}{c}\text { Dis- } \\
\text { charge. }\end{array}$ \\
\hline & & Feet. & $S q . f t$. & Ft.per sec. & Feet. & Sec.-ft. \\
\hline January $16 \ldots$ & R. J. Taylor... & 20 & 15.2 & 0.88 & 1.91 & 13.3 \\
\hline February $9 .$. & ....do... & 20 & 15.2 & .67 & 1.86 & 10.2 \\
\hline April 4..... & J. S. Evans. & 8 & 10.4 & .19 & 1.80 & 2.0 \\
\hline April 25.. & .....do... & 10 & 8.8 & .66 & 1.70 & 5.8 \\
\hline June $27 . . . . .$. & .....do... & 27 & 2.8 & 2.61 & 2.90 & 73 \\
\hline July $25 .$. . & .....do.. & 19 & 16.1 & 1.43 & 2.05 & 23 \\
\hline August $15 \ldots$. & .....do.. & 18 & 10.8 & .67 & 1.80 & 7.2 \\
\hline September 8. & .....do.. & 17 & 9.2 & .73 & 1.75 & 6.7 \\
\hline September 27. & .....do... & 17 & 8.1 & .77 & 1.78 & 6.2 \\
\hline November $2 \ldots$ & F. R. S. Buttemer. & 17 & 19.7 & .44 & 1.83 & 8.7 \\
\hline November 24 .. & .....do.. & 18 & 18.2 & .42 & 1.85 & 7.7 \\
\hline December $10 \ldots$ & .....do.. & 18 & 18.4 & .41 & 1.82 & 7.6 \\
\hline
\end{tabular}


Daily gage height, in feet, of Pine Creek near Round Valley, Cal., for 1905.

\begin{tabular}{|c|c|c|c|c|c|c|c|c|c|c|c|c|}
\hline Day. & Jan. & Feb. & Mar. & Apr. & May. & June. & July. & Aug. & Sept. & Oct. & Nov. & Dec. \\
\hline $1 .$. & 2.2 & 2.2 & 1.8 & 1.8 & 1.8 & 1.9 & 2.9 & 1.8 & 1.7 & 1.8 & 1.8 & \\
\hline 2. & 2.15 & 2.0 & 1.8 & 1.8 & 1.8 & 1.9 & 2.9 & 1.7 & 1.7 & 1.8 & 1.8 & \\
\hline 3.. & 2.1 & 2.0 & 1.8 & 1.8 & 1.75 & 1.9 & 2.85 & 1.7 & 1.7 & 1.8 & 1.8 & 1.9 \\
\hline $4 .$. & 1.95 & 1.9 & 1.8 & 1.8 & 1.75 & 1.9 & 2.8 & 1.7 & 1.7 & 1.85 & 1.8 & 1.9 \\
\hline 5.. & 1.95 & 1.9 & 1.8 & 1.8 & 1.75 & 1.95 & 2.8 & 1.7 & 1.7 & 1.85 & 1.85 & 1.9 \\
\hline $6 .$. & 1.95 & 1.8 & 1.8 & 1.8 & 1.7 & 1.95 & 2.8 & 1.7 & 1.7 & 1.85 & 1.85 & 1.9 \\
\hline 7.. & 1.9 & 1.8 & 1.8 & 1.75 & 1.7 & 2.05 & 2.9 & 1.7 & 1.7 & 1.85 & 1.85 & 1.9 \\
\hline $8 .$. & 1.9 & 1.85 & 1.8 & 1.75 & 1.7 & 2.1 & 2.95 & 1.7 & 1.65 & 1.85 & 1.85 & 1.9 \\
\hline 9. & 1.9 & 1.85 & 1.8 & 1.75 & 1.7 & 2.45 & 3.0 & 1.8 & 1.6 & 1.85 & 1.8 & 1.9 \\
\hline $10 .$. & 1.8 & 1.85 & 1.75 & 1.75 & 1.7 & 2.7 & 3.0 & 1.8 & 1.65 & 1.8 & 1.8 & 1.85 \\
\hline $11 .$. & 1.8 & 1.85 & 1.75 & 1.8 & .1 .75 & 2.6 & 2.9 & 1.75 & 1.65 & 1.8 & 1.8 & 1.85 \\
\hline $12 .$. & 1.8 & 1.85 & 1.8 & 1.8 & 1.8 & 2.8 & 2.85 & 1.7 & 1.65 & 1.7 & 1.8 & 1.85 \\
\hline $13 .$. & 1.75 & 1.85 & 1.8 & 1.8 & 1.8 & 3.3 & 2.8 & 1.7 & 1.65 & 1.75 & 1.8 & 1.8 \\
\hline 14.. & 1.75 & 1.85 & 1.8 & 1.8 & 1.8 & 3.3 & 2.7 & 1.7 & 1.65 & 1.75 & 1.8 & 1.8 \\
\hline $15 \therefore$ & 1.75 & 1.85 & 1.8 & 1.8 & 1.75 & 3.3 & 2.6 & 1.7 & 6 & 1.8 & 1.8 & 1.8 \\
\hline 16. & 1.8 & 1.8 & 1.85 & 1.8 & 1.75 & 3.3 & 2.5 & 1.7 & 1.6 & 1.8 & 1.8 & 1.8 \\
\hline $17 .$. & 1.8 & 1.8 & 1.85 & 1.8 & 1.75 & 3.25 & 2.4 & 1.7 & 1.65 & 1.8 & 1.8 & 1.8 \\
\hline 18. & 1.8 & 1.85 & 1.85 & 1.75 & 1.75 & 3.2 & 2.45 & 1.75 & 1.65 & 1.85 & 1.8 & 1.8 \\
\hline 19. & 1.8 & 1.85 & 1.8 & 1.75 & 1.75 & 3.2 & 2.5 & 1.75 & 1.7 & 1.85 & 1.8 & 1.8 \\
\hline 20. & 1.85 & 1.8 & 1.8 & 1.75 & 1.8 & 3.1 & 2.5 & 1.75 & 1.7 & 1.85 & 1.8 & 1.8 \\
\hline 21.. & 2.0 & 1.8 & 1.8 & 1.75 & 1.9 & 3.1 & 2.1 & 1.75 & 1.75 & 1.85 & 1.8 & 1.8 \\
\hline $22 .$. & 2.0 & 1.8 & 1.8 & 1.7 & 1.9 & 3.1 & 1.95 & 1.75 & 1.8 & 1.85 & 1.8 & 1.8 \\
\hline 23. & 1.9 & 1.8 & 1.8 & 1.7 & 1.9 & 3.0 & 1.95 & 1.75 & 1.8 & 1.85 & 1.8 & 1.8 \\
\hline 24.. & 1.9 & 1.8 & 1.8 & 1.7 & 2.0 & 2.9 & 2.0 & 1.7 & 1.8 & 1.85 & 1.85 & 1.8 \\
\hline 25. & 1.9 & 1.85 & 1.8 & 1.7 & 2.0 & 2.9 & 2.0 & 1.7 & 1.8 & 1.85 & 1.85 & 1.8 \\
\hline 26.. & 1.9 & 1.85 & 1.8 & 1.7 & 2.0 & 2.9 & 2.0 & 1.7 & 1.8 & 1.85 & 1.85 & 1.8 \\
\hline $27 .$. & 1.9 & 1.8 & 1.8 & 1.7 & 2.0 & 2.9 & 2.05 & 1.7 & 1.8 & 1.8 & 1.85 & 1.8 \\
\hline & 1.9 & 1.8 & 1.75 & 1.75 & 1.95 & 2.9 & 2.05 & 1.7 & 1.8 & 1.8 & 1.8 & 1.8 \\
\hline 29.. & 1.9 & & 1.75 & 1.75 & 1.9 & 2.9 & 2.0 & 1.7 & 1.8 & 1.8 & 1.8 & 1.8 \\
\hline & 1.85 & & 1.75 & 1.75 & 1.8 & 2.9 & 2.0 & 1.7 & 1.8 & 1.8 & 1.8 & 1.8 \\
\hline 31... & 1.85 & & 1.8 & $\cdots$ & 1.85 & & 1.9 & 1.7 & $\cdots$ & 1.8 & . & 1.8 \\
\hline
\end{tabular}

Station rating table for Pine Creek near Round Valley, Cal., from August 4, 1903, to December $31,1905$.

\begin{tabular}{|c|r||r|r|r|r|r|r|}
\hline $\begin{array}{c}\text { Gage } \\
\text { height. }\end{array}$ & Discharge. & $\begin{array}{c}\text { Gage } \\
\text { height. }\end{array}$ & Discharge. & $\begin{array}{c}\text { Gage } \\
\text { height. }\end{array}$ & Discharge. & $\begin{array}{c}\text { Gage } \\
\text { height. }\end{array}$ & Discharge. \\
\cline { 1 - 6 } Feet. & Second-feet. & Feet. & Second-feet. & Feet. & Second-feet. & Feet. & Second-feet. \\
1.60 & 1 & 2.10 & 23 & 2.60 & 52 & 3.10 & 95 \\
1.70 & -4 & 2.20 & 28 & 2.70 & 59 & 3.20 & 106 \\
1.80 & 8 & 2.30 & 33 & 2.80 & 67 & 3.30 & 119 \\
1.90 & 13 & 2.40 & 39 & 2.90 & 75 & & \\
2.00 & 18 & 2.50 & 45 & 3.00 & 85 & & \\
& & & & & & \\
\hline
\end{tabular}

Note.-The above table is based on 20 discharge measurements made during 1903-1905. It is fairly well defined between gage heights 1.7 feet and 3 feet. The table has been extended beyond these limits, being based on one measurement at 3.7 feet. 
Estimated monthly discharge of Pine Creek near Round Valley, Cal., for 1905.

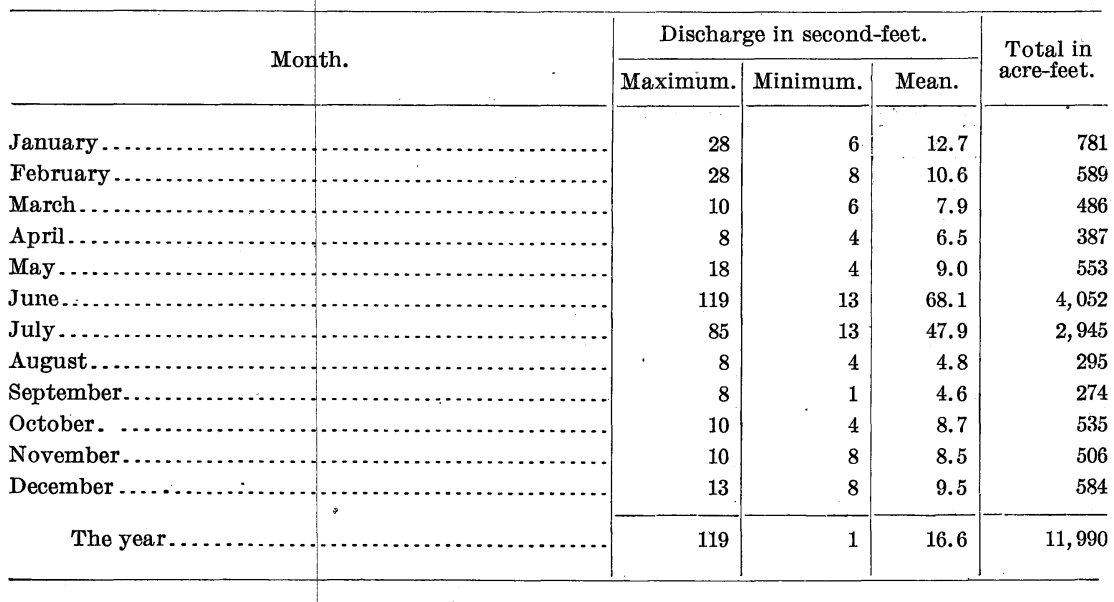

OWENS RIVER CANAL NEAR BISHOP, CAL.

This station was established August 5, 1903, by J. C. Clausen and R. S. Hawley. It is located at the footbridge near the quarter-section line which divides the north half of sec. 27 , T 6 S.., R. 32 E., of the Mount Diablo meridian.

The channel is straight for 300 feet above and 100 feet below the station. The current is sluggish. The right bank is high and rocky and will not overflow. The left bank is low and will overflow. The bed of the stream is composed of gravel and is permanent.

Discharge measurements are made from the footbridge. The initial point for soundings is on right bank of the canal.

The gage is a vertical rod nailed to the bridge. No regular observer could be obtained, but the ditch tender reads the rod when passing the station.

Information in regard to this station is contained in the following Water-Supply Paper of the United States Geological Survey:

Description: 100, pp 209-210; 134, p 211.

Discharge: 100, p 210; 134, p 211.

Discharge, monthly: $134, \mathrm{p} 213$.

Gage heights: 100, p 210; 134, p 212.

Rating table: 134, p 213.

Discharge measurements of Owens River Canal near Bishop, Cal., in 1905.

\begin{tabular}{|c|c|c|c|c|c|c|}
\hline Date. & Hydrographer. & Width. & $\begin{array}{l}\text { Area of } \\
\text { section. }\end{array}$ & $\begin{array}{c}\text { Mean } \\
\text { velocity. }\end{array}$ & $\begin{array}{c}\text { Gage } \\
\text { height. }\end{array}$ & $\begin{array}{c}\text { Dis- } \\
\text { charge. }\end{array}$ \\
\hline January $10 \ldots$ & R. J. Taylor & $\begin{array}{r}\text { Feet. } \\
11.5\end{array}$ & $\begin{array}{r}S q . f t . \\
4.9\end{array}$ & $\begin{array}{r}\text { Ft.per sec. } \\
0.92\end{array}$ & $\begin{array}{r}\text { Feet. } \\
1.55\end{array}$ & $\begin{array}{r}\text { Sec.-ft. } \\
4.5\end{array}$ \\
\hline February 4. & .....do. . & 11 & 4.1 & .80 & 1.49 & 3.3 \\
\hline April $6 . . .$. & J. S. Evans. & 14.5 & 16.4 & 1.71 & 2.40 & 28 \\
\hline April 24. & .....do. & 15.0 & 22 & 1.91 & 2.77 & 42 \\
\hline May $12 \ldots .$. & .....do.. & 14.5 & 24 & 1.79 & 2.78 & 43 \\
\hline June $1 \ldots$ & .....do.. & 14.5 & 21 & 1.76 & 2.62 & 38 \\
\hline July $7 . . .$. & .....do. & 14 & 16.8 & 1.90 & 2.30 & 32 \\
\hline August 4. & .....do. . & 14.5 & 23 & 1.87 & 2.75 & 43 \\
\hline September 11. & .....do . ... & 15 & 19.6 & 1.73 & 2.60 & 34 \\
\hline October 24. & F. R. S. Buttemer & 14.5 & 19.3 & 1.45 & 2.38 & 28 \\
\hline November 22 . & .....do... & 12 & 5.6 & .95 & 1.50 & 5.3 \\
\hline December 13 .. & .....do. & 13 & 6.6 & 1.17 & 1.63 & 7.7 \\
\hline
\end{tabular}

IRR $177-06-5$ 
Daily gage height, in feet, of Owens River canal near Bishop, Cal., for 1905.

\begin{tabular}{|c|c|c|c|c|c|c|c|}
\hline Day. & Apr. & May. & June. & July. & Aug. & Sept. & Oct. \\
\hline $1 \ldots \ldots$ & & 0.0 & 2.7 & 2.55 & 2.8 & 2.55 & 2.8 \\
\hline $2 \ldots$ & & .0 & 2.7 & 2.55 & 2.75 & 2.55 & 2.8 \\
\hline 3. & & .0 & 2.7 & 2.55 & 2.8 & 2.55 & 2.8 \\
\hline $4 .$. & & 2.4 & ...... & 2.55 & 2.75 & 2.55 & 2.8 \\
\hline 5 & & 2.7 & 2.65 & 2.55 & 2.8 & 2.55 & 2.8 \\
\hline 6. & & 2.7 & 2.65 & 2.55 & 2.85 & 2.75 & 2.75 \\
\hline 7. & & 2.7 & 2.6 & 2.55 & 2.9 & 2.8 & 2.75 \\
\hline $8 \ldots \ldots$ & & 2.65 & 2.6 & 2.55 & 2.85 & 2.8 & 2.8 \\
\hline $9, \ldots \ldots \ldots \ldots$ & & 2.7 & ......... & 2.55 & 2.85 & 2.8 & 2.8 \\
\hline $10 \ldots \ldots \ldots \ldots$ & & 2.5 & $\ldots \ldots \ldots$ & 2.55 & 2.9 & 2.8 & 2.8 \\
\hline $11 \ldots \ldots \ldots$ & & 2.7 & 2.65 & 2.55 & 2.9 & 2.75 & 2.7 \\
\hline 12. & & 2.8 & 2.7 & 2.55 & 2.5 & 2.8 & 2.7 \\
\hline $13 \ldots \ldots \ldots \ldots \ldots$ & & 2.8 & 2.65 & 2.55 & 2.5 & 2.8 & 2.7 \\
\hline $14:$. & & $\cdots$ & 2.7 & 2.55 & 2.5 & 2.8 & 2.75 \\
\hline $15 \ldots \ldots \ldots \ldots$ & & 2.75 & 2.55 & 2.55 & 2.5 & 2.75 & $\cdots$ \\
\hline 16. . & & 2.8 & 2.55 & 2.55 & 2.5 & 2.8 & \\
\hline 17. & & 2.75 & 2.55 & 2.5 & .0 & 2.75 & \\
\hline 18. & & 2.75 & 2.55 & 2.65 & .0 & 2.6 & \\
\hline $19 \ldots \ldots \ldots \ldots \ldots$ & & 2.7 & 2.5 & 2.7 & .0 & 2.6 & \\
\hline $20 \therefore \ldots \ldots \ldots \ldots$ & & 2.75 & 2.5 & 2.7 & 2.6 & 2.6 & \\
\hline 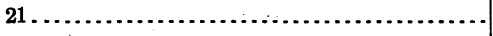 & & 2.75 & 2.5 & 2.65 & 2.6 & 2.6 & $\cdots$ \\
\hline $22 \ldots \ldots \ldots \ldots \ldots$ & & 2.7 & 2.55 & 2.65 & .0 & 2.6 & \\
\hline $23 . \ldots \ldots .$. & & 2.65 & 2.55 & 2.7 & .0 & 2.6 & ... \\
\hline $24 \ldots \ldots \ldots \ldots \ldots$ & & 2.65 & 2.55 & 2.7 & 2.6 & 2.6 & (........ \\
\hline $25 \ldots \ldots$ & & 2.7 & 2.55 & 2.7 & 2.6 & 2.65 & $\cdots$ \\
\hline $26 . \ldots \ldots \ldots \ldots \ldots$ & 2.8 & 2.7 & 2.55 & 2.7 & 2.8 & $=2.65$ & \\
\hline $27 \ldots \ldots \ldots$ & 2.8 & 2.75 & 2.55 & 2.7 & 2.85 & 2.7 & $\ldots$ \\
\hline $28 . \therefore \ldots \ldots$ & & …. & 2.55 & 2.75 & 2.85 & 2.65 & ... \\
\hline 29. & & $\ldots$ & 2.55 & 2.7 & 2.9 & 2.5 & $\cdots$ \\
\hline $30 . \ldots \ldots . .$. & 2.65 & 2.7 & 2.55 & 2.8 & 2.95 & 2.8 & $\ldots .$. \\
\hline $31 .$. & & 2.7 & . & 2.75 & 2.55 & & \\
\hline
\end{tabular}

BISHOP CREEK OANAL NEAR BISHOP, CAL.

This station was established August 5, 1903, by J.C.Clausen, assisted by R. S. Hawley. It is located at the footbridge below the waste gate near the house of A. Fitzgerald, $3 \frac{1}{2}$ miles northwest of Bishop, Cal.

The channel is straight for 50 feet above and 100 feet below this station. The current is swift. The right bank is high and the left bank is low. Neither bank is liable to overflow. The bed of the stream is composed of sand and gravel and is fairly permanent.

Discharge measurements are made from the footbridge. The initial point for soundings is on right bank.

The gage is a vertical rod fastened to the bridge anchor.

Information in regard to this station is contained in the following Water-Supply Papers of the United States Geological Survey:

Description: 100; pp 210-211; 134, p 214.

Discharge: 100, p 211; 134, p 214.

Discharge, monthly: 134, p 216.

Gage heights: 100, p 211; 134, p 215.

Rating table: 134, p 216. 
Discharge measurements of Bishop Creek canal near Bishop, Cal., in 1905.

\begin{tabular}{|c|c|c|c|c|c|c|}
\hline Date. & Hydrographer. & Width. & $\begin{array}{l}\text { Area of } \\
\text { section. }\end{array}$ & $\underset{\text { velocity. }}{\text { Mean }}$ & $\begin{array}{c}\text { Gage } \\
\text { height. }\end{array}$ & $\begin{array}{c}\text { Dis- } \\
\text { charge. }\end{array}$ \\
\hline March 17 & R. J. Taylor. . . . . . . . . . . . . . . . & $\begin{array}{r}\text { Feet. } \\
15\end{array}$ & $\begin{array}{r}S q \cdot f t . \\
10.1\end{array}$ & Ft. per sec. & $\begin{array}{r}\text { Feet. } \\
1.40\end{array}$ & $\begin{array}{r}\text { Sec.-ft. } \\
8.5\end{array}$ \\
\hline April $6 . . . . .$. & J. S. Evans... & 16 & 30 & 2.07 & 2.80 & 62 \\
\hline April $24 \ldots$ & .....do... & 16 & 47 & 2.23 & 3.81 & 105 \\
\hline May $12 \ldots$ & ....do... & 16 & 49 & 2.20 & 3.95 & 108 \\
\hline June $1 . . .$. & .....do. . & 16 & 50 & 2.08 & 4.00 & 105 \\
\hline July 7 .. & .....do. . & 16 & 44 & 1.75 & 3.70 & 77 \\
\hline August $4 . . .$. & .....do. . & 16 & 51 & 2.39 & 4.10 & 122 \\
\hline September 11 . & ......do .... & 16 & 44 & 2.20 & 3.85 & 97 \\
\hline October $24 . . .$. & F. R. S. Buttemer.. & 16 & 26 & 1.12 & 1.92 & 29 \\
\hline November 22 & .....do.. & 13 & 4.6 & .39 & 1.05 & 1.8 \\
\hline December $13 .$. & .....do.. & 13 & 5.1 & .31 & 1.06 & 1.6 \\
\hline December $21 .$. & ....do. & 14 & 15.6 & .79 & 1.21 & 12.4 \\
\hline
\end{tabular}

Daily gage height, in feet, of Bishop Creek canal near Bishop, Cal., for 1905.

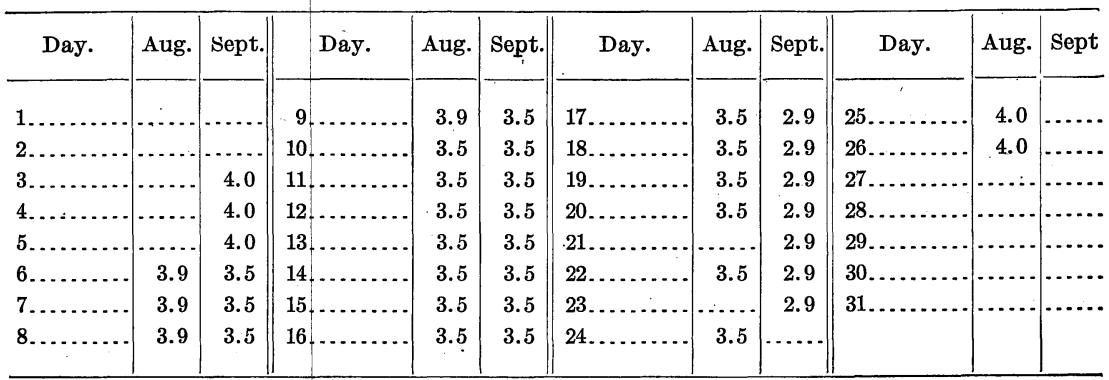

FARMERS' GANAL NEAR BISHOP, CAL.

This station was established August 6, 1903, by J. C. Clausen and R. S. Hawley. It is located at the footbridge near the house of Robert Love and 3 miles north of Bishop, Cal.

The channel is straight for 300 feet above and 50 feet below the station. The current is sluggish. Both banks are low and are liable to overflow. The bed of the stream is sandy and shifting.

Discharge measurements are made from the footbridge, to which the gage is attached. The initial point for soundings is on the footbridge, near the right bank.

The gage is a vertical rod fastened to the bridge pier.

Information in regard to this station is contained in the following Water-Supply Papers of the United States Geological Survey:

Description: 100, pp 211-212; 134, p 216.

Discharge: 100, p 212; 134, p 217.

Discharge, monthly: 134, p 219.

Gage heights: 100, p 212; 134, pp 217-218.

Rating table: 134, p 219. 
Discharge measurements of Farmers' canal near Bishop, Cal., in 1905.

\begin{tabular}{|c|c|c|c|c|c|c|}
\hline Date. & Hydrographer. & Width. & $\begin{array}{l}\text { Area of } \\
\text { section. }\end{array}$ & $\begin{array}{c}\text { Mean } \\
\text { velocity. }\end{array}$ & $\begin{array}{c}\text { Gage } \\
\text { height. }\end{array}$ & $\begin{array}{c}\text { Dis- } \\
\text { charge. }\end{array}$ \\
\hline January $10 \ldots$ & R. J. Taylor............................. & $\begin{array}{c}\text { Feet. } \\
13\end{array}$ & $\begin{array}{r}S q . f t . \\
9.0\end{array}$ & $\begin{array}{r}\text { Ft. per sec. } \\
0.69\end{array}$ & $\begin{array}{r}\text { Feet. } \\
2.20\end{array}$ & $\begin{array}{r}\text { Sec.-ft. } \\
6.2\end{array}$ \\
\hline February 4 & .....do............... & 13 & 9.4 & .74 & 2.16 & 7.0 \\
\hline March $7 .$. & .....do......... & 10 & 16.8 & 1.79 & 2.90 & 30 \\
\hline April 6... & J. S. Evans....... & 10.5 & 12.8 & 1.47 & 2.55 & 18.8 \\
\hline April $24 \ldots$ & .....do. . & 10 & 8.4 & .64 & 2.30 & 5.4 \\
\hline May $12 . . . . .$. & .....do... & 10.5 & 12.8 & 1.16 & 2.60 & 14.9 \\
\hline June 1... & .....do... & 10.5 & 14.6 & 1.37 & 2.60 & 20 \\
\hline July $7 \ldots .$. & .....do... & 9.5 & 15.4 & 1.56 & 2.75 & 24 \\
\hline August 4.. & ......do... & 10 & 9.0 & .53 & 2.00 & 4.8 \\
\hline September 11.. & .....do... & 10.5 & 15.7 & 1.04 & 2.55 & 16.4 \\
\hline November 22. & 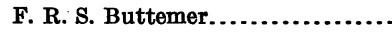 & $10 . \dot{5}$ & 10.8 & .70 & 2.35 & 7.6 \\
\hline December 13... & .....do......... & 10.5 & 10.8 & .59 & 2.36 & 6.4 \\
\hline
\end{tabular}

Daily gage height, in feet, of Farmers' canal near Bishop, Cal., for 1905.

\begin{tabular}{|c|c|c|c|c|c|c|c|c|}
\hline Day. & Jan. & Feb. & Mar. & Apr. & May. & June. & July. & Aug. \\
\hline 1.... & & & & & & & & 2.5 \\
\hline $2 \ldots \ldots \ldots \ldots \ldots$ & $\cdots \cdot$ & & $\ldots \ldots$ & . $\ldots \ldots . .$. & 2.8 & 2.7 & & ...... \\
\hline $3, \ldots \ldots \ldots \ldots$ & 2.2 & 2.15 & 3.0 & $\cdots$ & 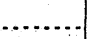 & & 2.6 & ...... \\
\hline $4 \ldots \ldots \ldots \ldots \ldots$ & .......... & 2.16 & $\therefore \therefore$. & 2.65 & ........ & $\cdots$ & 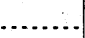 & 2.5 \\
\hline $5 \ldots \ldots \ldots \ldots$ & .......... & .......... & 2.9 & $\ldots . . .$. & 2.8 & ........ & $\therefore . . .$. & $\ldots$. \\
\hline $6 \ldots$ & 2.2 & & ..... & 2.55 & $\ldots$ & 2.7 & ........ & ..... \\
\hline $7 \ldots \ldots \ldots \ldots$ & $\ldots$ & & 2.9 & ......: & & & 2.75 & $\ldots .$. \\
\hline $8 \ldots$ & & & & 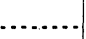 & $\cdots \cdot$ & & $\cdots$ & 2.6 \\
\hline $9 \ldots \ldots \ldots \ldots$. & 2.2 & & 2.9 & & 2.6 & 2.75 & & $\ldots \ldots$ \\
\hline $10 \ldots \ldots \ldots \ldots$ & 2.2 & & $\cdots$ & …....... & …..... & & & ...... \\
\hline $11 \ldots \ldots \ldots \ldots$ & $\cdots$ & 2.15 & & 1.8 & (2) & & & 2.6 \\
\hline $12 \ldots .$. & & & & 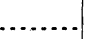 & 2.6 & $\cdots \cdot \cdot$ & 2.7 & $\cdots$ \\
\hline $13 . .$. & & & & 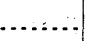 & 2.6 & 2.9 & :....... & \\
\hline $14 \ldots \ldots \ldots \ldots$ & 2.2 & 2.15 & 3.0 & 1.7 & & & 2.65 & $\ldots$. \\
\hline $15 \ldots \ldots \ldots \ldots$ & & & & & & & & 2.65 \\
\hline $16 \ldots+\ldots \ldots \ldots$ & ...... & & $\ldots .$. & & 2.65 & 2.95 & $\cdots$ & ..... \\
\hline 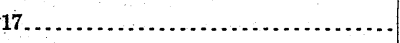 & 2.15 & & 2.9 & & & & & ........ \\
\hline $18 \ldots \ldots \ldots \ldots$ & 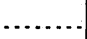 & & & 1.1 & $\ldots \ldots .$. & & 2.6 & 2.55 \\
\hline 19 & 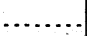 & & & 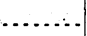 & 2.95 & $\cdots . .$. & ......... & ......... \\
\hline $20 \ldots \ldots \ldots \ldots$ & 2.15 & & & & $\ldots . .$. & 2.8 & ........ & ......... \\
\hline $21 \ldots \ldots \ldots \ldots$ & $\ldots$ & & & - & 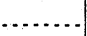 & $\cdots$ & 2.6 & 2.2 \\
\hline $22 \ldots \ldots \ldots \ldots$ & & & & 1.2 & & & & ..... \\
\hline $23 \ldots .$. & & 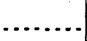 & 2.75 & $\ldots$ & 2.95 & 2.7 & ........ & $\ldots$. \\
\hline $24 \ldots \ldots \ldots \ldots \ldots$ & & & & 2.3 & & & & $\ldots$. \\
\hline $25 \ldots \ldots \ldots \ldots \ldots$, & 2.15 & & & 2.3 & $\ldots \ldots \ldots$ & $\ldots$ & 2.6 & 2.65 \\
\hline $26 \ldots \ldots \ldots \ldots \ldots$ & & & & $-\ldots$ & 2.9 & & & 2.65 \\
\hline $27 \ldots \ldots \ldots$ & $\cdots \cdots$ & & & & & 2.7 & & ..... \\
\hline $28 \ldots \ldots \ldots \ldots \ldots$ & 2.15 & $\ldots .$. & 2.75 & 2.7 & $\cdots .$. & $\cdots$ & 2.55 & ......... \\
\hline $29 . .$. & & & & & & $\therefore$ & & $\ldots \ldots$ \\
\hline $30 \ldots \ldots \ldots \ldots \ldots$ & $\ldots \ldots$ & $\ldots$ & $\ldots \ldots$ & $\ldots . .$. & 2.9 & 2.6 & $\ldots$. & (........ \\
\hline 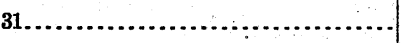 & 2.15 & & 2.75 & ...... & & $\ldots .$. & & \\
\hline
\end{tabular}


MCNALIY CANAL NEAR BISHOP, CAL.

This station was established July 31, 1903, by J: C. Clausen and R. S. Hawley. It is located at the head of the canal, $3 \frac{3}{4}$ miles north of Bishop, Cal.

The channel is straight for 50 feet below the gage. The current is swift. Both banks are high and will not overflow. The bed of the stream is rocky and permanent.

Discharge measurements are made from a footbridge. The initial point for soundings is marked on the footbridge, near the right bank.

The gage is a vertical board fastened to the headworks of the canal. No gage reader could be obtained for this station, but since the gage was installed there has been little variation in the amount of water flowing in the canal.

Information in regard to this station is contained in the following Water-Supply Papers of the United States Geological Survey:

Description: 100, p 213; 134, pp 219-220.

Discharge: 100, p $213 ; 134$, p 220.

Discharge, monthly: 134, p 221.

Gage heights: $134, \mathrm{pp} 220-221$.

Rating table: $134, \mathrm{p} 221$.

Discharge measurements of McNally canal near Bishop, Cal., in 1905.

\begin{tabular}{|c|c|c|c|c|c|c|}
\hline Date. & Hydrographer. & Width. & $\begin{array}{l}\text { Area of } \\
\text { section. }\end{array}$ & $\begin{array}{c}\text { Mean } \\
\text { velocity. }\end{array}$ & $\begin{array}{c}\text { Gage } \\
\text { height. }\end{array}$ & $\begin{array}{c}\text { Dis- } \\
\text { charge. }\end{array}$ \\
\hline & & Feet. & $S q . f t$. & Ft.per sec. & Feet. & Sec.-ft. \\
\hline March $17 .$. & R. J. Taylor.... & 20 & 11.2 & 1.06 & 0.50 & 11.9 \\
\hline April $6 \ldots$ & J. S. Evans.... & 20 & 14.2 & 1.76 & .70 & 25 \\
\hline April $24 \ldots . .$. & .... do . . . . . . & 21 & 18.2 & 2.09 & -1.00 & 38 \\
\hline May $12 \ldots . . .$. & .....do........... & 21 & 21 & 2.05 & 1.05 & 43 \\
\hline June $1 . . . . . .$. & .....do................ & 21 & 25 & 2.23 & 1.10 & 56 \\
\hline July $7 \ldots$ & . . . do................... & 22 & 32 & 2.69 & 1.30 & 86 \\
\hline August 4. . & .....do. .... & 22 & 32 & 1.66 & 1.50 & 53 \\
\hline September $11 .$. & .....do............. & 21 & 23 & 2.17 & 1.10 & 50 \\
\hline November 22 . & F. R, S. Buttemer.... & 21 & 17.5 & 1.14 & .70 & 20 \\
\hline December $13 a .$. & 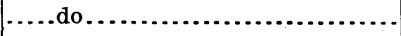 & & & ...... & .10 & 0 \\
\hline
\end{tabular}

$a$ Headgate shut down; no water in canal.

\section{GEORGE COLIINS CANAI NEAR BISHOP, CAL.}

This station was established August 17, 1903, by R. S. Hawley. It is located at the footbridge 3 miles east and one-half mile north of Bishop, Cal.

The channel is straight for 75 feet above and for 50 feet below the station. The current is sluggish. The right bank is low and the left bank is high. Neither bank is liable to overflow. The bed of the stream is composed of sand and is fairly permanent.

Discharge measurements are made from the bridge. The initial point for soundings is marked on the footbridge near the right bank of the canal.

The gage is a vertical rod fastened to the bridge near the house of Arthur Wines.

Information in regard to this station is contained in the following Water-Supply Papers of the United States Geological Survey:

Description: 100, p 213; 134, p 222.

Discharge: 100, p 214; 134, p 222.

Discharge, monthly: 134, p 225.

Gage heights: 100, p 214; 134, pp 223-224.

Rating table: 134, p 224. 
Discharge measurements of George Collins canal near Bishop, Cal., in 1905.

\begin{tabular}{|c|c|c|c|c|c|c|}
\hline Date. & Hydrographer. & Width. & $\begin{array}{l}\text { Area of } \\
\text { section. }\end{array}$ & $\begin{array}{c}\text { Mean } \\
\text { velocity. }\end{array}$ & $\begin{array}{c}\text { Gage } \\
\text { height. }\end{array}$ & $\begin{array}{c}\text { Dis- } \\
\text { charge. }\end{array}$ \\
\hline May 2.. & J. S. Evans.... & $\begin{array}{c}\text { Feet. } \\
11\end{array}$ & $\begin{array}{r}S q . f t . \\
10.8\end{array}$ & $\begin{array}{r}\text { Ft. per sec. } \\
1.12\end{array}$ & $\begin{array}{l}\text { Feet. } \\
1.10\end{array}$ & $\begin{array}{r}\text { Sec.-ft. } \\
12.1\end{array}$ \\
\hline May 25. . & .....do... & 14 & 13 & 1.13 & 1.35 & 14 \\
\hline June 28 . & ..... do........... & 11.5 & 7.3 & .71 & 1.00 & 5.2 \\
\hline July 26.: & .....do...... & 11.5 & - 9.1 & .65 & 1.00 & 5.9 \\
\hline August 9.. & .....do........... & 11.5 . & 8.0 & .50 & .60 & 4.0 \\
\hline September 5... & .....do.. & 12 & 8.4 & .57 & .60 & 4.8 \\
\hline September $14 .$. & .....do. & 11.6 & 8.0 & .65 & .50 & 5.2 \\
\hline October $3 . . . .$. & .....do... & 12 & 8.8 & 1.00 & .65 & 8.8 \\
\hline October $25 \ldots$. & F. R. S. Buttemer..... & 11 & 5.8 & .59 & .78 & 3.4 \\
\hline November $23 .$. & $\ldots$ do...................... & 11.5 & 5.2 & .65 & .82 & 3.4 \\
\hline December $11 \ldots$ & .....do................... & 10.7 & 4.7 & .53 & .80 & 2.5 \\
\hline
\end{tabular}

Daily gage height, in feet, of George Collins canal near Bishop, Cal., for 1905.

\begin{tabular}{|c|c|c|c|c|c|c|c|c|c|c|c|c|c|}
\hline Day. & May. & June. & July. & Aug. & Sept. & Oct. & Day. & May. & June. & July. & Aug. & Sept. & Oct. \\
\hline 1. & & 0.9 & $\cdots$ & 0.8 & $\cdots$ & 1.0 & 17. & 1.0 & 1.4 & 1.2 & 0.9 & & \\
\hline 2. & 0.9 & 1.0 & ....... & .8 & 0.8 & .9 & 18. & .1 .0 & 1.5 & & .9 & 1.2 & \\
\hline 3. & $\cdots$ & 1.0 & 0.8 & 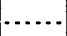 & .8 & & 19. & 1.0 & 1.4 & & & $\cdots$ & \\
\hline 4.. & .9 & $\ldots \ldots$ & 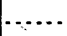 & $\ldots$ & ... & .9 & 20. & 1.0 & ...... & 1.0 & .... & 1.2 & \\
\hline 5. & $\cdots$ & 1.0 & .... & .5 & .8 & & 21. & … & 1.0 & $\cdots$ & .9 & 1.1 & \\
\hline 6. & $\ldots$ & 1.0 & .8 & & .... & .9 & $22 .$. & 1.0 & 1.0 & 1.0 & $\cdots$ & ...... & \\
\hline $7 .$. & 1.0 & 1.2 & .9 & .5 & .9 & & $23 .$. & & & & .8 & 1.1 & \\
\hline 8. & & 1.5 & $\therefore$ & $\cdots$ & $\cdots$ & & $24 \ldots$ & 1.0 & .9 & & .8 & 1.1 & \\
\hline & .8 & 1.5 & 1.4 & .5 & 1.2 & & 25. & & .9 & & & $\cdots$ & \\
\hline 10. & $\cdots$ & 1.4 & $\ldots$. & .5 & $\cdots$ & & 26. & $\cdots$ & 1.0 & .9 & $\cdots$ & 1.0 & \\
\hline 11. & .8 & .8 & 1.4 & ....... & 1.2 & & $27 .$. & .6 & $\ldots$ & & .9 & $\therefore \ldots$ & \\
\hline 12. & $\cdots \cdot$ & 1.5 & 1.4 & $\cdots$ & 1.2 & & 28. & .5 & & & .... & 1.0 & $\cdots$ \\
\hline 13. & ..... & 1.6 & ...... & .8 & & & $29 .$. & .5 & .8 & .9 & .9 & & \\
\hline 14. & .8 & 1.6 & 1.4 & .9 & & & 30. & .6 & .8 & & .9 & & \\
\hline & 1.0 & 1.4 & 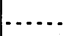 & $\cdots$ & 1.2 & & 31.. & & & & & & \\
\hline 16. & 1.0 & 1.4 & 1.2 & & & & & & & & & & \\
\hline
\end{tabular}

NoTe.-Water shut off October 6 to December 31.

\section{BISHOP CREEK NEAR BISHOP, OAL.}

This station was established August 10, 1903, by J. C. Clausen and R. S. Hawley. It is located at the wagon bridge on the Bishop road about $4 \frac{1}{1}$ miles from Bishop and about 2 miles from the point where the creek leaves the canyon. North Hillside canal, South Hillside canal, and Powers canal are taken out above the station.

The channel is straight for $\mathbf{1 0 0}$ feet above and for $\mathbf{5 0}$ feet below the station. The current is swift. Both banks are high and rocky and are not liable to overflow. The bed of the stream is rough and rocky and is permanent.

Discharge measurements are made from the wagon bridge. The initial point for soundings is marked on the bridge near the right bank of the stream.

The gage is a vertical rod fastened in the rocks and braced to the right bank just above the wagon bridge. During 1905 the gage was read once each day by A: S. Kilpatrick. The bench mark is a large flat granite bowlder on the right bank 40 feet above the bridge. 
Information in regard to this station is contained in the following Water-Supply Papers of the United States Geological Survey:

Description: 100, pp 214-215; 134, p 225 .

Discharge: 100, p 215; 134, p 226.

Discharge, monthly: 134, p 228.

Gage heights: 100, p 215; 134, pp 226-227.

Rating table: 134, p 228.

Discharge measurements of Bishop Creek near Bishop, Cal., in 1905.

\begin{tabular}{|c|c|c|c|c|c|c|}
\hline Date. & Hydrographer. & Width. & $\begin{array}{l}\text { Area of } \\
\text { section. }\end{array}$ & $\underset{\text { velocity. }}{\text { Mean }}$ & $\begin{array}{c}\text { Gage } \\
\text { height. }\end{array}$ & $\begin{array}{c}\text { Dis- } \\
\text { charge. }\end{array}$ \\
\hline & & Feet. & Sq. ft. & Ft.per sec. & Feet. & Sec.-ft. \\
\hline January $11 .$. & R. J Taylor. & 16 & 22 & 1.18 & 1.40 & 26 \\
\hline February 8 & .... do . . . . . . . & 16 & 24 & 1.25 & 1.55 & 30 \\
\hline March 4..... & .....do....... & 16 & 23 & 1.35 & 1.55 & 31 \\
\hline April 3..... & J. S. Evans....... & 16 & 20 & 2.10 & 1.65 & 42 \\
\hline April $18 \ldots$. & .....do.... & 16 & 22 & 1.50 & 1.57 & 33 \\
\hline May $5 . . . . . .$. & .....do........ & 16 & 34 & 2.76 & 2.30 & 94 \\
\hline June $21 . . . . .$. & .... do.... & 19 & 63 & 6.21 & 3.80 & 391 \\
\hline July $10 . . . . . .$. & ..... do..... & 18 & 61 & 5.54 & 3.60 & 338 \\
\hline July $27 . . .$. & .....do............. & 17 & 44 & 4.01 & 2.75 & 178 \\
\hline August $10 \ldots .$. & .....do. . & 16 & 37 & 3.35 & 2.50 & 124 \\
\hline September $7 .$. & .....do... & 16 & 28 & 2.07 & 2.00 & 58 \\
\hline September 22 . & .... do.... & 16 & 24 & 1.91 & 1.80 & 46 \\
\hline October $25 \ldots$. & F. R. S. Buttemer. & 15 & 25 & .84 & 1.35 & 21 \\
\hline November $23 .$. & ..... do... & 16 & 19.6 & 1.22 & 1.52 & 24 \\
\hline December $11 .$. & ......do... & 16 & 22 & 1.05 & 1.67 & 23 \\
\hline December $21 .$. & .....do........ & 14 & 15.6 & .79 & 1.21 & 12.4 \\
\hline
\end{tabular}


Daily gage height, in feet, of Bishop Creek near Bishop, Cal., for 1905.

\begin{tabular}{|c|c|c|c|c|c|c|c|c|c|c|c|c|}
\hline Day. & Jan. & Feb. & Mar. & Apr. & May. & June. & July. & Aug. & Sept. & Oct. & Nov. & Dec. \\
\hline $1 .$. & 1.4 & 1.5 & 1.55 & 1.8 & 2.5 & 2.9 & 3.4 & 2.5 & 2.1 & 1.9 & 1.4 & 1.5 \\
\hline $2 .$. & 1.4 & 1.5 & 1.55 & 1.8 & 2.5 & 2.9 & 3.3 & 2.3 & 2,1 & 1.9 & 1.4 & 1. 4 \\
\hline 3.. & 1.4 & 1.45 & 1.5 & 1.75 & 2.5 & 2.9 & 3.2 & 2.3 & 2.1 & 1.8 & 1.4 & 1.5 \\
\hline 4.. & 1.4 & 1.5 & 1.6 & 1. 75 & 2.3 & 2.9 & 3.3 & 2.3 & 2.0 & 1.9 & 1.4 & 1.4 \\
\hline 5. & 1.4 & 1.5 & 1.6 & 1.75 & 2.3 & 3.0 & 3. 3 & 2.2 & 2.0 & 1.8 & 1.4 & 1.4 \\
\hline 6.. & 1.4 & 1.5 & 1.6 & 1.8 & 2.1 & 3.1 & 3. 3 & 2.2 & 1.9 & 1.8 & 1.4 & 1.5 \\
\hline $7 .$. & 1.45 & 1.5 & 1.6 & 1.8 & 2.1 & 3. 3 & 3. 4 & 2.2 & 1.8 & 1.8 & 1.4 & 1.5 \\
\hline 8. & 1. 45 & 1.5 & 1.6 & 1.8 & 2. 1 & 3.4 & 3.4 & 2.3 & 1.9 & 1.8 & 1.5 & $\cdot 1.5$ \\
\hline 9.. & 1.4 & 1.5 & 1.6 & 1.75 & 2.0 & 3.3 & 3. 5 & 2.4 & 1.9 & 1.8 & 1.5 & 1.5 \\
\hline b.. & 1.4 & 1.55 & 1.6 & 1.75 & 2.0 & 3.4 & 3.6 & 2.5 & 1.9 & 1.8 & 1.4 & 1.4 \\
\hline $1 .$. & 1.4 & 1.5 & 1.6 & 1.7 & 2.0 & 3.5 & 3.7 & 2.6 & 1.8 & 1.8 & 1.4 & 1.4 \\
\hline 12. & 1.4 & 1.5 & 1.65 & 1.7 & 2.1 & 3.6 & 3. 7 & 2.5 & 1.7 & 1.7 & 1.5 & 1.5 \\
\hline 13.. & 1.4 & 1.45 & 1.65 & 1.7 & 2. 1 & 3.6 & 3.6 & 2.4 & 1.8 & 1.7 & 1.5 & 1.5 \\
\hline 14. & 1.4 & 1.45 & 1.7 & 1. 75 & 2.1 & 3.6 & 3.2 & 2.35 & 1.8 & 1.7 & 1.4 & 1.5 \\
\hline 15 .. & 1.45 & 1.45 & 1.7 & 1.75 & 2.2 & 3.7 & 3.0 & 2.3 & 1.7 & 1.7 & 1.5 & 1.5 \\
\hline 16. & 1.45 & 1.4 & 1.7 & 1.75 & 2.2 & 3.8 & 2.8 & 2.2 & 1.7 & 1.6 & 1.4 & 1.5 \\
\hline 17.. & 1. 45 & 1.4 & 1.65 & 1.75 & 2.5 & 3.8 & 2.7 & 2.2 & 1.9 & 1.6 & 1.5 & 1.4 \\
\hline 18.. & 1.5 & 1.4 & 1.65 & 1.7 & 2.5 & 3.8 & 2.6 & 2.2 & 1.8 & 1.6 & 1.5 & 1.4 \\
\hline 19.. & 1.5 & 1.4 & 1.7 & 1.7 & 2.6 & 3.8 & 2.6 & 2.2 & 1.9 & 1.6 & 1.6 & 1.5 \\
\hline .. & 1. 45 & 1.4 & 1.7 & 1.7 & 2.7 & 3.8 & 2.5 & 2.2 & 1.8 & 1.5 & 1.6 & 1.5 \\
\hline 21.. & 1.45 & 1.4 & 1.7 & 1.7 & 2.5 & 3.8 & 2.5 & 2.3 & 1.9 & 1.5 & 1.5 & 1.4 \\
\hline 22. & 1.4 & 1.4 & 1.7 & 1.7 & 2.5 & 3.8 & 2.5 & 2.2 & 1.8 & 1.5 & 1.5 & 1.5 \\
\hline 23. & 1.4 & 1.5 & 1.7 & 1.8 & 2.5 & 3.7 & 2.6 & 2.2 & 1.8 & 1.5 & 1.5 & 1.4 \\
\hline 4. & 1.4 & 1.5 & 1.7 & 1.8 & 2.6 & 3.6 & 2.6 . & 2.2 & 1.9 & .4 & 1.5 & 1.5 \\
\hline $25 \%$ & 1.4 & 1.5 & 1. 75 & 2.0 & 2.6 & 3. 3 & 2.7 & 2.2 & 1.9 & 1.4 & 1.5 & 1.4 \\
\hline 26. & 1.4 & 1.5 & 1. 75 & 2.4 & 2.6 & 3.3 & 2.7 & 2.2 & 1.9 & 1.4 & 1.5 & 1.4 \\
\hline 27 . & 1.4 & 1.5 & 1.8 & 2.4 & 2.7 & 3.2 & 2.8 & 2.2 & 1.9 & 1.4 & 1.5 & 1.5 \\
\hline 28.. & 1.4 & 1.5 & 1.8 & 2.5 & 2.8 & 3.2 & 2.8 & 2.1 & 1.8 & 1.4 & 1.5 & 1.5 \\
\hline 29. & 1.45 & & 1.8 & 2.5 & 2.8 & 3.2 & 2.7 & 2.1 & 1.8 & 1.4 & 1.6 & 1.6 \\
\hline & 1.45 & & 1.8 & 2.5 & 2.8 & 3. 3 & 2.6 & 2.1 & 1.7 & 1.4 & 1.6 & 1.7 \\
\hline 31.. & 1.45 & & 1.8 & & 2.8 & & 2.5 & 2.0 & & 1.4 & & 1.6 \\
\hline
\end{tabular}

NoтE.-Gage heights interpolated February 17-19.

Station rating table for Bishop Creek near Bishop, Cal., from January 1 to December 11, 1905.

\begin{tabular}{|r|r||r|r|r|r|r|r|}
\hline $\begin{array}{c}\text { Gage } \\
\text { height. }\end{array}$ & Discharge. & $\begin{array}{c}\text { Gage } \\
\text { height. }\end{array}$ & Discharge. & $\begin{array}{r}\text { Gage } \\
\text { height. }\end{array}$ & Discharge. & $\begin{array}{c}\text { Gage } \\
\text { height. }\end{array}$ & Discharge. \\
\cline { 1 - 5 } Feet. & Second-feet. & Feet. & Second-feet. & Feet. & Second-feet. & Feet. & Second-feet. \\
1.40 & 21 & 2.10 & 74 & 2.80 & 170 & 3.50 & 314 \\
1.50 & 27 & 2.20 & 84 & 2.90 & 188 & 3.60 & 339 \\
1.60 & 33 & 2.30 & 95 & 3.00 & 206 & 3.70 & 365 \\
1.70 & 40 & 2.40 & 108 & 3.10 & 225 & 3.80 & 391 \\
1.80 & 48 & 2.50 & 122 & 3.20 & 245 & & \\
1.90 & 56 & 2.60 & 137 & 3.30 & 267 & & \\
2.00 & 65 & 2.70 & 153 & 3.40 & 290 & & \\
& & & & & & \\
\hline
\end{tabular}

Note.-The above table is based on 14 discharge measurements made during 1905 and some older measurements. It is not very well defined. This table does not apply later than December 11, as conditions were changed at the station. 
Estimated monthly discharge of Bishop Creek near Bishop, Cal., for 1905.

\begin{tabular}{|c|c|c|c|c|}
\hline \multirow{2}{*}{ Month. } & \multicolumn{3}{|c|}{ Discharge in second-feet. } & \multirow{2}{*}{$\begin{array}{l}\text { Total in } \\
\text { acre-feet. }\end{array}$} \\
\hline & Maximum. & Minimum. & Mean. & \\
\hline 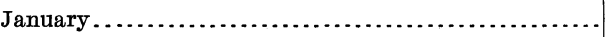 & 27 & 21 & 22.5 & 1,384 \\
\hline 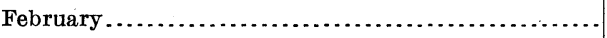 & 30 & 21 & 25.2 & 1,400 \\
\hline 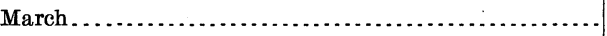 & 48 & 27 & 38.2 & 2,349 \\
\hline 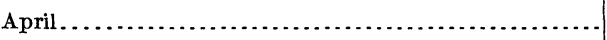 & 122 & 40 & 56.6 & 3,368 \\
\hline 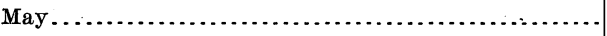 & 170 & 65 & 113 & 6,948 \\
\hline 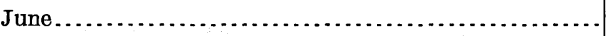 & 391 & 188 & 299 & 17,790 \\
\hline July . . . . . . . . . . . . & 365 & 122 & 215 & 13,220 \\
\hline August . . . . . . . . . . . . & 137 & 65 & 92.1 & 5,663 \\
\hline September............. & 74 & 40 & 53. 6 & 3,189 \\
\hline 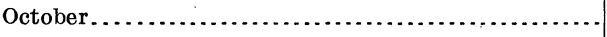 & 56 & 21 & 36.1 & 2,220 \\
\hline November $\ldots \ldots \ldots \ldots, \ldots \ldots \ldots \ldots \ldots \ldots \ldots \ldots \ldots \ldots \ldots$ & 33 & 21 & 25.6 & 1,523 \\
\hline 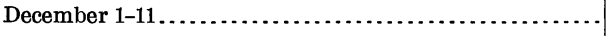 & 27 & 21 & 24.3 & 530 \\
\hline The period.. & & & - & 59,580 \\
\hline
\end{tabular}

RAWSON GANAL NEAR BISHOP, CAL.

This station was established August 7, 1903, by R. S. Hawley. It is located at the county bridge $2 \frac{1}{2}$ miles east of Bishop, Cal.

The channel is straight for 100 feet above and below the station and the current is swift. The right bank is high and the left bank is low. Neither bank is liable to overflow. The bed of the stream is composed of gravel and is permanent.

Discharge measurements are made from the bridge. The initial point for soundings is marked on the bridge near the right bank of the canal.

The gage is a vertical rod fastened to the bridge. During 1905 the gage was read by W. P. Parker.

Information in regard to this station is contained in the following Water-Supply Papers of the United States Geological Survey:

Description: 100, pp 215-216; 134, p 229.

Discharge: 100, p 216; 134, p 229.

Discharge, monthly: 134, p 231.

Gage heights: 100, p 216; 134, pp 230-231.

Rating table: 134, p 231.

Discharge measurements of Rawson canal near Bishop, Cal., in 1905.

\begin{tabular}{|c|c|c|c|c|c|c|}
\hline Date. & Hydrographer. & Width. & $\begin{array}{l}\text { Area of } \\
\text { section. }\end{array}$ & $\begin{array}{c}\text { Mean } \\
\text { velocity. }\end{array}$ & $\begin{array}{c}\text { Gage } \\
\text { height. }\end{array}$ & $\begin{array}{c}\text { Dis- } \\
\text { charge. }\end{array}$ \\
\hline March $14 \ldots . .$. & R. J. Taylor........ & $\begin{array}{r}\text { Feet. } \\
17\end{array}$ & $\begin{array}{r}S q . f t . \\
13.2\end{array}$ & $\begin{array}{r}\text { Ft.per sc. } \\
1.67\end{array}$ & $\begin{array}{r}\text { Feet. } \\
1.75\end{array}$ & $\begin{array}{r}\text { Sec.-ft. } \\
22\end{array}$ \\
\hline April 1... & J. S. Evans.. & 17 & 13.3 & 1.95 & 1.85 & 26 \\
\hline April $17 \ldots$ & .....do........ & 17 & 10.6 & 1.59 & 1.60 & 16.9 \\
\hline May 2... & ..... do... & 17 & 16.3 & 1.72 & 1.90 & 28 \\
\hline May $25 \ldots$ & ....do... & 17 & 17.4 & 1.55 & 1.90 & 27 \\
\hline June $28 . .$. & .....do..... & 17 & 14.8 & 1.22 & 1.70 & 18. 1 \\
\hline July $26 \ldots \ldots$. & . . . . do . . . . & 14 & 5.4 & .39 & 1.30 & 2.1 \\
\hline August 9 . & ..... do... & 17 & 14.6 & 1.00 & 1.55 & 14. 6 \\
\hline September 5. & .....do. & 16 & 9.8 & .72 & 1.45 & 7.0 \\
\hline September 14 & .....do... & 17 & 10.1 & 1.05 & 1.50 & 10.6 \\
\hline October 3.. & ..... do...... & 17 & 15.2 & 1.17 & 1.90 & 17.8 \\
\hline October $25 \ldots .$. & F. R. S. Buttemer... & 7 & 4.2 & .29 & 1.05 & 1.2 \\
\hline November 23 & ..... do.... & 6 & 1.6 & .69 & 1.15 & 1.1 \\
\hline December $11 \ldots$ & .....do........... & 7 & 4.2 & .31 & 1.08 & 1.3 \\
\hline
\end{tabular}


Daily gage height, in feet, of Rawson canal near Bishop, Cal., for 1905.

\begin{tabular}{|c|c|c|c|c|c|c|c|c|}
\hline Day. & Mar. & Apr. & May. & June. & July. & Aug. & Sept. & Oct. \\
\hline $1 .$. & & 1.8 & 1: 7 & 1.8 & 0.8 & 0.9 & 1,3 & 1.8 \\
\hline 2. & & $\ldots$ & 1.7 & 1.8 & .9 & .9 & $\ldots$. & 1.8 \\
\hline $3 .$. & $\cdots$ & 1.8 & 1.7 & 1.8 & .9 & .8 & 1.3 & 1.8 \\
\hline $4 .$. & $\ldots .$. & 1.8 & 1.9 & 1.8 & .9 & .8 & 1.5 & 1.8 \\
\hline 5. & ..... & 1.8 & ...... & 1.7 & .9 & .8 & 1.5 & 1.8 \\
\hline 6. & 1.4 & $\ldots$. & 1.9 & 1.9 & .8 & .8 & 1.5 & 1.8 \\
\hline$\ldots \ldots$ & 1.4 & 1.7 & 1.9 & 1.9 & .8 & .8 & 1.7 & 1.6 \\
\hline 8. & 1.5 & 1.7 & 1.8 & 1.9 & .8 & .8 & 1.7 & 1.4 \\
\hline $9 .$. & 1.5 & $\ldots$. & 1.8 & 1.8 & .8 & .8 & 1.7 & 1.4 \\
\hline 10. & 1.6 & 1.7 & 1.8 & 1.8 & .8 & .8 & 1.7 & 1.4 \\
\hline & 1.6 & 1.7 & 1.8 & 1.9 & .7 & .8 & 1.7 & 1.4 \\
\hline$\ldots \ldots \ldots$ & 1.6 & 1.7 & 1.8 & 1.8 & .7 & .8 & 1.7 & 1.6 \\
\hline $13 .$. & 1.8 & …. & 1.8 & 1.8 & .7 & .9 & 1.6 & 1.6 \\
\hline 14.: & 1.8 & 1.7 & 1.8 & 1.7 & .8 & .9 & 1.6 & \\
\hline 15. & & 1.6 & 1.9 & $=1.0$ & .8 & .0 & 1.6 & \\
\hline 16.. & & 1.6 & 1.9 & .9 & .8 & & 1.6 & \\
\hline 17. & & 1.6 & 1.9 & 1.4 & .8 & .9 & 1.6 & \\
\hline $18 .$. & & 1.6 & 1.9 & 1.4 & .8 & .9 & 1.6 & $\ldots$ \\
\hline …........ & & 1.6 & 1.9 & .......... & .8 & .9 & 1.6 & ....... \\
\hline $20 .$. & .. & 1.7 & 1.9 & ......... & .8 & $\ldots$ & 1.6 & $\ldots$ \\
\hline .......... & & 1.7 & 1.8 & ......... & .8 & .7 & 1.7 & $\ldots$ \\
\hline $22 .$. & & 1.7 & 1.9 & .6 & .7 & .7 & 1.7 & $\cdots$ \\
\hline 23.. & & $\cdots$ & 1.9 & .8 & .7 & .7 & $\cdots$ & \\
\hline 24. & & 1.7 & 1.8 & .9 & .7 & .7 & 1.7 & \\
\hline $25:$. & 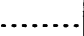 & 1.7 & 1.8 & .9 & .7 & .7 & 1.7 & $\cdots$ \\
\hline 26.. & & 1.7 & 1.8 & .9 & $\cdots$ & 1.3 & 1.7 & \\
\hline $27 .$. & $\cdots$ & 1.7 & 1.8 & .8 & $\cdots$ & 1.3 & 1.7 & ........ \\
\hline $28 .$. & $\ldots$ & 1.7 & 1.8 & .8 & $\cdots$. & 1.3 & 1.7 & \\
\hline $29 . \ldots \ldots$ & …...... & 1.7 & 1.7 & .8 & .9 & ..... & 1.7 & ....... \\
\hline $30 \ldots \ldots \ldots \ldots \ldots$ & 1.8 & 1.7 & 1.7 & .8 & .9 & 1.3 & & \\
\hline $31 \ldots .$. & 1.8 & ......... & 1.7 & & .9 & & & \\
\hline
\end{tabular}

Note.-Water shut off January 1 to March 5 and October 14 to December 31.

A. O. COLIINS GANAL NEAR BISHOP, CAL.

This station was established August 7, 1903, by R. S. Hawley. It is located at the county bridge, 3 miles east of Bishop, Cal.

The channel is straight for 100 feet above and $\mathbf{5 0}$ feet below the station. The current is sluggish. Both banks are high and are not liable to overflow. The bed of the stream is sandy and shifting.

Discharge measurements are made from the bridge. The initial point for soundings is marked on the foot $\log$ near the right bank of the canal.

The gage is a vertical rod fastened to the right bank just above the bridge, from which discharge measurements are made. During 1905 the gage was read by W. P. Parker.

Information in regard to this station is contained in the following Water-Supply Papers of the United States Geological Survey:

Description: 100, pp 216-217; 134, p 232.

Discharge: 100, p 217; 134, p 232.

Discharge, monthly: 134, p 235.

Gage heights: 100, p 217; 134, pp 233-234

Rating table: 134, p 234. 
Discharge measurements of A. O. Collins canal near Bishop, Cal., in 1905.

\begin{tabular}{|c|c|c|c|c|c|c|}
\hline Date. & Hydrographer. & Width. & $\begin{array}{l}\text { Area of } \\
\text { section. }\end{array}$ & $\underset{\text { velocity. }}{\text { Mean }}$ & $\begin{array}{c}\text { Gage } \\
\text { height. }\end{array}$ & $\begin{array}{c}\text { Dis- } \\
\text { charge. }\end{array}$ \\
\hline March 14 & R. J. Taylor. & $\begin{array}{c}\text { Feet. } \\
11\end{array}$ & $\begin{array}{r}S q . f t . \\
12.5\end{array}$ & $\begin{array}{r}\text { Ft. per sec. } \\
2.00\end{array}$ & $\begin{array}{l}\text { Feet. } \\
2.90\end{array}$ & $\begin{array}{r}\text { Sec.-ft. } \\
25\end{array}$ \\
\hline April 1... & J. S. Evans... & 8 & 2.8 & .75 & 2.00 & 2.1 \\
\hline May 2.. & .....do. . & 11.5 & 12.8 & 1.95 & 3.00 & 25 \\
\hline June $28 \ldots$ & .... do. do. & 10 & 10.8 & 1.70 & 2.75 & 18.4 \\
\hline July $26 \ldots . .$. & .....do... & 10 & 12.8 & 1.44 & 2.90 & 18.4 \\
\hline August $9 \ldots$. & .....do... & 10 & 8.8 & $\quad 1.59$ & 2.65 & 14.0 \\
\hline September 5. & .....do... & 10 & 6.0 & 1.22 & 2. 38 & 7.3 \\
\hline September 14 . & .....do... & 10 & 5.4 & 1.20 & 2.33 & .6 .5 \\
\hline October $3 \ldots . .$. & .....do & 10 & 10.4 & 1.47 & 2.80 & 15.3 \\
\hline October $25 \ldots$ & F. R. S. Buttemer. & & & & & 0 \\
\hline November 23 . & ....do. . . . . . & 10.5 & 7.0 & .70 & 2.25 & 4.9 \\
\hline December $11 .$. & .....do... & 10 & 3.8 & .45 & 1.92 & 1.7 \\
\hline
\end{tabular}

Daily gage height, in feet, of A. O. Collins canal near Bishop, Cal., for 1905.

\begin{tabular}{|c|c|c|c|c|c|c|c|c|c|}
\hline Day. & Feb. & Mar. & Apr. & May. & June. & July. & Aug. & Sept. & Oct. \\
\hline $1 .$. & & & & 2.6 & 1.8 & 2.5 & 2.5 & 2.4 & 2.5 \\
\hline $2 .$. & & & & 2.6 & 1.8 & 2.5 & 2.0 & $\ldots .$. & 2.5 \\
\hline $3 .$. & & & & 2,8 & 1.6 & 2.7 & ..... & 2.6 & 2.5 \\
\hline $4 .$. & & & & 3.0 & 1.9 & 2.7 & & 2.6 & 2.2 \\
\hline 5. & & & & ..... & 1.9 & 2.8 & 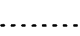 & 2.6 & 2.2 \\
\hline 6. & & & & 3.0 & 1.7 & 2.8 & & 2.6 & 2.2 \\
\hline $7 .$. & & & & 3.0 & 1.7 & 2.8 & ..... & 2.3 & 2.2 \\
\hline $8 \ldots$ & & & & 2.4 & 1.8 & 2.9 & $\ldots$. & 2.0 & 2.2 \\
\hline $9 .$. & & 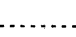 & $\ldots$. & 2.3 & 1.9 & 2.9 & $\ldots$. & 2.0 & 2.2 \\
\hline $10 .$. & & 2.4 & & 2.3 & 1.9 & 3.0 & 2.3 & 2.0 & 2.2 \\
\hline $11 .$. & & 2.4 & 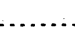 & 2.3 & 1.9 & 3.0 & 2.3 & 2.0 & 2.3 \\
\hline $12 \ldots \ldots \ldots$ & & 2.4 & & 2.3 & 1.9 & 3.0 & 2.3 & 2.0 & 2.3 \\
\hline $13 .$. & & 2.8 & $\cdots$ & 2.3 & 2.2 & 3.0 & 2.3 & 2.3 & 2.3 \\
\hline $14 \ldots \ldots$ & & 2.8 & $\ldots$ & 2.3 & 2.3 & 2.9 & 2.3 & 2.3 & $\ldots$. \\
\hline $15 \ldots \ldots$ & & 2.8 & & 2.3 & 2.3 & 2.9 & & 2.3 & ........ \\
\hline 16. & & 2.8 & & & 2.4 & 2.9 & $\ldots$. & 2.3 & $\ldots . .$. \\
\hline $17 \ldots \ldots$ & & 2.8 & & & $\cdots$ & 2.9 & 2.3 & 2.9 & ......... \\
\hline 18. & & 2.8 & & & .... & 2.9 & 2.1 & 2.9 & n...... \\
\hline $19 \ldots \ldots$ & & 2.8 & 2.6 & & ..... & 2.8 & 2.1 & 2.9 & $\ldots$ \\
\hline $20 \ldots \ldots$ & & 2.8 & 2.6 & & ..... & 2.8 & & 2.9 & .... \\
\hline $21 .$. & & 2.8 & 2.6 & $\ldots$ & $\ldots$. & 2.8 & 2.1 & 2.9 & $\ldots$ \\
\hline $22 \ldots \ldots \ldots$ & 2.3 & 3.0 & 2.6 & & 2.3 & 2.8 & 2.1 & 2.7 & $\cdots$ \\
\hline $23 \ldots$ & & 3.0 & & & 2.3 & 2.7 & 2.0 & $\ldots$. & ......... \\
\hline $24 .$. & & 3.0 & 2.5 & & 2.3 & 2.7 & 2.0 & 2.7 & $\ldots$ \\
\hline $25 \ldots$ & $\cdots$ & 2.7 & 2.5 & 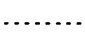 & 2.6 & 2.7 & 2.0 & 2.7 & ….... \\
\hline $26 \ldots \ldots$ & 2.3 & & 2.5 & & 2.6 & 2.7 & 2.0 & 2.7 & …..... \\
\hline $27 \ldots$ & & & 2.5 & & 2.6 & ..... & 2.0 & 2.5 & $\ldots$ \\
\hline $28 \ldots$ & & & 2.6 & & 2.6 & $\ldots .$. & 2.4 & 2.5 & …..... \\
\hline $29 .$. & & & 2.6 & & 2.6 & 2.7 & $\cdots$ & 2.5 & \\
\hline $30 \ldots$ & & & 2.6 & .... & 2.5 & 2.7 & 2.4 & $\cdots$ & $\ldots$ \\
\hline $31 \ldots$ & & & & 1.7 & & 2.5 & & 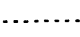 & $\cdots$ \\
\hline
\end{tabular}

NotE. - No flow on days of missing gage heights. 


\section{DELI OANAI NEAR BISHOP, CAI.}

This station was established August 24, 1903, by R. S. Hawley. It is located at a flume 3 miles from the head gate at a point where the canal crosses a slough in Sanders's field.

The channel is straight for $\mathbf{1 5 0}$ feet above and for 200 feet below the station. The current is sluggish at all times.

Discharge measurements are made from a bridge across the flume. The initial point for soundings is the edge of the flume at the right side.

The gage is a vertical rod fastened to the flume.

Information in regard to this station is contained in the following Water-Supply Papers of the United States Geological Survey:

Description: 100, pp 217-218; 134, p 235.

Discharge: 100, p 218; 134, pp 235-236.

Discharge, monthly: 134, p 237.

Gage heights: 100, p 218; 134, pp 236-237.

Discharge measurements of Dell canal near Bishop, Cal., in 1905.

\begin{tabular}{|c|c|c|c|c|c|c|}
\hline Date. & Hydrographer. & Width. & $\begin{array}{l}\text { Area of } \\
\text { section. }\end{array}$ & $\begin{array}{c}\text { Mean } \\
\text { velocity. }\end{array}$ & $\begin{array}{c}\text { Gage } \\
\text { height. }\end{array}$ & $\begin{array}{c}\text { Dis- } \\
\text { charge. }\end{array}$ \\
\hline & - & Feet. & $S q . f t$. & Ft. per sec. & Feet. & Sec.-ft. \\
\hline March 10.. & R. J. Taylor. & 7.5 & 10.1 & 2.38 & 1.40 & 24 \\
\hline April 14........ & J. S. Evans.. & 7.6 & 7.6 & 2.16 & 1.20 & 16.4 \\
\hline April 28.. & .....do... & 7.6 & 11.8 & 1.95 & 1.35 & 23 \\
\hline May $11 . .$. & .....do....... & 7.6 & 12.5 & 2.08 & 1.48 & 26 \\
\hline June $26 . .$. & .....do..... & 7.6 & 11.4 & 1.93 & 1.60 & 22 \\
\hline July $6 . . .$. & .... do .......... & 7.6 & 12.2 & 1.97 & 1.70 & 24 \\
\hline August 2. & .....do..... & 7.6 & 9.1 & 1.89 & 1.30 & 17.2 \\
\hline September $6 .$. & ....do do... & 7.6 & 9.1 & 1.85 & 1.31 & 16.8 \\
\hline September 28. & $\ldots . . d o, \ldots$. & 7.6 & 6.8 & 1.70 & 1.12 & 11.6 \\
\hline October $30 . . .$. & F. R. S. Buttemer. & 7.5 & 9.0 & 1.52 & 1.22 & 13.7 \\
\hline November $18 .$. & .....do............... & 7.5 & 9.7 & 1.71 & 1.35 & 16.6 \\
\hline December $5 . .$. & .....do...... & 7.3 & 4.4 & .68 & 62 & 3.0 \\
\hline
\end{tabular}

Daily gage height, in feet, of Dell canal near Bishop, Cal., for 1905.

\begin{tabular}{|c|c|c|c|c|c|c|c|c|c|c|c|}
\hline Day. & Feb. & Mar. & Apr. & Nov. & Dec. & Day. & Feb. & Mar. & Apr. & Nov. & Dec: \\
\hline & & ...... & 1.3 & & & & & & & & \\
\hline $2 \ldots$ & & 1.4 & & & 0.9 & $18 \ldots \ldots \ldots$ & & 1.4 & & 1.35 & \\
\hline 3. & & $\ldots$. & & & & $19 \ldots \ldots$ & & & & & \\
\hline $4 .$. & & 1.4 & & & .8 & $20 \ldots$ & . & 1.4 & & 1.3 & \\
\hline 5. & & & & & & $21 \ldots$. & & & & & \\
\hline $6 .$. & & 1.4 & & & .6 & 22. & & 1.4 & & 1.3 & \\
\hline $7 .$. & & $\cdots$ & & & & 23. & & & & & \\
\hline 8... & & 1.4 & & & .6 & 24. & & 1.2 & & 1.3 & \\
\hline 9. & & $\cdots$ & & & & 25. & & & & & \\
\hline 10. & & 1.4 & & & .6 & 26... & $1 . \dot{4}$ & 1.2 & & 1.3 & \\
\hline $11 .$. & & & & & & $27 \ldots \ldots$ & & & & & \\
\hline 12. & & 1.4 & & & .8 & $28 \ldots$. & 1.4 & 1.2 & & 1.4 & \\
\hline 13. & & & & & & $29 \ldots$ & & & & & \\
\hline 14.. & & 1.4 & & & .8 & 30. & ........ & 1.2 & & 1.35 & \\
\hline 15. & & & & & & & & & & & \\
\hline $6 .$. & & 1.4 & & & .8 & & & & & & \\
\hline
\end{tabular}




\section{BIG PINE AND OWENS RIVER CANAI NEAR BISHOP, CAL.}

This station was established by J. C. Clausen and R. S. Hawley, August 4, 1903 . It is located at a footbridge near the house of William Oliver, the observer. It is $7 \frac{1}{2}$ miles south and 3 miles east of Bishop, Cal.

The channel is straight for 600 feet above and 300 feet below the station, The current is sluggish. Both banks are high and are not liable to overflow. The bed of the stream is sandy and somewhat shifting.

Discharge measurements are made from the footbridge. The initial point for soundings is marked on the footbridge near the right bank of the canal.

The gage is a vertical rod securely nailed to the footbridge.

Information in regard to this station is contained in the following Water-Supply Papers of the United States Geological Survey:

Description: 100, pp 218-219; 134, p 238.

Discharge: 100, p 219; 134, p 238.

Discharge, monthly: $134, \mathrm{p} 240$.

Gage heights: 100, p 219; 134, pp 238-239.

Rating table: $134, \mathrm{p} 240$.

Discharge measurements of Big Pine and Owens River canal near Bishop, Cal., in 1905.

\begin{tabular}{|c|c|c|c|c|c|c|}
\hline Date. & Hydrographer. & Width. & $\begin{array}{l}\text { Area of } \\
\text { section. }\end{array}$ & $\begin{array}{c}\text { Mean } \\
\text { velocity. }\end{array}$ & $\begin{array}{c}\text { Gage } \\
\text { height. }\end{array}$ & $\begin{array}{c}\text { Dis- } \\
\text { charge. }\end{array}$ \\
\hline January $25 \ldots$ & R. J. Taylor......................... & $\begin{array}{r}\text { Feet. } \\
22\end{array}$ & $\begin{array}{r}S q . f t . \\
11.3\end{array}$ & $\begin{array}{r}\text { Ft.per sec. } \\
0.84\end{array}$ & $\begin{array}{l}\text { Feet. } \\
1.30\end{array}$ & $\begin{array}{r}\text { Sec.-ft. } \\
9 . b\end{array}$ \\
\hline February $13 \ldots$ & ..... do...... & 22 & 9.5 & .70 & 1.15 & 6.7 \\
\hline April $28 \ldots \ldots$. & J. S. Evans.... & 21 & 12.6 & .85 & 1.30 & 10.7 \\
\hline May $11 \ldots$. & .....do......... & 22 & 16 & 1.11 & 1.50 & 17. 7 \\
\hline June $26 \ldots$ & ......do..... & 22 & 27 & 1.11 & 1.90 & 30 \\
\hline July $6 \ldots \ldots \ldots$ & .....do. . . . . . & 22 & 18.8 & 1.05 & 1.75 & 19.6 \\
\hline August 2 . & ..... do. . & 22 & 10.8 & .58 & 1.20 & 6.3 \\
\hline September $6 .$. & .....do... & 16 & 10.0 & .59 & 1.17 & 5. 9 \\
\hline September $28 .$. & .....do..... & 14 & 7.2 & .36 & 1. 10 & 2.6 \\
\hline October $30 \ldots .$. & F. R. S. Buttemer. . & 22 & 41 & 1.00 & 2.53 & 41 \\
\hline November 18 & ......do... & 22 & 17.6 & .85 & 1.42 & 15 \\
\hline December $5 . .$. & ......do......... & 22 & 18. 2 & .89 & 1.45 & 16. 2 \\
\hline
\end{tabular}


Daily gage height, in feet, of Big Pine and Owens River canal near Bishop, Cal., for 1905.

\begin{tabular}{|c|c|c|c|c|c|c|c|c|c|c|c|c|}
\hline D & Jan. & Feb. & Mar. & Apr. & May. & June. & July. & Aug. & Sept. & Oċt. & Nov. & Dec. \\
\hline 1.. & 1.2 & 1.4 & 1.2 & 1.15 & 1.4 & 2.0 & 1.8 & .1 .1 & 1.2 & 1.4 & 2.55 & 1.4 \\
\hline 2. & 1.2 & 1.4 & 1.2 & 1.15 & 1.8 & 1.85 & 1.8 & 1.1 & 1.1 & 1.3 & 2.55 & 1. 45 \\
\hline 3.. & 1.2 & 1.4 & 1.15 & 1.15 & 1.8 & 1.8 & 1.8 & 1.1 & 1.2 & 1.4 & 2.55 & 1.5 \\
\hline 4.. & 1.2 & 1.3 & 1.15 & 1.15 & 1.9 & 1.8 & 1.8 . & 1.1 & 1.2 & 1.5 & 2.5 & 1.5 \\
\hline $5 .$. & 1. 2 & 1.25 & 1. 15 & 1.15 & 1.7 & 1.8 & 1.8 & 1.0 & 1.2 & 1.65 & 2.5 & 1. 45 \\
\hline $6 .$. & 1.25 & 1.25 & 1.15 & 1.15 & 1.7 & 1.7 & 1.7 & 1.1 & 1.2 & $1.9^{-}$ & 2.25 & 1.45 \\
\hline $7 .$. & 1.3 & 1.25 & 1.15 & 1.1 & 1.5 & 1.7 & 1.7 & 1.1 & 1.2 & .1 .9 & 2.3 & 145 \\
\hline $8 .$. & 1.3 & 1.15 & 1.15 & 1.1 & 1.5 & 1.7 & 2.0 & 1.1 & 1.2 & 1.8 & 2.3 & 1.45 \\
\hline 9. & 1.25 & 1.15 & 1.1 & (a) & 1.6 & 1.95 . & 2.05 & 1.2 & 1.2 & 2.0 & 2.3 & 1.45 \\
\hline $10 .$. & 1.25 & 1,2 & 1.1 & (a) & 1.55 & 2.15 & 2.2 & 1.2 & 1.2 & 2.1 & 2.55 & 1.4 \\
\hline $11 .$. & 1. 25 & 1.2 & 1.1 & (a) & 1.55 & $2.1^{\circ}$ & 2.25 & 1.2 & 1.3 & 2. 15 & 1.2 & 1.4 \\
\hline 12. & 1.25 & 1.2 & 1.15 & (a) & 1.5 & 2.3 & 2.35 & 1.2 & 1.3 & 2.2 & 1.2 & 1.4 \\
\hline $13 .$. & 1.25 & 1.2 & 1.5 & (a) & 1.5 & 2.45 & 2.4 & 1.2 & 1.2 & 2.2 & 1.2 & 1.4 \\
\hline $14 .$. & 1.25 & 115 & 1.75 & 1. 35 & 1.5 & 2.65 & 2.4 & 1.2 & 1.2 & 2.1 & 1.2 & . 1.4 \\
\hline $15 .$. & 1.3 & 1.15 & 1.55 & 1.4 & 1.5 & 2.7 & 2.25 & 1.2 & 1.3 & 2.15 & 1.2 & 1.4 \\
\hline $16 .$. & 1.4 & 1.2 & 1.55 & 1.35 & 1.5 & 2.8 & 2.1 & 1.2 & 1.3 & 2.15 & 1.2 & 1.4 \\
\hline $17 .$. & 1.4 & 1.2 & 1.4 & 1.35 & 1.55 & 1.8 & 2.05 & 1.2 & 1.3 & 2.3 & 1.3 & 1.4 \\
\hline 18.. & 1. 35 & 1.2 & 1.4 & 1. 45 & 1.6 & 1.8 & $1.8^{\circ}$ & 1.2 & 1.3 & 2.3 & 1.4 & 1.4 \\
\hline 19. & 1.35 & 1.2 & 1.35 & 1.3 & 1.8 & 1.85 & 1.7 & 1.1 & 1.1 & 2.35 & 1.4 & 1.4 \\
\hline $20 .$. & 1.4 & 1.2 & 1. 35 & 1.3 & 2.1 & 1.7 & 1.6 & 1.1 & 1.15 & 2.35 & 1.35 & 1.4 \\
\hline 21.. & 1.4 & 1.15 & 1.35 & 1.15 & 2.15 & 1.65 & 2.0 & 1.0 & 1.15 & 2.35 & 1.3 & 1.35 \\
\hline $22 .$. & 1. 35 & 1. 15 & 1. 35 & 1.15 & 2.25 & 1.7 & 1.9 & 1.0 & 1.1 & 2.35 & 1.25 & 1. 35 \\
\hline 23. & 1.35 & 1.15 & 1.1 & 1.1 & 2.2 & 1.8 & 2.0 & 1.5 & 1.1 & 2.35 & 1.25 & 1.35 \\
\hline 24. & 1.35 & 1.25 & 1.1 & 1.1 & 2.25 & 2.2 & 1.6 & 1.6 & 1.1 & 2.35 & 1.25 & 1.3 \\
\hline 25.. & 1.35 & 1.25 & 1.1 & 1.1 & 2.0 & 2.2 & 1.4 & 1.5 & 1.2 & 2.35 & 1.3 & 1.3 \\
\hline $26 .$. & 1. 35 & 1.25 & 1.1 & 1.45 & 1.8 & 1.9 & 1.3 & 1.4 & 1.2 & 2.35 & 1.3 & 1.4 \\
\hline $27 .$. & 1.3 & 1.3 & 1.2 & 1.4 & 2.25 & 1.8 & 1.3 & 1.4 & 1.15 & 2.35 & 1.4 & 1.4 \\
\hline $28 .$. & 1.3 & 1.2 & 1.2 & 1.4 & 2. 25 & 1.6 & 1.2 & 1.4 & 1.15 & 2.45 & 1.4 & 1.4 \\
\hline 29. & 1.3 & & 1.2 & 1.4 & 2. 45 & 1.5 & 1.1 & 1.2 & 1.15 & 2.45 & 1.4 & 1.3 \\
\hline $30 \ldots$ & 1. 25 & & 1.1 & 1.4 & 2.4 & 1.8 & 1.1 & 1.3 & 1.15 & 2.5 & 1.4 & 1.3 \\
\hline $31 .$. & 1.25 & & 1.1 & & 2.1 & ........ & 1.1 & 1.3 & & 2.55 & $\cdots \cdot$ & 1.3 \\
\hline
\end{tabular}

$a$ Water shut off A pril 9-13.

\section{SAYGER GANAL AT ALVORD, CAL.}

This station was established August 4, 1903, by J. C. Clausen and R. S. Hawley. It is located at the county road bridge, one-fourth mile east of the Southern Pacific Railroad station at Alvord, Cal.

The channel is straight for 300 feet above and for 100 feet below the station. The current is sluggish. Both banks are low and liable to overflow. The bed of the stream is shifting.

Discharge measurements are made from the bridge. The initial point for soundings is marked on the bridge near the left bank of the canal.

The gage is a vertical rod fastened to the bridge.

Information in regard to this station is contained in the following Water-Supply Papers of the United States Geological Survey:

Description: 100, pp 219-220; 134, pp 240-241.

Discharge: 100, p 220; 134, p 241.

Gage heights: 100, p 220; 134, pp 241-242. 
Discharge measurements of Sanger canal at Alvord, Cal., in 1905.

\begin{tabular}{|c|c|c|c|c|c|c|}
\hline Date. & Hydrographer. & Width. & $\begin{array}{l}\text { Area of } \\
\text { section. }\end{array}$ & $\frac{\text { Mean }}{\text { velocity. }}$ & $\begin{array}{c}\text { Gage } \\
\text { height. }\end{array}$ & $\begin{array}{l}\text { Dis- } \\
\text { charge. }\end{array}$ \\
\hline & & Feet. & $S q . f t$. & Ft. per sec. & Feet. & Sec.-ft. \\
\hline January $7 \ldots . .$. & R.J.Taylor $\ldots \ldots \ldots$ & 16 & 23 & 0.59 & 2.38 & 13.6 \\
\hline February $18 .$. & ... . do. . . . . . . . & 16 & -22 & .63 & 2.32 & 13.9 \\
\hline April 14....... & J.S. Evans. . . . . . . . . . . . . & 16 & 26 & .38 & 2.80 & 9.9 \\
\hline April 26.... & ..... do... & 16 & 29 & .30 & 2.88 & 8.8 \\
\hline May $19 \ldots . . .$. & .... do........... & 16 & 32 & .18 & 3.05 & 5.6 \\
\hline June $9 . \ldots . .$. & .....do... & 16 & 29 & .16 & 2.80 & 4.7 \\
\hline A.ugust 17...... & ..... do . . . & 16 & 18 & .22 & 2.10 & 4.0 \\
\hline September 13... & .....do.... & 16 & 16 & .18 & 2.10 & 2.8 \\
\hline October $30 . . .$. & F. R. S. Buttemer ... & 16 & 30 & .44 & 2.82 & 13.2 \\
\hline November $18 .$. & 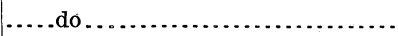 & 16 & 26 & .39 & 2.60 & 10.2 \\
\hline
\end{tabular}

Daily gage height, in feet, of Sanger canal at Alvord, Cal., for 1905.

\begin{tabular}{|c|c|c|c|c|c|c|c|c|c|c|c|}
\hline Day. & Jan. & $\mathrm{Fe}$. & Mar. & Apr. & May. & Day. & Jan. & Feb. & Mar. & Apr. & May. \\
\hline $1 \ldots \ldots \ldots$ & 2.2 & $\ldots \ldots$ & 1.2 & 2.7 & 2.9 & 17. & 1.9 & $\ldots$ & 2.2 & 2.8 & \\
\hline $2 \ldots \ldots \ldots$ & $\ldots . .$. & 1.7 & 1.2 & 2.7 & 2.9 & 18 . & $\ldots$ & 1. 3 & 2.2 & 2.8 & 3.5 \\
\hline $3 \ldots \ldots \ldots$ & 2.1 & {$\left[\begin{array}{lll}-1 & -1\end{array}\right.$} & $\ldots \ldots$ & $\ldots$. & $\ldots \ldots$ & 19 & 1.8 & 1.3 & 2.2 & $\ldots \ldots$ & $\ldots . .$. \\
\hline $4 \ldots$ & ....... & 1.6 & 1.2 & 2.8 & 2.9 & 20 & $\ldots$. & $\cdots$ & $\ldots \ldots$ & 2.8 & 3.5 \\
\hline $5 \ldots \ldots \ldots$ & 2.1 & 1.6 & 1.2 & $\ldots$. & $\ldots$. & 21. & 1.8 & 1.3 & 2.4 & $\ldots \ldots$ & $\cdots$ \\
\hline $6 \ldots \ldots$ & $\cdots$ & ..... & $\cdots \cdot$ & 2.8 & 3.0 & 22 & 1.8 & $\because \cdots$ & $\ldots \ldots$ & 2.8 & \\
\hline $7 \ldots \ldots . . . .$. & 2.1 & 1.5 & 1.2 & ..... & 3.0 & 23 & $\cdots$ & 1.3 & 2.4 & $\ldots \ldots$ & $\cdots$ \\
\hline $8 \ldots \ldots$ & 2.1 & $\cdots \ldots$ & $\cdots$ & 2.9 & ..... & 24 & 1.8 & $\ldots$ & $\ldots \ldots$ & 2.8 & \\
\hline $9 \ldots$ & $\ldots$ & 1.5 & 1.8 & 2.9 & 3.1 & 25 & - & 1.3 & 2.4 & ...... & \\
\hline $10 \ldots \ldots$ & 2.0 & $\ldots \ldots$ & $\ldots \ldots$ & $\cdots$ & …. & 26 & 1.8 & 1.3 & 2.4 & 2.8 & \\
\hline $11 \ldots$ & $\ldots . .$. & 1.4 & 2.0 & 2.9 & 3.3 & $27 \ldots$ & & . & $\ldots$. & & \\
\hline $12 \ldots \ldots \ldots$ & 2.0 & 1.4 & 2.1 & $\ldots \ldots$ & $\ldots \ldots$ & 28 & 1.7 & 1.2 & 2.6 & 2.8 & $\cdots$ \\
\hline $13 \ldots$ & ....... & 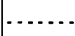 & $\cdots$ & 2.9 & 3.4 & 29 & 1.7 & & $\therefore .$. & 2.8 & \\
\hline $14 \ldots \ldots$ & 1.9 & 1.3 & 2.1 & . . . . . & 3.4 & 30 & & & 2.7 & 2.8 & $\ldots$ \\
\hline $15 \ldots \ldots$ & 1.9 & & ..... & 2.8 & 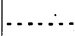 & 31 & 1.7 & & 2.7 & $\cdots$ & $\cdots$ \\
\hline $16 \ldots \ldots$ & $\ldots \ldots$ & 1.3 & 2.2 & 2.8 & 3.4 & & & & & & \\
\hline
\end{tabular}

EAST SIDE CANAL NEAR GITRUS, CAL.

This station was established August 27; 1903, by R. S. Hawley. It is located at the head gate of the canal.

At low stages the meter measurements are made by wading at a point below the head gate. High-stage measurements are made from Southern Pacific Railroad bridge, one-half mile below head gate.

The gage is a vertical rod fastened to the head gate at which discharge measurements are made. During 1905 the gage was read by J. Vaughn.

Information in regard to this station is contained in the following Water-Supply Papers of the United States Geological Survey:

Description: 100, p 220; 134, pp 242-243.

Discharge: 100, p 221; 134, p 243.

Discharge, monthly: 134, p 245.

Gage heights: $100, p$ 221; 134, pp 243-244.

Rating table: 134, p 245. 
Discharge measurements of East Side canal near Citrus, Cal., in 1905.

\begin{tabular}{|c|c|c|c|c|c|c|}
\hline Date. & Hydrographer. & Width. & $\begin{array}{l}\text { Area of } \\
\text { section. }\end{array}$ & $\underset{\text { velocity. }}{\text { Mean }}$ & $\begin{array}{c}\text { Gage } \\
\text { height. }\end{array}$ & $\begin{array}{c}\text { Dis- } \\
\text { charge. }\end{array}$ \\
\hline & & Feet. & Sq.ft. & Fit. per sec. & Feet. & Sec.-ft. \\
\hline February $2 \ldots .$. & R.J. Taylor & 20 & 21 & 1.48 & 2.30 & 31 \\
\hline May 29........ & J.S. Evans......... & 21 & 28 & 1.14 & 2.60 & 32 \\
\hline August $23 . . . .$. . & ....do....... & 24 & 35 & .63 & 3.10 & 22 \\
\hline September $20 .$. & .....do....... & 21 & 28 & .50 & 3.00 & 14 \\
\hline November 8... & F. R.S. Buttemer.... & 18 & 27 & .66 & 2.63 & 18 \\
\hline December $3 . .$. & .....do..................... & 11 & 3.7 & 1.30 & 1.90 & 4.8 \\
\hline
\end{tabular}

Daily gage height, in feet, of East Side canal near Citrus, Cal., for 1905.

\begin{tabular}{|c|c|c|c|c|c|c|c|c|c|c|c|c|}
\hline Day. & Jan. & Feb. & Mar. & Apr. & May. & June. & July. & Aug. & Sept. & oct. & Nor. & Dec. \\
\hline 1. & & & & & 2.5 & $\cdots$ & & & 3.0 & & & 2.0 \\
\hline 2. & & & & & & 2.4 & & & & 3.1 & & \\
\hline 3.. & $\ldots$ & 2.3 & 2.3 & 2.5 & & & 3.4 & .... & & & 3.0 & \\
\hline 4. & & & & & & & & 3.3 & 3.1 & & & 2.1 \\
\hline 5 & $\ldots . .$. & . & & & 2.5 & 2.6 & & & & & & \\
\hline 6.. & 2.3 & 2.0 & 2.3 & & & & & & & 3.1 & 2.9 & \\
\hline 7.. & ........ & . & & 2.5 & & & 3.3 & 3.3 & & & & \\
\hline 8.. & $\ldots$ & & & $\ldots$ & 2.5 & $\ldots$. & & & 3.1 & & & 2.1 \\
\hline 9 & 2.4 & & & & & 3.0 & & & & 3.1 & & \\
\hline 10. & ....... & 2.0 & 2.4 & 2.5 & & .. & 3.3 & $\cdots$ & ...... & & $2: 9$ & $\cdots$ \\
\hline $11 .$. & & & & & & & & 3.1 & 3.1 & & & 2.1 \\
\hline 12. & 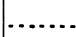 & &. & & 2.5 & 3.2 & & & & & & \\
\hline 13. & 2.2 & 2.0 & 2.6 & & & & & & & 3.1 & 2.8 & \\
\hline 14. & $\cdots$ & & $\cdots$ & 2.6 & & & 3.3 & 3.1 & & & & \\
\hline 15. & & & & $\cdots$ & 2.6 & & & & 3.1 & & & 2.1 \\
\hline 16.. & 2.3 & & & & & 3.2 & & & & 3.1 & & \\
\hline 17. & ..... & 2.1 & 2.5 & 2.6 & & & 3.2 & & & & 2.8 & \\
\hline 18. & $\cdots$ & & & & & & & 3.1 & 3.1 & & & 2.1 \\
\hline 19. & $\cdots$ & $\ldots$ & $\ldots$ & & 2.6 & 3.2 & & & & & & \\
\hline 20.. & 2.3 & 2.2 & 2.1 & & & & & & & 3.2 & 2.7 & \\
\hline $21 .$. & $\ldots . .$. & & - & 2.6 & $\ldots$ & $\ldots$ & 3.1 & 3.1 & & & & \\
\hline 22. & $\ldots$ & & & & 2.5 & & & & 3.1 & & & 2.1 \\
\hline 23. & 2.2 & & $\ldots$ & & & 3.2 & & & & 3.2 & & \\
\hline 24. & $\ldots$. & 2.2 & 2.2 & 2.6 & & & 3.2 & & & & 2.6 & \\
\hline 25. & $\cdots$ & & & & & & & 3.2 & 3.0 & & & 2.0 \\
\hline 26. & .... & & & & 2.5 & 3.2 & & & & & & \\
\hline $27 \ldots$ & 2.3 & 2.2 & 2.5 & & & & & & & 3.2 & 2.6 & $\ldots$. \\
\hline $28 .$. & $\therefore$ & & ... & 2.5 & $\cdots$ & $\ldots \ldots$ & 3.2 & 3.1 & & & & \\
\hline 29. & ....... & & & & 2.6 & & & & 3.1 & & & 1.9 \\
\hline $30 .$. & 2.3 & 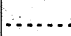 & $\ldots$ & & & 2.3 & & & & 3.0 . & & \\
\hline $31 .$. & $\cdots$ & & 2.5 & & & $\ldots$ & 3.2 & & & & $\ldots$. & $\ldots$. \\
\hline
\end{tabular}




\section{STEVENS GANAL NEAR CITRUS, CAL.}

This station was established August 27; 1903, by R. S. Hawley. It is located at the waste gate of the canal, $3 \frac{1}{2}$ miles north of Citrus, Cal.

The channel is straight for 300 feet above and 200 feet below the station. The current is sluggish. Both banks are high and are not liable to overflow. The bed of the stream is composed of gravel and earth and is fairly permanent.

Discharge measurements are made by wading. The initial point for soundings is on the right bank of the canal.

The gage is a vertical rod fastened to the waste gate. During 1905 the gage was read by J. Vaughn.

Information in regard to this station is contained in the following Water-Supply Papers of the United States Geological Survey:

Description: $100, \mathrm{p} 221 ; 134, \mathrm{p} 245$.

Discharge: 100, p 222; 134, p 246.

Discharge, monthly: 134, p 248.

Gage heights: $100, \mathrm{p} 222 ; 134$, pp 246-247

Rating table: 134, pp 247-248.

Discharge measurements of Stevens canal near Citrus, Cal., in 1905.

\begin{tabular}{|c|c|c|c|c|c|c|}
\hline Date. & Hydrographer. & Width. & $\begin{array}{l}\text { Area of } \\
\text { section. }\end{array}$ & $\begin{array}{c}\text { Mean } \\
\text { velocity. }\end{array}$ & $\begin{array}{c}\text { Gage } \\
\text { height. }\end{array}$ & $\begin{array}{l}\text { Dis- } \\
\text { charge. }\end{array}$ \\
\hline May 29. & J. S. Evans....... & $\begin{array}{r}\text { Feet. } \\
12\end{array}$ & $\begin{array}{c}S q . f t \\
29\end{array}$ & $\begin{array}{r}\text { Ft. per sec. } \\
1.28\end{array}$ & $\begin{array}{r}\text { Feet. } \\
2.70\end{array}$ & $\begin{array}{r}\text { Sec.-ft. } \\
37\end{array}$ \\
\hline August $23 \ldots$. & .....do.............. & 12 & 26 & .71 & 2.50 & 18.5 \\
\hline September 20. & . . . do do . . . . . . . . & 12 & 25 & .92 & 2.55 & 23 \\
\hline November $8 \ldots$ & F. R. S. Buttemer. & 13 & 11.6 & .92 & 1.80 & 10.7 \\
\hline December $3 \ldots$ & .....do...... & 11 & 9.6 & .76 & 1.65 & 7.3 \\
\hline
\end{tabular}

IRR $177-06-6$ 
Daily gage height, in feet, of Stevens canal near Oitrus, Cal., for 1905.

\begin{tabular}{|c|c|c|c|c|c|c|c|c|c|c|c|c|}
\hline Day. & Jan. & Feb. & Mar. & Apr. & May. & June. & July. & Aug. & Sept. & Oct: & Nov. & Dec. \\
\hline 1. & & 1.6. & 1.9 & & 2.1 & & & & 2.3 & & 2.8 & 2.5 \\
\hline 2. & 1.6 & $\cdots$ & .... & & & 2.8 & $\cdots$ & 2.5 & $\therefore$ & 2.6 & $\cdots$ & \\
\hline 3.. & ..... & 1.6 & 1.8 & 1.5 & 2.2 & $\cdots$ & 2.8 & & & $\cdots$ & 2.8 & \\
\hline 4. & 1.6 . & & & & & $\cdots$ & ..... & 2.5 & 2.4 & 2. 6 & & 1.8 \\
\hline 5. & $\cdots$ & & $\ldots$. & 1.5 & 2.3 & 2.8 & 2.9 & & & & & \\
\hline 6. & 1.5 & 1.5 & 1.9 & ... & & . & & & 2.4 & 2.6 & 2.8 & 1.8 \\
\hline 7. & $\ldots \ldots$ & $\cdots$ & .... & 1.5 & $\ldots$. & 2.8 & 2.8 & 2.5 & & & & \\
\hline 8.. & $\ldots$. & 1.5 & 2.3 & ..... & 2.2 & & & & 2.4 & ..... & 2.8 & 1.8 \\
\hline 9. & 1.5 & ..... & ....... & ..... & $\ldots .$. & 2.8 & ..... & 2.5 & & 2.7 & $\cdots$ & \\
\hline 10. & $\ldots . .$. & 1.5 & 2.3 & 1.5 & 2.2 & $\cdots$ & 2.9 & & & $\therefore .$. & 2.8 & \\
\hline 11. & 1.5 & & $\therefore \ldots \ldots$ & $\ldots$ & $\ldots$ & -. & $\cdots$ & 2.5 & 2.5 & 2.7 & & 1.7 \\
\hline 12. & $\cdots$ & $\cdots$ & $\ldots$ & 1.6 & 2.2 & 2.8 & $2: 9$ & & & $\cdots$ & & \\
\hline 13. & 1.5 & 1.5 & 2.4 & $\cdots$ & & & & $\therefore$ & 2.5 & 2.7 & 2.8 & 1.7 \\
\hline 14. & & & $\cdots$ & 1.6 & $\ldots$ & 2.8 & 2.9 & 2.7 & & & & \\
\hline 15. & & 1.5 & 2.5 & $\cdots$ & 2.2 & & & & 2.5 & $\cdots$ & 2.8 & 1.7 \\
\hline 16: & 1.5 & $\cdots$ & & & & 2.9 & & 2.7 . & & 2.8 & & \\
\hline 17. & $\cdots$ & 1.5 & 2.6 & 1.6 & 2.2 & 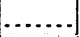 & 2.8 & & & & 2.8 & $\therefore$ \\
\hline 18. & 1.6 & & & $\ldots .$. & & & & 2.6 & 2.6 & 2.8 & & 1.7 \\
\hline 19. & $\ldots$. & $\ldots$ & $\therefore$. & 1.6 & $2: 2$ & 2.9 & 2.7 & & & & $\ldots$ & $\cdots$ \\
\hline 20. & 1.5 & 1.6 & 2.4 & ........ & ........ & $\cdots$ & & & 2.6 & 2.8 & 2.6 & 1.7 . \\
\hline 21. & & ..... & $\cdots$ & 1.6 & .... & 2.9 & 2.7 & 25 & & & $\cdots$ & $\cdots$ \\
\hline 22. & $\therefore$. & 1.6 & 2.0 & & 2.5 & $\ldots$ & & .... & 2.6 & ..... & 2.6 & 1.7 \\
\hline 23. & 1.5 & ..... & & & $\cdots \cdot$ & 2.9 & & 2.5 & & 2.9 & $\cdots$ & ..... \\
\hline 24. & $\ldots$. & 2.0 & 1.5 & 1.6 & & 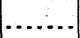 & 2.7 & ..... & & $\ldots$ & 2.5 & ...... \\
\hline 25. & 1.5 & & $\because \cdots$ & & $\cdots$ & $\cdots$ & & 2.5 & 2.6 & 2.9 & & 1.7 \\
\hline 26. & $\ldots$ & .......... & …... & 1.5 & 2.6 & 2.9 & 2.8 & & & & & \\
\hline $27 .$. & 1.5 & 1.9 & 1.5 & & $\cdots$ & $\cdots$ & & $\cdots$ & 2.6 & 2.9 & 2.5 & 1.7 \\
\hline 28.. & & & $\ldots$ & 2.1 & $\ldots .$. & 2.7 & 2.8 & 2.4 & & & & \\
\hline & & & 1.5 & & 2.7 & & & & 2.5 & & 2.5 & 1.7 \\
\hline 30.. & 1.5 & 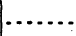 & $\cdots$ & & $\ldots$. & 2.8 & $\cdots$ & 2.4 & $\cdots$ & 2.8 & & - . . . \\
\hline 31. & & & 1.5 & & 2.8 & $\ldots$. & 2.5 & $\ldots$ & & $\cdots$ & & \\
\hline
\end{tabular}

OWENS RIVER NEAR OITRUS, CAL.

This station was established October 30,1903 , by $R$. S. Hawley. It is located at the county bridge 4 miles east of Independence, Cal., and 1 mile from the Southern Pacific Railroad station at Citrus, Cal. The station at this point shows the amount of waste water which is discharged into Owens Lake.

The channel is straight for 200 feet above and for 300 feet below the station. The current has a mean velocity of about 2 feet per second at ordinary stages. Both banks are high and are not liable to overflow. The bed of the stream is sandy and is liable to shift.

Discharge measurements are made by means of a cable and car located about 1,500 feet above the bridge. The cable has a span of 120 feet.

The gage is a vertical rod nailed to a pile on the upstream side of the middle pier of the bridge. During 1905 the gage was read by Milton Levy. The bench mark is a copper tack in the floor beam directly over the gage rod; elevation, 13.50 feet above the datum of the gage.

Information in regard to this station is contained in the following Water-Supply Papers of the United States Geological Survey:

Description: 100, p 222; 134, p 248.

Discharge: $100, \dot{\mathrm{p}} 223 ; 134, \mathrm{p} 249$.

Discharge, monthly: 134, p 251.

Gage heights: 100, p 223; 134, pp 249-250.

Rating table: 134, p. 251 . 
Discharge measurements of Owens River near Citrus, Cal., in 1905.

\begin{tabular}{|c|c|c|c|c|c|c|}
\hline Date & Hydrographer. & Width. & $\begin{array}{l}\text { Area of } \\
\text { section. }\end{array}$ & $\begin{array}{c}\text { Mean } \\
\text { velocity. }\end{array}$ & $\begin{array}{c}\text { Gage } \\
\text { height. }\end{array}$ & $\begin{array}{c}\text { Dis- } \\
\text { charge. }\end{array}$ \\
\hline & & Feet. & $S q . f t$ & Ft.per sec. & Feet. & Sec.-ft. \\
\hline February $2 \ldots$ & R. J. Taylor.... & 73 & 176 & 2.21 & 6.00 & 389 \\
\hline May $29 \ldots \ldots$. & J. S. Evans.................. & 47 & 40 & 1.52 & 3.60 & 61 \\
\hline July $21 \ldots \ldots$. & .....do.................. & 68 & 68 & 1.84 & 4.30 & 125 \\
\hline August $23 \ldots$. & 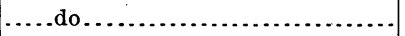 & 24 & 15.2 & .80 & 3.00 & 12.1 \\
\hline September $20 .$. & ....do.do. . . . . . & 25 & 20 & 1.05 & 3.15 & 12 \\
\hline November $8 .$. & F. R. S. Buttemer. . & 71 & 91 & 1.98 & 4.65 & 180 \\
\hline December 3.... & ....do............... & 73 & 131 & 2.21 & 5.20 & 290 \\
\hline Derember $15 .$. & .....do.... & 72 & 128 & 2.20 & 5.30 . & 282 \\
\hline
\end{tabular}

Daily gage height, in feet, of Ouiens River near Citrus, Cal., for 1905.

\begin{tabular}{|c|c|c|c|c|c|c|c|c|c|c|c|c|}
\hline Day. & Jan. & Feb. & Mar. & Apr. & May. & June. & July. & Aug. & Sept. & Oct. & Nov. & Dec. \\
\hline 1. & & & 5.85. & & & & 5.6 & & & 3.5 & 4.6 & 5.15 \\
\hline $2 .$. & & 6.0 & & & & & 5.35 & & & 3. 45 & 4.65 & 5.2 \\
\hline 3.. & ........ & 6.8 & & & & & 5.0 & & & 3.45 & 4.7 & 5.3 \\
\hline 4... & ....... & 6.8 & 5.8 & & & & 5.0 & & $\cdots$ & 3.5 & 4.75 & 5. 35 \\
\hline 5 & $\ldots \ldots$ & $\cdots$ & $\cdots$ & & & & 5.0 & & & 3.55 & 4.8 & 5.35 \\
\hline $6 .$. & 5.7 & 6.9 & 5.8 & 5.0 & & & 5.0 & & & 3.5 & 4.8 & 5.4 \\
\hline 7.. & ..5.65 & 6.8 & ...... & & & & 5.0 & & & 3.5 & 4.8 & 5.4 \\
\hline 8. & 5.65 & 6.85 & 5.75 & & & & 5.5 & & & 3.6 & 4.85 & 5.35 \\
\hline 9. & $\ldots$. & 6.8 & 5.45 & & & & 5.1 & & & 3.8 & 4.85 & 5.4 \\
\hline 10. & .. & 6.85 & 5. 4 & & & & 5.1 & & & 3.85 & 4.9 & 5.4 \\
\hline 11.. & & 6.9 & 5.45 & & & & 5.2 & & & 3.9 & 4.9 & 5.4 \\
\hline 12. & $\ldots$ & 6.9 & 5.4 & & & & 5.25 & & & 3.9 & 4.9 & 5. 35 \\
\hline $13 .$. & 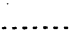 & ....... & 5.7 & & & & 5.3 & & & 4.0 & 4.9 & 5.4 \\
\hline $14 \ldots$ & 5.7 & 7.1 & $\ldots \ldots$ & 4.5 & & & 5.3 & & & 4.1 & $4.95^{\circ}$ & 5.4 \\
\hline $15 .$. & 5.8 & 7.15 & 5.8 & & & & 5.3 & & & 4.15 & 4.95 & 5.4 \\
\hline 16. & . & 7.2 & 6.3 & & & & 5.3 & & & 4.15 & 5.0 & 5.4 \\
\hline 17. & $\ldots$. & 7.2 & 6.4 & & & & 5.0 & & 3.1 & 4.2 & 5.0 & 5.5 \\
\hline 18. & .... & ...... & & & & & 4.8 & & 3.1 & 4. 25 & 5.0 & 5. 45 \\
\hline 19. & 5.8 & $\cdots$ & & & & & 4. 75 & & 3.2 & 4.3 & 5.0 & 5.4 \\
\hline 20. & 5.9 & $\cdots$ & & & & & 4.6 & & 3.3 & 4.3 & 5.0 & 5.5 \\
\hline $21 .$. & 5.85 & 5.8 & & & & & 4.4 & & 3.3 & 4. 4 & 5.0 & 5.4 \\
\hline 22. & $\ldots$ & 5.8 & & & & $\ldots$ & 4.1 & & 3.3 & 4.4 & 5.0 & 5.45 \\
\hline $23 .$. & 6.0 & 5.8 & & & & 6.0 & 3.5 & & 3.3 & 4.5 & 5.0 & 5.5 \\
\hline 24.. & .... & $\cdots$ & & & & 6.1 & 3.2 & & 3.2 & 4.5 & 5.0 & 5.5 \\
\hline 25. & $\ldots$. & $\ldots$ & & & & 6.3 & 3.0 & & 3.25 & 4.4 & 5.0 & 5.45 \\
\hline 26 . & 6.0 & 5.8 & & & & 6.4 & 2.8 & & 3.3 & 4. 45 & 5.0 & 5.45 \\
\hline 27. & 6.0 & 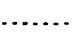 & & & & 6.35 & 2. 75 & & 3.35 & 4.5 & 5.0 & 5.4 \\
\hline 28. & $\cdots$ & & & & & 6.0 & 2.6 & & 3.4 & 4.5 & 5.0 & 5.5 \\
\hline $29 .$. & ..... & & & & & 5.6 & 2.5 & & 3.4 & 4.5 & 5.0 & 5.4 \\
\hline $30 .$. & 5.8 & & & & & 5.6 & & & 3.45 & 4. 55 & 5.0 & 5. 45 \\
\hline $31 .$. & . & & & & & & & & ...... & 4.6 & . & 5.45 \\
\hline
\end{tabular}


Station rating table for Owens River near Citrus, Cal., from January 1 to December 31, 1905.

\begin{tabular}{|r|r||r|r|r|r|r|r|}
\hline $\begin{array}{c}\text { Gage } \\
\text { height. }\end{array}$ & Discharge. & $\begin{array}{c}\text { Gage } \\
\text { height. }\end{array}$ & Discharge. & $\begin{array}{c}\text { Gage } \\
\text { height. }\end{array}$ & Discharge. & $\begin{array}{c}\text { Gage } \\
\text { height. }\end{array}$ & Discharge. \\
\hline Feet. & Second-feet. & Feet. & Second-feet. & Feet. & Second-feet. & Feet. & Second-feet. \\
2.50 & 0 & 3.70 & 63 & 4.90 & 217 & 6.10 & 440 \\
2.60 & 1 & 3.80 & 73 & 5.00 & 233 & 6.20 & 462 \\
2.70 & 3 & 3.90 & 83 & 5.10 & 249 & 6.30 & 485 \\
2.80 & 5 & 4.00 & 94 & 5.20 & 266 & 6.40 & 508 \\
2.90 & 8 & 4.10 & 105 & 5.30 & 283 & 6.50 & 532 \\
3.00 & 12 & 4.20 & 117 & 5.40 & 301 & 6.60 & 556 \\
3.10 & 18 & 4.30 & 130 & 5.50 & 319 & 6.70 & 581 \\
3.20 & 24 & 4.40 & 144 & 5.60 & 337 & 6.80 & 607 \\
3.30 & 31 & 4.50 & 158 & 5.70 & 356 & 6.90 & 634 \\
3.40 & 38 & 4,60 & 172 & 5.80 & 376 & 7.00 & 662 \\
3.50 & 46 & 4.70 & 187 & 5.90 & 397 & 7.10 & 690 \\
3.60 & 54 & 4.80 & 202 & 6.00 & 418 & 7.20 & 720 \\
& & & & & & \\
\hline
\end{tabular}

Note.-The above table is based on 11 discharge measurements made during 1904-5. It is fairly well defined between gage heights 3 feet and 7 feet.

Estimated monthly discharge of Owens River near Citrus, Cal., for 1905.

\begin{tabular}{|c|c|c|c|c|c|}
\hline & \multirow{2}{*}{, Month. } & \multicolumn{3}{|c|}{ Discharge in second-feet. } & \multirow{2}{*}{$\begin{array}{l}\text { Total in } \\
\text { acre-feet. }\end{array}$} \\
\hline & & Maximum. & Minimum. & Mean. & \\
\hline \multicolumn{2}{|c|}{ January $a_{\ldots}$} & 418 & 283 & 369 & 22,690 \\
\hline \multicolumn{2}{|c|}{ February $a, \ldots \ldots \ldots \ldots \ldots \ldots$} & 720 & 376 & 540 & 29,990 \\
\hline \multicolumn{2}{|c|}{ March 1-17 a $\ldots \ldots$} & 508 & 301 & 372 & 12,540 \\
\hline \multicolumn{2}{|c|}{ June $23-30 \ldots$} & 508 & 337 & 430 & 6,823 \\
\hline \multicolumn{2}{|c|}{ July. - } & 337. & 0 & 175 & 10,760 \\
\hline \multicolumn{2}{|c|}{ September $17-30$. } & 42 & 18 & 29.9 & 830 \\
\hline \multicolumn{2}{|c|}{ October. } & 172 & 42 & 107 & 6,579 \\
\hline \multicolumn{2}{|c|}{ November....... } & 233 & 172 & 219 & 13,030 \\
\hline \multicolumn{2}{|c|}{ 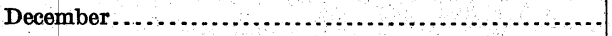 } & 319 & 292 & 301 & 18,510 \\
\hline & 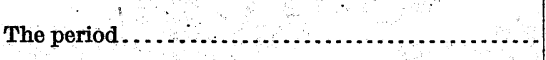 & & & & 121,800 \\
\hline
\end{tabular}

a Missing gage heights interpolated.

POWERS CANAI NEAR BISHOP, CAL,

This station was established August 19, 1903, by R. S. Hawley. It is located one-half mile above a mill on Bishop Creek, and $4 \frac{1}{2}$ miles west of Bishop, Cal.

The canal is straight for 20 feet above and 50 feet below the station. The bed of the canal is composed of gravel and is not subject to change.

Discharge measurements are made by wading.

The gage is a rod on the left bank of the canal. The bench mark is on granite bowlder 60 feet north of penstock of the mill pressure pipe; elevation, 3.50 feet above the datum of the gage.

Information in regard to this station is contained in the following Water-Supply Papers of the United States Geological Survey:

Description: 134, pp 251-252.

Discharge: 134, p 252.

Discharge, monthly: 134, p 253.

Gage heights: 134, p 252.

Rating table: 134, p 252. 
Discharge measurements of Powers canal near Bishop, Cal., in 1905.

\begin{tabular}{|c|c|c|c|c|c|c|}
\hline Date. & Hydrographer. & Width. & $\begin{array}{l}\text { Area of } \\
\text { section. }\end{array}$ & $\underset{\text { velocity. }}{\text { Mean }}$ & $\begin{array}{c}\text { Gage } \\
\text { height. }\end{array}$ & $\begin{array}{l}\text { Dis- } \\
\text { charge. }\end{array}$ \\
\hline April $3 . .$. & J. S. Evans.......... & $\begin{array}{c}\text { Feet. } \\
3\end{array}$ & $\begin{array}{l}S q \cdot f t . \\
0.7\end{array}$ & $\begin{array}{r}\text { Ft.per sec. } \\
1.00\end{array}$ & $\begin{array}{l}\text { Feet. } \\
1.10\end{array}$ & $\begin{array}{r}S e c_{.}-f t . \\
0.7\end{array}$ \\
\hline June $21 \ldots$. & ..... do ................ & 9 & 6.4 & 2.66 & 1.90 & 17.0 \\
\hline July 27 . & . . . do do . . . . . . . & 8 & 5.0 & 2.36 & 1.68 & 11.8 \\
\hline August 10 & ..... do ........... & 7 & 1.3 & 1.08 & 1.30 & 1.4 \\
\hline September $7 \ldots$ & .... do ........... & 7.5 & 2.1 & 1.33 & 1.40 & 2.8 \\
\hline November $23 \ldots$ & F. R. S. Buttemer... & 8 & 1.4 & .79 & 1.25 & 1.1 \\
\hline December $11 \ldots$ & . . do $\ldots \ldots \ldots \ldots \ldots$ & 8 & 3.6 & .89 & 1.58 & 3.2 \\
\hline December $21 \ldots$ & ...... do ..... & & & & 1.16 & 1.0 \\
\hline
\end{tabular}

\section{SOUTH HIILSIDE CANAL NEAR BISHOP, CAL.}

This station was established August 26, 1903, by R. S. Hawley. It is located just below waste gate at head of canal; 5 miles west of Bishop, Cal.

Discharge measurements are made by wading.

The gage is a rod on the left bank of the canal. The bench mark is on granite bowlder marked by ring of white paint and located on left bank 2 feet from gage; elevation, 2.99 feet above the datum of the gage.

Information in regard to this station is contained in the following Water-Supply Papers of the United States Geological Survey:

Description: 134, p 253.

Discharge: 134, p 253.

Discharge, monthly: 134, p 255.

"Gage heights: 134, p 254.

Rating table: 134, p 254 .

Discharge measurements of South Hillside canal near Bishop, Cal., in 1905.

\begin{tabular}{|c|c|c|c|c|c|c|}
\hline Date. & Hydrographer. & Width. & $\begin{array}{l}\text { Area of } \\
\text { section. }\end{array}$ & $\begin{array}{c}\text { Mean } \\
\text { velocity. }\end{array}$ & $\begin{array}{c}\text { Gage } \\
\text { height. }\end{array}$ & $\begin{array}{l}\text { Dis- } \\
\text { charge. }\end{array}$ \\
\hline January $21 \ldots$ & R. J. Taylor.... & $\begin{array}{r}\text { Feet. } \\
4.5\end{array}$ & $\begin{array}{r}\text { Sq. ft. } \\
0.9\end{array}$ & $\begin{array}{r}\text { Ft. per sec. } \\
1.11\end{array}$ & $\begin{array}{r}\text { Feet. } \\
1.60\end{array}$ & $\begin{array}{r}\text { Sec.-ft. } \\
1.0\end{array}$ \\
\hline February 8 & ..... do ............ & 4.5 & .9 & . 1.11 & 1.60 & 1.0 \\
\hline March $4 . . . .$. & .....do $\ldots . . . . . . . . . . . . . .$. & 4.5 & 1.3 & 1.46 & 1.66 & 1.9 \\
\hline April $18 \ldots$. & J. S. Evans............. & 4.8 & 1.6 & 1.88 & 1.70 & 3.0 \\
\hline May $5 . .$. & ..... do ..... & 4.8 & 3.2 & 2.66 & 2.00 & 8.5 \\
\hline July $27 \ldots$ & ..... do ..... & 4.5 & 2.4 & 3.29 & 1.98 & 7.9 \\
\hline August $10 \ldots$. & ..... do ... & 5.0 & 3.6 & 2.47 & 2.00 & 8.9 \\
\hline September: 22 . & ..... do ..... & 4.5 & 3.2 & 2.75 & 2.00 & 8.8 \\
\hline October $25 \ldots$ & F. R. S. Buttemer................ & 4.0 & 2.0 & 1.75 & 1.75 & 3.5 \\
\hline November 23 . & $\mid \ldots \ldots$ do $\ldots \ldots \ldots \ldots \ldots \ldots \ldots \ldots$ & 5.0 & 1.0 & .90 & 1.58 & .9 \\
\hline December $11 \ldots$ & 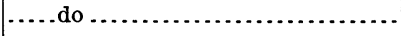 & 3.7 & .6 & .50 & 1.50 & .3 \\
\hline
\end{tabular}




\section{NORTH HILLSIDE CANAL NEAR BISHOP, CAL.}

This station was established September 3, 1903, by R. S. Hawley. It is located at head gate of the canal, 5 miles west of Bishop, Cal.

Discharge measurements are made from crosspiece of the flume on which the distances across are marked at each foot. The initial point for soundings is the end of crosspiece of flume at right bank.

The gage is a vertical rod fastened to the right side of flume or head gate. The bench mark is on a granite bowlder marked with white paint and located near gage; elevation, 2.96 feet above the datum of the gage.

Information in regard to this station is contained in the following Water-Supply Papers of the United States Geological Survey:

Description: 134, p 255.

Discharge: 134, p 255.

Discharge, monthly: 134, p 256.

Gage heights: 134, p 256.

Rating table: 134, p 256.

Discharge measurements of North Hillside Canal near Bishop, Cal., in 1905.

\begin{tabular}{|c|c|c|c|c|c|c|}
\hline Date. & Hydrographer. & Width. & $\begin{array}{l}\text { Area of } \\
\text { section. }\end{array}$ & $\begin{array}{c}\text { Mean } \\
\text { velocity. }\end{array}$ & $\begin{array}{c}\text { Gage } \\
\text { height. }\end{array}$ & $\begin{array}{c}\text { Dis- } \\
\text { charge. }\end{array}$ \\
\hline & & Feet. & sq. ft. & Ft.per sec. & Feet. & Sec.-ft. \\
\hline March 4.. & R. J. Taylor.. & 4.3 & 1.8 & 0.83 & 1.51 & 1.5 \\
\hline April $18 . . . .$. & J. S. Evans.. & 4 & 2.2 & 2.23 & 1.70 & 4.9 \\
\hline May $5 . . . . . .$. & .... do.......... & 4 & 3.6 & 3.33 & 2.00 & 12.0 \\
\hline June $21 . . . . .$. & .....do............. & 4 & 3.0 & 7.67 & 2.10 & 23.0 \\
\hline July $27 . . . . . .$. & . . . do............... & 4 & 2.4 & 7.83 & 2.05 & 18.8 \\
\hline August 10 . & .....do....... & 4 & 2.6 & 7.46 & 2.08 & 19.4 \\
\hline September $17 .$. & .....do..... & 4 & 3.2 & 3.70 & 1.98 & 11.8 \\
\hline September $22 .$. & .....do..... & 4.2 & 2.5 & 5.20 & 2.0 & 13.0 \\
\hline October $23 \ldots$. & .....do... & 4.0 & 2.0 & 1.95 & 1.70 & 3.9 \\
\hline November $23 .$. & .....do. . & 6 & 2.4 & 1.21 & 1.70 & 2.9 \\
\hline December $11 \ldots$ & .....do... & 4.3 & 2.2 & .32 & 1.52 & .7 \\
\hline December 21... & 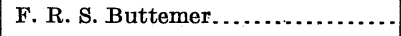 & 4.5 & .8 & .75 & 1.45 & .6 \\
\hline
\end{tabular}

\section{BIG PINE CREEK NEAR BIG PINE, CAL.}

This station was established December 5, 1903, by R. S. Hawley. It is located 3 miles southwest of Big Pine, Cal., at a point where the creek leaves the foothills.

The channel is straight for 30 feet above and 50 feet below the station. The current is swift at all stages. Both banks are high and rocky. The bed of the stream is rough and rocky, and is not subject to much change.

Discharge measurements are made from a footbridge. The initial point is at the right bank.

The gage is a veritical rod fastened to a tree on the left bank of the creek, 3 feet above the footbridge. During 1905 the gage was read by Mrs. J. M. Randle. Bench marks were established as follows: (1) On a granite bowlder marked by a ring of white paint, 40 feet southeast of the south end of the footbridge; elevation, 8.54 feet; (2) a point marked with a ring of white paint, 45 feet southwest of the south end of the footbridge; elevation, 10.44 feet. Elevations refer to the datum of the gage.

Information in regard to this station is contained in the following Water-Supply Papers of the United States Geological Survey:

Description: 134, p 257.

Discharge: $134, \mathrm{pp} 257,260$.

Discharge, monthly: 134, p.259.

Gage heights: 134, p 258.

Rating table: 134, p 258. 
Discharge measurements of Big Pine Creek near Big Pine, Cal., in 1905.

\begin{tabular}{|c|c|c|c|c|c|c|}
\hline Date. & Hydrographer. & Width. & $\begin{array}{l}\text { Area of } \\
\text { section. }\end{array}$ & $\begin{array}{c}\text { Mean } \\
\text { velocity. }\end{array}$ & $\begin{array}{c}\text { Gage } \\
\text { height. }\end{array}$ & $\begin{array}{c}\text { Dis- } \\
\text { charge. }\end{array}$ \\
\hline & & Feet. & $S q . f t$ & Ft.per sec. & Feet. & Sec.-ft. \\
\hline January $18 \ldots$ & R. J. Taylor. & 13 & 9.9 & 2.52 & 1.95 & \\
\hline February 18 . & ......do.... & 12.5 & 8.5 & 2.71 & 1.85 & 23 \\
\hline April $26 \ldots . .$. & J. S. Evans... & 13 & 10.8 & 2.78 & 2.20 & 30 \\
\hline May $19 . \ldots \ldots$. & .....do........ & 13 & 13.8 & 3.70 & 2.38 & 51 \\
\hline June $9 . . . . . .$. & $\ldots$ do........ & 13 & 15.8 & 3.86 & 2.48 & 61 \\
\hline July $16 . \ldots \ldots \ldots$ & .....do......... & 13.5 & 21.0 & 5.71 & 2.85 & 120 \\
\hline August $17 \ldots$. & .....do... & 13 & 18.4 & 4. 77 & 2.60 & 88 \\
\hline September 13. & .....do...... & 13 & 13.0 & 3.15 & 2.30 & 41 \\
\hline November $16 .$. & F. R. S. Buttermer. & 10 & 6.6 & 1.61 & 1.85 & 10.6 \\
\hline December $1 \ldots$. & .....do........ & 13.5 & 8.8 & 1.62 & 2.00 & 14.3 \\
\hline December $16 \ldots$ & .....do.. & 13.5 & 12.6 & 0.83 & 2.20 & 10.5 \\
\hline December $16 \ldots$ & .... do...... & 13.5 & 12.5 & 0.98 & 2.20 & 12.2 \\
\hline
\end{tabular}

$a$ Channel changed.

Daily gage height, in feet, of Big Pine Creek near Big Pine, Cal., for 1905.

\begin{tabular}{|c|c|c|c|c|c|c|c|c|c|c|c|c|}
\hline Day. & Jan. & Feb. & Mar. & Apr. & May. & June. & July. & Aug. & Sept. & Oct. & Nov. & Dec. \\
\hline & 1.85 & 1.9 & 1.85 & 1.8 & 2.5 & 2.4 & 2.95 & 2.9 & 2.7 & 225 & 1.8 & \\
\hline 2. & 1.85 & 1.9 & 1.9 & 1.9 & 2.5 & 2.4 & 3.0 & 2.9 & 2.6 & 2.15 & & 1.9 \\
\hline 3. & 1.85 & 1.9 & 1.9 & 1.85 & 2.5 & 2.4 & 3.0 & 2.9 & 2.5 & 2.15 & & \\
\hline $4 .$. & 1.85 & 2.0 & 1.9 & 1.9 & 2.5 & 2.4 & 3.0 & 2.9 & 2.4 & 2.15 & & 1.9 \\
\hline 5. & 1.85 & 20 & 1.9 & 1.9 & 2.4 & 2.4 & 3.0 & 2.9 & 2.4 & 2.1 & & \\
\hline $6 \ldots$ & 1.85 & 2.0 & 1.9 & 1.85 & 2.4 & 2.45 & 3.0 & 2.9 & 2.35 & 2.0 & & \\
\hline $7 .$. & 1.85 & 1.95 & 1.9 & 1.85 & 2.4 & 2.45 & 3.0 & 2.8 & 2.25 & 2.0 & $\cdots$ & \\
\hline 8. & 1.85 & 1.95 & 1.9 & 1.9 & 2.3 & 2.5 & 3.0 & 2.8 & 2.25 & 2.0 & 1.8 & \\
\hline 9. & 1.9 & 1.9 & 1.9 & 1.95 & 2.0 & 2.5 & 3.2 & 2.9 & 2.2 & 2.0 & & 1.9 \\
\hline $10 .$. & 1.9 & 1.9 & 1.85 & 1.95 & 2.0 & 2.5 & 3.5 & 3.0 & 2.3 & 2.0 & ........ & \\
\hline $11 .$. & 1.85 & 1.9 & 1.85 & 1.95 & 2. 0 & 2.7 & 3.5 & 3.0 & 2.4 & 2.0 & 1.85 & 2.0 \\
\hline $12 .$. & 1.8 & 1.9 & 1.9 & 1.95 & 2.0 & 2.8 & 3.5 & 3.0 & 2.4 & 2.0 & & \\
\hline 13. & 1.8 & 1.9 & 1.9 & 1.95 & 2.1 & 2.9 & 3.3 & 2.9 & 2.45 & 2.0 & & \\
\hline $14 .$. & 1.8 & 1.9 & 1.9 & .1 .95 & 2.2 & 2.9 & 3.3 & 2.9 & 2.45 & 2.0 & & \\
\hline $15 .$. & 1.8 & 1. 85 & 1.9 & 1.95 & 2.3 & 2.9 & 3.3 & 2.9 & 2.45 & 1.85 & 1.85 & 2.0 \\
\hline 16. & 1.85 & 1.85 & 1.9 & 1.95 & 2.3 & 2.9 & 3.05 & 2.9 & 2.45 & 1.85 & & \\
\hline $17 .$. & 1.85 & 1.85 & 1.9 & 1.95 & 2.3 & 2.95 & 3.2 & 2.9 & 2.35 & 1.8 & & \\
\hline $18 .$. & 1.85 & 1.85 & 1.9 & 195 & 2.4 & 3.0 & 3.1 & 2.8 & 2.4 & 1.8 & 1.8 & \\
\hline $19 \ldots$ & 1.85 & 1.85 & 1.9 & 1.95 & 2.4 & 3.0 . & 3.0 & 2.8 & 2.45 & 1.8 & & \\
\hline $20 .$. & 1.85 & 1. 85 & 1.9 & 1.95 & 2.4 & 3.0 & 3.0 & 2.9 & 2. 45 & 1.8 & & 2.2 \\
\hline $21 \ldots$ & 1.85 & 1.85 & 1.9 & 1.95 & 2.5 & 3.2 & 3.0 & 2.8 & 2.4 & 1.8 & & \\
\hline $22 \ldots$ & 1.8 & 1.85 & 1.9 & 1.95 & 2.5 & 3.3 & 3.0 & 2.8 & 2.4 & 1.8 & & \\
\hline $23 .$. & 1.85 & 1.85 & 1.9 & 2.0 & 2.4 & 3.4 & 3.1 & 2.7 & 2.4 & 1.8 & 1.85 & 2.15 \\
\hline $24 \ldots$ & 1.85 & 1.85 & 1.9 & 2.0 & 2.4 & 3.2 & 3.2 & 2.7 & 2.35 & 1.8 & & \\
\hline $25 \ldots$ & 1.85 & 1.85 & 1.9 & 2.0 & 2.4 & 2.8 & 3.3 & 2.7 & 2.3 & 1.8 & & \\
\hline $26 \ldots$ & 1.85 & 1.85 & 1.9 & 2.2 & 2.4 & 2.8 & 3.4 & 2.7 & 2.3 & 1.8 & & 2.3 \\
\hline $27 \ldots$ & 1.85 & 1.85 & 1.9 & 2.3 & 2.4 & 2.8 & 3.3 & 2.7 & 2.3 & 1.8 & & \\
\hline $28 .$. & 1.85 & 1.85 & 1.8 & 2.3 & 2.4 & 2.9 & 3.2 & 2.7 & 2.3 & 1.8 & 1.85 & \\
\hline $29 \ldots$ & 1.85 & 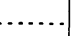 & 1.8 & 2.4 & 2.4 & 2: 9 & 3.1 & 2.7 & 2.3 & 1.8 & & \\
\hline $30 \ldots$ & 1.85 & & 1.8 & 2.5 & 2.4 & 2.9 & 3.1 & 2.7 & 2.3 & 1.8 & & \\
\hline 31... & 1.85 . & ....... & 1. 8 & $\ldots$ & 2.4 & 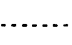 & 3.0 & 2.7 & …... & 1.8 & ......... & \\
\hline
\end{tabular}


Station rating table for Big Pine Creek near Big Pine, Cal., from March 21 to December 15, 1905.

\begin{tabular}{|c|c|c|c|c|c|c|c|}
\hline $\begin{array}{c}\text { Gage } \\
\text { height. }\end{array}$ & Discharge. & $\begin{array}{c}\text { Gage } \\
\text { height. }\end{array}$ & Discharge. & $\begin{array}{c}\text { Gage } \\
\text { height. }\end{array}$ & Discharge. & $\begin{array}{l}\text { Gage } \\
\text { height. }\end{array}$ & Discharge. \\
\hline $\begin{array}{r}\text { Feet. } \\
1.80\end{array}$ & $\mid \begin{array}{r}\text { Second-feet. } \\
9\end{array}$ & $\begin{array}{r}\text { Feet. } \\
2.30\end{array}$ & $\begin{array}{r}\text { Second-feet. } \\
40\end{array}$ & $\begin{array}{l}\text { Feet. } \\
2.80\end{array}$ & $\begin{array}{c}\text { Second-feet. } \\
115\end{array}$ & $\begin{array}{l}\text { Feet. } \\
\text { 3. } 30\end{array}$ & $\begin{array}{c}\text { Second-feet. } \\
220\end{array}$ \\
\hline 1.90 & $\therefore \quad 11$ & 2.40 & 52 & 2.90 & 134 & 3.40 & 244 \\
\hline 2.00 & 15 & 2.50 & 66 & 3.00 & 154 & 3.50 & 268 \\
\hline 2.10 & 21 & 2.60 & 81 & 3.10 & 175 & & \\
\hline 2.20 & 30 & 2.70 & 97 & 3.20 & 197 & & \\
\hline
\end{tabular}

Note.-The above table is based on eight discharge measurements made during 1905 . It is fairly well defined between gage heights 2 feet and 2.8 feet. The table has been extended beyond these limits, : being based on extension of area and mean-velocity curves.

Estimated monthly discharge of Big Pine Creek near Big Pine, Cal., for 1905.

\begin{tabular}{|c|c|c|c|c|}
\hline \multirow{2}{*}{ Month. } & \multicolumn{3}{|c|}{ Discharge in second-feet. } & \multirow{2}{*}{$\begin{array}{l}\text { Total in } \\
\text { acre-feet. }\end{array}$} \\
\hline & Maximum. & Minimum. & Mean. & \\
\hline January.. & 24 & 19 & 21.7 & 1,334 \\
\hline 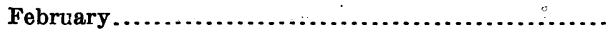 & 30 & $\cdot 22$ & 23.9 & 1,327 \\
\hline March... & 24 & 9 & 18.9 & 1,162 \\
\hline April............. & 66 & 9 & 17.9 & . $\quad 1,065$ \\
\hline May ........... & 66 & 15 & 46. 7 & 2,872 \\
\hline June.................. & 244 & 52 & 118 & 7,021 \\
\hline July ................ & 268 & 144 & 187 & 11,500 \\
\hline August...................... & 154 & 97 & 122 & 7,501 \\
\hline September............ & 97 & 30 & 51.1 & 3,041 \\
\hline October............... & 35 & 9 & 13.7 & 842 \\
\hline November............ & 10 & 9 & 9.6 & \\
\hline December 1-15 .......... & 15 & 11 & 12.5 & 372 \\
\hline The period & & & & 38,610 \\
\hline
\end{tabular}

Note.-Discharge January 1 to March 20 based on discharge measurements directly. Rating table used March 21 to December 15. Because of a change in channel the rating table does not apply after December 15.

Discharges for days in November and December, when gage heights are not recorded, were interpolated.

\section{BIRCH OREEK NEAR TINEMAHA, CAL.}

This station was established June 14, 1905, by J. S. Evans. It is located 6 miles southwest of Big Pine and 500 feet west of ranch of Charles Peterson.

The channel is straight for 25 feet above and below the station. The water is swift at all stages. Both banks are low, but not subject to overflow. The bed of the stream is composed of coarse gravel and is not subject to material change. The stream flows in one channel.

Discharge measurements are made from a footbridge, which consists of a cottonwood tree placed across the stream. The initial point for soundings is a nail in footbridge on the left bank.

The gage is a vertical staff nailed to a willow tree on the left bank. During 1905 the gage was read by Charles Peterson. The bench mark is a nail in the top of a.willow stump on the left bank; elevation, 2.00 feet above the datum of the gage. 
Discharge measurements of Birch Creek near Tinemaha, Cal., in 1905.

\begin{tabular}{|c|c|c|c|c|c|c|}
\hline Date. & Hydrographer. & Width. & $\begin{array}{l}\text { Area of } \\
\text { section. }\end{array}$ & $\begin{array}{c}\text { Mean } \\
\text { velocity. }\end{array}$ & $\begin{array}{c}\text { Gage } \\
\text { height. }\end{array}$ & $\begin{array}{l}\text { Dis- } \\
\text { charge. }\end{array}$ \\
\hline June 14.. & J. S. Evans............... & $\begin{array}{r}\text { Feet. } \\
7\end{array}$ & $\begin{array}{r}S q . f t \\
9.6\end{array}$ & $\begin{array}{r}\text { Ft. per sec. } \\
2.19\end{array}$ & $\begin{array}{l}\text { Feet. } \\
1.00\end{array}$ & $\begin{array}{r}\text { Sec.-ft. } \\
21\end{array}$ \\
\hline July $17 \ldots$ & ....do........... & 8 & 8.0 & 1.35 & .71 & 10.8 \\
\hline August $24 \ldots .$. & .....do...$\ldots \ldots \ldots \ldots$ & 8 & 7.6 & 1.00 & .58 & 7.6 \\
\hline November 17 .. & F. R. S. Buttemer............... & 8 & 6.2 & .35 & .55 & 2.2 \\
\hline December 2.... & 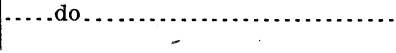 & 8 & $6 . \overline{0}$ & .42 & .55 & 2.5 \\
\hline
\end{tabular}

Daily gage height, in feet, of Birch Creek near Tinemaha, Cal., for 1905.

\begin{tabular}{|c|c|c|c|c|c|c|c|}
\hline Day. & June. & July. & Aug. & Sept. & Oct. & Nov. & Dec. \\
\hline 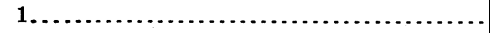 & & 0.8 & 0.65 & 0.6 & 0.5 & 0.35 & 0.8 \\
\hline $2 \ldots \ldots \ldots \ldots \ldots$ & . & .75 & .65 & .55 & .4 & .35 & .55 \\
\hline $3 \ldots \ldots \ldots \ldots$ & & .75 & .65 & .6 & .4 & .35 & .55 \\
\hline $4 \ldots \ldots \ldots \ldots \ldots \ldots$ & & .8 & .65 & .55 & .35 & .45 & .55 \\
\hline 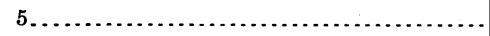 & ... & .8 & .6 & .55 & .35 & .5 & .5 \\
\hline 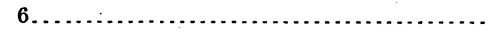 & -. & .8 & .55 & .5 & .3 & .5 & .5 \\
\hline 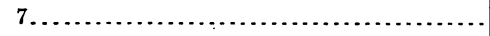 & $\cdots$ & .85 & .6 & .5 & .3 & .5 & .5 \\
\hline $8 \ldots \ldots \ldots \ldots \ldots \ldots \ldots$ & & .9 & .65 & .45 & .3 & .5 & .5 \\
\hline $9 \ldots \ldots \ldots, \ldots$ & & .9 & .75 & .45 & .35 & .5 & .5 \\
\hline 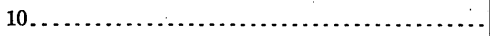 & - & .85 & .8 & .5 & .35 & .5 & $\ldots \ldots$ \\
\hline $11 \ldots \ldots \ldots \ldots$ & & .9 & .65 & .5 & .35 & .5 & .......... \\
\hline 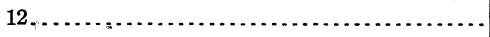 & ... & .9 & .6 & .5 & .35 & .5 & . $\ldots \ldots$ \\
\hline $13 \ldots \ldots \ldots \ldots \ldots \ldots$ & - & .85 & .6 & .45 & .35 & .5 & ......... \\
\hline $14 \ldots \ldots \ldots \ldots \ldots \ldots$ & 1.0 & .75 & .6 & .45 & .35 & .5 & (......... \\
\hline 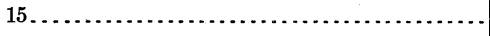 & $\ldots \ldots$ & .7 & .6 & .45 & .35 & .5 & $\ldots \ldots$ \\
\hline $16 \ldots \ldots \ldots \ldots \ldots \ldots$ & $\ldots$ & .7 & .55 & .4 & .35 & .5 & $\ldots \ldots$ \\
\hline 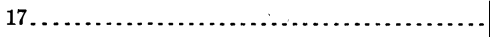 & $\ldots$ & .7 & .55 & .4 & .35 & .5 & ......... \\
\hline 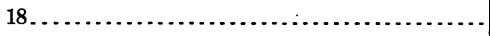 & .9 & .7 & .55 & .4 & .35 & .5 & $\ldots \ldots$ \\
\hline 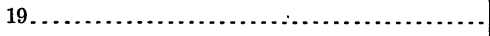 & .9 & .65 & .5 & .4 & .35 & .5 & ......... \\
\hline 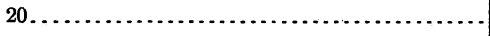 & .9 & .65 & .6 & .4 & .35 & .5 & ......... \\
\hline 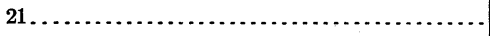 & .8 & .65 & .65 & .4 & .35 & .5 & ......... \\
\hline 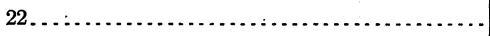 & 1.0 & -.65 & .65 & .4 & .35 & .5 & ........ \\
\hline 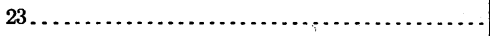 & .8 & .7 & .55 & .4 & .35 & .5 & \\
\hline $24 \ldots \ldots \ldots \ldots \ldots \ldots \ldots$ & .9 & .7 & .55 & .4 & .35 & .5 & $\ldots$ \\
\hline 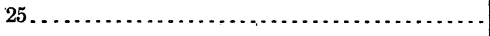 & .75 & .75 & .55 & .4 & .35 & .5 & ...... \\
\hline $26 \ldots \ldots \ldots$ & .75 & .8 & .55 & .4 & .35 & .5 & $\ldots \ldots$ \\
\hline $27 \ldots \ldots \ldots \ldots$ & .75 & .8 & .6 & .4 & .35 & .5 & $\ldots \ldots$ \\
\hline $28 \ldots \ldots \ldots \ldots$ & .75 & .75 & .6 & .4 & .35 & .65 & \\
\hline $29 \ldots \ldots \ldots \ldots \ldots \ldots \ldots \ldots \ldots \ldots \ldots$ & .8 & .7 & .65 & .45 & .35 & .7 & $\ldots \ldots$ \\
\hline 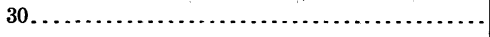 & .85 & .65 & .65 & .45 & .35 & .6 & ......... \\
\hline $31 \ldots \ldots \ldots \ldots \ldots \ldots$ & ......... & .65 & .65 & ......... & .35 & (......... & -........ \\
\hline
\end{tabular}

\section{INDEPENDENCE CREEK NEAR INDEPENDENCE, CAL.}

This station was established June 15, 1905, by J. S. Evans. · It is located about 1 mile west of the town of Independence near intake of the city waterworks of Independence.

The channel is straight for 25 feet above and below the gaging station. Both banks are low, but not subject to overfiow. The bed of the stream is composed of gravel and rocks. The approximate depth of water is 1.2 feet. The stream discharges in one channel.

Discharge measurements are made by wading. The initial point for soundings is on the right bank. 
The gage is a vertical timber nailed to a willow tree on the right bank. During 1905 the gage was read by Milton Levy. The bench mark is a cross on large rock on right bank of the stream; elevation, 1.70 feet above the datum of the gage.

Discharge measurements of Independence Creek near Independence, Cal., in 1905.

\begin{tabular}{|c|c|c|c|c|c|c|}
\hline Date. & Hydrographer. & Width. & $\begin{array}{l}\text { Area of } \\
\text { section. }\end{array}$ & $\begin{array}{c}\text { Mean } \\
\text { velocity. }\end{array}$ & $\begin{array}{l}\text { Gage } \\
\text { height. }\end{array}$ & $\begin{array}{c}\text { Dis- } \\
\text { charge. }\end{array}$ \\
\hline June $15 . . . \ldots$. & J. S. Evans $\ldots \ldots \ldots \ldots \ldots \ldots \ldots \ldots \ldots$ & Feet. & $\begin{array}{r}S q \cdot f t . \\
13.6\end{array}$ & $\begin{array}{r}\text { Ft. per sec. } \\
3.46\end{array}$ & $\begin{array}{l}\text { Feet. } \\
1.10\end{array}$ & $\begin{array}{r}\text { Sec.-ft. } \\
47\end{array}$ \\
\hline July $21 . . . \ldots$. & ....do......... & 12 & 8.0 & 2.42 & .72 & 19.4 \\
\hline August 23. . & . ...do ................. & 11 & 5.0 & 1.46 . & .47 & 7.3 \\
\hline November 11 .. & F. R. S. Buttemer........ & 11 & 3.9 & .79 & .35 & 3.1 \\
\hline December 3.... & .....do................... & 11 & 4.6 & .80 & .37 & 3.7 \\
\hline
\end{tabular}

Daily gage height, in feet, of Independence Creek near Independence, Cal., for 1905.

\begin{tabular}{|c|c|c|c|c|c|c|c|}
\hline Day. & June. & July. & Aug. & Sept. & Oct. & Nov. & Dec. \\
\hline & & 0.9 & 0.65 & 0.45 & 0.35 & 0.35 & 0.4 \\
\hline $2 \ldots$ & $\cdots$ & $-\quad .9$ & .65 & .45 & .35 & .35 & .4 \\
\hline 3... & & .85 & .65 & .45 & .35 & .35 & .4 \\
\hline 4... & $\because$ & .85 & .6 & .45 & .35 & .35 & .4 \\
\hline 5. & & .85 & .6 & .45 & .35 & .35 & .4 \\
\hline $6 \ldots$ & 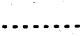 & .85 & .6 & .45 & .35 & .35 & .4 \\
\hline • $7 \ldots$ & .. & .9 & .55 & .45 & .35 & .35 & .94 \\
\hline $8 .$. & .... & .85 & .55 & .45 & .35 & .35 & .35 \\
\hline $9 \ldots$ & & .85 & .55 & .45 & .3 & .35 & .4 \\
\hline 10. & . & .85 & .55 & .35 & .35 & .35 & .4 \\
\hline 11.. & ..... & .9 & .55 & .35 & .35 & .35 & .4 \\
\hline 12. & 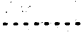 & .9 & .55 & .35 & .35 & .35 & .3 \\
\hline 13.. & . & .9 & .55 & .4 & $: 35$ & .35 & .35 \\
\hline 14.. & $\ldots$ & .85 & .55 & .4 & .35 & .35 & .35 \\
\hline 15. & 1.1 & .8 & .55 & .35 & .4 & .35 & .4 \\
\hline $16 \ldots \ldots \ldots \ldots$ & 1.1 & .8 & .55 & .35 & .4 & .35 & .4 \\
\hline $17 \ldots \ldots \ldots \ldots \ldots$ & 1.1 & .75 & .55 & .4 & .35 & .35 & .4 \\
\hline ........... & 1.1 & .75 & .55 & .4 & .35 & .35 & .35 \\
\hline 19. & 1.1 & .75 & .55 & .4 & .35 & .35 & .4 \\
\hline $20 .$. & 1.1 & .75 & .55 & .35 & .35 & .35 & .4 \\
\hline $21 \ldots \ldots$ & 1.1 & .7 & .55 & .35 & .4 & .35 & .3 \\
\hline 22. & 1.05 & .7 & .55 & .35 & .35 & .35 & .3 \\
\hline $23 \ldots$ & 1.05 & .7 & .55 & .4 & .35 & .4 & .35 \\
\hline $24 .$. & 1.1 & .7 & .55 & .4 & .35 & .4 & .35 \\
\hline 25. & .95 & .7 & .55 & .35 & .35 & .4 & .3 \\
\hline $26 \ldots$ & .95 & .7 & .55 & .35 & .35 & .4 & .3 \\
\hline $27 \ldots$ & .95 & .7 & .5 & .35 & .35 & .4 & .3 \\
\hline $28 \ldots \ldots$ & .95 & .7 & .5 & .35 & .35 & .4 & .35 \\
\hline $29 . \ldots \ldots \ldots$ & .9 & .7 & .5 & .35 & .35 & .4 & .35 \\
\hline $30 \ldots \ldots \ldots \ldots \ldots \ldots$ & .9 & .7 & .5 & .35 & .35 & .4 & .35 \\
\hline & & .65 & .45 & $\ldots \ldots \ldots$ & .35 & ......... & .35 \\
\hline
\end{tabular}

Note.-Gage heights interpolated June 16-20; October 29 to November 10; November 12 to December 2. 
Station rating table for IndependenceCreek near Independence, Cal., from June 15 to December $31,1905$.

\begin{tabular}{|c|c||c|c||c|c||c|c|}
\hline $\begin{array}{c}\text { Gage } \\
\text { height. }\end{array}$ & Discharge. & $\begin{array}{c}\text { Gage } \\
\text { height. }\end{array}$ & Discharge. & $\begin{array}{c}\text { Gage } \\
\text { height. }\end{array}$ & Discharge. & $\begin{array}{c}\text { Gage } \\
\text { height. }\end{array}$ & Discharge. \\
\hline Feet. & Second-feet. & Feet. & Second-feet. & Feet. & Second-feet. & Feet. & Second-feet. \\
0.30 & 1.6 & 0.60 & 12.9 & 0.80 & 24 & 1.00 & 39 \\
0.40 & 4.8 & 0.70 & 18 & 0.90 & 31 & 1.10 & 47 \\
0.50 & 8.5 & & & & & \\
\hline
\end{tabular}

Note.-The above table is based on five discharge measurements made during 1905. It is well defind between gage heights. 0.4 foot and 1.1 feet.

Estimated monthly discharge of Independence Creek near Independence, Cal., for 1905.

\begin{tabular}{|c|c|c|c|c|}
\hline \multirow{2}{*}{ Month. } & \multicolumn{3}{|c|}{ Discharge in second-feet. } & \multirow{2}{*}{$\begin{array}{l}\text { Total in } \\
\text { acre-feet. }\end{array}$} \\
\hline & Maximum. & Minimum. & Mean. & \\
\hline June $15-30$. & 47 & 31 & 41.5 & 1,317 \\
\hline July $. . . . . \ldots \ldots \ldots . . . . . .$. & 31 & 15.4 & 23.5 & 1,445 \\
\hline August.................... & 15.4 & 6.6 & 10.9 & 670 \\
\hline September............. & 6.6 & 3.1 & 4.6 & 274 \\
\hline October................ & 4.8 & 1.6 & 3.2 & 198 \\
\hline November............. & 4.8 & 3.1 & 3.6 & 214 \\
\hline \multirow{2}{*}{ 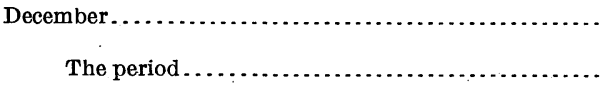 } & 4.8 & 1.6 & 3.6 & 223 \\
\hline & & & & 4,341 \\
\hline
\end{tabular}

OAK CREEK NEAR INDEPENDENCE, CAL.

This station was established June 15, 1905, by J. S. Evans. It is located about 1 mile west of old Fort Independence and 3 miles northwest of the town of Independence.

The channel is straight for a short distance above and below the station. Both banks are low and subject to slight overflow in high water. The bed of the stream is composed of gravel and rocks, with high velocity at all stages. The stream flows in one channel and has an average depth of 1.2 feet.

Discharge measurements are usually made by wading, although a plank placed across the stream is used as a footbridge for higher stages of the water. The initial point for soundings is a nail in the footbridge on the left bank.

The gage is a staff nailed to a stake driven on the left bank of the stream. During 1905 the gage was read by A. M. Bell. The bench mark is a nail driven in the top of a stake set firmly in the ground on the right bank of the stream near the end of the footbridge; elevation, 1.30 feet above the datum of the gage.

Discharge measurements of Oak Creek near Independence, Cal., in 1905.

\begin{tabular}{|c|c|c|c|c|c|c|}
\hline . Date. & Hydrographer: & Width. & $\begin{array}{l}\text { Area of } \\
\text { section. }\end{array}$ & $\begin{array}{c}\text { Mean } \\
\text { velocity. }\end{array}$ & $\begin{array}{c}\text { Gage } \\
\text { height. }\end{array}$ & $\begin{array}{l}\text { Dis- } \\
\text { charge. }\end{array}$ \\
\hline June $15 .$. & J. S. Evans.. & $\begin{array}{r}\text { Feet. } \\
6\end{array}$ & $\begin{array}{r}S q . f t . \\
8.4\end{array}$ & Ft.per sec. & $\begin{array}{l}\text { Feet. } \\
\quad 1.10\end{array}$ & $\begin{array}{r}\text { Sec.-ft. } \\
32\end{array}$ \\
\hline July $17 . . . \ldots$ & .....do...$\ldots \ldots \ldots \ldots \ldots$ & 5 & 4.4 & 4.00 & .63 & 17.6 \\
\hline August 23.. & .....do....... & 5 & 2.8 & 4.18 & .30 & 11.7 \\
\hline November $8 .$. & F. R. S. Buttemer ........ & 5 & 3.4 & 3.24 & .35 & $11: 0$ \\
\hline December 4 .. & ....do..... & 6 & 3.1 & 1.87 & .25 & 5.8 \\
\hline
\end{tabular}


Daily gage height, in feet, of Oak Creek near Independence, Cal., for 1905.

\begin{tabular}{|c|c|c|c|c|c|c|c|}
\hline Day. & June. & July. & Aug. & Sept. & Oct. & Nov. & Dec. \\
\hline 1.. & & 1.0 & 0.6 & 0.4 & 0.3 & 0.3 & 0.3 \\
\hline 2.. & .. & 1.0 & .5 & .4 & .3 & .3 & .3 \\
\hline 3.. & ... & 1.0 & .5 & .4 & .3 & .3 & .25 \\
\hline 4.. & $\cdots$ & .9 & .5 & .4 & .3 & .3 & .25 \\
\hline 5.. & $\cdots$ & 1.0 & .5 & .4 & .2 & .3 & .3 \\
\hline 6.. & & 1.0 & .5 & .3 & .2 & .4 & .3 \\
\hline 7.. & & 1.0 & .5 & .3 & .2 & .4 & .25 \\
\hline $8 \ldots$ & & 1.0 & .5 & .3 & .3 & .3 & .25 \\
\hline $9 . .$. & $\cdots$ & 1.0 & .5 & .3 & .3 & .4 & .25 \\
\hline $10 .$. & $\cdots$ & 1.1 & .5 & .3 & .3 & .3 & .25 \\
\hline $11 .$. & $\cdots$ & 1.1 & .5 & .3 & .3 & .3 & .25 \\
\hline ......... & -. & 1.1 & .5 & .3 & .3 & .3 & .25 \\
\hline $13 .$. & $\cdots$ & 1.0 . & .5 & .3 & .3 & .3 & .3 \\
\hline $14 .$. & ...... & .9 & .4 & .3 & .2 & .3 & .3 \\
\hline $15 .$. & $\cdots$ & .9 & .4 & .3 & .3 & .3 & .3 \\
\hline $16 .$. & $\cdots$ & .8 & .4 & .2 & .2 & .3 & .3 \\
\hline $17 .$. & $\ldots$. & .8 & .4 & .2 & .2 & .3 & .25 \\
\hline ……..... & 1.3 & .7 & .4 & .2 & .3 & .3 & .25 \\
\hline $19 .$. & 1.3 & .7 & .4 & .2 & .3 & .3 & .25 \\
\hline $20 .$. & 1.3 & .6 & .4 & .2 & .3 & .3 & .25 \\
\hline $21 .$. & 1.4 & .7 & .4 & .2 & .3 & .3 & .25 \\
\hline $22 .$. & 1.2 & .6 & .4 & .2 & .3 & .3 & .25 \\
\hline $23 .$. & 1.2 & .6 & .4 & .2 & .3 & .3 & .25 \\
\hline$\ldots \ldots \ldots$ & 1.1 & .7 & .4 & .2 & .3 & .3 & .25 \\
\hline $25 \ldots \ldots \ldots \ldots$ & 1.0 & .7 & .4 & .2 & .3 & .3 & .25 \\
\hline $26 \ldots \ldots \ldots \ldots \ldots$ & 1.0 & .7 & .4 & .2 & .3 & .3 & .25 \\
\hline $27 \ldots \ldots \ldots \ldots$ & 1.0 & .7 & .4 & .2 & .3 & .3 & .25 \\
\hline $28 \ldots$ & .9 & .7 & .4 & .2 & .3 & .3 & .25 \\
\hline $29 \ldots, \ldots \ldots \ldots \ldots \ldots$ & 1.0 & .6 & .4 & .2 & .3 & .3 & .25 \\
\hline $30 \ldots \ldots \ldots \ldots \ldots \ldots$ & 1.0 & .6 & .4 & .3 & .3 & .3 & .25 \\
\hline 31.. & & .6 & .4 & & .3 & & .25 \\
\hline
\end{tabular}

MISCELLANEOUS MEASUREMENTS IN OWENS RIVER DRAINAGE BASIN.

The following is a list of the miscellaneous discharge measurements made in the Owens River drainage basin during 1905:

Ash Creek near Lone Pine, Cal.-This stream discharges into Owens Lake from the eastern slope of the Sierra Nevada. The following measurements were made during 1905 at a point where the stream leaves the foothills and enters the valley:

June 19: Width, 10 feet; area, 5.4 square feet; mean velocity, 1.96 feet per second; discharge, 10.6 second-feet.

July 19: Width, 4 feet; area, 1 square foot; mean velocity, 1.80 feet per second; discharge, 1.8 second-feet.

Birch Creek near Bishop, Cal.-This stream is tributary to Owens River above Bishop, Cal. A measurement was made August 1, 1905, by J. S. Evans at the point where the stream leaves the foothills and enters the valley.

Width, 6 feet; area, 4.4 square feet; mean velocity, 2.72 feet per second; discharge, 12 second-feet.

Clear Creek near Big Pine, Cal.-This stream is tributary to Owens River from the eastern slope of the Sierra Nevada. The following measurements were made during 1905 at a point where the stream leaves the foothills and enters the valley:

June 14: Width, 7 feet; area, 5.6 square feet; mean velocity, 1.86 feet per second; discharge, 10.4 second-feet. 
July 17: Width, 6 feet; area, 3.4 square feet; mean velocity, 1.61 feet per second; discharge, 5.5 second-feet.

Cottonwood Creek near Olancha, Cal.- - This stream discharges into Owens Lake from the eastern slope of the Sierra Nevada. The gage rod is a 1 by 3 inch timber graduated to feet and tenths and nailed to a cottonwood tree on the right bank of the stream. The following measurements were made during 1905 at a point where the stream leaves the foothills and enters the valley:

June 16: Width, 28 feet; area, 30 square feet; mean velocity, 3.03 feet per second; gage height, 0.80 foot; discharge, 91 second-feet.

July 20: Width, 20 feet; area, 16.2 square feet; mean velocity, 1.86 feet per second; gage height, 0.33 foot; discharge, 30 second-feet.

November 10: Width, 8 feet; area, 5 square feet; mean velocity, 0.86 foot per second; discharge, 4.3 second-feet.

Division Creek near Independence, Cal.-This stream is tributary to Owens River from the eastern slope of the Sierra Nevada. The following discharge measurements were made during 1905 at a point where the.stream leaves the foothills and enters the valley:

June 14: Width, 4 feet; area, 1.9 square feet; mean velocity, 1.74 feet per second; discharge, 3.3 second-feet.

July 17: Width, 4 feet; area, 1.2 square feet; mean velocity, 1.75 feet per second; discharge, 2.1 second-feet.

December 2: Width, 6.5 feet; area, 2.9 square feet; mean velocity, 1.86 feet per second; discharge, 5.4 second-feet.

Eight Mile Creek near Independence, Cal.-This stream is tributary to Owens River from the eastern slope of the Sierra Nevada. A measurement was made December 4, 1905, at a point where the stream leaves the foothills and enters the valley:

Width, 4.5 feet; area, 2.2 square feet; mean velocity, 1.45 feet per second; discharge, 3.2 second-feet.

Fish Springs near Big Pine, Cal.-A measurement was made of the water discharging from these springs on December 4, 1905:

Width, 7 feet; area, 20 square feet; mean velocity, 1.45 feet per second; discharge, 29 second-feet.

Goodale Creek near Tibbetts, Cal.-This stream is a tributary to Owens River from the eastern slope of the Sierra Nevada. The following measurements were made during 1905 at a point where the stream leaves the foothills and enters the valley:

June 14: Width, 4.5 feet; area, 2.6 square feet; mean velocity, 2.27 feet per second; discharge, 5.9 second-feet.

July 17: Width, 3.5 feet; area, 1.8 square feet; mean velocity, 1.94 feet per second; discharge, 3.5 second-feet.

August 22: Width, 3 feet; area, 1.5 square feet; mean velocity, 2.26 feet per second; discharge, 3.4 second-feet.

November 17: Width, 3.7 feet; area, 2.2 square feet; mean velocity, 2.09 feet per second; discharge, 4.6 second-feet.

Georges Creek near Independence, Cal.- -This stream is tributary to Owens River from the eastern slope of the Sierra Nevada. A measurement was made June 15, 1905, at a point where the stream leaves the foothills and enters the valley:

Width, 11 feet; area, 9.2 square feet; mean velocity, 2.61 feet per second; discharge, 24 second-feet.

Lone Pine Creek near Lone Pine, Cal.-This stream is tributary to Owens River from the eastern slope of the Sierra Nevada. The following measurements were made during 1905 at a point where the stream leaves the foothills and enters the valley:

June 15: Width, 13 feet; area, 14.2 square feet; mean velocity, 2.61 feet per second; discharge, 37 second-feet.

July 19: Width, 13 feet; area, 13 square feet; mean velocity, 2.31 feet per second; discharge, 30 second-feet. 
November 9: Width, 12.5 feet; area, 4.2 square feet; mean velocity, 0.83 foot per second; discharge, 3.5 second-feet.

Moffett Creek near Independence, Cal.-Whis stream is tributary to Owens River from the eastern slope of the Sierra Nevada. A measurement was made on June 15 at a point where the stream leaves the foothills and enters the valley:

Width, 7 feet; area, 4 square feet; mean velocity, 2.25 feet per second; discharge, 9 secondfeet.

$M c G e e$ Creek near Bishop, Cal.-This stream is tributary to Owens River from the eastern slope of the Sierra Nevada. A measurement was made on August 1, 1905, at a point where the stream leaves the foothills and enters the valley:

Width, 4 feet; area, 4 square feet; mean velocity, 2.18 feet per second; discharge, 8.7 second-feet.

Shepherds Creek near Independence, Cal.-This stream is tributary to Owens River from the eastern slope of the Sierra Nevada. The following measurements were made during 1905 at a point where the stream leaves the foothills and enters the valley:

June 15: Width, 6 feet; area, 6 square feet; mean velocity, 2.53 feet per second; discharge, 15.2 second-feet.

July 18: Width, 6 feet; area, 2.7 square feet; mean velocity, 2.37 feet per second; discharge, 6.4 second-feet.

Taboose Creek near Tibbetts, Cal.-This stream is tributary to Owens River from the eastern slope of the Sierra Nevada. The gage rod is a 1 by 3 inch timber graduated to feet and tenths and nailed to a willow tree on the left bank of the stream. The bench mark is a nail in willow tree on the left bank, to which gage is fastened. Its elevation is 1.60 feet above the zero of the gage. The following measurements were made during 1905 at a point where the stream leaves the foothills and enters the valley:

June 14: Width, 10 feet; area, 10.6 square feet; mean velocity, 1.68 feet per second; gage height, 0.60 foot; discharge, 17.8 second-feet.

July 17: Width, 10 feet; area, 6.6 square feet; mean velocity, 1.82 feet per second; gage height, 0.40 foot; discharge, 12 second-feet.

August 24: Width, 10 feet; area, 3 square feet; mean velocity, 1.67 feet per second; gage height, 0.10 foot; discharge, 5 second-feet.

November 17: Width, 7 feet; area, 3.8 square feet; mean velocity, 1 foot per second; gage height, 0.10 foot; discharge, 3.8 second-feet.

December 2: Width, 7 feet; area, 4.6 square feet; mean velocity, 1 foot per second; gage height, 0.15 foot; discharge, 4.6 second-foot.

Tuttle Creek near Lone Pine, Cal.-This stream is tributary to Owens River from the eastern slope of the Sierra Nevada. The following measurements were made during 1905 at the point where the stream leaves the foothills and enters the valley:

June 16: Width, 12 feet; area, 6 square feet; mean velocity, 1.80 feet per second; discharge, 10.8 second-feet.

July 19: Width, 10 feet; area, 4.5 square feet; mean velocity, 2.40 feet per second; discharge, 10.8 second-feet.

Tinemaha Creek near Tinemaha, Cal.-This stream is tributary to Owens River from the eastern slope of the Sierra Nevada. The following measurements were made during 1905 at the point where the stream leaves the foothills and enters the valley:

June 14: Width, 8 feet; area, 10.5 square feet; mean velocity, 1.86 feet per second; discharge, 19.5 second-feet.

July 17: Width, 6 feet; area, 4.4 square feet; mean velocity, 3.36 feet per second; discharge, 14.8 second-feet.

December 2: Width, 7 feet; area, 5.2 square feet; mean velocity, 0.60 foot per second; discharge, 3.1 second-feet. 


\section{MOHA VE RIVER DRAINAGE BASIN.}

\section{DESCRIPTION OF BASIN,}

The Mohave River rises on the northern slope of the San Bernardino Mountains, and,flowing in a northerly direction, finally disappears in the sands of the Mohave Desert. This stream has few tributaries, the only ones of importance being West Fork and Deep Creek, which have their source in the higher elevations of the San Bernardino Mountains. The formation is of granite, with good covering of soil. On.the higher elevations there is a .considerable growth of timber, which diminishes as one approaches the lower reaches, changing to a light growth of brush and grass, finally merging into the barren desert. During the greater portion of the year the stream bed is dry below the junction of West Fork and Deep Creek, where the waters disappear in the sand and gravelly bed of the stream. Water again rises at a point lower down on the river above Victorville, where the gaging station is located. Water is diverted above and below the gaging station, but is again returned to the river channel. There are several artesian wells along the river above the gaging station, the water being used for irrigation. This stream does not discharge in any large quantity except during an extremely heavy rainfall in the winter months. The precipitation throughout this basin is very light, with the possible exception of the higher elevation of * the San Bernardino Mountains, where there is a considerable fall of snow during the winter months, which melts in the early spring.

\section{MOHAVE RIVER AT VICTORVILLE, CAL.}

This station was established February 27, 1899, by Bert Cole. It is located in the town of Victorville, a station on the Atchison, Topeka and Santa Fe Railroad, where the Mohave River passes through a narrow gorge locally known as the "Narrows." This place has been under investigation as a possible dam site, and soundings for the depth of bed rock were made by the United States Geological Survey during the season of 1899 . The greatest depth of bed rock was found to be 54 feet. The diamond drill showed the rock to be a fine granite. A more detailed account of this exploration will be found in the Twenty-first Annual Report, part 4. Above the "Narrows" the valley broadens into a large reservoir site, but as no surveys of it have been made the capacity is unknown.

The channel is straight for 300 feet above and below the section where the rod is located. Both banks are high and rocky and not subject to overflow. The channel is composed of sand, which is constantly shifting.

During médium and low stages discharge measurements are made from a low foot bridge or by wading. During floods discharge measurements are made from the county bridge. The mean estimated monthly discharge is obtained by averaging the discharge measurements made during the year.

Gage readings were discontinued in 1902. On March 1, 1905, a new inclined rod was fastened to the rock on the left bank about 300 feet above the county bridge. This gage was established as a matter of interest in connection with the discharge of the stream at high stages. There is no relation between gage readings and the discharge, as the bed of the stream is constantly changing at different stages of the river. During 1905 discharge measurements were made twice each week, and the gage was read by P. H. Leahy. The bench mark is a bolt sulphured in solid rock on the left bank, about 15 feet from the Santa Fe Railroad track and 40 feet west of the gage rod. It is 20.45 feet above the datum of the gage. Elevation, 2,726.52 feet above sea level, as established by connection with a standard United States Geological Survey bench mark at Victorville.

Information in regard to this station is contained in Water-Supply Papers Nos. 81, 100, and 134 of the United States Geological Survey. 
Discharge measurements of Mohave River at Victorville, Cal., in 1905.

\begin{tabular}{|c|c|c|c|c|c|c|c|}
\hline Date. & Hydrographer. & $\begin{array}{c}\text { Gage } \\
\text { height. }\end{array}$ & $\begin{array}{l}\text { Dis- } \\
\text { charge. }\end{array}$ & Date. & Hydro & $\begin{array}{c}\text { Gage } \\
\text { height. }\end{array}$ & $\begin{array}{c}\text { Dis- } \\
\text { charge. }\end{array}$ \\
\hline & & Feet. & Sec.-ft. & & & & Sec.-ft. \\
\hline January 3 & P. H. I & & 56 & June 27 & P. H. & & 30 \\
\hline January 6 & ..do & & 58 & June 30. & ....do.do. & & 3 \\
\hline January 10 & do. & & 70 & July 3 . & .....do. & & 1 \\
\hline January 13 & do & & $71^{\circ}$ & July 7 . & ....do. & & 3 \\
\hline January 17 . & ..do. & 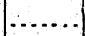 & 59 & July $11 \ldots$ & ....do. & & $31^{\circ}$ \\
\hline January & .do & & 59 & July $14 \ldots$ & $\ldots \ldots .0$ & & 27 \\
\hline January & .. do & & 56 & July & $\ldots \ldots c$ & & 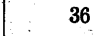 \\
\hline January & ..d & & 53 & July & $\therefore$. & & 32 \\
\hline Januar & ..d & 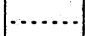 & 56 & July $25 \ldots$ & $\ldots \ldots$ & & 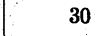 \\
\hline Februa & ..do & & .822 & July 2 & $\ldots . . d$ & & 34 \\
\hline Februa &.$d$ & & 82 & Augus & $\ldots$. & & \\
\hline Februar & ..d & & 90 & Augu & $\ldots . . c$ & & 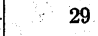 \\
\hline Februe & $\ldots \mathrm{d}$ & & 2,032 & Augu & $\ldots . .0$ & & 5 \\
\hline Februa & $\ldots \mathrm{dc}$ & $\ldots$ & 97 & Augus & $\ldots .$. & & 29 \\
\hline Febru & $\ldots d$ & $\because \cdots$ & 95 & Aug & $\ldots \ldots c$ & & St \\
\hline Marc & $\ldots . . d$ & 3.96 & 98 & r $1 .$. & d & & \\
\hline Marc & E. C. I & 3.92 & 90 & Sept & $\ldots$ & & 5 \\
\hline Marc & P. H. Le & 3.85 & 91 & Sept & $\ldots .$. & & \\
\hline March & $\ldots . \mathrm{dc}$ & 3.77 & 74 & Sept & $\cdots . c$ & & 3 \\
\hline Marc & $\therefore d$ & 9.90 & 5,410 & er 15 . & $\cdots$ & & \\
\hline March & $\ldots . d c$ & 4.50 & 820 & er 19. & $\ldots$. & & \\
\hline Marc & o. W & 4.95 & 2,350 & er 22 . & $\ldots$. & & \\
\hline Marc & P. H. & 4.06 & 980 & er 26 . & ..... & & 4 \\
\hline Marc & $\therefore \ldots . d c$ & 4.04 & 740 & er 29. & $\ldots$. & & 4 \\
\hline $\mathrm{Ma}$ & $\ldots . . d$ & $\ldots$. & 460 & 3..... & $\ldots$ & & \\
\hline Mar & $\ldots . d$ & 4.0 & 340 & Oc & $\ldots$ & & 4 \\
\hline $\mathrm{Ma}$ & $\ldots . . d$ & 3.91 & 290 & $0 . .$. & & & \\
\hline Apr & R. S. & 3.75 & 133 & $13 . .$. & $\ldots . . c$ & & 4 \\
\hline Apr & P. H. & 3.75 & 133 & $17 \ldots$ & $\ldots$. & & 4 \\
\hline Apri & ..... do & 3.7 & 123 & $20 \ldots$ & $\therefore$. & & 5 \\
\hline Apri & ....do & 3.66 & 104 & $24 \ldots$ & $\ldots . c$ & & 5 \\
\hline Apr & $\ldots . . d c$ & 3.58 & 93 & $27 \ldots$ & $\ldots .$. & & 5 \\
\hline Apri & ....do & 3.5 & 60 & $31 . .$. & $\ldots . .$. & 4.20 & 4 \\
\hline April & R. S. H & 3.5 & 38 & per $3 .$. & $\ldots$ & 4.20 & 4 \\
\hline April & Р. $\mathrm{H}$. & 3.45 & 58 & er $7 \ldots$ & $\ldots \ldots$ & 4.29 & 8 \\
\hline May 3 & $\therefore$ do. & 3.76 & 151 & er 10. & $\therefore . . . c$ & 4.21 & $\theta$ \\
\hline Ma & $\ldots d$ & 3.98 & 218 & r 14.. & $\ldots .$. & 4.20 & 6 \\
\hline May 10. & $\therefore d d$ & 4.60 & 327 & nber 17. & $\ldots \ldots$ & 4.16 & 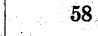 \\
\hline May 1 & $\ldots . d$ & & 260 & ber 22. & $\therefore \ldots$ & 4.15 & 6 \\
\hline May 1 & $\ldots . . d$ & ....... & 152 & tber 24 .. & $\ldots . . c$ & 4.16 & 7 \\
\hline May 20. & ...do & 3.98 & 80. & November 28. . & $\ldots . d$ & 4.15 & 6 \\
\hline May 2 & ....do & & 65 & December $1 \ldots$ & $\ldots . . d$ & 4.16 & 6 \\
\hline May 27 & ....do & & 55 & December $5 .$. & :...do. & 4. 09 & 7 \\
\hline May 29 & ...do & & 48 & December $8 .$. & .....do. & 4.09 & 6 \\
\hline June $2 .$. & $\therefore$ do & & 63 & December 12. & $\ldots . d$ & 4.02 & 6 \\
\hline June 6. & ...do.. & & 51 & December 15. & .....do. & 4.02 & 7 \\
\hline Junie 10. & $d$ & & 46 & December $19 .$. & .....do... & 4.05 & 7 \\
\hline June 13 . & $\ldots$. do & & 39 & December 22 .. & .....do.. & 4.05 & 67 \\
\hline & ...do. & & 43 & December 26 & $\therefore$...do... & 4.03 & 6 \\
\hline June 20 . . & ...do. & & 43 & December $30 .$. & ....do.do.. & 4.03 & 58 \\
\hline
\end{tabular}


Daily gage height, in feet, of Mohave River at Victorville, Cal., for 1905.

\begin{tabular}{|c|c|c|c|c|c|c|c|c|c|c|}
\hline Day. & Mar. & Apr. & May. & June. & ‘uly. & Aug. & Sept. & Oct. & Nov. ${ }^{2}$ & Dec. \\
\hline & 4.0 & 3.9 & 3.45 & 4.0 & 4.05 & 4.0 & 4.0 & 4.1 & 4.2 & 4.15 \\
\hline 2. & 3.95 & 3.9 & 3.5 & 4.0 & 4.0 & 4.0 & 4.0 & 4.1 & 4.2 & 4.15 \\
\hline 3.. & ....... & 3.85 & 3.65 & 4.0 & 4.0 & 4.0 & 4.0 & 4.1 & 4.2 & 4.15 \\
\hline 4.. & 3.95 & 3.9 & 3.85 & 4.0 & 4.0 & 4.0 & 4.0 & 4.1 & 4.2 & 4.15 \\
\hline $5 \ldots$ & 3.85 & 3.8 & 3.95 & 4.0 & 4.0 & 4.0 & 4.0 & 4.1 & 4.2 & 4.15 \\
\hline $6 .$. & 3.85 & 3.75 & 4.0 & 4.0 & 4.0 & 4.0 & 4.0 & 4.15 & 4.2 & 4.1 \\
\hline $7 \ldots$ & 3.8 & 3.75 & 4.85 & 4.0 & 4.0 & 4.0 & 4.0 & 4.15 & 4.3 & 4.1 \\
\hline $8 .$. & 3.8 & 3.4 & 4.85 & 4.0 & 4.0 & 4.0 & 4.0 & 4.15 & 4.3 & 4.1 \\
\hline $9 .$. & 3.8 & 3.4 & ..... & 4.0 & 4.0 & 4.0 & 4.0 & 4.15 & 4.25 & 4.1 \\
\hline $10 \ldots$ & 3.75 & 3.65 & 4.6 & 4.0 & 4.0 & 4.0 & 4.0 & 4.15 & 4.2 & 4.05 \\
\hline $11 .$. & 3.75 & 3.65 & 4.6 & 4.0 & 4.0 & 4.0 & 4.0 & 4.15 & 4.2 & 4.05 \\
\hline $12 .$. & 3.75 & 3.65 & 4.2 & 4.0 & 4.0 & 4.0 & 4.0 & 4.15 & 4.2 & 4.0 \\
\hline 13. & 9.95 & 3.65 & 4.2 & 4.05 & 4.0 & 4.0 & 4.0 & 4.15 & 4.2 & 4.0 \\
\hline $14 .$. & 5.0 & 3.65 & 4.1 & 4.05 & 4.0 & 4.0 & 4.0 & 4.15 & 4.2 & 4.0 \\
\hline $15 \ldots$ & 5.0 & 3.6 & 4.1 & 4.05 & 4.0 & 4.0 & 4.0 & 4.15 & 4.2 & 4.0 \\
\hline $16 .$. & 5.05 & 3.6 & 4.05 & 4.05 & 4.0 & 4.0 & 4.0 & 4.15 & 4. 15 & 4.0 \\
\hline $17 \ldots$ & 4.1 & 3.6 & 4.0 & 4.05 & 4.0 & 4.0 & 4.0 & 4.2 & 4.15 & 4.0 \\
\hline $18 .$. & 4.05 & 3.6 & 4.0 & 4.05 & 4.0 & 4.0 & 4.0 & 4.2 & 4. 15 & 4.05 \\
\hline $19 . \ldots$. & 4.05 & 3.55 & 4.0 & 4.05 & 4.0 & 4.0 & 4.0 & 4.2 & 4.15 & 4.05 \\
\hline $20 \ldots$ & 4.05 & 3.65 & 4.0 & 4.05 & 4.0 & 4.0 & 4.0 & 4.2 & 4.15 & 4.05 \\
\hline $21 .$. & 4.05 & 3.6 & 4.0 & 4.05 & 4.0 & 4.0 & 4.0 & 4.2 & 4.15 & 4.05 \\
\hline $22 \ldots$ & 4.05 & 3.6 & 4.0 & 4.05 & 4.0 & 4.0 & 4.0 & 4.2 & 4.15 & 4.05 \\
\hline $23 .$. & 4.0 & 3.5 & 4.0 & 4.05 & 4.0 & 4.0 & 4.0 & 4.2 & 4. 15 & 4.05 \\
\hline $24 \ldots$ & 4.0 & 3.5 & 4.0 & 4.05 & 4.0 & 4.0 & 4.0 & 4.2 & 4.15 & 4.05 \\
\hline $25 .$. & 4.0 & 3.5 & 3.95 & 4.05 & 4.0 & 4.0 & 4.0 & 4.2 & 4.2 & 4.05 \\
\hline $26 .$. & 4.0 & 3.5 & 3.95 & 4.05 & 4.0 & 4.0 & 4.0 & 4.2 & 4.2 & 4.05 \\
\hline $27 \ldots$ & 4.0 & 3.45 & 4.0 & 4.05 & 4.0 & 4.0 & 4.0 & 4.2 & 4.15 & 4.05 \\
\hline $28 \ldots$ & 3.85 & 3.45 & 4.2 & 4.05 & 4.0 & 4.0 & 4.0 & 4.2 & 4.15 & 4.05 \\
\hline $29 . .$. & 3.8 & 3.45 & 4. 1 & 4.05 & 4.0 & 4.0 & 4.0 & 4.2 & 4.15 & 4.05 \\
\hline $30 \ldots$ & 4.2 & 3.45 & 4.1 & 4.05 & 4.0 & 4.0 & 4.0 & 4.2 & 4.15 & 4.05 \\
\hline $31 \ldots \ldots \ldots \ldots \ldots$ & 3.9 & $\cdots \cdots$ & 4.0 & ...... & 4.0 & 4.0 & 4.0 & 4.2 & ..... & 4.05 \\
\hline
\end{tabular}

IRR $177-06-7$ 
Daily discharge, in feet, of Mohave River at Victorville, Cal., for 1905.

\begin{tabular}{|c|c|c|c|c|c|c|c|c|c|c|c|c|}
\hline Day. & Jan. & Feb. & Mar. & Apr. & May. & June. & July. & Aug. & Sept. & Oct. & Nov. & Dec. \\
\hline 1. & 56 & .60 & 100 & 263 & 60 & 61 & 35 & 30 & a 47 & 40 & 48 & $a 65$ \\
\hline 2. & 56 & 325 & $a 98$ & 237 & 100 & $a 63$ & 36 & $a 30$ & 44 & 40 & 47 & 66 \\
\hline 3. & a 56. & 605 & $a 90$ & 211 & $a_{151}$ & 60 & $a 36$ & 30 & 41 & a 40 . & $a 46$ & 68 \\
\hline 4. & 57 & $713^{\circ}$ & 90 & 185 & 172 & 57 & 35 & 29 & 38 & 40 & 55 & 70 \\
\hline 5. & 58 & $a 822$ & 91 & 159 & 195 & 54 & 34 & a 29 & $a 35$ & 44 & 64 & a 72 \\
\hline 6. & $a 58$ & 576 & $a 91$ & a 133 & a 218 & $a 51$ & 33 & 29 & 35 & a 49 & 72 & 67 \\
\hline 7. & 61 & 328 & 88 & 133 & 246 & 50 & $a 32$ & 29 & 35 & 47 & $a 81$ & 67 \\
\hline 8. & 64 & $a 82$ & 84 & $a 133$ & 272 & 49 & 32 & 29 & $a 35$ & 45 & 76 & $a 67$ \\
\hline 9. & 67. & 84 & 81 & 133 & 300. & 47 & 32 & 29 & 35 & 43 & 71 & 67 \\
\hline 10. & a 70 & 87 & 77 & 128 & $a 327$ & $a 46$ & 31 & 29 & 36 & $a 41$ & $-a 66$ & 66 \\
\hline 11. & 70 & $a 90$ & a 74 & $a 123$ & 296 & 44 & $a 31$ & 29 & $a 37$ & 41 & 65 & 66 \\
\hline 12. & 71 & 90 & 74 & 118 & 273 & 41 & 29 & 29 & 38 & 41 & 64 & $a 65$ \\
\hline 13. & a 71 & 90 & $a 5,410$ & 110 & $a 260$ & $a 39$ & 28 & 29 & 39 & $a 44$ & 63 & 67 \\
\hline 14. & 68 & 90 & 2,295 & a 104 & 224 & 40 & $a 27$ & 31 & 40 & 44 & $a 62$ & 70 \\
\hline 15. & 65 & $a 2,032$ & a 820 & 103 & 188 & 41 & 29 & 31 & $a 42$ & 44 & 61 & a 73 \\
\hline 16. & 62 & 1,387 & $a 2,250$ & 98 & a 152 & $a 43$ & 31 & 33 & 42 & 44 & 59 & 73 \\
\hline 17. & $a_{59}$ & 150 & 1,665 & 95 & 134 & 43 & 34 & 33 & 42 & a 44 & $a 58$ & 72 \\
\hline 18. & 59 & $a 97$ & a 980 & $a 93$ & 116 & 43 & e. 36 & 33 & 42 & 47 & 60 & 71 \\
\hline 19. & 59 & 96 & 920 & 86 & 98 & 43 & 35 & 33 & $a 43$ & 50 & 61 & a 71 \\
\hline 20. & $a 59$ & 95 & 860 & 79 & $a 80$ & $a 43$ & 33 & 33 & 43 & $a_{54}$ & 62 & 70 \\
\hline 21. & 59 & $a 95$ & 800 & 75 & 75 & 41 & a 32 & 36 & 43 & 53 & 64 & 68 \\
\hline 22. & 59 & 95 & $a 740$ & 70 & 70 & 39 & 32 & 26 & $a 43$ & 52 & $a 65$ & $a 67$ \\
\hline 23. & 58 & 95 & 647 & 65 & $a 65$ & 37 & 32 & 36 & 42 & 51 & 69 & 67 \\
\hline 24. & 57 & 95 & 553 & $a 60$ & 63 & 35 & 31 & 36 & 42 & $a 51$ & a 73 & 67 \\
\hline 25. & $a 56$ & 95 & $a 460$ & a 38 & 60 & 33 & $a 50$ & $a 36$ & 42 & 51 & 71 & 67 \\
\hline 26. & 55 & 95 & 450 & 43 & 58 & 31 & 32 & 33 & $a 42$ & 51 & 70 & $a 66$ \\
\hline 27. & a 53 & 95 & $a 340$ & 48 & a.55 & $a 30$ & 33 & 31 & 42 & a 50 & 69 & 64 \\
\hline 28. & 54 & 95 & 327 & 53 & 52 & 31 & $a 34$ & $\dot{a} 29$ & 42 & 50 & $a 67$ & : 62 \\
\hline 29. & 54 & $\therefore$ & 315 & a 58 & $a 48$ & 33 & 32 & 31 & $a 42$ & 50 & 67 & 60 \\
\hline & 55 & .... & 302 & 60 & 53 & a. 35 & 32 & 33 & 42 & 49 & 66 & $a .58$ \\
\hline $31 \ldots$ & a 56 & $\ldots \ldots$ & 290 & & 57 & & 32 & $a 35$ & & $a 49$ & & 58 \\
\hline
\end{tabular}

a Taken from discharge measurements.

Nots.-Daily discharge obtained by interpolation between discharge measurements.

Estimated monthly discharge of Mohave River at Victorville, Cal., for 1905.

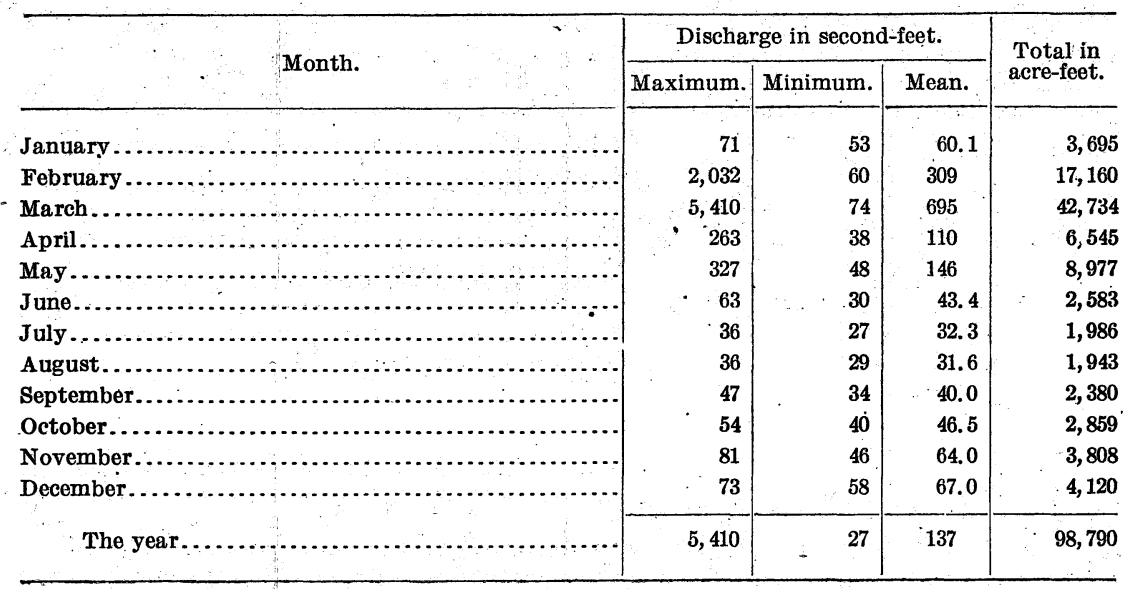




\section{SOUTHERN PACIFĨC OCEAN DRAINAGE.}

\section{GENERAL FEATURES.}

The Southern Pacific Ocean drainage includes those streams south of San Francisco Bay whose waters, in times of flood at least, reach the Pacific Ocean.

\section{SAN LUIS REY RIVER DRAINAGE BASIN.}

\section{DESCRIPTION OF BASIN.}

The San Luis Rey River rises on the western slope of the Coast Range in the northern portion of San Diego County, and, flowing in a westerly direction, discharges its waters into the Pacific Ocean near the town of Oceanside. It has numerous small tributaries, none of which have their sources at elevations above 5,000 feet. On the upper reaches of this stream the country is rolling, with several small valleys which are under cultivation; being used principally for the raising of grain and the pasturage of stock. At a point below what is known as Warner's ranch reservoir site the river flows through a deep, narrow canyon, with a heavy grade for a distance of about 10 miles, below which point the grade is light and the discharge is over a sandy and gravelly bed, where the water soon disappears, again rising in small quantities near the town of Pala, where the gaging station is located. Below this point it flows for a distance of about 25 miles on a light grade to the Pacific Ocean. There is a good soil covering throughout this basin, with a considerable growth of brush and grass, and with small areas of timber on the extreme higher elevations. The water is diverted at several points along this stream for irrigation, a considerable quantity being diverted from the canyon above the gaging station and used in the vicinity of Escondido, which lies in an entirely separate drainage basin. This stream is torrential in its character, the discharge being very light except during the winter season, in times of heavy rainfall. The mean precipitation varies from 10 to 20 inches and falls principally in the form of rain, there being only a light fall of snow on the extreme higher elevations, which soon melts and only adds to the flood discharge.

SAN LUIS REY RIVER NEAR PALA, CAL.

This station was established October 9, 1903, by W. B. Clapp. It is located at Sickler's mill, 4 miles above Pala, Cal. It is reached by driving from Fallbrook or Temecula, stations on the Southern California Railway, 18 and 13 miles distant, respectively.

The channel is straight for about 800 feet above and 2,000 feet below the station. The grade of the stream is 0.60 foot in 100 feet. The current is swift. The right bank rises abruptly about 15 feet beyond the oak tree to which the cable is fastened and is not liable to overflow. The left bank is low, but is not liable to overflow. It was once a portion of the river channel, but is now well above high-water marks. The bed of the stream is rocky in portions of the flood channel, but the low-water channel is clear of rocks. There is a considerable growth of small timber in the channel, but this has been cleared the entire width of the cross section for a distance of 100 feet above and 50 feet below the station. This timber growth is not permanent, being washed out by floods every few years.

Discharge measurements are usually made by wading. During high water they are made from a car suspended from a cable stretched across the river at the gage. The initial point for soundings is the base of the oak tree to which the left end of the cable is fastened.

The gage is an inclined staff fastened to tree stumps and stakes at the left bank of the river. During 1905 the gage was read once each day by M. M. Sickler. The bench mark is a United States standard bronze-capped iron post set flush with the ground on the right bank of the river and the north side of the wagon road, and about 50 feet west from the line of the cable prolonged. Its elevation is 557 feet above mean sea level and 26.98 feet above the datum of the gage. 
Information in regard to this station is contained in Water-Supply Papers Nos. 100 and 134 of the United States Geological Survey.

Discharge measurements of San Luis Rey River near Pala, Cal., in 1905.

\begin{tabular}{|c|c|c|c|c|c|c|}
\hline Date. & Hydrographer. & Width. & $\begin{array}{l}\text { Area of } \\
\text { section. }\end{array}$ & $\underset{\text { velocity. }}{\text { velty }}$ & $\begin{array}{c}\text { Gage } \\
\text { height. }\end{array}$ & $\begin{array}{c}\text { Dis- } \\
\text { charge. }\end{array}$ \\
\hline February $8 . .$. & E.C. La Rue... & $\begin{array}{c}\text { Feet. } \\
44\end{array}$ & $\underset{43}{S q . f t}$ & $\begin{array}{r}\text { Ft.per sec. } \\
2.51\end{array}$ & $\begin{array}{r}\text { Feet. } \\
2.25\end{array}$ & $\begin{array}{c}\text { Sec.-ft. } \\
108\end{array}$ \\
\hline February 17... & F.M. Sickler... & 51 & 70 & 2.69 & 2.72 & 188 \\
\hline February 18 . & .....do... & 100 & 204 & 3.60 & 4.75 & 735 \\
\hline March 17....... & .....do........ & 188 & 679 & 7.14 & 7.50 & 5,180 \\
\hline March 22.... & R.S. Hawley:... & 67 & 100 & 3.05 & 2.64 & 305 \\
\hline March $22 . . . . .$. & F. M. Sickler... & 68 & 96 & 3.11 & 2.64 & 299 \\
\hline April 11... & .....do.. & 44 & 42 & 2.48 & 1.80 & 104 \\
\hline May $15 . . .$. & .....do....... & 41 & 26 & 1.88 & 1.20 & 49 \\
\hline May $17 . . .$. & ....do...... & 40 & 21 & 1.90 & 1.10 & 40 \\
\hline May $20 . . . . . . . .$. & ......do....... & 43 & 25 & 2.28 & 1.40 & $\dot{5} 7$ \\
\hline July $6 \ldots$ & .....do...... & 3.0 & 1.7 & 2.06 & .55 & 3.5 \\
\hline August $8 . . .$. & R.S. Hawley. & 3.6 & 1.7 & 1.74 & (a) & 3 \\
\hline Augúst $19 . \ldots .$. & M. M. Sickler.... & 3.5 & 1.6 & 2.00 & ........... & 3.2 \\
\hline August $26 . . . .$. & ......dq. & 3.5 & 1.6 & 2.06 & .... & 3.3 \\
\hline September $2 .$. & .....do... & 3.5 & 1.5 & 1.80 & & 2.7 \\
\hline September $9 .$. & .....do.. & 3.5 & 1.5 & 1.80 & & 2.7 \\
\hline September $16 .$. & ......do... & 3.5 & 1.4 & 1.78 & & 2.5 \\
\hline September $21 .$. & D. W.Murph & 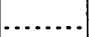 & $\cdots$ & 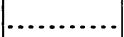 & & 2.1 \\
\hline September $23 .$. & F. M. Sickler... & 3.5 & 1.4 & 2.00 & .42 & 2.8 \\
\hline October $28 . . .$. & .....do.... & 3.5 & 1.6 & 2.25 & .53 & 3.6 \\
\hline December $27 \ldots$ & ......do..... & 7 & 4.1 & 3.15 & .63 & 12.9 \\
\hline December $29 \ldots$ & .....do...... & 10.9 & 6.7 & 1.40 & .61 & 9.4 \\
\hline
\end{tabular}

$a$ Channel so uncertain from July 11 to September 23 that no readings were made. 
Estimated monthly discharge of San Luis Rey River near Pala, Cal., for 1903-1905.

\begin{tabular}{|c|c|c|c|c|}
\hline \multirow[b]{2}{*}{ · } & \multicolumn{3}{|c|}{ Discharge in second-feet. } & \multirow{2}{*}{$\begin{array}{l}\text { Total in } \\
\text { acre-feet. }\end{array}$} \\
\hline & Maximum. & Minimum. & Mean. & \\
\hline 1903. & & & & \\
\hline October (23 days) ........... & 1.5 & 1.0 & 1.1 & 50 \\
\hline November.............. & 1.5 & 1.0 & 1.4 & 83 \\
\hline December............. & 1.5 & 1.0 & 1.2 & 74 \\
\hline The period.... & & & $\ldots$ & 207 \\
\hline 1904. & & & & \\
\hline January................ & 1.5 & 1.5 & 1.5 & 92 \\
\hline February ............... & 2.5 & 1.5 & 2.0 & 115 \\
\hline March.......... & 348 & 1.5 & 41.6 & 2,558 \\
\hline April.................... & 104 & 20 & 43.6 & 2,595 \\
\hline May............. & 35 & 12 & 16.2 & 996 \\
\hline June.................... & 12 & 9 & 10.8 & 643 \\
\hline July.................. & 9 & 3 & 3.8 & 234 \\
\hline August............... & 2 & 2 & 2.0 & 123 \\
\hline September.............. & 2 & 2 & 2.0 & 119 \\
\hline October............... & 13 & 2 & 2.5 & 154 \\
\hline November............. & 3 & 2 & 2.4 & 143 \\
\hline December............... & 5.5 & 2 & 3.7 & 227 \\
\hline The year.. & 348 & 1.5 & 11.0 & 7,999 \\
\hline 1905. & & & & \\
\hline January..... & 66 & 3 & 19.1 & 1,174 \\
\hline February ................ & 711 & 13 & 151 & 8,386 \\
\hline March............... & 4,265 & 2 & 336 & 20,660 \\
\hline April..... & 139 & 27 & 64.9 & 3,862 \\
\hline May..................... & 282 & 27 & 88.1 & 5,417 \\
\hline June........... & 35 & 11 & 23 & 1,369 \\
\hline July.................. & 9 & 3 & 4.1 & 252 \\
\hline August................. & 3.5 & 3 & 3 & 184 \\
\hline September............ & 3.0 & 2.5 & 2.6 & 155 \\
\hline October................. & 3.8 & 2.8 & 3.2 & 197 \\
\hline November.............. & 96.0 & 3.8 & 17.8 & 1,059 \\
\hline 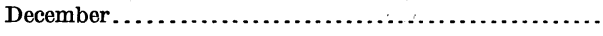 & 47.0 & 10 & 18.3 & 1,125 \\
\hline The year. & 4,265 & 2.0 & 60.9 & 43,840 \\
\hline
\end{tabular}

NotE.-The estimated monthly discharge is approximate only. 


\section{SANTA ANA RIVER DRAINAGE BASIN.}

DESCRIPTION OF BASIN.

Santa Ana River has its source in the southern slope of the San Bernardino Mountains and, flowing in a southwesterly direction, traverses San Bernardino Valley, below which it breaks in a narrow canyon through the Santa Ana Mountains, finally discharging its waters through Santa Ana Valley into the Pacific Ocean below the town of Santa Ana. This stream has numerous tributaries which rise in the southern slope of the San Bernardino Mountains, the surface flow of most of which only reach Santa Ana River, where it traverses San Bernardino Valley in times of flood discharge. The topography on the higher elevations is rough and rugged, reaching elevations of from 10,000 to 12,000 feet, the formation being of granite with good soil covering and considerable growth of timber. On the lower elevations the topography is less rough and the soil covering is principally of brush. A gaging station is located on this stream at a point known as Warm Springs, a distance of about 8 miles above Redlands. Below this station the river leaves the mountainous country and discharges over a sandy and gravelly bed through San Bernardino Valley. During the summer months the entire flow of the stream is diverted above this gaging station and used for power development at the mouth of the canyon, below which point it is again taken out and used for irrigation on the higher elevations of San Bernardino Valley along the base of the mountains where the country is under a high state of cultivation, used principally for the raising of citrus fruits. The water rises to the surface in San Bernardino Valley near the city of San Bernardino and is diverted and used extensively for irrigation in the neighborhood of Riverside. In addition to this surface flow a large number of wells have been sunk in this territory, many of which are artesian, and from others the water is being pumped. This developed water is also used extensively for irrigation in the vicinity of San Bernardino and Riverside. The water is again forced to the surface by bed-rock obstructions at Riverside Narrows below the city of Riverside, and gradually increases in volume until it reaches Santa Ana Canyon, where it is diverted for irrigation on the lower valley lands in the vicinity of Santa Ana and Fullerton. During the summer months measurements are made of the flow at Rincon, Cal., at the head of Santa Ana Canyon. There are only occasional flood discharges of this river which flow continuously from the mountain to the sea. The mean precipitation throughout this basin is from 15 to 30 inches, which falls in the form of rain except on the higher elevations of the San Bernardino Mountains, where there is a considerable snowfall, which usually remains on the extreme high elevations until midsummer. A storage reservoir has been constructed on Bear Creek, a tributary of this stream, and is known as the Bear Valley reservoir. This stored water is held until the summer months and used for irrigation in San Bernardino Valley.

\section{SANTA ANA RIVER NEAR MENTONE, CAL.}

This station was established in June, 1896. It is located 5 miles northeast of Mentone, Cal., three-fourths of a mile below the headworks of the Mentone Power Company's canal, and opposite the warm springs in the canyon.

The Edison Electric Company diverts the greater portion of the water from Santa Ana River above the gaging station, but also returns all of it above the station. They, however, allow only limited portions of the water to pass out of their conduits during certain hours of the day, holding back the water for the purpose of obtaining additional power when the greatest demand exists.

The Mentone Power Company"s canal, formerly called the Santa Ana canal, diverts water above the station, all of which is returned below the point of measurement. During the low-water season the entire flow of the river is diverted by the canals.

The channel is straight for 100 feet above and below the station, and has a width of 22 feet at low and 125 feet at high stages. The current is swift at all stages. The right bank is low and is liable to overflow at flood stages for about 100 feet. The left bank is low but is 
not liable to overflow. Both banks are overgrown with alders. The bed of the stream is composed of firm sand and small bowlders. It is subject to considerable change during flood stages.

Discharge measurements are made by means of a cable and car. The initial point for soundings is the bench-mark spike set in the north side of a cottonwood tree on the left bank, 30 feet west of the tree to which the cable is fastened. At flood stages the velocity is so high that measurements can be made only by means of floats.

The gage is an inclined timber fastened to a large bowlder on the left bank 100 feet above the cable. The channel was deepened by a flood March 31, 1903, and the gage was accordingly lowered to reach low-water stages June 30, 1903. During 1905 the gage was read once each day by A. Laird. The bench mark is a spike in the north side of the cottonwood tree on the left bank, 30 feet west of the tree to which the cable is fastened; elevation, 7.29 feet above the datum of the gage.

Information in regard to this station is contained in Water-Supply Papers Nos. 81, 100, and 134 of the United States Geological Survey.

Discharge measurements of Santa Ana River near Mentone, Cal., in 1905.

\begin{tabular}{|c|c|c|c|c|c|}
\hline \multirow[b]{2}{*}{ Date. } & \multirow[b]{2}{*}{ Hydrographer. } & \multirow[b]{2}{*}{$\begin{array}{c}\text { Gage } \\
\text { height of } \\
\text { river. }\end{array}$} & \multicolumn{3}{|c|}{ Discharge. } \\
\hline & & & River. & $\mid \begin{array}{c}\text { Mentone } \\
\text { Power } \\
\text { Com- } \\
\text { pany's } \\
\text { canal. }\end{array}$ & $\begin{array}{l}\text { Total for } \\
\text { River. }\end{array}$ \\
\hline February $13 \ldots$ & Clapp and La Rue........ & $\begin{array}{r}\text { Feet. } \\
1.15\end{array}$ & $\begin{array}{r}\text { Sec.-ft. } \\
10\end{array}$ & $\begin{array}{r}\text { Sec.-ft. } \\
40\end{array}$ & $\begin{array}{r}\text { Sec.-ft. } \\
50\end{array}$ \\
\hline February $21 . .$. & 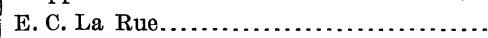 & 1.70 & 17 & 74 & 91 \\
\hline February $22 \ldots$ & .....do............ & 1.65 & 10 & 71 & 81 \\
\hline February $23 . .$. & .....do........... & 1.65 & 11 & 63 & 74 \\
\hline March 14 a.m.. & ....do............. & 2.60 & 188 & 0 & 188 \\
\hline March 14 p.m.. & ....do $\ldots \ldots \ldots \ldots \ldots \ldots \ldots$ & 2.50 & 138 & 50 & 188 \\
\hline March 15 a.m.. & ....do........................ & 2.30 & 132 & 57. & 189 \\
\hline March 15 p.m.. & .....do.......... & 2.10 & 113 & 57 & 170 \\
\hline $\operatorname{March} 30 \ldots . . .$. & R. S. Hawley..$\ldots \ldots \ldots \ldots \ldots \ldots \ldots \ldots \ldots \ldots \ldots \ldots$ & 1.90 & 81 & 46 & 127 \\
\hline April 14, 1 p.m. & .....do... & 1.95 & 86 & 1 & 87 \\
\hline May $17 . . . . . .$. & .....do.... & 2.25 & 111 & 37 & 148 \\
\hline June $7 . . . . . .$. & .....do..... & 2.20 & 79 & 0 & 79 \\
\hline June $26 . . . . . . .$. & .....do...... & 1.43 & 4 & $66^{\circ}$ & 70 \\
\hline July $6 . . . . . . .$. & .....do...... & 1.40 & 3 & 61 & 64 \\
\hline
\end{tabular}


- Daily gage height, in feet, of Santa Ana River near Mentone, Cal., for 1905.

\begin{tabular}{|c|c|c|c|c|c|c|c|c|c|c|c|c|}
\hline Da & Jan. & Feb. & Mar. & Apr. & May. & Jüne. & July. & Aug. & Sept. & Oct. & Nov. & Dec. \\
\hline $1 .$. & 0.85 & 0.8 & 1.3 & 1.7 & 1.3 & 2.3 & 1.4 & 1.3 & 1.3 & 1.3 & 1.3 & 1.5 \\
\hline 2. & .85 & 2.5 & 1.3 & 2.05 & 2.2 & 2.3 & 1.4 & 1.3 & 1.3 & 1.3 & 1.3 & 1.5 \\
\hline 3... & .85 & 2.3 & 1.3 & 2.05 & 2.0 & 2.3 & 1.4 & 1.3 & 1.3 & 1.3 & 1.3 & 1.4 \\
\hline 4.. & .85 & 2.8 & 1.3 & 1.35 & 2.0 & 2.3 & 1.4 & 1.3 & 1.3 & 1.3 & 1.3 & 1.4 \\
\hline 5.. & .85 & 3.0 & 1.3 & 1.35 & 1.9 & 2.3 & 1.4 & 1.3 & 1.3 & 1.3 & 1.3 & 1.4 \\
\hline 6.. & .85 & 1.9 & 1.25 & 1.35 & 1.9 & 2.25 & 1.4 & 1.3 & 1.3 & 1.3 & 1.3 & 1.4 \\
\hline $7 .$. & .85 & 1.9 & 1.25 & 1.35 & 2.0 & 2.25 & 1.4 & 1.3 & 1.3 & 1.3 & 1.35 & 1.4 \\
\hline 8.. & .85 & 1.75 & 1.25 & 1.35 & 2.3 & 2.2 & 1.4 & 1.3 & 1.3 & 1.3 & 1.4 & 1.4 \\
\hline 9.. & 2.0 & 1.5 & 1.25 & 1.35 & 2.2 & 2.2 & 1.4 & 1.3 & 1.3 & 1.3 & 1.35 & 1.4 \\
\hline $10 .$. & 1.0 & 1.5 & 1.25 & 1.35 & 2.2 & 2.2 & 1.4 & 1.3 & 1.3 & 1.3 & 1.3 & 1.4 \\
\hline 11.. & .85 & 1.35 & 1.25 & 1.35 & 2.2 & 2.15 & 1.4 & 1.3 & 1.3 & 1.3 & 1.3 & 1.4 \\
\hline 12. & .85 & 1.25 & $1.3^{\circ}$ & 1.35 & 2.2 & 1.6 & 1.4 & 1.3 & 1.3 & 1.3 & 1.3 & 1.4 \\
\hline 13. & .85 & 1.15 & 3.4 & 1.3 & 2.2 & 1.75 & 1.4 & 1.3 & 1.3 & 1.3 & 1.3 & 1.4 \\
\hline 4.. & .85 & 1.1 & 2.6 & 1.3 & 2.5 & 1.6 & 1.4 & 1.3 & 1.3 & 1.3 & 1.3 & 1.4 \\
\hline 15.. & .85 & 1.1 & 2.35 & 1.3 & 2.5 & 1.45 & 1.4 & 1.3 & 1.3 & 1.3 & 1.3 & 1.4 \\
\hline 16. & .8 & 2.8 & 2.4 & 1.3 & 2.6 & 1.45 & 1.35 & 1.3 & 1.3 & 1.3 & 1.3 & 1.4 \\
\hline 17.. & .8 & 2.1 & 2.5 & 2.2 & 2.6 & 1.6 & 1.35 & 1.3 & 1.3 & 1.3 & 1.3 & 1.4 \\
\hline 18. & .8 & 1.9 & 2.6 & 2.0 & $2.5^{\cdots}$ & 1.6 & 1.35 & 1.3 & 1.3 & 1.3 & 1.3 & 1.4 \\
\hline 19. & .8 & 1.9 & 2.5 & 2.1 & 2.6 & 1.55 & 1.35 & 1.3 & 1.3 & 1.3 & 1.35 & 1.6 \\
\hline 20. & .8 & 1.8 & 2.6 & 1.2 & 2.6 & 1.55 & 1.35 & 1.3 & 1.3 & 1.3 & 1.35 & 2.2 \\
\hline 21.. & 1.65 & 1.7 & 2.5 & 1.15 & 2.5 & 1.45 & 1.35 & 1.3 & 1.3 & 1.3 & 1.4 & 1.6 \\
\hline 22. & .85 & 1.7 & 2.4 & 1.2 & 2.2 & 1.45 & 1.35 & 1.3 & 1.3 & 1.3 & 1.35 & 1.5 \\
\hline 23. & .85 & 1.5 & 2.0 & 1.4 & 2.2 & 1.45 & 1.35 & 1.3 & 1.3 & 1.3 & 1.35 & 1.5 \\
\hline $24 .$. & .85 & 1.3 & 1.9 & 1.4 & 2.1 & 1.45 & 1.3 & 1.3 & 1.3 & 1.3 & 1.35 & 1.5 \\
\hline 25. & .85 & 1.85 & 1.9 & 1.3 & 2.3 & 1.45 & 1.3 & 1.3 & 1.3 & 1.3 & 1.35 & 1.5 \\
\hline 26. & .85 & 1.25 & 1.9 & 1.3 & 2.2 & 1.45 & 1.3 & 1.3 & 1.3 & 1.3 & 1.8 & 1.5 \\
\hline 27.. & .85 & 1.35 & 1.8 & 1.3 & 2.2 & 1.45 & 1.3 & 1.3 & 1.3 & 1.3 & 2.5 & 1.5 \\
\hline 28.. & .85 & 1.3 & 1.65 & 1.25 & 2.4 & 1.4 & 1.3 & 1.3 & 1.3 & 1.3 & 2.9 & 1.5 \\
\hline 29. & .8 & 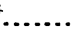 & 1.5 & 1.2 & 2.4 & 1.4 & 1.3 & 1.3 & 1.3 & 1.3 & 1.7 & 2.0 \\
\hline 30.. & .8 & & 2.0 & 1.2 & 2.4 & 1.4 & 1.3 & 1.3 & 1.3 & 1.3 & 1.5 & 1.6 \\
\hline 31......... & .8 & ........ & 1.8 & ... & 2.4 & $\ldots \ldots$ & 1.3 & 1.3 & s & 1.3 & & 1.5 \\
\hline
\end{tabular}

Station rating table for Santa Ana River near Mentone, Cal., from January 1 to February 17, 1905.

\begin{tabular}{|r|r||r|r|r|r|r|r|}
\hline $\begin{array}{c}\text { Gage } \\
\text { height. }\end{array}$ & Discharge. & $\begin{array}{c}\text { Gage } \\
\text { height. }\end{array}$ & Discharge. & $\begin{array}{c}\text { Gage } \\
\text { height. }\end{array}$ & Discharge. & $\begin{array}{c}\text { Gage } \\
\text { height. }\end{array}$ & Discharge. \\
\hline Feet. & Second-feet. & Feet. & Second-feet. & Feet. & Second-feet. & Feet. & Second-feet. \\
0.80 & 1 & 1.40 & 23 & 2.00 & 80 & 2.60 & 170 \\
0.90 & 2 & 1.50 & 29 & 2.10 & 93 & 2.70 & 186 \\
1.00 & 5 & 1.60 & 37 & 2.20 & 106 & 2.80 & 202 \\
1.10 & 8 & 1.70 & 47 & 2.30 & 122 & 2.90 & 218 \\
1.20 & 12 & 1.80 & 57 & 2.40 & 138 & 3.00 & 234 \\
1.30 & 17 & 1.90 & 68 & 2.50 & 154 & & \\
& & & & & & & \\
\hline
\end{tabular}


SANTA ANA RIVER DRAINAGE BASIN.

Station rating table for Santa Ana River near Mentone, Cal., from February 18 to March 12 and from June 1 to December 31, 1905.

\begin{tabular}{|c|c|c|c|c|c|c|c|}
\hline $\begin{array}{c}\text { Gage } \\
\text { height. }\end{array}$ & Discharge. & $\begin{array}{c}\text { Gage } \\
\text { height. }\end{array}$ & Discharge. & $\begin{array}{c}\text { Gage } \\
\text { height. }\end{array}$ & Discharge. & $\begin{array}{c}\text { Gage } \\
\text { height. }\end{array}$ & Discharge. \\
\hline Feet. & Second-feet. & Feet. & Second-feet. & Feet. & Second-feet. & Feet. & Second-feet. \\
\hline 1.30 & 1 & 2.00 & 48 & 2.70 & 164 & 3.40 & 290 \\
\hline 1.40 & 3 & 2.10 & 62 & 2.80 & 182 & 3.50 & 308 \\
\hline 1.50 & 6 & 2.20 & 78 & 2.90 & 200 & 3.60 & 326 \\
\hline 1.60 & 11 & 2.30 & 94 & 3.00 & 218 & 3.70 & 344 \\
\hline 1.70 & 17 & 2.40 & 110 & 3.10 & 236 & 3.80 & 362 \\
\hline 1.80 & 25 . & 2.50 & 128 & 3.20 & 254 & 3.90 & 380 \\
\hline 1.90 & 35 & 2.60 & 146 & 3.30 & 272 & & \\
\hline
\end{tabular}

Station rating table for Santa Ana River near Mentone, Cal, from March 13 to May 31, 1905.

\begin{tabular}{|c|r||r|r||r|r||r|r|}
\hline $\begin{array}{c}\text { Gage } \\
\text { height. }\end{array}$ & Discharge. & $\begin{array}{c}\text { Gage } \\
\text { height. }\end{array}$ & Discharge. & $\begin{array}{c}\text { Gage } \\
\text { height. }\end{array}$ & Discharge. & $\begin{array}{c}\text { Gage } \\
\text { height. }\end{array}$ & Discharge. \\
\cline { 2 - 6 } Feet. & Second-feet. & Feet. & Second-feet. & Feet. & Second-feet. & Feet. & Second-feet. \\
0.80 & 1 & 1.60 & 46 & 2.40 & 151 & 3.20 & 287 \\
0.90 & 2 & 1.70 & 56 & 2.50 & 168 & 3.30 & 304 \\
1.00 & 4 & 1.80 & 68 & 2.60 & 185 & 3.40 & 321 \\
1.10 & 8 & 1.90 & 80 & 2.70 & 202 & 3.50 & 338 \\
1.20 & 13 & 2.00 & 92 & 2.80 & 219 & 3.60 & 355 \\
1.30 & 20 & 2.10 & 106 & 2.90 & 236 & 3.70 & 362 \\
1.40 & 28 & 2.20 & 120 & 3.00 & 253 & 3.80 & 379 \\
1.50 & 36 & 2.30 & 135 & 3.10 & 270 & & \\
\hline
\end{tabular}


Daily discharge, in second-feet, of Santa Ana canal near Mentone, Cal., for 1905.

\begin{tabular}{|c|c|c|c|c|c|c|c|c|c|c|c|c|}
\hline Day. & Jan. & Feb. & Mar. & Apr. & May. & June. & July. & Aug. & Sept. & Oct. & Nov. & Dec. \\
\hline 1. & 22 & 25 & 60 & 57 & 71 & 0 & 63 & 53 & 66 & 51 & 46 & 34 \\
\hline 2. & 22 & 64 & 57 & 0 & 66 & 0 & 61 & 53 & 63 & 51 & 32 & 32 \\
\hline 3.. & 21 & 64 & 60 & 0 & 66 & 0 & 61 & 51 & 63 & 56 & 32 & 34 \\
\hline 4.. & 21 & 0 & 60 & 54 & 56 & 0 & 61 & 61 & 63 & 56 & 32 & 32 \\
\hline 5.. & 21 & 0 & 60 & 63 & 58 & 0 & 61 & 61 & 66 & 51 & 32 & 32 \\
\hline 6.. & 22 & 22 & 57 & 63 & 73 & 0 & 61 & 61 & 66 & 46 & 36 & 32 \\
\hline $7 .$. & 21 & 42 & 57 & 63 & 51 & 0 & 58 & 63 & 66 & 46 & 41 & 32 \\
\hline 8.. & 20 & 37 & 54 & 72 & 56 & 0 & 58 & 63 & 61 & 41 & 46 & 32 \\
\hline 9. & 0 & 37 & 51 & 78 & 66 & 0 & 56 & 63 & 61 & 56 & 41 & 32 \\
\hline 10 & 61 & 35 & 48 & 78 & 76 & 0 & 56 & 63 & 63 & 46 & 29 & 30 \\
\hline 11. & 54 & 43 & 48 & 72 & 76 & 0 & 58 & 63 & 73 & 46 & 29 & 32 \\
\hline 12. & 41 & 26 & 51 & 66 & 76 & 66 & 61 & 63 & 76 & 61 & 30 & 32 \\
\hline 13. & 41 & 28 & 0 & 66 & 76 & 43 & 63 & 66 & 76 & 66 & 32 & 29 \\
\hline 14. & 31 & 29 & 0 & 63 & 0 & 71 & 66 & 68 & 78 & 56 & 30 & 29 \\
\hline 15. & 31 & 35 & 0 & 57 & 0 & 71 & 66 & 68 & 78 & 61 & 30 & 32 \\
\hline 16. & 27 & 29 & 97 & 43 & 71 & 71 & 63 & 71 & 78 & 56 & 29 & 30 \\
\hline 17. & 29 & 32 & 78 & 0 & .71 & 68 & 63 & 71 & 78 & 51 & 29 & 30 \\
\hline 18. & 33 & 42 & 0 & 0 & 71 & 68 & 63 & 81 & 78 & 46 & 29 & 29 \\
\hline 19. & 25 & 70 & 0 & 0 & 36 & 68 & 61 & 81 & 73 & 43 & 29 & 29 \\
\hline 20. & 27 & 70 & 0 & 71 & 36 & 68 & 61 & 56 & 68 & 41 & 29 & 29 \\
\hline 21. & 66 & 70 & 0 & 66 & 32 & 68 & 61 & 56 & 68 & 41 & 34 & 32 \\
\hline 22. & 61 & 74 & 78 & 66 & 29 & 68 & 61 & 56 & 61 & 46 & 32 & 25 \\
\hline 23. & 49 & 74 & 66 & 66 & 29 & 68 & 61 & 56 & 68 & 41 & 30 & 25 \\
\hline $24 \therefore$ & 40 & 66 & 78 & 66 & 29 & 68 & 61 & 73 & 68 & 41 & 29 & 25 \\
\hline 25. & 31 & 66 & 78 & 71 & 0 & 68 & 61 & 73 & 51 & 46 & 30 & 25 \\
\hline 26 .. & 29 & 58 & 60 & 71 & 0 & 68 & 61 & 73 & 56 & 46 & 30 & 25 \\
\hline $27 .$. & 27 & 51 & 85 & 66 & 0 & 68 & 61 & 76 & 56 & 41 & 41 & 29 \\
\hline $28 .$. & 25 & 58 & 85 & 66 & 0 & 63 & 61 & 76 & 66 & 41 & 50 & 28 \\
\hline $29 .$. & 25 & $\cdots$ & 78 & .71 & 0 & 63 & 61 & 76 & 66 & 41 & 41 & 30 \\
\hline $30 .$. & 25 & ....... & 42 & 76 & 0 & 63 & 56 & 73 & 66 & 41 & 32 & 29 \\
\hline $31 \ldots$. & 25 & ... & 57 & ........ & 0 & .... & 56 & 73 & $\ldots$ & 41 & ........... & 27 \\
\hline
\end{tabular}

Estimated monthly discharge of Santa Ana a River near Mentone, Cal., for 1905.

[Drainage area, 182 square miles.]

\begin{tabular}{|c|c|c|c|c|c|c|}
\hline \multirow{2}{*}{ Month. } & \multicolumn{3}{|c|}{ Discharge in second-feet. } & \multirow[b]{2}{*}{$\begin{array}{l}\text { Total in } \\
\text { acre-feet. }\end{array}$} & \multicolumn{2}{|c|}{ Run-off. } \\
\hline & Maximum. & Minimum. & Mean. & & $\begin{array}{l}\text { Second-feet } \\
\text { per square } \\
\text { mile. }\end{array}$ & $\begin{array}{l}\text { Depth } \\
\text { in inches. }\end{array}$ \\
\hline January.. & 108 & 22 & 36.6 & 2,251 & 0.201 & 0.232 \\
\hline February.. & 234 & 26 & 97.6 & 5,420 & .536 & .558 \\
\hline March ..... & 321 & 48 & 130 & 7,993 & .714 & .823 \\
\hline April... & 120 & 63 & 90.5 & 5,385 & .497 & .554 \\
\hline May .......... & 256 & 91 & 172 & 10,580 & .945 & 1.09 \\
\hline June........ & 94 & 64 & 77.7 & 4,624 & .427 & .476 \\
\hline July .......... & 69 & 57 & $62.9^{\circ}$ & 3,867 & .346 & .399 \\
\hline August....... & 82 & 52 & 66.8 & 4,107 & $\cdot 367$ & .423 \\
\hline September....... & 79 & 52 & 68.3 & 4,064 & .375 & .418 \\
\hline October......... & 67 & 42 & 49.2 & 3,025 & .270 & .311 \\
\hline November...................... & 250 & 30 & 47.5 & 2,827 & .261 & .291 \\
\hline December.............. & 107 & 31 & 38.4 & 2,362 & .211 & .243 \\
\hline The year. & 321 & 22 & 78.1 & 56,500 & .429 & 5.82 \\
\hline
\end{tabular}




\section{SEEPAGE MEASUREMENTS.}

In the vicinity of Colton and San Bernardino large quantities of water are developed in addition to the natural surface flow. This water is used for the irrigation of land in the vicinity of.San Bernardino, Colton, and Riverside, and also for domestic supply for these towns. Much of this water returns to Santa Ana River below Riverside, above a point known as Slover Mountain and is again diverted and used for irrigation on the lower lands below Riverside, and above what is known as Riverside Narrows. Below this point there are still further diversions which irrigate the lower lands along the river bottom, much of this water again returning to the river above Rincon. Measurements were made during the summer of 1905 to. determine the amount of water, including the natural flow and developed water, above Colton, Cal. Also measurements were made of natural flow and developed water below Slover Mountain and above Riverside Narrows, this all being return water from irrigated lands on the higher elevations. Measurements were also made of diversion ditches and Santa Ana River below Riverside Narrows and above what is known as the Auburndale Bridge. The following tabulations show the result of these measurements which were made by $K$. Sanborn, of Riverside, Cal.

Natural flow of return water to Santa Ana River in second-feet; compared with developed water in San Bernardino Valley above Colton, Cal., 1905.

[Measurements by K. Sanborn, engineer Riverside Water Company:]

\begin{tabular}{|c|c|c|c|c|}
\hline Date. & Location. & Developed. & Natural. & Total. \\
\hline & & $S e c-. f t$ & Sec.-ft. & Sec.-ft. \\
\hline June 9. & Barnhill pumping plant.. & 1.10 & & 1.10 \\
\hline September 27.. & ..... do...................... & .80 & & .80 \\
\hline June $10 \ldots \ldots$. & Beam ditch............. & ...... & 0.00 & .00 \\
\hline September 2... & ....do........................... & $\ldots$. & .00 & .00 \\
\hline June $16 \ldots \ldots . .$. & Bloomington pumping plants..... & 6.62 & & 6.62 \\
\hline September $27 .$. & .....do... & 7.20 & & 720 \\
\hline June $10 \ldots . .$. & City of San Bernardino, Sixth street pumping plant. & 1.99 & & 1.99 \\
\hline September $27 .$. & 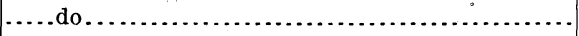 & 4.80 & & 4.80 \\
\hline June $19 \ldots \ldots$. & Cty of San Bernardino, Lytle Creek ....... & 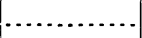 & 2.04 & 2.04 \\
\hline September 27.. & …do & $\cdots \cdot$ & 1.90 & 1.90 \\
\hline June $9 . \ldots \ldots .$. & City of Colton pumping plant (total).. & 3.81 & -. & 3.81 \\
\hline September 27.. & City of Colton (water used for irrigation) ...... & 1.20 & & 1.20 \\
\hline September 27.. & City of Colton pumping plant (total).. & 2.70 & & 2.70 \\
\hline May $31 \ldots . . .$. & Camp Carlton ditch............. & 2.60 & & 2.60 \\
\hline August 14 & .....do. & 1.20 & & 1.20 \\
\hline June $10 . . .$. & Carr pumping plant. & .72 & & .72 \\
\hline August 14 & ....do......................... & .60 & & .60 \\
\hline June 10. & Daley ditch... & 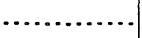 & .00 & .00 \\
\hline September 4 . & .....do. . & .... & .00 & .00 \\
\hline May 30 . & Excelsior Land and Water Co.. & .65 & & .6 \\
\hline September 28. & ....do $\ldots \ldots \ldots \ldots \ldots \ldots$ & .40 & & .40 \\
\hline May 31 . & Grand Terrace Pumping Co. pumping plant.. & .54 & & .5 \\
\hline August 14 . & ....do do................................. & .00 & & .00 \\
\hline May $31 \ldots$ & Gage canal, Palm avenue weir......... & 28.83 & & 28.83 \\
\hline September $2 \ldots$ & 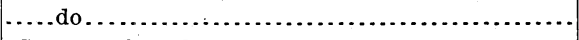 & 32.70 & & 32.70 \\
\hline May $26 \ldots$ & Gage canal intake, Santa Ana River.... & & .00 & .00 \\
\hline September $4 . .$. & .....do..... & $\cdots$ & .00 & .00 \\
\hline June $2 .$. & Haws \& Talmadge ditch... & $\cdots$ & .00 & .00 \\
\hline September 5. . & ......do.. & ….... & .00 & .00 \\
\hline June $6 . . . \ldots .$. & Hunter pumping plant...... & 1.09 & $\cdots$ & 1.09 \\
\hline September $4 . .$. & .....do.......... & 1.60 & & 1.60 \\
\hline June $9 . . . .$. & Johnson \& Hubbard pumping plant. & .59 & & .59 \\
\hline
\end{tabular}


Natural flow of return water to Santa Ana River in second-feet, compared with developed water in San Bernardino Valley above Colton, Cal., 1905-Continued.

\begin{tabular}{|c|c|c|c|c|}
\hline Date. & Location. & Developed. & $\begin{array}{c}\text { Natural. } \\
-\end{array}$ & Total. \\
\hline Șeptember $4 . .$. & Johnson \& Hubbard pumying plant.... & $\begin{array}{l}\text { Sec.-ft. } \\
\quad 0.30\end{array}$ & $\begin{array}{c}\text { Sec.-ft. } \\
\ldots . . . . . . .\end{array}$ & $\begin{array}{l}\text { Sec.-ft. } \\
0.30\end{array}$ \\
\hline June $9 . . . . . .$. & Lamb pumping plant........ & 30 & & .30 \\
\hline September 27. & .....do................. & .00 & & .00 \\
\hline June $9 . . . . . .$. & Lawson Well Co. pumping plant.. & .62 & & .62 \\
\hline September 27. & 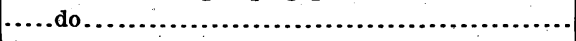 & .60 & & .60 \\
\hline June $10 \ldots . . .$. & Logsdon \& Farrell ditch........... & $\ldots .$. & 0.00 & .00 \\
\hline September 2. & .....do................................. & $\ldots \ldots \ldots$ & .00 & .00 \\
\hline June 10........ & Lytle Creek Water and Improvement Co.. & .00 & .. & .00 \\
\hline September 27. & …do............................ & .00 & & .00 \\
\hline May $30 \ldots . . .$. & Merryfield pumping plant.... & .67 & & .67 \\
\hline September 28. & ....do........................... & .40 & & .40 \\
\hline June 2......... & McKenzie ditch........ & $\therefore \ldots$ & .00 & .00 \\
\hline September 5. & ....do.............................. & & .00 & .00 \\
\hline June $9 . . . . . .$. & Meeks \& Daley ditch.......... & & 15.70 & 15.70 \\
\hline September $2 . .$. & ....do do........................ & $\cdots$ & 15.50 & 15.50 \\
\hline June 16.... & McIntyre ditch.... & & .00 & .00 \\
\hline September 28 . & ....do.............. & $\cdots$ & .00 & .00 \\
\hline June $9 . . . . . .$. & Orange Land and Water Co. pumping plant. & .00 & & .00 \\
\hline September 27. & .....do................ & 1.60 & & 1.60 \\
\hline June $16 \ldots \ldots$. & Riverside Highland Water Co., Lytle Creek ... & 7.05 & & 7.05 \\
\hline September 28. & ....do............................ & 8.00 & & 8.00 \\
\hline May $31 \ldots . . .$. & Riverside Highland Water Co., Santa Ana River. & 6.36 & & 6.36 \\
\hline September $4 . .$. & 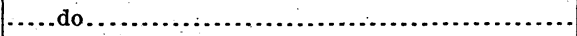 & 7.60 & .40 & 8.00 \\
\hline June 16....... & Rancheria pumping plant........ & 1.24 & & 1.94 \\
\hline September 27. & …do........................... & 1.10 & -. & 1.10 \\
\hline June 2... & Rabel ditch... & ....... & .00 & .00 \\
\hline September & .....do................ & $\cdots$ & .00 & .00 \\
\hline May $31 .$. & Riverside Water Co., upper canal... & 25.33 & 24.85 & 50.18 \\
\hline August 31 . & ....do................................. & 21.20 & 12.90 & 34.10 \\
\hline May 31.... & Riverside Water Co., mill pumping plant.. & .92 & $\cdots$ & .92 \\
\hline August 31 .. & ....do.................... & 1.30 & 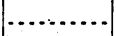 & 1.30 \\
\hline May $31 \ldots .$. & Riverside Water Co., mill flume....... & & 2.24 & 2.24 \\
\hline August 31 & 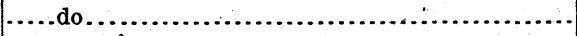 & ...... & .00 & .00 \\
\hline May $26 \ldots$. & Riverside Water Co., flume pump No. $1 . . . .$. & 3.95 & $\cdots \cdot$ & 3.95 \\
\hline August 31 . & .....do... & 3.60 & $\cdots$ & 3.60 \\
\hline May $26 \ldots$. & Riverside Water Co., flume pump No. $2 .$. & 3.18 & $\cdots$. & 3.18 \\
\hline August 31 . & ......do.... & 3.00 & $\because$ & 3.00 \\
\hline June 6......... & Rosedale Water Co. pumping plant. & .30 & & .30 \\
\hline September $28 .$. & ......do.... & .00 & .... & .00 \\
\hline June 6......... & Rogers pumping plant.. & & .00 & .00 \\
\hline August 31 & .....do.......................... & .00 & $\cdots$ & .00 \\
\hline June 2: $: .$. & Shay or Stout Dam ditch.. & $\cdots$ & .00 & .00 \\
\hline September 5... & ....do....................... & .... & .00 & .00 \\
\hline May 31 & Swamp ditch..... & & .47 & .47 \\
\hline August 14 . & .....do........... & .... & .40 & .40 \\
\hline June 6.... & West Riverside 350-inch Water Co. pumping plant . & 2.00 & & 2.00 \\
\hline September 28. . & ....do.................. & 5.60 & …. & 5.60 \\
\hline June $19 . . . . .$. & Whitlock ditch... & & .00 & .00 \\
\hline September 2... & .....do.............. & & .00 & .00 \\
\hline June 6......... & Whiting ditch..... & & .00 & .00 \\
\hline September $4 . .$. & 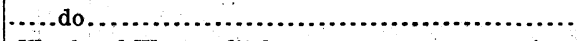 & $\cdots \cdot$ & .00 & .00 \\
\hline May $31 \ldots . . .$. & 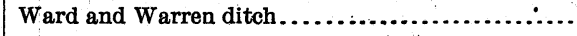 & .00 & $\cdots$ & .00 \\
\hline September 4 & ....do. & & .00 & .00 \\
\hline
\end{tabular}


Return waters in San Bernardino Valley below Slover Mountain and above Riverside Narrows, 1905.

[Measurements by K. Sanborn, engineer Riverside Water Company.]

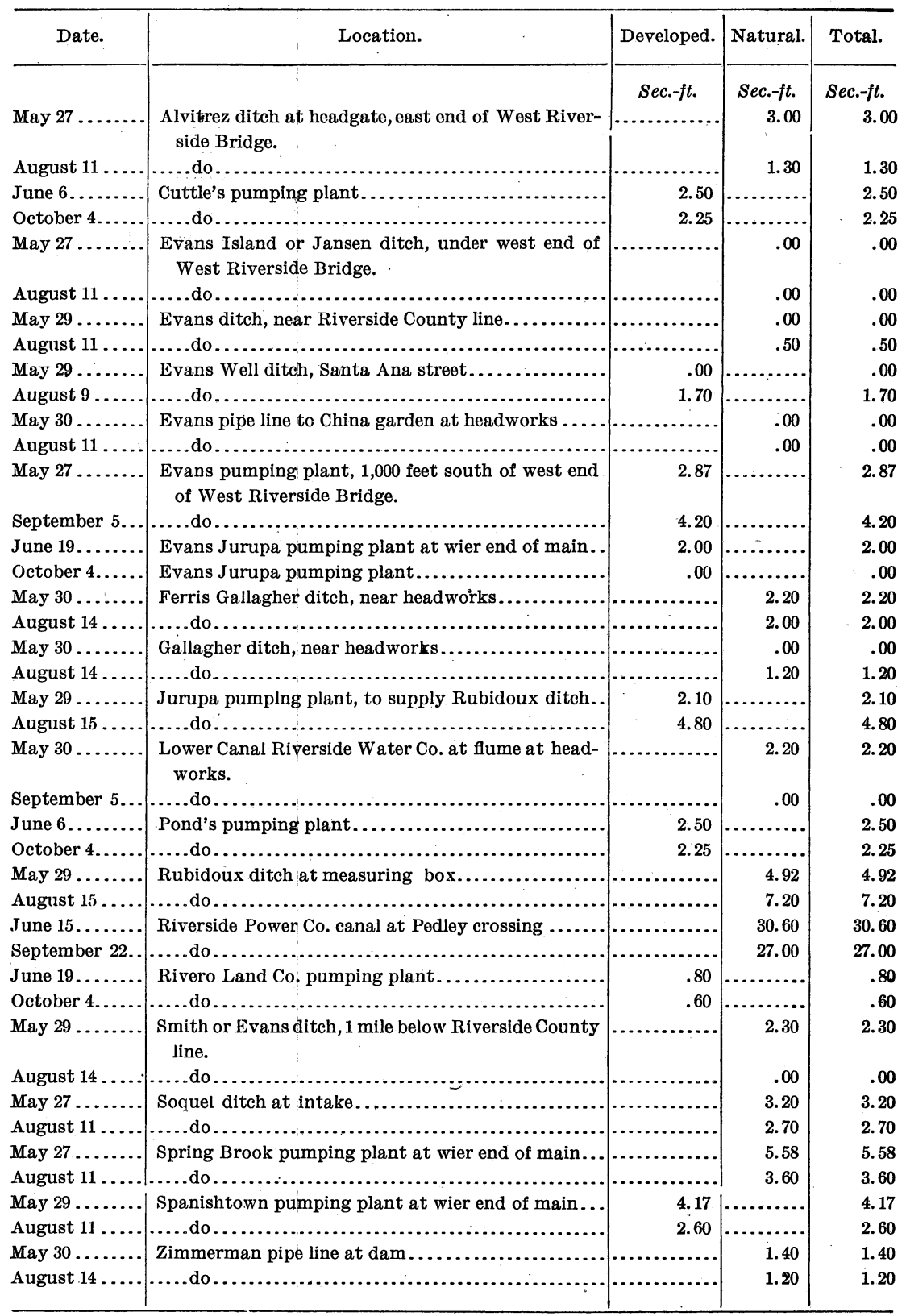


Discharge measurements of canals between the Riverside Narrows and the Auburndale bridge having their source in the Santa Ana River, 1905.

\begin{tabular}{|c|c|c|}
\hline Date. & Location. & Discharge. \\
\hline June $15 . . . . . .$. & 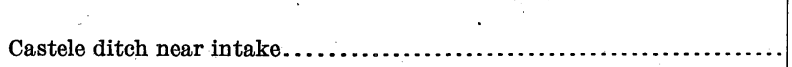 & $\begin{array}{r}\text { Sec. }-\mathrm{ft} . \\
0.00\end{array}$ \\
\hline September $22 .$. & ....do............... & .00 \\
\hline June $15 \ldots . . .$. & Durkee ditch at Auburndale road crossing........ & 4. 10 \\
\hline September 22.. & .....do.................. & .00 \\
\hline June $15 \ldots . . .$. & Fuller ditch at waste gate... & 5.60 \\
\hline September $22 .$. & ....do................. & 5.40 \\
\hline June $15 \ldots . . .$. & Gilliland ditch at Auburndale road crossing. . & 1.40 \\
\hline September $22 .$. & .....do...................... & .30 \\
\hline June $15 . . . . .$. & Newton ditch near intake.................. & 2.30 \\
\hline September $22 \therefore$ & 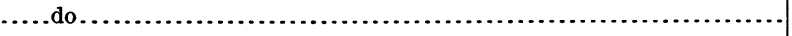 & 1.70 \\
\hline June $15 \ldots . . .$. & Newberry ditch at Auburndale road crossing.. & .00 \\
\hline September $22 \therefore$ & ....do................................. & .00 \\
\hline June $15 \ldots \ldots$. & Roberts or Le Gay ditch near intake Santa Ana River. . & 1.00 \\
\hline September $22 .$. & .....do..................... & 1.70 \\
\hline June $15 \ldots . . .$. & Wilbur ditch at Rogers pipe trestle crossing Santa Ana River.. & 6. 10 \\
\hline September $22 .$. & 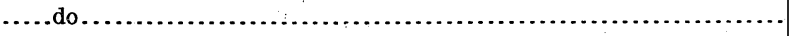 & 6. 40 \\
\hline June 15. & Santa Ana River, Auburndale Bridge....................... & 63.60 \\
\hline Do .. & Santa Ana River, Auburndale Bridge, including ditches........ & 71.13 \\
\hline September $22 .$. & 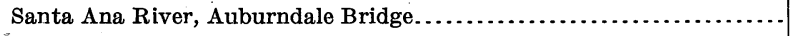 & 57.00 \\
\hline Do... & Santa Ana River, Auburndale Bridge, including ditches.... & 57.80 \\
\hline
\end{tabular}

\section{MISCELLANEOUS MEASUREMENTS IN SANTA ANA RIVER DRAINAGE BASIN.}

The following is a list of miscellaneous discharge measurements made in the Santa Ana River drainage basin during 1905:

City Creek near Highlands, Cal.-This stream is a tributary of Santa Ana River. A measurement was made September 23 by W. B. Clapp in diversion canal at mouth of canyon. Measurement made over weir.

Discharge, 1.16 second-feet.

Chino Creek near Rincon, Cal.- This stream is a tributary of Santa Ana River. The following measurements were made during 1905 at the wagon bridge at Rincon road crossing, one-fourth mile above junction of Chino Creek with the Santa Ana river.

February 24: Width, 16 feet; area, 22 square feet; mean velocity, 2.09 feet per second; discharge, 46 second-feet.

April 17: Width, 7 feet; area, 5.6 square feet; mean velocity, 4.64 feet per second; discharge, 26 second-feet.

May 16: Width, 5 feet; area, 4 square feet; mean velocity, 4.25 feet per second; discharge, 17 second-feet.

June 6: Width, 4 feet; area, 1.7 square feet; mean velocity, 3.53 feet per second; discharge, 6.0 second-feet.

July 29: Width, 2 feet; area, 0.4 square foot; mean velocity, 2.75 feet per second; discharge, 1.1 second-feet.

September 21: Width, 3 feet; area, 2 square feet; mean velocity, 0.95 foot per second; discharge, 1.9 second-fieet.

Cable Canyon Creek near Glen Helen, Cal.-This stream is a tributary of the Santa Ana River. A measurement was made July 13 by K. Sanborn. Measurement made over weir.

Discharge, 1.66 second-feet.'

Devil Canyon near Irvington Station, Cal.-This stream is a tributary of the Santa Ana River. A measurement was made July 13 by $\mathrm{K}$. Sanborn at the mouth of the canyon, 100 feet below head of M. L. \& W. Co.'s ditch. 
Discharge, 1.26 second-feet.

East Twin Creek near Arrowhead Springs, Cal.-This stream is a tributary of the Santa Ana River. A measurement was made September 23 by W. B. Clapp at the mouth of the canyon. Measurement made over weir.

Discharge, 0.54 second-foot.

Lytle Creek near Rialto, Cal.-Thïs stream is one of the principal tributaries of the Santa Ana River. A measurement was made June 16 by K. Sanborn at the head of Fontella Development Company's canal at the mouth of the canyon. Measurement made over weir.

Discharge, 23 second-feet.

Mill Creek near Mentone, Cal.-This stream is one of the principal tributaries of the Santa Ana River. The following measurements were made during 1905:

At road crossing between Mentone and Santa Ana Canyon, April 14: Width, 16 feet; area, 11.2 square feet; mean velocity, 4.11 feet per second; discharge, 46 second-feet. This. is waste water and does not include water diverted by Crafton Zanja.

At head of Crafton Zanja, September 23: Width, 7.7 feet; area, 4.7 square feet; mean velocity, 4.33 feet per second; discharge, 20 second-feet.

Morton Canyon Creek near Mentone, Cal.-This stream is a small tributary of Santa Ana River. A measurement was made September 22 by W. B. Clapp at the mouth of the canyon, 500 feet above its junction with Santa Ana River.

Width, 0.8 foot; area, 0.096 square foot; mean velocity, 1.67 feet per second; discharge, 0.16 second-foot.

Plunge Creek near East.Highlands, Cal.-This stream is a tributary of the Santa Ana River. The following measurements were made during 1905:

At road crossing Orange avenue between Redlands and Highlands, April 15: Width, 16. feet; area, 5.1 square feet; mean velocity, 1.94 feet per second; discharge, 9.9 second-feet.

At cement ditch at the mouth of the canyon, September 22: Width, 2 feet; area, 0.6 square foot; mean velocity, 1.67 foot per second; discharge, 1.0 second-foot.

Redlands tunnel near Mentone, Cal.-This is developed water from tunnel in the bed of the Santa Ana River at the mouth of the canyon. A measurement was made September 22 over weir at the mouth of the tunnel.

Discharge, 1.1 second-feet.

Santa Ana River near Redlands, Cal.-A measurement of this stream was made at the road crossing at Orange avenue between Redlands and Highlands April 14.

Width, 55 feet; area, 34 square feet; mean velocity, 2.35 feet per second; discharge, 76 second-feet.

Santa Ana.River near Rincon, Cal.-The following measurements were made during 1905 at the Rincon wagon bridge, at the lower end of San Bernardino Valley, and the head of the lower Santa Ana Canyon. These measurements, with the addition of those of Chino Creek, show the total discharge of the Santa Ana River below all diversions in San Bernardino Valley, and show the amount of water which is used for irrigation in the vicinity of Orange, Santa Ana, Anaheim, and Fullerton, Cal., diversions being made below this point of measurement.

The gage is a 1 by 4 inch timber, graduated to feet and tenths and bolted to the first caisson from the south end of the Rincon wagon bridge. There is no bench mark.

February 24: Width, 40 feet; area, 68 square feet; mean velocity, 3.01 feet per second; gage height, 3.63 feet; discharge, 205 second-feet.

April 17: Width, 48 feet; area, 68 square feet; mean velocity, 2.53 feet per second; gage height, 3.60 feet; discharge, 172 second-feet.

May 16: Width, 45 feet; area, 50 square feet; mean velocity, 2.30 feet per second; gage height, 3.35 feet; discharge, 115 second-feet.

June 6: Width, 45 feet; area, 44 square feet; mean velocity, 1.98 feet per second; gage height, 3.32 feet; discharge, 87 second-feet.

July 29: Width, 48 feet; area, 34 square feet; mean velocity, 2.06 feet per second; gage height, 3.25 feet; discharge, 70 second-feet. 
September 21: Width, 46 feet; area, 39 square feet; mean velocity, 1.95 feet per second; gage height, 3.33 feet; discharge, 76 second-feet.

Waterman Canyon Creek or West Twin Creek, near Arrowhead Springs, Cal.-This stream is a tributary of Santa Ana River. A measurement was made July 13 by K. Sanborn at the bridge on the road to Waterman's ranch at the mouth of the canyon.

Discharge, 1.02 second-feet.

\section{SAN GABRIEL RIVER DRAINAGE BASIN.}

\section{DESCRIPTION OF BASIN.}

The San Gabriel River rises in the Sierra Madre Mountains and, flowing in a southwesterly direction through the San Gabriel and Los Angeles valleys, discharges its waters into the Pacific Ocean near Long Beach, Cal. In the upper reaches of this basin there are numerous tributaries, which have their source in the higher elevations of the Sierra Madre Range. The topography in the upper reaches of this basin is rough and rugged, with deep and narrow canyons, while on the lower elevations the country is rolling, with large areas of valley land. The formation on the higher mountain elevation is of granite, with a light soil covering, with sparse timber growth. As one approaches the middle elevations the covering is brush, with scattering timber, while in the foothill country there is nothing but a growth of grass. The gaging station on this stream is located at a point where the stream leaves the higher mountains in the vicinity of Azusa. Below this point the river enters San Gabriel Valley, where the stream has a comparatively light grade, the bed being composed of bowlders, gravel, and sand, in which the water quickly disappears, except in times of flood discharge. The waters of this stream again appear on the surface at the lower end of San Gabriel Valley, at the discharge from the foothills, where an obstruction to the underground passage forces the water to the surface, on which it flows for a short distance and again disappears in the sands of the flat country below the foothills. The entire flow of this stream during the summer months is diverted at a point about 5 miles above the gaging station and is used for power purposes at the mouth of the canyon. From this point it is carried in ditches and used for irrigation in San Gabriel Valley. The water is again diverted where it appears on the surface at the lower end of this valley and is used for irrigation on the lower levels below this point. The mean precipitation in this basin varies from 15 to 30 inches and is principally in the form of rain. On small areas in the higher mountain elevations the precipitation is in the form of snow, which melts in the early spring months.

\section{SAN GABRIEL RIVER AND CANALS NEAR AZUSA, CAL.}

Owing to the numerous diversions, it has been difficult to obtain accurate discharge measurements at Azusa, but during 1898 the San Gabriel Electric Company completed its system, and measurements are now obtained with greater ease and hence with greater accuracy. The headworks of this company are located about 6 miles above the mouth of the canyon. The water is carried around the left side by a series of tunnels and conduits, and a head of 400 feet is obtained where the electric power is generated. Weirs are placed on the conduit of the electric company and the water is measured at this point. The capacity of the conduit is 80 second-feet.

The cable and gage are located about 1 mile from Azusa. During the season of low water for a period of from six to eight months the canals above the station divert the entire flow and there is no running water at the station. The total flow of the river is obtained by adding the daily discharge for the river to the figures for the corresponding dates for the canals.

The channel is straight for 100 feet above and 500 feet below the cable and has a width of 280 feet at high water. At low stages there are two channels having different elevations, and accurate measurements are difficult to obtain. The bed of the stream is composed of cobblestones and bowlders, and the current is swift.

Discharge measurements are made by means of a cable and car. The gage is a vertical staff. During 1905 the gage was read by Don. D. Morgan.

Information in regard to this station is contained in Water-Supply Papers Nos. 81, 100, and 134 of the United States Geological Survey. 
Discharge measurements of San Gabriel River and canals near Azusa, Cal., in 1905.

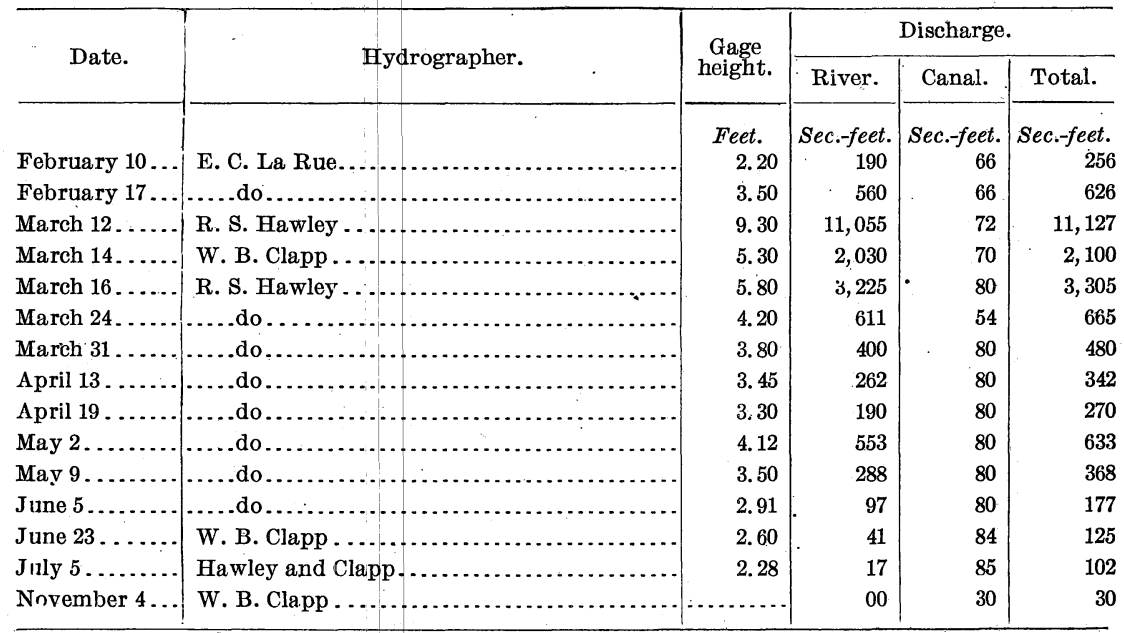

Daily gage height, in feet, of San Gabriel River near Azusa, Cal., for 1905.

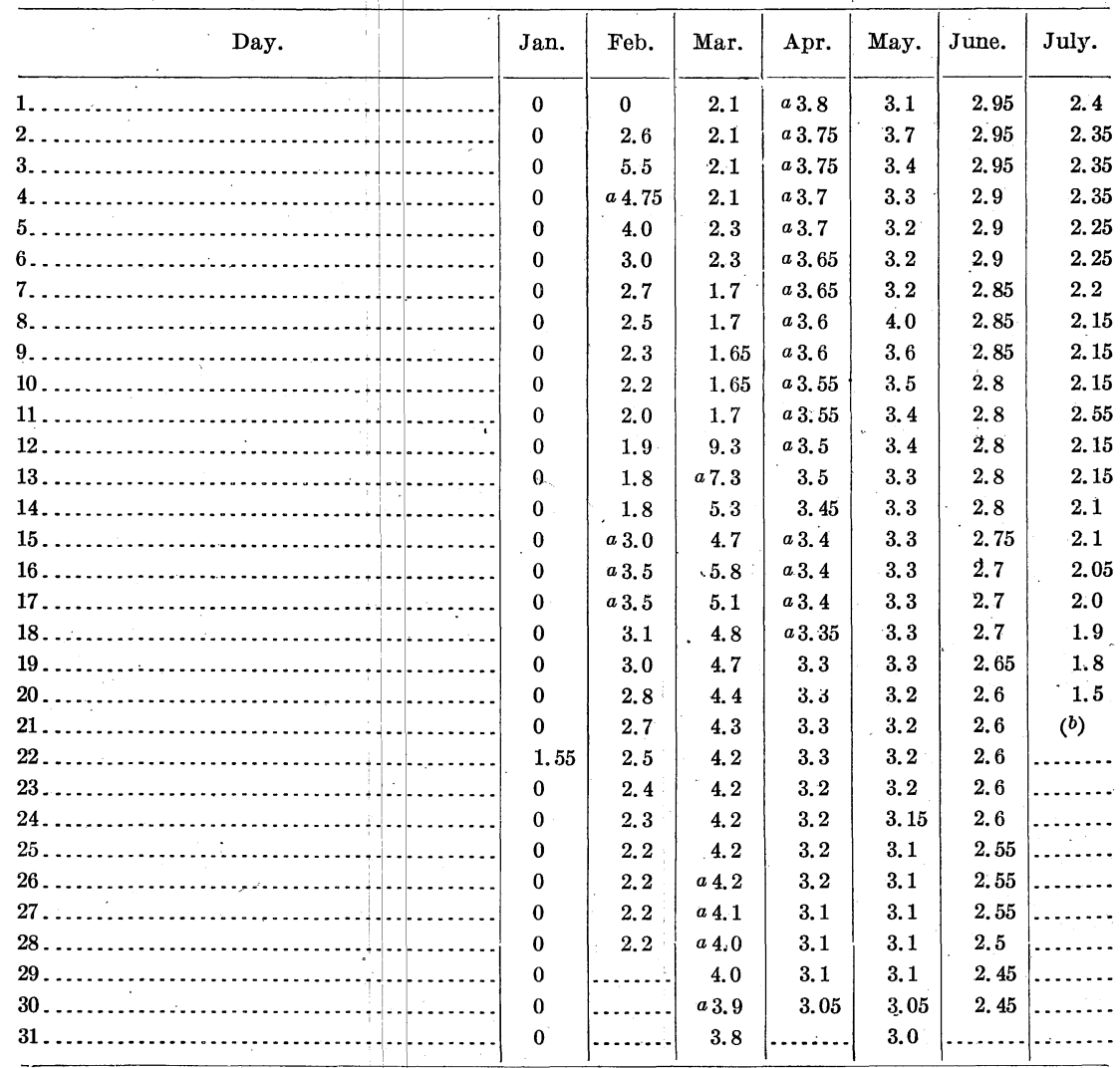

$a$ Estimated.

$b$ Dry from July 21 to December 31 .

NoтE.-Gage washed out on March 12; replaced April 19. Gage heights taken with a level between March 12 and April 19. 
Station rating table for San Gabriel River near Azusa, Cal., from February 2. to March 11, 1905.

\begin{tabular}{|r|r||r|r|r|r|r|r|}
\hline $\begin{array}{c}\text { Gage } \\
\text { height. }\end{array}$ & Discharge. & $\begin{array}{c}\text { Gage } \\
\text { height. }\end{array}$ & Discharge: & $\begin{array}{c}\text { Gage } \\
\text { height. }\end{array}$ & Discharge. & $\begin{array}{c}\text { Gage } \\
\text { height. }\end{array}$ & Discharge. \\
\hline Freet. & Second-feet. & Freet. & Second-feet. & Feet. & Second-feet. & Feet. & Second-feet. \\
1.10 & 0 & 2.50 & 207 & 3.60 & 610 & 4.70 & 1,620 \\
1.50 & 55 & 2.60 & 231 & 3.70 & 670 & 4.80 & 1,750 \\
1.60 & 65 & 2.70 & 259 & 3.80 & 730 & 4.90 & 1,900 \\
1.70 & 76 & 2.80 & 289 & 3.90 & 800 & 5.00 & 2,050 \\
1.80 & 88 & 2.90 & 320 & 4.00 & 870 & 5.10 & 2,220 \\
1.90 & 101 & 3.00 & 351 & 4.10 & 950 & 5.20 & 2,390 \\
2.00 & 115 & 3.10 & 390 & 4.20 & 1,040 & 5.30 & 2,570 \\
2.10 & 130 & 3.20 & 430 & 4.30 & 1,140 & 5.40 & 2,750 \\
2.20 & 147 & 3.30 & 470 & 4.40 & 1,250 & 5.50 & 2,940 \\
2.30 & 165 & 3.40 & 513 & 4.50 & 1,370 & & \\
2.40 & 185 & 3.50 & 560 & 4.60 & 1,490 & & \\
& & & & & & \\
\hline
\end{tabular}

Nore.-The above table is applicable only for open-channel conditions. It is based on discharge measurements made during first part of 1905; 1904 table applies from January 1 to February 1, inclusive.

Station rating table for San Gabriel River near Azusa, Cal., from March 12 to December 31, 1905.

\begin{tabular}{|r|r||r|r|r|r|r|r|}
\hline $\begin{array}{c}\text { Gage } \\
\text { height. }\end{array}$ & Discharge. & $\begin{array}{c}\text { Gage } \\
\text { height. }\end{array}$ & Discharge. & $\begin{array}{c}\text { Gage } \\
\text { height. }\end{array}$ & Discharge. & $\begin{array}{c}\text { Gage } \\
\text { height. }\end{array}$ & Discharge. \\
\hline Feet. & Second-feet. & Feet. & Second-feet. & Feet. & Second-feet. & Feet. & Second-feet. \\
1.70 & 1 & 3.20 & 170 & 4.70 & 1,040 & 6.40 & 4,499 \\
1.80 & 2.5 & 3.30 & 202 & 4.80 & 1,160 & 6.60 & 4,951 \\
1.90 & 4.5 & 3.40 & 237 & 4.90 & 1,300 & 6.80 & 5,403 \\
2.00 & 7 & 3.50 & 273 & 5.00 & 1,460 & 7.00 & 5,855 \\
2.10 & 10 & 3.60 & 311 & 5.10 & 1,640 & 7.20 & 6,307 \\
2.20 & 14 & 3.70 & 351 & 5.20 & 1,830 & 7.40 & 6,759 \\
2.30 & 18 & 3.80 & 394 & 5.30 & 2,030 & 7.60 & 7,211 \\
2.40 & 24 & 3.90 & 441 & 5.40 & 2,245 & 7.80 & 7,663 \\
2.50 & 32 & 4.00 & 492 & 5.50 & 2,470 & 8.00 & 8,115 \\
2.60 & 42 & 4.10 & 550 & 5.60 & 2,695 & 8.20 & 8,567 \\
2.70 & 55 & 4.20 & 616 & 5.70 & 2,920 & 8.40 & 9,019 \\
2.80 & 71 & 4.30 & 687 & 5.80 & 3,145 & 8.60 & 9,471 \\
2.90 & 90 & 4.40 & 762 & 5.90 & 3,370 & 8.80 & 9,923 \\
3.00 & 113 & 4.50 & 841 & 6.00 & 3,595 & 9.00 & 10,375 \\
3.10 & 140 & 4.60 & 935 & 6.20 & 4,047 & 9.20 & 10,828 \\
& & & & & & & \\
\hline
\end{tabular}

Noтk.-The above table is applicable only for open-channel conditions. It is based on 11 discharge measurements made during 1905. 
Daily discharge, in second-feet, of San Gabriel canals near Azusa, Cal., for 1905.

\begin{tabular}{|c|c|c|c|c|c|c|c|c|c|c|c|c|}
\hline Day. & Jan. & Feb. & Mar. & Apr. & May. & June. & July. & Aug. & Sept. & Oct. & Nov. & Dec. \\
\hline $1 \ldots$ & 30 & 34 & 67 & 80 & 80 & 80 & 85 & 55 & 34 & 30 & 29 & 50 \\
\hline $2 .$. & 23 & 67 & 67 & 80 & 80 & 80 & 85 & 54 & 33 & 29 & 29 & 47 \\
\hline $3 .$. & 21 & 70 & 67 & 80 & 80 & 80 & 86 & 54 & 32 & 28 & 29 & 45 \\
\hline 4... & 20 & 78 & 67 & 80 & 80 & 80 & 85 & 53 & 33 & 28 & 29 & 44 \\
\hline $5 \ldots$ & 19.5 & 68 & 0 & 80 & 80 & 80 & 85 & 52 & 34 & 28 & 29 & 44 \\
\hline $6 \ldots$ & 19 & 78 & 67 & 80 & 80 & 80 & 86 & 50 & 35 & 28 & 32 & 42 \\
\hline $7 \ldots$ & 18.5 & 69 & 72 & 80 & 80 & 80 & 86 & 47 & 34 & 28 & 44 & 42 \\
\hline 8... & 18.5 & 86 & 72 & 80 & 48 & 80 & 85 & 46 & 33 & 28 & 95 & 41 \\
\hline $9 .$. & 41. & 74 & 72 & 80 & 80 & 80 & 85 & 45 & 32 & 27 & 53 & 39 \\
\hline $10 .$. & 59 & 66 & 72 & 80 & 80 & 80 & 85 & 45 & 32 & 27 & 42 & 37 \\
\hline $11 .$. & 40 & 66 & 72 & 80 & 80 & 73 & 71 & 46 & 32 & 27 & 38 & 37 \\
\hline $12 \ldots$ & 35 & 66 & 72 & 80 & 80 & 73 & 74 & 44 & 32 . & 27 & 36 & 37 \\
\hline $13 .$. & 31 & 66 & 0 & 80 & 80 & 76 & 75 & 44 & 32 & 28 & 36 & 38 \\
\hline $14 \ldots$ & 29 & 66 & 70 & 80 & 80 & 76 & 74 & 43 & 31 & 29 & 34 & 39 \\
\hline $15 .$. & 27 & 66 & 79 & 80 & 80 & 0 & 74 & 43 & 31 & 29 & 34 & 39 \\
\hline $16 .$. & 30 & 67 & 80 & 80 & 80 & 78 & 74 & 42 & 30 & 29 & 45 & 39 \\
\hline $17 .$. & 30 & 66 & 80 & 80 & 80 & 78 & 74 & 41 & 31 & $-\quad 30$ & 53 & 39 \\
\hline 18.. & 28 & 68 & ' 80 & 80 & 80 & 78 & 74 & 40 & 31 & 30 & 43 & 39 \\
\hline $19 .$. & 28 & 69 & 79 & 80 & 80 & 78 & 73 & 41 & 30 & 30 & 40 & 38 \\
\hline $20 \ldots$ & 27 & 68 & 80 & 80 & 80 & 78 & 73 & 41 & 29 & 29 & 42 & 46 \\
\hline 21.. & 55 & 68 & 80 & 80 & 80 & 78 & 70 & 40 & 29 & 29 & 41 & 42 \\
\hline $22 .$. & 67 & 68 & 78 & 80 & 80 & 88 & 69 & 40 & 28 & 31 & 39 & 39 \\
\hline $23 .$. & 67 & 68 & 57 & 80 & 80 & 84 & 68 & 39 & 30 & 32 & 38 & 38 \\
\hline $24 \ldots$ & 57 & 67 & 54 & 80 & 80 & 84 & 67 & 38 & 30 & 31 & 37 & 37 \\
\hline $25 \ldots$ & 46 & 68 & 69 & 80 & 80 & 84 & 64 & 38 & 30 & 30 & 37 & 38 \\
\hline 26... & 41 & 67 & 80 & 80 & 80 & 84 & 62 & 36 & 29 & 29 & 37 & 38 \\
\hline $27 \ldots$ & 40 & 67 & 80 & 80 & 80 & 84 & 64 & 36 & 30 & 29 & 97 & 38 \\
\hline $28 \ldots$ & 38 & 67 & 80 & 80 & 80 & 84 & 62 & 35 & 31 & 28 & 87 & 38 \\
\hline 29... & 37 & $\cdots$ & 80 & 80 & 80 & 84 & 60 & 34 & 32 & 28 & 62 & 37 \\
\hline 30... & 36 & $\ldots$. & 80 & 80 & 80 & 84 & 68 & 33 & 31 & 29 & 53 & 37 \\
\hline $31 \ldots$ & 34 & & 80 & $\ldots$ & 80 & & 56 & 33 & $\ldots$ & 28 & & 38 \\
\hline
\end{tabular}

Estimated monthly discharge of San Gabriel River a near Azusa, Cal., for 1905.

[Drainage area, 222 square miles.]

\begin{tabular}{|c|c|c|c|c|c|c|}
\hline \multirow[b]{2}{*}{ Month. } & \multicolumn{3}{|c|}{ Discharge in second-feet. } & \multirow[b]{2}{*}{$\begin{array}{l}\text { Total in } \\
\text { acre-feet. }\end{array}$} & \multicolumn{2}{|c|}{ Run-off. } \\
\hline & Maximum. & Minimum. & Mean. & & $\begin{array}{l}\text { Second-feet } \\
\text { per square } \\
\text { mile. }\end{array}$ & $\begin{array}{l}\text { Depth } \\
\text { in inches. }\end{array}$ \\
\hline January... & 108 & 18.5 & 36.6 & 2,251 & 0.165 & 0.190 \\
\hline February.......... & 3,010 & 34 & 466 & 25,880 & 2.10 & 2.19 \\
\hline March.......... & 11,130 & 142 & 1,222 & 75,140 & 5.50 & 6.34 \\
\hline 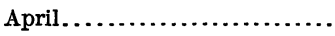 & 474 & 206 & 329 & 19,580 & 1.48 & 1.65 \\
\hline May.......... & 540 & 193 & 278 & 17,090 & 1.25 & 1.44 \\
\hline June............ & 182 & 63 & 139 & 8,271 & .626 & .698 \\
\hline July.......... & 109 & 56 & 83.0 & 5,103 & .374 & .431 \\
\hline August.... & 55 & 33 & 42.8 & 2,631 & .193 & .222 \\
\hline September....... & 35 & 28 & 31.4 & 1,869 & .141 & .157 \\
\hline October...... & 32 & 27 & 28.8 & 1,771 & .130 & .150 \\
\hline November....................... & 97 & 29 & 44.7, & 2,660 & .201 & .224 \\
\hline 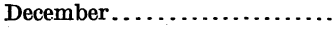 & 50 & 37 & 40.1 & 2,466 & .181 & .209 \\
\hline The year. & 11,130 & 18.5 & 2,284 & 164,700 & 1.03 & 13.90 \\
\hline
\end{tabular}




\section{MISCELLANEOUS MEASUREMENTS IN SAN GABRIEL RIVER DRAINAGE BASIN.}

The following is a list of miscellaneous discharge measurements made in the San Gabriel River drainage basin during 1905:

Baldwin ditch near El Monte, Cal.-This ditch diverts water from the Rio Hondo River and is used for irrigation of land in the vicinity of the Old Mission. A measurement was made October 6 by W. B. Clapp at Old Mission Bridge, 4 miles below El Monte, Cal.

Width, 1.2 feet; area, 1.4 square feet; mean velocity, 2.14 feet per second; discharge, 3.0 second-feet.

Cate ditch near El Monte, Cal.- This ditch diverts water from the San Gabriel River and is used for the irrigation of land in the vicinity of Rivera, Cal. A measurement was made October 6 by W. B. Clapp in flume at road crossing near county road between El Monte and Whittier and about 5 miles below El Monte.

Width, 4 feet; area, 7.7 square feet; mean velocity, 1.45 feet per second; discharge, 11.2 second-feet.

Ranchito or Standerford ditch near El Monte, Cal.-This ditch diverts water from the San Gabriel River and is used for the irrigation of land in the vicinity of Rivera and Downey, Cal. A measurement was made October 6 by W. B. Clapp 25 feet below head-gate and about 5 miles below El Monte, Cal.

Width, 11.8 feet; area, 11 square feet; mean velocity, 1.42 feet per second; discharge, 15.6 second-feet.

Los Nietos or Banta ditch.-This ditch diverts water from the San Gabriel River and is used for the irrigation of land in the vicinity of Los Nietos, Cal. A measurement was made October 6 by W. B. Clapp 100 feet below head-gate of Ranchito ditch.

Width, 18 feet; area, 14.8 square feet; mean velocity, 1.14 feet per second; discharge, 16.8 second-feet.

Rio Hondo near El Monte, Cal.-This stream constitutes one branch of the San Gabriel River. A measurement was made October 6 by W. B. Clapp at Old Mission Bridge, 4 miles below El Monte and below the diversion of the Baldwin ditch.

Width, 13 feet; areạ, 12 square feet; mean velocity, 1.75 feet per second; discharge, 21 second-feet.

Rincon ditch near El Monte, Cal.-This ditch diverts water from the San Gabriel River 2 miles below Southern Pacific Railroad bridge and is used for irrigation of land in the vicinity of Rincon, Cal. A measurement was made October 6 by W. B. Clapp at road crossing, one-half mile east of the Durfee ranch.

Width, 6 feet; area, 3.2 square feet; mean velocity, 1.06 feet per second; discharge, 3.4 second-feet.

Sheep Creek ditch near El Monte, Cal.-Whis ditch diverts water from Sheep Creek, a tributary of San Gabriel River. A measurement was made October 6 by W. B. Clapp in flume at road crossing, 1 mile east of Durfee ranch.

Width, 3 feet; area, 2.1 square feet; mean velocity, 2.19 feet per second; discharge, 4.6 second-feet.

Santa Anita Creek near Sierra Madre, Cal.-This stream is a tributary of the San Gabriel River. A measurement was made October 23 by W. B. Clapp at the mouth of the canyon above Baldwin diversion.

Width, 3 feet; area, 1.0 square foot; mean velocity, 1.50 feet per second; discharge, 1.5 second-feet.

San Gabriel River near El Monte, Cal.-A measurement was made of this stream on April 13 by R. S. Hawley at wagon bridge, 2 miles east of El Monte, Cal.

Width, 56 feet; area, 20 square feet; meản velocity, 2.35 feet per second; discharge, 47 second-feet. 


\section{LOS ANGELES RIVER DRAINAGE BASIN.}

\section{DESCRIPTION OF BASIN.}

The Los Angeles River is formed by the Tujunga, Pacoima, and other small creeks which have their source in the Sierra Madre Range of mountains to the northeast of the city of Los Angeles. These streams leave the mountains at a point about 25 miles above the city and enter the comparatively flat country of San Fernando Valley, where, except in times of excessive flood, the waters disappear in the sand and gravel washes of the valley. These waters again make their appearance at the lower end of this valley, where a secondary range of hills, extending from east to west, forces the waters to the surface in what is known as Los Angeles River. Below this point the river discharges through the flat country of Los Angeles Valley, finally entering the Pacific Ocean near the town of Long Beach, Cal. During the summer months the entire flow of Los Angeles River is diverted at a point about 5 miles above Los Angeles for the supply of the city, and only a small amount of water passes this point except during flood discharge of the river. The topography is rough in the upper reaches of this drainage basin, the streams discharging in deep, narrow canyons. In this portion of the drainage basin the formation is of granite, with good soil covering and light timber and heavy brush growth. There is a considerable area of foothill country within this basin, lying between the base of the Sierra Madre Range and Los Angeles Valley, which has a light covering of brush and grass. The soil of San Fernando Valley consists principally of river wash, coarse sand, and gravel, except along the base of the mountains and foothills, where the soil is of good depth and is under a high state of cultivation. The soil of Los - Angeles Valley, below the city of Los Angeles, consists of a light sandy loam and is under a high state of cultivation. The mean precipitation throughout this basin is from 15 to 30 inches and falls in the form of rain, except on small areas on the higher mountain elevations.

\section{MISCELIANEOUS MEASUREMENTS IN LOS ANGELES RIVER DRAINAGE BASIN.}

The following is a list of miscellaneous discharge measurements made in Los Angeles River drainage basin during 1905:

Arroyo Seco near Pasadena, Cal.-This stream is a tributary of Los Angeles River. The following measurements were made on this stream during 1905 by W. B. Clapp:

At mouth of canyon, 5 miles above Pasadena, Cal., April 11: Width, 13.5 feet; area, 8.1 square feet; mean velocity, 2.11 feet per second; discharge, 17.1 second-feet.

At Devils Gate, 3 miles above Pasadena, Cal., April 11: Width, 8 feet; area, 8.7 square feet; mean velocity, 1.44 feet per second; discharge, 12.5 second-feet.

At point 1,000 feet above submerged dam of Pasadena Land and Water Company, at Pasadena, Cal., April 11: Width, 8 feet; area, 2 square feet; mean velocity, 1.30 feet per second; discharge, 2.6 second-feet.

At sycamore grove, near Morgan's south line, Los Angeles, Cal., by J. B. Lippincott, October 27: Width, 1.1 feet; area, 0.16 square foot; mean velocity, 0.64 foot per second; discharge, 0.10 second-foot.

At sycamore grove, 600 feet above Morgan's south line, October 27: Width, 1.2 feet; area, 0.20 square foot; mean velocity, 1.14 feet per second; discharge, 0.23 second-foot.

Big Tejunga Creek near Sunland, Cal.-This stream is a tributary of the Los Angeles River. The following measurements were made during 1905 by R. S. Hawley:

At mouth of canyon, 1 mile above Sunland, Cal., April 12: Width, 25 feet; area, 13.7 square feet; mean velocity, 4.45 feet per second; discharge, 61 second-feet.

At Southern Pacific Company's railroad crossing near Pacoima, Cal., April 12: Width, 25 feet; area, 10.3 square feet; mean velocity, 2.82 feet per second; discharge, 29 secondfeet.

Two miles below Southern Pacific Railroad bridge at Pacoima, Cal., April 12: No discharge. 
Little Tejunga Creek near Sunland, Cal.-This stream is a tributary of Los Angeles River and discharges into Big Tejunga Creek below Sunland. A measurement was made April 11 by $\mathrm{R}$. S. Hawley at mouth of canyon two-thirds of a mile above the junction with Big Tejunga Creek.

Width, 6 feet; area, 1.6 square feet; mean velocity, 2.06 feet per second; discharge, 3.3 second-feet.

Pacoima Creek near Fernando, Cal.-This stream is a tributary of Los Angeles River. The following measurements were made during 1905 by R. S. Hawley:

At mouth of canyon, 5 miles above Fernando, Cal., April 12: Width, 20 feet; area, 7.3 square feet; mean velocity, 4.11 feet per second; discharge, 30 second-feet.

At Southern Pacific Railroad bridge, 1 mile east of Fernando, Cal., April 12: Width, 10 feet; area, 6.2 square feet; mean velocity, 2.84 feet per second; discharge, 17.6 second-feet.

Three miles below Southern Pacific Railroad bridge, near Fernando, Cal., April 12: No discharge.

\section{MISCELLANEOUS MEASUREMENTS ON LOS ANGELES RIVER.}

The following measurements were made on Los Angeles River at Los Angeles, Cal., during 1905:

At Fourth Street Bridge.-March 13: Width, 120 feet; area, 446 square feet; mean velocity, 10.15 feet per second; discharge, 4,525 second-feet.

At Huron street.-April 12: Width, 38 feet; area, 20 square feet; mean velocity, 2.15 feet per second; discharge, 43 second-feet.

At Ninth Street Bridge.-April 12: Width, 13 feet; area, 5.8 square feet; mean velocity, 2.19 feet per second; discharge, 12.7 second-feet.

During the summer of 1905 measurements were made to determine the amount of water diverted by the city of Los Angeles for domestic supply and also to determine the amount of water which passes the city's diversion point. The amount of water diverted for the city's supply is shown in measurenents made in the 44-inch conduit and the main supply conduit. The amount of water discharging in the river below these points of diversion is shown by measurements made at Huron street. The following measurements were made during 1905 at these points:

Measurements on Los Angeles River in Los Angeles.

\begin{tabular}{|c|c|c|c|c|c|c|}
\hline \multirow[b]{2}{*}{ Date. } & \multirow{2}{*}{$\begin{array}{c}\text { 44-inch } \\
\text { conduit } \\
\text { dischárge. }\end{array}$} & \multirow{2}{*}{$\begin{array}{c}\text { Main } \\
\text { supply } \\
\text { conduit } \\
\text { discharge. }\end{array}$} & \multicolumn{4}{|c|}{ Huron street. } \\
\hline & & & Width. & Area. & $\begin{array}{c}\text { Mean } \\
\text { velocity. }\end{array}$ & $\begin{array}{c}\text { Dis- } \\
\text { charge. }\end{array}$ \\
\hline & Sec.-ft. & Sec.-ft. & Feet. & $s q . f t$. & Ft.per sec. & Sec.-ft. \\
\hline May $15 . . . . . .$. & 42 & 11 & 24 & 7.2 & 1.47 & 10.6 \\
\hline June $22 \ldots . . .$. & .. 38 & 9.9 & 16 & 3.2 & 1.03 & 3.3 \\
\hline July $28 . \ldots \ldots \ldots \ldots \ldots \ldots$ & 37 & 8.4 & 6 & 2.2 & 1.77 & 4.6 \\
\hline September $19 . \ldots \ldots \ldots \ldots \ldots \ldots$ & 35.3 & 8.7 & 6 & 2.2 & 1.55 & 3.4 \\
\hline
\end{tabular}




\section{MALIBU CREEK DRATNAGE BASIN.}

\section{DESCRIPTION OF BASIN.}

Malibu Creek rises in the Santa Monica Mountains and enters the Pacific Ocean about 15 miles above the town of Santa Monica. This stream is formed by Triunfo and Las Virgenes creeks, which drain the northern portion of the Santa Monica Range and the lower foothill country to the north. The formation throughout this basin is shale, sandstone, and conglomerate, with good soil covering. There is a sparse growth of timber on the higher elevations, but a greater portion of this area has a covering of brush and grass used extensively for pasturage, with limited areas of cultivated land for the raising of grain. A reservoir has been constructed on the upper reaches of the Triunfo Creek and the waters are used for irrigation within the basin during the summer months. This reservoir covers an area of about 300 acres when filled.

The mean precipitation in this basin amounts to about 25 inches and falls wholly in the form of rain.

\section{MALIBU OREEK NEAR OALABASAS, CAL.}

This station was established November 29,1901 , by S. G. Bennett. It is located at Chapman's ranch, 40 miles from Los Angeles, by wagon road, and 8 miles southwest of Calabasas, about one-fourth mile below the mouth of Las Virgenes Creek.

The channel section is poor and subject to change during high water, but is at the only point where an observer could be secured. The excessive cost of visiting the station has made it impossible to obtain as many meter measurements as desired, but the observer is instructed to take float velocities at various gage heights, and these data, with cross sections and slope, are used in addition to meter measurements for computing discharges for use in constructing rating curves and tables. The estimated discharge is a rough approximation only.

The channel is straight for about 600 feet above the station and curved for about 300 feet below. The current is swift. Both banks are high. The right bank is rocky, and the bed of the stream is composed of rock and gravel.

The initial point for soundings is on the right bank. The gage rod is a vertical staff fastened to an alder tree on the right bank. During 1905 the gage was read by J. G. Chapman. The bench mark is a cross on a small projection on a rock bluff about 10 feet southwest of the gage rod; elevation, 5.43 feet above the datum of the gage.

Information in regard to this station is contained in Water-Supply Papers Nos. 100 and 134 of the United States Geological Survey.

Discharge measurements of Malibu Creek near Calabasas, Cal., in 1905.

\begin{tabular}{|c|c|c|c|c|c|c|}
\hline Date. & Hydrographer. & Width. & $\begin{array}{l}\text { Area of } \\
\text { section. }\end{array}$ & $\begin{array}{c}\text { Mean } \\
\text { velocity. }\end{array}$ & $\begin{array}{c}\text { Gage } \\
\text { height. }\end{array}$ & $\begin{array}{c}\text { Dis- } \\
\text { charge. }\end{array}$ \\
\hline & . & Feet. & Sq. ft. & Ft. per sec. & Feet. & Sec.-ft. \\
\hline April 20. & R. S. Hawley... & 9 & 6.2 & 1.5 & 0.9 & 9.3 \\
\hline May $12 .$. & 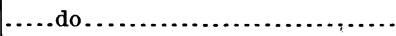 & 9 & 6.3 & 1.35 & .82 & 8.5 \\
\hline
\end{tabular}


Daily gage height, in feet, of Malibu Creek near Calabasas, Cal., for 1905.

\begin{tabular}{|c|c|c|c|c|c|c|c|c|c|c|c|c|}
\hline Day. & Jan. & Feb. & Mar. & Apr. & May. & June. & July. & Aug. & Sept. & Oct. & Nov. & Dec. \\
\hline 1.. & 1.2 & 1.2 & 1.1 & 1.5 & 0.8 & 0.6 & 0.5 & 0.5 & 0.5 & 0.4 & 0.45 & 0.5 \\
\hline $2 \ldots$ & 1.2 & 1.3 & 1.1 & 1.25 & .8 & .6 & .5 & .5 & .5 & .4 & .45 & .5 \\
\hline $3 .$. & 1.2 & 1.6 & 1.1 & 1.2 & .8 & .6 & .5 & .5 & .5 & .4 & .45 & .5 \\
\hline $4 . .$. & 1.2 & 2.85 & 1.1 & 1.2 & .8 & .6 & .5 & .5 & .5 & .4 & .45 & .5 \\
\hline $5 \ldots$ & 1.2 & 1.85 & 1.1 & 1.2 & .8 & .6 & .5 & .5 & .5 & .4 & .55 & .5 \\
\hline 6... & 1.2 & 2.1 & 1.1 & 1.25 & .8 & .6 & .5 & .5 & .5 & .4 & .55 & .5 \\
\hline 7.. & 1.2 & 1.3 & 1.1 & 1.25 & 1.3 & .6 & .5 & .5 & .5 & .4 & .55 & .5 \\
\hline $8 \ldots$ & 1.2 & 1.2 & 1.1 & 1.25 & .9 & .6 & .5 & .5 & .5 & .4 & .55 & .5 \\
\hline $9 \ldots$ & 1.2 & 1.15 & 1.1 & 1.2 & .9 & .6 & .6 & .5 & .5 & .4 & .55 & .5 \\
\hline $10 \ldots$ & 1.2 & 1.15 & 1.1 & 1.2 & .9 & .6 & .6 & .5 & -5 & .4 & .55 & .6 \\
\hline $11 .$. & 1.2 & 1.15 & 1.0 & 1.2 & .9 & .6 & .6 & .5 & .45 & .4 & .55 & .6 \\
\hline .......... & 1.2 & 1.15 & 7.6 & 1.1 & .9 & .6 & .6 & .5 & .45 & .4 & .6 & .6 \\
\hline $13 .$. & 1.2 & 1.15 & 4.2 & 1.25 & .9 & .6 & .6 & .5 & .45 & .4 & .6 & .6 \\
\hline $14 .$. & 1.2 & 1.1 & 3.25 & 1.0 & .7 & .6 & .6 & .5 & .45 & .4 & .6 & .6 \\
\hline 15.. & 1.2 & 1.1 & 3.25 & 1.0 & .7 & .6 & .6 & .5 & .45 & .4 & .6 & .6 \\
\hline $16 \ldots$ & 1.2 & 1.8 & 4.75 & 1.0 & .7 & .6 & .6 & .5 & .45 & .4 & .6 & .6 \\
\hline $17 \ldots$ & 1.2 & 1.65 & 3.55 & 1.0 & .7 & .6 & .6 & .5 & .45 & .4 & .6 & .6 \\
\hline $18 \ldots$ & 1.2 & 1.55 & 2.8 & .9 & .7 & .6 & .6 & .5 & .4 & .4 & .6 & .6 \\
\hline ........... & 1.22 & 1.4 & 2.5 & .9 & .7 & .6 & .6 & .5 & .4 & .4 & .6 & .6 \\
\hline ......... & 1.25 & 1.3 & 2.4 & .9 & .7 & .6 & .6 & .5 & .4 & .4 & .6 & .6 \\
\hline 21.. & 1.3 & 1.2 & 2.4 & .9 & .7 & .6 & .6 & .5 & .4 & .4 & .6 & .6 \\
\hline .. & 1.24 & 1.2 . & 2.3 & .9 & .7 & .6 & .6 & .5 & .4 & .4 & .6 & .6 \\
\hline $23 .$. & 1.2 & 1.15 & 2.2 & .9 & .7 & .6 & .5 & .5 & .45 & .4 & .6 & .6 \\
\hline $24 .$. & 1.2 & 1.1 & 2.2 & .9 & .7 & .6 & .5 & .5 & .45 & .4 & .6 & .6 \\
\hline $25 .$. & 1.2 & 1.1 & 1.0 & .9 & .7 & .5 & .5 & .5 & .45 & .4 & .6 & .6 \\
\hline $26 \ldots$ & 1.2 & 1.1 & 1.6 & .9 & .7 & .5 & .5 & .5 & .45 & .4 & .5 & .6 \\
\hline $27 \ldots$ & 1.2 & 1.1 & 1.45 & .9 & .7 & .5 & .5 & .5 & .45 & .4 & .5 & .6 \\
\hline 28. . & 1.2 & 1.1 & 1.55 & .8 & .6 & .5 & .5 & .5 & .45 & .4 & .5 & .6 \\
\hline $29 .$. & 1.2 & .. & 1.7 & .8 & .6 & .5 & .5 & .5 & .45 & .45 & .5 & .6 \\
\hline $30 \ldots$ & 1.2 & & 1.7 & .8 & .6 & .5 & .5 & .5 & .45 & .45 & .5 & .6 \\
\hline $31 \ldots \ldots . .$. & 1.2 & 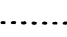 & 1.55 & & .6 & & .5 & .5 & & .45 & & .6 \\
\hline
\end{tabular}


Estimated monthly discharge of Malibu Creek near Calabasas, Cal., for 1905.

[Drainage area, 97 square miles.]

\begin{tabular}{|c|c|c|c|c|c|c|}
\hline \multirow[b]{2}{*}{ Month. } & \multicolumn{3}{|c|}{ Discharge in second-feet. } & \multirow{2}{*}{$\begin{array}{l}\text { Total in } \\
\text { acre-feet. }\end{array}$} & \multicolumn{2}{|c|}{ Run-off. } \\
\hline & Maximum. & Minimum. & Mean. & & $\begin{array}{l}\text { Second-feet } \\
\text { per square } \\
\text { mile. }\end{array}$ & $\begin{array}{l}\text { Depth } \\
\text { in inches. }\end{array}$ \\
\hline January ..... & 10 & 5 & 5.4 & 332 & 0.056 & 0.065 \\
\hline February... & 560 & 2 & 36.1 & 2,005 & .372 & .387 \\
\hline March...... & 6,800 & 1 & 484. & 29,760 & 4.99 & 5.75 \\
\hline Aprii...... & 41 & 8 & 14.4 & 857 & .148 & .165 \\
\hline May ........ & 24 & 4 & 7.3 & 449 & .075 & .086 \\
\hline June......... & 4 & 3 & 3.8 & 226 & .039 & .044 \\
\hline July ........ & 3 & 3 & 3.0 & 184 & .031 & .036 \\
\hline August. ............. & 3 & 3 & 3.0 & 184 & .031 & .036 \\
\hline September. . & 3 & 1 & 2.1 & 125 & .022 & .024 \\
\hline October.... & 2 & 1 & 1.1 & 68 & .011 & .013 \\
\hline 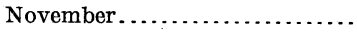 & 4 & 2 & 3.6 & 214 & .037 & .041 \\
\hline 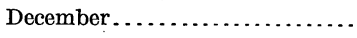 & 4 & 3 & 3.7 & 228 & .038 & .044 \\
\hline The year. & 6,800 & 1 & 47.3 & 34,630 . & .488 & 6.69 \\
\hline
\end{tabular}

NoTE.-The estimated monthly discharge is only an approximation.

\section{TRIUNFO CREEK NEAR CALABASAS, CAL.}

This station is located 8 miles southwest of Calabasas, Cal., about one-half mile above the mouth of Las Virgenes Creek.

The channel section is poor and subject to change during high water, but is at the only point where an observer could be secured. The excessive cost of visiting the station has made it impossible to obtain as many meter measurements as desired, but the observer is instructed to take float velocities during floods at various gage heights, and these data, with cross sections and grade of stream, are used in addition to meter measurements for computing discharges for use in constructing rating curves and tables. The estimated discharge from this stream is a rough approximation only.

The channel is straight for about 400 feet above and 800 feet below the station, and the water is swift. Both banks are high and rocky. The bed of the stream is composed of gravel and sand and is shifting.

The initial point for soundings is on the right bank. The gage rod is a vertical staff bolted to rock cliff on right bank. During 1905 the gage was read by J. G. Chapman. The bench mark is a cross on a point of rock 3.5 feet above the bed of the creek on the right bank; elevation, 4.53 feet above the datum of the gage.

Information in regard to this section is contained in Water-Supply Papers Nos. 100 and 134 of the United States Geological Survey. 
Discharge measurements of Triunfo Creek near Calabasas, Cal., in 1905.

\begin{tabular}{|c|c|c|c|c|c|c|}
\hline Date. & Hydrographer. & width. & $\begin{array}{l}\text { Area of } \\
\text { section. }\end{array}$ & $\begin{array}{c}\text { Mean } \\
\text { velocity. }\end{array}$ & $\begin{array}{c}\text { Gage } \\
\text { height. }\end{array}$ & $\begin{array}{c}\text { Dis- } \\
\text { charge. }\end{array}$ \\
\hline April 20. & R. S. Hawley ........ & Feet. & $\underset{7.1}{S q . f t .}$ & $\mid \begin{array}{r}\text { Ft. per sec. } \\
1.13\end{array}$ & $\begin{array}{c}\text { Feet. } \\
1.60\end{array}$ & $\begin{array}{r}\text { Sec. }-f t . \\
8.0\end{array}$ \\
\hline May 12. & .....do............ & 17 & 6.3 & 1.13 & 1.44 & 7.1 \\
\hline
\end{tabular}

Daily gage height, in feet, of Triunfo Creek near Calabasas, Cal., for 1905.

\begin{tabular}{|c|c|c|c|c|c|c|c|}
\hline Day. & Jan. & Feb. & Mar. & Apr. & May. & June. & .July: \\
\hline $1 .$. & & 0.00 & 0.5 & 1.3 & & 1.3 & 1.0 \\
\hline $2 \ldots$ & & .75 & .5 & 1.5 & $\cdots$ & 1.3 & 1.1 \\
\hline 3.. & & .9 & .5 & 1.5 & .. & 1.3 & 1.1 \\
\hline $4 .$. & & 2.15 & .5 & 1.5 & $\ldots$ & 1.3 & 1.1 \\
\hline $5 .$. & & 1.65 & .5 & 1.5 & $\ldots$ & 1.3 & 1.1 \\
\hline $6 \ldots$ & & 2.2 & .5 & 1.5 & …...... & 1.3 & 1.1 \\
\hline $7 .$. & & 1.65 & .5 & 1.6 & 1.4 & 1.3 & 1.1 \\
\hline 8.. & & 1.0 & .5 & 1.6 & .8 & 1.3 & 1.1 \\
\hline $9 \ldots$ & & .9 & .5 & 1.6 & .4 & 1.3 & 1.2 \\
\hline ....... & & .7 & .45 & 1.5 & & 1.3 & 1.2 \\
\hline $11 .$. & $\ldots$ & .55 & .45 & 1.55 & $\cdots$ & 1.25 & 1.2 \\
\hline ....... & ...... & .5 & 6.0 & 1.5 & ......... & 1.25 & 1.2 \\
\hline 13.. & & .5 & 2.55 & 1.5 & $\ldots$. & 1.25 & 1.2 \\
\hline $14 .$. & & .3 & 2.15 & 1.5 & 1.2 & 1.25 & 1.2 \\
\hline & & .3 & 2.05 & 1.5 & 1.2 & 1.25 & 1.2 \\
\hline 16.. & & 1.65 & 3.3 & 1.5 & 1.2 & 1.25 & 1.2 \\
\hline 17.. & & 1.45 & 3.1 & 1.5 & 1.2 & 1.25 & 1.2 \\
\hline …... & $\ldots . .$. & 1.3 & 2.6 & 1.5 & 1.2 & 1.2 & 1.2 \\
\hline $19 .$. & $\cdots$ & 1.2 & 2.25 & 1.4 & 1.2 & 1.2 & 1.2 \\
\hline $20 .$. & & 1.1 & 1.2 & 1.4 & 1.2 & 1.2 & 1.2 \\
\hline $21 .$. & & .9 & 1.2 & 1.4 & 1.2 & 1.2 & 1.2 \\
\hline $22 .$. & & .85 & 1.2 & 1.4 & 1.2 & 1.2 & 1.2 \\
\hline $23 .$. & & .8 & 1.1 & & 1.2 & 1.2 & 1.1 \\
\hline $24 .$. & ... & .8 & 2.2 & $\cdots$ & 1.2 & 1.2 & 1.1 \\
\hline …............ & $\ldots$ & .7 & 1.6 & $\ldots$ & 1.2 & 1.0 & 1.1 \\
\hline & & .7 & 1.4 & …..... & 1.3 & 1.0 & 1.1 \\
\hline $27 .$. & & .7 & 1.4 & $\cdots$ & 1.3 & 1.0 & 1.1 \\
\hline $28 \ldots$ & & .6 & 1.3 & & 1.3 & 1.0 & 1.1 \\
\hline . & & $\cdots$ & 1.4 & …..... & 1.3 & 1.0 & 1.1 \\
\hline$\cdots$ & & $\ldots$ & 1.4 & ........ & 1.3 & 1.0 & 1.0 \\
\hline 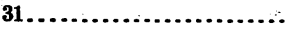 & & $\cdots$ & 1.4 & ......... & 1.3 & $\cdots$ & 0.0 \\
\hline
\end{tabular}

Note.-Creek dry Jan 2 to Feb. 1, Apr. 23 to May 6, May 10-13, Aug. 1 to Dec. 31. 
Estimated monthly discharge of Triunfo Creek near Calabasas, Cal., for 1905.

[Drainage area, 72 square miles.]

\begin{tabular}{|c|c|c|c|c|c|c|}
\hline \multirow[b]{2}{*}{ Month. } & \multicolumn{3}{|c|}{ Discharge in second-feet. } & \multirow[b]{2}{*}{$\begin{array}{l}\text { Total in } \\
\text { acre-feet. }\end{array}$} & \multicolumn{2}{|c|}{ Run-off. } \\
\hline & Maximum. & Minimum. & Mean. & & $\begin{array}{l}\text { Second-feet } \\
\text { per square } \\
\text { mile. }\end{array}$ & $\begin{array}{l}\text { Depth } \\
\text { in inches. }\end{array}$ \\
\hline January $\ldots . . .6 \ldots \ldots \ldots \ldots$ & $\mathbf{0}$ & 0 & 0.0 & 0 & 0.0 & $\therefore 0.0$ \\
\hline February $. . . \ldots \ldots \ldots \ldots \ldots \ldots . . . .$. & 410 & 0 & 26.2 & 1,455 & .364 & .379 \\
\hline March . . ....................... & 5,000 & 1 & 353.0 & 21,700 & 4.90 & 5.65 \\
\hline April $\ldots \ldots \ldots \ldots \ldots \ldots \ldots \ldots \ldots$ & 40 & 0 & 11.2 & 666 & .156 & .174 \\
\hline 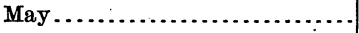 & 6 & 0 & 1.0 & 61 & .014 & .016 \\
\hline 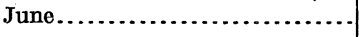 & 2 & 1 & 1.3 & 77 & .018 & .020 \\
\hline July . . . . . . . . . . . . . . . . . . & 1 & 0 & .97 & 60 & .013 & .015 \\
\hline August. . . . . . . . . . . . . & 0 & 0 & .0 & 0 & .0 & .0 \\
\hline September. ...................... & 0 & 0 & .0 & 0 & .0 & .0 \\
\hline October................. & 0 & 0 & .0 & 0 & .0 & .0 \\
\hline November................. & 0 & 0 & .0 & 0 & .0 & .0 \\
\hline 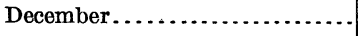 & 0 & 0 & .0 & 0 & .0 & .0 \\
\hline The year .................. & 5,000 & 0 & 32.8 & 24,020 & .455 & 6.25 \\
\hline
\end{tabular}

'Note.-The estimated monthly discharge is only an approximation.

\section{SANTA CLARA RIVER DRAINAGE BASIN.}

\section{DESCRIPTION OF BASIN.}

The Santa Clara River rises in the Coast Range in the northwestern part of Los Angeles County and flows in a westerly direction, discharging its waters into the Pacific Ocean near Ventura, Cal. It has numerous tributaries from the north, all of which are torrential in their character, having a heavy discharge during the winter months. This stream has a comparatively flat grade, flowing over a sandy and gravelly bed, into which it sinks during a greater portion of the year. At numerous points along its course the water rises to the surface during the summer and flows for a short distance, again disappearing in the sand. At points where the waters make their appearance on the surface numerous diversions are made for irrigation along the valley, where the soil is deep and is under the highest state of cultivation, this being especially the case on the lower reaches of the river below the town of Santa Paula. The principal tributaries of this stream are Piru Creek, Sespe Creek, and Santa Paula Creek, all of which produce a very heavy flood discharge. of short duration during the rainy season, but during the summer months have a light discharge. On the upper reaches of the principal tributaries of this stream the topography is rough and broken, the streams discharging through deep canyons. This is especially noticeable on the Sespe Creek. On the upper reaches of the main stream the country is rolling and has a sparse covering of timber with a considerable growth of brush and grass, and is used extensively for pasturage. The formation is of shale, sandstone, and conglomerate throughout the entire basin. The precipitation is extremely light in the upper reaches of this basin, but increases on the lower reaches nearer the Pacific Ocean and falls in the form of rain.

\section{MISCELLANEOUS MEASUREMENTS IN SANTA CLARA RIVER DRAINAGE BASIN,}

The following is a list of miscellaneous discharge measurements made in the Santa Clara River drainage basin during 1905:

Camulos ditch near Camulos, Cal.-This ditch diverts water from the Santa Clara River 1 mile above Camulos, Cal. The water is used for the irrigation of land at Camulos. A measurement was made October 19 by W. B. Clapp in a flume about 1,000 feet below the head of the canal. 
Width, 3.5 feet; area, 3.5 square feet; mean velocity, 2.17 feet per second; discharge, 7.6 second-feet.

Farmers' canal near Santa Paula, Cal.-This canal diverts water from the Santa Clara River at a point 1 mile above Santa Paula, Cal. The water is used for irrigation of land between Santa Paula and Saticoy, Cal. A measurement was made October 21 by W. B. Clapp in flume 200,feet below where canal crosses Santa Clara River, about 1 mile above the town of Santa Paula, Cal.

Width, 4 feet; area, 4.8 square feet; mean velocity, 1.50 feet per second; discharge, 7.2 second-feet:

Grees ditch near Santa Paula, Cal.-This ditch diverts water from the Santa Clara River about 1 mile above the town of Santa Paula, Cal. The water is used for the irrigation of land between Santa Paula and Saticoy. A measurement was made on October 21 by W. B. Clapp at a point 200 feet below head of ditch and about 1 mile above the town of Santa Paula, Cal.

Width, 8 feet; area, 6.6 square feet; mean velocity, 1.39 feet per second; discharge, 9.2 second-feet.

Piru Land and Water Company's upper diversion near Esperanza, Cal.-This water is diverted from Piru Creek for domestic and irrigation purposes in the town of Piru, Cal. A measurement was made October 20 by W. B. Clapp at point of diversion at head of pipe line, 1 mile above the town of Esperanza, Cal.

Width, 5 feet; area, 1.5 square feet; mean velocity, 0.93 foot per second; discharge, 1.4 second-feet.

Piru Creek near Esperanza, Cal.-This stream is a tributary of the Santa Clara River. A measurement was made October 20 by W. B. Clapp at point 1 mile above Esperanza, Cal., and below the upper diversion of the Piru Land and Water Company.

Width, 8 feet; area, 2.4 square feet; mean velocity, 1.42 feet per second; discharge, 3.4 second-feet. For total flow of Piru Creek at this point add upper diversion of Piru Land and Water Company.

Piru Creek near Piru City, Cal.-A measurement was made October 20 by W. B. Clapp 1 mile above Piru City and above lower diversion of Piru Land and Water Company.

Width, 8 feet; area, 2.8 square feet; mean velocity, 1.90 feet per second; discharge, 5.3 second-feet. This measurement shows total flow of Piru Creek at this point and includes the discharge of Piru Creek 1 mile above Esperanza, Cal. The increase is caused by seepage water along the creek.

Sespe Land and Water Company's canal near Sespe, Cal.-This canal diverts water from Sespe Creek and is used for irrigation of land in the vicinity of Sespe. A measurement was made October 20 by W. B. Clapp one-half mile below head of canal.

Width, 5.2 feet; area, 4.5 square feet; mean velocity, 1.47 feet per second; discharge, 6.6 second-feet.

Sespe Creek near Sespe, Cal:- -This creek is a tributary of the Santa Clara River. A measurement was made October 20 by W. B. Clapp one-half mile below heading of Sespe Land and Water Company's canal.

Width, 4 feet; area, 2.9 square feet; mean velocity, 1.66 feet per second; discharge, 4.8 second-feet. For total flow of Sespe Creek add discharge of Sespe Land and Water Company's canal as shown by measurement made on this date.

San Francisquito Creek near Saugus, Cal.- This creek is a tributary of the Santa Clara River. A measurement was made October 19 by W. B. Clapp at road crossing near Newhall ranch, $3 \frac{1}{2}$ miles below Saugus, Cal., and 500 feet above junction with Santa Clara River

Width, 5 feet; area, 2.8 square feet; mean velocity, 0.46 foot per second; discharge, 1.3 second-feet.

Santa Paula Creek near Santa Paula, Cal.-This stream is a tributary of the Santa Clara River. A measurement was made October 21 by W. B. Clapp 3 miles above Santa Paula and above diversion of the Santa Paula Land and Water Company.

Width, 11 feet; area, 5.6 square feet; mean velocity, 0.88 foot per second; discharge, 4.9 second-feet. This measurement shows total discharge of Santa Paula Creek on this date. 
Santa Clara River near Saugus, Cal.--A measurement was made October 19 by W. B. Clapp at road crossing near Newhall ranch, $3 \frac{1}{2}$ miles below Saugus, Cal.

Width, 6 feet; area, 3.8 square feet; mean velocity, 1.39 feet per second; discharge, 5.3 second-feet.

Santa Clara River near Camulos, Cal.-A measurement was made October 19 by W. B. Clapp at a point 1 mile above Camulos and 1,000 feet below diversion of Camulos ditch.

Width, 11 feet; area, 4.9 square feet; mean velocity, 2.06 feet per second; discharge, 10.1 second-feet. For total discharge of Santa Clara River at this point add discharge of Camulos ditch.

Santa Clara River near Santa Paula, Cal.-A measurement was made October 21 by W. B. Clapp 1 mile above Santa Paula and about 500 feet below diversion of Farmers and Grees canals.

Width, 33 feet; area, 17.4 square feet; mean velocity, 1.55 feet per second; discharge, 27 second-feet. For total diversion of Santa Clara River at this point add discharge of Farmers and Grees canals.

Santa Clara River near Saticoy, Cal.-A measurement was made October 21 by W. B. Clapp at a point opposite Saticoy and 200 feet below head of Santa Clara Water and Irrigation Company's canal.

Width, 14 feet; area, 14.7 square feet; mean velocity, 1.63 feet per second; discharge, 24 second-feet. For total discharge of Santa Clara River at this point add discharge of Santa Clara Water and Irrigation Company's canal.

Santa Clara Water and Irrigation Company's canal near Saticoy, Cal.-This canal diverts water from the Santa Clara River and is used for the irrigation of Santa Clara ranch near Oxnard, Cal.

Measurement made over weir. Discharge equals 16.7 second-feet.

\section{SANTA YNEZ RIVER DRAINAGE BASIN.}

\section{DESCRIPTION OF BASIN.}

Santa Ynez River rises in the mountains of Santa Barbara and Ventura counties and flows westerly with a flat grade to the Pacific Ocean, having a length of approximately 75 miles. The Santa Ynez Range of mountains, varying in elevation from 3,000 to 4,000 feet, forms the southern boundary of this drainage basin. The northern divide ranges from 4,500 to 5,500 feet in elevation, culminating in Mount Pinos, the elevation of which is 8,826 feet. The northern part of the watershed is drained by streams running in a southerly direction and uniting with the Santa Ynez River proper, which runs close to the northerly base of the Santa Ynez Mountains, flowing westerly and paralleling the Coast Range. The principal tributary, Mono Creek, enters from the north.

The formation throughout the entire drainage basin consists chiefly of shale and sandstone, the strike being parallel to the coast and the dip nearly vertical, inclining somewhat to the south.

The greater portion of the drainage is sparsely covered with brush and small trees, only a small area on the higher elevations having any considerable growth of timber.

The mean annual precipitation is estimated at 25 inches for the entire area and falls almost entirely in the form of rain.

There are several reservoir sites on Santa Ynez and its tributaries which have been surveyed.

\section{SANTA YNEZ RIVER NEAR SANTA BARBARA, CAL.}

The original station, at which measurements were made during the greater part of 1903 , was located about 1 mile above the mouth of Mono Creek. On November 1, 1903, the old station was abandoned and a new station established by L. M. Hyde. It is located at the Gibraltar dam site, 5 miles below the original station, and is below the mouth of Mono Creek. It is 9 miles above the San Marcus ranch and halfway between the old quicksilver mines. 
The channel is straight for 700 feet above and 600 feet below the station. The right bank is low, but is not liable to overflow. The left bank rises abruptly about 20 feet beyond the tree to which the cable is attached. It is not liable to overflow. The bed of the stream is composed of sand and gravel, free from vegetation and bowlders. The cross section is regular and is permanent. The current is swift.

Discharge measurements are made at high water by means of a cable. Measurements can can usually be made by wading. The initial point for soundings is a blaze at the base of the cottonwood tree on the right bank, to which the cable is attached.

The gage is an inclined timber spiked to a cottonwood tree on the right bank. The tree is blazed and graduated above the gage rod for recording stages above the gage. The bench mark is a cross on a bench of a ledge of rock on the left bank, about 100 feet below the cable. Elevation, 13.54 feet above the datum of the gage. The approximate elevation above sea level, as estimated from topographic maps, is 1,200 feet.

Information in regard to this station is contained in Water Supply Papers Nos. 81, 100, and 134 of the United States Geological Survey.

Daily gage height, in feet, of Santa Ynez River near Santa Barbara, Cal., for 1905.

\begin{tabular}{|c|c|c|c|c|c|c|c|c|c|c|c|c|}
\hline $\mathrm{Da}$ & Jan. & Feb. & Mar. & Apr. & May. & June. & July. & Aug. & Sept. & Oct. & Nov. & Dec. \\
\hline 1.. & 2.3 & 2.5 & 3.1 & 3.25 & 2.65 & 2.4 & 2.05 & 2.0 & 1.9 & 1.8 & 1.8 & 2.05 \\
\hline $2 .$. & 2.3 & 3.6 & 3.05 & 3.25 & 2.7 & 2.4 & 2.05 & 2.0 & 1.9 & 1.8 & 1.8 & 2.05 \\
\hline 3.. & 2.25 & 8.4 & 3.0 & 3.2 & 2.65 & 2.4 & 2.05 & 2.0 & 1.85 & 1.8 & 1.8 & 2.05 \\
\hline 4.. & 2.25 & 6.8 & 3.0 & 3.15 & 2.6 & 2.4 & 2.05 & 2.0 & 1.85 & 1.8 & 1.8 & 2.05 \\
\hline $5 .$. & 2.25 & 4.0 & 3.05 & 3.15 & 2.6 & 2.4 & 2.05 & 2.0 & 1.85 & 1.8 & 1.8 & 2.05 \\
\hline 6.. & 2.25 & 3.9 & 3.1 & 3.1 & 2.6 & 2.4 & 2.05 & 2.0 & 1.85 & 1.8 & 1.85 & 2.05 \\
\hline 7... & 2.2 & 3.75 & 3.0 & 3.1 & 2.6 & 2.4 & 2.05 & 2.0 & 1.85 & 1.8 & 1.85 & 2.05 \\
\hline $8 .$. & 3.8 & 3.7 & 2.9 & 3.05 & 2.6 & 2.4 & 2.05 & 2.0 & 1.85 & 1.8 & 1.85 & 2.05 \\
\hline $9 .$. & 3.7 & 3.65 & 2.9 & 3.05 & 2.6 & 2.35 & 2.05 & 2.0 & 1.85 & 1.8 & 1.85 & 2.05 \\
\hline 10. & 2.6 & 3.65 & 2.85 & 3.05 & 2.6 & 2.35 & 2.05 & 2.0 & 1.85 & 1.8 & 1.85 & 2.05 \\
\hline $1 .$. & 2.55 & 3.6 & 2.9 & 3.0 & 2.6 & 2.35 & 2.05 & 2.0 & 1.85 & 1.8 & 1.85 & 2.05 \\
\hline $12 .$. & 2.5 & 3.6 & 4.7 & 3.05 & 2.6 & 2.3 & 2.05 & 2.0 & 1.85 & 1.8 & 1.9 & 2.05 \\
\hline 13. & 2.45 & 3.6 & 8.9 & 3.0 & 2.55 & 2.3 & 2.05 & 2.0 & 1.85 & 1.8 & 1.9 & 2.05 \\
\hline $14 .$. & 2.4 & 3.55 & 5.65 & 2.95 & 2.55 & 2.25 & 2.05 & 2.0 & 1.85 & 1.8 & 1.9 & 2.05 \\
\hline 15. & 2.45 & 3.4 & 4.65 & 2.95 & 2.55 & 2.25 & 2.05 & 2.0 & 1.85 & 1.8 & 1.9 & 2.05 \\
\hline 16.. & 2.5 & 4.45 & 5.3 & 2.9 & 2.55 & 2.2 & 2.05 & 1.95 & 1.85 & 1.8 & 1.9 & 2.05 \\
\hline 7.. & 2.45 & 6.55 & 4.65 & 2.9 & 2.55 & 2.2 & 2.05 & 1.95 & 1.85 & 1.8 & 1.9 & 2.05 \\
\hline 18.. & 2.4 & 4.0 & 4.4 & 2.9 & 2.5 & 2.2 & 2.05 & 1.95 & 1.85 & 1.8 & 1.9 & 2.05 \\
\hline 9. & 2.4 & 3.75 & 4. 15 & 2.85 & 2.5 & 2.15 & 2.05 & 1.95 & 1.85 & 1.8 & 1.9 & 2.05 \\
\hline $20 .$. & 2.4 & 3.7 & 4.0 & 2.85 & 2.5 & 2.15 & 2.05 & 1.95 & 1.85 & 1.8 & 1.9 & 2.05 \\
\hline $1 .$. & 4.25 & 3.65 & 3.8 & 2.85 & 2.5 & 2.15 & 2.05 & 1.95 & 1.85 & 1.8 & 1.9 & 2.05 \\
\hline 22. & 3.15 & 3.5 & 3.75 & 2.8 & 2.5 & 2.1 & 2.05 & 1.95 & 1.85 & 1.8 & 1.9 & 2.05 \\
\hline 23.. & 2.9 & 3.4 & 3.65 & 2.8 & 2.5 & 2.1 & 2.05 & 1.95 & 1.8 & 1.8 & 1.9 & 2.05 \\
\hline $24 .$. & 2.8 & 3.3 & 3.6 & 2.8 & 2.5 & 2.1 & 2.05 & 1.9 & 1.8 & 1.8 & 1.9 & 2.05 \\
\hline 25. & 2.75 & 3.2 & 3.55 & 2.75 & 2.45 & 2.1 & 2.05 & 1.9 & 1.8 & 1.8 & 1.9 & 2.05 \\
\hline 26.. & 2.65 & 3.15 & 3.5 & 2.75 & 2.45 & 2.1 & 2.0 & 1.9 & 1.8 & 1.8 & 2.0 & 2.05 \\
\hline $27 .$. & 2.65 & 3.1 & 3.4 & 2.85 & 2.45 & 2.1 & 2.0 & 1.9 & 1.8 & 1.8 & 2.1 & 2.05 \\
\hline 28.. & 2.6 & 3.1 & 3.4 & 2.75 & 2.45 & 2.1 & 2.0 & 1.9 & 1.8 & 1.8 & 2.1 & 2.05 \\
\hline $29 .$. & 2.6 & ......... & 3.6 & 2.7 & 2.45 & 2.1 & 2.0 & 1.9 & 1.8 & 1.8 & 2.1 & 2.05 \\
\hline $30 .$. & 2.5 & $\ldots \ldots \ldots$ & 3.4 & 2.7 & 2.45 & 2.1 & 2.0 & 1.9 & 1.8 & 1.8 & 2.05 & 2.05 \\
\hline $31 \ldots$ & 2.5 & $\therefore \ldots \ldots$ & 3.3 & 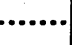 & 2.45 & $\ldots$ & 2.0 & 1.9 & . & 1.8 & a........ & 2.05 \\
\hline
\end{tabular}


SANTA YNEZ RIVER DRAINAGE BASIN.

Station rating table for Santa Ynez River near Santa Barbara, Cal., from January 1 to February 2, 1905 .

\begin{tabular}{|c|c|c|c|c|c|c|c|}
\hline $\begin{array}{c}\text { Gage } \\
\text { height. }\end{array}$ & Discharge. & $\begin{array}{c}\text { Gage } \\
\text { height. }\end{array}$ & Discharge. & $\begin{array}{c}\text { Gage } \\
\text { height. }\end{array}$ & Discharge. & $\begin{array}{c}\text { Gage } \\
\text { height. }\end{array}$ & Discharge. \\
\hline Feet. & Second-feet. & Feet. & Second-feet. & Feet. & Second-feet. & Feet. & Second-feet. \\
\hline 2.00 & 0.4 & 2.60 & 38 & 3.20 & 154 & 3.80 & 401 \\
\hline 2.10 & 2.8 & 2.70 & 50 & 3.30 & 186 & 3.90 & 451 \\
\hline 2.20 & 6.4 & 2.80 & 65 & 3.40 & 222 & 4.00 & 502 \\
\hline 2.30 & 11.6 & 2.90 & 82 & 3.50 & 263 & & \\
\hline 2.40 & 18.5 & 3.00 & 103 & 3.60 & 306 & & \\
\hline 2.50 & 28 & 3.10 & 126 & 3.70 & 353 & & \\
\hline
\end{tabular}

Station rating table for Santa Ynez River near Santa Barbara, Cal., from February 3 to December $31,1905$.

\begin{tabular}{|r|r||r|r|r|r||r|r|}
\hline $\begin{array}{c}\text { Gage } \\
\text { height. }\end{array}$ & Discharge. & $\begin{array}{c}\text { Gage } \\
\text { height. }\end{array}$ & Discharge. & $\begin{array}{c}\text { Gage } \\
\text { height. }\end{array}$ & Discharge. & $\begin{array}{c}\text { Gage } \\
\text { height. }\end{array}$ & Discharge. \\
\cline { 1 - 6 } Feet. & Second-feet. & Feet. & Second-feet. & Feet. & Second-feet. & Feet. & Second-feet. \\
1.80 & 1 & 3.00 & 153 & 4.20 & 875 & 5.80 & 2,790 \\
1.90 & 2 & 3.10 & 184 & 4.30 & 965 & 6.00 & 3,070 \\
2.00 & 3 & 3.20 & 220 & 4.40 & 1,065 & 6.20 & 3,360 \\
2.10 & 5 & 3.30 & 265 & 4.50 & 1,175 & 6.40 & 3,650 \\
2.20 & 10 & 3.40 & 315 & 4.60 & 1,285 & 6.60 & 3,940 \\
2.30 & 19 & 3.50 & 370 & 4.70 & 1,395 & 6.80 & 4.230 \\
2.40 & 30 & 3.60 & 430 & 4.80 & 1,510 & 7.00 & 4,520 \\
2.50 & 44 & 3.70 & 495 & 4.90 & 1,630 & 7.50 & 5,245 \\
2.60 & 60 & 3.80 & 565 & 5.00 & 1,750 & 8.00 & 5,970 \\
2.70 & 79 & 3.90 & 635 & 5.20 & 1,990 & 8.50 & 6,695 \\
2.80 & 100 & 4.00 & 710 & 5.40 & 2,250 & 9.00 & 7,420 \\
2.90 & 125 & 4.10 & 790 & 5.60 & 2,510 & & \\
& & & & & & \\
\hline
\end{tabular}

Note.-The above table is applicable only for open-channel conditions. is based on discharge. measurements made during January, February, and March, 1906.

Estimated monthly discharge of Santa Ynez River near Santa Barbara, Cal., for 1905.

[Drainage area, 207 square miles.]

\begin{tabular}{|c|c|c|c|c|c|c|}
\hline \multirow[b]{2}{*}{ Month. } & \multicolumn{3}{|c|}{ Discharge in second-feet. } & \multirow[b]{2}{*}{$\begin{array}{l}\text { Total in } \\
\text { acre-feet. }\end{array}$} & \multicolumn{2}{|c|}{ Run-off. } \\
\hline & Maximum. & Minimum. & Mean. & & $\begin{array}{l}\text { Second-feet } \\
\text { per square } \\
\text { mile. }\end{array}$ & $\begin{array}{c}\text { Depth } \\
\text { in inches. }\end{array}$ \\
\hline January....$\ldots \ldots \ldots \ldots \ldots \ldots$ & 635 & 6.4 & 73.8 & 4,538 & 0.357 & 0.412 \\
\hline February...$\ldots \ldots$ & 6,550 & 28.0 & 905 & 50,260 & 4.37 & 4.55 \\
\hline March.................. & 7,275 & 112 & 797 & 49,010 & 3.85 & 4.44 \\
\hline April................... & 242 & 79 & 143 & 8,509 & .691 & .771 \\
\hline May .................. & 100 & 37 & 52.5 & 3,228 & .254 & .293 \\
\hline June......................... & 30 & 5 & 15.9 & 946 & .077 & .086 \\
\hline July ............ & 4 & 3 & 3.8 & 234 & .018 & .021 \\
\hline August.......... & 3 & 2 & 2.6 & 160 & .013 & .015 \\
\hline September................... & 2 & 1 & 1.4 & 83 & .0068 & .0076 \\
\hline October........... & 1 & 1 & 1.0 & 61 & .0048 & .0055 \\
\hline November....... & 5 & 1 & 2.1 & 125 & .010 & .011 \\
\hline 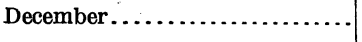 & 4 & 4 & 4.0 & 246 & .019 & .022 \\
\hline The year... & 7,275 & 1.0 & 167 & 117,400 & .806 & 10.63 \\
\hline
\end{tabular}


SANTA MARIA RIVER DRAINAGE BASIN.

DESCRIPTION OF BASIN.

The Santa Maria River drains the northern slope of the San Rafael Mountains and a limited area of foothill country to the north of this range. It flows in a westerly direction, finally discharging its waters into the Pacific Ocean at Guadalupe about 25 miles south of San Luis Obispo. Its flow is torrential in character, subject to floods of short duration during the rainy period, but being practically dry during the summer months. It has numerous tributaries, the most important of which is the Sisquoc, which enters it about 12 miles above the town of Santa Maria. The gaging station is located about 25 miles above the town of Santa Maria and above most of its important tributaries. The country throughout this basin consists of rolling foothills, with the exception. of the higher elevations of the San Rafael Mountains, which reach an elevation of 6,000 to 8,000 feet. The river breaks from the foothills at the point where it is joined by the Sisquoc and flows through the flat country of the Santa Maria Valley for a distance of about 25 miles until it joins the Pacific Ocean at Guadalupe. The formation throughout this basin is of shale, sandstone, and conglomerate, with a good covering of heavy clay soil. There is considerable growth of timber on the higher elevations of the San Rafael Mountains, but over most of the area the growth of timber is light, with large areas of brush and grass. The pasturage of stock is carried on extensively throughout the basin. There are no diversions along this stream for irrigation although tunnel work has been attempted above Santa Maria for the development of underground water, with poor results. There are numerous wells in the vicinity of Santa Maria which produce considerable water for the irrigation of land in that locality, the soil being very deep and of exceptional quality, susceptible to the highest state of cultivation. The mean precipitation in this drainage basin is probably about 25 inches. The greatest rainfall occurs on the lower elevations near the coast. The higher elevations receive some snowfall; which melts early in the spring and does not tend to keep up the flow of the stream through the summer.

SANTA MARIA RIVER NEAR SANTA MARIA, CAL.

This station was established October 22,1903 , by W. B. Clapp. It is located near the ranch house on Dutard's ranch, 21 miles above Santa Maria, Cal., a station on the Pacific Coast Railway. It is reached by driving from Santa Maria.

The channel is slightly curved for 300 feet above and curved for 1,000 feet below the station. The water is swift at medium and flood stages.

The right bank is high and rocky, and not liable to overflow. The left bank is low, covrered with scattering poplar trees, but not liable to overflow. The bed of the stream is composed of sand and gravel. A portion of the bed is covered with a light growth of low brush. The channel is not liable to much change.

At low and medium water discharge measurements are made with meter by wading. During high water velocities are measured by means of floats. For this purpose two wires are stretched across the stream 254 feet apart. The rise in the channel above the upper wire is 0.40 foot in 100 feet, and below the upper wire it is 0.57 foot in 100 feet. The initial points for soundings are blazes on the poplar trees on the left bank, to which the wires are attached.

The gage is an inclined staff, fastened to a rock ledge at the right bank. During 1905 the gage was read once each day by James A. Thompson. The bench mark is a spike driven near the ground into the south side of the poplar tree to which the upper wire is fastened on the left bank of the stream; elevation, 9.65 feet above the datum of the gage.

Information in regard to this station is contained in Water-Supply Papers Nos. 100 and 134 of the United States Geological Survey. 
Discharge measurements of Santa Maria River near Santa Maria, Cal., in 1905.

\begin{tabular}{|c|c|c|c|c|c|c|}
\hline Date. & Hyd'rographer. & Width. & $\begin{array}{l}\text { Area of } \\
\text { section. }\end{array}$ & $\underset{\text { Mean }}{\text { velocity. }}$ & $\begin{array}{l}\text { Gage } \\
\text { height. }\end{array}$ & $\begin{array}{c}\text { Dis- } \\
\text { charge }\end{array}$ \\
\hline February $2 \ldots$ & E. C. La Rue.. & $\begin{array}{r}\text { Feet. } \\
34\end{array}$ & $\begin{array}{r}S q \cdot f t \\
30\end{array}$ & $\begin{array}{r}\text { Ft. per sec. } \\
2.67\end{array}$ & $\begin{array}{l}\text { Feet. } \\
\quad 3.30\end{array}$ & ${ }_{80}^{\text {Sec.-ft. }}$ \\
\hline March 6........ & O. W. Peterson.. & 24 & 22 & 2.14 & 2.70 & 47 \\
\hline March $21 . . . .$. & .....do............. & 40 & 28 & 2.39 & 1.20 & 67 \\
\hline
\end{tabular}

Daily gage height, in feet, of Santa Maria River near Santa Maria, Cal., for 1905.

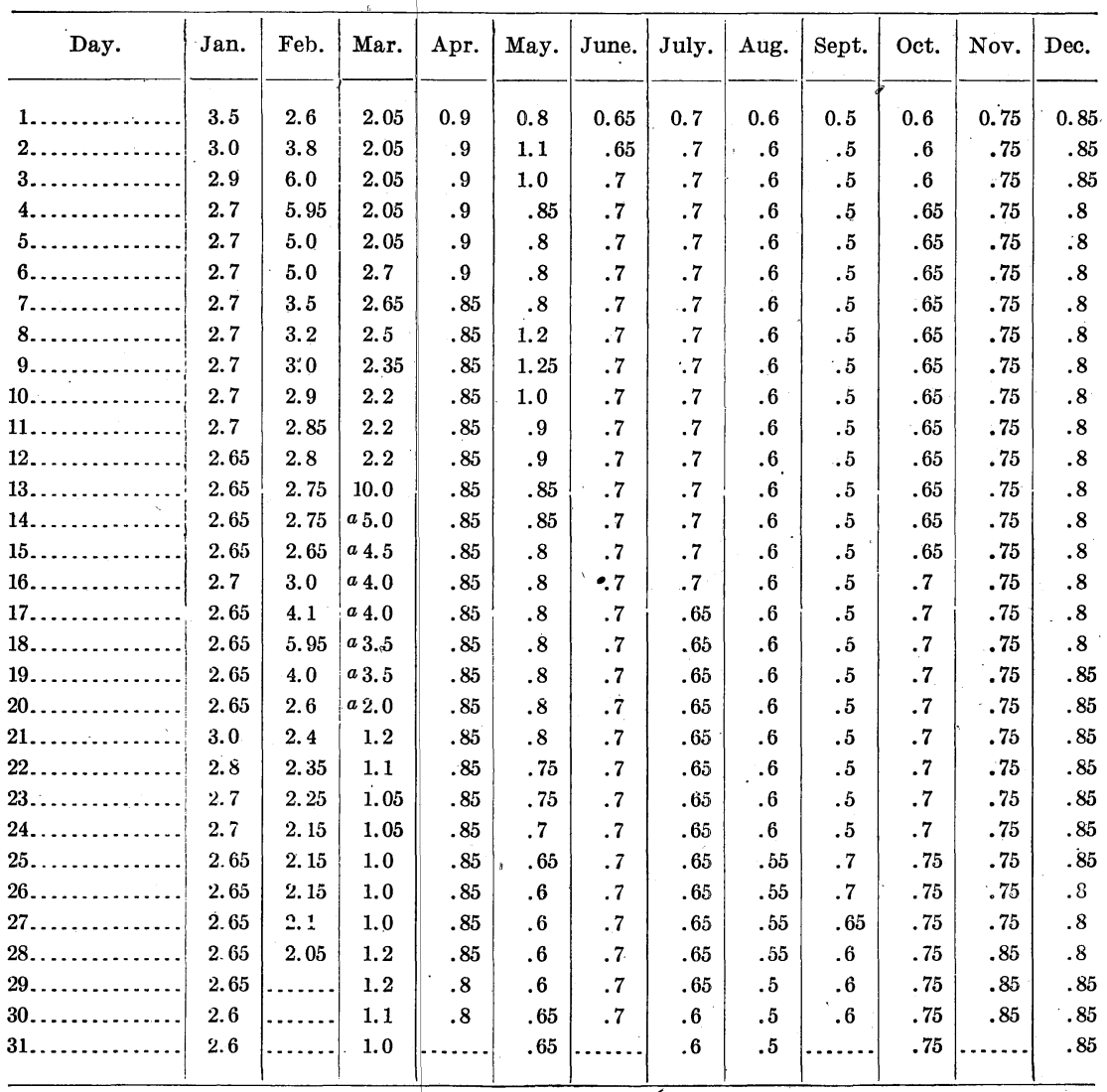

IRR $177-06-9$ 
Station rating table for Santa Maria River near Santa Maria, Cal., from January 1 to February 2, 1905.

\begin{tabular}{|r|r||r|r||r|r||r|r|}
\hline $\begin{array}{c}\text { Gage } \\
\text { height. }\end{array}$ & Discharge. & $\begin{array}{c}\text { Gage } \\
\text { height. }\end{array}$ & Discharge. & $\begin{array}{c}\text { Gage } \\
\text { height. }\end{array}$ & Discharge. & $\begin{array}{c}\text { Gage } \\
\text { height. }\end{array}$ & Discharge. \\
\hline Feet. & Second-feet. & Feet. & Second-feet. & Feet. & Second-feet. & Feet. & Second-feet. \\
2.00 & 1 & 2.60 & 13 & 3.20 & 67 & 3.70 & 178 \\
2.10 & 2 & 2.70 & 18 & 3.30 & 82 & .3 .80 & 213 \\
2.20 & 3 & 2.80 & 25 & 3.40 & 100 & 3.90 & 250 \\
2.30 & 5 & 2.90 & 33 & 3.50 & 122 & 4.00 & 290 \\
2.40 & 7 & 3.00 & 43 & 3.60 & 148 & 4.10 & 330 \\
2.50 & 10 & 3.10 & 54 & & & & \\
\hline
\end{tabular}

Station rating table for Santa Maria River near Santa Maria, Cal., from February 3 to. March 12, 1905.

\begin{tabular}{|r|r||r|r|r|r||r|r|}
\hline $\begin{array}{c}\text { Gage } \\
\text { height. }\end{array}$ & Discharge. & $\begin{array}{c}\text { Gage } \\
\text { height. }\end{array}$ & Discharge. & $\begin{array}{c}\text { Gage } \\
\text { height. }\end{array}$ & Discharge. & $\begin{array}{c}\text { Gage } \\
\text { height. }\end{array}$ & Discharge. \\
\hline Feet. & Second-feet. & Feet. & Second-feet. & Feet. & Second-feet. & Feet. & Second-feet. \\
1.70 & 1 & 2.70 & 48 & 3.70 & 285 & 4.70 & 915 \\
1.80 & 2 & 2.80 & 59 & 3.80 & 335 & 4.80 & 990 \\
1.90 & 3 & 2.90 & 72 & 3.90 & 385 & 4.90 & 1,065 \\
2.00 & 5 & 3.00 & 87 & 4.00 & 440 & 5.00 & 1,140 \\
2.10 & 8 & 3.10 & 105 & 4.10 & 500 & 5.20 & 1,290 \\
2.20 & 12 & 3.20 & 125 & 4.20 & 565 & 5.40 & 1,440 \\
2.30 & 17 & 3.30 & 147 & 4.30 & 630 & 5.60 & 1,595 \\
2.40 & 23 & 3.40 & 173 & 4.40 & 700 & 5.80 & 1,755 \\
2.50 & 30 & 3.50 & 205 & 4.50 & 770 & 6.00 & 1,915 \\
2.60 & 38 & 3.60 & 242 & 4.60 & 840 & & \\
\hline
\end{tabular}

Stationrating table for Santa Maria River near Santa Maria, Cal., from March 13 to . December $31,1905$.

\begin{tabular}{|r|r||r|r||r|r|r|r|}
\hline $\begin{array}{c}\text { Gage } \\
\text { height. }\end{array}$ & Discharge. & $\begin{array}{c}\text { Gage } \\
\text { height. }\end{array}$ & Discharge. & $\begin{array}{c}\text { Gage } \\
\text { height. }\end{array}$ & Discharge. & $\begin{array}{c}\text { Gage } \\
\text { height. }\end{array}$ & Discharge. \\
\hline Feet. & Second-feet. & Feet. & Second-feet. & Feet. & Second-feet. & Feet. & Second-feet. \\
0.50 & 1 & 2.00 & 250 & 4.00 & 1,070 & 7.00 & 3,260 \\
0.60 & 3 & 2.10 & 280 & 4.20 & 1,185 & 7.20 & 3,450 \\
0.70 & 6 & 2.20 & 310 & 4.40 & 1,305 & 7.40 & 3,650 \\
0.80 & 10 & 2.30 & 340 & 4.60 & 1,435 & 7.60 & 3,870 \\
0.90 & 18 & 2.40 & 375 & 4.80 & 1,565 & 7.80 & 4,100 \\
1.00 & 31 & 2.50 & 410 & 5.00 & 1,700 & 8.00 & 4,350 \\
1.10 & 47 & 2.60 & 445 & 5.20 & 1,840 & 8.20 & 4,640 \\
1.20 & 65 & 2.70 & 480 & 5.40 & 1,980 & 8.40 & 4,970 \\
1.30 & 84 & 2.80 & 520 & 5.60 & 2,130 & 8.60 & 5,340 \\
1.40 & 105 & 2.90 & 560 & 5.80 & 2,280 & 8.80 & 5,750 \\
1.50 & 127 & 3.00 & 600 & 6.00 & 2,430 & 9.00 & 6,200 \\
1.60 & 150 & 3.20 & 690 & 6.20 & 2,585 & 10.00 & 10,000 \\
1.70 & 175 & 3.40 & 780 & 6.40 & 2,745 & & \\
1.80 & 200 & 3.60 & 875 & 6.60 & 2,910 & & \\
1.90 & 225 & 3.80 & 975 & 6.80 & 3,080 & & \\
& & & & & & & \\
\hline
\end{tabular}

NoTE.-The upper part of the rating curve is based on slope measurements. 
Estimated monthly discharge of Santa Maria River near Santa Maria, Cal., for 1905.

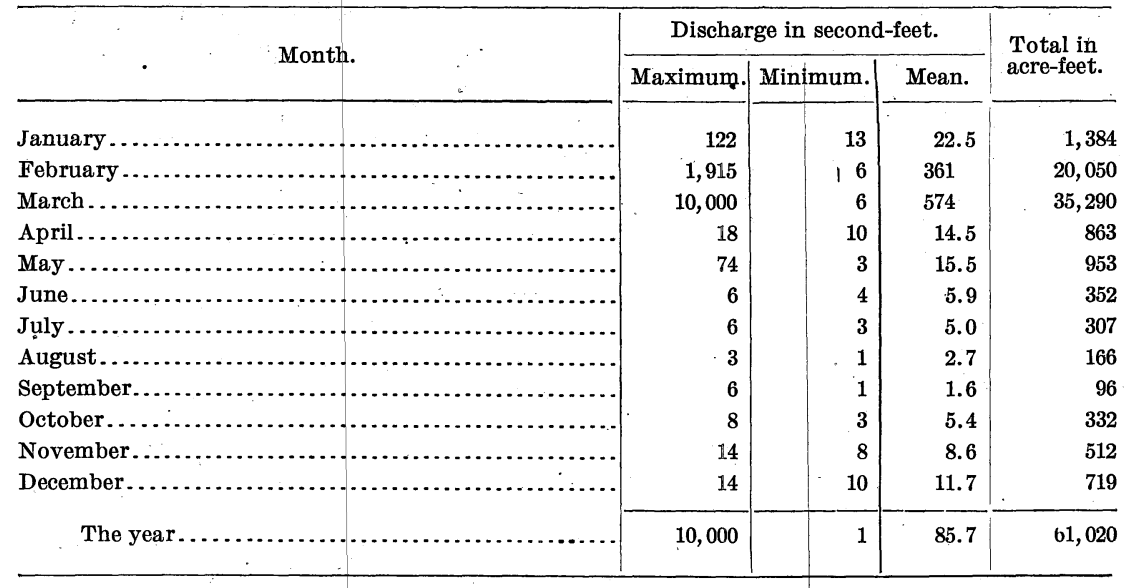

Note.-Estimates during high-water periods are approximate only.

\section{SALINAS RIVER DRAINAGE BASIN.}

\section{DESCRIPTION OF BASIN.}

The Arroyo Seco is the most northern tributary of any size of the Salinas River, and rises on the slopes of the highest portion of the Santa Lucia Range, one of the ranges that go to make up the Coast Range of California extending in a general southeast direction from San Francisco Bay. The upper valleys of this stream are far back in the range, surrounded by high mountains.

The drainage area of the Arroyo Seco is almost entirely made up of sharp ridges and Vshaped canyons. The western portion is well covered with brush and trees of medium size. Toward the east this growth decreases until at the Salinas Valley the country is bare. The stream beds of this area fall rapidly, the Arroyo Seco rising at an elevation of nearly 6,000 feet and discharging into the Salinas at an elevation of $\mathbf{1 7 0}$ feet.

Below the gaging station several canals divert water from this stream before it reaches the broad wash of sand and gravel on the flat floor of the Salinas Valley, into which it sinks during the dry season and from which it receives its name "Arroyo Seco."

There are five reservoir sites on the stream and its tributaries, of more or less value as possible storage reservoirs, that have already been surveyed.

This portion of the range undoubtedly receives as great rainfall as any other locality in this region, and it is estimated that the average annual precipitation is from 30 to 50 inches and falls almost entirely in the form of rain.

\section{ARROYO SECO NEAR SOLEDAD, CAL.}

The original gaging station on this stream was established by W. W. Cockins, jr., in December, 1900, at Foster's ranch, near Piney, Cal. High water of January, 1901, enlarged an old side channel, dividing the stream into two channels. The gaging station was then removed to Pettitt's ranch, 4 miles below the old station.

The channel is straight for 400 feet above and below the station. The right bank is low, wooded, and subject to overflow. The left bank is high, rocky, and not subject to overflow. The bed of the stream is composed of gravel and is not subject to much change.

Discharge measurements are made by means of a cable and ear. The initial point for soundings is on the left bank. 
The gage rod is in two sections. The low-water section is placed 400 feet above the cable on the right bank; the high-water section is fastened to a sycamore tree above the cable and about 250 feet from the right bank of the low-water channel. During 1905 the gage was read by Mrs. Charles Pettitt. The bench mark is a spike in the tree to which the high-water section of the gage is fastened; elevation, 15.00 feet above the datum of the gage.

Information in regard to this station is contained in Water-Supply Papers Nos. 81, 100, and 134. of the United States Geological Survey.

Discharge measurements of Arroyo Seco River near Soledad, Cal., in 1905.

\begin{tabular}{|c|c|c|c|c|c|c|}
\hline Date. & Hydrographer. & Width. & $\begin{array}{l}\text { Area of } \\
\text { section. }\end{array}$ & $\begin{array}{c}\text { Mean } \\
\text { velocity. }\end{array}$ & $\begin{array}{c}\text { Gage } \\
\text { height. }\end{array}$ & $\begin{array}{c}\text { Dis- } \\
\text { charge. }\end{array}$ \\
\hline January 31 & La Rue and Pettitt.................. & $\begin{array}{r}\text { Feet. } \\
122\end{array}$ & $S q \cdot f t$. & $\begin{array}{r}\text { Ft.per sec. } \\
1.04\end{array}$ & $\begin{array}{l}\text { Feet. } \\
\mathbf{5 . 9 1}\end{array}$ & $\begin{array}{r}\text { Sec.-ft. } \\
128\end{array}$ \\
\hline February 2 & Charles Pettitt & 130 & 410 & 3.84 & 8.80 & 1,574 \\
\hline February 3 & .....do. & 124 & 281 & 3.05 & 7.50 & 856 \\
\hline February 5 & .....do. & 125 & 254 & 3.06 & 7.35 & 778 \\
\hline February 16 & .....do.. & 125 & 212 & 2.29 & 6.82 & 486 \\
\hline February 1 & .....do. & 133 & 423 & 4.32 & 8.95 & 1,829 \\
\hline March 11 & .....do.. & 120 & 135 & 1.05 & 5.90 & 142 \\
\hline March 13. & .....do. . & 133 & 484 & 4.65 & 10.05 & 2,253 \\
\hline March 16 & .....do.. & 128 & 327 & 4.02 & 8.00 & 1,315 \\
\hline March 17. & ...do. & 128 & 288 & 2.91 & 7.50 & 838 \\
\hline March 19 & ...do.. & 133 & 455 & 4.90 & 9.30 & 2,233 \\
\hline March 29. & ...do.. & 125 & 248 & 2.36 & 7.05 & 585 \\
\hline April 9. & ...do. & 123 & 158 & 1.57 & 6.10 & 248 \\
\hline April 16 & ....do & 123 & 150 & 1.40 & 6.09 & 210 \\
\hline April 19 & ...do & 123 & 151 & 1.59 & 6.16 & 240 \\
\hline April 29. & $\ldots$ do & 122 & 117 & 1.07 & 5.95 & 125 \\
\hline May $7 .$. & $\ldots$. do & 130 & 351 & 3.75 & 8.30 & 1,317 \\
\hline May 8.. & ...do & 125 & 241 & 2.86 & 7.12 & 689 \\
\hline May 28. . & .....do... & 123 & 107 & 1.15 & 5.93 & 123 \\
\hline June 4 & ...do. & 123 & 113 & $1 . \dot{0} 3$ & 5.83 & 116 \\
\hline June 12 . & ...do... & 122 & 95 & .82 & 5.75 & 78 \\
\hline June 18. & ...do... & 122 & 89 & .76 & 5.70 & 68 \\
\hline Do. & ...do. . & 48 & 63 & 1.24 & 5.70 & 78 \\
\hline June 24. & ...do.. & 43 & 54 & 1.00 & 5.62 & 54 \\
\hline July $3 . .$. & ...do & 41 & 49 & .80 & 5.55 & 39 \\
\hline July $9 .$. & .....do.. & 40 & 46 & .61 & 5.48 & 28 \\
\hline July $15 \ldots$ & ......do. & 38 & 42 & .57 & 5.45 & 24 \\
\hline July $23 .$. & .....do. & 35 & 39 & .54 & 5.42 & 21 \\
\hline July 29. & ......do. & 36 & 37 & .43 & 5.40 & 16 \\
\hline August 6 . . & .....do. & 34 & 35 & .37 & 5.38 & 13 \\
\hline August 10 & .....do......... & 27 & 24 & .33 & 5.33 & 8 \\
\hline
\end{tabular}


Daily gage height, in feet, of Arroyo Seco River near Soledad, Cal., for 1905.

\begin{tabular}{|c|c|c|c|c|c|c|c|c|c|c|c|c|}
\hline Day. & Jan. & Feb. & Mar. & Apr. & May. & June. & July. & Aug. & Sept. & Oct, & Nov. & Dec. \\
\hline 1. & 6.1 & 8.4 & 6.15 & 6.5 & 5.9 & 5.85 & 5.6 & 5.4 & 5.2 & 5.25 & 5.35 & 5.65 \\
\hline 2. & 5.9 & 8.6 & 6.1 & 6.45 & 6.55 & 5.85 & 5.55 & 5.4 & 5.2 & 5.25 & 5.35 & 5.6 \\
\hline 3. & 5.8 & 7.45 & 6.05 & 6.4 & 6.2 & 5.8 & 5.5 & 5.4 & 5.2 & 5.25 & 5.35 & 5.55 \\
\hline 4. & 5.75 & 7.4 & 6.0 & 6.35 & 6.05 & 5.85 & 5.5 & 5.4 & 5.2 & 5.25 & 5.35 & 5.5 \\
\hline 5. & 5.7 & 7.35 & 6.0 & 6.3 & 6.0 & 5.8 & 5.5 & 5.4 & 5.2 & 5.25 & 5.35 & 5.5 \\
\hline 6. & 5.7 & 7.2 & 6.0 & 6.25 & 6.0 & 5.8 & 5.5 & 5.4 & 5.2 & 5.25 & 5.35 & 5.5 \\
\hline 7. & 5.65 & 7.0 & 5.95 & 6.25 & 7.9 & 5.8 & 5.5 & 5.35 & 5.2 & 5.25 & 5.35 & 5.5 \\
\hline 8.. & 5.6 & 6.8 & 5.9 & 6.2 & 7.2 & 5.8 & 5.5 & 5.35 & 5.2 & 5.25 & 5.35 & 5.5 \\
\hline 9. & 5.8 & 6.65 & 5.9 & 6.1 & 6.8 & 5.8 & 5.5 & 5.35 & 5.2 & 5.25 & 5.35 & 5.5 \\
\hline $10 .$. & 6.0 & 6.55 & 5.9 & 6.15 & 6.6 & 5.8 & 5.5 & 5.35 & 5.2 & 5.25 & .35 & 5.5 \\
\hline 11.. & 5.9 & 6.45 & 5.9 & 6.1 & 6.45 & 5.75 & 5.45 & 5.35 & 5.2 & 5.25 & 5.35 & 5.5 \\
\hline $12 .$. & 5.8 & 6.35 & 6.55 & 6.1 & 6.4 & 5.75 & 5.45 & 5.35 & 5.2 & 5.25 . & 5.35 & 5.5 \\
\hline 13.. & 5.8 & 6.3 & 10.1 & 6.1 & 6.3 & 5.7 & 5.45 & 5.35 & 5.2 & 5.25 & 5.35 & 5.5 \\
\hline $14 .$. & 5.9 & 6.25 & 7.85 & 6.1 & 6.2 & 5.7 & 5.45 & 5.35 & 5.2 & 5.25 & 5.35 & 5.5 \\
\hline $15 .$. & 5.9 & 6.2 & 7.5 & 6.05 & 6.2 & 5.7 & 5.45 . & 5.35 & 5.2 & 5.25 & 5.35 & 5.5 \\
\hline 16.. & 6.95 & 6.8 & 8.0 & 6.1 & 6.15 & 5.7 & 5.45 & 5.3 & 5.25 & 5.25 & 5.35 & 5.5 \\
\hline 17.. & 6.3 & 8.5 & 7.6 & 6.05 & 6.1 & 5.7 & 5.45 & 5.3 & 5.25 & 5.3 & 5.4 & 5.5 \\
\hline 18. & 6.1 & 7.45 & 7.3 & 6.0 & 6.1 & 5.7 & 5.45 & 5.3 & 5.25 & 5.3 & 5.4 & 5.55 \\
\hline $19 .$. & 6.2 & 7.05 & 9.1 & 6.2 & 6.05 & 5.7 & 5.45 & 5.3 & 5.25 & 5.3 & $\dot{5} .4$ & 5.6 \\
\hline $20 .$. & 6.1 & 6.9 & 7.6 & 6.1 & 6.05 & 5.7 & 5.4 & 5.3 & 5.2 & 5.3 & 5.4 & 5.6 \\
\hline 21. & 6.5 & 6.6 & 7.35 & 6.05 & 6.0 & 5.7 & 5.4 & 5.3 & 5.2 & 5.3 & 5.4 & 5.6 \\
\hline 22. & 6.2 & 6.5 & 7.1 & 6.05 & 6.0 & 5.65 & 5.4 & 5.3 & 5.2 & 5.3 & 5.4 & 5.6 \\
\hline 23. & 6.1 & 6.4 & 6.9 & 6.0 & 5.95 & 5.65 & 5.4 & 5.3 & 5.2 & 5.35 & 5.4 & 5.6 \\
\hline $24 .$. & 6.05 & 6.35 & 6.75 & 6.0 & 5.9 & 5.65 & 5.4 & 5.3 & 5.2 & 5.35 & 5.5 & 5.55 \\
\hline $25 .$. & 6.1 & 6.3 & 6.7 & 6.0 & 5.9 & 5.6 & 5.4 & 5.3 & 5.2 & 5.35 & 5.65 & 5.55 \\
\hline $26 \ldots$ & 6.0 & 6.25 & 6.65 & 6.0 & 5.95 & 5.6 & 5.4 & 5.3 & 5.2 & 5.35 & 5.5 & 5.5 \\
\hline $27 \ldots$ & 6.0 & 6.2 & 6.55 & 6.0 & 5.95 & 5.6 & 5.4 & 5.25 & 5.2 & 5.35 & 5.5 & 5.5 \\
\hline $28 .$. & 6.0 & 6.2 & 6.5 & 6.0 & 5.95 & 5.6 & 5.4 & 5.25 & 5.2 & 5.35 & 5.75 & 5.5 \\
\hline $29 .$. & 5.95 & & 7.5 & 5.95 & 5.9 & 5.6 & 5.4 & 5.25 & 5.2 & 5.35 & 5.6 & 5.55 \\
\hline $30 \ldots$ & 5.9 & & 6.65 & 5.95 & 5.9 & 5.6 & 5.4 & 5.25 & 5.2 & 5.35 & 5.9 & 5.5 \\
\hline $31 \ldots$ & 5.9 & & 6.6 & ..... & 5.85 & . & 5.4 & 5.25 & $\cdots$ & 5.3 & & 5.5 \\
\hline
\end{tabular}

Station rating table for Arroyo Seco River near Soledad, Cal., from January 1 to December 31, 1905.

\begin{tabular}{|r|r||r|r||r|r|r|r|}
\hline $\begin{array}{c}\text { Gage } \\
\text { height. }\end{array}$ & Discharge. & $\begin{array}{c}\text { Gage } \\
\text { height. }\end{array}$ & Discharge. & $\begin{array}{c}\text { Gage } \\
\text { height. }\end{array}$ & Discharge. & $\begin{array}{c}\text { Gage } \\
\text { height. }\end{array}$ & Discharge. \\
\cline { 1 - 6 } Feet. & Second-feet. & Feet. & Second-feet. & Feet. & Second-feet. & Feet. & Second-feest. \\
5.20 & 2 & 6.30 & 265 & 7.40 & 800 & 9.00 & 1,900 \\
5.30 & 6 & 6.40 & 305 & 7.50 & 860 & 9.20 & 2,060 \\
5.40 & 15 & 6.50 & 345 & 7.60 & 920 & 9.40 & 2,225 \\
5.50 & 30 & 6.60 & 390 & 7.70 & 980 & 9.60 & 2,400 \\
5.60 & 50 & 6.70 & 435 & 7.80 & 1,045 & 9.80 & 2,580 \\
5.70 & 70 & 6.80 & 480 & 7.90 & 1,110 & 10.00 & 2,760 \\
5.80 & 95 & 6.90 & 530 & 8.00 & 1,175 & 10.20 & 2,945 \\
5.90 & 125 & 7.00 & 580 & 8.20 & 1,310 & 10.40 & 3,135 \\
6.00 & 155 & 7.10 & 635 & 8.40 & 1,450 & & \\
6.10 & 190 & 7.20 & 690 & 8.60 & 1,595 & & \\
6.20 & 225 & 7.30 & 745 & 8.80 & 1,745 & & \\
\hline
\end{tabular}

NotE.-The above table is applicable only for open-channel conditions. It is based on 31 discharge measurements made during 1905 . It is well defined between gage heights 5.3 feet and 9.5 feet. 
Estimated monthly discharge of Arroyo Seco River near Soledad, Cal., for 1905

[Drainage area, 215 square miles.]

\begin{tabular}{|c|c|c|c|c|c|c|}
\hline \multirow[b]{2}{*}{ Month. } & \multicolumn{3}{|c|}{ Discharge in second-feet. } & \multirow[b]{2}{*}{$\begin{array}{l}\text { Total in } \\
\text { acre-feet. }\end{array}$} & \multicolumn{2}{|c|}{ Run-off. } \\
\hline & Maximum. & Minimum. & Mean. & & $\begin{array}{l}\text { Second-feet } \\
\text { per square } \\
\text { mile. }\end{array}$ & $\begin{array}{l}\text { Depth } \\
\text { in inches. }\end{array}$ \\
\hline Januarj்.. & 555 & 50 & 160 & 9,838 & 0.744 & 0.858 \\
\hline February ........ & 1,595 & 225 & 556 & 30,880 & 2.59 & 2.70 \\
\hline March...... & 2,850 & 125 & 586 & 36,030 & 2.73 & 3.15 \\
\hline April.................. & 345 & 140 & 202 & 12,020 & .940 & 1.05 \\
\hline May......... & 1,110 & 110 & 246 & 15,130 & 1.14 & 1.31 \\
\hline June............ & 110 & 50 & 75.6 & 4,499 & .352 & .393 \\
\hline July....... & 50 & 15 & 22.8 & 1,402 & .106 & .122 \\
\hline August................ & 15 & 4 & 8.6 & 529 & .040 & .046 \\
\hline September.............. & 4 & 2 & 2.3 & 137 & .011 & .012 \\
\hline October............ & 10 & 4 & 6.1 & 375 & .028 & .032 \\
\hline November............... & 125 & 10 & 22.4 & 1,333 & .104 & .116 \\
\hline 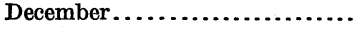 & 60 & 30 & 36.5 & 2,245 & .170 & .196 \\
\hline The year..... & 2,850 & 2 & 160 & 114,400 & .746 & 9.98 \\
\hline
\end{tabular}

MISCELLANEOUS MEASUREMENTS IN SOUTHERN CALIFORNIA.

The following is a list of miscellaneous discharge measurements made in Riverside and San Diego counties during 1905:

Cottonwood Creek near Jamul, Cal.-This stream rises in the Coast Range in the southeastern part of San Diego County, and flowing in a southwesterly direction discharges into Tiajuana River below the California-Mexico boundary line. The following discharge measurements were made at the Barrett Dam. The gage is a 2-by-4 inch vertical timber painted white and graduated to feet and hundredths. It is nailed to the timber wall on the left bank of the stream at the concrete dam. No bench mark was established, as the zero of the rod is set level with the top of the concrete dam.

November 18: Width, 2.7 feet; area, 0.5 square foot; mean velocity, 1.00 foot per second; discharge, 0.5 second-foot.

December 14: Width, 5 feet; area, 1.6 square feet; mean velocity, 0.69 foot per second; gage height, 0.05 foot; discharge, 1.1 second-feet.

Temecula Creek near Temecula, Cal.-This creek rises in the Coast Range in the southeastern part of Riverside County, and flowing in a southwesterly direction discharges into the Pacific Ocean near Oceanside, Cal. The following discharge measurements were made on this stream during 1905:

At Pauba dam site, 12 miles above Temecula, Cal., November 14: Width, 7.5 feet; area, 2 square feet; mean velocity, 1.25 feet per second; discharge, 2.5 second-feet.

At Temecula Bridge, 1 mile below Temecula, Cal., November 14: Width, 11 feet; area, 5.4 square feet; mean velocity, 1.07 feet per second; gage height, 3.10 feet; discharge, 5.8 second-feet.

December 30: Width, 9 feet; area, 3.5 square feet; mean velocity, 1.32 feet per second; gage height, 2.98 feet; discharge, 4.6 second-feet.

A gage was placed on the Temecula Bridge consisting of a 2-by-6 inch vertical timber, graduated to feet and hundredths. It is fastened to the downstream pier near the right bank of the stream. No bench mark was established.

San Diego River near Lakeside, Cal.-This stream rises in the Coast Range in the eastern part of San Diego County, and flowing in a southwesterly direction discharges in the Pacific Ocean near San Diego, Cal. A measurement was made at the concrete dam of the San Diego Flume Company, at the head of that company's flume. 
November 23: Width, 6 feet; area, 1.0 square foot; mean velocity, 1.90 feet per second; discharge, 1.9 second-feet.

Santa Y sabel River near San Pasqual, Cal.-This stream rises in the eastern part of San Diego County, and flowing in a southwesterly direction discharges into the Pacific Ocean near Del Mar, Cal. The lower portion of this river is known as the San Dieguito River. The following measurements were made during 1905 at the upper end of the San Pasqual Valley. The gage consists of a 2 -by-4 inch inclined timber, graduated to feet and hundredths, and bolted to a large granite bowlder on the left bank of the stream. The bench mark is on top of the bowlder to which the gage is bolted. It is marked with a circle of white paint. Its elevation is $\mathbf{1 1 . 6 6}$ feet above the zero of the gage.

November 21: Width, 5 feet; area, 2.2 square feet; mean velocity, 2.05 feet per second; discharge, 4.5 second-feet.

November 28: Width, 83 feet; area, 24 square feet; mean velocity, 1.70 feet per second; gage height, 3.72 feet; discharge, 41 second-feet.

December 21: Width, 24 feet; area, 7.2 square feet; mean velocity, 1.44 feet per second; gage height, 3.55 feet; discharge, 10.4 second-feet.

Sweetwater River near Descanso, Cal.-This stream rises in the southeastern part of San Diego County, and flowing in a southwesterly direction discharges into San Diego Bay near National City. The following measurements were made during 1905, 1 mile below Descanso. The gage is a $2-b y-6$ inch vertical timber, graduated to feet and hundredths. It is bolted to a granite bowlder on the left bank of the stream. The bench mark is the highest point on the large bowlder to which the gage is fastened. It is marked with a circle of white paint, and its elevation is 5 feet above the zero of the gage.

September 11: Measured by Weir; discharge, 0.37 second-foot.

November 21: Width, 3 feet; area, 0.9 square foot; mean velocity, 1.22 feet per second; gage height, 3.54 feet; discharge, 1.1 second-feet.

December 10: Width, 6 feet; area, 1.9 square feet; mean velocity, 1.35 feet per second; gage height, 3.62 feet; discharge, 2.5 second-feet.

\section{SAN FRANCISCO BAY DRAINAGE BASIN.}

\section{GENERAL FEATURES.}

Sacramento River, rising in northern California and flowing south, and San Joaquin River, rising in the southern Sierras and flowing north, drain the western slope of the Sierra Nevada and the eastern slope of the Coast Range north of San Francisco. They meet near Suisun Bay, finally discharging their waters into the Pacific Ocean through San Francisco Bay.

\section{SACRAMENTO RIVER.}

\section{DESCRIPTION OF RIVER.}

Sacramento River is the principal river of California, and drains all of the territory south of Mount Shasta and between the Coast Range and Trinity Range on the west and the Sierra Nevadas on the east. The portion of the drainage basin above the gaging station which is located near Red Bluff, Cal., extends from the Trinity Mountains on the west to Warner Mountains near the California-Nevada State line on the east. The watershed on the west from the Trinity Mountains is comparatively narrow, being only from 10 to 35 miles in width, and furnishes a very small proportion of the discharge of this river, but from the east Pit River, which is the most important tributary, drains a large area extending about 120 miles east from the Sacramento River and between Mount Shasta on the north and Lassen Peak on the south. The greater portion of this basin is composed of lava, and shows other.evidences of volcanic activity, such as volcanic cones and craters. Nearly all the streams tributary to Pit River have their origin in large springs, many of which discharge several hundred second-feet. The most important tributary of the Pit is the McCloud 
River, draining the southeastern slope of Mount Shasta. It derives its waters principally from the melting of the snow on the high elevations of this mountain. The western portion of the watershed extending along the Trinity Range is well timbered, as is also that portion of the drainage area in the Sierra Nevadas lying between Mount Shasta and Lassen Peak. Farther east, however, there is little or no forest covering, and the country is used extensively for pasturage. The rainfall is very unequally distributed, varying from less than 10 inches in the eastern portion of the basin to 50 inches along the northern and western portion. Below the gaging station the river enters the Sacramento Valley, through which it flows on a comparatively light grade until it reaches Suisun Bay. During the winter months, when the Sacramento and its tributaries are in flood, large areas of the Sacramento Valley are overflowed. The floods which occur in the latter part of the rainy season, and after these large overflow areas or basins are filled, cause great damage in the lower portion of the valley.

\section{SACRAMENTO RIVER NEAR RED BLUFF, CAL.}

The gaging station at Jellys Ferry, which is located about 12 miles above the town of Red Bluff, was established April 30,1895. The right bank of the river is high, but the left bank is liable to overflow when the river rises above the 25-foot mark. The river has been known to reach the 35-foot mark. Because of the liability to overflow it was deemed advisable to select a new gaging station, where the water at flood stage could be more confined. A point in Iron Canyon, where the river had been gaged by the State engineering department in 1879 and by commissioner of public works in 1893-94, was chosen as a new gaging station.

The river at this point in lower portion of Iron Canyon, 4 miles above Red Bluff, has a direct course for 2 or 3 miles. The width between banks at low water is about 500 feet. The depth of water at low stages averages 6 feet, with a maximum depth of 9 feet. The banks are steep and firm. The river flows in a bed of coarse gravel and cobbles, with here and there a small bowlder. The bed rock is lava.

Discharge measurements are made from a cable 600 feet in span, which is anchored in the lava rock which forms the wall of the canyon.

The river stage rod used by commissioner of public works was still in place, and has been used in making river height observations since January 28,1902 , the date upon which the observations were begun. A second set of gage rods were placed on the right balnk 3,200 feet below the gaging station January 1, 1904, as no observer could be obtained to continue readings of the station gage. By synchronous readings of the lower gages and the station gage the actual readings for this lower gage have been converted into equivalent readings for the station gage. On September 28, 1904, it was necessary to move the lower gage rods to the left bank about 4,000 feet below the gaging station. The actual readings of this set of gage rads have also been converted into equivalent readings for the station gage. During 1905 the gage was read twice each day by Fred Weeks. All reported gage heights are those determined for the station gage. Bench marks were established as follows: (1) A nail in the crotch of sycamore tree to which the third section of the gage is fastened; elevation, 8.355 feet. (2) Point of rock marked with heavy ring of white paint 7.1 feet south of eyebolt on left bank; elevation, 47.75 feet. - (3) A point marked with ring of white paint 45 feet north of 10-foot mark on cable; elevation 38.01 feet. (4) Eye-bolt on left bank; elevation 44.80 feet. (5) Point on rock under left cable support marked with circle of white paint; elevation, 27.59 feet. Elevations refer to the datum of the gage.

Information in regard to this station is contained in Water-Supply Papers Nos. 81, 100, and 134 of the United States Geological Survey. 
Discharge measurements of Sacramento River near Red Bluff, Cal., in 1905.

\begin{tabular}{|c|c|c|c|c|c|c|}
\hline Date. & Hydrographer. & Width. & $\begin{array}{l}\text { Area of } \\
\text { section. }\end{array}$ & $\begin{array}{c}\text { Mean } \\
\text { velocity. }\end{array}$ & $\begin{array}{c}\text { Gage } \\
\text { height. }\end{array}$ & $\begin{array}{l}\text { Dis- } \\
\text { charge. }\end{array}$ \\
\hline August 5....... & Peterson and Lee. & $\begin{array}{r}\text { Feet. } \\
448\end{array}$ & $\begin{array}{r}S q \cdot f t . \\
3,520\end{array}$ & $\begin{array}{r}\text { Ft. per sec. } \\
1.48\end{array}$ & $\begin{array}{r}\text { Feet. } \\
1.49\end{array}$ & $\begin{array}{l}\text { Sec.-ft. } \\
\quad 5,208\end{array}$ \\
\hline August $30 \ldots . .$. & c. H. Lee.............................. & 497 & 3,404 & 1.48 & 1.34 & 5,043 \\
\hline
\end{tabular}

Daily gage height, in feet, of Sacramento River near Red Bluff, Cal., for 1905.

\begin{tabular}{|c|c|c|c|c|c|c|c|c|c|c|c|c|}
\hline Day. & Jan. & Feb. & Mar. & Apr. & May. & June. & July. & Aug. & Sept. & Oct. & Nov. & Dec. \\
\hline 1. & 7.2 & 8.9 & 4.85 & 8.05 & 4.05 & 3.0 & 1.95 & 1.45 & 1.25 & 1.3 & 1.3 & 1.75 \\
\hline $2 .$. & 6.15 & 15.85 & 4.75 & 7.8 & 4.3 & 3.0 & 1.95 & 1.45 & 1.25 & 1.3 & 1.3 & 1.55 \\
\hline $3 .$. & 4.8 & 13.3 & 4.6 & 7.55 & 4.1 & 3.0 & 1.9 & 1.45 & 1.25 & 1.3 & 1.3 & 1.55 \\
\hline $4 .$. & 4.7 & 11.35 & 4.55 & 7.1 & 3.9 & 3.0 & 1.9 & 1.45 & 1.25 & 1.25 & 1.3 & 1.55 \\
\hline 5. & 4.3 & 10.15 & 4.5 & 6.8 & 3.7 & 3.0 & 1.9 & 1.45 & 1.25 & 1.25 & 1.3 & 1.55 \\
\hline $6 .$. & 4.0 & 8.95 & 4.4 & 6.45 & 3.7 & 3.0 & 1.8 & 1.45 & 1.25 & 1.25 & 1.4 & 1.55 \\
\hline 7.. & 3.75 & 8.15 & 4.3 & 6.25 & 4.1 & 2.85 & 1.75 & 1.4 & 1.25 & 1.3 & 1.4 & 1.55 \\
\hline $8 .$. & 3.55 & 7.4 & 4.2 & 5.95 & 5.5 & 2.9 & 1.7 & 1.4 & 1.25 & 1.3 & 1.4 & 1.55 \\
\hline $9 .$. & 3.45 & 6.8 & 4.15 & 5.75 & 4.95 & 2.85 & 1.75 & 1.4 & 1.25 & 1.3 & 1.4 & 1.55 \\
\hline $10 .$. & 3.35 & 6.4 & 4.05 & 5.45 & 4.6 & 2.85 & 1.75 & 1.4 & 1.25 & 1.3 & 1.4 & 1.55 \\
\hline 11. & 3.2 & 6.0 & 4.05 & 5.25 & 3.85 & 2.7 & 1.7 & 1.4 & 1.25 & 1.3 & 1.4 & 1.55 \\
\hline $12 \ldots$ & 3.1 & 5.55 & 4.9 & 5.0 & 4.15 & 2.7 & 1.7 & 1.3 & 1.25 & 1.3 & 1.45 & 1.55 \\
\hline $13 .$. & 3.85 & 5.2 & 10.25 & 4.85 & 4.15 & 2.65 & 1.7 & 1.3 & 1.25 & 1.25 & 1.45 & 1.7 \\
\hline $14 .$. & 14.35 & 4.9 & 13.55 & 4.75 & 3.9 & 2.6 & 1.7 & 1.3 & 1.25 & 1.3 & 1.45 & 1.55 \\
\hline $15 .$. & 9.8 & 4.75 & 11.15 & 4.7 & 3.85 & 2.55 & 1.7 & 1.3 & 1.25 & 1.3 & 1.45 & 1.55 \\
\hline 16. & 9.5 & 4.55 & 10.7 & 5.45 & 3.85 & 2.5 & 1.7 & 1.3 & 1.25 & 1.3 & 1.45 & 1.55 \\
\hline 17. & 7.95 & 4.55 & 9.7 & 4.95 & 4.0 & 2.4 & 1.7 & 1.3 & 1.25 & 1.3 & 1.45 & 1.7 \\
\hline 18. & 6.85 & 4.5 & 8.25 & 4.75 & 3.85 & 2.35 & 1.6 & 1.3 & 1.25 & 1.3 & 1.45 & 1.8 \\
\hline $19 .$. & 7.3 & 5.25 & 11.4 & 5.2 & 3.75 & 2.35 & 1.6 & 1.3 & 1.25 & 1.3 & 1.45 & 2.1 \\
\hline $20 .$. & 7.05 & 8.2 & 10.3 & 5.0 & 3.6 & 2.3 & 1.55 & 1.3 & 1.25 & 1.3 & 1.55 & 2.1 \\
\hline $21 .$. & 9.2 & 7.95 & 11.1 & 4.85 & 3.5 & 2.3 & 1.55 & 1.3 & 1.25 & 1.3 & 1.55 & 1.9 \\
\hline $22 .$. & 14.3 & 7.2 & 11.2 & 4.7 & 3.4 & 2.25 & 1.55 & 1.3 & 1.25 & 1.3 & 1.55 & 1.7 \\
\hline $23 .$. & 20.3 & 6.6 & 9.0 & 4.55 & 3.35 & 2.25 & 1.55 & 1.3 & 1.25 & 1.3 & 1.55 & 1.55 \\
\hline $24 \ldots$ & 15.55 & 6.0 & 9.8 & 4.4 & 3.3 & 2.2 & 1.5 & 1.3 & 1.25 & 1.3 & 1.55 & 1.55 \\
\hline $25 .$. & 15.45 & 5.7 & 8.45 & 4.4 & 3.2 & 2.25 & 1.5 & 1.25 & 1.25 & 1.3 & 1.55 & 1.55 \\
\hline $26 .$. & 12.75 & 5.45 & 9.0 & 4.4 & 3.2 & 2.2 & 1.45 & 1.25 & 1.25 & 1.3 & 1.55 & 1.55 \\
\hline $27 \ldots$ & 9.8 & 5.3 & 8.9 & 4.3 & 3.2 & 2.1 & 1.45 & 1.25 & 1.25 & 1.3 & 1.6 & 1.7 \\
\hline $28 \ldots$ & 8.95 & 5.0 & 8.2 & 4.15 & 3.2 & 2.0 & 1.45 & 1.25 & 1.3 & 1.3 & 1.7 & 1.7 \\
\hline $29 .$. & 7.5 & & 15.5 & 4.05 & 3.2 & 2.0 & 1.45 & 1.25 & 1.3 & 1.3 & 1.8 & 1.9 \\
\hline $30 \ldots$ & 6.85 & & 9.85 & 4.0 & 3.15 & 1.95 & 1.45 & 1.25 & 1.3 & 1.3 & 1.95 & 1.8 \\
\hline $31 \ldots$ & 6.55 & 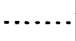 & 8.7 & & 3.05 & ....... & 1.45 & 1,25 & & 1.3 & …. & 1.9 \\
\hline
\end{tabular}


Station rating table for Sacramento River near Red Bluff, Cal., from January 1, 1904, to December 31, 1905.

\begin{tabular}{|c|c|c|c|c|c|c|c|}
\hline $\begin{array}{c}\text { Gage } \\
\text { height. }\end{array}$ & Discharge. & $\begin{array}{c}\text { Gage } \\
\text { height. }\end{array}$ & Discharge. & $\begin{array}{c}\text { Gage } \\
\text { height. }\end{array}$ & Discharge. & $\begin{array}{c}\text { Gage } \\
\text { height. }\end{array}$ & Discharge. \\
\hline Feet. & Second-feet. & Feet. & Second-feet. & Feet. & Second-feet. & Feet. & Second-feet. \\
\hline 1.00 & 4,450 & 3.10 & 10,380 & 5.40 & 18,700 & 9.60 & 37,180 \\
\hline 1.10 & 4,690 & 3. 20 & 10,710 & 5.60 & 19,500 & 9.80 & 38,140 \\
\hline 1.20 & 4,930 & 3.30 & 11,040 & 5.80 & 20,300 & 10.00 & 39,100 \\
\hline 1.30 & 5,170 & 3.40 & 11,370 & 6.00 & 21,100 & 10.50 & $=41,600$ \\
\hline 1.40 & 5,410 & 3.50 & $.11,700$ & 6.20 & 21,940 & 11.00 & 44,200 \\
\hline 1.50 & 5,650 & 3.60 & 12,040 & $6.40^{-}$ & 22,780 & 11.50 & 46,900 \\
\hline 1.60 & 5,920 & 3.70 & 12,380 & 6.60 & 23,620 & $\cdot 12.00$ & 49,700 \\
\hline 1.70 & 6,190 & 3.80 & 12,720 & 6.80 & 24,460 & 12.50 & 52,600 . \\
\hline 1.80 & 6,460 & 3.90 & 13,060 & 7.00 & $25 ; 300$ & 13.00 & 55,600 \\
\hline 1.90 & 6,730 & 4.00 & 13,400 . & 7.20 & 26,180 & 13.50 & 58,600 \\
\hline 2.00 & 7,000 & $4: 10$ & 13,760 & 7.40 & 27,060 & 14.00 & 61,700 \\
\hline 2.10 & 7,300 & 4.20 & 14,120 & 7.60 & 27,940 & 14.50 & 64,900 \\
\hline 2.20 & 7,600 & 4.30 & 14,480 & 7.80 & 28,820 & $15.00^{\circ}$ & $.68,200$ \\
\hline 2.30 & 7,900 & 4.40 & 14,840 & 8.00 & 29,700 & 16.00 & 75,100 \\
\hline 2.40 & 8,200 & 4.50 & 15,200 & 8.20 & 30,620 & 17.00 & 82,200 \\
\hline 2.50 & 8,500 & 4.60 & $15 ; 580$ & 8.40 & 31,540 & 18.00 & 89,700 \\
\hline 2.60 & 8,810 & 4.70 & 15,960 & 8.60 & 32,460 & 19.00 & 97,600 \\
\hline 2.70 & 9,120 & 4.80 & 16,340 & 8.80 & 33,380 & 20.00 & 105,900 \\
\hline 2.80 & 9,430 & 4.90 & i6, 720 & 9.00 & 34,300 & . & \\
\hline 2.90 & 9,740 & $\cdot 5.00$ & 17,100 & 9.20 & 35,260 & $\cdots$ & \\
\hline 3.00 & 10,050 & 5.20 & - 17,900 & 9.40 . & 36,220 & & \\
\hline
\end{tabular}

Note.-The above table is applicable only for open-channel conditions. It is based on discharge measurements made during 1902-1905. It is fairly well defined.

Estimated monthly discharge of Sacramento River near Red Bluff, Cal., for 1905.

[Drainage area, 9,295 square miles.]

\begin{tabular}{|c|c|c|c|c|c|c|}
\hline \multirow{2}{*}{ Month. } & \multicolumn{3}{|c|}{ Discharge in second-feet. } & \multirow{2}{*}{$\begin{array}{c}\text { Total in } \\
\text { acre-feet. }\end{array}$} & \multicolumn{2}{|c|}{ Run-off. } \\
\hline & Maximum. & Minimum. & Mean. & & $\begin{array}{c}\text { Second-feet } \\
\text { per square } \\
\text { mile. }\end{array}$ & $\begin{array}{l}\text { Depth } \\
\text { in inches. }\end{array}$ \\
\hline January. & 108,500 . & $10,380^{\circ}$ & 31,830 & $1,957,000$ & 3.42 & 3.94 \\
\hline February .: & 74,050 & 15,200 & 26,800 & $1,488,000$ & 2.88 & 3.00 \\
\hline March.... & 71,600 & 13,580 & 30,920 & $1,901,000$ & 3. 33 & $3.84^{\circ}$ \\
\hline April..... & 29,930 & 13,400 & 18,680 & $1,112,000$ & 2.01 & 2.24 \\
\hline May...... & 19,100 & 10,220 & 12,750 & 784,000 & 1.37 & 1.58 \\
\hline June...... & 10,050 & 6,865 & 8,623 & 513,100 & .928 & 1.04 \\
\hline July...... & $6 ; 865$ & 5,530 & 6,075 & 373,500 & .654 & .754 \\
\hline August.... & 5,530 & 5,050 & 5,251 & 322,900 & .565 & .651 \\
\hline September.... & 5,170 & 5,050 & 5,062 & 301,200 & .545 & .608 \\
\hline October... & 5,170 & 5,050 & 5,155 & 317,000 & .555 & .640 \\
\hline November.............. & 6,865 & 5,170 & 5,616 & 334,200 & .604 & · . .674 \\
\hline December: ..... & 7,300 & 5,785 & 6,100 & 375,100 & .656 & .756 \\
\hline The $y$ & 108,500 & 5,050 & 13,570 & $9,779,000$ & 1.46 & 19.72 \\
\hline
\end{tabular}




\section{SACRAMENTO RIVER AT SACRAMENTO, CAL.}

The following gage heights were observed at this station during 1905:

Daily gage height, in feet, of Sacramento River at Sacramento, Cal., for 1905.

\begin{tabular}{|c|c|c|c|c|c|c|c|}
\hline Day. & Jan. & Feb. & Mar. & Apr. & May. & June. & July. \\
\hline & 18.5 & 20.9 & 19.7 & 21.8 & 19.6 & 17.4 & 11.4 \\
\hline $2 .$. & 18.0 & 21.6 & 19.7 & 21.8 & 19.8 & 17.3 & 11.2 \\
\hline $3 \ldots$ & 17.7 & 21.8 & 19.6 & 21.7 & 19.6 & 17.2 & 11.0 \\
\hline $4 \ldots$ & 18.1 & 21.8 & 19.5 & 21.7 & 19.6 & 17.0 & 10.9 \\
\hline $5 \ldots \ldots \ldots \ldots \ldots$ & 18.4 & 21.9 & 19.5 & 21.6 & 19.0 & 16.9 & 10.8 \\
\hline $6 \ldots$ & 18.4 & 22.0 & 19.4 & 21.5 & 18.9 & 16.4 & 10.7 \\
\hline $7 \ldots \ldots$ & 18.2 & 21.9 & 19.3 & 21.5 & 19.0 . & 16.3 & 10.6 \\
\hline 8. & 18.1 & 21.9 & 19.0 & 21.5 & 19.0 & 16.2 & 10.5 \\
\hline $9 \ldots \ldots \ldots \ldots \ldots$ & 18.0 & 21.8 & 18.8 & 21.6 & 19.0 & 16.1 & 10.4 \\
\hline 10. & 17.8 & 21.8 & 18.7 & 21.6 & 19.3 & 16.0 & 10.3 \\
\hline $11 .$. & 17.5 & 21.6 & 18.2 & 21.2 & 18.9 & 16.0 & 10.0 \\
\hline $12 .$. & 17.1 & 21.4 & 18.1 & 21.0 & 18.8 & 16.0 & 9.9 \\
\hline $13 \ldots \ldots \ldots \ldots$ & 16.7 & 21.2 & 18.0 & 20.9 & 18.5 & 15.9 & 9.8 \\
\hline $14 \ldots \ldots \ldots \ldots \ldots \ldots$ & 16.7 & 20.9 & 18.9 & 20.8 & 18.5 & 15.8 & 9.7 \\
\hline $15 \ldots$ & 17.4 & 20.8 & 18.9 & $=20.7$ & 18.6 & 15.4 & 9.7 \\
\hline $16 \ldots \ldots \ldots$ & 18.0 & 20.5 & 19.0 & 20.6 & 18.8 & 15.1 & 9.6 \\
\hline $17 .$. & 18.2 & 20.4 & 19.2 & 20.6 & 19.3 & 15.0 & 9.5 \\
\hline $18 \ldots \ldots \ldots$ & 18.5 & 20.4 & 19.4 & 20.2 & 18.9 & 14.7 & 9.4 \\
\hline $19 .$. & 18.8 & 20.4 & 20.1 & 20.4 & 18.9 & 14.5 & 9.3 \\
\hline $20 \ldots$ & 19.0 & 20.4 & 21.0 & 20.3 & 18.9 & 14.0 & 9.2 \\
\hline $21 \ldots \ldots$ & 19.0 & 20.4 & 20.8 & 20.2 & 18.8 & 13.8 & 9.1 \\
\hline 22. & 20.0 & 20.2 & 21.1 & 20.0 & 18.8 & 13.6 & 9.0 \\
\hline $23 \ldots \ldots \ldots$ & 20.5 & $20.1^{\circ}$ & 21.0 & 19.9 & 18.6 & 13.3 & 8.9 \\
\hline $24 \ldots \ldots \ldots \ldots \ldots \ldots$ & 20.2 & 20.0 & 21.2 & 19.8 & 18.5 & 13.0 & 8.8 \\
\hline $25 \ldots \ldots \ldots \ldots \ldots \ldots$ & 20.4 & 20.0 & 21.2 & 19.7 & 18: 0 & 12.7 & 8.7 \\
\hline $26 \ldots$. & 20.5 & 20.0 & 21.3 & 19.8 & 17.9 & 12.4 & 8.6 \\
\hline $27 \ldots$ & 20.7 & 20.0 & 21.7 & 20.1 & 18.0 & 12.0 & 8.4 \\
\hline 28. & 20.8 & 19.9 & 21.6 & 20.0 & 17.9 & 11.9 & 8.2 \\
\hline $29 \ldots$ & 20.8 & $\cdots$ & $21: 8$ & 19.9 & 17.8 & 11.8 & 8.2 \\
\hline $30 \ldots$ & 20.9 & & 21.8 & 19.8 & 17.7 & 11.6 & \\
\hline $31, \ldots \ldots \ldots \ldots \ldots$ & 20.9 & & 21.9 & & 17.5 & & \\
\hline
\end{tabular}

\section{MISCELLANEOUS MEASUREMENTS IN SACRAMENTO RIVER DRAINAGE} BASIN.

Sacramento River at Baird Station, on Southern Pacific Railway, Gregory post-office, Cal.A measurement of this stream was made August 28, 1905, from wagon bridge by W. B. Clapp.

Width, 116 feet; area, 308 square feet; mean velocity, 1.14 feet per second; discharge, 352 second-feet.

Clear Creek near Stella post-office; Cal.-A measurement was made of this stream August 26, 1905, 500 feet above mouth of Brandy Creek by W. B. Clapp.

Width, 28 feet; area, 31 square feet; mean velocity, 1.26 feet per second; discharge, 39. second-feet.

North Fork Cottonwood Creek near Gas Point, Cal.-A measurement of this stream was made August 28, 1905, 40 feet below the highway bridge above the junction of the North and Middle forks by C. H. Lee. Several diversions are made above the point of measurement; their total-discharge is reported to be about 8 second-feet.

Width, 15.5 feet; area, 9.0 square feet; mean velocity, 0.66 foot per second; discharge, 5.9 second-feet. 
Middle Fork Cottonwood Creek near Gas Point, Cal.-A measurement of this stream was made August 28, 1905, 300 feet above its junction with the North Fork by C. H. Lee. Three small diversions are made above the point of measurement.

Width, 35 feet; area, 11.3 square feet; mean velocity, 0.81 foot per second; discharge, 9.1 second-feet.

Pit River near Copper City, Cal.-This stream is the largest tributary of the Sacramento River, joining it about 11 miles north of Redding, Cal. It has a well-sustained summer flow. A measurement of this stream was made August 25, 1905, by C. H. Lee from the downstream side of the Delamar Mining Company's toll bridge above the mouth of Squaw Creek.

Width, 135 feet; area, 465 square feet; mean velocity, 6.68 feet per second; discharge, 3,107 second-feet.

Squaw Creek near Copper City, Cal.-A measurement of this stream was made 500 feet below bridge near Copper City by C. H. Lee on August 26, 1905.

Width, 39 feet; area, 95 square feet; mean velocity, 0.21 foot per second; discharge, 20 second-feet.

Cow Creek (above Clover Creek) at Millville, Cal.-A measurement of this stream was made August 26, 1905, at the highway bridge south of Millville by C. H. Lee.

Width, 39.2 feet; area, 35 square feet; mean velocity, 1.75 feet per second; discharge, 61 second-feet.

Clover Creek at Millville, Cal.-A measurement of this stream was made August 26, 1905, about 500 feet below the highway bridge above its junction with Cow Creek by C. H. Lee.

Width, 15 feet; area, 7.8 square feet; mean velocity, 0.40 foot per second; discharge, 3.1 second-feet.

Clover Creek ditch.-A measurement of this ditch was made on August 27, 1905, in flume at its heading, 3 miles above Millville by C. H. Lee.

Width, 2.15 feet; area, 1.8 square feet; mean velocity, 1.33 feet per second; discharge, 2.4 second-feet. This water is diverted from Clover Creek.

Little Cow Creek near Palocedro, Cal.-A measurement of this stream was made on August 27, 1905, 800 feet downstream from the highway bridge east of Palocedro by C. H. Lee. There are several small diversions from this stream above this point.

Width, 11.2 feet; area, 9.0 square feet; mean velocity, 0.50 foot per second; discharge, 4.5 second-feet.

Cow Creek near Palocedro, Cal.-A measurement of this stream was made August 27, 1905, 150 feet below its junction with Little Cow Creek by C. H. Lee.

Width, 41.5 feet; area, 58 square feet; mean velocity, 1.05 feet per second; discharge, 61 second-feet.

Battle Creek near Balls Ferry, Cal.-This stream is an important tributary of the Sacramento River. A measurement was made August 29, 1905, at a point about 2 miles above the wagon bridge on main road from Red Bluff to Balls Ferry by C. H. Lee.

Width, 88 feet; area, 171 square feet; mean velocity, 1.92 feet per second; discharge, 329 second-feet.

Mill ditch near Balls Ferry, Cal.-This ditch heads in Battle Creek $1 \frac{1}{2}$ miles above the wagon bridge on the main road from Red Bluff to Balls Ferry. A measurement was made

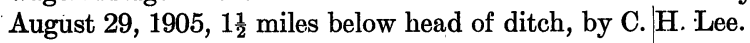

Width, 22 feet; area, 19.3 square feet; mean velocity, 1.33 feet per second; discharge, 26 second-feet.

Antelope Creek near Red Bluff, Cal.-A measurement of this stream was made August 31, 1905, 600 feet above the Antelope Creek and Red Bluff Water Company's dam, about 8 miles east of Red Bluff, by C. H. Lee.

Width, 36 feet; area, 83 square feet; mean velocity, 0.54 foot per second; discharge, 45 second-feet.

Mill Creek near Tehama, Cal.-A measurement of this stream was made September 1, 1905, 300 feet above the head-gate of the Los Molinas ditch and about 6 miles east of Tehama, by C. H. Lee. 
Width, 40.2 feet; area, 91 square feet; mean velocity, 1.34 feet per second; discharge, 122 second-feet.

Millrace canal near Tehama, Cal.-This canal diverts water from Mill Creek about $1 \frac{1}{2}$ miles above its junction with Sacramento River. A measurement was made in flume at head of canal September 1, 1905, by C. H. Lee.

Width, 11.8 feet; area, 40 square feet; mean velocity, 2.25 feet per second; discharge, 90 second-feet.

Deer Creek near Vina, Cal.-A measurement of this stream was made September 1, 1905, from the downstream side of the bridge at upper main road crossing, about 4 miles from Vina, by C. H. Lee. Two or three small ditches divert water from creek above this point of measurement.

Width, 57.7 feet; area, 77 square feet; mean velocity, 1.08 feet per second; discharge, 83 second-feet.

Valley Counties Power Company's canal near Centerville, Cal.-This canal diverts water from Butte Creek. A measurement was made May 19 in flume at town of Centerville.

Width, 6 feet; area, 13.2 square feet; mean velocity, 1.74 feet per second; discharge, 23 second-feet.

\section{PIT RIVER DRAINAGE BASIN.}

\section{DESCRIPTION OF BASIN.}

Pit River has its source in the Warner Mountains in the extreme northeast part of California. It flows in a southwesterly direction, discharging its waters into Sacramento River a few miles above Redding, Cal. It has numerous tributaries, the larger of which have their source in large springs, which discharge from crevices in the lava formation. About 50 per cent of the area of this drainage basin is barren of timber and composed, principally, of lava with a light soil covering, being used extensively for pasturage and the raising of stock. There are numerous small valleys with light grades, which hold the water throughout the summer months principally in the state of swamps. These areas are used, mainly, as meadow land and for the raising of stock feed. Pit River does not discharge in any great volume until it reaches a point near Fall River Mills, which lies about midway between the point where the Pit River enters the Sacramento and its source. Fall River, which is the principal tributary of the Pit from the north, receives its water supply from large perennial springs which discharge 1,500 second-feet. Hat Creek and Burney Creek are also large tributaries from the south and drain the northern slope of Lassen Peak. Their principal sources are also from large perennial springs in the lava formation.

West Valley Creek is a tributary of South Fork of Pit River. Ash Creek flows into Clear Creek, through which it enters Pit River from the south. There is considerable timber scattered throughout this drainage basin, the principal growth lying in the southern portion of the basin and also in that section lying north of Pit River and between Fall River and the upper Sacramento. There are numerous reservoir sites on the upper reaches of this stream, all of which have been or are being surveyed. Several gaging stations are maintained on Pit River and tributaries at points where surveys have been made for the construction of storage reservoirs. The rainfall throughout this basin is very unevenly distributed, ranging from 10 inches in the eastern portion to 50 to 75 inches in the western and northwestern portion. About 50 per cent of the precipitation falls in the form of snow, but does not remain any length of time except on the higher elevation of Mount Shasta and Lassen Peak.

McCloud River drains the southeastern slope of Mount Shasta. Its drainage area is comparatively small, covering 676 square miles. It is long and narrow, extending from north to south. There are few tributaries. Its main water supply comes from Mount Shasta, on which the snow remains during the entire year. It is also fed by numerous large springs scattered throughout the drainage basin. The precipitation is very heavy and is principally in the form of rain, except on the higher elevations of Mount Shasta. The discharge of this stream seldom falls below 1,200 second-feet. It discharges into the Pit River a few miles above the junction of the Pit with the Sacramento. The entire basin is well timbered. 


\section{PIT RIVER NEAR CANBY, CAL.}

This station was established December 26, 1903, by H. E. Green and J. S. Evans. It is located at the wagon bridge, $3 \frac{1}{2}$ miles southwest of Canby, Cal.

The channel is straight for 150 feet above and 200 feet below the station. The current is moderate at all stages. The banks are high and are subject to overflow. The channel is rocky and is not sübject to much change.

Discharge measurements are made from the bridge. The initial point for soundings is a nail in the railing post at the end of the bridge on the left bank.

The gage is a vertical plank securely fastened to the first bridge pier from the left bank of the stream. During 1905 the gage was read twice each day by Ernest M. Hess. The bench mark is a painted point on a large bowlder at the bend of the wagon road near the south end of the bridge. Elevation, 24.90 feet above the datum of the gage.

A description of this station, with gage height and discharge data, is contained in WaterSupply Paper No. 134, United States Geological Survey.

Discharge measurements of Pit River near Canby, Cal., in 1905.

\begin{tabular}{|c|c|c|c|c|c|c|}
\hline Date. & Hydrographer. & Width. & $\begin{array}{l}\text { Area of } \\
\text { section. }\end{array}$ & $\begin{array}{l}\text { Mean } \\
\text { velocity. }\end{array}$ & $\begin{array}{c}\text { Gage } \\
\text { height. }\end{array}$ & $\begin{array}{l}\text { Dis- } \\
\text { charge. }\end{array}$ \\
\hline January 5 & J. Y. Toler. & $\begin{array}{r}\text { Feet. } \\
99\end{array}$ & $\underset{187}{S q . f t}$ & $\begin{array}{r}\text { Ft. per sec. } \\
2.55\end{array}$ & $\begin{array}{r}\text { Feet. } \\
4.20\end{array}$ & $\begin{array}{c}\text { Sec. }-f t . \\
476\end{array}$ \\
\hline January 9. & .....do. . & 87 & 127 & 1.70 & 3.70 & 216 \\
\hline January 20 & ....do. & 99 & 186 & 2.53 & 4,18 & 470 \\
\hline January 26 & ....do. & 117 & 228 & 2.96 & 4.50 & 675 \\
\hline February 7 & .....do. & 116 & 220 & 2.91 & 4.40 & 641 \\
\hline February 13 & .....do. & 87 & 131 & 1.91 & 3.70 & 250 \\
\hline February 22 & ...do. . & 119 & 235 & 3.03 & 4.60 & 712 \\
\hline February 27 & .....do..... & 93 & 147 & 2.11 & 3.90 & 309 \\
\hline March $7 .$. & ....do... & 87 & 128 & 1.86 & 3.65 & 238 \\
\hline March $9 . .$. & .....do. & 87 & 127 & 1.83 & 3.65 & 233 \\
\hline March 14 & .....do. do. & 87 & 135 & 1.80 & 3.70 & 243 \\
\hline March 22. & .....do. & 109 & .227 & 2.90 & 4.60 & 659 \\
\hline March 26 . & ....do. & 109 & 230 & 2.93 & 4.65 & 674 \\
\hline April 4.... & .....do. & 138 & 292 & 3.33 & 5.00 & 971 \\
\hline April 5... & .....do. & 138 & 289 & 3.30 & 5.00 & 955 \\
\hline April $10 . .$. & .....do. & 114 & 206. & 2.81 & $4.40^{\circ}$ & 579 \\
\hline April 16... & .....do. & 93 & 146 & 2.27 & 4.00 & 331 \\
\hline April 19.... & .....do.. & 93 & 136 & 2.07 & 3.90 & 282 \\
\hline May $1 . . .$. & .....do. & 63 & 83 & 1.08 & 3: $\mathbf{3 0}$ & 90 \\
\hline May $2 \ldots$ & .....do. & 63 & 83 & 1.10 & 3.30 & 91 \\
\hline May $5 . .$. & .....do & 61 & 54 & .57 & 2.90 & 31 \\
\hline May $16 \ldots$ & .....do & 90 & 120 & 1.85 & 3.70 & 222 \\
\hline May $20 \ldots$ & .....do. & 90 & 115 & 1.84 & 3.70 & 212 \\
\hline June 9... & .....do. & 87 & 123 & 1.64 & 3.60 & 201 \\
\hline July $17 \ldots$ & .....do... & 52 & 40 & .60 & 2.90 & 24 \\
\hline July $22 \ldots$ & .....do. & 50 & 37 & .62 & 2.85 & 23 \\
\hline August 8 ... & ....do.do. & 60 & 39 & .37 & 2.80 & 14.5 \\
\hline August 10 & ...do. & 60 & 37 & .38 & 2.75 & 14.1 \\
\hline August 21 . & .....do. & 9 & 2.9 & 1.27 & 2.50 & 3.7 \\
\hline August 28 . & .....do. & 7 & 1.8 & 1.17 & 2.45 & 2. 1 \\
\hline August 31 & ....do... & 7 & 2.0 & 1.15 & 2.45 & 2.3 \\
\hline September & ....do. & 8 & 2.6 & 1.19 & 2.50 & 3.1 \\
\hline september & .....do. & 9 & 3.4 & 1.68 & 2.60 & 5.7 \\
\hline October 6 . . & ... . .do. & 35 & 21 & 1.00 & 2.80 & 21 \\
\hline October 19 & .....do. & 55 & 57 & .91 & 3.10 & 52 \\
\hline October 25 . & . $\therefore$ do do & 58 & 58 & $\cdot .86$ & 3.20 & 50 \\
\hline October 28. & .....do. & 58 & 61 & .90 & 3.20 & 55 \\
\hline November 7 & .....do...... & 60 & 67 & 1.19 & 3.30 & 80 \\
\hline November 1 & ....do... & 60 & 70 & 1.19 & 3.30 & 83 \\
\hline
\end{tabular}


PIT RIVER DRAINAGE BASIN.

Daily gage height, in feet, of Pit River near Canby, Cal., for 1905.

\begin{tabular}{|c|c|c|c|c|c|c|c|c|c|c|c|c|}
\hline Day. & Jan. & Feb. & Mar. & Apr. & May. & June. & July. & Aug. & Sept. & Oct. & Nov. & Dec. \\
\hline $1 .$. & 4.55 & 4.6 & 3.8 & 5.5 & 3.4 & 3.6 & 3.3 & 2.9 & 2.5 & 2.8 & 3.2 & 3.5 \\
\hline $2 .$. & 4.4 & 5.05 & 3.8 & 5.6 & 3.4 & 3.7 & 3.25 & 2.9 & 2.5 & 2.8 & 3.25 & 3.5 \\
\hline $3 \ldots$ & 4.1 & 5.25 & 3.75 & 5.5 & 3.3 & 3.7 & 3.25 & 2.9 & 2.5 & 2.8 & 3.25 & 3.5 \\
\hline $4 \ldots$ & 4.0 & 5.65 & 3.7 & 5.4 & 3.0 & 3.7 . & 3.25 & 2.9 & 2.5 & 2.8 & 3.3 & 3.5 \\
\hline 5. & 3.9 & 4.85 & 3.7 & 5.5 & 3.0 & 3.7 & 3.25 & 2.9 & 2.5 & 2.8 & 3.3 & 3.5 \\
\hline 6. & 3.8 & 4. 65 & 3.7 & 4.8 & 3.0 & 3.5 & 3.25 & 2.5 & 2.5 & 2.8 & 3.3 & 3.5 \\
\hline $7 \ldots$ & 3.8 & 4.25 & 3.65 & 4.8 & 3.6 & 3.6 & 3.2 & 2.5 & 2.5 & 2.8 & 3.3 & 3.5 \\
\hline $8 .$. & 3.8 & 4.1 & 3.65 & 4.7 & 3.6 & 3.7 & 3.2 & 2.5 & 2.5 & 2.8 & 3.3 & 3.5 \\
\hline $9 .$. & 3.7 & 4.05 & 3.65 & 4.5 & 3.3 & 3.65 & 3.2 & 2.5 & 2.5 & 3.05 & 3.3 & 3.5 \\
\hline 10. & 3.6 & 4.0 & 3.65 & 4.3 & 3.15 & 3.65 & 3.2 & 2.5 & 2.5 & 3.05 & 3.3 & 3.4 \\
\hline $11 \ldots$ & 3.55 & 3.9 & 3.65 & 4.2 & 3.2 & 3.6 & 3.2 & 2.5 & 2.5 & 3.05 & 3.3 & 3.4 \\
\hline $12 \ldots$ & 3.45 & 3.7 & 3.7 & 4.1 & 3.2 & 3.6 & 3.2 & 2.5 & 2.5 & 3.05 & 3.3 & 3.35 \\
\hline $13 \ldots$ & 3.5 & 3.7 & 3.7 & 4.1 & 3.2 & 3.6 & 3.2 & 2.5 & 2.5 & 3.05 & 3.3 & 3.3 . \\
\hline 14. & 3.7 & 3.75 & 3.7 & 4.0 & 3.6 & 3.5 & 3.2 & 2.5 & 2.5 & 3.05 & 3.3 & 3.3 \\
\hline 15. & 3.95 & 3.7 & 3.7 & 4.0 & 3.8 & 3.5 & 3.2 & 2.5 & 2.5 & 3.1. & 3.3 & 3.3 \\
\hline 16. & 4.25 & 3.65 & 3.8 & 4.0 & 3.8 & 3.4 & 3.2 & 2.5 & 2.5 & 3.1 & 3.25 & 3.3 \\
\hline 17. & 4.3 & 3.75 & 4.1 & 4.0 & 3.8 & 3.4 & 3.2 & 2.5 & 2.5 & 3.15 & 3.25 & 3.3 \\
\hline $18 .$. & 4.3 & 3.8 & 4.35 & 4.0 & 3.8 & 3.4 & 3.2 & 2.5 & 2.5 & 3.15 & 3.25 & 3.3 \\
\hline $19 .$. & 4.3 & 4.2 & 4.4 & 4.0 & 3.8 & 3.4 & 3.2 & 2.5 & 2.6 & 3.2 & 3.25 & 3.35 \\
\hline 20. & 4.2 & 4.95 & 4.5 & 3.95 & 3.8 & 3.4 & 3.2 & 2.4 & 2.6 & 3.2 & 3.3 & 3.4 \\
\hline $21 \ldots$ & 4.1 & 4.8 & 4.95 & 3.85 & 3.75 & 3.4 & 3.2 & 2.4 & 2.6 & 3.2 & 3.3 & 3.4 \\
\hline 22. & 4.35 & 4.6 & 4.8 & 3.8 & 3.7 & $3 . \dot{4}$ & 3.2 & 2.4 & 2.6 & 3.2 & 3.3 & 3.4 \\
\hline 23. & 4.5 & 4.3 & 4.45 & 3.3 & 3.65 & 3.4 & 3.1 & 2.4 & 2.6 & 3.2 & 3.35 & 3.4 \\
\hline $24 \ldots$ & 5.0 & 4.2 & 4.5 & 3.3 & 3.8 & 3.4 & 3.1 & 2.4 & 2.6 & 3.2 & 3.35 & 3.4 \\
\hline 25. & 5.35 & 4.1 & 4.6 & 3.3 & 3.7 & 3.4 & 3.1 & 2.4 & 2.6 & 3.2 & 3.4 & 3.4 \\
\hline 26. & 4.6 & 4.0 & 4.7 & 3.3 & 3.6 & 3.4 & 3.1 & 2.4 & 2.6 & 3.2 & 3.4 & 3.4 \\
\hline 27. & 4.2 & 4.0 & 4.8 & 3.4 & 3.5 & 3.3 & 3.1 & 2.5 & 2.7 & 3.2 & 3.4 & 3.4 \\
\hline 28. & 4.05 & 3.9 & 4.85 & 3.4 & 3.5 & 3.3 & 3.1 & 2.5 & 2.8 & 3.2 & 3.4 & 3.4 \\
\hline $29 .$. & 4.0 & ....... & 4.85 & 3.5 & 3.5 & 3.3 & 3.1 & 2.5 & 2.8 & 3.2 & 3.45 & 3.4 \\
\hline $30 .$. & 4.0 & & 4.8 & 3.4 & 3.6 & 3.3 & 3.1 & 2.5 & 2.8 & 3.2 & 3.5 & 3.4 \\
\hline $31 .$. & 4.15 & & 5.0 & 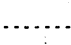 & 3.6 & $\ldots . .$. & 3.1 & 2.5 & & 3.2 & ..... & 3.4 \\
\hline
\end{tabular}

- Station rating table for Pit River near Canby, Cal., from January 1 to December 31, 1905.

\begin{tabular}{|c|c||c|r|r|r||r|r|}
\hline $\begin{array}{c}\text { Gage } \\
\text { height. }\end{array}$ & Discharge. & $\begin{array}{c}\text { Gage } \\
\text { height. }\end{array}$ & Discharge. & $\begin{array}{c}\text { Gage } \\
\text { height. }\end{array}$ & Discharge. & $\begin{array}{c}\text { Gage } \\
\text { height. }\end{array}$ & Discharge. \\
\cline { 1 - 5 } Feet. & Second-feet. & Feet. & Second-feet. & Feet. & Second-feet. & Feet. & Second-feet. \\
2.40 & 1.5 & 3.30 & 87 & 4.20 & 475 & 5.10 & 1,028 \\
2.50 & 3.5 & 3.40 & 115 & 4.30 & 533 & 5.20 & 1,091 \\
2.60 & 6 & 3.50 & 148 & 4.40 & 593 & 5.30 & 1,154 \\
2.70 & 10 & 3.60 & 186 & 4.50 & 655 & 5.40 & 1,217 \\
2.80 & 16 & 3.70 & 226 & 4.60 & 717 & 5.50 & 1,280 \\
2.90 & 24 & 3.80 & 269 & 4.70 & 779 & 5.60 & 1,343 \\
3.00 & 34 & 3.90 & 316 & 4.80 & 841 & & \\
3.10 & 47 & 4.00 & 366 & 4.90 & 903 & & \\
3.20 & 64 & 4.10 & 419 & 5.00 & 965 & & \\
\hline
\end{tabular}

Note. - The above table is applicable only for open-channel conditions. It is based on thirty-nine discharge measurements made during 1905 . It is well defined between gage heights 2.4 feet and 5 feet. 
Estimated monthly discharge of Pit River near Canby, Cal., for 1905.

[Drainage area, 1,500 square miles.]

\begin{tabular}{|c|c|c|c|c|c|c|}
\hline \multirow{2}{*}{ Month. } & \multicolumn{3}{|c|}{ Discharge in second-feet. } & \multirow[b]{2}{*}{$\begin{array}{l}\text { Total in } \\
\text { acre-feet. }\end{array}$} & \multicolumn{2}{|c|}{ Run-off. } \\
\hline & Maximum. & Minimum. & Mean. & & $\begin{array}{c}\text { Second-feet } \\
\text { per square } \\
\text { mile. }\end{array}$ & $\begin{array}{l}\text { Depth } \\
\text { in inches. }\end{array}$ \\
\hline January.. & 1,186 & 132 & 443 & 27,240 & 0.295 & 0.340 \\
\hline February........ & 1,374 & 206 & 533 & 29,600 & .355 & .370 \\
\hline March..... & 965 & 206 & 479 & 29,450 & .319 & .368 \\
\hline April....... & 1,343 & 87 & 510 & 30,350 & .340 & .379 \\
\hline May........ & 269 & 34 & 165 & 10,140 & .110 & .127 \\
\hline June............. & 226 & 87 & 151 & 8,985 & .101 & .113 \\
\hline July ....... & 87 & 47 & 61.7 & 3,794 & .041 & .047 \\
\hline August.......... & 24 & 1.5 & 6.4 & 394 & .0043 & .0050 \\
\hline September........ & 16 & 3.5 & 5.6 & 333 & .0037 & .0041 \\
\hline October......... & 64 & 16 & 45.4 & 2,792 & .030 & .035 \\
\hline November.........., $\ldots \ldots \ldots \ldots$ & 148 & 64 & 92.2 & 5,486 & .061 & .068 \\
\hline December.................... & $1^{148}$ & 87 & 118 & 7,256 & .079 & .091 \\
\hline The year... & 1,374 & 1.5 & 218 & 155,800 & .145 & 1.95 \\
\hline
\end{tabular}

PIT RIVER NEAR BIEBER, OAL.

This station was established January 22, 1904, by J. S. Evans and William Busch. It is located 12 miles below Bieber, Cal., near Muck Valley.

The channel is straight for 200 feet above and 300 feet below the station. The current is very sluggish at low-water stage. The banks are high and not subject to overflow. The channel is very rocky and rough, but is not subject to change.

Discharge measurements are made from a cable and car. The initial point for soundings is the foot of the platform at end of the cable on the right bank of the stream.

The gage is a heavy wooden rod fastened to a large bowlder on the right bank of the stream. During 1905 the gage was read once each day by F. H. Holabird. The bench mark is on a large bowlder on the right bank 50 feet east of the pine tree to which the cable is fastened; elevation $\mathbf{1 2 . 0 0}$ feet above the datum of the gage.

A description of this station and gage height and discharge data are contained in WaterSupply Paper No. 134, United States Geological Survey. 
Discharge measurements of Pit River near Bieber, Cal., in 1905.

\begin{tabular}{|c|c|c|c|c|c|c|}
\hline Date. & Hydrographer. & Width. & $\begin{array}{l}\text { Area of } \\
\text { section. }\end{array}$ & $\begin{array}{c}\text { Méan } \\
\text { velocity. }\end{array}$ & $\begin{array}{c}\text { Gage } \\
\text { height. }\end{array}$ & $\begin{array}{c}\text { Dis- } \\
\text { charge. }\end{array}$ \\
\hline January 8 . & J. Y. Toler . . . . . . . & $\begin{array}{r}\text { Feet. } \\
104\end{array}$ & $\begin{array}{c}S q \cdot f t \\
343\end{array}$ & $\begin{array}{r}\text { Ft. per sec. } \\
0.99\end{array}$ & $\begin{array}{l}\text { Feet. } \\
\quad 3.70\end{array}$ & $\begin{array}{r}\text { Sec.-ft. } \\
340\end{array}$ \\
\hline January 22 . & .....do. & 210 & 729 & 1.96 & 5.25 & 1,430 \\
\hline January 23 . & ....do. . & 218 & 915 & 2.46 & 6.00 & 2,254 \\
\hline Do..... & .....do. & 217 & 889 & 2.50 & 5.94 & 2,220 \\
\hline February 9. & ......do. & 204 & 630 & 1.68 & 4.80 & 1,060 \\
\hline February 24 . & ...... do. & 208 & 673 & 1.93 & 5.08 & 1,296 \\
\hline March 11. & .....do. & 144 & 389 & .97 & 3.70 & 378 \\
\hline March $24 \ldots$ & .....do. & 212 & 743 & 2.22 & 5.40 & 1,650 \\
\hline March 23 & ......do. & 213 & 767 & 2.11 & 5.51 & 1,620 \\
\hline April 7... & .....do. & 210 & 739 & 2.02 & 5.30 & 1,492 \\
\hline April 6..... & ....do. . & 212 & 757 & 2.18 & 5.40 & 1,648 . \\
\hline April 17... & .....do. & 199 & 504 & 1.32 & 4.30 & 665 \\
\hline May $3 \ldots . .$. & .....do. . & 126 & 232 & .61 & 2.88 & 141 \\
\hline May $18 \ldots$ & .....do. & 134 & 309 & .79 & 3.50 & 244 \\
\hline June $12 \ldots$ & .....do. & 126 & 215 & .51 & 2.80 & 110 \\
\hline July $7 \ldots$ & .....do. & 75 & 143 & .24 & 2.30 & 34 \\
\hline July $19 . \ldots$. & ..... do. & 79 & 144 & .29 & 2.40 & 42 \\
\hline August $9 . .$. & .....do... & 58 & 105 & .22 & 1.85 & 23 \\
\hline August $18 \ldots$ & ....do. & 34 & 18.8 & 1.02 & 1.80 & 19.1 \\
\hline August $29 \ldots$ & ..... do . & 30 & 12.6 & .78 & 1.70 & 9.9 \\
\hline September 10 . & .....do. & 6 & 2.4 & .46 & 1.40 & 1.1 \\
\hline September 20 . & .....do. . & 7 & 2.0 & .60 & 1.40 & 1.2 \\
\hline October 7... & .....do. & 6 & 2.8 & .61 & 1.50 & 1.7 \\
\hline October $17 \ldots$ & ....do. & 31 & 12.7 & .83 & 1.70 & 10.5 \\
\hline October $26 . .$. & .....do. do. & 45 & 22.5 & 1.80 & 2.20 & 41 \\
\hline November 8 & ......do... & 80 & 168 & .42 & 2.60 & 71 \\
\hline
\end{tabular}

IRR $177-06-10$ 
Daily gage height, in feet, of Pit River near Bieber, Cal., for 1905.

\begin{tabular}{|c|c|c|c|c|c|c|c|c|c|c|c|c|}
\hline Day. & Jan. & Feb. & Mar & Apr. & May. & June. & July. & Aug. & Sept. & Oct. & Nov. & Dec. \\
\hline $1 .$. & 4.8 & 5.4 & 4.6 & 6.2 & 3.0 & 3.2 & 2.5 & 2.1 & 1.5 & $a 1.5$ & $a_{2.3}$ & $a_{2.4}$ \\
\hline 2. & 5.0 & 5.5 & 4.5 & 6.3 & 3.0 & 3.2 & 2.45 & 2.1 & 1.5 & $a 1.5$ & $a_{2.4}$ & $a_{2.4}$ \\
\hline 3. & 4.9 & 5.3 & 4.3 & 6.15 & 2.9 & 3.1 & 2.4 & 1.9 & 1.5 & a 1.5 & $a_{2.4}$ & $a_{2.4}$ \\
\hline 4.. & 4.5 & 5.0 & 4.0 & 5.9 & 2.9 & 3.1 & 2.4 & 1.8 & 1.4 & $a 1.5$ & $a_{2.5}$ & $a_{2.4}$ \\
\hline 5.. & 4.2 & 4.8 & 4.0 & 5.7 & 2.9 & 3.1 & 2.4 & 1.8 & 1.4 & $a 1.5$ & $a_{2.5}$ & $a_{2.4}$ \\
\hline 6.. & 3.9 & 4.6 & 4.0 & 5.4 & 3.0 & 3.0 & 2.3 & 1.8 & 1.4 & $a 1.5$ & $a_{2.5}$ & $a 2.4$ \\
\hline $7 .$. & 3.7 & 6.0 & 3.9 . & 5.2 & 3.0 & 3.0 & 2.3 & 1.75 & 1.4 & 1.5 & a 2.5 & $a_{2.4}$ \\
\hline 8.. & 3.7 & 5.5 & 3.9 & 5.1 & 3.0 & $2: 9$ & 2.3 & 1.75 & 1.35 & $a 1.5$ & 2.6 & $a_{2.4}$ \\
\hline 9. & 3.5 & 4.8 & 3.8 & 5.0 & 2.9 & 2.9 & 2.4 & 1.85 & 1.35 & $a 1.5$ & $a_{2.6}$ & $a_{2.4}$ \\
\hline $10 .$. & 3.4 & 4.6 & 3.7 & 4.9 & $2.9^{\prime}$ & 2.8 & 2.35 & 1.8 & 1.4 & $a 1.5$ & $a_{2.6}$ & $a_{2.4}$ \\
\hline 11.. & 3.4 & 4.3 & 3.7 & 4.7 & 2.8 & 2.8 & 2.45 & 1.8 & 1.4 & $a 1.5$ & $a_{2.6}$ & $a_{2.4}$ \\
\hline 12. & 3. 3 & 3.9 & 3.7 & 4.6 & 2.8 & 2.8 & 2.45 & 1.5 & 1.4 & $a 1.6$ & $a_{2.6}$ & $a_{2.3}$ \\
\hline 13. & $3 . \grave{4}$ & 3.7 & 3.7 & 4.4 & 2.8 & 2.8 & 2.4 & 1.5 & 1.4 & $a 1.6$ & $a .2 .6$ & $a_{2.3}$ \\
\hline $14 .$. & 3.4 & 3.7 & 3.8 & 4.3 & 2.8 & 2.7 & 2.4 & 1.5 & $a 1.4$ & $a 1.6$ & $a 2.6$ & $a_{2.3}$ \\
\hline 15. & 3.6 & 3.9 & 3.8 & 4.2 & 2.8 & 2.7 & 2.7 & 1.5 & $a_{1.4}$ & $a_{1.6}$ & $a_{2.7}$ & $a 2.3$ \\
\hline 16. & 3.9 & 4.0 & 4.0 & 4.3 & 3.0 & 2.7 & 2.7 & 1.7 & $a_{1.4}$ & $a 1.6$ & $a_{2.7}$ & $a 2.3$ \\
\hline 17.. & 4.95 & 4.1 & 4.0 & 4.3 & 3.4 & 2.7 & 2.7 & 1.7 & $a 1.4$ & 1.7 & $a_{2.7}$ & $a 2.3$ \\
\hline & 5.1 & 4.3 & 5.0 & 4.3 & 3.5 & 2.7 & 2.3 & 1.8 & $a_{1.4}$ & 2.0 & $a_{2.7}$ & $a_{2.3}$ \\
\hline 19. & 5.0 & 4.6 & 5.4 & 4.2 & 3.5 & 2.5 & 2.4 & 1.8 & $a_{1.4}$ & 2.0 & $a_{2.7}$ & $a 2.3$ \\
\hline 20. & 5.0 & 4.8 & 5.5 & 4.0 & 3.4 & 2.5 & 2.4 & 1.7 & $a_{1.4}$ & 2.0 & $a_{2.7}$ & $a 2.3$ \\
\hline $21 .$. & 5.0 & 5.0 & 5.5 & 4.0 & 3.4 & 2.5 & 2.3 & 1.7 & a 1.4 & 2.0 & $a 2.7$ & $3: 3$ \\
\hline 22. & 5.25 & 5.8 & 5.8 & 3.9 & | 3.2 & 2.4 & 2.2 & 1.5 & a 1.4 & 2.0 & $a_{2.7}$ & 3.3 \\
\hline 23. & 5.95 & 5.2 & 5.55 & 3.9 & 3.2 & 2.4 & 2.2 & 1.5 & $a 1.4$ & 2.0 & $a_{2.6}$ & 2.2 \\
\hline 24. & 6.5 & 5.05 & 5.45 & 3.7 & 3.2 & 2.4 & 2.6 & 1.5 & a 1.4 & $a_{2.0}$ & $a_{2.6}$ & 2.2 \\
\hline 25. & 6.0 & 5.0 & 5.4 & 3.6 & 3.1 & 2.3 & 2.4 & 1.5 & $a 1.4$ & $a 2.0$ & $a 2.6$ & 2.2 \\
\hline 26.. & 5.8 & 5.0 & 5.7 & 3.4 & 3.1 & 2.3 & 2.1 & 1.5 & $a_{1.4}$ & 2.2 & $a_{2.6}$ & 2.2 \\
\hline 27. & 5.5 & 4.8 & 5.9 & 3.1 & 3.0 & 2.3 & 2.1 & 1.7 & $a 1.4$ & a 2.2 & $a_{2.6}$ & 2.2 \\
\hline 28. & 5.0 & 4.8 & 5.8 & 3.1 & 3.1 & 2.7 & 2.1 & 1.7 & 1.5 & $a_{2.2}$ & a 2.6 & 2.2 \\
\hline 29.: & 4.8 & & 5.8 & 3.1 & 3.2 & 2.7 & 2.2 & 1.7 & 1.5 & $a_{2.2}$ & $a_{2.6}$ & 2.2 \\
\hline 30.. & 5.1 & & 5.8 & 3.0 & 3.3 & 2.7 & 2.2 & 1.5 & $a_{1.5}$ & $a_{2.3}$ & a 2.5 & 2.2 \\
\hline 31.. & 5.4 & ....... & 6.0 & ...... & 3.3 & $\cdots .$. & 2.25 & 1.5 & $\cdots$ & $a_{2.3}$ & . & 2.2 \\
\hline
\end{tabular}

a Estimated.

NoTE.-River frozen over December 23-31.

Station rating table for Pit River near Bieber, Cal., from January 1 to December 31, 1905.

\begin{tabular}{|c|c||c|r|r|r|r|r|}
\hline $\begin{array}{c}\text { Gage } \\
\text { height. }\end{array}$ & Discharge. & $\begin{array}{c}\text { Gage } \\
\text { height. }\end{array}$ & Discharge. & $\begin{array}{c}\text { Gage } \\
\text { height. }\end{array}$ & Discharge. & $\begin{array}{c}\text { Gage } \\
\text { height. }\end{array}$ & Discharge. \\
\hline Feet. & Second-feet. & Feet. & Second-feet. & Feet. & Second-feet. & Feet. & Second-feet. \\
1.30 & 0.5 & 2.50 & 64 & 3.70 & 349 & 4.90 & 1,130 \\
1.40 & 1.0 & 2.60 & 77 & 3.80 & 396 & 5.00 & 1,215 \\
1.50 & 2.2 & 2.70 & 92 & 3.90 & 448 & 5.20 & 1,400 \\
1.60 & 5.8 & 2.80 & 109 & 4.00 & 505 & 5.40 & 1,595 \\
1.70 & 9.6 & 2.90 & 127 & 4.10 & 565 & 5.60 & 1,810 \\
1.80 & 13.6 & 3.00 & 147 & 4.20 & 630 & 5.80 & 2,035 \\
1.90 & 18 & 3.10 & 168 & 4.30 & 695 & 6.00 & 2,270 \\
2.00 & 23 & 3.20 & 190 & 4.40 & 760 & 6.20 & 2,525 \\
2.10 & 29 & 3.30 & 213 & 4.50 & 830 & 6.40 & 2,810 \\
2.20 & 36 & 3.40 & 239 & 4.60 & 900 & & \\
2.30 & 44 & 3.50 & 270 & 4.70 & 975 & & \\
2.40 & 53 & 3.60 & 307 & 4.80 & 1,050 & & \\
& & & & & & & \\
\hline
\end{tabular}

Note.-The above table is applicable only for open-channel conditions. . It is based on 26 discharge measurements made during 1905. It is well defined between gage heights 3.5 feet and 6 feet. Below 3.5 feet the table is not so well defined. 
Estimated monthly discharge of Pit River near Bieber, Cal., for 1905.

[Drainage area, 2,948 square miles.]

\begin{tabular}{|c|c|c|c|c|c|c|}
\hline \multirow[b]{2}{*}{ Month. } & \multicolumn{3}{|c|}{ Discharge in second-feet. } & \multirow{2}{*}{$\begin{array}{l}\text { Total in } \\
\text { acre-feet. }\end{array}$} & \multicolumn{2}{|c|}{ Run-off. } \\
\hline & Maximum. & Minimum. & Mean. & & $\begin{array}{l}\text { Second-feet } \\
\text { per square } \\
\text { mile. }\end{array}$ & $\begin{array}{l}\text { Depth } \\
\text { in inches. }\end{array}$ \\
\hline January .... & 2,960 & 213 . & 1,036 & 63,700 & 0.351 & 0.405 \\
\hline February .............. & 2,270 & 349 & 1,083 & 60,150 & .367 & .382 \\
\hline March............ & 2,270 & 349 & 1,101 & 67,700 & .373 & .430 \\
\hline April............ & 2,665 & 147 & 950 & 56,530 & .322 & .359 \\
\hline May......... & 270 & 109 & 166 & 10,210 & .056 & .065 \\
\hline June............ & 190 & 44 & 103 & 6,129 & .035 & .039 \\
\hline July ......... & 92 & 29 & 51.8 & 3,185 & .018 & .021 \\
\hline August........ & $29^{\circ}$ & 2.2 & 9.73 & 598 & .0033 & .0038 \\
\hline September..... & 2.2 & .8 & 1.23 & 73.2 & .00042 & .00047 \\
\hline October......... & 44 & 2.2 & 15.4 & 947 & .0052 & .0060 \\
\hline November...................... & 92 & 44 & 76.1 & 4,528 & .026 & .029 \\
\hline December $1-22 \ldots \ldots \ldots \ldots \ldots$ & 213 & 44 & 63.9 & 2,788 & .022 & .025 \\
\hline The period. & & & & 276,500 & & \\
\hline
\end{tabular}

NoTE.-Discharge interpolated for missing gage heights in September, October, November, and December.

\section{SOUTH FORK OF PIT RIVER NEAR IVY, CAL.}

This station was established January 11, 1904, by H. E. Green and J. S. Evans. It is located 3 miles west of Ivy post-office, at the outlet of Jess Valley.

The channel is straight for 200 feet above and 80 feet below the station. The current is sluggish at low-water stage. The right bank is low and subject to overflow in high water. The bed of the stream is composed of earth and is filled with vegetation at low-water stage.

Discharge measurements are made from a cable and car. The initial point for soundings is the foot of the post used for fastening the car on the left bank of the stream. .

The gage is a vertical plank fastened to a tree on the left bank 50 feet above the station. It is read only at times discharge measurements are made, as it is impossible to get an observer on account of its isolated location. The bench mark is on a large bowlder 150 feet east of cabin on the right bank and below the station; elevation, 12.00 feet above the datum of the gage.

A description of this station, with gage height and discharge data, is contained in WaterSupply Paper No. 134, United States Geological Survey. 
Discharge measurements of South Fork of Pit River near Ivy, Cal., in 1905.

\begin{tabular}{|c|c|c|c|c|c|c|}
\hline Date. & Hydrographer. & Width. & $\begin{array}{l}\text { Area of } \\
\text { section. }\end{array}$ & $\begin{array}{c}\text { Mean } \\
\text { velocity. }\end{array}$ & $\begin{array}{c}\text { Gage } \\
\text { height. }\end{array}$ & $\begin{array}{c}\text { Dis- } \\
\text { charge. }\end{array}$ \\
\hline January $1 .$. & J. Y. Toler.. & $\begin{array}{c}\text { Feet. } \\
\quad 32\end{array}$ & $\begin{array}{c}\text { Sq. feet. } \\
63\end{array}$ & $\begin{array}{r}\text { Ft. per sec. } \\
0.92\end{array}$ & $\begin{array}{r}\text { Feet. } \\
\quad 4.90\end{array}$ & $\begin{array}{c}\text { Sec.-feet. } \\
58\end{array}$ \\
\hline January 13 .. & .....do.. & 28 & 37. & .62 & 3.80 & 23 \\
\hline February 1. & .....do... & 30 & 47 & 1.04 & 4.18 & 49 \\
\hline February 17 & .....do.... & 28 & 42 & 1.00 & 4.00 & 42 \\
\hline March 4... & .....do.... & 28 & 42 & 1.05 & 4.05 & 44 \\
\hline March $17 .$. & .....do.... & 31 & 63 & 1.46 & 4.72 & 93 \\
\hline March 30 & $\ldots .$. do............. & 30 & 51 & 1.18 & 4.30 & 60 \\
\hline April 13:. & .....do........... & 33 & 61 & 1.41 & 4.60 & 86 \\
\hline April 22. . & .....do..... & 33 & 62 & 1.37 & 4.60 & 85 \\
\hline May $12 \ldots$ & .....do. . . . . . . & 66 & 104 & 1.52 & 5.33 & 158 \\
\hline May $13 . .$. & .....do... & 66 & 101 & 1.53 & 5.33 & 155 \\
\hline May 25.. & .....do.... & 85 & 112 & 1.41 & 5.40 & 158 \\
\hline May 26. & ......do.. & 98 & 118 & 1.40 & 5.44 & 165 \\
\hline June $5 . .$. & $\therefore$...do.. & 107 & 145 & 1.34 & 5.68 & 195 \\
\hline June 6 ..... & .....do.... & 97 & 126 & 1.31 & 5.50 & 165 \\
\hline June $17 \ldots$ & .....do..... & 39 & 80 & 1.32 & 5.05 & 106 \\
\hline June $19 \ldots$ & .....do.... & 38 & 78 & 1.28 & 5.00 & 100 \\
\hline July $1 . .$. & .....do.... & 24 & 22 & 1.86 & 4.15 & 41 \\
\hline July $13 \ldots$ & .....do...... & 23 & 19.5 & 1.79 & 4.05 & 35 \\
\hline July $14 \ldots$ & .....do.... & 23 & 18.3 & 1.75 & 4.00 & 32 \\
\hline July 26 ... & .....do. & 15 & 20 & 1.60 & 4.00 & 32 \\
\hline July $27 \ldots$ & .....do. . & 15 & 20 & 1.60 & 4.00 & 32 \\
\hline August 15. . & .....do. . & 16 & 19.5 & 1.44 & 4.10 & 28 \\
\hline August 16 . . & .....do. & 16 & 19.8 & 1.41 & 4.10 & 28 \\
\hline August $24 .$. & ...do. & 16 & 17.2 & 1.16 & 3.70 & 19.9 \\
\hline September 5 & .....do. . & 6 & 8.6 & 1.42 & 3.20 & 12.2 \\
\hline September 14 & .....do... & 14 & 8.6 & 1.95 & 3.30 & 16.8 \\
\hline October $3 . .$. & .....do.... & 15 & 7.2 & 1.97 & 3.20 & 14.2 \\
\hline October $12 .$. & .....do... & 15 & 7.2 & 1.97 & 3.20 & 14.2 \\
\hline October 13. & .....do....... & 15 & 7.2 & 2.00 & 3.20 & 14.4 \\
\hline October $21 .$. & .....do.... & 16 & 6.8 & 2.03 & 3.20 & 13.8 \\
\hline November 1 & .....do. & 15 & 7.2 & 2.03 & 3.20 & 14.6 \\
\hline November 2 & .....do. & 16 & 8.0 & 2.09 & 3.20 & 16.7 \\
\hline November 14 & ....do. . & 16 & 8.4 & 2.13 & 3.20 & 17.9 \\
\hline November 15 & ....do...... & 16 & 7.6 & 2.12 & 3.20 & 16.1 \\
\hline
\end{tabular}

WEST VALLEY CREEK NEAR ITKELY, CAL.

This station was established January 7, 1904, by H. E. Green and J, S. Evans. It is located 7 miles east of Likely, Cal., at the outlet of West Valley.

The channel is straight for 200 feet above and 100 feet below the station. The banks are high and not subject to overflow. The bed of the stream is rocky and not subject to change. The current is swift.

Discharge measurements are made from a cable and car, or by wading at low-water stage. The initial point for soundings is a juniper stump on the left bank, which is used for a tieing post for the car. After July 1, 1905, gagings were made at the ranch house near the upper rod. These gagings give a much less discharge than at the regular station. The gage is a vertical plank nailed to a juniper tree on the right bank of the creek. As no one could be obtained to read the station gage, a gage was placed 2 miles above the station near the ranch house, where an observer could be obtained. The gage as read at this point is not considered satisfactory for use in constructing a discharge curve for the gaging station, but only gives a general idea of the fluctaations in the flow of the stream. 
Numerous springs and swamps appear in the valley between the gaging station and the ranch house, giving a greater discharge at the gaging station than at this point. During 1905 the gage was read by Jasper L. Fountain. The bench mark is a point marked with paint on a large bowlder 30 feet from the creek on the left bank; elevation, 10.00 feet above the datum of the gage.

A description of this station, with gage height and discharge data, is contained in WaterSupply Paper No. 134, United States Geological Survey.

Discharge measurements of West Valley Creek near Likely, Cal., in 1905.

\begin{tabular}{|c|c|c|c|c|c|c|}
\hline Date. & Hydrographerer. & Width. & $\begin{array}{l}\text { Area of } \\
\text { section. }\end{array}$ & $\begin{array}{c}\text { Mean } \\
\text { velocity. }\end{array}$ & $\begin{array}{c}\text { Gage } \\
\text { height. }\end{array}$ & $\begin{array}{c}\text { Dis- } \\
\text { charge. }\end{array}$ \\
\hline & & Feet. & $S q . f t$. & Ft.per sec. & Feet. & Sec.-ft. \\
\hline January 12 . & J. Y. Toler. & 15 & 22 & 1.28 & 3.30 & 28 \\
\hline January 31 & .....do. & 15 & 23 & .96 & 3.20 & 22 \\
\hline February 2. & .....do. & 15 & 22 & .93 & 3.20 & - 20 \\
\hline February 16 & .....do. & 15 & 24 & 1.09 & 3.22 & 26 \\
\hline March 4. & ......do. & 15 & 24 & 1.00 & 3.20 & 24 \\
\hline March 5... & ...do. & 15 & 24 & 1.00 & 3.20 & 24 \\
\hline March $16 .$. & ...do.. & 15 & 24 & 1.02 & 3.22 & 25 \\
\hline March $17 \ldots$ & ...do. & 15 & 32 & 2.16 & 3.70 & 69 \\
\hline March 29 & ....do. & 15 & 21 & 1.43 & 3.30 & 30 \\
\hline March 31. & ...do & 15 & 21 & 1.42 & 3.30 & 30 \\
\hline April 13.. & ...do.. & 15 & 22 & 1.18 & 3.25 & 26 \\
\hline April 22. . & ...do... & 15 & 19 & 1.19 & 3.20 & 23 \\
\hline May $12 \ldots$ & ...do. & 15 & 25 & 1.40 & 3.32 & .35 \\
\hline May $13 . .$. & ..do.. & 15 & 26 & 1.46 & 3.35 & 38 \\
\hline May $25 \ldots$ & ...do.. & 15 & 21 & 1.05 & 3.22 & 22 \\
\hline May 26. & ...do. & 15 & 22 & .95 & 3.19 & 21 \\
\hline June 4.... & $\ldots$. do & 15 & 28 & 1.68 & 3.50 & 47 \\
\hline June 5... & $\ldots . d c$ & 15 & 26 & 1.50 & 3.40 & 39 \\
\hline June $6 . .$. & $\ldots . \mathrm{dc}$ & 15 & 25 & 1.28 & 3.30 & 32 \\
\hline June 17... & ...do... & 15 & 22 & 1.18 & 3.20 & 26 \\
\hline June 19... & ...do... & 15 & 22 & 1.09 & 3.15 & $24^{\circ}$ \\
\hline July $1 \ldots$ & ......do. & 3 & 4.4 & 3.84 & 3.10 & 16.9 \\
\hline July $13 \ldots$ & $\ldots . . \mathrm{dc}$ & 8 & 10.0 & 1.74 & 3.20 & 17.4 \\
\hline July $14 . .$. & $\ldots . d$ & 8 & 10.0 & 1.74 & 3.20 & 17.4 \\
\hline July $26 .$. & $\ldots$. d & 8 & 9.6 & 1.72 & 3.15 & 16.5 \\
\hline August 15. & $\ldots d$ & 8 & 10.0 & 1.70 & 3.15 & 17 \\
\hline August 24. . & $\ldots d$ & 16 & 7.2 & 1.43 & 3.00 & 10.3 \\
\hline Septembe & $\ldots d c$ & 21 & 13 & 1.02 & 3.10 & 13.2 \\
\hline September 6. & $\ldots . \mathrm{dc}$ & 21 & 13.7 & 1.04 & 3.10 & 14.2 \\
\hline September 14. & $\ldots d c$ & 17 & 7.4 & 1.53 & 3.10 & 11.3 \\
\hline September 15. & ...do.. & 17 & 7.8 & 1.49 & 3.10 & 11.6 \\
\hline October 3... & $\ldots . . d$ & 18 & 8.4 & 1.65 & 3.10 & 13.9 \\
\hline October $12 .$. & ......do... & 19 & 10.1 & 1.59 & 3.20 & 17.1 \\
\hline October $13 . .$. & ...do.. & 19 & 10.4 & 1.68 & 3.20 & 17.5 \\
\hline October 21... & $\ldots . d$ & 18 & 7.6 & 1.72 & 3.10 & 13.1 \\
\hline October $22 . . .$. & ...do.. & 18 & 7.8 & 1.73 & 3.10 & 13.5 \\
\hline November $1 \ldots$ & ....do. & 13 & 11.4 & 1.24 & 3.20 & 14.0 \\
\hline November 14 & ....do. . & 13 & 11.0 & 1.32 & 3.20 & 14.5 \\
\hline November 15. & .....do.... & 13 & 11.0 & 1.30 & 3.20 & 14.3 \\
\hline
\end{tabular}

NOTE.-After July 1 gagings were made near upper gage. 
Daily gage height, in feet, of West Valley Creek near Likely, Cal., for 1905.

\begin{tabular}{|c|c|c|c|c|c|c|c|c|c|c|c|c|}
\hline Day. & Jan. & Feb. & Mar. & Apr. & May. & June. & July. & Aug. & Sept. & oct. & Nov. & Dec. \\
\hline & 3.3 & 3.2 & 3.2 & 4.5 & 3.15 & 3.2 & 3.2 & 0.2 & 3.0 & a 3.1 & 3.15 & 3.1 \\
\hline 2. & 3.3 & 3.2 & 3.2 & 3.4 & 3.15 & 3.2 & 3.2 & 3.2 & 3.0 & a 3.1 & 3. 15 & $\therefore 1$ \\
\hline & 3.25 & 3.2 & 3.2 & 3.3 & 3.15 & 3.2 & 3.2 & 3.2 & 3.0 & a 3.1 & 3.15 & 3.15 \\
\hline 4. & 3.25 & 3.2 & 3.15 & 3.3 & 3.15 & 3.2 & 3.2 & 3.2 & 3.1 & a 3.1 & 3.2 & 3.15 \\
\hline 5. & 3.25 & 3.2 & 3.15 & 3.3 & 3.15 & 3.6 & 3.2 & 3.2 & 3.1 & a.3.1 & 3.2 & 3.15 \\
\hline & 3.2 & 3.2 & a 3.15 & 3.3 & 3.15 & 3.5 & 3.2 & 3.2 & 3.1 & a 3.1 & 3.2 & 3.15 \\
\hline 7. & 3.2 & 3.2 & a 3.15 & 3.25 & 3.15 & 3.45 & 3.2 & 3.2 & 3.1 & a 3.1 & 3.2 & 3. 15 \\
\hline 8. & 3.2 & 3.2 & $a 3.15$ & 3.25 & 3.3 & 3.55 & 3.2 & 3.2 & 3.1 & a 3.2 & 3.2 & 3.1 \\
\hline 9 & 3.2 & 3.2 & $a 3.1$ & 3. 25 & 3.2 & 3.55 & 3.15 & 3.2 & 3.1 & $a 3.2$ & 3.2 & 3.15 \\
\hline 10. & 3.2 . & 3.2 & a 3.1 & 3.25 & 3.4 & 3.3 & 3.15 & 3.2 & 3.1 & $a 3.2$ & 3.2 & 3.15 \\
\hline 11. & 3.2 & 3.2 & 3.1 & 3.25 & 3.3 & 3.3 & 3.15 & 3.2 & 3.1 & a 3.2 & 3.2 & 3.1 \\
\hline 12. & 3.2 & 3.2 & 3.1 & 3.25 & 3.25 & 3.25 & 3.15 & 3.2 & 3.2 & a 3,2 & 3.2 & 3.15 \\
\hline 13. : & 3.2 & 3. 2 . & 3.15 & 3.25 & 3.2 & 3.25 & 3.15 & 3.2 & 3.2 & $a 3.2$ & 3.2 & 3.1 \\
\hline 14. & 3.2 & 3.2 & 3.2 & 3.25 & 3.2 & 3.2 & 3.15 & 3.0 & 3.2 & a.3.2 & 3.2 & 3. 25 \\
\hline 15. & 3.2 & 3.2 & 3.2 & ·3. 3 & 3.2 & 3.2 & 3.15 & 3.0 & 3.2 & a 3.2 & 3.2 & 3.15 \\
\hline 16. & 3.2 & 3.2 & 3.2 & 3.3 & 3.2 & 3.2 & 3.15 & 3.0 & 3.2 & a 3.2 & 3.2 & 3.1 \\
\hline 17. & $3.25^{\circ}$ & 3.2 & 3.65 & 3.3 & 3.2 & 3.2 & 3.15 & 3.0 & $a 3.2$ & a 3.2 & 3.2 & 3.15 \\
\hline 18. & 3.25 & 3.2 & 3.4 & 3.25 & 3.2 & 3.2 & 3.15 & 3.0 & a 3.2 & a 3.1 & 3.2 & 3.1 \\
\hline 19 & 3.3 & 3.2 & 3.5 & 3.2 & 3.2 & 3.2 & 3.15 & 3.0 & $a 3.2$ & a 3.1 & 3.2 & 3.1 \\
\hline 20. & 3.3 . & 3.2 & 3. 35 & 3.2 & 3.2 & 3.2 & 3.15 & 3.0 & $a 3.2$ & a 3.1 & 3.6 & 3.15 \\
\hline 21. & 3.3 & 3.2 & 3.5 & 3.2 & 3.2 & 3.2 & 3.15 & 3.0 & $a 3.2$ & a 3.1 & 3.4 & 3.1 \\
\hline 22. & 3.25 & 3.2 & 3.5 & 3.2 & 3.2 & 3.2 & 3.15 & 3.0 & $a 3.2$ & 3.1 & 3.8 & 3.1 \\
\hline 23. & 3.2 & 3.2 & 3.35 & 3.2 & 3.2 & 3.2 & 3.15 & 3.0 & $a 3.2$ & 3.1 & 3.25 & 3.15 \\
\hline 24. & 3.2 & 3.2 & 3.3 & 3.2 & 3.2 & 3.2 & 3.15 & 3.0 & a 3.2 & 3.1 & 3.1 & 3.1 \\
\hline 25. & 3.2 & 3.2 & 3.3 & 3.2 & 3.2 & 3.2 & 3.15 & 3.0 & $a 3.2$ & 3.1 & 3.1 & 3.15 \\
\hline 26. & 3.2 & 3.2 & 3.4 & 3.15 & 3.2 & 3.2 & 3.15 & 3.0 & $a 3.2$ & 3.1 & 3.1 & 3.1 \\
\hline 27. & 3.2 & 3.2 & 3.7 & 3. 15 & 3.2 & 3.2 & 3.15 & 3.0 & a 3.2 & 3.1 & 3.15 & 3.1 \\
\hline 28. & 3.2 & 3.2 & 3.4 & 3.15 & 3.2 & 3.2 & 3.15 & 3.0 & a 3.2 & 3.1 & 3.2 & 3.1 \\
\hline 29. & 3.2 & & 3. 35 & 3.15 & 3.2 & 3.2 & 3.15 & 3.0 & a 3.2 & 3.1 & 3.1 & 3.15 \\
\hline 30. & 3.2 & & 3.4 & 3.15 & 3.2 & 3.2 & 3.15 & 3.0 & a 3.2 & 3.1 & 3.1 & 3.1 \\
\hline 31. & 3.2 & . & 3.5 & ...... & 3.2 & ..... & 3.2 & 3.0 & $\cdots \cdots$ & 3.1 & $\ldots$ & 3.6 \\
\hline
\end{tabular}


Daily discharge, in second-feet, of West Valley-Creek near Likely, Cal., for 1905.

\begin{tabular}{|c|c|c|c|c|c|c|c|c|c|c|c|}
\hline Day. & Jan. & Feb. & Mar. & Apr. & May. & June. & July. & Aug. & Sept. & Oct. & Nov. \\
\hline 1. & 28 & 21 & 24 & 188 & 22 & 23 & 20 & 20 & 10 & 16 & 12 \\
\hline 2. & 28 & 20 & 24 & 38 & 22 & 23 & 20 & 20 & 9 & 15 & 12 \\
\hline $3 .$. & 25 & 20 & 24 & 30 & 22 & 24 & 20 & 20 & 9 & 14 & 12 \\
\hline $4 .$. & 25 & 20 & 24 & 30 & 22 & 24 & 19 & 20 & 13 & 14 & 14 \\
\hline 5. & 25 . & 21 & 24 & 30 & 23 & 57 & 19 & 20 & 13 & 14 & 14 \\
\hline 6. & 22 & 21 & 21 & 30 & 23 & 47 & 19 & 20 & 14 & 15 & 14 \\
\hline $7 \ldots$ & 22 & 21 & 21 & 26 & 23 & 43 & 18 & 20 & 14 & 15 & 14 \\
\hline $8 .$. & 22 & 22 & 21 & 26 & 32 & 52 & 18 & 20 & 13 & 16 & 14 \\
\hline $9 .$. & 22 & 22 & 19 & 26. & 26 & 52 & 16 & 20 & 13 & 16 & 15 \\
\hline $10 .$. & 22 & 22 & 19 & 26 & 42 & 32 & 16 & 20 & 12 & 16 & 15 \\
\hline $11 \ldots \ldots$. & 22 & 23 & 19 & 26 . & $\cdot 33$ & 32 & 15 & 20 & 12 & 17 & 15 \\
\hline $12 \ldots \ldots \ldots$ & 22 & 23 & 19 & 26 & 30 & 29 & 15 & 20 & 26 & 17 & 15 \\
\hline $13 .$. & 22 & 24 & 21 & 26 & 27 & 29 & 15 & 20 & 26 & 18 & 14 \\
\hline $14 \ldots \ldots$ & 22 & 24 & 24 & 26 & 27 & 26 & 15 & 11 & 26 & 18 & 14 \\
\hline 15. & 22 & 25 & 25 & 30 & 27 & 26 & 15 & 11 & 26 & 17 & 14 \\
\hline $16:$. & 22 & 26 & 25 & 30 & 26 & 26 & 15 & 11 & 26 & 16 & 14 \\
\hline $17 .$. & 25 & 26 & 63 & 30 & 26 & $26^{\circ}$ & 15 & 10 & 26 & 16 & 14 \\
\hline $18 \ldots \ldots \ldots$ & 25 & 26 & 38 & 26 & 26 & 26 & 15 & 10 & 25 & 15 & 14 \\
\hline $19 \ldots$ & 28 & 26 & 48 & 23 & 24 & 26 & 16 & 10 & 24 & 14 & 14 \\
\hline $20 .$. & 28 & 26 & 34 & 23 & 24 & 26 & 16 & 10 & 24 & 14 & \\
\hline $21 . \ldots \ldots \ldots \ldots \ldots \ldots \ldots$ & 28 & 26 & 48 & 23 & 24 & 25 & 16 & 10 & 23 & 13 & \\
\hline 22. & 25 & 26 & 48 & 23 & 23 & 25 & 16 & 10 & 22 & 14 & $\ldots \ldots$ \\
\hline $23 .$. & 22 & 25 & 34 & - 23 & 23 & 24 & 16 & 10 & 21 & 14 & $\therefore \cdots$ \\
\hline $24 \ldots$ & 22 & 25 & 30 & 23 & 21 & 24 & 16 & 10 & 21 & 13 & $\cdots \ldots$ \\
\hline $25 \ldots$ & 22 & 25 & 30 & 23 & 21 & 23 & 16 & 10 & 20. & 13 & ....... \\
\hline 26. & 22 & 25 & 38 & 21 & 21 & 23 & 17 & 10 & 20 & 12 & $\ldots .$. \\
\hline $27 \ldots$ & 22 & 25 & 69 & 21 & 21 & 22 & 17 & 10 & 19 & 12 & ...... \\
\hline $28 .$. & 22 & 25 & 38 & 21 & 21 & 22 & 17 & 10 & 18 & 11 & ....... \\
\hline $29 .$. & 22 & ... & 34 & 21 & 22 & 21 & 17 & 10 & 18 & 11 & ...... \\
\hline $30 \ldots$ & 22 & ..... & 38 & 21 & 22 & 21 & 17 & 10 & 17 & 10 & ....... \\
\hline $31 \ldots \ldots \ldots$ & 22 & ......... & 48 & & $\begin{array}{r}23 \\
-\quad-\end{array}$ & $\cdots$ & 20 & 10 & $\cdots \cdot \cdot$ & 10 & $\therefore$ \\
\hline
\end{tabular}

NoTE.-Daily discharge obtained by indirect method for shifting channel, as no measurements were made later than November 19. No estimate has been made for the remainder of the year. 
Estimated monthly discharge of West Valley Creek near Likely, Cal., for 1905.

\begin{tabular}{|c|c|c|c|c|}
\hline \multirow{2}{*}{ Month. } & \multicolumn{3}{|c|}{ Discharge in second-feet. } & \multirow{2}{*}{$\begin{array}{l}\text { Total in } \\
\text { acre-feet. }\end{array}$} \\
\hline & Maximum. & Minimum. & Mean. & \\
\hline January ......... & 28 & 22 & 23.5 & 1,445 \\
\hline February... & 26 & 20 & 23.6 & 1,311 \\
\hline March...... & 69 & 19 & 32.0 & 1,968 \\
\hline April........ & 188 & 21 & 31.2 & 1,856 \\
\hline May .......... & 42 & 21 & 24.8 . & 1,525 \\
\hline June.......... & 57 & 21 & 29.3 & $1,7.44$ \\
\hline July .......... & 20 & 15 & 16.8 & 1,033 \\
\hline August............................ & 20 & 10 & 14.3 & 879 \\
\hline September.................. & 26 & 9 & 18.7 & 1,113 \\
\hline October........................ & 18 & 10 & 14.4 & 885 \\
\hline 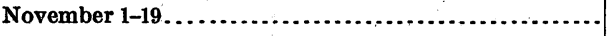 & 15 & 12 & 13.9 & .527 \\
\hline The period. & & & & 14,290 \\
\hline
\end{tabular}

\section{ASH OREEK AT ADIN, CAL.}

This station was established March 13, 1904, by J. S. Evans and William Busch. It was originally located one-fourth of a mile above the town of Adin. During the summer the closing of the waste gates in the dam at Adin interfered with the discharge at this point to such an extent that on August 15, 1904, the station was reestablished at i. point 100 feet below the wagon bridge in the town of Adin, Cal., which is about 500 feet below the dam.

The channel is straight for 200 feet above and 200 feet below the station. The left bank is high, but the right bank is subject to overflow from the side channel which, in flood diverts water from above the station. The bed of the stream is gravelly end not subject to change.

Discharge measurements are made from a suspension footbridge constr 1cted with onehalf inch cables.

The gage is a vertical plank fastened to a tree on the left bank of the s ream. During 1905 the gage was read once each day by $\mathrm{H}$. Williams.

A description of this station, with gage heights and discharged data, is contained in Water Supply Paper No. 134, United States Geological Survey. 
Discharge measurements of Ash Creek River at Adin, Cal., in 1905.

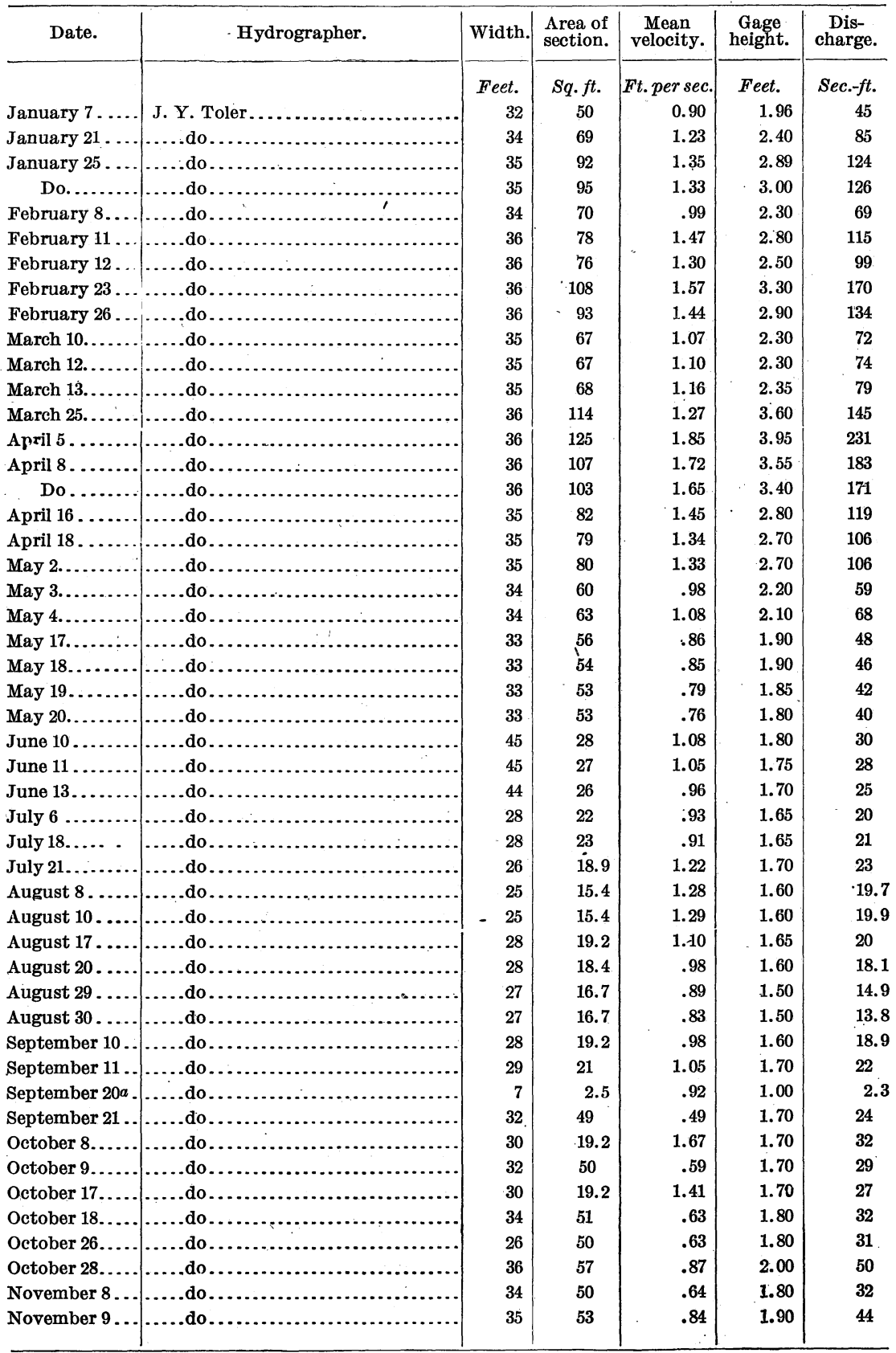


Daily gage height, in feet, of Ash Creek at Adin, Cal., for 1905.

\begin{tabular}{|c|c|c|c|c|c|c|c|c|c|c|c|c|}
\hline Day. & Jan. & Feb. & Mar. & Apr. & May. & June. & July. & Aug. & Sept. & Oct. & Nov. & Dec. \\
\hline $1 .$. & 2.5 & 3.4 & 2.5 & 4.1 & 2.3 & 1.9 & 1.7 & 1.5 & 1.6 & 1.7 & 1.9 & 1.8 \\
\hline $2 \ldots$ & 2.4 & 5.9 & 2.5 & 6.0 & $2: 2$ & 1.9 & 1.7 & 1.6 & 1.7 . & 1.7 & 1.9 & 1.8 \\
\hline 3.. & 2.2 & 3.5 & 2.5 & 6.0 & 2.1 & 1.9 & 1.7 & 1.6 & 1.7 & 1.7 & 1.9 & 1.8 \\
\hline $4 .$. & 1.9 & 3.4 & 2.5 & 4.6 & 2.1 & 1.9 & 1.6 & 1.6 & 1.7 & 1.7 & 1.9 & 1.8 \\
\hline 5.. & 1.9 & 3.4 & 2.5 & 4.6 & 2.1 & 1.9 & 1.5 & 1.6 & 1.7 & 1.7 & 1.9 & 1.8 \\
\hline $6 \ldots$ & 1.9 & 3.4 & 2.5 & 4.0 & 2.1 & $1: 8$ & 1.5 & 1.6 & 1.8 & 1.7 & 1.9 & 1.8 \\
\hline $7 \ldots$ & 1.9 & 3.4 & 2.5 & 3.7 & 2.1 & 1.8 & 1.5 & 1.6 & 1.8 & 1.7 & 1.9 & 1.8 \\
\hline 8... & 1.9 & 3.2 & 2.5 & 3.7 & 2.1 & 1.8 & 1.5 & 1.6 & 1.8 & 1.7 & 1.9 & 1.8 \\
\hline $9 .$. & 1.9 & 2.5 & 2.4 & 3.2 & 2.1 & 1.8 & 1.5 & 1.6 & 1.8 & 1.7 & 1.9 & 1.8 \\
\hline $10 .$. & 1.9 & 2.4 & 2.4 & 3.2 & 2.2 & 1.8 & 1.5 & 1.6 & 1.9 & 1.8 & 1.9 & 1.8 \\
\hline $11 .$. & 1.9 & 2.3 & 2.4 & 3.1 & 2.2 & 1.8 & 1.5 & 1.6 & 1.9 & 1.8 & 1.9 & 1.8 \\
\hline 12. & 1.9 & 2.3 & 2.4 & 3.1 & 2.2 & 1.8 & 1.5 & 1.6 & 1.8 & 1.9 & 1.9 & 1.8 \\
\hline 3.. & 2.0 & 2.2 & 2.4 & 3.0 & 2.2 & 1.8 & 1.5 & 1.6 & 1.8 & 1.9 & 1.9 & 1.8 \\
\hline $4 .$. & 2.0 & 2.1 & 2.4 & 3.0 & 2.2 & 1.8 & 1.5 & 1.6 & 1.8 & 1.9 & 1.9 & 1.8 \\
\hline 5... & 2.0 & 2.1 & 2.4 & 3.0 & 2.1 & 1.8 & 1.5 & 1.6 & 1.8 & 1.9 & 1.9 & 1.8 \\
\hline 6... & 4.0 & 2.1 & 2.4 & 3.0 & 2.1 & 1.8 & 1.5 & 1.6 & 1.8 & 1.9 & 1.8 & 1.8 \\
\hline $17 \ldots$ & 2.5 & 2.2 & 3.1 & 2.8 & 2.1 & 1.8 & 1.5 & 1.6 & 1.7 & 1.7 & 1.8 & 1.8 \\
\hline 18.. & 2.0 & 3.1 & 5.1 & 2.8 & .2 .2 & 1.8 & 1.5 & 1.6 & 1.7 & 1.7 & 1.8 & 1.8 \\
\hline 19.. & 2.8 & 5.9 & 6.2 & 2.8 & 2.2 & 1.8 & 1.5 & 1.6 & 1.7 & 1.7 & 1.8 & 1.8 \\
\hline 20 .. & 2.4 & 6.0 & 4.3 & 2.8 & 2.3 & 1.8 & 1.5 & 1.6 & 1.6 & 1.7 & 1.8 & 1.8 \\
\hline 1.. & 2.4 & 3.4 & 4.6 & 2.8 & 2.3 & 1.7 & 1.5 & 1.6 & 1.6 & 1.7 & 1.8 & 1.8 \\
\hline 2.. & 4.1 & 3.3 & 4.6 & 2.8 & 2.3 & 1.7 & 1.5 & 1.6 & 1.6 & 1.7 & 1.8 & 1.8 \\
\hline 23... & 4.6 & 3.3 & 3.8 & 2.7 & 2.1 & 1.7 & 1.5 & 1.6 & 1.6 & 1.7 . & 1.8 & 1.8 \\
\hline $24 \ldots$ & 4.6 & 2.6 & 5.4 & 2.7 & 2.1 & 1.7 & 1.5 & 1.6 & $1: 6$ & 1.7 & 1.8 & 1.8 \\
\hline $25 .$. & 3.0 & 2.6 & 3.6 & 2.7 & 1.9 & 1.7 & 1.5 & 1.7 & 1.6 & 1.7 & 1.8 & 1.8 \\
\hline $26 .$. & 2.4 & 2.3 & 9.0 & 2.7 & 1.9 & 1.7 & 1.5 & 1.7 & 1.6 & 1.7 & 1.8 & 1.8 \\
\hline 27. & 2.3 & 2.3 & 4.6 & 2.7 & 1.9 & 1.7 & 1.5 & 1.7 & 1.6 & 1.8 & 1.8. & 1.8 \\
\hline $3 .$. & 2.3 & 2.5 & 4.1 & 2.8 & 1.9 & 1.7 & 1.5 & 1.6 & 1.6 & 1.8 & 1.8 & 1.8 \\
\hline 29. & 2.3 & & 4.2 & 2.8 & 1.9 & 1.7 & 1.5 & 1.6 & 1.6 & 1.8 & 1.8 & 1.8 \\
\hline 30.. & 4.5 & $\cdots$ & 4.2 & 2.8 & 1.9 & 1.7 & 1.5 & 1.6 & 1.6 & 1.8 & 1.8 & 1.8 \\
\hline 31. & 4.5 & 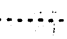 & 4.1 & & 1.9 & & 1.5 & 1.6 & $\cdots$ & 1.9 & & 1.8 \\
\hline
\end{tabular}

Station rating table for Ash Creek at Adin, Cal., from January 1 to December 31, 1905.

\begin{tabular}{|c|c|c|c|c|c|c|c|}
\hline $\begin{array}{c}\text { Gage } \\
\text { height. }\end{array}$ & Discharge. & $\begin{array}{c}\text { Gage } \\
\text { height. }\end{array}$ & Discharge. & $\begin{array}{c}\text { Gage } \\
\text { height. }\end{array}$ & Discharge. & $\begin{array}{c}\text { Gage } \\
\text { height. }\end{array}$ & Discharge. \\
\hline Feet. & Second-feet. & Feet. & Second-feet. & Feet. & Second-feet. & Feet. & Second-feet. \\
\hline 1.50 & 14 & 2.60 & 97 & 3. 70 & 205 & 4.80 & 342 \\
\hline : 1.60 & 20 & 2. 70 & 106 & 3. 80 & 216. & 4.90 & 356 \\
\hline 1.70 & 27 & 2.80 & 115 & 3.90 & 227 & 5.00 & 370 \\
\hline 1.80 & 34 & 2.90 & 124 & 4.00 & 238 & 5. 20 & 400 \\
\hline 1.90 & 41 & 3.00 & 134 & 4.10 & 250 & 5.40 & $430^{\circ}$ \\
\hline 2.00 & 48 & 3. 10 & 144 & 4.20 & 262 & 5.60 & 462 \\
\hline 2.10 & 56 & 3.20 & 154 & 4.30 & 274 & 5.80 & 494 \\
\hline 2.20 & 64 & 3.30 & 164 & 4.40 & 287 & 6.00 & .526 \\
\hline 2.30 & 72 & 3.40 & 174 & 4.50 & 300 & 6.20 & 560 \\
\hline 2.40 & 80 & 3.50 & 184 & 4.60 & 314 & & \\
\hline 2.50 & 88 & 3.60 & 194 & 4. 70 & 328 & $t$ & \\
\hline
\end{tabular}

NotE.-The above table is applicable only for open-channel conditions. It is based on 49 discharge measurements made during 1905. It is well defined between gage heights 1.5 feet and 4 feet. Above 4 feet the table is a rough approximation. 
Estimated monthly discharge of Ash Creek at Adin, Cal., for 1905.

[Drainage area, 260 square miles.]

\begin{tabular}{|c|c|c|c|c|c|c|}
\hline \multirow[b]{2}{*}{ Month. } & \multicolumn{3}{|c|}{ Discharge in second-feet. } & \multirow[b]{2}{*}{$\begin{array}{l}\text { Total in } \\
\text { acre-feet. }\end{array}$} & \multicolumn{2}{|c|}{ Run-off. } \\
\hline & Maximum. & Minimum. & Mean. & & $\begin{array}{l}\text { Second-feet } \\
\text { per square } \\
\text { mile. }\end{array}$ & $\begin{array}{l}\text { Depth } \\
\text { in inches. }\end{array}$ \\
\hline January..... & 314 & 41 & 107 & 6,579 & 0.412 & 0.475 \\
\hline February ......... & 526 & 56 & 158 & 8,775 & .608 & .633 \\
\hline March......... & $-1,120$ & 80 & 214 & 13,160 & .823 & .949 \\
\hline April............. & 526 & 106 & 176 & 10,470 & .677 & .755 \\
\hline May ............... & 72 & 41 & 56.7 & 3,486 & .218 & .251 \\
\hline June................ & 41 & 27 & 32.8 & 1,952 & .126 & .141 \\
\hline July . . . .... & 27 & 14 & 15.5 & 953 & .060 & .069 \\
\hline August...... & 27 & 14 & 20.5 & 1,260 & .079 & .091 \\
\hline September....... & 41 & 20 & 27.2 & 1,618 & .105 & .117 \\
\hline October $\ldots \ldots \ldots \ldots$ & 41 & 27 & 31.1 & 1,912 & .120 & .138 \\
\hline November............... & 41 & 34 & 37.5 & 2,231 & .144 & .161 \\
\hline December...$\ldots \ldots \ldots \ldots$ & 34 & 34 & 34 & 2,091 & .131 & .151 \\
\hline The year. & - 1,120 & 14 & 75.9 & 54,490 & .292 & 3.93 \\
\hline
\end{tabular}

\section{MCCLOUD RIVER NEAR GREGORY, CAL.}

This station was established March 23, 1902, in cooperation with the McCloud River Electric Company. It is located at John's Camp, near Hirze Mountain, 14 miles east of Gregory Post-office, Cal. Baird Station, on the Southern Pacific Railroad, is just across Sacramento River from Gregory.

The channel is straight for 300 feet above and 600 feet below the gaging station. The current is swift at all stages. The banks are high and wooded and not liable to overflow, The bed is composed of limestone on the sides, with some large river gravel and bowlders in the center of the channel.

Measurements are made from a car suspended from a cable. The initial point for soundings is at the gage rod on the right bank of the stream. A line is placed 150 feet below the cable, marking a course for float measurements during floods.

The gage rod is a timber nailed and wired to a tree on the right bank. The bench mark is a point marked with a ring of white paint on the top of a bowlder 10 feet upstream from the 10-foot mark on the cable; elevation, 21.19 feet above the datum of the gage.

Information in regard to this station is contained in Water-Supply Papers Nos. 100 and 134 of the United States Geological Survey. 
Discharge measurements of McCloud River near Gregory, Cal., in 1905.

\begin{tabular}{|c|c|c|c|c|c|c|}
\hline Date. & Hydrographer. & Width. & $\begin{array}{l}\text { Area of } \\
\text { section. }\end{array}$ & $\begin{array}{c}\text { Mean } \\
\text { velocity. }\end{array}$ & $\begin{array}{c}\text { Gage } \\
\text { height. }\end{array}$ & $\begin{array}{c}\text { Dis- } \\
\text { charge. }\end{array}$ \\
\hline February 21 . & o. W. Peterson & $\begin{array}{r}\text { Feet. } \\
114\end{array}$ & $\begin{array}{r}S q . f t . \\
830\end{array}$ & $\begin{array}{r}\text { Ft.per sec } \\
6.43\end{array}$ & $\begin{array}{r}\text { Feet. } \\
4.58\end{array}$ & $\begin{array}{c}\text { Sec. } f t . \\
\quad 5,337\end{array}$ \\
\hline February 22 . & .....do.,$\ldots$ & 114 & 759 & 5.47 & 3.95 & 4,155 \\
\hline February 22 . & .....do. & 114 & 766 & 5.38 & 3.98 & 4,120 \\
\hline August 6 . & Peterson \& Lee. & 103 & 543 & 2.55 & 1.63 & 1,386 \\
\hline
\end{tabular}

Daily gage height, in feet, of McCloud River near Gregory, Cal., for 1905.

\begin{tabular}{|c|c|c|c|c|c|c|c|c|c|c|c|c|}
\hline De & Jan. & Feb. & Mar. & Apr. & May. & June. & July. & Aug. & Sept. & Oct. & Nov. & Dec \\
\hline 1. & .9 & 3.85 & 2.9 & 3.4 & 8 & .1 & 1.8 & 1.65 & 1.55 & 1.55 & 1.5 & 1.55 \\
\hline 2. & 2.6 & 4.5 & 2. & 3.3 & .7 & 2 & 1.8 & 65 & 1.55 & 55 & 1.5 & 1.55 \\
\hline 3. & 2.45 & 3.85 & 2. & 3.25 & 2.65 & 2.1 & 1.8 & .65 & 1.55 & .55 & .5 & 1.55 \\
\hline $4 .$. & 2.3 & 3.7 & 2.75 & 3.2 & 2.6 & 2.1 & 1.8 & 1.65 & 1.55 & .55 & 1.5 & 1.55 \\
\hline 5. & 2.2 & 3.6 & 2. & 3. & 2.5. & 2.1 & 1.8 & 6 & 1.55 & .55 & 1.5 & 1.55 \\
\hline $6 .$. & $2: 2$ & 3.6 & 2. & 3 & 2.6 & 2.05 & 1.8 & 65 & 1.55 & .55 & .5 & 1.55 \\
\hline 7. & 2.15 & 3. & 2. & 2. & 2.7 & 05 & 1.8 & $1: 6$ & 55 & 1.6 & .5 & 1.55 \\
\hline 8. & 2.1 & 3. & 2.65 & 2.85 & 2.8 & .0 & 1.8 & 1.6 & 55 & 55 & 1.5 & 1.55 \\
\hline 9. & 0 & 3 & 2. & 2.8 & 2.7 & 2.0 & 8 & 1. & 55 & 55 & .5 & 1.55 \\
\hline & 2.0 & 3. & & 2.7 & 2.7 & 0 & 1.8 & & 55 & 55 & .5 & 1.55 \\
\hline . & 2.0 & 3. & & 2 & 2.7 & 0 & 8 & & .55 & .55 & 1.5 & 1.55 \\
\hline . & 1.95 & 2.9 & & 2 & 2.6 & 2.0 & 1.75 & & .55 & .55 & .5 & 1.55 \\
\hline & 2.0 & 2.7 & & 2.6 & 2.6 . & 9 & 1.75 & 1. & .55 & 55 & 1.5 & 1.5 \\
\hline 1. & 3.1 & 2. & & 2.55 & 2.55 & 1.95 & 1.75 & 1.6 & 1.55 & 55 & 1.5 & 1.55 \\
\hline 5. & 25 & 2. & 5.25 & 2.6 & 2.5 & 1.9 & 1.75 & $1.6=$ & .55 & 55 & 1.5 & 1.55 \\
\hline 6. & 3.3 & 2. & 4. & 2.8 & 2.5 & 1.9 & 1. & 1.6 & 1.55 & .55 & 1.5 & 1.55 \\
\hline 7. & 3.1. & 2. & 4.15 & 2 & 2.5 & 1.9 & .1 .7 & 1.6 & .55 & .55 & .5 & 1.55 \\
\hline 8. & 3.0 & 2. & 4. & 2.75 & 2.5 & 1.9 & 1.7 & & 55 & 55 & .55 & 1.55 \\
\hline & 3.0 & 3 & & & 2.4 & & 1.7 & & 55 & 55 & 5 & 1.6 \\
\hline & 2 & 5. & & 2. & 2.4 & & 1.7 & & 65 & 55 & 65 & 1. \\
\hline & 4.1 & 4. & & 2 & 3 & 1. & 1.7 & 1. & 1.55 & .55 & 1.55 & 1.55 \\
\hline 2. & 8.05 & 3. & 4. & 2.8 & 2.25 & 1.85 & 1.7 & 1.6 & 1.55 & .55 & 1.55 & 1.55 \\
\hline 3. & 7.4 & 3. & 4. 35 & 2. & 3 & 1.85 & 1.7 & & .55 & 55 & .55 & 1.55 \\
\hline 24 & 6.2 & 3. & 4.0 & 2. & 2.2 & 1.85 & 1.7 & & 55 & 55 & .5 & 1.55 \\
\hline 5. & 6.6 & 3. & 3. & 2. & 2.2 & 15 & 1.7 & 1.6 & 55 & 55 & .55 & 1.55 \\
\hline & 5 & 3 & 4. & 2. & 2.2 & 5 & 5 & & 55 & 55 & .55 & 1.55 \\
\hline & 4.2 & 3. & & & & & & & 55 & 5 & 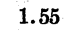 & 1.55 \\
\hline & 3.8 & 2.9 & 3. & 2 & 2.2 & 1. & 1. & & .55 & 55 & .55 & 1.55 \\
\hline & 3.45 & & 3 & 2 & 2.2 & 1. & 1.65 & & 1.55 & 55 & 1.55 & 1.55 \\
\hline & 3.25 & & 3.6 & 2.7 & 2.2 & 1.8 & 1.65 & 1.55 & 1.55 & 1.55 & 1.55 & 1.55 \\
\hline & 3.25 & & 3.6 & & 2.1 & & 1.65 & 1.55 & & 1.5 & & 1. \\
\hline
\end{tabular}


Station rating table for $M$ cCloud River near Gregory, Cal., from September 23, 1902, to December $31,1905$.

\begin{tabular}{|r|r||r|r||r|r|r|r|}
\hline $\begin{array}{c}\text { Gage } \\
\text { height. }\end{array}$ & Discharge. & $\begin{array}{c}\text { Gage } \\
\text { height. }\end{array}$ & Discharge. & $\begin{array}{c}\text { Gage } \\
\text { height. }\end{array}$ & Discharge. & $\begin{array}{r}\text { Gage } \\
\text { height. }\end{array}$ & Dischargə. \\
\cline { 1 - 5 } Feet. & Second-feet. & Feet. & Second-feet. & Feet. & Second-feet. & Feet. & Second-feet. \\
1.00 & 1,090 & 2.40 & 2,042 & 3.80 & 3,860 & 5.40 & 7,170 \\
1.10 & 1,132 & 2.50 & 2,140 & 3.90 & 4,030 & 5.60 & 7,630 \\
1.20 & 1,178 & 2.60 & 2,242 & 4.00 & 4,210 & 5.80 & 8,110 \\
1.30 & 1,228 & 2.70 & 2,349 & 4.10 & 4,395 & 6.00 & 8,600 \\
1.40 & 1,282 & 2.80 & 2,461 & 4.20 & 4,585 & 6.20 & 9,120 \\
1.50 & 1,340 & 2.90 & 2,578 & 4.30 & 4,785 & 6.40 & 9,650 \\
1.60 & 1,402 & 3.00 & 2,700 & 4.40 & 4,990 & 6.60 & 10,190 \\
1.70 & 1,468 & 3.10 & 2,825 & 4.50 & 5,200 & 6.80 & 10,740 \\
1.80 & 1,538 & 3.20 & 2,955 & 4.60 & 5,410 & 7.00 & 11,300 \\
1.90 & 1,612 & 3.30 & 3,090 & 4.70 & 5,625 & 7.20 & 11,870 \\
2.00 & 1,690 & 3.40 & 3,230 & 4.80 & 5,840 & 7.40 & 12,450 \\
2.10 & 1,772 & 3.50 & 3,380 & 4.90 & 6,060 & 7.60 & 13,040 \\
2.20 & 1,858 & 3.60 & 3,535 & 5.00 & 6,280 & 7.80 & 13,640 \\
2.30 & 1,948 & 3.70 & 3,695 & 5.20 & 6,720 & 8.00 & 14,250 \\
& & & & & & & \\
\hline
\end{tabular}

Note.-The above table is applicable only for open-channel conditions. It is based on 10 discharge measurements made during 1902-1905. It is fairly well defined between gage heights 1.5 feet and 4.6 feet. The table has been extended beyond these limits. Above 8 feet the discharge is a rough approximation. 
Estimated monthly discharge of McCloud River near Gregory, Cal., for 1902-1905.

[Drainage, 608 square miles.]

\begin{tabular}{|c|c|c|c|c|c|c|}
\hline \multirow[b]{2}{*}{ Month. } & \multicolumn{3}{|c|}{ Discharge in second-feet. } & \multirow[b]{2}{*}{$\begin{array}{l}\text { Total in } \\
\text { acre-feet. }\end{array}$} & \multicolumn{2}{|c|}{ Run-off. } \\
\hline & Maximum. & Minimum. & Mean. & & $\begin{array}{l}\text { Second-feet } \\
\text { per square } \\
\text { mile. }\end{array}$ & $\begin{array}{c}\text { Depth } \\
\text { in inches. } \\
-\end{array}$ \\
\hline $\begin{array}{cc}1902 \\
\text { September } 23 \text { to } 30\end{array}$ & 1,340 & 1,282 & 1,333 & 21,150 & 2.19 & 0.651 \\
\hline October.. & 2,140 & 1,228 & 1,372 & 84,360 & 2.26 & 2.61 \\
\hline November. & 12,740 & 1,282 & 2,799 & 166,600 & 4.60 & 5.13 \\
\hline December. . & 6,060 & 1,538 & 2,576 & 158,400 & 4.24 & 4.89 \\
\hline 1903 & & & & & & \\
\hline January ......... & $13 ; 340$ & 1,538 & 2,847 & 175,100 & 4.68 & 5.40 \\
\hline February... & 2,578 & 1,772 & 2,046 & 113,600 & 3.37 & 3.51 \\
\hline March...... & 11,870 & 2,042 & 3,723 & 228,900 & 6.12 & 7.06 \\
\hline April....... & 6,060 & 2,242 & 2,819 & 167,700 & 4.64 & 5.18 \\
\hline May ....... & 2,242 & 1,612 & 1,913 & 117,600 & 3.15 & 3.63 \\
\hline June....... & 1,690 & 1,468 & 1,545 & 91,930 & 2.54 & 2.83 \\
\hline July . . ..... & 1,468 & 1,340 & 1,373 & 84,420 & 2.26 & 2.61 \\
\hline Aúgust.... & 1,352 & 1,311 & 1,323 & 81,350 & 2.18 & 2.51 \\
\hline September. & 1,311 & 1,282 & 1,305 & 77,650 & 2.15 & 2.40 \\
\hline October.... & 1,435 & 1,282 & 1,317 & 80,980 & 2.17 & 2.50 \\
\hline November. . & 14,560 & 1,282 & 3,475 & 206,800 & 5. 72 & 6.38 \\
\hline December.. & 2,263 & 1,455 & 1,829 & 112,500 & 3.01 & 3.47 \\
\hline The y & 14,560 & 1,282 & 2,126 & $1,539,000$ & 3.50 & 47.48 \\
\hline 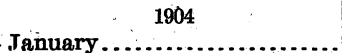 & 2,042 & 1,503 & 1,652 & 101,600 & 2.72 & 3.14 \\
\hline February......... & 39,500 & 1,468 & 6,153 & 353,900 & 10.12 & 10.92 \\
\hline March..... & 41,000 & 3,380 & $9, \mathbf{3 1 0}$ & 572,400 & 15.31 & 17.65 \\
\hline April....... & 7,285 & 4,120 & 5,468 & 325,400 & 8.99 & 10.03 \\
\hline May ........ & 4,490 & 3,090 & 3,756 & 230,900 & 6.18 & 7.12 \\
\hline June........ & 2,890 & 1,858 & 2,218 & 132,000 & 3.65 & 4.07 \\
\hline July ....... & 1,858 & 1,612 & 1,746 & 107,400 & 2.87 & 3.31 \\
\hline August.... & 1,612 & 1,503 & 1,572 & 96,660 & 2.59 & 2.99 \\
\hline September. & 1,815 & 1,468 & 1,512 & 89,970 & 2.49 & 2.78 \\
\hline October.. & 9,785 & 1,503 & 2,697 & 165,800 & 4.44 & 5.12 \\
\hline November. & $2 ; 140$ & 1,503 & 1,640 & 97,590 & 2.70 & 3.01 \\
\hline December. & 4,585 & 1,575 & 1,844 & 113,400 & 3.03 & 3.49 \\
\hline The year & 41,000 & 1,468 & 3,297 & $2,387,000$. & 5.42 & 73.63 \\
\hline January 1905 . & 14,400 & 1,651 & 854 & 237,000 & 6.34 & 7.31 \\
\hline February ......... & 6,945 & 2,042 & 3,284 & 182,400 & 5.40 & 5.62 \\
\hline March....... & 10,740 & 2,242 & 4,062 & 249,800 & 6.68 & 7.70 \\
\hline April...... & 3,230 & 2,191 & 2,490 & 148,200 & 4.10 & 4.57 \\
\hline May....... & 2,461 & 1,772 & 2,112 & 129,900 & 3.47 & 4.00 \\
\hline June...... & 1,858 & 1,538 & 1,648 & 98,060 & 2.71 & 3.02 \\
\hline July ....... & 1,538 & 1,435 & 1,491 & $\backslash 91,680$ & 2.45 & 2.82 \\
\hline August......... & 1,435 & 1,371 & 1,402 & 86,210 & 2.31 & 2.66 \\
\hline September....... & 1,371 & 1,371 & 1,371 & 81,580 & 2.25 & 2.51 \\
\hline October......... & 1,402 & 1,340 & 1,371 & 84,300 & 2.25 & 2.59 \\
\hline November. & 1,371 & 1,340 & 1,352 & 80,450 & 2.22 & 2.48 \\
\hline December.... & 1,402 & 1,371 & 1,372 . & 84,360 & 2.26 & 2.61 \\
\hline The year.. & 14,400 & 1,340 & 2,151 & $1,554,000$ & 3.54 & 47.89 \\
\hline
\end{tabular}




\section{MISCELLANEOUS MEASUREMENTS IN PIT RIVIR DRATNAGE BASTN.}

The following miscellaneous measurements were made in the Pit River drainage basin during 1905:

Cottonwood Creek, near Lakeview, Oreg.-This stream is tributary to Goose Lake. A measurement was made June 28,1905 , by S. G. Bennett and J. Y. Toler, one-half mile below Wilshire's sawmill and 10 miles above Lakeview, Oreg.

Width, 12.7 feet; area, 4.7 square feet; mean velocity, 1.55 feet per second; discharge, 7.3 second-feet.

Corporation ditch, near Likely, Cal.-This ditch diverts water from the South Fork of Pit River above Likely, Cal. The following measurements were made below head-gate, 2 miles east of Likely, Cal.:

June 7: Width, 5.7 feet; area, 16.3 square feet; mean velocity, 1.42 feet per second; discharge, 23 second-feet.

June 20: Width, 5.6 feet; area, 16.6 square feet; mean velocity, 1.41 feet per second; discharge, 24 second-feet.

September 6: Width, 7.0 feet; area, 19.9 square feet; mean velocity, 1.46 feet per second; discharge, 29 second-feet.

Duke's ditch, near Likely, Cal.-This ditch diverts water from the South Fork of Pit River. The following measurements were made in flume, 400 yards below head-gate, by John Y. Toler:

June 7: Width, 3 feet; area, 2.88 square feet; mean velocity, 4.83 feet per second; discharge, 13.9 second-feet.

June 20: Width, 3 feet; area, 2.3 square feet; mean velocity, 4.25 feet per second; discharge, 9.8 second-feet.

Fitz Hugh Creek, near Alturaš, Cal.-This creek is tributary to Pit River. The following measurements were made at Doten's ranch, 10 miles south of Alturas, by John Y. Toler:

July 2: Width, 8.5 feet; area, 2.8 square feet; mean velocity, 0.64 foot per second; discharge, 1.8 second-feet.

July 25: Width, 8.4 feet; area, 2.7 square feet; mean'velocity, 0.52 foot per second; discharge, 1.4 second-feet.

September 7: 'Width, 7.0 feet; area, 2.8 square feet; mean velocity, 0.64 foot per second; discharge, 1.8 second-feet.

September 16: Width, 8 feet; area, 3.6 square feet; meøn velocity, 0.78 foot per second; discharge, 2.8 second-feet.

Ganstad's ditch, near Likely, Cal.-This ditch diverts water from the South Fork of the Pit River. The following measurements were made at head-gate, 2 miles east of Likely, Cal., by John Y. Toler:

June 8: Width, 6.6 feet; area, 10.5 square feet; mean velocity, 0.66 foot per second; discharge, 6.9 second-feet.

July 2: Width, 6.6 feet; area, 10.5 square feet; mean velocity, 0.83 foot per second; discharge, 8.7 second-feet.

Gooch's ditch, near Lookout, Cal.-This ditch diverts water from Pit River. A measurement was made 250 yards above bridge at Lookout on June 13 by John Y. Toler.

Width, 14.5 feet; area, 25 square feet; mean velocity, 0.48 foot per second; discharge; 12 second-feet.

E. Lauer and Son's ditch near Alturas, Cal.-This ditch diverts water from Pit River above the town of Alturas, Cal. A measurement was made in flume at XL ranch, 9 miles north of the town of Alturas, Cal., on May 23, by Jno. Y. Toler.

Width, 4.1 feet; area, 0.89 square foot; mean velocity, 1.60 feet per second; discharge, 1.42 second-feet.

Pine Creek near Alturas, Cal.- This stream is a tributary of South Fork of Pit River. The following measurements were made in flume 100 feet below GGibbins \& Mulkey's power house, 7 miles east of the town of Alturas, Cal. 
August 11: Width, 2.2 feet; area, 2.8 square feet; mean velocity, 4.43 feet per second; discharge, 12.4 second-feet.

August 22: Width, 2.15 feet; area, 2.6 square feet; mean vclocity, 4.35 feet per second; discharge, 11.3 second-feet.

October 1: Width, 2.3 feet; area, 3.1 square feet; mean velocity, 3.94 feet per second; discharge, 12.2 second-feet.

Pit River at Lookout, Cal.-The following measurements were made during 1905 at bridge on county road east of town of Lookout, Cal.

June 13: Width, 87 feet; area, 217 square feet; mean velocity, 0.71 foot per second; discharge, 155 second-feet.

July 7: Width, 67 feet; area, 112 square feet; mean velocity, 0.51 foot per second; discharge, 57 second-feet.

July 20: Width, 40 feet; area, 71 square feet; mean velocity, 0.41 foot per second; discharge, 29 second-feet.

August 19: Width, 8 feet; area, 4.8 square feet; mean velocity, 2.06 feet per second; discharge, 9.9 second-feet.

Rush Creek near Adin, Cal.-This stream is a tributary to Ash Creek. The following measurements were made in canyon above upper end of Round Valley north of the town of Alturas, Cal.

July 18: Width, 13 feet; area, 9.7 square feet; mean velocity, 0.56 foot per second; discharge, 5.6 second-feet.

September 22: Width, 9 feet; area, 5.0 square feet; mean velocity, 1.16 feet per second; discharge, 5.8 second-feet.

Von Loam's ditch near.Likely, Cal.-This ditch diverts water from the South Fork of Pit River. The following measurements were made below head-gate, 2 miles east of the town of Likely, Cal.

June 8: Width, 7.8 feet; area, 16.9 square feet; mean velocity, 2.09 feet per second; discharge, 35 second-feet.

June 20: Width, 8.5 feet; area, 14.9 square feet; mean velocity, 1.68 feet per second; discharge, 25 second-feet.

Willow Creek near Adin, Cal.-This stream is tributary to Ash Creek. The following measurements were made at bridge on county road, 5 miles south of the town of Adin, Cal.

July 19: Width, 8 feet; area, 5.2 square feet; mean velocity, 1.09 feet per second; discharge, 5.7 second-feet.

September 21: Width, 8 feet; area, 4.4 square feet; mean velocity, 1.00 foot per second; discharge, 4.4 second-feet.

\section{STONY CREEK DRAINAGE BASIN.}

\section{DESCRIPTION OF BASIN.}

Stony Creek drains a portion of the Coast Range and flowing in a northerly direction discharges its waters into the Sacramento River near Orland, Cal. It has numerous tributaries, all of which are torrential in their character. The formation on the higher elevations is of granite with good soil covering and is heavily timbered. In the lower portion of the drainage basin the formation is shale, sandstone, and conglomerate, with heavy growth of brush and grass. This portion of the basin is used extensively for pasturage, the soil being heavy, it packs readily, producing a large per cent of run-off. The mean average rainfall on the higher elevation is about 40 inches; while on the lower reaches it is 20 inches. The precipitation is almost wholly in the form of rain, with some snow on the upper reaches which soon melts and only adds to the flood discharge.

The gaging station on this stream is located near the point where it emerges from the foothills and enters the Sacramento Valley. 
STONY CREEK NEAR FRUTO, CAI.

This station was established on January 30, 1901, by Burt Cole. It is located at Julian's . ranch, 7 miles northwest of Fruto, and $1 \frac{3}{4}$ miles above the proposed mill-site dam.

The channel is straight for 200 feet above and below the cable. The current is very swift at high water and sluggish at low water. Neither bank is subject to overflow, but at high stages of the creek the water spreads to the right for several hundred feet. The bed of the stream is of gravel and is subject to some change.

Discharge measurements are made from a car and cable. The initial point for soundings is the eyebolt on the left bank to which the cable is attached.

The gage is in two sections. During 1905 the gage was read twice each day by W. H. Julian. The bench mark is the head of an iron bolt set in the rock near the upper gage; elevation, 14.00 feet above the datum of the gage.

Information in regard to this station is contained in Water-Supply Papers Nos. 81, 85, 100, and 134 of the United States Geological Survey.

Discharge measurements of Stony Creek near Fruto, Cal., in 1905.

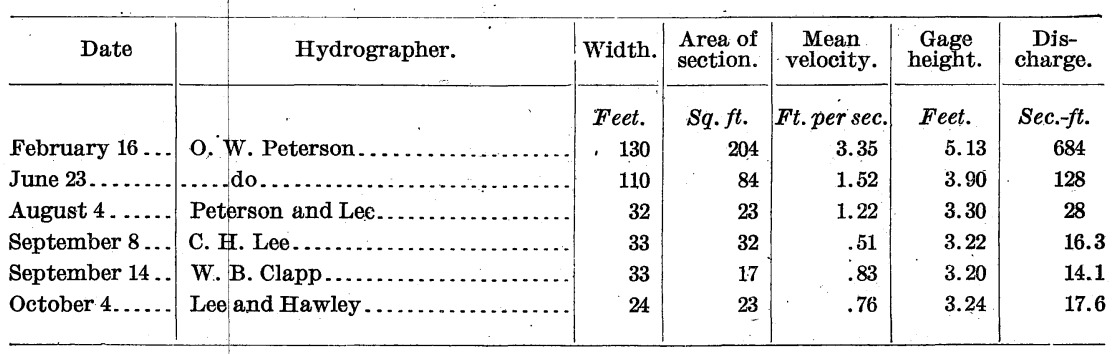

IRR $177-06-11$ 
Daily gage height, in feet, of Stony Creek near Fruto, Cal., for 1905.

\begin{tabular}{|c|c|c|c|c|c|c|c|c|c|c|c|c|}
\hline Day. " & Jan. & Feb. & Mar. & Apr. & May. & June. & July. & Aúg. & Sept. & Oct. & Nov. & Dec. \\
\hline 1.. & 7.0 & 10.0 & 5.2 & 6.1 & 4.7 & 4.6 & 3.7 & 3.2 & 3.1 & 3.2 & 3.3 & 3.6 \\
\hline 2. & 6.8 & 8.75 & 5.2 & 6.0 & -5.0 & 4.5 & 3.6 & 3.2 & 3.1 & 3.2 & 3.3 & 3.6 \\
\hline 3.. & 6.6 & 7.35 & 5.1 & 6.0 & 5.0 & 4.5 & 3.6 & 3.2 & 3.2 & 3.2 & 3.3 & 3.6 \\
\hline $4 .$. & 6.3 & 6.7 & 5.1 & 5.9 & 5.0 & 4.6 & 3.6 & 3.2 & 3.2 & 3.2 & 3.3 & 3.6 \\
\hline 5. & 6.2 & 6.2 & 5.0 & 5.9 & 4.9 & 4.5 & 3.6 & 3.2 & 3.2 & 3.2 & 3.3 & 3.6 \\
\hline $6 \ldots$ & 6.1 & 6.0 & 5.0 & 5.8 & 4.8 & 4.5 & 3.5 & 3.2 & 3.2 & 3.2 & 3.3 & 3.6 \\
\hline $7 .$. & 5.0 & 5.8 & 5.0 & 5.8 & 6.3 & 4. 4 & 3.5 & 3.2 & 3.2 & 3.2 & 3.3 & 3.6 \\
\hline 8... & 4.9 & 5.6 & 4.9 & 5.7 & 5.75 & 4.4 & 3.5 & 3.2 & 3.2 & 3.2 & 3.3 & .3. 6 \\
\hline $9 .$. & 4.9 & 5.5 & 4.9 & 5.6 & 5.5 & 4.3 & 3.5 & 3.2 & 3.2 & 3.2 & 3.3 & 3.6 \\
\hline $0 .$. & 4.8 & 5.4 & 4.9 . & 5.5 & 5.5 & 4.3 & 3.5 & 3.2 & 3.2 & 3.2 & 3.3 & 3.6 \\
\hline 1... & 4.8 & 5.4 & 4.8 & 5.4 & 5.4 & 4.2 & 3.5 & 3.2 & 3.2 & 3.2 & 3.3 & 3.6 \\
\hline $2 .$. & 4.8 & 5.3 & 6.4 & 5.3 & 5.4 & 4.2 & 3.4 & 3.2 & 3.2 & 3.2 & 3.3 & 3.6 \\
\hline $13 .$. & 4.7 & 5.3 & 8.25 & 5.3 & 5.3 & 4.2 & 3.4 & 3.2 & 3.2 & 3.2 & 3.3 & 3.6 \\
\hline 4.. & 9.5 & 5.2 & 7.75 & 5.2 & 5.3 & 4. 1 & 3.4 & 3.2 & 3.2 & 3.2 & 3.3 & 3.6 \\
\hline $5 \ldots$ & 7.75 & 5.2 & 8.5 & 5.2 & 5.2 & 4.1 & 3.4 & 3.2 & 3.2 & 3.2 & 3.3 & 3.6 \\
\hline 16. & 8.5 & 5.1 & 8.5 & 5.3 & 5.2 & 4.1 & 3.3 & 3.2 & 3.2 & 3.2 & 3.3 & 3.6 \\
\hline $17 .$. & 7.8 & 5.5 & 7.7 & 5.3 & 5.1 & 4.1 & 3.3 & 3.2 & 3.2 & 3.2 & 3.3 & 3.6 \\
\hline $18 \ldots$ & 7.7 & 5.8 & 7.15 & 5.3. & 5.0 & 4.1 & 3.3 & 3.2 & 3.2 & 3.2 & 3.3 & 3.6 \\
\hline $19 \ldots$ & 7.4 & 5.7 & 9.25 & 5.2 . & 4.9 & 4.0 & 3.3 & 3.2 & 3.2 & 3.2 & 3.3 & 3.6 \\
\hline 20. & 6.8 & 6.0 & 8.25 & 5.2 & 49 & 4.0 & 3.3 & 3.1 & 3.2 & 3.2 & 3.3 & 3.6 \\
\hline 21.. & 6.8 & 5.8 & 7. & 5.0 & 4.8 & 4.0 & 3.3 & 3.1 & 3.2 & 2 & .3 & 3.6 \\
\hline $2 .$. & 9.75 & 5.6 & 6.75 & 5.0 & 4.8 & 3.9 & 3.3 & 3.1 & 3.2 & 3.2 & 3.3 & 3.6 \\
\hline 23. & 9.25 & 5.5 & 6.55 & 5.0 & 4.7. & 3.9 & 3.3 & 3.1 & 3.2 & 3.2 & 3.3 & 3.6 \\
\hline $24 .$. & 9.5 & 5.4 & 7.25 & 4. 9. & 4.7 & 3.9 & 3.3 & 3.1 & 3.2 & 3.3 & 3.3 & 3.6 \\
\hline $25 \ldots$ & 7.7 & 5.4 & 0.6 & 4.5 & 4.6 & 3.8 & 3.3 & 3.1 & 3.2 & 3.3 & 3.4 & 3.6 \\
\hline $26 \ldots$ & 6.9 & 5.3 & 6.55 & 4.9 & 4.: & 3.8 & 3.3 & 3.1 & 3.2 & 3.3 & 3.4 & 3.6 \\
\hline $27 .$. & 6.45 & 5.3 & 6.3 & 4.8 & 5.25 & 3.8 & 3.3 & 3.1 & 3.2 & 3.3 & 3.4 & 3.8 \\
\hline 28. & 6.3 & 5.2 & 6.1 & 4.8 & 4.8 & 3.7 & 3.3 & 3.1 & 3.2 & 3.3 & 3.4 & 3.8 \\
\hline $29 \ldots$ & 6.1 & & 7.35 & 4.8 & 4.8 & .3 .7 & 3.3 & 3.1 & 3.2 & 3.3 & 3.6 & 3.8 \\
\hline 30.. & 6.0 & & 6.5 & 4.7. & 4.7 & 3.7 & 3.2 & 3.1 & 3.2 & 3.3 & 3.6 & 3.8 \\
\hline 31.. & 6.0 & & 6.15 & $\cdots$ & 4.6 & & 3.2 & 3.1 & & 3.3 & & 3.8 \\
\hline
\end{tabular}

Station rating table for Stony Creek near Fruto, Cal., from January 1 to December 31, 1905.

\begin{tabular}{|rr||r|r||r|r|r|r|}
\hline $\begin{array}{r}\text { Gage } \\
\text { height. }\end{array}$ & Discharge. & $\begin{array}{c}\text { Gage } \\
\text { height. }\end{array}$ & Discharge. & $\begin{array}{c}\text { Gage } \\
\text { height. }\end{array}$ & Discharge. & $\begin{array}{r}\text { Gage } \\
\text { height. }\end{array}$ & Discharge. \\
\hline Feet. & Second-feet. & Feet. & Second-feet & Feet. & Second-feet. & Feet. & Second-feet. \\
3.10 & 10 & 4.50 & 350 & 5.90 & 1,310 & 7.60 & 3,150 \\
3.20 & 14 & 4.60 & 400 & 6.00 & 1,400 & 7.80 & 3,410 \\
3.30 & 23 & 4.70 & 450 & 6.10 & 1,490 & 8.00 & 3,690 \\
3.40 & 34 & 4.80 & 500 & 6.20 & 1,580 & 8.20 & 3,980 \\
3.50 & 47 & 4.90 & 560 & 6.30 & 1,680 & 8.40 & 4,280 \\
3.60 & 62 & 5.00 & 620 & 6.40 & 1,780 & 8.60 & 4,600 \\
3.70 & 80 & 5.10 & 685 & 6.50 & 1,880 & 8.80 & 4,930 \\
3.80 & 100 & 5.20 & 755 & 6.60 & 1,980 & 9.00 & 5,270 \\
3.90 & 120 & 5.30 & 830 & 6.70 & 2,090 & 9.20 & 5,630 \\
4.00 & 150 & 5.40 & 905 & 6.80 & 2,200 & 9.40 & 6,000 \\
4.10 & 180 & 5.50 & 980 & 6.90 & 2,310 & 9.60 & 6,400 \\
4.20 & 210 & 5.60 & 1,060 & 7.00 & 2,420 & 9.80 & 6,800 \\
4.30 & 250 & 5.70 & 1,140 & 7.20 & 2,660 & 10.00 & 7,280 \\
4.40 & 300 & 5.80 & 1,220 & 7.40 & 2,900 & & \\
& & & & & & & \\
\hline
\end{tabular}

Note.-The above table is based on 22 discharge measurements made during 1903-1905 and is fairly well defined throughout. Above 3.7 feet the table is the same as for 1904. 
Estimated monthly discharge of Stony Creek near Fruto, Cal., for 1905.

[Drainage area, 760 square miles.]

\begin{tabular}{|c|c|c|c|c|c|c|}
\hline \multirow[b]{2}{*}{ Month. } & \multicolumn{3}{|c|}{ Discharge in second-feet. } & \multirow[b]{2}{*}{$\begin{array}{l}\text { Total in } \\
\text { acre-feet. }\end{array}$} & \multicolumn{2}{|c|}{ Run-off. } \\
\hline & Maximum. & Minimum. & Mean. & & $\begin{array}{l}\text { Second-feet } \\
\text { per square } \\
\text { mile. }\end{array}$ & $\begin{array}{l}\text { Depth } \\
\text { in inches. }\end{array}$ \\
\hline January.. & 6,700 & 450 & 2,420 & 148,800 & . $\quad 3.18$ & 3.67 \\
\hline February... & 7,280 & 685 & 1,471 & 81,700 & 1.94 & 2.02 \\
\hline March............ & 5,720 & 500 & 2,050 & 126,000 & 2.70 & 3.11 \\
\hline April...... & 1,490 & 450 & 870 & 51,770 & 1.14 & 1.27 \\
\hline May....... & 1,680 & 400 & 675 & 41,500 & .888 & 1.02 \\
\hline June........ & 400 & 80 & 206 & 12,260 & .271 & .302 \\
\hline July............ & 80 & 14 & 36.5 & 2,244 & .048 & .055 \\
\hline August.... & 14 & 10 & 12.4 & 762 & .016 & .018 \\
\hline September. . & 14 & 10 & 13.7 & 815 & .018 & .020 \\
\hline October.... & 23 & 14 & 16.3 & 1,002 & .021 & .024 \\
\hline November... & 62 & 23 & 27.1 & 1,613 & .036 & .040 \\
\hline December......................... & 100 & 62 & 68.1 & 4,187 & .090 & .104 \\
\hline The year.... & 7,280 & 10 & 656 & 472,700 & .862 & 11.62 \\
\hline
\end{tabular}

\section{FEATHER RIVER DRAINAGE BASIN.}

\section{DESCRIPTION OF BASIN.}

Feather River drains a portion of the western slope of the Sierra Nevada extending east nearly to the Nevada State line, a distance of about 75 miles and north and south for a distance of from 30 to 40 miles.

The greater portion of the watershed is rough and mountainous and has numerous tributaries which drain the slopes of the higher mountains. The formation in the southern and eastern part of the basin is of granite, with a comparatively deep soil corering. There is also a considerable area composed of lava and other volcanic matter in the northern part of the basin. Numerous meadows and valleys also exist, which tend to maintain a steady flow during the dry season. The soil is generally porous and absorbs the moisture readily. The entire watershed is well covered with a growth of brush and timber, much of which is large enough to make lumbering a profitable industry, with the exception of the meadow lands and valleys, which are used for stock ranges and grazing lands. There are numerous large springs, especially in the lava districts, which supply a more or less steady flow throughout the year. These are especially noticeable on North Fork, where there are perennial springs discharging from 50 to 100 second-feet. There is little artificial storage in the drainage area, and the water used for irrigation in the valleys is taken from the natural flow of the streams.

The mean annual precipitation is probably from 40 to 60 inches and is well distributed over the area. It falls largely in the form of snow, but disappears in the early part of the summer.

\section{FEATHER RIVER AT OROVILLE, CAL.}

This station was established January 1, 1902, by S. G. Bennett. It is located at the northeast edge of the town of Oroville, Cal., where Feather River breaks from the foothills on the western slope of the Sierra Nevada into Sacramento Valley. The drainage area is 3,640 square miles.

The current at the station is sluggish at low water and swift at high water. The channel is rough, rocky, and not subject to much change. 
Discharge measurements at low and ordinary stages were made by means of a cable and boat 500 feet above the wagon bridge. High-water measurements were made by means of floats. In February, 1905, a cable with car was placed about 1,000 feet above the bridge. The cable has a span of about 400 feet. The initial point of soundings is the eyebolt on the left bank.

The original gage was previously placed by the United States Weather Bureau and used by them for a number of years. Readings were taken on this rod and reported by the Weather Bureau when there was danger of an overflow on the lower Feather and Sacramento rivers. On August 11, 1904, a new gage rod was put in. The zero of this rod was placed 2 feet lower than that of the old Weather Bureau rod to avoid negative readings at low stages of the river. This rod is placed at the wagon bridge on the left bank of the stream and is in four sections. Three of the lower sections are bolted to rock, and the fourth section, for registering the flood stages, is nailed to the bridge pier. 'In December, 1905, a staff gage was placed at the station 50 feet below the cable on the left bank. During 1905 the gage was read by D. G. Page, readings being taken from the gage at the bridge. The bench marks are as follows: (1) A point on rock marked with white paint, one-half the distance from the low-water gage to the high-water gage on the bridge pier; elevation, 12.25 feet. (2) A point on rock marked with white paint, 35 feet southwest of low-water gage; elevation, 14.86 feet. (3) A circle of white paint on the point of a rock 15 feet upstream from the 70-foot mark on the cable; elevation, 5.05 feet. (4) A point on rock marked with white paint, 4 feet downstream from 15-foot mark on cable; elevation, 27.16 feet. The elevations of bench marks Nos. 1 and 2 refer to the datum of the gage at the bridge; elevations of bench marks Nos. 3 and 4 refer to the datum of the gage established in December, 1905.

Information in regard to this station is contained in Water-Supply Papers Nos. 81, 85, 100, and 134 of the United States Geological Survey.

Discharge measurements of Feather River at Oroville, Cal., in 1905.

\begin{tabular}{|c|c|c|c|c|c|c|}
\hline Date. & Hydrographer. & Width. & $\begin{array}{l}\text { Area of } \\
\text { section. }\end{array}$ & $\underset{\text { velocity. }}{\text { Mean }}$ & $\begin{array}{l}\text { Gage } \\
\text { height. }\end{array}$ & $\begin{array}{l}\text { Dis- } \\
\text { charge. }\end{array}$ \\
\hline February $11 \ldots$ & O. W. Peterson. & $\begin{array}{r}\text { Feet. } \\
281\end{array}$ & $\begin{array}{r}S q . f t \\
3,424\end{array}$ & $\begin{array}{r}\text { Ft.per sec. } \\
2.22\end{array}$ & $\begin{array}{l}\text { Feet. } \\
6.45\end{array}$ & $\begin{array}{r}\text { Sec.-ft. } \\
7,618\end{array}$ \\
\hline February $18 .$. & .....do.............. & 279 & 3,320 & 2.08 & 6.10 & 6,911 \\
\hline May $16 . . . . . .$. & W. B. Clapp....... & 281 & 3,728 & 2.28 & 6.65 & 8,509 \\
\hline June $26 . . . \ldots$. & O.W. Peterson...... & 273 & 2,419 & 1.13 & 2.75 & 2,744 \\
\hline August $1 . . .$. & Peterson and Lee.. & 270 & 2,078 & .72 & 1.28 & 1,495 \\
\hline September $2 \ldots$ & c. H. Lee............ & 268 & 2,005 & .62 & .86 & 1,242 \\
\hline October $7 . . . .$. & Hawley and Lee... & 272 & 2,013 & .60 & .90 & 1,210 \\
\hline November 17. & R. S. Hawley................... & 270 & 1,969 & .62 & .92 & 1,212 \\
\hline
\end{tabular}


Daily gage height, in feet, of Feather River at Oroville, Cal., for 1905.

\begin{tabular}{|c|c|c|c|c|c|c|c|c|c|c|c|c|}
\hline Day & Jan. & Feb. & Mar. & Apr. & May. & June. & July. & Aug. & Sept. & Oct. & Nov. & Dec. \\
\hline 1.. & 10.0 & 9.5 & 6.9 & 8.3 & 7.15 & 5.4 & 2.1 & 1.3 & 0.95 & 1.0 & 1.0 & 1.4 \\
\hline $2 .$. & 8.3 & 11.3 & 6.9 & 8.05 & 7.05 & 5.25 & 2.1 & 1.25 & .9 & 1.0 & 1.0 & 1.35 \\
\hline 3.. & 7.4 & 10.25 & 6.95 & 8.15 & 6.95 & 5.2 & 2.1 & 1.25 & .9 & .95 & 1.0 & 1.2 \\
\hline 4.. & 6.65 & 9.05 & 7.5 & 8.1 & 6.85 & 5.2 & 2.05 & 1.2 & .9 & .95 & 1.0 & 1.15 \\
\hline 5. & 5.8 & 8.9 & 7.75 & 8.05 & 6.75 & 5.15 & 2.0 & 1.15 & .9 & .95 & .95 & 1.1 \\
\hline 6. & 5.5 & 8.95 & 7.4 & 8.0 & 6.55 & 5.0 & 2.0 & 1.15 & .95 & 1.0 & .95 & 1.0 \\
\hline $7 .$. & 5.5 & 7.85 & 7.0 & 8.0 & 6.45 & 4.95 & 1.95 & 1.15 & .95 & 1.0 & .95 & 1.0 \\
\hline 8.. & 5.1 & 7.4 & 6.8 & 8.05 & 6.35 & 4.95 & 1.9 & 1.1 & 1.0 & 1.0 & .95 & .95 \\
\hline 9.. & 4.5 & 6.95 & 6.75 & 8.0 & 6.25 & 4.9 & 1.9 & 1.1 & 1.0 & 1.1 & .9 & .95 \\
\hline$\because$ & 4.2 & 6.7 & 6.6 & 7.9 & 6.15 & 4.8 & 1.85 & 05 & 1.0 & .05 & .9 & .95 \\
\hline.. & 3.4 & 6.5 & 6.7 & 7. & 6. & 4.7 & 1.8 & 1.05 & 1.0 & 1.05 & .85 & .95 \\
\hline$\cdots$ & 3.3 & 6.25 & 7.85 & 7.7 & $6 .($ & 4. & 1.8 & 1.05 & .95 & 1.0 & .85 & 1.0 \\
\hline $3 .$. & 3.2 & 5.9 & 10.25 & 7.5 & 5.85 & 4.45 & 1.75 & 1.05 & .95 & 1.0 & .85 & 1.0 \\
\hline 4.. & $\mid 10.15$ & 5.6 & 9.95 & 7.5 & 5.9 & 4.2 & 1.75 & 1.0 & .9 & .95 & .85 & 1.1 \\
\hline 15. & 10.25 & 5.4 & 9.45 & 7.5 & 6.15 & 4.1 & 1.7 & 1.0 & .9 & .95 & .9 & 1.2 \\
\hline $16 .$. & 7.65 & 5.4 & 8.8 & 7.4 & 6.45 & 3.9 & 1.7 & 1.0 & .9 & .95 & .9 & 1.15 \\
\hline 7.. & 5.6 & 6.35 & 8.4 & 7.4 & 6.6 & 3.8 & 1.65 & 1.05 & .85 & .95 & .9 & $a_{1.15}$ \\
\hline 3... & 5.5 & 6.35 & 8.9 & 7.45 & 6.5 & 3.7 & 1.65 & 1.05 & .85 & .95 & .9 & $a_{1} 1.15$ \\
\hline 19.. & 7.4 & 6.1 & 12.5 & 7.55 & 6.45 & 3.6 & 1.6 & 1.05 & .85 & 1.0 & .9 & $a_{1} 1.15$ \\
\hline D... & 6.65 & 7.8 & 11.35 & 7.35 & 6.4 & 3.45 & 1.6 & 1.1 & .9 & 1.0 & 1.0 & $a_{1.15}$ \\
\hline 1.. & 7.35 & 8.25 & 11.35 & 6.95 & 6.4 & 3.2 & 1.6 & 1.1 & .9 & 1.0 & 1.4 & $a_{1.1}$ \\
\hline 22. & 11.8 & 7.75 & 10.95 & 6.7 & 6.3 & 3.15 & 1.55 & 1.1 & .9 & 1.0 & 1.3 & $a .1 .1$ \\
\hline 23.. & 11.95 & 7.4 & 10.8 & 6.8 & 6.3 & 3.05 & 1.5 & 1.2 & .95 & 1.0 & 1.3 & $a_{1.1}$ \\
\hline 24. & 10.5 & 7.3 & 10.7 & 6.95 & 6.3 & 3.0 & 1.5 & 1.2 & 95 & 1.0 & 1.25 & 1.1 \\
\hline 5.. & 9.8 & 7.2 & 9.95 & 7.15 & 6.25 & 2.9 & 1.5 & 1.1 & .9 & .95 & 1.2 & 1.15 \\
\hline 26 .. & 8.65 & 7.1 & 9.3 & 7.3 & 6.2 & 2.8 & 1.45 & 1.0 & .9 & .95 & 1.2 & 1.15 \\
\hline $27 .$. & 7.9 & 7.1 & 9.05 & 7.45 & $\cdots$ & 2.6 & 1.45 & 1.0 &. & .95 & 1.1 & 1.2 \\
\hline 28. & 7.45 & 7.0 & 9. & 7.5 & 6.0 & 2.5 & 1.4 & 1.0 & 1.7 & .95 & 1.1 & 1.2 \\
\hline 29. & 7.1 & & 10.65 & 7.4 & 5.95 & 2.4 & 1.4 & .95 & 1.3 & 1.0 & 1.3 & 1.3 \\
\hline & 6.85 & - & 10.05 & 7.3 & 5.85 & 2.3 & 1.35 & 1.0 & 1.0 & 1.0 & 1.4 & 1.35 \\
\hline & 6.75 & & 8.95 & & 5.65 & & 1.35 & 1.0 & & 1.0 & & $a_{1.35}$ \\
\hline
\end{tabular}

$a$ Estimated. 
Station rating table for Feather River at Oroville, Cal., from January 1 to December 31, 1905.

\begin{tabular}{|r|r||r|r|r|r|r|r|}
\hline $\begin{array}{c}\text { Gage } \\
\text { height. }\end{array}$ & Discharge. & $\begin{array}{c}\text { Gage. } \\
\text { height. }\end{array}$ & Discharge. & $\begin{array}{c}\text { Gage } \\
\text { height. }\end{array}$ & Discharge. & $\begin{array}{c}\text { Gage } \\
\text { height. }\end{array}$ & Discharge. \\
\hline Feet. & Second-feet. & Feet. & Second-feet. & Feet. & Second-feet. & Feet. & Second-feet. \\
S.90 & 1,230 & 2.70 & 2,590 & 4.40 & 4,570 & 7.20 & 8,640 \\
1.00 & 1,295 & 2.80 & 2,690 & 4.50 & 4,700 & 7.40 & 9,000 \\
1.10 & 1,360 & 2.90 & 2,790 & 4.60 & 4,830 & 7.60 & 9,400 \\
1.20 & 1,430 & 3.00 & 2,900 & 4.70 & 4,960 & 7.80 & 9,800 \\
1.30 & 1,500 & 3.10 & 3,010 & 4.80 & 5,090 & 8.00 & 10,260 \\
1.40 & 1,570 & 3.20 & 3,120 & 4.90 & 5,220 & 8.20 & 10,740 \\
1.50 & 1,640 & 3.30 & 3,230 & 5.00 & 5,350 & 8.40 & 11,260 \\
1.60 & 1,710 & 3.40 & 3,340 & 5.20 & 5,630 & 8.60 & 11,780 \\
1.70 & 1,780 & 3.50 & 3,450 & 5.40 & 5,910 & 8.80 & 12,340 \\
1.80 & 1,850 & 3.60 & 3,570 & 5.60 & 6,190 & 9.00 & 12,900 \\
1.90 & 1,925 & 3.70 & 3,690 & 5.80 & 6,470 & 9.50 & 14,400 \\
2.00 & 2,000 & 3.80 & 3,810 & 6.00 & 6,750 & 10.00 & 16,150 \\
2.10 & 2,075 & 3.90 & 3,930 & 6.20 & 7,050 & 10.50 & 18,100 \\
2.20 & 2,155 & 4.00 & 4,050 & 6.40 & 7,350 & 11.00 & $20 ; 100$ \\
2.30 & 2,235 & 4.10 & 4,180 & 6.60 & 7,660 & 11.50 & 22,500 \\
2.40 & 2,315 & 4.20 & 4,310 & 6.80 & 7,980 & 12.00 & 25,100 \\
2.50 & 2,400 & 4.30 & 4,440 & 7.00 & 8,300 & 12.50 & 28,100 \\
2.60 & 2,490 & & & & & & \\
& & & & & & \\
\hline
\end{tabular}

NotE. - The above table is based on 21 discharge measurements made during 1904-5. It is well defined between gage heights 0.85 foot and 8 feet. Above gage height 3.5 feet the table is the same as for 1904 .

Estimated monthly discharge of Feather River at Orovitle, Cal., for 1905.

[Drainage area, $3,640 a$ square miles.]

\begin{tabular}{|c|c|c|c|c|c|c|}
\hline \multirow{2}{*}{ Month. } & \multicolumn{3}{|c|}{ Discharge in second-feet. } & \multirow{2}{*}{$\begin{array}{l}\text { Total in } \\
\text { acre-feec. }\end{array}$} & \multicolumn{2}{|c|}{ Run-off. } \\
\hline & Maximum. & Minimum. & Mean. & & $\begin{array}{l}\text { Second-feet } \\
\text { per square } \\
\text { mile. }\end{array}$ & $\begin{array}{l}\text { Depth } \\
\text { in inches. }\end{array}$ \\
\hline January .. & 24,800 & 3,120 & 9,860 & 606,300 & 2.71 & 3.12 \\
\hline February . & 21,500 & 5,910 & 9,612 & 533,800 & 2.64 & 2.75 \\
\hline March.... & 28,100 & 7,660 & 13,560 & 833,800 & 3.72 & 4.29 \\
\hline April.. & 11,000 & 7,820 & 9,403 & 559,500 & 2.58 & 2.88 \\
\hline May.... & 8,550 & 6,260 & 7,254 & 446,000 & 1.99 & 2.29 \\
\hline June.. & 5,910 & 2,235 & 4,098 & 243,800 & 1.13 & 1.26 \\
\hline July... & 2,075 & 1,535 & 1,790 & 110,100 & .492 & .567 \\
\hline August - & 1,500 & 1,262 & 1,354 & 83,250 & .372 & .429 \\
\hline September. & 1,780 & 1,200 & 1,273 & 75,750 & .350 & .390 \\
\hline October. & 1,360 & 1,262 & 1,286 & 79,070 & .353 & .407 \\
\hline November. & 1,570 & 1,200 & 1,321 & 78,610 & .363 & .405 \\
\hline December. & 1,570 & 1,262 & 1,385 & 85,160 & .380 & .438 \\
\hline The year... & 28,100 & 1,200 & 5,183 & 3,$735 ; 000$ & 1.42 & 19.23 \\
\hline
\end{tabular}

a Revised since previous reports. 


\section{MISCELLANEOUS MEASUREMENTS IN FEATHER RIVER DRAINAGE BASIN.}

The following miscellaneous measurements were made in the Feather River drainage basin during 1905:

Mohawk Creek near New Mohawk, Cal.-A measurement was made on this stream on September 10, 1905, just above its junction with Middle Fork of Feather River by H. A. Campbell.

Width, 12.9 feet; area, 16.9 square feet; mean velocity, 1.18 feet per second; discharge, 20 second-feet.

Middle Fork of Feather River at Mohawk Valley, Cal.-A measurement was made just. above mouth of Mohawk Creek on September 10, 1905, by H. A. Campbell.

Width, 10.0 feet; area, 8.8 square feet; mean velocity, 0.17 foot per second; discharge, 1.5 second-feet.

Feather River at Prattville, Cal.-A measurement of this stream was made November 25 at lower end of Big Meadows, 4 miles southeast of Prattville, Cal.

Width, 61 feet; area, 320 square feet; mean velocity, 1.88 feet per second; discharge, 601 second-feet.

Grizzly Creek near Beckwith, Cal.- - This stream is tributary to Middle Fork of Feather River. A measurement was made December 17 one-half mile above its junction with Middle Fork and about $2 \frac{1}{2}$ miles west of the town of Beckwith, Cal.

Width, 4 feet; area, 1.8 square feet; mean velocity, 1.72 feet per second; discharge, 3.1 second-feet.

Indian Creek near Crescent Mills, Cal.-This stream is tributary to North Fork of Feather River. A measurement was made December 14 at lower end of Indian Valley, one-half mile below highway bridge on Crescent Mill and Taylorsville road, about $1 \frac{1}{2}$ miles below the town of Crescent Mills.

Width, 45 feet; area, 66 square feet; mean velocity 1.15 feet per second; discharge, 76 second-feet.

\section{YUBA RIVER DRAINAGE BASIN.}

\section{DESCRIPTION OF BASIN.}

Yuba River is a tributary of Feather River, which it enters at Marysville, 30 miles above the junction of Feather and Sacramento rivers. The entire drainage area of this river is about 1,327 square miles, of which about 1,220 square miles are above the gaging station at Smartsville. Its extreme length is about 60 miles, and extreme width 56 miles. In the lower stretches of the river, at the location of the present gaging station and in the valley below, the channel has been filled to a considerable depth with débris from hydraulic mining.

The drainage basin is subdivided into 5 small basins, namely: North Fork, with a drainage area of 491.6 square miles; Middle Fork, with a drainage area of 218 square miles; South Fork, with a drainage area of 360 square miles; Deer Creek, with a drainage area of 89.6 square miles, and Dry Creek, with a drainage area of 105.5 square miles. The latter tributary discharges into the main river about 5 miles below the gaging station. The watershed rises gently in rounded and broken mountains, to the crest of the Sierra Nevada, which at the headwaters of the Yuba has a mean elevation of about 8,200 feet; with peaks rising to a height of 9,100 feet. From Mount Lincoln-a peak common to the watersheds of the Yuba, American, and Truckee rivers-to a point about $2 \frac{1}{2}$ miles northeast of Mount Weber, the summit of the Sierra Nevada divides the watershed of Yuba River from that of Truckee River, which discharges into Humboldt Basin. Farther north from Mount Weber there is a secondary crest which divides the watersheds of Yuba and Feather rivers, the watershed of the latter stream reaching farther east to a less elevated divide in which the passes are lower than those of the easterly crest. 
The western and lower portions of the Yuba drainage basin are composed of slate and kindred rock, very much eroded and merging into the gravel and alluvial deposits of the Sacramento Valley. The upper portions of the basin are composed principally of lavas and granites, all deeply eroded, particularly the lavas. A stratum of serpentine traverses the watershed of the Yuba River in a direction generally parallel with the crest of the Sierras. The North Fork rises in lavas which vary much in composition and hardness, but which generally have a deep soil covering, with timber and brush growth. The Middle Fork rises in similar lavas and granite. The main and tributary streams fall rapidly, and their canyons head well up in the mountains. The sides of these canyons are covered with timber and brush, which, with the deep soil, retain the moisture and feed numerous perennial springs. In the case of the North Fork this is particularly noticeable. The forests of its watersheds make a reliable and constant stream. The mean annual precipitation for the basins of North and Middle forks is about $\mathbf{5 4}$ inches. Warm rains on soft snow sometimes give high flood discharge, but snow remains on the higher peaks until midsummer. The headwaters of South Fork lie upon a broad granite surface into which the streams have not cut deeply until the main stream reaches a point 16 miles from the summit, where it drops rapidly into a deeply eroded canyon. This part of the basin has a precipitation annually of about 60 inches. The entire drainage area of the Yuba contains nearly 100 small glacial lakes.

\section{YUBA RIVER NEAR SHARTSVIIIE, CAL.}

This station was established June 2, 1903, by. W. H. Stearns. It is located at what is called "The Narrows," 1 mile from Smartsville, Cal., 18 miles from the Southern Pacific Railroad station at Wheatland, Cal., and 20 miles from Marysville, Cal.

The channel is straight for 200 feet above and 300 feet below the station, and the current is swift at all stages. In the 150 feet above the cable the stream has a fall of 0.2 foot and of 0.9 foot in the 200 feet below. Both banks are high and rocky and are not subject to overflow. The banks widen out considerably just below the station. The bed of the stream is composed of gravel and sand-tailings from hydraulic mining-and is constantly shifting. After the rains of 1904 it was found that the bed of the stream had been lowered for an average depth of 2 feet.

Discharge measurements are made from a car and cable. One auxiliary cable is stretched parallel to and 100 feet upstream from the main cable, and a second one is located 150 feet below the station cable for float measurements. The initial point for soundings is on the left bank at the eyebolt to which the cable is fastened. Frequent discharge measurements are made on account of continual changes of the river bed. These changes, however, do not materially affect discharge measurements for the same gage height.

The gage is in two sections. The lower one is bolted to the rock wall on the left bank of the river and the upper one to the right bank. During 1905 the gage was read once each day by J. R. McKeel. Bench marks were established as follows: (1) A point on a rock marked with white paint, 12 feet upstream and about 15 feet to the left of the right eyebolt; elevation, 39.34 feet. (2) A bolt in shelf of rock marked with white paint, 12 feet from high-water gage on right bank; elevation, 13.75 feet. (3) A bolt in the face of rock 20 feet upstream from the cable on right bank; elevation, 16.60 feet. Elevations refer to the datum of the gage. 
Information in regard to this station is contained in Water-Supply Papers Nos. 81, 100, and 134, United States Geological Survey.

Discharge measurements of Yuba River near Smartsville, Cal., in 1905.

\begin{tabular}{|c|c|c|c|c|c|c|}
\hline Date. & Hydrographer. & Width. & $\begin{array}{l}\text { Area of } \\
\text { section. }\end{array}$ & $\begin{array}{c}\text { Mean } \\
\text { velocity. }\end{array}$ & $\begin{array}{c}\text { Gage } \\
\text { height. }\end{array}$ & $\begin{array}{c}\text { Dis- } \\
\text { charge. }\end{array}$ \\
\hline January $20 . . .$. & J.R.McKeel......... & $\begin{array}{r}\text { Feet. } \\
180\end{array}$ & $\begin{array}{r}S q . f t . \\
688\end{array}$ & $\begin{array}{r}\text { Ft. per sec. } \\
5.09\end{array}$ & $\begin{array}{l}\text { Feet. } \\
6.70\end{array}$ & $\begin{array}{r}\text { Sec.-ft. } \\
3,500\end{array}$ \\
\hline February $12 \ldots$ & $\ldots \ldots$ do......... & 175 & 671 & 4.69 & 6.30 & 3,147 \\
\hline February 13.. & O. W. Peterson.. & 178 & 665 & 4.39 & 6.25 & 2,920 \\
\hline February 26. & J.R.McKeel. . & 180 & 936 & 5.76 & 7.00 & 5,397 \\
\hline March 5.... & .....do....... & 179 & 836 & 5.40 & 6.60 & 4,512 \\
\hline March $15 \ldots$ & ....do... & 180 & 982 & 6.02 & 6.90 & 5,914 \\
\hline April 8... & .....do.... & 170 & 1,060 & 6.56 & 7.00 & 6,959 \\
\hline April 24. . & ......do.... & 170 & 968 & 6.64 & 6.30 & 6,423 \\
\hline May $4 . . .$. & .....do.... & 180 & 868 & 6.01 & 5.80 & 5,221 \\
\hline May $13 . . .$. & ....do... & 180 & 864 & 5.94 & 5.50 & 5,132 \\
\hline May $28 . .$. & .....do.... & 183 & 883 & 6.31 & 5.75 & 5,570 \\
\hline June $4 . .$. & .....do... & 180 & 732 & 5.40 & 5.00 & 3,955 \\
\hline June $16 . . . . .$. & .....do.... & 180 & 652 & 5.09 & 4.50 & 3,318 \\
\hline July $7 \ldots \ldots$ & .....do.... & 155 & 286 & 3.58 & 2.40 & 1,025 \\
\hline July $20 \ldots$ & .....do.... & 74 & 165 & 4.22 & 2.00 & 697 \\
\hline July $29 . .$. & .....do........ & 73 & 158 & 4.01 & 1.60 & 634 \\
\hline July $31 \ldots$ & O. W. Peterson, C.H. Lee. & 76 & 137 & 3.38 & 1.64 & 463 \\
\hline August $7 \ldots$. & J.R.McKeel. ...... & 68 & 137 & 3.83 & 1.50 & 525 \\
\hline August $15 . . .$. & .....do.. & -62 & 126 & 3.78 & 1.40 & 476 \\
\hline August $21 . . .$. & ....do... & 68 & 140 & 3.80 & 1.50 & 532 \\
\hline August $28 \ldots . .$. . & .....do.... & 62 & 120 & 3.68 & 1.30 & 442 \\
\hline September 5... & .....do.... & 62 & 120 & 3.66 & 1.30 & 439 \\
\hline September $25 .$. & ... do.......... & 57 & 113 & 3.56 & 1.20 & 402 \\
\hline October $8 . .$. & Hawley and Lee.. & 74 & 115 . & 3.43 & 1.45 & 395 \\
\hline October $20 .$. & J.R.McKeel. ...... & 66 & 126 & 3.62 & 1.40 & 456 \\
\hline October $30 \ldots$ & .....do.. & 66 & 125 & 3.55 & $1.35^{\circ}$ & 444 \\
\hline November $9 \ldots$ & .....do... & 63 & 121 & 3.48 & 1.30 & 420 \\
\hline November $16 .$. & .....do. do. & 63 & 117 & 3.44 & 1.20 & 403 \\
\hline November $23 . .$. & ....do.. & 63 & 123 & 3.56 & 1.35 & 438 \\
\hline November $30 .$. . & ....do.. & 150 & 224 & 3.70 & 2.20 & 830 \\
\hline December $6 \ldots$. & .....do... & 70 & 140 & 3.80 & 1.60 & 533 \\
\hline December $13 \ldots$ & .....do.... & 68 & 133 & 3.65 & 1.50 & 486. \\
\hline December $29 \ldots$ & .....do...... & 84 & 167 & 3.96 & 1.90 & 661 \\
\hline
\end{tabular}


Daily gage height, in feet, of Yuba River near Smartsville, Cal., for 1905.

\begin{tabular}{|c|c|c|c|c|c|c|c|c|c|c|c|c|}
\hline Day. & Jan. & Feb. & Mar. & Apr. & May. & June. & July. & Aug. & Sept. & Oct. & Nov. & Dec. \\
\hline & 9.0 & 7.5 & 6.7 & 7.0 & 6.5 & 5.5 & 2.7 & 1.6 & 1.3 & 1.4 & 1.4 & 2.0 \\
\hline $2 .$. & 8.4 & 9.4 & 6.7 & 7.0 & 6.3 & 5.2 & 2.7 & 1.6 & 1.3 & 1.4 & 1.4 & 1.7 \\
\hline 3.. & 7.6 & 8.5 & 6.6 & 7.1 & 6.1 & 5.1 & 2.6 & 1.6 & $a_{1.3}$ & 1.4 & 1.4 & 1.7 \\
\hline $4 \ldots$ & 7.2 & 7.9 & 6.7 & 7.1 & 5.8 & 5.0 & $a_{2.5}$ & 1.6 & 1.3 & 1.4 & 1.4 & 1.7 \\
\hline $5 \ldots$ & 6.9 & 8.5 & 6.6 & 7.0 & 5.7 & 4.8 & 2.5 & 1.6 & 1.3 & 1.4 & $a_{1.4}$ & 1.6 \\
\hline $6 .$. & 6.8 & 7.6 & 6.6 & 7.1 & 5.7 & 4.9 & 2.4 & $a_{1.6}$ & 1.3 & 1.3 & 1.4 & 1.6 \\
\hline 7. & 6.4 & 7.2 & 6.5 & 7.3 & 5.6 & 5.0 & 2.4 & 1.5 & 1.3 & 1.3 & 1.4 & 1.6 \\
\hline 8.. & 6.4 & 7.0 & 6.5 & 7.0 & 6.0 & 4.9 & 2.3 & 1.5 & 1.3 & 1.4 & 1.3 & 1.6 \\
\hline $9 .$. & 6.3 & 6.9 & 6.4 & 6.9 & 6.2 & 4.9 & 2.3 & 1.5 & 1.3 & $a_{1.4}$ & 1.3 & 1.5 \\
\hline $10 .$. & 6.2 & 6.6 & 6.4 & 6.9 & 5.7 & 4.9 & 2.2 & 1.5 & $a_{1.3}$ & 1.4 & 1.3 & $a_{1.5}$ \\
\hline $11 .$. & 6.1 & 6.6 & 6.3 & 6.5 & 5.7 & 5.2 & 2.2 & 1.5 & 1.3 & 1.4 & 1.3 & 1.5 \\
\hline $12 .$. & 6.1 & 6.3 & 6.4 & 6.3 & 5.5 & 5.0 & 2.2 & 1.5 & 1.2 & 1.4 & $a_{1.3}$ & 1.5 \\
\hline 13.. & 6.1 & 6.2 & 7.3 & 6.3 & 5.5 & 5.0 & 2.1 & $a_{1.5}$ & 1.2 & 1.4 & 1.3 & 1.5 \\
\hline $14 .$. & 8.2 & 6.1 & 7.7 & 6.2 & $a_{5.8}$ & 4.7 & 2.1 & 1.5 & 1.2 & 1.4 & 1.3 & 1.5 \\
\hline $15 .$. & 7.7 & 6.1 & 6.9 & 6.2 & 6.1 & 4.7 & 2.1 & 1.4 & 1.2 & $a_{1.3}$ & 1.3 & 1.5 \\
\hline $16 \ldots$ & 7.3 & 6.0 & 6.8 & 6.4 & 6.9 & 4.5 & 2.1 & 1.4 & 1.2 & 1.3 & 1.2 & 1.6 \\
\hline $17 .$. & 6.9 & 7.0 & 6.6 & 6.0 & 7.0 & 4.5 & 2.0 & 1.4 & $a_{1.2}$ & 1.3 & 1.2 & 1.6 \\
\hline 18. . & 6.3 & 7.0 & 7.3 & 7.2 & 6.9 & 4.3 & 2.0 & 1.4 & 1.2 & 1.4 & 1.2 & 2.0 \\
\hline $19 .$. & $a_{6.5}$ & 6.9 & 11.3 & 6.3 & 6.9 & 4.2 & 2.0 & 1.4 & $a_{1.2}$ & 1.4 & $a_{1.4}$ & 2.0 \\
\hline $20 \ldots$ & 6.7 & 8.5 & 8.8 & 6.0 & 6.8 & 4.0 & 2.0 & $a_{1.4}$ & 1.2 & 1.4 & 1.5 & 2.4 \\
\hline $1 .$. & 7.5 & 7.4 & 9.4 & 6.0 & 7.0 & 3.8 & 1.9 & 1.4 & 1.2 & 1.4 & 1.6 & 2.0 \\
\hline $22 .$. & 10.4 & 7.1 & 8.5 & 6.0 & 6.3 & 3.6 & 1.9 & 1.5 & 1.2 & $a_{1.4}$ & 1.4 & 1.8 \\
\hline $23 .$. & 10.0 & 6.9 & 7.9 & 6.1 & 6.2 & 3.4 & 1.8 & 1.4 & 1.2 & 1.4 & 1.4 & 1.6 \\
\hline $24 .$. & 8.9 & 6.8 & 8.4 & 6.3 & 6.0 & 3.3 & 1.8 & 1.4 & $\begin{array}{ll}a & 1.2\end{array}$ & 1.4 & 1.3 & 1.6 \\
\hline $25 .$. & 8.2 & 6.9. & 8.0 & 6.6 & 6.2 & 3.2 & 1.8 & 1.4 & 1.2 & 1.4 & 1.4 & 1.6 \\
\hline $26 \ldots$ & 7.8 & 7.0 & 8.1 & 7.0 & 6.5 & 3.1 & 1.7 & 1.4 & 1.2 & 1.5 & $a_{1.6}$ & 1.6 \\
\hline $27 \ldots$ & 7.4 & 6.9 & 8.0 & 7.4 & 5.8 & 3.0 & 1.7 & $\iota 1.4$ & 1.5 & 1.4 & 1.8 & 1.6 \\
\hline $28 \ldots$ & 7.1 & 6.8 & 7.5 & 7.1 & 5.7 & 2.9 & 1.7 & 1.3 & 1.5 & $a_{1.4}$ & 1.6 & 1.8 \\
\hline $29 .$. & 6.9 & & 8.4 & 7.0 & 5.6 & 2.8 & 1.6 & 1.3 & 1.4 & $a_{1.4}$ & 1.8 & 1.9 \\
\hline 30. & 6.8 & & 8.0 & 7.1 & 5.5 & 2.8 & 1.6 & 1.3 & 1.4 & 1.4 & 2.2 & 1.8 \\
\hline $31 \ldots$ & 6.7 & & 7.3 & $\ldots .$. & 5.5 & .... & 1.6 & 1.3 & & 1.4 & & 1.8 \\
\hline
\end{tabular}

$a$ Gage heights estimated. 
Daily discharge, in second-feet, of Yuba River near Smartsville, Cal., for 1905.

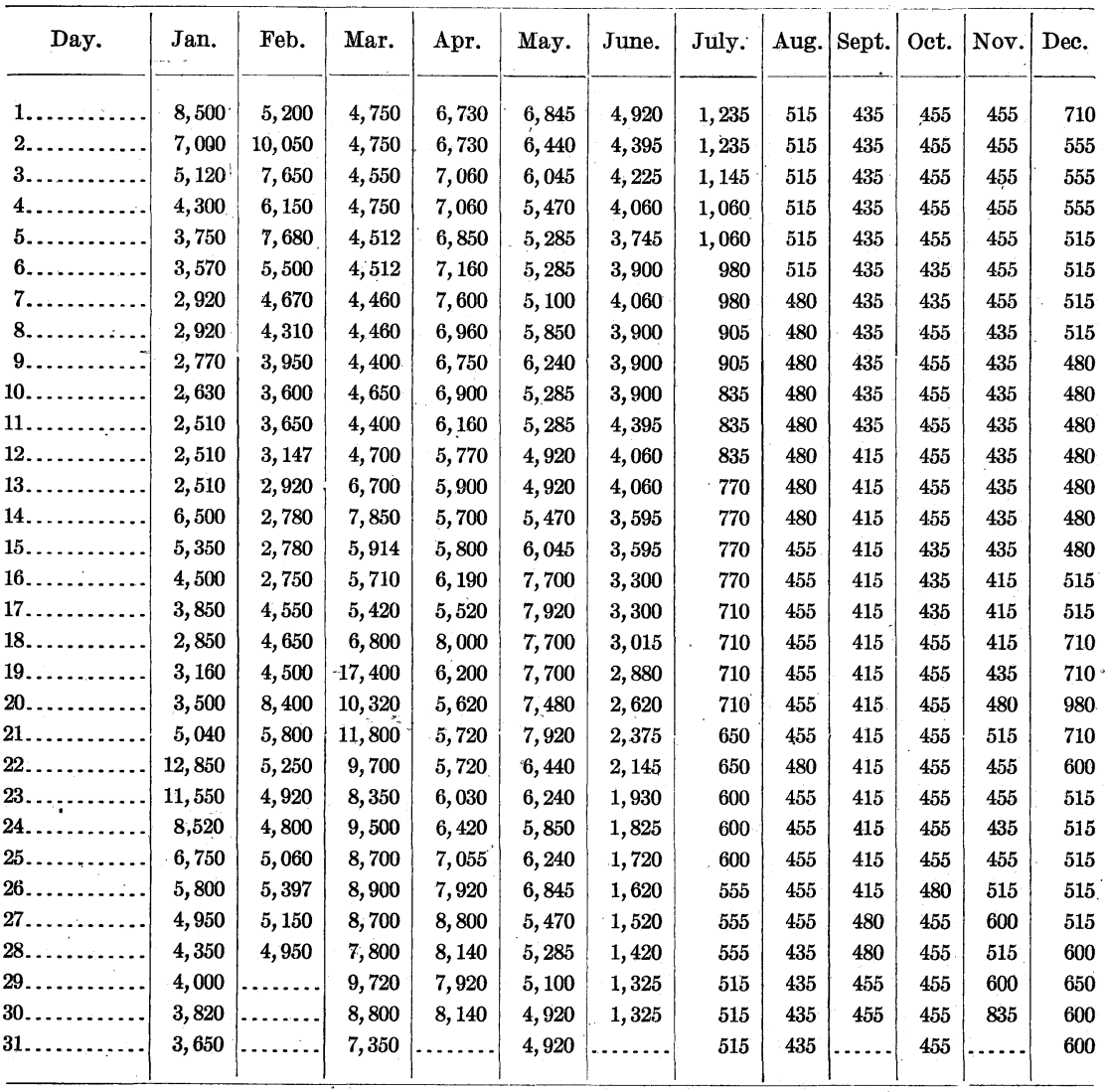

Note.-January 1 to April 24 the daily discharge was obtained indirectly. From April 25 to December 31 the daily discharge was obtained from a rating table based on measurements subsequent to April 24. 
Estimated monthly discharge of Yuba River near Smartsville, Cal., for 1905.

[Drainage area, 1,220 square miles.]

\begin{tabular}{|c|c|c|c|c|c|c|}
\hline \multirow[b]{2}{*}{ Month. } & \multicolumn{3}{|c|}{ Discharge in second-feet. } & \multirow[b]{2}{*}{$\begin{array}{l}\text { Total in } \\
\text { acre-feet. }\end{array}$} & \multicolumn{2}{|c|}{ Run-off. } \\
\hline & Maximum. & Minimum. & Mean. & & $\begin{array}{l}\text { Second-feet } \\
\text { per square } \\
\text { mile. }\end{array}$ & $\begin{array}{l}\text { Depth } \\
\text { in inches. }\end{array}$ \\
\hline January... & 12,850 & 2,510 & 4,903 & 301,500 & 4.02 & 4.64 \\
\hline February.......... & 10,050 & 2,750 & 5,008 & 278,100 & 4.10 & 4.27 \\
\hline $\operatorname{March} . . . . . . . .$. & 17,400 & 4,400 & 7,107 & 437,000 & 5.83 & 6.72 \\
\hline April....... & 8,800 & 5,520 & 6,751 & 401,700 & 5.53 & 6.17 \\
\hline May....... & 7,920 & 4,920 & 6,071 & 373,300 & 4.98 & 5.74 \\
\hline June........ & 4,920 & 1,325 & 3,101 & 184,500 & 2.54 & 2.83 \\
\hline July ........ & 1,235 & 515 & 782 & 48,080 & .641 & .739 \\
\hline August...... & 515 & 435 & 471 & 28,960 & .386 & .445 \\
\hline September... & 480 & 415 & 429 & 25,530 & .352 & .393 \\
\hline October.... & 480 & 435 & 453 & 27,850 & .371 & .428 \\
\hline November. . & 835 & 415 & 474 & 28,200 & .389 & .434 \\
\hline December. . & 980 & 480 & 566 & 34,800 & .464 & .535 \\
\hline The year. & 12,850 & 415 & 3,010 & $2,170,000$ & 2.47 & 33.34 \\
\hline
\end{tabular}

\section{MISCELLANEOUS MEASUREMENTS, YUBA RIVER DRAINAGE BASIN.}

Bay Counties Power Company flume near Nevada City, Cal.-A measurement was made in the flume opposite Purdon bridge over the South Fork of Yuba River on September 3, 1905, by H. A. Campbell.

Width, 6 feet; area, 13.5 square feet; mean velocity, 5.11 feet per second; discharge, 69 second-feet.

Middle Fork of Yuba River near Nevada City, Cal.-A measurement was made one-half mile upstream from Freeman's bridge and about 3 miles north of Nevada City on September 4,1905 , by H. A. Campbell.

Width, 22 feet; area, 31 square feet; mean velocity, 2.06 feet per second; discharge, 64 second-feet.

North Fork of Yuba River near Goodyears bar, Cal.-A measurement was made on this stream September 5, 1905, from the downstream side of the wagon bridge at Goodyears bar by H. A. Campbell.

Width, 44 feet; area, 96 square feet; mean velocity, 1.54 feet per second; discharge, 149 second-feet.

North Fork of North Fork of Yuba River near Downieville, Cal.--This stream enters the North Fork of Yuba River at Downieville, Cal. A measurement was made $1 \frac{1}{2}$ miles north of Downieville and 100 yards above its junction with Middle Fork of North Fork on September 6,1905 , by H. A. Campbell.

Width, 10 feet; area, 18.5 square feet; mean velocity, 0.36 foot per second; discharge, 6.7 second-feet.

A measurement was made 30 yards below junction of Middle Fork of North Fork of Yuba River, about $1 \frac{1}{2}$ miles north of Downieville, Cal., on September 6, 1905, by H. A. Campbell.

Width, 26 feet; area, 37 square feet; mean velocity, 0.73 foot per second; discharge, 27 second-feet.

Wheeler flume near Downieville, Cal.-This flume takes water from the East Fork of the North Fork of Yuba River. A measurement was made on Septembër 6, 1905, at the headgate three-fourths mile north of Downieville by H. A. Campbell.

Width, 3.1 feet; area, 2.3 square feet; mean velocity, 1.91 feet per second; discharge, 4.4 second-feet. 
Davis ditch near Downievitle, Cal.--This ditch is taken out of East Fork of North Fork of Yuba River about a mile above Downieville. A measurement was made one-half mile below the head-gate on September 6, 1905, by H. A. Campbell.

Width, 3.0 feet; area, 1.9 square feet; mean velocity, 1.47 feet per second; discharge, 2.8 second-feet.

East Fork of North Fork of Y Y uba River at Downieville, Cal.-A measurement was made on September 6, 1905, 10 yards above its junction with North Fork of North Fork of Yuba River at Downieville by $\mathrm{H}$ A. Campbell.

Width, 22 feet; area, 29 square feet; mean velocity, 0.59 foot per second; discharge, 17 second-feet.

North Fork of North Fork of Yuba River at Downieville, Cal.-A measurement was made on September 6, 1905, on this stream 10 yards above its junction with North Fork of Yuba River by H. A. Campbell.

Width, 28 feet; area, 52 square feet; mean velocity, 0.77 foot per second; discharge, 40 second-feet.

North Fork of Yuba River at Downieville, Cal.-A measurement was made from the upstream side of the wagon bridge at Downieville on September 6, 1905, by H. A. Campbell.

Width, 40 feet; area, 184 square feet; mean velocity, 0.76 foot per second; discharge, 139 second-feet.

South Fork of North Fork of Yuba River near Sierra City, Cal.--A measurement was made on September 7, 1905, by H. A. Campbell, about one-third of a mile above its junction with the North Fork of the North Fork and about $1 \frac{1}{4}$ miles east of Sierra City.

Width, 2.7 feet; area, 2.5 square feet; mean velocity, 0.68 foot per second; discharge, 1.7 second-feet.

Mining company's flume near Sierra City, Cal.-A measurement was made on September 7,1905 , by $\mathrm{H}$. A. Campbell, about 1 mile below heading.

Width, 2.5 feet; area, 2.2 square feet; mean velocity, 4.50 feet per second; discharge, 9.9 second-feet.

North Fork of North Fork of Yuba River near Sierra City, Cal.-A measurement was made on September 7, 1905, by H. A. Campbell, about one-half mile above its junction with the South Fork of the North Fork and about 1 mile east of Sierra City.

Width, 25 feet; area, 41 square feet; mean velocity, 0.56 foot per second; discharge, 23 second-feet.

\section{BEAR RIVER DRAINAGE BASIN.} DESCRIPTION OF BASIN.

Bear River drains an area of 287 square miles between the Yuba and American rivers. Its headwaters do not reach back to the crest of the range so that it seldom receives precipitation in the form of lasting snow. It is torrential in character, having no forested areas except in its upper portion. The rainfall records kept by the Central Pacific from Auburn to Emigrant Gap are indicative of the precipitiation in the southern part of its basin. A 28-year record at Grass Valley in the northern portion of its watershed gives a mean of 49.41 inches.

BEAR RIVER ABOVE WHEATLAND, CAL.

This station was established by $\mathrm{O}$. W. Peterson on October 8,1904 . It is located about 800 feet below McCourtney Crossing and 8 miles above Wheatland.

The channel is straight for 350 feet both above and below the station. At ordinary stages the velocity is moderate. Neither bank is subject to overflow. The bed of the stream is composed of gravel and is not subject to any material change.

Discharge measurements are made from a car and cable. An auxiliary cable is located 150 feet downstream and parallel to the large one, so that float measurements can be made at very high water. The white-oak tree to which the right end of the cable is fastened is the initial point for soundings.

The gage rods are 300 feet above the station, on the left bank of the river. Two of the sections are bolted to the rock and the upper section is nailed to a tree. During 1905 the 
gage was read once each day by Hermann Ernestus. A standard United States Geological Survey bench mark is sulphured in a hole drilled in the rock between the two upper sections of the gage; elevation, 12.26 feet above the datum of the gage.

A description of this station with gage height and discharge data is contained in WaterSupply Paper No. 134, United States Geological Survey.

Discharge measurements of Bear River above Wheatland, Cal., in 1905.

\begin{tabular}{|c|c|c|c|c|c|c|}
\hline Date. & Hydrographer. & Width. & $\begin{array}{l}\text { Area of } \\
\text { section. }\end{array}$ & $\begin{array}{c}\text { Mean } \\
\text { velocity. }\end{array}$ & $\begin{array}{c}\text { Gage } \\
\text { height. }\end{array}$ & $\begin{array}{l}\text { Dis- } \\
\text { charge. }\end{array}$ \\
\hline & & Feet: & $S q . f t$ & Ft.per sec. & Feet. & Sec.-ft. \\
\hline February $8 . .$. & F. R. S. Buttemer... & 142 & 225 & 4.21 & 5.10 & 948 \\
\hline February $13 . .$. & & 140 & 165 & 3.62 & 4. 57 . & 598 \\
\hline February $19 \ldots$ & ......do... & 139 & 172 & 3.66 & 4. 66 & 629 \\
\hline February $24 .$. & …..do................ & 139 & 151 & 3. 50 & 4,50 & 528 \\
\hline June $27 . . . . . .$. & o. W. Peterson...... & 42 & 47 & 1.89 & 3.42 & 89 \\
\hline July $30 .$. & Peterson and Lee... & 27 & 39 & .95 & 3.10 & 37 \\
\hline September 3... & c. H. Lee............... & 31 & 44 & 1.43 & 3.24 & 63 \\
\hline October $10 . .$. & Hawley and Lee.. & 26 & 20 & 2.15 & 3.00 & 43 \\
\hline
\end{tabular}

Daily gage height, in feet, of Bear River above Wheatland, Cal., for 1905.

\begin{tabular}{|c|c|c|c|c|c|c|c|c|c|c|c|c|}
\hline Day. & Jan. & Feb. & Mar. & Apr. & May. & June. & July. & Aug. & Sept. & Oct. & Nov. & Dec. \\
\hline $1 .$. & 5.0 & 5.6 & 4.3 & a 5.45 & $a 4.1$ & $a 4.1$ & 3. 2 & 3.1 & 3.2 & 3. 1 & .3 .1 & 3.4 \\
\hline $2 .$. & 4.8 & 7.0 & 4.2 & 5.3 & a 4.2 & $a 4.1$ & 3.2 & 3.1 & 3.2 & 3.1 & 3.0 & 3.3 \\
\hline $3 \ldots$ & 4. 3 & 4.9 & 4. 2 & 5.0 & 4.5 & $a_{4.11}$ & 3.2 & 3.1 & 3.2 & 3. 1 & 3.0 & 3.2 \\
\hline 4.. & 4.2 & 6.7 & 4.2 & 4.9 & 4. 2 & $a 4.1$ & 3.2 & 3.1 & 3. 2 & 3. 1 & 3.0 & 3. 2 \\
\hline $5 \ldots$ & 4.2 & 7.0 & 4. 2 & 4.8 & $a 4.4$ & $a 4.1$ & 3.2 & 3.1 & 3.4 & 3.1 & 3.0 & 3.2 \\
\hline $6 \ldots$ & 4. 1 & 5.5 & 4.2 & 4.7 & $a 4.5$ & $a 4.0$ & 3.2 & 3.1 & 3. 2 & 3.1 & 3.0 & 3,1 \\
\hline $7 \ldots$ & 4.0 & 5.1 & 4. 2 & 4.6 & 4.6 & $a 4.0$ & 3.1 & 3.1 & 3.2 & 3.1 & 3.0 & 3.1 \\
\hline 8. & 3.9 & 5.2 & 4.1 & 4.5 & $a 4.7$ & $a 3.8$ & 3.1 & 3.1 & 3.2 & 3.1 & 3.0 & 3.1 \\
\hline $9 \ldots$ & 4.4 & 5.0 & 4.0 & 4.4 & 4.8 & $a 3.8$ & 3.1 & 3.1 & 3. 2 & 3.1 & 3.0 & 3.1 \\
\hline $10 \ldots$ & 4.2 & 4.8 & 4.0 & 4.4 & a 4.7 & $a 3.8$ & 3.1 & 3.1 & 3.1 & 3.1 & 3.0 & 3.0 \\
\hline $11 .$. & 4.0 & 4.9 & 4.0 & 4.4 & a 4.7 & $a 3.8$ & 3.1 & 3.1 & 3.1 & 3.1 & 3.0 & 3.0 \\
\hline $12 \ldots$ & 3.9 & 4.6 & 4. 2 & 4.4 & $a 4.7$ & $a 3.8$ & 3.1 & 3.1 & 3.1 & 3. 1 & 3.0 & 3.0 \\
\hline 13.. & 3.8 & 4.5 & 4.7 & 4.3 & $a 4.7$ & 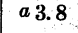 & 3.1 & 3.35 & 3.1 & 3.1 & 2.9 & 3.0 \\
\hline $14 .$. & 5.0 & 4.4 & 4. 6 & 4. 3 & $a 4.7$ & a.8 & 3.1 & 3.1 & 3.1 & 3.1 & 2.9 & 3.0 \\
\hline 15. & 4.5 & 4.3 & 4. 4 & 4.3 & a 5.1 & $a 3.8$ & 3.2 & 3.4 & 3.1 & 3.1 & 2.9 & 3.0 \\
\hline $16 .$. & 4.8 & 4.7 & 4. 4 & 4. 5 & a 5.5 & $a 3.6$ & 3. 1 & 3. 1 & 3.1 & 3.1 & 2.9 & 3.1 \\
\hline $17 .$. & 4. 4 . & 5.0 & 4. 4 & 4.4 & a 5.5 & $a 3.6$ & 3.1 & 3.1 & 3.1 & 3.1 & 2.9 & 3.0 \\
\hline $18 .$. & 4.3 & 4.8 & 4,9 & 4. 9 & a 5.2 & a 3.6 & 3.2 & 3. 1 & 3.0 & 3.1 & 2.9 & 3.0 \\
\hline 19. & 4.9 & 4.7 & 10.5 & 4.9 & a 5.0 & $a 3.6$ & 3.1 & 3.1 & 3.0 & 3.1 & 2.9 & (3.2 \\
\hline $20 .$. & 4. 6 & 5.1 & 6.8 & 4. 6 & a 5.0 & $a 3.6$ & 1 & 3.1 & 0 & 3.1 & 0 & 3.4 \\
\hline$\cdots$ & 6.5 & 4.9 & 6.8 & 4.7 & $a 4.8$ & a 3.6 & 3.1 & 3. 1 & 3.0 & 3.1 & 3.1 & 3.3 \\
\hline 22. & 8.5 & 4.7 & 5.9. & 4.7 & $a 4.6$ & $a 3.6$ & 3.1 & 3.1 & 3.0 & 3.1 & 3.0 & 3.2 \\
\hline $23 \ldots$ & 7.5 & 4.6 & 5.4 & 4. 5 & $a 4.5$ & $a 3.5$ & 3.1 & 3. 1 & 3. 0 & 3.0 & 3.0 & 3.1 \\
\hline $24 .$. & 5.7 & 4.5 & 5.9 & 4.4 & $n 4.4$ & $a 3.5$ & 3.1 & 3.1 & 3.0 & 3. 1 & 3.0 & 3.0 \\
\hline $25 .$. & 5.2 & 4.5 & 5. 3 & 4.3 & $a 4.3$ & $a 3.5$ & 3.1 & 3.1 & 3.2 & 3.1 & 3.0 & 3.0 \\
\hline $26 \ldots$ & 5.0 & 4.4 & 5.5 & 4.2 & $a 4.3$ & $a 3.5$ & 3.1 & 3.1 & 3.1 & 3.1 & 3.0 & 3.0 \\
\hline $27 .$. & 5.8 & 4.4 & 5.7 & $a 4.2$ & $a 4.2$ & 3.5 & 3.1 & 3.1 & 3.1 & 3.0 & 3. 35 & 3.0 \\
\hline 28. & 5.6 & 4.3 & 5.4 & $a 4.2$ & $a 4.2$ & 3.5 & 3.1 & 3.1 & 3.1 & 3.1 & 3.2 & 3.0 \\
\hline 3. & 5.5 & & 7.0 & $a 4.1$ & a4. 2 & 3.4 & 3.1 & 3.1 & 3. 2 & 3.1 & 3.2 & 3.4 \\
\hline 30. & 5,5 & & 6.2 & 4. 1 & $a 4.2$ & 3.3 & 3.1 & 3.3 & 3.1 & 3.1 & 3.5 & 3. 2 \\
\hline 31. & 5,8 & & 5.6 & & 04.2 & & 3.1 & 3. 2 & & 3.1 & $\cdots$ & 3.3 \\
\hline
\end{tabular}

a Estimated. 
Station rating table for Bear River above Wheatland, Cal., from October 9, 1904, to December $31,1905$.

\begin{tabular}{|r|r|r|r|r|r|r|r|}
\hline $\begin{array}{c}\text { Gage } \\
\text { height. }\end{array}$ & Discharge. & $\begin{array}{c}\text { Gage } \\
\text { height. }\end{array}$ & Discharge. & $\begin{array}{c}\text { Gage } \\
\text { height. }\end{array}$ & Discharge. & $\begin{array}{c}\text { Gage } \\
\text { height. }\end{array}$ & Discharge. \\
\cline { 1 - 5 } Feet. & Second-feet. & Feet. & Second-feet. & Feet. & Second-feet. & Feet. & Second-feet. \\
2.90 & 10 & 4.10 & 328 & 5.30 & 1,105 & 7.00 & 3,250 \\
3.00 & 20 & 4.20 & 375 & 5.40 & 1,200 & 7.20 & 3,575 \\
3.10 & 33 & 4.30 & 425 & 5.50 & 1,300 & 7.40 & 3,910 \\
3.20 & 49 & 4.40 & 475 & 5.60 & 1,405 & 7.60 & 4,265 \\
3.30 & 69 & 4.50 & 530 & 5.70 & 1,515 & 7.80 & 4,635 \\
3.40 & 92 & 4.60 & 585 & 5.80 & 1,625 & 8.00 & 5,020 \\
3.50 & 118 & 4.70 & 645 & 5.90 & 1,740 & 8.20 & 5,410 \\
3.60 & 146 & 4.80 & 710 & 6.00 & 1,860 & 8.40 & 5,810 \\
3.70 & 177 & 4.90 & 780 & 6.20 & 2,105 & 8.60 & 6,220 \\
3.80 & 210 & 5.00 & 855 & 6.40 & 2,365 & 8.80 & 6,640 \\
3.90 & 246 & 5.10 & 930 & 6.60 & 2,640 & 9.00 & 7,060 \\
4.00 & 285 & 5.20 & 1,015 & 6.80 & 2,935 & & \\
\hline
\end{tabular}

Note.-The above table is based on 11 discharge measurements made during 1904-5. It is well defined between gage heights 3.1 feet and 5.1 feet. Above 6 feet the table is a rough approximation.

Estimated monthly alischarge of Bear River above Wheatland, Cal., for 1904 and 1905.

[Drainage area, 263 square miles.]

\begin{tabular}{|c|c|c|c|c|c|c|}
\hline \multirow[b]{2}{*}{ Month. } & \multicolumn{3}{|c|}{ Discharge in second-feet. } & \multirow[b]{2}{*}{$\begin{array}{l}\text { Total in } \\
\text { acre-feet. }\end{array}$} & \multicolumn{2}{|c|}{ Run-off. } \\
\hline & Maximum. & Mínimum. & Mean. & & $\begin{array}{l}\text { Second-feet } \\
\text { per square } \\
\text { mile. }\end{array}$ & $\begin{array}{l}\text { Depth } \\
\text { in inches. }\end{array}$ \\
\hline 1904. & & & & & & \\
\hline October $9-31 . . . .$. & 7,060 & 92 & 559 & 25,500 & 2.13 & 1.82 \\
\hline November........ & 1,300 & 92 & 223 & 13,270 & .848 & .946 \\
\hline December...... & 6,640 & 118 & 504 & 30,990 & 1.92 & 2.21 \\
\hline 1905. & & & & & & \\
\hline January ............ & 6,015 & 210 & 1,058 & 65,060 & 4.02 & 4.64 \\
\hline February ...... & 3,250 & 425 & 975 & 54,150 & 3.71 & 3.86 \\
\hline March... & 10,210 & 285 & 1,300 & 79,930 & 4.94 & 5.70 \\
\hline April.... & 1,250 & 328 & 576 & 34,270 & 2.19 & 2.44 \\
\hline May.......... & 1,300 & 328 & 615 & 37,820 & 2.34 & 2.70 \\
\hline June..... & 328 & 69 & 193 & 11,480 & .734 & .819 \\
\hline July ....... & 49 & 33 & 37.1 & 2,281 & .141 & .163 \\
\hline August.... & 92 & 33 & 38.1 & 2,343 & .145 & .167 \\
\hline September..... & 92 & 20 & 37.3 & 2,220 & .142 & .158 \\
\hline October... & 33 & 20 & 32.2 & 1,980 & .122 & .141 \\
\hline November... & 118 & 10 & 23.8 & 1,416 & .090 & .100 \\
\hline December........... & 92 & 20 & 39.8 & 2,447 & .151 & .174 \\
\hline The year...... & 10,210 & 10 & 410 & 295,400 & 1.56 & 21.06 \\
\hline
\end{tabular}

NoTE. -Discharge estimated for missing gage heights. 


\section{MISCELLANEOUS MEASUREMENTS IN BEAR RIVER DRAINAGE BASIN.}

South Yuba Mining Company's ditch near Colfax, Cal.-This ditch is taken out of Bear River $2 \frac{1}{2}$ miles east of Colfax. A measurement was made September 1, 1905, one-eighth of a mile below this heading in flume, by H. A. Campbell.

Width, 6.0 feet; area, 11.8 square feet; mean velocity, 254 feet per second; discharge, 30 second-feet.

Bear River near Colfax, Cal.-A measurement was made September 1, 1905, one-eighth of a mile below intake of the South Yuba Mining Company's canal and $2 \frac{1}{2}$ miles northeast of Colfax, by H. A. Campbell.

Width, 29 feet; area, 17.8 square feet; mean velocity, 1.45 feet per second; discharge, 26 second-feet.

Green Horn River near Colfax, Cal.-A measurement was made 75 yards above the junction of Big Horn and Bear rivers and about $3 \frac{1}{2}$ miles north of Colfax on September 1, 1905, by H. A. Campbell.

Width, 10 feet; area, 6.0 square feet; mean velocity, 1.13 feet per second; discharge, 6.8 second-feet.

Bear River near Colfax, Cal.-A measurement was made on this stream on September 1, 1905, about 100 yards above mouth of Green Horn River and $3 \frac{1}{2}$ miles north of Colfax.

Width, 24 feet; area, 21 square feet; mean velocity, 2.05 feet per second; discharge, 43 second-feet.

\section{CACHE CREEK DRAIN AGE BASIN.}

DESCRIPTION OF BASIN.

Cache Creek drains that portion of the eastern slope of the Coast Range directly north from the Puta Creek basin. This basin is long and narrow, extending from northwest to southeast; it has numerous tributaries, of which North Fork is the largest. . Most of these tributaries are torrential in their character, but the flow of the main stream is regulated largely by its discharge from Clear Lake, which is fed by numerous creeks having their source in the higher portion of the drainage basin. The lake covers an area of 65 square miles, and has a drainage area of 417 square miles. The streams which enter Cache Creek below Clear Lake are practically dry during the summer months. There are large cultivated areas on the west side of Clear Lake, a greater portion of which is meadow land used for stock raising. There are two gaging stations located on this stream-one at Lower Lake directly at the point where the stream discharges from the lake and one at Yolo a short distance below where it emerges from the foothills. There are numerous diversions above the gaging station at Yolo which take practically the entire flow during the summer months. This water is used for irrigation in the vicinity of Woodland and Yolo, where the soil is rich and deep and susceptible of the highest state of cultivation.

\section{CLEAR LAKE AT LAKEPORT, CAL.}

This station was established in January, 1901. It embraces èvaporation for both lake and land. The lake pan is held in place by a triangular raft placed in a protected arm of the lake and anchoredo in such a manner that it has a clear swing, adjusting itself to the wind so one of its angles will cut the water. The pan is submerged to within an inch or so of the water surface. The land pan is located in a clear open space and set in the ground so that its top is flush with the ground surface, it being protected by a small wire fence that it may not be disturbed. These pans are the regulation type, as referred to in Circular No. 4 of instructions for observing evaporation. The record of this station from January, 1901, to December, 1904, was published in Water-Supply Paper No. 134. The observer is Mr. D. C. Rumsey, at Lakeport, Cal. 
Evaporation record of Clear Lake at Lakeport, Cal., 1905.

\begin{tabular}{|c|c|c|c|c|c|}
\hline \multirow{2}{*}{ Month. } & \multicolumn{2}{|c|}{$\begin{array}{c}\text { Evaporation in } \\
\text { inches. }\end{array}$} & \multirow{2}{*}{ Month. } & \multicolumn{2}{|c|}{$\begin{array}{c}\text { Evaporation in } \\
\text { inches. }\end{array}$} \\
\hline & Lake. & Land. & & Lake. & Land. \\
\hline January.... & 0.60 & 0.60 & 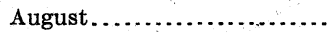 & 7.25 & $\therefore 8.45$ \\
\hline February .......... & .90 & 1.10 & September........ & 5.95 & 6.65 \\
\hline March........... & 1.05 & 1.15 & October.......... & 3.10 & 3.55 \\
\hline April..... & 2.45 & 2.85 & November........ & 1.30 & 1.55 \\
\hline May...... & 3.45 & 3. 70 & December..... & .95 & 1.05 \\
\hline June....... & 6.50 & 7.00 & & & \\
\hline July .... & 7.70 & 8.90 & Annual.. & 41.20 & . 46.55 \\
\hline
\end{tabular}

\section{CACHE CREEK AT LOWER LAKE, CAI.}

This station was established January 1,1900 , by S. G. Bennett. It is located threefourths mile from Lower Lake, Cal.

The channel is straight for 150 feet above and 300 feet below the station. The current has a moderate velocity at ordinary stages. The right bank is low, and will overflow at a gage height of about 10 feet. It is covered with a thick growth of willow and oak trees for 100 feet back from the water's edge. The left bank is high and rocky, and is not liable to overflow. The bed of the stream is composed of firm gravel, and changes only slightly. Gravel is sometimes washed in from Siegler Creek 300 feet below the cable.

Discharge measurements are made from a cable 300 feet above the wagon bridge. The initial point for soundings is a small tree in line with the cable on the left bank, 28 feet from the tree to which the cable is attached.

The present gage is a vertical plank nailed to a timber driven into the bed of the river and fastened to a large willow tree on the left bank 100 feet above the cable. On March 25,1903 , when the new gage was put in place, the reading was 5.7 feet. The old gage read 4.4 feet on the same date. During 1905 the gage was read once each day by Mr. J. R. Anderson. The bench mark is a nail in the root of the oak tree, to which cable is fastened on the left bank; elevation, 8.43 feet above the datum of the gage.

IRR $177-06-12$ 
Information in regard to this station is contained in Water-Supply Papers Nos. 81, 85, 100, and 134 of the United States Geological Survey.

Discharge measurements of Cache Creek at Lower Lake, Cal., in 1905.

\begin{tabular}{|c|c|c|c|c|c|c|}
\hline Date. & Hydrographer. & Width. & $\begin{array}{l}\text { Area of } \\
\text { section. }\end{array}$ & $\begin{array}{c}\text { Mean } \\
\text { velocity. }\end{array}$ & $\begin{array}{c}\text { Gage } \\
\text { height. }\end{array}$ & $\begin{array}{c}\text { Dis- } \\
\text { charge. }\end{array}$ \\
\hline January $1 . . .$. & 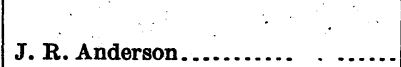 & $\begin{array}{r}\text { Feet. } \\
51\end{array}$ & $S q \cdot f t$. & $\begin{array}{r}\text { Ft. per sec. } \\
1.86\end{array}$ & $\begin{array}{l}\text { Feet. } \\
4.10\end{array}$ & $\begin{array}{r}\text { Sec.-ft. } \\
257\end{array}$ \\
\hline January 7 .. & .....do... & 51 & -146 & 2.05 & 4.20 & 300 \\
\hline January $14 a$. & .....do. & 52 & 159 & 2.08 & 4.50 & 331 \\
\hline January 15. . & ...do.. & 52 & 159 & 2.35 & 4.50 & 373 \\
\hline January 22 . & ....do... & 52 & 178 & 2.61 & 4.90 & $46 i$ \\
\hline January $23 a$. & .....do... & 56 & 222 & 1.35 & 5.60 & 299 \\
\hline January 24 . & .....do... & 52 & 206 & 2.86 & 5.40 & 589 \\
\hline January 26. & ......do... & $5 \mathbf{3}$ & 218 & 3.09 & 5.60 & 673 \\
\hline January 31 . . & ....do... & 53 & 229 & 3.27 & 5.80 & 748 \\
\hline February $1 a \ldots$ & .....do... & 60 & 273 & 2.06 & 6.48 & 562 \\
\hline February $2 a \ldots$ & ...do... & 56 & 254 & 3.11 & 6.20 & 790 \\
\hline February 9 & ...do. & 56 & 266 & 3.63 & 6.40 & 965 \\
\hline February 15 . & .....do... & 56 & 254 & 3.34 & 6.20 & 848 \\
\hline February $21 .$. & ....do.. & 56 & 258 & 3.44 & 6.30 & 888 \\
\hline February $27 .$. & .....do... & 56 & 256 & 3.38 & 6.20 & 864 \\
\hline March 5... & .....do. . & 56 & 249 & 3.33 & 6.10 & 828 \\
\hline March $11 \ldots$ & .....do.. & 56 & 234 & 3.24 & 5.80 & 757 \\
\hline March $13 a$ & .....do...... & 60 & 286 & 2.18 & 6.68 . & 625 \\
\hline March $14 \ldots . .$. & .....do.. & 56 & 248 & 3.28 & 6.08 & 814 \\
\hline March 20... & .....do...... & 59 & 271 & 3.52 & 6.52 & 955 \\
\hline March 25.. & ....do. & 60 & 296 . & 3.69 & 6.90 & 1,092 \\
\hline March $31 \ldots . .$. & ....do,... & 60 & 308 & 3.76 & 7.10 & 1,159 \\
\hline April 7... & $\therefore$ do. & 60 & 305 & 3.63 & 7.00 & 1,108 \\
\hline April 15... & .....do & 56 & 277 & 3.55 & 6.60 & 984 \\
\hline April 22... & .do & 56 & 259 & 3.42 & 6.35 & 886 \\
\hline April $29 . \ldots \ldots$ & .... do & 56 & 248 & 3. 34 & 6.10 & 829 \\
\hline May 6.... & .....do & 56 & 233 & 3.24 & 5.80 & 755 \\
\hline July $11 \ldots \ldots . .$. & O. W. Peter & 54 & 153 & 2.53 & 4.30 & 387 \\
\hline September 29 & C. H. Lee. & 53 & 86 & .93 & 2.83 & 80 \\
\hline October 8...... & J. R. Anderson. & 48 & 73 & 86 & 2.70 & 63 \\
\hline October 14..... & .....do $:$ & 48 & 73 & .82 & 2.70 & 60 \\
\hline October $21 \ldots$. & .....do... & 48 & 68 & .70 & 2.60 & 48 \\
\hline November $5 .$. & .....do. & 48 & 62 & .50 & 2.50 & 31 \\
\hline November $11 .$. & ....do.do. & 48 & 61 & .46 & 2.45 & 28 \\
\hline November $22 .$. & $\therefore$. do & .48 & 58 & .41 & 2.40 & 24 \\
\hline November $27 .$. & .....do... & 48 & 54 & .37 & 2.30 & 20 \\
\hline
\end{tabular}

a Backwater from siegler Creek. 
Daily gage height, in feet, of Cache Creek at Lower Lake, Cal., for 1905.

\begin{tabular}{|c|c|c|c|c|c|c|c|c|c|c|c|c|}
\hline Day. & Jan. & Feb. & Mar. & Apr. & May. & June. & July. & Aug. & Sept. & Oct. & Nov. & Dec. \\
\hline 1.. & 4.1 & 6.5 & 6.2 & .7 .3 & 5.9 & 5.3 & 4.5 & 3.8 & 3.25 & 2.8 & 2.5 & 2.5 \\
\hline 2. & 4.1 & 6.2 & 6.15 & 7.1 & 6.0 & .3 & 4.5 & 3.8 & 3.2 & 2.8 & 2.5 & 2.5 \\
\hline 3.. & 4.15 & 6.25 & 6.15 & 7.05 & 6.0 & 5.25 & 4. 45 & 3.75 & 3.2 & 2.8 & 2.5 & 2. 45 \\
\hline $4 .$. & 4.2 & 6.25 & 6.1 & 7.0 & 5.95 & 5.25 & 4.4 & 3.75 & 3.2 & 2.8 & 2.5 & 2. 45 \\
\hline 5. & 4.2 & 6.35 & 6.1 & 7.0 & 5.90 & 5.2 & 4.4 & 3.7 & 3.2 & 2.8 & 2.5 & 2.45 \\
\hline 6. & 4.2 & 6.4 & 6.1 & 7.0 & 5.8 & 5.2 & 4.4 & 3.7 & 3.15 & 2.8 & 2.5 & 2.4 \\
\hline 7.. & 4.2 & 6.4 & 6.05 & 7.0 & 6.05 & 5.15 & 4.4 & 3.7 & 3.15 & 2.75 & .2 .45 & 2.4 \\
\hline 8.. & 4.2 & 6.4 & 6.0 & 7.0 & 6.0 & 5.15 & 4.4 & 3.7 & 3.15 & 2.75 & 2.45 & 2.4 \\
\hline 9.. & 4.2 & 6.4 & 6.0 & 6.9 & 5.9 & 5.1 & 4. 35 & 3.7 & 3.1 & 2.75 & 2. 45 & 2.4 \\
\hline $10 .$. & 4.2 & 6.4 & 5.9 & $6.8^{-}$ & 5.85 & 5.1 & 4. 35 & 3.7 & 3. & 7 & 2.45 & 2.35 \\
\hline 11.. & 4.2 & 6.3 & 5.8 & 6.8 & 5.8 & 5.05 & 4.3 & 3.65 & 3.1 & 2.7 & 2.45 & 2.35 \\
\hline $12 .$. & 4.2 & 6.3 & 6.2 & 6.85 & 5.8 & 5.0 & 4.3 & 3.65 & 3.05 & 2.7 & 2. 45 & 2.35 \\
\hline $13 .$. & 4.3 & 6.3 & 6.45 & 6.75 & 5.8 & 4.95 & 4.25 & 3.65 & 3.05 & 2.7 & 2.4 & 2.35 \\
\hline 14.. & 4.5 & 6.3 & 6.2 & $6: 6$ & 5.75 & 4.95 & 4. 25 & 3.6 & 3.05 & 2.7 & 2.4 & 2.35 \\
\hline $15 .$. & 4.5 & 6.2 & 6.2 & 6.65. & 5.75 & 4.9 & 4.2 & 3.6 & 3.05 & 2.7 & 2.4 & 2. 45 \\
\hline 16.. & 4.7 & 6.2 & 6.3 & 6.55 & 5.8 & 4.9 & 4. 15 & 3.6 & 3.0 & 2.7 & 2.4 & 2.45 \\
\hline $17 .$. & 4.75 & 6.3 & 6.4 & 6.45 & 5.7 & 4.85 & 4. 15 & 3. 55 & 3.0 & 2.65 & 2.4 & 2.35 \\
\hline 18.. & 4.75 & 6.3 & 6.3 & 6.4 & 5.7 & 4.85 & 4.1 & 3. 55 & 3.0 & 2.65 & 2.4 & 2.45 \\
\hline $19 .$. & 4.8 & 7.0 & 6.6 & 6.5 & 5.7 & 4.8 & 4.1 & 3.5 & 2.95 & 2.6 & 2.4 & 2.5 \\
\hline $20 \ldots$ & 4.8 & 6.3 & 6.55 & 6.3 & 5.6 & 4.8 & 4.1 & 3.5 & 2.95 & 2.6 & 2.5 & 2.5 \\
\hline 21.. & 4.9 & 6.3 & 6.7 & 6.35 & 5.6 & 4.75 & 4.05 & 3.5 & 2.9 & 2.6 & 2.35 & 2. 45 \\
\hline 22. & 5.85 & 6.35 & 6.8 & 6.35 & 5.5 & 4.75 & 4.05 & 3:45 & 2.9 & .6 & 2.4 & 2.4 \\
\hline 23. & 5.55 & 6.35 & 6. & 6.3 & 5.5 & 4.7 & 4.0 & 3.45 & 2. & 6 & 2.25 & 2.5 \\
\hline 24. & 5.7 & 6.3 & 6.95 & 6.25 & 5.5 & 4. & 4.0 & 3. 45 & 2.9 & 2.6 & 2.3 & 2.5 \\
\hline 25. & 5.65 & 6.25 & 6.95 & 6.25 & 5.45 & 4.7 & 4.0 & 3.4 & 2.9 & 2.6 & 2.3 & 2.5 \\
\hline 26 . & 5.65 & 6.25 & 7.1 & 6.2 & 5.4 & 4.65 & 4.0 & 3.4 & 2.9 & 2.6 & 2.35 & 2.5 \\
\hline 27. & 5.75 & 6.2 & 6.95 & 6.2 & 5.4 & 4.6 & 4.0 & 3.35 & 2.9 & 2.6 & 2.3 & 2. 45 \\
\hline 28. & 5.8 & 6.2 & 6.75 & 6.15 & 5.4 & 46 & 3.95 & 3.35 & 2.85 & 2.55 & 2.65 & 2.4 \\
\hline 29 . & 5.8 & $\ldots$ & 7.2 & 6.1 & 5.4 & 4.55 & 3.9 & 3.35 & 2.85 & 2.55 & 2.5 & 2.4 \\
\hline 30. & 5.8 & & 7.2 & 6.05 & 5.45 & 4.5 & 3.9 & 3.3 & 2.8 & 2.55 & 2.5 & 2.5 \\
\hline & 5.8 & & 7.1 & & 5.4 & & 3.85 & 3.3 & & 2.5 & & 2.55 \\
\hline
\end{tabular}

Station rating table for Cache Creek at Lower Lake, Cal., from January 1 to December 31, 1905.

\begin{tabular}{|r|r||r|r|r|r|r|r|}
\hline $\begin{array}{c}\text { Gage } \\
\text { height. }\end{array}$ & Discharge. & $\begin{array}{c}\text { Gage } \\
\text { height. }\end{array}$ & Discharge. & $\begin{array}{c}\text { Gage } \\
\text { height. }\end{array}$ & Discharge. & $\begin{array}{c}\text { Gage } \\
\text { height. }\end{array}$ & Discharge. \\
\cline { 1 - 5 } Feet. & Second-feet. & Feet. & Second-feet. & Feet. & Second-feet. & Feet: & Second-feet. \\
2.30 & 17 & 3.40 & 149 & 4.50 & 365 & 5.60 & 670 \\
2.40 & 27 & 3.50 & 163 & 4.60 & 390 & 5.70 & 701 \\
2.50 & 37 & 3.60 & 178 & 4.70 & 416 & 5.80 & 732 \\
2.60 & 48 & 3.70 & 194 & 4.80. & 442 & 5.90 & 763 \\
2.70 & 59 & 3.80 & 212 & 4.90 & 469 & 6.00 & 795 \\
2.80 & 71 & 3.90 & 232 & 5.00 & 496 & 6.20 & 859 \\
2.90 & 83 & 4.00 & 252 & 5.10 & 524 & 6.40 & 923 \\
3.00 & 95 & 4.10 & 274 & 5.20 & 552 & 6.60 & 988 \\
3.10 & 108 & 4.20 & 296 & 5.30 & 581 & 6.80 & 1,054 \\
3.20 & 121 & 4.30 & 318 & 5.40 & 610 & 7.00 & 1,120 \\
3.30 & 135 & 4.40 & 341 & 5.50 & 640 & 7.20 & 1,188 \\
\hline
\end{tabular}

NotE.-The above table is based on 28 discharge measurements made during 1905 and during the latter part of 1904. It is well defined throughout. 
Estimated monthly discharge of Cache Creek near Lower Lake, Cal., for 1905.

[Drainage area, 500 square miles.]

\begin{tabular}{|c|c|c|c|c|c|c|}
\hline \multirow{2}{*}{ Month. } & \multicolumn{3}{|c|}{ Discharge in second-feet. } & \multirow[b]{2}{*}{$\begin{array}{l}\text { Total in } \\
\text { acre-feet. }\end{array}$} & \multicolumn{2}{|c|}{ Run-off. } \\
\hline & Maximum. & Minimum. & Mean. & & $\begin{array}{l}\text { Second-feet } \\
\text { per square } \\
\text { mile. }\end{array}$ & $\begin{array}{l}\text { Depth } \\
\text { in inches. }\end{array}$ \\
\hline January. & 748 & 274 & 448 & 27,550 & 0.896 & 1.03 \\
\hline February.... & 1,120 & 562 & 884 & 49,100 & 1.77 & 1.84 \\
\hline March....... & 1,188 & 610 & 933 & 57,370 & 1.87 & 2.16 \\
\hline April.. & 1,222 & 811 & 991 & 58,970 & 1.98 & 2.21 \\
\hline May... & 811 & 610 & 702 & 43,160 & 1.40 & 1.61 \\
\hline June... & $581^{\circ}$ & 365 & 476 & 28,320 & 952 & 1.06 \\
\hline July.... & 365 & 222 & 294 & 18,080 & .588 & .678 \\
\hline August... & 212 & 135 & 173 & 10,640 & 346 & .399 \\
\hline September. & 128 & 71 & 98.5 & 5,861 & .197 & .220 \\
\hline October....... & 71 & 37 & 56.0 & 3,443 & .112 & .129 \\
\hline November........ & 54 & 12 & 30.1 & 1,791 & .060 & .067 \\
\hline December. & 42 & 22 & $3 n .7$ & 1,888 & .061 & .070 \\
\hline The year. & 1,222 & 12 & 426 & 306,200 & .853 & 11.47 \\
\hline
\end{tabular}

CACHE OREEK NEAR YOLO, CAL.

This station was established January 1,1903 , by S. G. Bennett. It is located at the wagon bridge on the road from Woodland to Yolo, about 1,000 feet above the Southern Pacific Railroad bridge. A new wagon bridge, which greatly improves the channel conditions, was erected during 1904 . The station was reestablished on the new bridge Decem. ber 4, 1904.

Numerous diversions are made from Cache Creek above this station which take practically all of the summer flow. The channel is straight for 1,000 feet above and below the station. The current is swift at ordinary and high stages. The banks are steep and wooded and their height has been increased by levees. They are said to overflow at extreme high water. The bed of the stream is composed of earth and gravel, with a little sand, and is not subject to any material change.

Discharge measurements are made from the downstream side of the bridge. The initial point for soundings is the end of the bridge on the right bank.

The gage is a staff in four sections, three of which are above the bridge and the fourth is bolted to the face of the concrete abutment on the right bank. During 1905 the gage was read by John Woodard. The bench mark is corner of top of concrete abutment to which the high-water section of gage is fastened and directly over gage; elevation, 31.68 feet above the datum of the gage. 
Information in regard to this station is contained in Water-Supply Papers Nos. 100 and 134 of the United States Geological Survey.

Discharge measurements of Cache Creek near Yolo, Cal., in 1905.

\begin{tabular}{|c|c|c|c|c|c|c|}
\hline Date. & Hydrographer. & Width. & $\begin{array}{l}\text { Area of } \\
\text { section. }\end{array}$ & $\underset{\text { velocity. }}{\text { Mean }}$ & $\begin{array}{c}\text { Gage } \\
\text { height. }\end{array}$ & $\begin{array}{c}\text { Dis- } \\
\text { charge. }\end{array}$ \\
\hline Tebruary $2 \ldots$ & O W. Peterson. & $\begin{array}{r}\text { Feet. } \\
114\end{array}$ & $\begin{array}{l}S q . f t . \\
1,255\end{array}$ & $\begin{array}{r}\text { Ft. per sec. } \\
6.07\end{array}$ & $\begin{array}{l}\text { Feet. } \\
11.75\end{array}$ & $\begin{array}{c}\text { Sec.-ft. } \\
7,624\end{array}$ \\
\hline February 3... & ....do.... & 107 & 833 & 5.83 & 8.30 & 4,860 \\
\hline Do..... & ....do.... & 108 & .890 & 5.87 & 8.80 & 5,227 \\
\hline February 4... & .....do... & 102 & 630 & 6.03 & 7.05 & 3,802 \\
\hline February $15 \ldots$ & .....do.......... & 94 & 345 & 4.39 & 4.85 & 1,516 \\
\hline May $16 \ldots . . . .$. & W. B. Clapp......... & 91 & 275 & 3.73 & 4.05 & 1,027 \\
\hline June $5 . . . . . .$. & Peterson and Rodman...... & 89 & 215 & 3.25 & 8.40 & 699 \\
\hline June $24 \ldots . . .$. & O. W. Peterson............ & 89 & 151 & 2.37 & 2.60 & 358 \\
\hline August $3 \ldots .$. & . ...do. . . & 87 & 88 & 1.47 & 1.79 & 129 \\
\hline September 7... & C. H. Lee......... & 32 & 19 & .67 & 1.26 & 13.0 \\
\hline September $13 .$. & 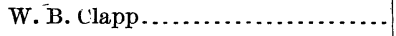 & 17 & 7.3 & .64 & 1.15 & 4.7 \\
\hline October $3 . . . .$. & Lee and Hawley .......... & 2 & .5 & .80 & 1.09 & .4 \\
\hline
\end{tabular}

Daily gage height, in feet, of Cache Creek near Yolo, Cal., for 1905.

\begin{tabular}{|c|c|c|c|c|c|c|c|c|c|c|}
\hline Day. & Jan. & Feb. & Mar. & Apr. & May. & June. & July. & Aug. & Sept. & Oct. \\
\hline $1 .$. & 5.0 & 6.0 & 4.7 & 5.9 & 4.2 & 3.6 & 2.55 & 1.8 & 1.4 & 1.0 \\
\hline 2. & 4.05 & 12.4 & 4.6 & 5.8 & 4.2 & 3.6 & 2.5 & 1.8 & 1.4 & 1.0 \\
\hline 3.. & 3.65 & 9.05 & 4.6 & 5.7 & 4.2 & 3.5 & 2.5 & 1.75 & 1.4 & 1.0 \\
\hline 4.. & 3.45 & 7.35 & 4.5 & 5.6 & 4.15 & 3.5 & 2.5 & 1.75 & 1.35 & 1.1 \\
\hline $5 .$. & 3.25 & 6.4 & 4.5 & 5.5 & 4.15 & 3.45 & 2.45 & 1.7 & 1.3 & 1.1 \\
\hline $6 \ldots$ & 3.1 & 6.0 & 4. 45 & 5.4 & 4.15 & 3.4 & 2.45 & 1.7 & 1.3 & 1.1 \\
\hline $7 \ldots$ & 3.1 & 5.6 & 4.4 & 5.3 & 4.1 & 3.35 & 2.4 & 1.7 & 1.25 & 1.1 \\
\hline $8 .$. & 3.05 & 5.35 & 4.4 & 5.2 & 4.75 & 3.3 & 2.4 & 1.75 & 1.25 & 1.1 \\
\hline $9 \ldots$ & 3.0 & 5.2 . & 4. 35 & 5.2 & 4.5 & 3.25 & 2.4 & 1.8 & 1.2 & 1.1 \\
\hline $10 \ldots$ & 3.0 & 5.1 & 4.35 & 5.1 & 4.4 & 3.2 & 2.35 & 1.8 & 1.15 & 1.05 \\
\hline $11 .$. & 3.0 & 5.0 & 4.3 & 5.1 & 4.3 & 3.15 & 2.3 & 1.75 & 1.15 & 1.05 \\
\hline 12. & 2.95 & 4.95 & 4.3 & 5.05 & 4. 25 & 3.1 & 2.25 & 1. 75 & 1.1 & 1.05 \\
\hline 13. & 3.2 & 4.9 & 5.65 & 5.0 & 4.2 & 3.1 & 2.2 & 1.7 & 1.1 & 1.1 \\
\hline 14. & 9.95 & 4.8 & 6.05 & 5.0 & 4.2 & 3.05 & 2.2 & 1.7 & 1.1 & 1.1 \\
\hline $15 .$. & 6.4 & 4.8 & 5.85 & 4.9 & 4. 15 & 3.05 & 2.15 & 1.65 & 1.15 & 1.1 \\
\hline $16 \ldots$ & 5.3 & 4.75 & 6.4 & 4.9 & 4.15 & 3.0 & 2.15 & 1.65 & 1.1 & 1.1 \\
\hline 17. & 7.4 & 5.3 & 6.2 & 4.85 & 4.1 & 3.0 & 2.1 & 1.65 & 1.1 & \\
\hline $18 \ldots$ & 7.0 & 5.25 & 5.7 & 4.8 & 4.1 & 3.0 & 2.1 & 1.6 & 1.1 & \\
\hline $19 .$. & 6.55 & 5.2 & 6.0 & 4.75 & 4.1 & 2.95 & 2.0 & 1.6 & $1: 15$ & \\
\hline $20 \ldots$ & 5.3 & 5.1 & 6.0 & 4.7 & 4.05 & 2.9 & 2.0 & 1.6 & 1.15 & \\
\hline $21 \ldots$ & 5.05 & 5.0 & 5.95 & 4.65 & 4.05 & 2.9 & 1.95 & 1.6 & 1.1 & \\
\hline $22 \ldots$ & 8.25 & 5.0 & 5.9 & 4.6 & 4.0 & 2.85 & 1.9 & 1.65 & 1.1 & \\
\hline $2 \lambda \ldots$ & 15.55 & 4.95 & 6.3 & 4.55 & 4.0 & 2.8 & 1.9 & 1.55 & 1.05 & \\
\hline $24 .$. & 8.8 & 4.9 & 6.2 & 4.5 & 3.95 & 2.8 & 1.85 & 1.55 & 1.05 & \\
\hline 25. & 9.25 & 4.85 & 6.1 & 4.45 & 3.95 & 2.75 & 1.8 & 1.55 & 1.05 & \\
\hline $26 \ldots$ & 7.0 & 4.8 & 6.05 & 4.4 & 3.9 & 2.7 & 1.8 & 1.5 & 1.05 & \\
\hline $27 \ldots$ & 6.2 & 4.8 & 6.0 & 4.35 & 3.9 & 2.7 & 1.75 & 1.5 & 1.05 & - \\
\hline $28 \ldots$ & 5.8 & 4.7 & 5.9 & 4.3 & 3.8 & 2.65 & 1.75 & 1.5 & 1.05 & . \\
\hline $29 \ldots$ & 5.4 & & 7.1 & 4.25 & 3.8 & 2.6 & 1. 6 & 1.45 & 1.0 & \\
\hline $30 \ldots$ & 5.15 & & 6.85 & 4.2 & 3.7 & 2.6 & 1.6 & 1.45 & 1.0 & $\cdots$ \\
\hline $31 \ldots$ & 5.0 & & 6.1 & $\ldots$. & 3.7 & 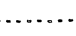 & 1.6 & 1.4 & & \\
\hline
\end{tabular}

NoTE.-Creek dry October 17 to December 31. 
Station rating table for Cache Creek near Yolo, Cal., from January 1, to December 31, 1905.

\begin{tabular}{|c|c|c|c|c|c|c|c|}
\hline $\begin{array}{c}\text { Gage } \\
\text { height. }\end{array}$ & Discharge. & $\begin{array}{r}\text { Gage } \\
\text { height. }\end{array}$ & Discharge. & $\begin{array}{l}\text { Gage } \\
\text { height. }\end{array}$ & Discharge. & $\begin{array}{c}\text { Gage } \\
\text { height. }\end{array}$ & Discharge. \\
\hline $\begin{array}{r}\text { Feet. } \\
1.00\end{array}$ & $\mid \begin{array}{r}\text { Second-feet. } \\
0\end{array}$ & $\begin{array}{r}\text { Feet. } \\
2.60\end{array}$ & \begin{tabular}{|} 
Second-feet. \\
361
\end{tabular} & $\begin{array}{r}\text { Feet. } \\
4.40\end{array}$ & $\begin{array}{c}\text { Second-feet. } \\
1,230\end{array}$ & $\begin{array}{r}\text { Feet. } \\
7.60\end{array}$ & $\begin{array}{c}\text { Second-feet } \\
3,635\end{array}$ \\
\hline 1.10 & 1 & 2.70 & 395 & 4.60 & 1,350 & 7.80 & $3,815 \cdot$ \\
\hline 1.20 & 7 & 2.80 & 430 & 4.80 & 1,480 & 8.00 & 4,000 \\
\hline 1.30 & 17 & 2.90 & 467 & 5.00 & 1,610 & 8.50 . & 4,475 \\
\hline 1.40 & 32 & 3.00 & 505 & 5.20 & 1,745 & $9.00^{\circ}$ & 4,970 \\
\hline 1.50 & 53 & 3.10 & 550 & 5. 40 & 1,885 & 9.50 & 5,470 \\
\hline 1.60 & 76 & 3.20 & 595 & 5.60 & 2,025 & 10.00 & 5,980 \\
\hline 1.70 & 100 & 3.30 & 640 & 5.80 & 2,170 & 10.50 & 6,505 \\
\hline 1.80 & 125 & 3: 40 & 690 & 6.00 & 2,320 . & 11.00 & 7,050 \\
\hline 1.90 & 151 & 3.50 & 740 & 6.20 & 2,470 & 11.50 & 7,600 \\
\hline 2.00 & 178 & 3.60 & 790 & $6.40^{\circ}$ & 2,625 & 12.00 & 8,150 \\
\hline 2.10 & 206 & 3. 70 & 840 & 6.60 & 2,785 & 13.00 & 9,300 \\
\hline 2.20 & 235 & 3.80 & 890 & 6.80 & 2,945 . & 14.00 & 10,500 \\
\hline 2.30 & 265 & 3.90 & 945 & 7.00 & 3,110 & 15.00 & $\quad 11,700$ \\
\hline 2.40 & 296 & 4.00 & 1,000 & 7.20 & 3,280 & 16.00 & 12,950 \\
\hline 2.50 & 328 & 4.20 & 1,110 & 7.40 & 3,455 & & \\
\hline
\end{tabular}

Note.-The above table is based on 12 discharge measurements made during 1905. It is well defined between gage heights 0 and 5 feet.

Estimated monthly discharge of Cache Creek near Yolo, Cal., for 1905.

[Drainage area, 1,280 square miles.]

\begin{tabular}{|c|c|c|c|c|c|c|}
\hline \multirow{2}{*}{ Month. } & \multicolumn{3}{|c|}{ Discharge in second-feet. } & \multirow{2}{*}{$\begin{array}{l}\text { Total in } \\
\text { acre-feet. }\end{array}$} & \multicolumn{2}{|c|}{ Run-off. } \\
\hline & Maximum. & Minimum. & Mean. & & $\begin{array}{l}\text { Second-feet } \\
\text { per square } \\
\text { mile. }\end{array}$ & $\begin{array}{l}\text { Depth } \\
\text { in inches. }\end{array}$ \\
\hline January ... & 12,390 & 486 & 2,313 & 142,200 & 1.81 & 2.09 \\
\hline February... & 8,610 & 1,415 & 2,142 & 119,000 & 1.67 & 1.74 \\
\hline March.............. & 3,195 & 1,170 & 1,969 & 121,100 & 1.54 & 1.78 \\
\hline April...... & 2,245 & 1,110 & 1,576 & 93,780 & 1.23 & 1.37 \\
\hline May ........ & 1,448 & 840 & 1,058 & 65,050 & .827 & .953 \\
\hline June....... & 790 & 361 & 542 & 32,250 & .423 & .472 \\
\hline July . . . . . . . . . & 344 & 76 & 214 & 13,160 & .167 & .192 \\
\hline August ......... & 125 & 32 & 86.5 & 5,319 & .068 & .078 \\
\hline September. & 32 & $\mathbf{0}$ & 7.1 & 423 & .0055 & .0061 \\
\hline October.... & 1 & $\mathbf{0}$ & .32 & 20 & .00025 & .00029 \\
\hline November.. & 0 & 0 & 0 & 0 & 0 & 0 \\
\hline December .... & 0 & 0 & 0 & 0 & 0 & 0 \\
\hline The year.. & 12,390 & 0 & 826 & 592,300 & .645 & 8. 68 \\
\hline
\end{tabular}

Note.-Estimates January 1 to February 10 are subject to considerable error owing to the scouring and filling at this section during high water. 


\section{MISCELLANEOUS MEASUREMENTS IN CACHE CREEK DRAINAGE BASIN,}

Clover Creek near Upper Lake, Cal.--This stream discharges into Clear Lake. A measurement was made July 12 by $\mathrm{O}$. W. Peterson, one-third of a mile above town of Upper Lake.

Width, 9.2 feet; area, 5.5 square feet; mean velocity, 0.49 foot per second; discharge, 2.7 second-feet.

Cole Creek at Kelseyville, Cal.-This stream discharges into Clear Lake. A measurement. was made July 11, 1905, by O. W. Peterson, near Kelseyville and Lower Lake road, threefourths of a mile below Kelseyville.

Width, 11.5 feet; area,4.3 square feet; mean velocity, 1.00 foot per second; discharge, 4.3 second-feet.

Capay ditch at Capay, Cal.-This ditch diverts water from Cache Creek. The following measurements were made at head of ditch at Capay by $\mathrm{O}$. W. Peterson.

June 19: Width, 18.8 feet; area, 34 square feet; mean velocity, 1.80 feet per second; discharge, 61 second-feet.

August 2: Width, 18.8 feet; area, 21 square feet; mean velocity, 1.57 feet per second; gage-height, 1.10 feet; discharge, 33 second-feet.

Kelsey Creek near Kelseyville, Cal.-This stream discharges into Clear Lake.'A measurement was made July 11 by O. W. Peterson, $1 \frac{1}{4}$ miles above Kelsey Creek Mill, about $3 \frac{1}{2}$ miles above the town of Kelseyville.

Width, 16.5 feet; area, 9 square feet; mean velocity, 1.29 feet per second; discharge, 11.6 second-feet.

Middle Crcek near Upper Lake, Cal.--This stream discharges into Clear Lake. A measurement was made July 12 by O. W. Peterson, $1 \frac{1}{2}$ miles above the town of Upper Lake in sec. 31 , T. $15 \mathrm{~N}$, R. 9 W, M. D. M.

Width, 9.9 feet; area, 8.3 square feet; mean velocity, 0.48 foot per second; discharge, 4.0 second-feet.

Moore's ditch near Woodland, Cal.-The following measurements were made at Walker Bridge, one-half mile below canal heading:

June 3: Width, 21.6 feet; area, 89 square feet; mean velocity, 0.90 foot per second; discharge, 80 second-feet.

June 3: Width, 20 feet; area, 102 square feet; mean velocity, 1.69 feet per second; discharge, 172 second-feet.

June 17: Width, 24.5 feet; area, 72 square feet; mean velocity, 0.83 foot per second; gage height, 4.20 feet; discharge, 60 second-feet.

June 17: Width, 26 feet; area, 89 square feet; mean velocity, 1.13 feet per second; gage height, 4.80 feet; discharge, 101 second-feet.

June 17: Width, 35 feet; area, 119 square feet; mean velocity, 1.36 feet per second; gage height, 5.55 feet; discharge, 162 second-feet.

June 21: Width, 25.2 feet; area, 74 square feet; mean velocity, 1.65 feet per second; gage height, 5.45 feet; discharge, 122 second-feet.

August 2: Width, 19.8 feet; area, 87 square feet; mean velocity, 1.14 feet per second; gage height, 4.40 feet; discharge, 99 second-feet.

\section{AMERICAN RIVER DRAINAGE BASIN.}

\section{DESCRIPTION OF BASIN.}

American River drains an area of about 2,000 square miles of the western slope of the Sierra Nevada. This drainage basin lies between those of the Bear and Yuba rivers on the north and that of Consumnes River on the south. It has three main forks, heading in the summit of the range, which reaches an elevation of about 9,000 feet. The country lying between these main forks is drained by numerous small tributaries. The formation in the higher and greater portions of this basin is of granite, with a considerable timber 
growth. The flow is rather torrential during the winter months, due to the large area of barren and sparsely timbered country in the lower portion of the watershed. The precipitation on the higher elevations is in the form of snow, which usually melts late in the spring. Rainfall records have been kept along the line of the Central Pacific Railroad, which follows the ridge to the north of North Fork.

The mean annual rainfall at Auburn is 33.40 inches, that at Colfax 47.4 inches, and at Cisco and Emigrant Gap about 50 inches. At Georgetown, between North and Middle forks, a 30-year record has an average of 56.72 inches, and at Placerville, above South Fork, another of about the same length shows 43.58 inches.

There are several small lakes in the upper reaches of this basin, the storage capacity of a few having been increased by the construction of low dams at their outlets. This stored water is used for mining purposes during the low-water flow and is used entirely within the drainage basin.

\section{AMERICAN RIVER NEAR FAIROAKS, CAL.}

This station was established November 3, 1904, by O. W. Peterson. It is located at Fairoaks Bridge, near Fairoaks.

The channel is straight for 400 feet above and below the station. At ordinary stages the velocity is sluggish. The right bank is not subject to overflow. At times of very high water the left bank is subject to overflow and a second channel is formed. The bed of the stream is composed of gravel, and is subject to slight changes at times of high water. At ordinary stages the river is about 210 feet in width and averages over 4 feet in depth.

Discharge measurements are made from the downstream side of the bridge. The vertical face of the right abutment is 4 feet from the initial point for soundings.

The gage is a staff nailed to one of the piles at the upper side at the right end of Fairoaks Bridge. During 1905 the gage was read twice each day by W. F. Bailey, jr. The bench mark is a nail driven in the guard rail over the upstream center pier; elevation, 31.00 feet above the datum of the gage.

A description of this station, with gage height and discharge data, is contained in Water-Supply Paper No. 134 of the United States Geological Survey.

Discharge measurements of American River near Fairoaks, Cal., in 1905.

\begin{tabular}{|c|c|c|c|c|c|c|}
\hline Date. & Hydrographer. & Width. & $\begin{array}{l}\text { Area of } \\
\text { section. }\end{array}$ & $\begin{array}{c}\text { Mean } \\
\text { velocity. }\end{array}$ & $\begin{array}{c}\text { Gage } \\
\text { height. }\end{array}$ & $\begin{array}{c}\text { Dis- } \\
\text { charge. }\end{array}$ \\
\hline January $26 \ldots$ & F. R. S. Buttemer.. & $\begin{array}{r}\text { Feet. } \\
356\end{array}$ & $\begin{array}{r}S q . f t . \\
1,648\end{array}$ & $\begin{array}{r}\text { Ft. per sec. } \\
2.41\end{array}$ & $\begin{array}{l}\text { Freet. } \\
\quad 4.52\end{array}$ & $\begin{array}{r}\text { Sec. }-f t . \\
\quad 3,971\end{array}$ \\
\hline January $26 \ldots$ & .....do... & 356 & 1,648 & 2.42 & 4. 49 & 3,994 \\
\hline February 2... & .....do $\ldots . . . . .$. & 373 & 2,891 & 4.35 & 7.55 & 12,580 \\
\hline February $11 \ldots$ & ......do... & 353 & 1,505 & 2.09 & 4.08 & 3,151 \\
\hline February $17 \ldots$ & ......do.... & 354 & 1,544 & 2.19 & 4.22 & 3,378 \\
\hline February $22 .$. & .....do........... & 355 & 1,776 & 2.67 & 4.90 & 4,742 \\
\hline May $17 \ldots . .$. & W. B. Clapp....... & 370 & 2,864 & 4.34 & 7.35 & 12,340 \\
\hline June $28 \ldots . .$. & 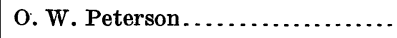 & 260 & 993 & 1.53 & 2.88 & 1,520 \\
\hline July $29 . \ldots \ldots$ & Peterson and Lee................. & 167 & 694 & .56 & 1.46 & 392 \\
\hline September $4 .$. & C. H. Lee........... & 165 & 599 & .20 & .88 & 119 \\
\hline
\end{tabular}


Daily gage height, in feet, of American River near Fairoaks, Cal., for 1905.

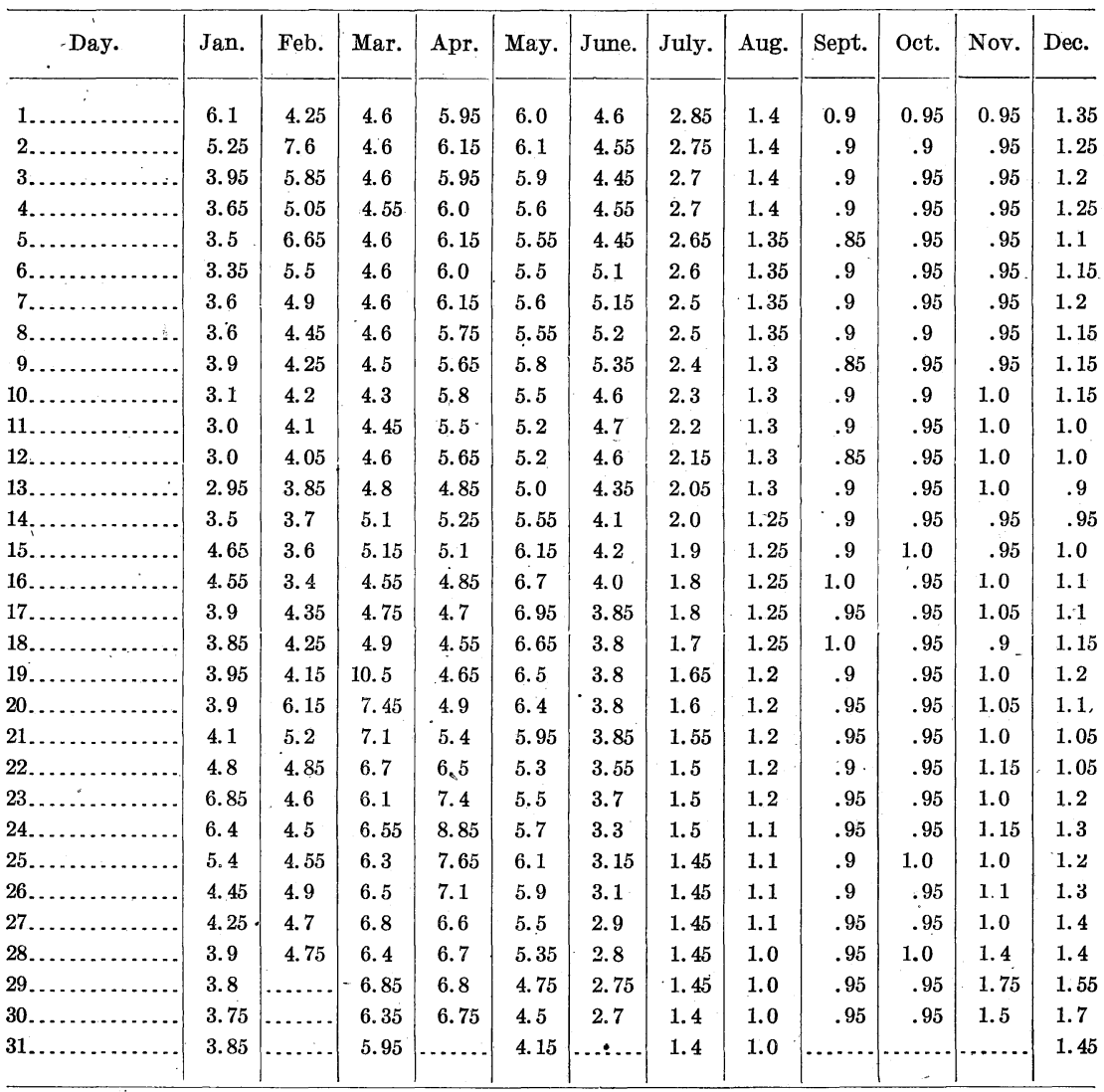

NoтE.-Gage heights estimated July 2-28; July 30 to September 2; November 1-9.

Station rating table for. American River near Fairoaks, Cal., from November 4 to December 31, 1904.

\begin{tabular}{|c|c||c|c|c|c||c|c|}
\hline $\begin{array}{c}\text { Gage } \\
\text { height. }\end{array}$ & Discharge. & $\begin{array}{c}\text { Gage } \\
\text { height. }\end{array}$ & Discharge. & $\begin{array}{c}\text { Gage } \\
\text { height. }\end{array}$ & Discharge. & $\begin{array}{c}\text { Gage } \\
\text { height. }\end{array}$ & Discharge. \\
\hline Feet. & Second-feet. & Feet. & Second-feet. & Feet. & Second-feet. & Feet. & Second-feet. \\
2.40 & 700 & 2.70 & 990 & 3.00 & 1,300 & 3.30 & 1,680 \\
2.50 & 790 & 2.80 & 1,090 & 3.10 & 1,410 & & \\
2.60 & 890 & 2.90 & 1,190 & 3.20 & 1,540 & & \\
& & & & & \\
\hline
\end{tabular}

NoтE.-The above table is based on 2 discharge measurements made during 1904, and on the 1905 curve. 
Station rating table for American River near Fairoaks, Cal., from January 1 to December 31, 1905.

\begin{tabular}{|r|r||r|r||r|r||r|r|}
\hline $\begin{array}{r}\text { Gage } \\
\text { height. }\end{array}$ & Discharge. & $\begin{array}{c}\text { Gage } \\
\text { height. }\end{array}$ & Discharge. & $\begin{array}{c}\text { Gage } \\
\text { height. }\end{array}$ & Discharge. & $\begin{array}{c}\text { Gage } \\
\text { height. }\end{array}$ & Discharge. \\
\cline { 1 - 6 } Feet. & Second-feet. & Feet. & Second-feet. & Feet. & Second-feet. & Feet. & Second-feet. \\
0.80 & 90 & 2.20 & 840 & 3.60 & 2,410 & 6.00 & 7,450 \\
0.90 & 120 & 2.30 & 920 & 3.70 & 2,555 & 6.20 & 8,040 \\
1.00 & 155 & 2.40 & 1,005 & 3.80 & 2,705 & 6.40 & 8,670 \\
1.10 & 195 & 2.50 & 1,095 & 3.90 & 2,860 & 6.60 & 9,340 \\
1.20 & 240 & 2.60 & 1,195 & 4.00 & 3,020 & 6.80 & 10.040 \\
1.30 & 285 & 2.70 & 1,300 & 4.20 & 3,365 & 7.00 & 10,770 \\
1.40 & 335 & 2.80 & 1,410 & 4.40 & 3,730 & 7.20 & 11,530 \\
1.50 & 390 & 2.90 & 1,525 & 4.60 & 4,115 & 7.40 & 12,310 \\
1.60 & 445 & 3.00 & 1,640 & 4.80 & 4,520 & 7.60 & 13,090 \\
1.70 & 505 & 3.10 & 1,760 & 5.00 & 4,950 & 7.80 & 13,890 \\
1.80 & 565 & 3.20 & 1,880 & 5.20 & 5,405 & 8.00 & 14,700 \\
1.90 & 630 & 3.30 & 2,005 & 5.40 & 5,880 & & \\
2.00 & 695 & 3.40 & 2,135 & 5.60 & 6,375 & & \\
2.10 & 765 & 3.50 & 2,270 & 5.80 & 6,900 & &. \\
\hline
\end{tabular}

Note.-The above table is based on 10 discharge measurements made during 1905 . It is fairly well defined between gage heights 0.8 foot and 5 feet.

Estimated monthly discharge of American River near Fairoaks, Cal., for 1904 and 1905.

\begin{tabular}{|c|c|c|c|c|}
\hline \multirow{2}{*}{ Month. } & \multicolumn{3}{|c|}{ Discharge in second-feet. } & \multirow{2}{*}{$\begin{array}{l}\text { Tótal in } \\
\text { acre-feet. }\end{array}$} \\
\hline & Maximum. & Minimum. & Mean. & \\
\hline 1904. & & & & $\therefore$ \\
\hline November $4-30, \ldots \ldots$ & 1,355 & 700 & 896 & 47,980 \\
\hline December........... & 13,600 & 700 & 1,404 & 86,330 \\
\hline 1905. & . & & & \\
\hline January ... & 10,220 & 1,582 & 3,549 & 218,200 \\
\hline February...$\ldots$ & 13,090 & 2,135 & 4,630 & $257,100^{\circ}$ \\
\hline March . . . ......... & 25,900 & 3,545 & 6,924 & 425,700 \\
\hline April......... & 18,300 & . 4,018 & 7,736 & 460,300 \\
\hline May ............ & 10,580 & 3,278 & 6,717 & 413,000 \\
\hline June............. & 5,760 & 1,300 & 3,231 & 192,300 \\
\hline July ........... & 1,468 & 335 & 719 & 44,210 \\
\hline August.......... & 335 & 155 & 255 & 15,680 \\
\hline Septembcr........ & 155 & 105 & 126 & 7,498 \\
\hline October............ & 155 & 120 & 138 & 8,485 \\
\hline November......... & 535 & 120 & 181 & 10,770 \\
\hline December............ & -505 & 120 & 242 & 14,880 \\
\hline The year..... & 25,900 & 105 & 2,874 & $2,068,000$ \\
\hline
\end{tabular}

MISCELLANEOUUS MEASUREMENTS IN AMERICAN RIVER DRATNAGE BASIN.

South Fork of American River near Placerville, Cal.-A measurement was made August 20, 1905, at Chillie Bar, 3 miles north of Placerville and 400 yards upstream from wagon bridge, by H. A. Campbell. The distance to water surface from top of outer bar, of lower chord in the middle panel, on the upstream side of bridge was 23.5 feet.

Width, 28 feet; area, 42 square feet; mean velocity, 1.80 feet per second; discharge, 76 second-feet. 
North Fork of the American River near Auburn, Cal.-A measurement was made about 400 yards above junction of North and Middle forks and about 2 miles northeast of Auburn on August 21, 1905, by H. A. Campbell.

Width, 28 feet; area, 44 square feet; mean velocity, 1.48 feet per second; discharge, 65 second-feet.

Middle Fork of the American River near Auburn, Cal.-A measurement was made on this stream about 400 yards above junction of the North and Middle forks and about 2 miles northeast of Auburn, Cal., on August 21, 1905, by H. A. Campbell.

Width, 55 feet; area, 101 square feet; mean velocity, 0.97 foot per second; discharge, 98 second-feet.

El Dorado ditch.-This ditch is taken out of South Fork of the American River about 1 mile below Slippery Ford. A measurement was made at the heading on August 24, 1905, by H. A. Campbell.

Width, 13.9 feet; area, 45 square feet; mean velocity, 1.24 feet per second; discharge, 56 second-feet.

Silver Fork of American River.-This stream enters South Fork of American River about 1 mile below Slippery Ford. A measurement was made on August 24, 1905, just above mouth, by $\mathrm{H}$. A. Campbell.

Width, 11 feet; area, 18.3 square feet; mean velocity, 1.07 feet per second; discharge, 19.6 second-feet.

South Fork of American River.-A measurement was made one-fourth of a mile above Silver Fork and three-fourths mile below Slippery Ford on August 25, 1905, by H. A. Campbell.

Width, 26 feet; area, 63 square feet; mean velocity, 0.54 foot per second; discharge, 34 second-feet.

South Fork of Silver Creek.-A measurement was made on this stream August 26, 1905, about 200 feet above the mouth by H. A. Campbell:

Width, 16.5 feet; area, 21 square feet; mean velocity, 0.30 foot per second; discharge, 6.3 second-feet.

Silver Creek.-A measurement was made August 26, 1905, 100 feet above the mouth of South Fork of Silver Creek by H. A. Campbell.

Width, 11 feet; area, 16.2 square feet; mean velocity, 0.92 foot per second; discharge, 14.9 second-feet.

\section{PUTA CREEK DRAINAGE BASIN. DESCRIPTION OF BASIN.}

Puta Creek drains a portion of the eastern slope of the Coast Range, its waters discharging into Sacramento River through what is known as the Yolo basin in the vicinity of Davis, Cal. This basin is rather long and narrow, extending from west to east; it has numerous tributaries which have a heavy flood discharge during the winter months, but are practically dry during the summer. This stream is torrential in its flow. It has a comparatively small drainage basin with an exceptionally heavy rainfall, especially on the higher elevations in the vicinity of Mount St. Helena. A five-year rainfall record at Helen Mine on the northern slope of Mount St. Helena gives an average of 99.52 inches. The precipitation decreases as we approach the lower elevations. The upper reaches of this basin are well timbered, but the lower portion is comparatively barren of timber, though it has a considerable growth of brush extending to a point where the stream leaves the foothills. The areas in the lower portion of the basin are used principally for pasturage. The topography of the country is rough and precipitous. The underlying rock is an impervious slate and serpentine with a thin soil covering. There is comparatively little tilled land in this drainage basin above the point where the stream emerges from the foothills at Winters. Below this point the soil is deep and susceptible to high cultivation, and at present is used for the raising of grain and fruit. 


\section{PUTA CREEK NEAR GUENOC, CAL.}

This station was established February 12, 1904. It is located about 2 miles below the old town of Guenoc, near the Asbill ranch house and at the Guenoc dam site. The nearest post-office is at Middletown, Cal.

The bed of the stream is gravelly, with a few bowlders above and below the section. Bed rock is laid bare on the right side of the channel. The lift bank is high and rocky; while the right bank is rather low and flat and subject to overflow in very high water. The velocity is moderate for ordinary stages of flow, but high in flood periods. The range is about 12 feet, representing discharges of from 7 to 17,000 second-feet. The channel is straight for several hundred feet upstream, but has several large bowlders in it. Downstream it is straight for about 100 feet. The section is located in a narrow gorge at the lower end of a long, flat valley, which is flooded during high water. The channel will not allow the water to run off through the gorge fast enough to prevent backwater forming during flood periods.

Meter measurements during high water are made from a car and cable. . At first the cable was located directly opposite the ranch house, but as the location proved very unfavorable it was moved November 15, 1904, to a point about 1,000 feet below. It now has a clear span of about 200 feet. The initial point for soundings is the eyebolt to which the cable is fastened on the left bank. An auxiliary cable has been placed parallel to the large one, so that float measurements can be made in time of very high water. During low water measurements are made by wading.

The gage, which was established near the old section, is still read, although a new one was placed near the cable in its present position. The old gage is a staff in two sections, both being fastened to trees. The section which is read in low water is about 600 feet upstream from the ranch stable, and the high-water section is directly opposite the stable. The new rod is made similarly to the other one. Both sections are located a few feet above the cable. One is bolted to a large bowlder on the left bank and the other nailed to a tree on the right bank. During 1905 the gage was read once each day during ordinary stages and twice a day during high water by Miss Agnes Asbill. The bench mark is the top of the rock to which the low-water section of the new gage is fastened; datum of the new gage.

A description of this station and gage height and discharge data are contained in WaterSupply Paper No. 134, United States Geological Survey.

Discharge measurements of Puta Creek near Guenoc, Cal., in 1905.

\begin{tabular}{|c|c|c|c|c|c|c|}
\hline Date. & Hydrographer. & Width. & $\begin{array}{l}\text { Area of } \\
\text { section. }\end{array}$ & $\begin{array}{c}\text { Mean } \\
\text { velocity. }\end{array}$ & $\begin{array}{c}\text { Gage } \\
\text { height. }\end{array}$ & $\begin{array}{l}\text { Dis- } \\
\text { charge. }\end{array}$ \\
\hline January $1 . . .$. & F. R. S. Buttemer. & $\begin{array}{r}\text { Feet. } \\
81\end{array}$ & $\begin{array}{c}S q . f t . \\
433\end{array}$ & $\begin{array}{r}\text { Ft.per sec. } \\
1.37\end{array}$ & $\begin{array}{l}\text { Feet. } \\
5.58\end{array}$ & $\begin{array}{c}\text { Sec.-ft. } \\
592\end{array}$ \\
\hline January $1 . .$. & ... do do.......... & 76 & 390 & 1.36 & 5.42 & 530 \\
\hline January 2. & .....do.... & 66 & 342 & 1.13 & 5.14 & 386 \\
\hline January $2 a \ldots$ & .....do...... & 66 & 321 & 1.12 & 5.12 & 359 \\
\hline January $3 . . .$. & ..... do.... & 66 & 322 & 0.90 & 4.94 & 289 \\
\hline Januạry $4 . .$. . & .....do....... & 64 & 316 & 0.78 & 4.82 & 247 \\
\hline January $14 . .$. & ....do............. & 110 & 624 & 2.94 & 7.04 & 1,834 \\
\hline January $15 \ldots$ & .....do............ & 95 & 478 & 1.83 & 5.96 & 876 \\
\hline January $16 \ldots$ & .....do......... & 94 & 429 & 2.04 & 5.86 & 875 \\
\hline January $16 \ldots$ & .....do............. & 95 & 467 & 2.08 & 5.98 & 972 \\
\hline July $8 . . . . . .$. & o. W. Peterson.................... & 16 & 12 & 1.58 & 3.72 & 19 \\
\hline September 29 . & 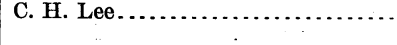 & 8 & 4.1 & 1.66 & 3.56 & 6.8 \\
\hline
\end{tabular}


PUTA CREEK DRAINAGE BASIN.

Daily gage height, in feet, of Puta Creek near Guenoc, Cal., for 1905.

\begin{tabular}{|c|c|c|c|c|c|c|c|c|c|c|c|c|}
\hline Day. & Jan. & Feb. & Mar. & Apr. & May. & June. & July: & Aug. & Sept. & Oct. & Nov. & Dec. \\
\hline 1. & 6.8 & 7.0 & 4.5 & 5.2 & 4. 3 & $a 4.0$ & 3.6 & 3.6 & 3. 6 & 3.7 & 3.6 & 3.6 \\
\hline 2. & $x 6.0$ & 6.5 & 4.4 & 5.2 & 4.3 & 4.0 & 3.7 & 3.6 & 3.6 & 3.7 & 3.6 & 3.0 \\
\hline 3. & $a_{5.4}$ & 0.2 & 4.3 & 5.2 & 4.3 & 3.9 & 3.7 & 3.6 & 3.6 & 3.7 & 3.6 & 3.6 \\
\hline 4. & 4.8 & 6.0 & 4.2 & 5.1 & 4.4 & 5.4 & 3.7 & 3.6 & 3.6 & 3.7 & 3.6 & 3.6 \\
\hline 5. & 4.7 & 6.3 & 4.4 & 5.1 & 4.3 & 5.6 & 3.7 & 3.6 & 3.6 & 3.7 & 3.6 & 3.6 \\
\hline 6.. & 4.4 & 6.0 & 4.4 & 5.1 & 4.3 & 5.0 & 3.7 & 3.6 & 3.6 & 3.7 & 3.6 & 3,6 \\
\hline $7 .$. & 4.4 & 5.8 & 4.3 & 5.0 & 5.0 & 4.2 & 3.7 & 3.6 & 3.6 & 3.7 & 3.6 & 3.6 \\
\hline 8. & 4.5 & 5.5 & 4.3 & 5.0 & 5.2 & 4.2 & 3.7 & 3.6 & $a 3.6$ & 3.7 & 3.6 & 3.6 \\
\hline 9. & 4.7 & 5.4 & 4.2 & 5.1 & 4.8 & 4.1 & 3.7 & 3.6 & 3.6 & 3.7 & 3.6 & 36 \\
\hline 10. & 4.6 & 5.1 & - 4.2 & 5.1 & 4.6 & 4.1 & 3.7 & $a 3.6$ & 3.6 & 3.7 & 3.6 & 3.6 \\
\hline 11. & 4.5 & 5.0 & 4.2 . & 5.0 & 4.4 & 4.0 & 3.7 & 3.6 & 3.6 & 3.7 & 3.6 & 3.6 \\
\hline 12. & 4.5 & 4.9 & 4.5 & 5.0 & 4.4 & 4.0 & 3.7 & 3.6 & a 3.6 & 3.7 & 3.6 & 3.6 \\
\hline 13. & 6.1 & 4.8 & 5.5 & 5.0 & 4.4 & 3.9 & 3.7 & 3.6 & 3.6 & 3.7 & 3.6 & 3.6 \\
\hline 14. & 7.45 & 4.7 & 6.5 & 5.0 & 4.4 & 3.9 & 3.7 & 3.5 & 3.6 & 3.7 & 3.6 & .6 \\
\hline 15. & 5.9 & 4.6 & 6.0 & 5.1 & 4.3 & 3.9 & 3.6 & 3.5 & 3.6 & a 3.7 & 3.6 & 3.7 \\
\hline 16. & 5.9 & 4.9 & 6.2 & 5.2 & 4.3 & 3.9 & 3.6 & 3.5 & 3.6 & 3.7 & 3.6 & 3.8 \\
\hline 17. & 4.4 & 5.1 & 6.0 & 5.2 & 4.3 & 3.9 & 3.6 & 3.5 & 3.6 & 3.7 & 3.6 & 3.8 \\
\hline 18. & 4.5 & 5.4 & 6.5 & 5.1 & 4.2 & 3.8 & 3.6 & 3.5 & a 3.6 & 3.7 & 3.6 & 3.8 \\
\hline 19. & 6.45 & 6.3 & b. 85 & 5.0 & 4.2 & 3.8 & 3.5 & 3.5 & 3.6 & 3.7 & 3.6 & 3.8 \\
\hline 20. & 5.4 & 6.0 & 6.1 & 4.9 & 4.2 & 3.8 & 3.5 & 3.6 & 3.6 & 3.7 & 3.6 & 3.8 \\
\hline 21.. & 6.8 & 5.9 & 5.9 & 4.9 & 4.2 & 3.8 & 3.5 & a 3.6 & 3.6 & 3.6 & 3.6 & 3.7 \\
\hline 22. & 13.5 & 5.7 & 5.8 & 4.8 & 4.1 & 3.8 & 3.5 & 3.6 & 3.6 & 3.6 & 3.6 & 3.7 \\
\hline 23. & 8.9 & 5.4 & 5.8 & 4.8 & 4.1 & 3.8 & 3.5 & 3.6 & 3.6 & 3.6 & 3.6 & 3.7 \\
\hline 24. & 7.2 & 5.2 & 5.7 & 4.7 & 4.1 & 3.8 & 3.6 & 3.6 & 3.6 & 3.6 & 3. 6 & 4.0 \\
\hline 25. & 6.7 & 5.0 & 5.4 & 4.6 & 4.1 & 3.7 & 3.6 & 3.6 & 3.6 & 3.6 & 3.6 & 4.1 \\
\hline 26 . & 6.0 & 4.8 & 5.0 & 4.5 & 4.0 & 3.7 & 3.6 & 3.6 & 3.6 & 3.6 & 3.6 & 3.9 \\
\hline 27. & 5.9 & 4.7 & 5.8 & 4.4 & 4.0 & 3.7 & $a 3.6$ & 3.6 & 3.6 & 3.6 & 3.6 & 3.9 \\
\hline $28 \ldots$ & 5.7 & 4.6 & 5.8 & 4.3 & 4.0 & 3.7 & 3.6 & 3.6 & 3.6 & 3.6 & 3.6 & a 3.9 \\
\hline 29. & 5.6 & & 7.5 & 4.3 & 4.0 & 3.7 & 3.6 & 3.6 & 3.6 & 3.6 & 3.6 & 3.8 \\
\hline 30. & 5.4 & & 5.8 & $a 4,3$ & 4.0 & 3.6 & 3.6 & 3.6 & 3.6 & .3 .6 & $a 3.6$ & 3.8 \\
\hline $31 .$. & 5.8 & & 5.5 & & 4,0 & & 3.6 & 3. & & 3.6 & & 3.7 \\
\hline
\end{tabular}


Station rating table for Puta Creek near Guenoc, Cal., from January 1 to December 31, 1905.

\begin{tabular}{|r|r||r|r|r|r|r|r|}
\hline $\begin{array}{c}\text { Gage } \\
\text { height. }\end{array}$ & Discharge. & $\begin{array}{c}\text { Gage } \\
\text { height. }\end{array}$ & Discharge. & $\begin{array}{c}\text { Gage } \\
\text { height. }\end{array}$ & Discharge. & $\begin{array}{c}\text { Gage } \\
\text { height. }\end{array}$ & Discharge. \\
\hline Feet. & Second-feet. & Feet. & Second-feet. & Feet. & Second-feet. & Feet. & Second-feet. \\
3.50 & 4 & 5.00 & 310 & 6.50 & 1,360 & 9.00 & 5,000 \\
3.60 & 10 & 5.10 & 350 & 6.60 & 1,450 & 9.20 & 5,400 \\
3.70 & 18 & 5.20 & 395 & 6.70 & 1,545 & 9.40 & 5,800 \\
3.80 & 28 & 5.30 & 445 & 6.80 & 1,640 & 9.60 & 6,230 \\
3.90 & 42 & 5.40 & 500 & 6.90 & 1,740 & 9.80 & 6,690 \\
4.00 & 58 & 5.50 & 560 & 7.00 & 1,840 & 10.00 & 7,150 \\
4.10 & 75 & 5.60 & 625 & 7.20 & 2,060 & 10.50 & 8,300 \\
4.20 & 93 & 5.70 & 695 & 7.40 & 2,290 & 11.00 & 9,450 \\
4.30 & 112 & 5.80 & 770 & 7.60 & 2,540 & 11.50 & 10,600 \\
4.40 & 133 & 5.90 & 850 & 7.80 & 2,820 & 12.00 & 11,800 \\
4.50 & 156 & 6.00 & 930 & 8.00 & 3,130 & 12.50 & 13,000 \\
4.60 & 181 & 6.10 & 1,010 & 8.20 & 3,460 & 13.00 & 14,200 \\
4.70 & 208 & 6.20 & 1,095 & 8.40 & 3,810 & 14.00 & 16,700 \\
4.80 & 238 & 6.30 & 1,180 & 8.60 & 4,200 & & \\
4.90 & 272 & 6.40 & 1,270 & 8.80 & 4,600 & & \\
& & & & & & & \\
\hline
\end{tabular}

Note.-The above table is based on 17 discharge measurements made during 1904-5. It is well defined between gage heights 3.5 feet and 7 feet. Above 7 feet the table depends on 2 measurements at 12 feet and 13.7 feet. Above 9 feet the table is the same as for 1904.

Estimated monthly discharge of Puta Creek near Guenoc, Cal., for 1905.

[Drainage area, 91 square miles.] 。

\begin{tabular}{|c|c|c|c|c|c|c|}
\hline \multirow[b]{2}{*}{ - Month. } & \multicolumn{3}{|c|}{ Discharge in second-feet. } & \multirow[b]{2}{*}{$\begin{array}{l}\text { Total in } \\
\text { acre-feet. }\end{array}$} & \multicolumn{2}{|c|}{ Run-off. } \\
\hline & Maximum. & Minimum. & Mean. & & $\begin{array}{c}\text { Second-feet } \\
\text { per square } \\
\text { mile. }\end{array}$ & $\begin{array}{l}\text { Depth } \\
\text { in inches. }\end{array}$ \\
\hline January . . . . . & 15,450 & 133 & 1,344 & 82,640 & 14.77 & 17.03 \\
\hline February... & 1,840 & 181 & 619 & 34,380 & 6.80 & 7.08 \\
\hline March..... & 1,690 & 93 & 630 & 38,740 & 6.92 & 7.98 \\
\hline April... & 395 & 112 & 288 & 17,140 & 3.16 & 3.53 \\
\hline May....... & 395 & 58 & 120 & 7,379 & 1.32 & 1.52 \\
\hline June.......... & $625^{\circ}$ & 10 & 85.0 & 5,058 & .934 & 1.04 \\
\hline July ........... & 18 & 4 & 12.4 & 762 & .136 & .157 \\
\hline August..... & 10 & 4 & 8.8 & 541 & .097 & .112 \\
\hline September... & 10 & 10 & i0.0 & 595 & .110 & .123 \\
\hline October... & 18 & 10 & 15,2 & 935 & .167 & .192 \\
\hline November. & 10 & 10 & 10.0 & 595 & .110 & .123 \\
\hline December........ & 75 & 10 & 22.1 & 1,359 & .243 & $\begin{array}{r}.280 \\
\times \quad .\end{array}$ \\
\hline The year. - & 15,450 & 4 & 264 & 190,100 & 2.90 & 39.17 \\
\hline
\end{tabular}

\section{PUTA OREEK AT WINTERS, CAI.}

This station was established September 26,1905 , by R. S. Hawley. It is located about 450 feet below the Southern Pacific Railroad bridge and about 800 feet southeast of the depot at Winters, Cal.

The channel is straight for about 500 feet above and 700 feet below the cable section. The bed of the stream is composed of coarse gravel and is liable to some change at very high stages, but at ordinary stages it is not subject to much change. The current is sluggish at low water, but is swift during flood stages. There is a growth of willows and cottonwoods with wild grapevines along the banks, but the main portion of the channel is clear. 
At very high flood stages the water spreads out over the left bank for about 150 feet, reaching nearly to the foot of the left cable support. At ordinary stages, however, the water remains within the high banks.

Measurements are made during low water by wading at a point about 400 feet above the bridge, but for higher stages a cable and car are used. The cable has a span of 280 feet. For float measurements, a course 250 feet long has been marked off by setting posts painted white, 250 feet above and parallel with the cable. Floats can be dropped from the railroad bridge above and timed from these posts to the cable. The initial point of soundings is a bolt in the trunk of the oak tree to which the cable is fastened on the right bank.

The gage consists of a series of timbers painted white and located under the cable. The first or-low-water section is on the right bank, and is nailed vertically to the trunk of a cottonwood tree. It has a range of about 6 feet. The second section is on the left bank and is nailed vertically to the stump of a cottonwood tree. The third section is an inclined rod anchored on the left bank with posts. The fourth section is on an eucalyptus tree on top of left bank. During 1905 the gage was read once each day by Roy Wyatt. Bench marks were established as follows: (1) A standard United States Geological Survey bench mark, located 150 feet south of the depot. Its elevation as marked on the copper plate is 131 feet, 39.23 feet above the datum of the gage. (2) A square-headed bolt in concrete, 3 feet south of the left cable support; elevation, 37.27 feet above the datum of the gage.

Discharge measurements of Puta Creek at Winters, Cal., in 1905.

\begin{tabular}{|c|c|c|c|c|c|c|}
\hline Date. & Hydrographer. & Width. & $\begin{array}{l}\text { Area of } \\
\text { section. }\end{array}$ & $\underset{\text { Melocity. }}{\text { Mean }}$ & $\begin{array}{c}\text { Gage } \\
\text { height. }\end{array}$ & $\begin{array}{c}\text { Dis- } \\
\text { charge. }\end{array}$ \\
\hline September $12 \ldots$ & W. B. Clapp. . & $\begin{array}{r}\text { Feet. } \\
16\end{array}$ & $\begin{array}{r}S q \cdot f t . \\
8.0\end{array}$ & $\begin{array}{r}\text { Ft. per sec. } \\
1.09\end{array}$ & $\begin{array}{c}\text { Feet. } \\
\end{array}$ & $\begin{array}{r}\text { Sec.-ft. } \\
8.7\end{array}$ \\
\hline September $26 .$. & Hawley and Lee. & 16 & 8.8 & .97 & 4.39 & 8.5 \\
\hline November 15 . & R. S. Hawley .... & 16 & 8.8 & 1.33 & 4. 42 & 11.7 \\
\hline
\end{tabular}

Daily gage height, in feet, of Puta Creek at Winters, Cal., for 1905.

\begin{tabular}{|c|c|c|c|c|c|c|c|c|c|}
\hline Day. & Sept. & Oct. & Nov. & Dec. & Day. & Sept. & Oct. & Nov. & Dec. \\
\hline $1 \ldots \ldots \ldots \ldots$ & $\ldots \ldots$ & 4.4 & $4: 4$ & 4.6 & $17 \ldots$ & & 4.4 & 4.45 & 4.55 \\
\hline $2 \ldots \ldots \ldots \ldots \ldots$ & $\ldots \ldots$ & 4.4 & 4. 4 & 4.65 & $18 \ldots \ldots \ldots \ldots \ldots$ & $\ldots \ldots$ & 4.4 & 4. 45 & 4. 6 \\
\hline $3 \ldots$. & & 4.4 & 4.4 & 4.6 & 19. & & 4.4 & 4.45 & 4.6 \\
\hline $4 \ldots \ldots \ldots \ldots$ & & 4. 4 & 4. 4 & 4.6 & $20 \ldots$ & & 4.4 & 4.45 & 4.6 \\
\hline $5 \ldots \ldots \ldots \ldots$ & $\ldots \ldots$ & 4.4 & 4.4 & 4.6 & $21 \ldots \ldots \ldots \ldots$ & $\ldots \ldots$ & 4.4 & 4.45 & 4.6 \\
\hline $6 \ldots \ldots \ldots$ & & 4.4 & 4. 4 & 4.6 & $22 \ldots$ & & 4.4 & 4.45 & 4. 6 \\
\hline $7 \ldots \ldots \ldots \ldots$ & $\ldots$ & 4. 4 & 4. 4 . & 4.55 & $23 \ldots \ldots \ldots \ldots \ldots$ & $\ldots \ldots$ & 4. 4 & 4.5 & 4.6 \\
\hline $8 \ldots$. & & 4. 4 & 4. 4 & 4.55 & 24. & & 4. 4 & 4.5 & 4.6 \\
\hline $9 \ldots$ & & 4. 4 & 4. 4 & 4.55 & $25 \ldots$ & $\ldots .$. & 4. 4 & 4.5 & 4.6 \\
\hline $10 \ldots \ldots \ldots$ & & 4. 4 & 4. 4 & 4.5 & $26 \ldots \ldots \ldots \ldots$ & 4. 4 & 4.4 & 4.5 & 4.6 \\
\hline $11 \ldots$ & & 4.4 & 4. 4 & 4.5 & $27 \ldots \ldots \ldots \ldots$ & 4.4 & 4.4 & 4.5 & 4. 6 \\
\hline 12. & & 4. 4 & 4. 45 & 4.5 & $28 \ldots \ldots \ldots \ldots \ldots$ & 4. 4 & 4. 4 & 4.5 & 4.6 \\
\hline $13 . .$. s. . & .. & 4. 4 & 4. 45 & 4.55 & $29 \ldots \ldots \ldots \ldots \ldots$ & 4. 45 & 4. 4 & 4.6 & 4.6 \\
\hline $14 \ldots .$. & & 4.4 & 4. 45 & 4.55 & $30 \ldots$ & 4.4 & 4. 4 & 4.6 & 4.6 \\
\hline $15 \ldots \ldots \ldots \ldots$ & $\ldots \ldots$ & 4. 4 & 4. 45 & 4.55 & $31 \ldots$ & $\ldots . . .$. & 4.4 & $\ldots \ldots$ & 4.6 \\
\hline $16 \ldots \ldots$ & $\ldots \ldots$ & 4. 4 & 4. 45 & 4.55 & & & & & \\
\hline
\end{tabular}




\section{SAN JOAQUIN RIVER.}

\section{DESCRIPTION OF RIVER.}

San Joaquin River is divided into two distinct parts. The valley portion forms the central drainage line of San Joaquin Valley, and during the spring is navigable for 100 miles or more. Stanislaus, Tuolumne, Merced, and Kings rivers are the largest streams in this portion of the drainage basin. The waters of Kings, Kaweah, Tule, and Kern rivers, which are located in the portion of San Joaquin Valley lying to the south and east of Fresno, although forming a portion of the drainage of San Joaquin River, seldom reach this stream. Their entire flow, except in extreme flood, is diverted and used for irrigation at points where they emerge from the foothills. The valley is fertile and almost destitute of timber. The mountainous portion of the stream drains the western slope of the Sierra Nevada between the Merced River on the north and Kings River on the south, the crest of its divide reaching an elevation of 13,000 feet in Mount Lyell and an elevation of 14,000 feet in Mount Goddard. There are numerous tributaries in this portion of the drainage basin, many of which have their source in the high elevations. The formation is of granite, which in the upper reaches is bare and sharply marked by glacial action. The middle reaches of the basin are well timbered, the timber diminishing on the lower foothills, which have a covering of brush and grass. The precipitation takes the form of snow on the higher elevations. The fall of the river is rapid, with many favorable locations for power development. There are numerous lakes in the upper reaches of the basin. A storage reservoir has been constructed on North Fork, which will tend to further regulate the flow of the river.

\section{SAN JOAQUIN RIVER AT HERNDON, CAL.}

The gage rod at this station was established by the engineering department of the Southern Pacific Railroad Company in 1879. The old trestle bridge was torn down by the railroad company during 1899 and a new iron structure was erected in its place. A new gage rod, set to the datum of the old gage, was bolted to the western side of the central concrete pier. The bench mark is a nail in a post at the south end of the bridge on the west side, 0.2 foot above the ground, and marked "B. M." It is at an elevation of 24.12 feet above gage datum.

The channel for some distance above and below the bridge is straight, and the water has a uniform velocity. The right bank is high, rocky, and steep. The bed of the stream is composed of small gravel and shifting sand. Bécause of the continual changes in the cross section, which were increased by a side channel breaking through the gravel pits on the left bank of the river just above the gaging station, meter measurements were discontinued at this station at the end of 1901 .

The river stage record for 1905 has been furnished by William Hood, chief engineer of the Southern Pacific Railroad Company. G. G. Nelson was the observer. 
Information in regard to this station is contained in Water-Supply Papers Nos. 81, 85, 100, and 134 of the United States Geological Survey.

Daily gage height, in feet, of San Joaquin River at Herndon, Cal., for 1905.

\begin{tabular}{|c|c|c|c|c|c|c|c|c|c|c|c|c|}
\hline Day. & Jan. & Feb. & Mar. & Apr. & May. & June. & July. & Aug. & Sept. & Oct. & Nov. & Dec. \\
\hline 1. & 3.5 & 3.0 & 3.9 & 4.5 & 7.0 & 6.75 & 6.1 & 3.5 & 3.15 & 2.5 & 2.4 & 2.6 \\
\hline $2 .$. & 3.5 & 3.15 & 4.1 & 4.5 & 6.75 & 6.6 & 6.0 & 3.5 & 3.1 & 2.5 & 2.4 & 2.65 \\
\hline 3.. & 3.3 & 4.6 & 4.25 & 4.5 & 6.6 & 6.6 & 5.75 & 3.5 & 3.0 & 2.5 & 2.4 & 2.65 \\
\hline 4.. & 3.25 & 4.4 & 4.25 & 4.5 & 6.0 & 6.75 & 6.0 & 3.5 & 3.0 & 2.5 & 2.4 & 2.6 \\
\hline 5.. & 3.15 & 4.3 & 4.25 & 5.0 & 5.5 & 6.75 & 6.0 & 3.5 & 2.9 & 2.5 & 2.4 & 2.6 \\
\hline 6.. & 3.15 & 4.75 & 4.15 & 5.25 & 5.5 & 6.4 & 5.75 & 3.5 & 2.75 & 2.5 & 2.4 & 2.6 \\
\hline 7.. & 3.15 & 4.5 & 4.15 & 5.3 & 5.65 & 6.3 & 5.5 & 3.4 & 2.75 & 2.5 & 2.4 & 2.6 \\
\hline 8.. & 3.15 & 4.0 & 4.25 & 5.5 & 5.65 & 7.15 & 5.3 & 3.4 & 2.75 & 2.5 & 2.4 & 2.6 \\
\hline 9.. & 3.1 & 3.75 & 4.3 & 6.65 & 6.0 & 7.4 & 5.3 & 3.4 & 2.75 & 2.5 & 2.4 & 2.5 \\
\hline 10. & 3.1 & 3.5 & 4.3 & 5.75 & 5.5 & 7.4 & 5.2 & 3.4 & 2.75 & 2.5 & 2.4 & 2.5 \\
\hline $11 .$. & 3.1 & 3.5 & 4.5 & 5.5 & 5.5 & 7.5 & 5.15 & 3.4 & 2.75 & 2.5 & 2.4 & 2.5 \\
\hline $12 .$. & 3.1 & 3.5 & 4.5 & 5.5 & 5.5 & 8.3 & 5.1 & 3.3 & 2.75 & 2.5 & 2.4 & 2.5 \\
\hline $13 .$. & 3.1 & 3.4 & 4.5 & 5.3 & 5.5 & 8.75 & 5.0 & 3.3 & 2.75 & 2.5 & 2.4 & 2.5 \\
\hline 14. & 3.1 & 3.3 & 7.15 & 4.75 & 5.5 & 8.0 & 4.75 & 3.3 & 2.65 & 2.5 & 2.4 & 2.5 \\
\hline 15. & 3.1 & 3.3 & 5.5 & 4.7 & 6.0 & 8.0 & 4.75 & 3.3 & 2.65 & 2.5 & 2.4 & 2.5 \\
\hline 16. & 3.3 & 3.25 & 5.0 & 5.1 & 7.0 & 7.75 & 4.65 & 3.25 & 2.65 & 2.5 & 2.4 & 2.5 \\
\hline 17. & 3.15 & 3.15 & 5.0 & 5.25 & 8.5 & 8.0 & 4.65 & 3.25 & 2.65 & 2.5 & 2.4 & 2.5 \\
\hline 18. & 3.1 & 3.15 & 5.0 & 5.0 & 8.75 & 8.25 & 4.65 & 3.25 & 2.65 & 2.5 & 2.4 & 2.5 \\
\hline 19. & 3.0 & 3.15 & 5.0 & 5.0 & 9.65 & 8.75 & 4.65 & 3.25 & 2.65 & 2.5 & 2.4 & 2.5 \\
\hline $20 \ldots$ & 3.0 & 3.15 & 6.5 & 5.0 & 8.25 & 8.3 & 4.5 & 3.25 & 2.65 & 2.5 & 2.4 & 2.6 \\
\hline $1 \ldots$ & 3.0 & 3.15 & 5.0 & 4.75 & 8.6 & 7.75 & 4.5 & 3.25 & 2.65 & 2.5 & 2.4 & 2.75 \\
\hline $22 .$. & 3.0 & 3.15 & 5.0 & 5.0 & 8.25 & 8.0 & 4.5 & 3.25 & 2.65 & 2.5 & 2.4 & 2.75 \\
\hline 23.. & 3.0 & 3.15 & 5.0 & 5.0 & 8.3 & 7.5 & 4.5 & 3.15 & 2.65 & 2.5 & 2.4 & 2.75 \\
\hline 24.. & 3.0 & 3.15 & 5.0 & 5.0 & 8.1 & 7.15 & 4.3 & 3.15 & 2.6 & 2.4 & 2.4 & 2.75 \\
\hline $25 .$. & 3.0 & 3.15 & 5.0 & 5.0 & 8.25 & 6.5 & 4.3 & 3.15 & 2.6 & 2.4 & 2.4 & 2.75 \\
\hline $26 .$. & $3.0^{\circ}$ & 3.15 & 5.0 & 5.5 & 8.5 & 6.65 & 4.3 & 3.15 . & 2.6 & 2.4 & 2.4 & 2. 75 \\
\hline $27 \ldots$ & 3.0 & 3.15 & 5.25 & 5.5 & 8.3 & 6.5 & 4.25 & 3.15 & 2.6 &, 2.4 & 2.4 & 2.75 \\
\hline $28 \ldots$ & 3.0 & 3.15 & 5.0 & 6.0 & 7.65 & 6.5 & 4.0 & 3.15 & 2.6 & 2.4 & 2.4 & 2.75 \\
\hline $29 \ldots$ & 3.0 & & 5.0 & 6.0 & 7.65 & 6.5 & 3.75 & 3.15 & 2.6 & 2.4 & 2.4 & 2.75 \\
\hline $30 \ldots$ & 3.0 & & 4.75 & 6.15 & 6.6 & 6.6 & 3.6 & 3.15 & 2.6 & 2.4 & 2.4 & 2.75 \\
\hline $31 \ldots$ & 3.0 & & 4.7 & 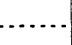 & 6.75 & & 3.6 & 3.15 & $\ldots \ldots$ & 2.4 & 2.6 & 2.65 \\
\hline
\end{tabular}

NoтE:-Gage heights reduced to feet and tenths from feet and inches as furnished by Southern Pacific Railroad Company.

\section{MISCELLAN EOUS MEASUREMENTS IN SAN JOAQUIN RIVER DRAINAGE BASIN.}

Pitman Creek.-This stream is tributary to the San Joaquin River through Big Creek. An estimate was made of the discharge below the mouth of Tamarack Creek on September 2,1905 , by R. S. Hawley.

Discharge, 1.5 second-feet.

Home Camp Creek.-This stream is tributary to the San Joaquin River through Big Creek, which it enters at Home Camp meadow. A measurement was made on this creek on September 2, 1905, 100 feet above its mouth, by R. S. Hawley.

Width, 3.0 feet; area, 0.8 square foot; mean velocity, 1.12 feet per second; discharge, 0.90 second-foot.

Big Creek.-This stream is tributary to San Joaquin River from the east. A measurement was made 100 feet above the mouth of Home Camp Creek on September 2, 1905, by R. S. Hawley. 
Width, 4.5 feet; area, 1.9 square feet; mean velocity, 2.53 feet per second; discharge, 4.8 second-feet.

Discharge below the mouth of Home Camp Creek was 5.7 second-feet.

Small Creek.-This is a small creek which enters the South Fork of the San Joaquin from the south at Mono crossing. A measurement was made at the Mono trail crossing about 1,000 feet above its mouth on September 4,1905 , by R. S. Hawley.

Width, 2.8 feet; area, 1.1 square feet; mean velocity, 1.18 feet per second; discharge, 1.3 second-feet.

South Fork of San Joaquin River.-This is the principal tributary of the San Joaquin River from the south. A measurement was made September 4, 1905, at the Mono crossing by R. S. Hawley.

Width, 60 feet; area, 94 square feet; mean velocity, 1.11 feet per second; discharge, 104 second-feet.

Mono Creek.-This stream is tributary to South Fork of San Joaquin River from the east. A measurement was made at the Mono Trail crossing, about 2 miles above its mouth, on September 4, 1905, by R. S. Hawley.

Width, 44.5 feet; area, 37 square feet; mean velocity, 1.17 feet per second; discharge, 43 second-feet.

Fish Creek.-This stream is.the principal tributary of the Middle Fork of the San Joaquin from the south about 10 miles above the junction of the Middle and South forks. A measurement was made on September 6, 1905, at the trail crossing, about 3 miles above its mouth, by R. S. Hawley.

Width, 29 feet; area, 36 square feet; mean velocity, 1.42 feet per second; discharge, 51 second-feet.

Middle Fork of San Joaquin River.-This stream drains the northeastern portion of the San Joaquin watershed. A measurement was made on September 6, 1905, at the Devils Post Pile at the trail crossing by R. S. Hawley.

Width, 42 feet; area, 41 square feet; mean velocity, 0.90 foot per second; discharge, 37 second-feet.

Reds Creek.-This stream enters Middle Fork of San Joaquin River one-half mile below the Devils Post Pile. A measurement was made September 6, 1905, at the Mammoth trail crossing, one-fourth mile above its mouth, by R. S. Hawley.

Width, 9 feet; area, 4.2 square feet; mean velocity, 0.93 foot per second; discharge, 3.9 second-feet.

King Creek.-This stream enters Middle Fork of San Joaquin River near Rainbow Falls. An estimate was made of the discharge one mile above its mouth, at Mammoth trail crossing, on September 7, 1905, by R. S. Hawley.

Discharge, 3.5 second-feet.

Mugler Creek.-This stream enters the San Joaquin River from the north. On September 7,1905 , an estimate of the discharge was made at the trail crossing, one-half mile below Mugler Meadow, by R. S. Hawley.

Discharge, 3.0 second-feet.

Stevenson Creek.-This stream enters the San Joaquin River 4 miles northwest of Shaver post-office. A measurement was made one-fourth mile above the head of Shaver Lake on September 2,1905 , by R. S. Hawley.

Width, 8 feet; area, 3.2 square feet; mean velocity, 1.22 feet per second; discharge, 3.9 second-feet.

North Fork of the San Joaquin River.-This stream enters the San Joaquin River 4 miles below the junction of the Middle and South forks. A measurement was made on September 7, 1905, 150 feet below the bridge, at the trail crossing, 3 miles above its mouth.

Width, 31 feet; area, 27 square feet; , mean velocity, 1.33 feet per second; discharge, 36 second-feet. 
North Fork of Willow Creek.-This stream enters the San Joaquin from the north. A measurement was made on September 9, 1905, at "The Pines," $1 \frac{1}{2}$ miles above reservoir dam, by R. S. Hawley.

Width, 7 feet; area, 3.6 square feet; mean velocity, 1.19 feet per second; discharge, 4.3 second-feet.

San Joaquin River at the San Joaquin power house.-A measurement was made on this stream on September 9, 1905, one-half mile below the power house, by R. S. Hawley. This measurement includes practically the total flow from the whole drainage area of the San Joaquin River about this point.

Width, 148.5 feet; area, 226 square feet; mean velocity, 1.37 feet per second; discharge, 310 second-feet.

\section{KERN RIVER DRAINAGE BASIN.}

\section{DESCRIPTION OF BASIN.}

Kern River drains 2,345 square miles of the western slope of the Sierra Nevada at its extreme southern limits. This drainage basin is the largest of any stream discharging into the San Joaquin Valley, it having an area 600 square miles greater than that of the Kings River. It has its source in the highest elevations of the Sierra Nevada, draining the western and southern slopes of Mount Whitney and numerous other high granite peaks grouped about it, which reach elevations of over 14,000 feet. Its general direction is south for about 65 miles when it turns and flows in a southwesterly direction, discharging into the San Joaquin Valley east of Bakersfield, Cal. Extending, as it does in its upper reaches, for some distance parallel with the Sierra Nevada, it receives waters not only from the main crest on the east, but also from a somewhat lowerdivide on the west behind the basins of the Kaweah and Tule rivers. It has numerous tributaries, the principal ones entering from the east, which drain the higher elevations of the main crest of the Sierra Nevada. The topography is extremely rough and broken in the upper reaches of this basin, becoming less rugged in the middle portion in the vicinity of Kernville, where there is quite an extensive valley with considerable cultivated land; below this point the stream enters a rough canyon, finally discharging into the flat country of the San Joaquin Valley. The entire flow, except during extreme flood stages, is diverted and used for irrigation at points where streams emerge from the foothills.

The formation is of granite, which, above the 10,000 -foot contour, is practically bare of timber growth. Between elevations of 3,000 and 10,000 feet there is a good depth of soil, with timber and brush covering; the lower reaches havea light covering of brush and grass.

There are several lakes and marshes scattered throughout this basin, but they are less numerous than in the basins farther to the north. Several power plants are located on this stream, none of which, however, receive water from storage reservoirs, the diversions being made from the natural flow of the river and again returned to the river channel. The precipitation is very light throughout this basin, with the possible exception of the high elevations surrounding Mount Whitney, where the snow remains through the summer months.

\section{KERN RIVER NEAR BAKERSFIELD, CAL,}

This station, established in 1893 by Walter James, chief engineer of the Kern County Land Company, is located at what is known as "first point of measurement," 5 miles above Bakersfield and at the mouth of the canyon of the river.

Regular meter measurements are taken, and an automatic gage records daily fluctuations of the river heights. A. K. Warren, the engineer in charge of this work for the Kern County Land Company, attends to the discharge measurements with accuracy and precision and furnishes the Geological Survey with the final results. 
Information in regard to this station is contained in Water-Supply Papers Nos. 81, 85, 100, and 134 of the United States Geological Survey.

Daily discharge, in second-feet, of Kern River near Bakersfield, Cal., for 1905.

\begin{tabular}{|c|c|c|c|c|c|c|c|c|c|c|c|c|}
\hline Day. & Jan. & Feb. & Mar. & Apr. & May. & June. & July. & Aug. & Sept. & Oct. & Nov. & Dec. \\
\hline & 280 & 305 & 435 & 799 & $, 1,700$ & 1,953 & 1,520 & 427 & 233 & 205 & 230 & 262 \\
\hline 2. & 285 & 326 & 458 & 848 & 1,713 & 1,979 & 1,422 & 413 & 229 & 226 & 227 & 281 \\
\hline & 272 & 435 & 480 & 934 & 1,743 & $1 ; 932$ & 1,313 & 403 & 224 & 227 & 221 & 276 \\
\hline 4. & 278 & 486 & 500 & 997 & 1,633 & 1,931 & 1,226 & 394 & 213 & 208 & 218 & 285 \\
\hline 5: & 290 & 517 & 512 & 1,047 & 1,542 & 1,919 & 1,215 & 376 & 216 & 196 & 215 & 277 \\
\hline 6.. & 281 & 503 & 530 & 1,070 & 1,517 & 1,879 & 1,218 & 362 & 215 & 190 & 216 & 271 \\
\hline 7.. & 269 & 452 & 545 & 1,103 & 1,453 & 1,997 & 1,171 & 345 & 217 & 181 & 231 & 267. \\
\hline 8. & 272 & 391 & 593 & 1,138 & 1,531 & 2,154 & 1,157 & 356 & 220 & 175 & 217 & 275 \\
\hline 9 & 270 & 364 & 616 & 1,185 & 1,461 & 2,150 & 1,115 & 358 & 218 & 176 & 225 & 278 \\
\hline $0 .$. & 276 & 365 & 609 & 1,119 & 1,418 & 2,066 & 1,130 & 351 & 207 & 186 & 227 & 284 \\
\hline 11. & 280 & 351 & 613 & 1,186 & 1,367 & 2,200 & 1,125 & 349 & 202 & 192 & 230 & 264 \\
\hline 12. & 272 & 338 & 631 & 1,180 & 1,300 & 2,567 & 1,092 & 349 & 208 & 199 & 232 & 278 \\
\hline 13. & 266 & 334 & 785 & 1,052 & 1,298 & 2,923 & 1,038 & 352 & 218 & 206 & 233 & 265 \\
\hline 14. & 246 & 317 & 1,154 & 971 & 1,317 & 2,978 & 958 & 352 & 225 & 206 & 228 & 241 \\
\hline 15. & 255 & 317 & 974 & 952 & 1,425 & 3,039 & 886 & 345 & 221 & 202 & 223 & 244 \\
\hline 16 . & 255 & 361 & 996 & 991 & 1,649 & 2,933 & 799 & 338 & 218 & 199 & 234 & 240 \\
\hline 17. & 260 & 398 & 1,143 & 995 & 1,949 & 2,948 & 748 & 327 & 215 & 203 & 240 & 238 \\
\hline 18. & 269 & 434 & 1,140 & 1,052 & 2,312 & 2,747 & 717 & 319 & 215 & 194 & 257 & 246 \\
\hline 19. & 277 & 417 & 1,009 & 1,121 & 2,359 & 2,569 & 665 & 318 & 208 & 198 & 263 & 259 \\
\hline 20. & 273 & 413 & 1,309 & 1,058 & 2,528 & 2,555 & 628 & 308 & 203 & 215 & 262 & 266 \\
\hline 21. & 305 & 401 & 1,073 & 992 & 2,549 & 2,547 & 634 & 286 & 197 & 218 & 247 & 285 \\
\hline 22. & 329 & 386 & 957 & 964 & 2,625 & 2,539 & 597 & 279 & 194 & 217 & 243 & 272 \\
\hline 3. & 343 & 390 & 888 & 920 & 2,558 & 2,408 & 578 & 267 & 195 & 221 & 239 & 255 \\
\hline 44. & 311. & 397 & 871 & 895 & 2,412 & 2,154 & 558 & 269 & 196 & 230 & 227 & 233 \\
\hline 25. & 301 & 410 & 900 & 936 & 2,456 & 1,835 & 557 & 286 & 201 & 226 & 237 & 221 \\
\hline 26. & 298 & 420 & 929 & 1,033 & 2,560 & 1,690 & 548 & 285 & 204 & 218 & 239 & 239 \\
\hline 27. & 281 & 432 & 999 & 1,070 & 2,598 & 1,685 & 536 & 282 & 214 & 217 & 253 & 255 \\
\hline 28.. & 276 & 434 & 978 & 1,067 & 2,378 & 1,617 & 532 & 271 & 200 & 221 & 259 & 262 \\
\hline 29.. & 271 & 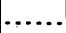 & 919 & 1,157 & 2,120 & 1,584 & 520 & $270^{\circ}$ & $\bullet 205$ & 222 & 263 & 271 \\
\hline 30. & 276 & & 1,051 & 1,451 & 1,962 & 1,563 & 488 & 265 & 205 & 224 & 245 & 255 \\
\hline 31.. & 284 & $\cdots$ & 913 & & 1,930 & & 451 & 250 & & 226 & & 257 \\
\hline
\end{tabular}


Estimated monthly discharge of Kern River near Bakersfield, Cal., for 1905.

[Drainage area, 2,345 square miles.]

\begin{tabular}{|c|c|c|c|c|c|c|}
\hline \multirow[b]{2}{*}{ Month. } & \multicolumn{3}{|c|}{ Discharge in second-feet. } & \multirow[b]{2}{*}{$\begin{array}{l}\text { Total in } \\
\text { acre-feet. }\end{array}$} & \multicolumn{2}{|c|}{ Run-off. } \\
\hline & Maximum. & Minimum. & Mean. & & $\begin{array}{l}\text { Second-feet } \\
\text { per square } \\
\text { mile. }\end{array}$ & $\begin{array}{c}\text { Depth } \\
\text { in inches. }\end{array}$ \\
\hline January .. & 343 & 246 & 281 & 17,280 & 0.120 & 0.138 \\
\hline February... & 517 & 305 & 396 & 21,990 & .169 & .176 \\
\hline March.. & 1,309 & 435 & 823 & 50,600 & .351 & .405 \\
\hline April. . & 1,451 & 799 & 1,043 & 62,060 & .445 & .496 \\
\hline May .... & 2,625 & 1,298 & 1,915 & 117,800 & .817 & .942 \\
\hline June............ & 3,039 & 1,563 & 2,235 & 133,000 & .953 & 1.06 \\
\hline July . ......... & 1,520 & 451 & 876 & 53,860 & .374 & .431 \\
\hline August ... & 427 & 250 & 327 & 20,110 & .139 & .160 \\
\hline September... & 233 & 194 & 211 & 12,560 & .090 & .100 \\
\hline October...... & 230 & 175 & 207 & 12,730 & .088 & .101 \\
\hline November..... & 263 & 215 & 236 & 14,040 & .101 & .113 \\
\hline December.. & 285 & 221 & 261 & 16,050 & .111 & .128 \\
\hline The year. & 3,039 & 175 & 734 & 532,100 & .313 & 4.25 \\
\hline
\end{tabular}

TULE RIVER DRAINAGE BASIN.

DESCRIPTION OF BASIN.

Tule River rises in the Sierra Nevada, and drains the country between the Kaweah River on the north and the Kern River on the south and east. Its drainage area is much less than that of the Kaweah River, although of the same general character. It has numerous small tributaries, few of which have their source at elevations above 8,000 feet: Its drainage basin does not extend back to the main divide, being cut off by the Kern River, which reaches to the north and drains the higher portion of Sierra Nevada to the east of Tule River. There is good timber and brush covering on the higher and middle elevations, with grass and scattering timber on the lower elevations, where the soil is extensively cultivated. Below the gaging station the water is diverted by several canals and used for the irrigation of land in the vicinity of Portersville, which is especially adapted for the raising of citrus fruits, such as oranges and lemons. During the flood period the water discharges through an old channel, and either sinks in the sand or finds its way to the old bed of Tulare Lake.

The mean precipitation in this watershed is probably not more than 20 inches, and falls principally in the form of rain.

TULE RIVER NEAR PORTERSVILLE, CAL.

This station was established April 8, 1901. It is located about 8 miles east of Portersville near the McFarland ranch, 100 feet below wagon bridge and about 1 mile above the mouth of the South Fork of Tule River.

The channel is straight for 200 feet above and below the cable section. The right bank is high and not subject to overflow. The left bank is subject to overflow at flood stages. The bed of the stream is composed of gravel, with some large rocks, but is not subject to much change.

Discharge measurements during medium and high water are made from a car and cable; at low water measurements are made by wading.

The gage is a staff in two sections on the right bank under the cable The lower section is inclined and fastened to posts set in the ground. The higher section is vertical and nailed to the tree through which the cable passes. During 1905 the gage was read by Adah 
McFarland. Bench marks were established as follows:(1) A cross on the top of a bowlder on the right bank of the river between the cable and the bridge; elevation, 13.10 feet. (2) A point marked with white paint on a dark granite bowlder 55 feet south of the rock pier of the bridge; elevation, 10.05 feet. Elevations refer to the datum of the gage.

Information in regard to this station is contained in Water-Supply Papers Nos. 81, 85. 100, and 134 of the United States Grological Survey.

Discharge measurements of Tule River near Portersville, Cal., in 1905.

\begin{tabular}{|c|c|c|c|c|c|c|}
\hline Date. & Hydrographer. & Width. & $\begin{array}{l}\text { Area of } \\
\text { section. }\end{array}$ & $\underset{\text { velocity. }}{\text { Mean }}$ & $\begin{array}{c}\text { Gage } \\
\text { height. }\end{array}$ & $\begin{array}{c}\text { Dis- } \\
\text { charge. }\end{array}$ \\
\hline & & Feet. & Sq. ft. & Ft. per sec. & Feet. & Sec.-ft. \\
\hline March 22.. & F. R. S. Buttemer.................. & 71 & 107 & 2.05 & 2.50 & 219 \\
\hline May $19 .$. & R. S. Hawley ........... & 77 & 152 & 2.34 & 3.00 & 355 \\
\hline June 13 . & .....do .............. & 69 & 97 & 1.68 & 2.27 & 163 \\
\hline July 26 .. & .....do...... & 33 & 26 & .96 & 1.16 & 25 \\
\hline September 18. & c. H. Lee........... & 26 & 17 & .58 & .92 & 10 \\
\hline September 26 . & Clapp and Holley... & 24 & 16 & .50 & .91 & 8.0 \\
\hline October $25 . .$. & Hawley and Lee................... & 30 & 22 & .70 & 1.10 & 15.5 \\
\hline
\end{tabular}

Daily gage height, in feet, of Tule River near Portersville, Cal., for 1905.

\begin{tabular}{|c|c|c|c|c|c|c|c|c|c|c|c|c|}
\hline Day. & Jan. & Feb. & Mar. & Apr. & May. & June. & July. & Aug. & Sept. & Oct. & Nov. & Dec. \\
\hline 1.. & 1.7 & 1.45 & 1.7 & 2.3 & 2.5 & 2.55 & 1.7 & 1.05 & 0.9 & 1.1 & 1.05 & 1.9 \\
\hline $2 .$. & 1.7 & 2.30 & 1.7 & 2.4 & 3.0 & 2.55 & 1.7 & 1.05 & .9 & 1.05 & 1.1 & 1.85 \\
\hline $3 .$. & 1.65 & 2.0 & 1.75 & 2.5 & .2 .9 & 2.5 & 1.65 & 1.05 & .9 & 1.05 & 1.1 & 1.85 \\
\hline 4. & 1.6 & 2.0 & 1.75 & 2.6 & 2.8 & 2.45 & .1 .6 & 1.05 & .9 & 1.0 & 1.1 & 1.8 \\
\hline 5. & 1.6 & 1.9 & 1.75 & 2.55 & 2.5 & 2.45 & 1.55 & 1.05 & .9 & 1.0 & 1.1 & 1.8 \\
\hline $6 .$. & 1.6 & 1.8 & 1.8 & 2.55 & 2.5 & 2.45 & 1.5 & 1.0 & .9 & 1.0 & 1.1 & 1.8 \\
\hline 7.. & 1.55 & 1.75 & 1.8 & 2.55 & 3.05 & 2.4 & 1.45 & 1.0 & 9 & 1.0 & 1.1 & 1.8 \\
\hline 8.. & 1.55 & 1.7 & 1.8 & 2.5 & 3.2 & 2.35 & 1.4 & 1.0 & .9 & 1.0 & 1.15 & $1 . S$ \\
\hline 9. & 1.5 & 1.65 & 1.75 & 2.5 & 2.95 & 2.35 & 1.4 & 1.0 & .9 & 1.05 & 1.15 & 1.8 \\
\hline 10. & 1.5 & $1.6^{\circ}$ & 1.75 & 2.4 & 2.85 & 2.3 & 1.4 & 1.0 & .95 & 1.05 & 1.15 & 1.8 \\
\hline 11.. & 1.5 & 1.6 & 1.7 & 2.35 & 2.8 & 2.3 & 1.4 & 1.0 & .95 & 1.0 & 1.15 & 1.8 \\
\hline 12. & 1.45 & 1.6 & 1.75 & 2.3 & 2.75 & 2.4 & 1.35 & .95 & .95 & 1.0 & 1.15 & 1.8 \\
\hline 13.. & 1.45 & 1.6 & 3.1 . & 2.35 & 2.7 & 2.35 & 1.35 & .95 & .95 & .95 & 1.15 & 1.8 \\
\hline 14. & 1.4 & 1.6 & 2.8 & 2.35 & 2.9 & 2.3 & 1.3 & .95 & 1.0 & 1.0 & 1.15 & 1.8 \\
\hline 15. & 1.4 & 1.7 & 2.6 & 2.4 & 3.0 & 2.25 & 1.3 & .95 & .95 & 1.0 & 1.2 & 1.8 \\
\hline 16. & 1.4 & 1.75 & 3.0 & 2.35 & 3.2 & 2.25 & 1.3 & .95 & .95 & 1.0 & 1.2 & 1.8 \\
\hline 17. & 1.4 & 1.75 & 3.0 & 2.3 & 3.3 & 2.1 & 1.25 & .95 & .95 & 1.0 & 1.3 & 1.8 \\
\hline 18. & 1.4 & 1.8 & 2.9 & 2.3 & 3.1 & 2.0 & 1.25 & .95 & .95 & 1.0 & 1.3 & 1.9 \\
\hline 19. & 1.4 & 1.8 & 4.1 & 2.5 & 3.0 & 1.95 & 1.2 & .95 & .95 & 1.0 & 1.3 & $1.9^{\circ}$ \\
\hline 20. & 1.4 & 1.75 & 3.7 & 2.45 & 3.0 & 1.9 & 1.2 & .95 & .95 & 1.0 & 1.7 & 1.9 \\
\hline 21. & 1.6 & 1.75 & 3.5 & 2.45 & 2.95 & 1.85 & 1.2 & .95 & .95 & 1.0 & 1.7 & 1.9 \\
\hline 22. & 1.6 & 1.75 & 3.1 & 2.4 & 2.8 & 1.8 & 1.15 & .95 & .95 & 1.0 & 1.65 & 1.9 \\
\hline 23. & 1.5 & 1.7 & 2.8 & 2.4 & 2.7 & 1.8 & 1.15 & .95 & .95 & 1.0 & 1.65 & 1.9 \\
\hline 24. & 1.5 & 1.7 & 2.5 & 2.35 & 2.75 & 1.8 & 1.1 & .95 & .95 & 1.0 & 1.65 & 1.85 \\
\hline 25. & 1.5 & 1.7 & 2.3 & 2.4 & 2.8 & 1.9 & 1.1 & .95 & .95 & 1.0 & 1.6 & 1.85 \\
\hline 26.. & 1.5 & 1.7 & 2.3 & 2.4 & 2.8 & 1.85 & 1.15 & .95 & .95 & 1.0 & 1.6 & 1.9 \\
\hline $27 .$. & 1.5 & 1.7 & 2.3 & 2.45 & 2.8 & 1.85 & 1.1 & $: 95$ & .95 & 1.0 & 2.1 & 2.0 \\
\hline 28. & 1.5 & 1.7 & 2.25 & 2.5 & $2: 75$ & 1.8 & 1.1 & .9 & 1.0 & 1.0 & 1.95 & 2.0 \\
\hline 29. & 1.5 & $\because \cdot$ & 2.9 & 2.6 & 2.7. & 1.8 & 1.15 & .9 & 1.0 & 1.0 & 1.85 & 2.1 \\
\hline 30. & 1.45 & $\ldots$ & 2.4 & 2.7 & 2.65 & 1.75 & 1.05 & .9 & 1.0 & 1.05 & 1.9 & .2 .1 \\
\hline $31 .$. & 1.4 & ....... & 2.3 & $\ldots \ldots \ldots$ & 2.6 & ....... & 1.05 & .9 & . & 1.05 & 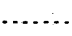 & 2.1 \\
\hline
\end{tabular}


Station rating table for Tule River near Portersville, Cal., from January 1 to December 31, 1905.

\begin{tabular}{|c|r||r|r||r|r|r|r|}
\hline $\begin{array}{c}\text { Gage } \\
\text { height. }\end{array}$ & Discharge. & $\begin{array}{c}\text { Gage } \\
\text { height. }\end{array}$ & Discharge. & $\begin{array}{c}\text { Gage } \\
\text { height. }\end{array}$ & Discharge. & $\begin{array}{c}\text { Gage } \\
\text { height. }\end{array}$ & Discharge. \\
\hline Feet. & Second-feet. & Feet. & Second-feet. & Feet. & Second-feet. & Feet. & Second-feet. \\
0.90 & 7 & 1.80 & 82 & 2.70 & 270 & 3.60 & 581 \\
1.00 & 12 & 1.90 & 98 & 2.80 & 297 & 3.70 & 626 \\
1.10 & 18 & 2.00 & 115 & 2.90 & 325 & 3.80 & 672 \\
1.20 & 24 & 2.10 & 133 & 3.00 & 355 & 3.90 & 720 \\
1.30 & 31 & 2.20 & 152 & 3.10 & 386 & 4.00 & 770 \\
1.40 & 38 & 2.30 & 173 & 3.20 & 419 & 4.10 & 822 \\
1.50 & 46 & 2.40 & 195 & 3.30 & 455 & & \\
1.60 & 56 & 2.50 & 219 & 3.40 & 494 & & \\
1.70 & 68 & 2.60 & 244 & 3.50 & 537 & & \\
\hline
\end{tabular}

Note.-The above table is based on seven discharge measurements made during 1905 . It is we!1 defined between gage heights 0.9 foot and 3 feet.

Estimated monthly discharge of Tule River near Portersville, Cal., for 1905.

[Drainage area, 437 square miles.]

\begin{tabular}{|c|c|c|c|c|c|c|}
\hline \multirow{2}{*}{ Month. } & \multicolumn{3}{|c|}{ Discharge in second-feet. } & \multirow[b]{2}{*}{$\begin{array}{l}\text { Total in } \\
\text { acre-feet. }\end{array}$} & \multicolumn{2}{|c|}{ Run-off. } \\
\hline & Maximum. & Minimum. & Mean. & & $\begin{array}{l}\text { Second-feet } \\
\text { per square } \\
\text { mile. }\end{array}$ & $\begin{array}{c}\text { Depth } \\
\text { in inches. }\end{array}$ \\
\hline January. & 68 & 38 & 47.4 & 2,914 & 0.108 & 0.124 \\
\hline February..... & 173 & 42 & 75.9 & 4,215 & .174 & .181 \\
\hline March........ & 822 & 68 & 230 & 14,140 & .526 & .606 \\
\hline April......... & 270 & 173 & 205 & 12,200 & .469 & .523 \\
\hline May.......... & 455 & 219 & 313 & 19,250 & .716 & .826 \\
\hline June............. & 232 & 75 & 146 & 8,688 & .334 & .373 \\
\hline July . . ........... & 68 & 15 & 32.9 & 2,023 & .075 & .086 \\
\hline August........ & 15 & 7 & 9.8 & 603 & .022 & .025 \\
\hline September.. & 12 & 7 & 8.2 & 488 & .019 & .021 \\
\hline October. . & 18 & 8 & 12.6 & 775 & .029 & . . .033 \\
\hline Novemter...... & 133 & 15 & 42.4 & 2,523 & .097 & .108 \\
\hline 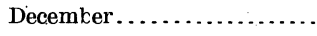 & 133 & 82 & 94.2 & 5,792 & .216 & .249 \\
\hline The year.... & 822 & 7 & 101 & 73,610 & .232 & 3.16 \\
\hline
\end{tabular}

MISCELLANEOUS MEASUREMENTS' IN TULE RIVER DRAINAGE BASIN.

South Fork of Tule River near Success, Cal.--The following measurements were made at a point one-haif mile above junction of South Fork with Main Tule River.

May 19: Width, 28 feet; area, 32 feet; mean velocity, 2.86 feet per second; discharge, 90 second-feet.

September 26, stream dry.

North Fork of Tule River near Springville, Cal.-A measurement was made September 26 at road crossing one-half mile above junction with Middle Fork of Kings River.

Water standing in pools; discharge, 00 second-feet.

Middle Fork of Tule River near Springville, Cal.-This stream is the principal tributary of Kaweah River and furnishes practically all the water of this river during low-water flow. A measurement was made September 27 one-fourth mile above clubhouse and 2 miles above the town of Springville.

Width, 33.5 leet; area, 36 square feet; mean velocity, 0.64 foot per second; discharge, 23 second-feet. 
South Fork of Middle Fork of Tule River near Springville, Cal.-This stream is a tributary to Middle Fork of Kaweah River. A measurement was made September 27 at a point 100 feet above its junction with Middle Fork.

Width, 8.4 feet; area, 4.0 square feet; mean velocity, 2.20 feet per second; discharge, 8.8 second-feet.

North Fork of Middle Fork of Tule River near Springville, Cal.-This stream is tributary to Middle Fork of Kaweah River. A measurement was made September 27100 feet above its junction with Middle Fork.

Width, 7.9 feet; area, 5.9 square feet; mean velocity, 2.19 feet per second; discharge, 12.9 second-feet.

\section{KAWEAH RIVER DRAINAGE BASIN. DESCRIPTIQN OF BASIN.}

Kaweah River drains the western slope of the Sierra Nevada between the basins of Kings River on the north and Kern and Tule rivers on the south. This is an important stream, but its watershed is only about one-third that of Kings River and is much less elevated and snow covered than those of the Kings and Kern rivers. It has a number of tributaries which have their source in numerous lakes and meadows on the higher elevations. The formation is of granite and similar in every way to that in the Kings River basin. The greater part of the area of 619 square miles above the gaging station is well covered with brush and timber. In this basin is situated the Sequoia National Park, where, the largest grove of big trees (Sequoia gigantea) of the Sierra Nevada is found. This grove is known as the "Giant Forest," and is one of the many points of interest of the Sierra Nevadas. There are two power plants on this stream owned by the Mount Whitney Power Company, which divert water from the Middle and East forks. This company has constructed several small storage reservoirs on the upper reaches of this basin. This has been done by building low dams at the outlet of some of the larger lakes. The stored water is held back for use during the low-water flow of the stream, and is of great benefit to the irrigators in the valley during the late summer months. About 6 miles below the gaging station the river leaves the foothills and flows across San Joaquin Valley in a general southwesterly direction to the old bed of Tulare Lake. After it leaves the foothills many canals divert water for the purpose of irrigating land in Tulare County, which is especially adapted to the raising of fruits.

The mean annual precipitation in the basin above the gaging station is from 20 to 40 inches, which falls in the form of snow over probably one-half the area.

\section{KAWEAH RIVER BELOW THREE RIVERS, GAL.}

This station was established April 29, 1903, by W. H. Stearns. It is located at a point three-fourths of a mile below the confluence of the North, Middle, and South forks. It is 10 miles from the Southern Pacific Railroad station at Lemon Cove, Tulare County, Cal., and one-fourth of a mile west of the wagon road from Exeter to Three Rivers.

The channel is straight for 400 feet above and below the station. The current is swift at high stages, but sluggish at low water. There are rapids about 400 feet above and 500 feet below the cable. The right bank is low and subject to overflow at high stages. The left bank is high enough to be above overflow. There are willow trees along the water's edge on both banks, and a line of willows, sycamores, and cottonwoods back from the water's edge on the left bank. The bed of the stream is composed of sand, gravel, and large bowlders. The section is probably permanent.

Discharge measurements are made from a cable and car. The initial point for soundings is a sycamore tree on the left bank of the stream, to which the cable is fastened.

The low-water gage is an inclined timber on the left bank. The high-water gage is a timber securely nailed to a willow tree on the left bank. During 1905 the gage was read by J. O. Carter. Bench marks were established as follows: (1) A point marked "B. M." with black paint on a large rock 10 feet upstream from the tree to which the cable is attached; 
elevation, 13.95 feet. (2) A point on rock marked with white paint 7 feet upstream from the 75-foot mark on the cable; elevation, 15.89 feet. Elevations refer to the datum of the gage.

Information in regard to this station is contained in Water-Supply Papers Nos. 100 and 134 of the United States Geological Survey.

Discharge measurements of Kaweah River below Three Rivers, Cal., in 1905.

\begin{tabular}{|c|c|c|c|c|c|c|}
\hline Date. & Hydrographer? & Width. & $\begin{array}{l}\text { Area of } \\
\text { section. }\end{array}$ & $\begin{array}{c}\text { Mean } \\
\text { velocity. }\end{array}$ & $\begin{array}{c}\text { Gage } \\
\text { height. }\end{array}$ & $\begin{array}{c}\text { Dis- } \\
\text { charge. }\end{array}$ \\
\hline March $18 . . . . .$. & F. R. S. Buttemer .. & $\begin{array}{r}\text { Feet. } \\
157\end{array}$ & $\begin{array}{r}S q \cdot f t \\
.464\end{array}$ & $\begin{array}{r}\text { Ft.per sec. } \\
1.24\end{array}$ & $\begin{array}{l}\text { Feet. } \\
\quad 5.94\end{array}$ & $\begin{array}{r}\text { Sec.-ft. } \\
577\end{array}$ \\
\hline March 20. . & ....do.......... & 160 & 526 & 1.55 & 6.26 & 815 \\
\hline May $20 . . .$. & R. S. Hawley ....... & 175 & 647 & 2.70 & 7.25 & 1,746 \\
\hline June $14 \ldots . . .$. & . . . do . . . . . . . . & 170 & 653 & 2.27 & 7.00 & 1,480 \\
\hline July $25 \ldots \ldots$. & .................... & 140 & 306 & 0.44 & 4.83 & 136 \\
\hline September 17 .. & C. H. Lee................ & 29 & 44 & 0.73 & 4.25 & 32 \\
\hline October $24 a \ldots$ & Hawley and Lee... & 56 & 61 & 0.80 & 4.30 & 49 \\
\hline
\end{tabular}

$a$ Section 400 feet below cable.

Daily gage height, in feet, of Kaweah River below Three Rivers, Cal., for 1905.

\begin{tabular}{|c|c|c|c|c|c|c|c|c|c|c|c|c|}
\hline $\mathrm{Da}$ & Jan. & Feb. & Mar. & Apr. & May. & June. & July. & Aug. & Sept. & Oct. & Nov. & Dec. \\
\hline 1. & 4.9 & 4.9 & 5.4 & 5.8 & 6.8 & 7.05 & 6.05 & 4.65 & 4.3 & 4.3 & 4.2 & 4.6 \\
\hline 2. & 4.85 & 5.95 & 5.45 & 5.9 & 6.7 & 7.0 & 6.0 & 4.65 & 4.3 & 4.35 & 4.2 & 4.6 \\
\hline 3. & 4.8 & 5.4 & 5.5 & 6.0 & 6.55 & 7.0 & 5.9 & 4.65 & 4.2 & 4.3 & 4.25 & 4.55 \\
\hline 4.. & 4.75 & 5.2 & 5.5 & 6.1 & 6.4 & 7.3 & 5.9 & 4.65 & 4.3 . & 4.25 & 4.25 & 4.5 \\
\hline $5 .$. & 4.7 & 5.65 & 5.5 & 6.2 & 6.3 & 6.8 & 5.8 & 4.6 & 4.3 & 4.3 & 4.25 & 4.6 \\
\hline $6 .$. & 4.7 & 5.2 & 5.4 & 6.2 & 6.4 & 7.45 & 5.75 & 4.6 & 4.3 & 4.2 & 4.3 & 4.5 \\
\hline 7. & 4.7 & 5.15 & 5.45 & 6.3 & 6.55 & 7.05 & 5.75 & 4.6 & 4.3 & 4.25 & 4.3 & 4.6 \\
\hline 8. & 4.7 & 5.15 & 5.5 & 6.25 & 6.6 & 6.95 & 5.7 & 4.55 & 4.3 & 4.2 & 4.3 & 4.55 \\
\hline 9. & 4.7 & 5.15 & 5.5 & 6.25 & 6.5 & 6.8 & 5.7 & 4.55 & 4.3 & 4.3 & 4.3 & 4.55 \\
\hline 10. & 4.7 & 5.0 & 5.45 & 6.2 & 6.35 & 7.05 & 5.65 & 4.55 & 4.2 & 4.25 & 4.3 & 4.55 \\
\hline 11. & 4.7 & 5.1 & 5.55 & 6.15 & 6.3 & 7.15 & 5.6 & 4.55 & 4.25 & 4.2 & 4.3 & $4.5^{\circ}$ \\
\hline 12. & 4.6 & 5.0 & a 5.6 & 6.0 & b. 3 & 7.35 & 5.5 & 4.55 & 4.25 & 4.2 & 4.25 & 4.5 \\
\hline 13. & 4.7 & 5.1 & 6.9 & 6.0 & 6.35 & 7.25 & 5.4 & 4.55 & 4.25 & 4.2 & 4.25 & 4.5 \\
\hline 14. & 4.6 & 5.0 & 5.9 & 6.0 & 6.55 & 7.15 & 5.3 & 4.6 & 4.25 & 4.2 & 4.3 & 4.55 \\
\hline 15. & 4.6 & 5.0 & 5.8 & 6.1 & 6.9 & 7.15 & 5.25 & 4.6 & 4.25 & 4.2 & 4.25 & 4.55 \\
\hline 16. & 4.65 & 5.45 & 6.1 & 6.2 & 7.3 & 7.15 & 5.2 & 4.6 & 4.25 & 4.25 & 4.3 & 4.55 \\
\hline 17. & 4.7 & 5.35 & 6.0 & 6.2 & 7.4 & 7.1 & 5.2 & 4.45 & 4.2 & 4.25 & 4.3 & 4.5 \\
\hline 18. & 4.65 & 5.65 & 5.95 & 6.35 & 7.35 & 7.0 & 5.1 & 4.4 & 4.25 & $a 4.25$ & 4.4 & 4.5 \\
\hline 19. & 4.7 & 5.3 & 7.0 & 6.3 & 7.4 & 6.95 & 5.1 & 4.4 & 4.2 & 4.25 & 4.3 & 4. 55 \\
\hline 20. & 4.7 & 5.2 & 6.2 & 6.25 & 7.3 & 6.9 & 5.0 & 4.35 & 4.25 & 4.25 & 4.45 & 4.6 \\
\hline 21. & 4.95 & 5.2 & 6.1 & 6.15 & 7.45 & 6.8 & 5.0 & 4.4 & 4.25 & 4.2 & 4.5 & 4.6 \\
\hline 22. & 4.95 & 5.15 & 5.9 & 6.15 & 7.4 & 6.7 & 5.0 & 4.4 & 4.2 & 4.2 & 4.4 & 4.5 \\
\hline 23. & 4.8 & 5.1 & a 5.9 & 6.15 & 7.3 & 6.6 & 4.95 & 4.4 & 4.2 & 4.2 & 4.4 & 4.5 \\
\hline 24 & 4.8 & 5.15 & 5.9 & 6.2 & 7.4 & 6.4 & 4.9 & 4.4 & 4.2 & 4.25 & 4.4 & 4.5 \\
\hline 25 & 4.75 & 5.2 & 5.9 & 6.35 & 7.5 & 6.30 & 4.9 & 4.4 & 4.2 & 4.3 & 4.4 & 4.6 \\
\hline 26. & 4.7 & 5.2 & 6.1 & 6.3 & 7.35 & 6.35 & 4.85 & 4.4 & 4.2 & 4.25 & 4.4 & 4.5 \\
\hline 27. & 4.7 & 5.1 & 6.0 & 6.35 & 7.1 & 6.0 & 4.85 & 4.4 & 4.2 & 4.25 & 4.55 & 4.5 \\
\hline 28 & 4.7 & 5.4 & 5.85 & 6.5 & 6.9 & 6.2 & 4.8 & 4.35 & 4.2 & 4.2 & 4.6 & 4.6 \\
\hline 29. & 4.7 & & 6.2 & 6.75 & 6.75 & 6.15 & 4.7 & 4.3 & 4.4 & 4.2 & 4.6 & 4.6 \\
\hline 30. & 4.7 & & 5.9 & 6.85 & 6.85 & 6.15 & 4.7 & 4. 3 & 4.45 & 4.25 & 4.6 & 4.6 \\
\hline & 4.7 & & 5.7 & ...... & 6.95 & & 4.7 & 4.25 & 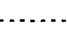 & 4.2 & $\cdots$ & 4.65 \\
\hline
\end{tabular}


Station rating table for Kaweah River below Three Rivers, Cal., from January 1 to December 31, 1905.

\begin{tabular}{|c|r||r|r|r|r||r|r|}
\hline $\begin{array}{c}\text { Gage } \\
\text { height. }\end{array}$ & Discharge. & $\begin{array}{c}\text { Gage } \\
\text { height. }\end{array}$ & Discharge. & $\begin{array}{c}\text { Gage } \\
\text { height. }\end{array}$ & Discharge. & $\begin{array}{c}\text { Gage } \\
\text { height. }\end{array}$ & Discharge. \\
\hline Feet. & Second-feet. & Feet. & Second-feet. & Feet. & Second-feet. & Feet. & Second-feet. \\
4.20 & 40 & 5.10 & 210 & 6.00 & 625 & 6.90 & 1,375 \\
4.30 & 49 & 5.20 & 240 & 6.10 & 690 & 7.00 & 1,480 \\
4.40 & 61 & 5.30 & 274 & 6.20 & 760 & 7.10 & 1,590 \\
4.50 & 75 & 5.40 & 312 & 6.30 & 835 & 7.20 & 1,700 \\
4.60 & 91 & 5.50 & 350 & 6.40 & 915 & 7.30 & 1,815 \\
4.70 & 109 & 5.60 & 395 & 6.50 & 1,000 & 7.40 & 1,935 \\
4.80 & 130 & 5.70 & 445 & 6.60 & 1,090 & 7.50 & 2,060 \\
4.90 & 154 & 5.80 & 500 & 6.70 & 1,180 & & \\
5.00 & 180 & 5.90 & 560 & 6.80 & 1,275 & & \\
\hline
\end{tabular}

Note.-The above table is based on seven discharge measurements made during 1905 . It is well defined throughout.

Estimated monthly discharge of Kaweah River below Three Rivers, Cal., for 1905.

[Drainage area, 520 square miles.]

\begin{tabular}{|c|c|c|c|c|c|c|}
\hline \multirow[b]{2}{*}{ Month. } & \multicolumn{3}{|c|}{ Discharge in second-feet. } & \multirow[b]{2}{*}{$\begin{array}{l}\text { Total in } \\
\text { acre-feet. }\end{array}$} & \multicolumn{2}{|c|}{ Run-off. } \\
\hline & Maximum. & Minimum. & Mean. & & $\begin{array}{l}\text { Second-feet } \\
\text { per square } \\
\text {.mile. }\end{array}$ & $\begin{array}{l}\text { Depth } \\
\text { in inches. }\end{array}$ \\
\hline January.. & 167 & 91. & 116 & 7,133 & 0.223 & 0.257 \\
\hline February... & 592 & 154 & 258 & 14,330 & .496 & .516 \\
\hline March... & 1,480 & 312 & 553 & 34,000 & 1.06 & 1.22 \\
\hline April..... & 1,325 & 500 & 783 & 46,590 & 1.51 & 1.68 \\
\hline May...... & 2,060 & 835 & 1,386 & 85,220 & 2.67 & 3.08 \\
\hline June....... & 1,998 & 625 & 1,348 & 80,210 & 2.59 & 2.89 \\
\hline July ....... & 658 & 109 & 303 & 18,630 & .583 & .672 \\
\hline August........ & 100 & 44 & 76.6 & 4,710 & .147 & .170 \\
\hline September..... & 68 & 40 & 45.2 & 2,690 & .087 & .097 \\
\hline October...... & 55 & 40 & 43.4 & 2,669 & .083 & .096 \\
\hline November....... & 91 & 40 & 56.6 & 3,368 & .109 & .122 \\
\hline December....... & 100 & 75 & 83.0 & 5,103 & .160 & .184 \\
\hline The year. & 2,060 & 40 & 421 & 304,700 & .810 & 10.98 \\
\hline
\end{tabular}

\section{MISCELLANEOUS MEASUREMENTS IN KAWEAH RIVER DRAINAGE BASIN.}

Marble Fork in Sequoia National Park, Cal.-This stream is one of the principal tributaries of the Kaweah River. A measurement was made October 12 by W. B. Clapp at a point 100 yards above its junction with the Middle Fork of Kaweah River.

Width, 8.1 feet; area, 6.0 square feet; mean velocity, 0.83 foot per second; discharge, 5.0 second-feet.

Middle Fork of Kaweah River in Sequoia National Park, Cal.-This stream is one of the principal tributaries of Kaweah River. A measurement was made October 12 by W. B. Clapp at a point 100 yards above its junction with Marhle Fork.

Width, 24.5 feet; area, 32.2 square feet; mean velocity; 0.51 foot per second; discharge, 16.3 second-feet.

Sonth Fork of Kaweah River near Three Rivers, Cal.-A measurement of this stream was made October 11 by W. B. Clapp, 2 miles above junction of Grouse Creek with South Fork, about 8 miles above the town of Three Rivers. 
Width, 3.5 feet; area, 1.7 square-feet; mean velocity, 2.53 feet per second; discharge, 4.3 second-feet.

East Fork of Kaweah River at power house of Mount Whitney Power Company.-Estimates of discharge of this stream were made by W. B. Clapp at the power house of Mount Whitney Power Company, 3 miles above the town of Three Rivers, Cal. The estimated discharge was calculated from discharge of nozzles at Mount Whitney Power Company's Plant.

September 28: Operating three Doble wheels. Wheel No. 1, 11-inch nozzle; wheel No. 2, 2-inch nozzle; wheel No. 3, 21-inch nozzle; effective head 1,290 feet; coefficient used, 0.98 ; discharge, 17.4 second-feet.

October 13: Two wheels running. Wheel No. 2, 2-inch nozzle; wheel No. 3, 21-inch nozzle; wheel No. 1, not running; effective head, 1,290 feet; coefficient used, 0.98; discharge, 14 second-feet.

Mount Whitney Power Company's flume, 100 feet below head-gate at boundary line Sequoia National Park.-The following measurements were made during 1905 by W. B. Clapp.

September 28: Width, 6.6 feet; area, 7.9 square feet; mean velocity, 2.18 feet per second; discharge, 17.2 second-feet.

October 12: Width, 4.75 feet; area, 6.4 square feet; mean velocity, 2.95 feet per second; discharge, 18.9 second-feet.

These measurements give total flow of Middle Fork of Kaweah River, including Marble Fork.

North Fork Kaweah River near Three Rivers, Cal.-A measurement of this stream was made October 13 by W. B. Clapp at a point 5 miles above junction of South Fork with Middle Fork of Kaweah River.

Width, 12 feet; area, 3.2 square feet; mean velocity, 1.50 feet per second; discharge, 4.8 second-feet.

\section{KINGS RIVER DRAINAGE BBASIN.}

\section{DESCRIPTION OF BASIN.}

Kings River rises on the western slope of the Sierra Nevada and drains the country located between the San Joaquin River on the north and the Kaweah and Kern rivers on the south. The crest of the Sierra Nevada at the head of this basin reaches elevations of over 14,000 feet, and here is the most rugged portion of the range; the sharp and precipitous peaks characteristic of this part of the Sierra Nevada produce the grandest scenery to be found in the United States. The main tributaries of this stream flow through great canyons cut in the granite, with high precipitous walls. The Kings River Canyon on the South Fork and Tehipite Valley on the Middle Fork rival the famed Yosemite Valley for grandeur of scenery. There are numerous tributaries, many of which have their sources in perpetual snow banks on the higher elevation. A large number of small lakes on the higher elevations are fed by small streams from perpetual snow banks or glaciers, and in them many of the tributaries have their source. The formation is of granite, which above an elevation of 10,000 feet is bare, with scanty vegetation, being carved by the action of glaciers; below the 10,000-foot contour is a heavy covering of timber and underbrush. Extensive groves of big trees are scattered throughout this basin. On the lower elevations along the foothills the soil covering is light with a grass growth used for pasturage. Fully 80 per cent of the drainage area is now included in the boundaries of the Sierra Forest Reserve which is patrolled for the prevention of fires and illegal herding. Below the gaging station, which is

- located at the point where the river leaves the foothills, canals divert the water for use in the valley lands of Fresno, Kings, and Tulare counties, where the climate and soil are especially adapted to the raising of grapes, fruits, etc., and the soil is under a high state of cultivation. During the period of flood discharge some water passes these canals and finds its way across Kings River delta in the natural channel to the old bed of Tulare Lake, which is now but an intermittent lake due largely to the diversion of water for irrigation purposes from the streams which drain into it. 
There is a drainage area of 1,742 square miles above the Red Mountain gaging station.

The mean annual precipitation for this area varies from about 30 to 60 inches, which over a greater portion of the basin falls in the form of snow. The greater discharge of this stream is in the spring months when the snow is melting.

\section{KTHGS RIVER TEAR SATGER, CAL.}

This station was established September 3, 1895, by J. B. Lippincott. It is located 15 miles east of Sanger, Cal., near the mouth of the canyon, and is above all diversions.

The channel is nearly straight for 300 feet above and below the station, and has a width of 180 feet at ordinary stages. The bed of the stream is composed of gravel and small bowlders and changes but little. The right bank is high and not subject to overflow. The left bank is subject to overflow during extreme high water. The current is swift.

Discharge measurements are made by means of a cable and car. The initial point for soundings is an eyebolt embedded in concrete on the right bank of river. An automatic river stage register was installed April 18, 1903. There is also an inclined wooden gage near by from which readings were formerly taken and which is now used in checking the selfrecording gage: $\mathrm{Mr}$. O. G. Williams reads the gage once each day and also examines the automatic register to see that it is in proper working order. The mean daily gage height is determined from the register sheets by the use of planimeter. The bench mark is a cross marked on a rock which is 24 feet upstream from the self-recording register; elevation, 18.00 feet above the datum of the gage.

Information in regard to this station is contained in Water-Supply Papers Nos. 81, 85, 100, and 134 of the United States Geological Survey.

Discharge measurements of Kings River near Sanger, Cal., in 1905.

\begin{tabular}{|c|c|c|c|c|c|c|}
\hline Date. & Hydrographer. & Width. & $\begin{array}{l}\text { Area of } \\
\text { section. }\end{array}$ & $\underset{\text { velocity. }}{\text { Mean }}$ & $\begin{array}{c}\text { Gage } \\
\text { height. }\end{array}$ & $\begin{array}{c}\text { Dis- } \\
\text { charge. }\end{array}$ \\
\hline & & Feet. & Sq.ft. & Ft. per sec. & Feet. & Sec.-ft. \\
\hline March 21. & F. R. S. Buttemer. & 217 & 883 & 2.73 & 6.91 & 2,406 \\
\hline May $22 \ldots \ldots$ & R. S. Hawley ....... & 317 & 1,827 & 4.95 & 10.10 & 9,046 \\
\hline June 15. . & .....do....... & 314 & 1,808 & 4.67 & 10.10 & 8,446 \\
\hline July $24 \ldots$. & $\ldots \ldots$ do $\ldots . . .$. & 169 & 584 & 1.69 & 5.42 & 986 \\
\hline August 19 & .....do...... & 145 & 390 & .97 & 4.33 & 382 \\
\hline September 16.. & C. H. Lee......... & 126 & 317 & .63 & 3.82 & 199 \\
\hline October 21..... & Hawley and Lee................ & 113 & 128 & 1.53 & 3.79 & 186 \\
\hline
\end{tabular}


Mean daily gage height, in feet, of Kings River near Sanger, Cal., for 1905.

\begin{tabular}{|c|c|c|c|c|c|c|c|c|c|c|c|c|}
\hline Day. & Jan. & Feb. & Mar. & Apr. & May. & June. & July. & Aug. & Sept. & Oct. & Nov. & Dec. \\
\hline 1. & 4.65 & 5.0 & 5.5 & 6.25 & 8.9 & 9.0 & 7.8 & 4.95 & 4.1 & 4.05 & 3.8 & 4.10 \\
\hline 2. & 4.55 & 5.65 & 5.7 & 6.5 & 8.35 & 9.05 & 7.6 & 4.85 & 4.05 & 4.0 & 3.8 & 4.05 \\
\hline $3 .$. & 4.45 & 5.6 & 5.85 & 6.6 & 8.05 & 9.15 & 7.5 & 4.8 & 4.0 & 4.0 & 3.8 & 4.0 \\
\hline $4 .$. & 4.45 & 5.45 & 5.95 & 6.7 & 7.7 & 8.85 & 7.4 & 4.8 & 4.0 & 3.9 & 3.8 & 4.1 \\
\hline $5 .$. & 4.4 & 5.7 & 5.9 & 6.9 & 7.55 & 8.7 & 7.3 & 4.7 & 4.0 & 3.85 & 3.8 & 4.15 \\
\hline $6 .$. & 4.4 & 5.65 & 5.9 & 7.05 & 7.65 & 9.05 & 7.25 & 4.65 & 4.0 & 3.85 & 3.8 & 4.1 \\
\hline 7... & $a 4.4$ & 5.4 & 5.9 & 7.2 & 7.75 & 9.35 & 7.25 & 4.6 & 4.0 & 3.8 & 3.8 & 4.1 \\
\hline 8.. & a 4.4 & 5.3 & 5.95 & 7.2 & 7.9 & 9.3 & 7.2 & 4.6 & 3.95 & 3.8 & 3.8 & 4.1 \\
\hline $9 .$. & 4.4 & 5.25 & 5.95 & 7.25 & 7.7 & 9.1 & 7.2 & 4.6 & 3.95 & 3.8 & .8 & 4.05 \\
\hline $10 .$. & 4. 35 & 5.15 & 5.9 & 7.25 & 7.4 & 9.4 & 7.2 & 4.65 & 3.9 & 3.8 & 3.8 & 4.0 \\
\hline $11 .$. & 4.4 & 5.05 & 5.9 & 7.0 & 7.4 & 9.8 & 7.15 & 4.6 & 3.9 & 3.75 & $a 3.8$ & 4.0 \\
\hline $12 .$. & 4.3 & 5.0 & 5.95 & 6.75 & 7.4 & 10.25 & 7.0 & 4.55 & 3.9 & 3.75 & 3.8 & 4.0 \\
\hline $13 .$. & 4.2 & 4.85 & 7.15 & 6.6 & 7.45 & 10.3 & 6.7 & 4.55 & 3.9 & 3.75 & 3.8 & 4.0 \\
\hline 14. & 4.35 & 4.85 & 7.35 & 6.6 & 7.95 & 10.2 & 6.4 & 4.5 & 3.9 & .7 & 8 & 4.0 \\
\hline 15. & a 4.35 & 4.85 & 6.65 & 6.9 & 8.8 & 10.0 & 6.2 & 4.45 & 3.9 & 3.7 & 3.8 & 4.0 \\
\hline 16. & 4.35 & 5.0 & 6.55 & 7.05 & 9.6 & 9.9 & 6.0 & 4.45 & 3.85 & 3.7 & 3.8 & 4.0 \\
\hline 17. & 4.4 & 5.4 & 6.75 & 7.0 & 10.25 & 9.85 & 5.85 & 4.4 & 3.8 & 3.7 & 3.8 & 4.0 \\
\hline $18 .$. & 4.4 & 5.25 & 6.75 & 7.3 & 10.15 & 9.6 & 5.75 & 4.4 & 3.8 & 3.75 & 3.8 & 4.0 \\
\hline 19. & 4. 35 & 5.2 & 7.0 & 7.2 & 10.15 & 9.4 & 5.65 & 4.35 & 3.8 & 3.75 & 3.9 & 4.0 \\
\hline $20 \ldots$ & 4.45 & 5.15 & 7.2 & 6.9 & 10.05 & 9.4 & 5.6 & 4.3 & 3.8 & 3.8 & 3.95 & 4.05 \\
\hline $21 .$. & 4.7 & 5.1 & 6.9 & 6.85 & 10.15 & 9.45 & 5.55 & 4.3 & 3.8 & 3.8 & 4.0 & 4.1 \\
\hline 22. & 4.8 & 5.1 & 6.55 & 6.8 & 10.15 & 9.4 & 5.55 & 4.3 & 3.75 & 3.8 & 3.9 & 4.0 \\
\hline 23. & 4.6 & 5.15 & 6.4 & 6.8 & 9.95 & 8.95 & 5.5 & 4.3 & 3.75 & 3.8 & 3.9 & 3.9 \\
\hline 24. & 4.6 & 5.2 & 6.5 & 7.1 & 9.95 & 8.45 & 5.5 & 4.25 & 3.75 & 3.8 & 3.8 & 3.9 \\
\hline 25. & 4.55 & 5.25 & 6.6 & 7.5 & 10.15 & 8.2 & 5.5 & 4.2 & 3.75 & 3.7 & 3.8 & a 3.9 \\
\hline 26. & 4.5 & 5.3 & 6.8 & 7.5 & 10.15 & 8.25 & 5.5 & 4.2 & 3.7 & 3.7 & 3.85 & 3.9 \\
\hline $27 .$. & 4.45 & 5.3 & 6.7 & 7.75 & 9.4 & 8.05 & 5.45 & 4.2 & 3.7 & 3.7 & 3.9 & 4.0 \\
\hline 28.. & 4. 45 & 5.35 & 6.4 & 8.2 & 8.85 & 7.95 & 5.4 & 4.15 & 3.7 & 3.7 & 4.1 & 4.0 \\
\hline 29. & 4.45 & & 6.55 & 8.75 & 8.5 & 8.0 & 5.25 & 4.1 & 3.75 & 3.75 & 4.0 & 4.0 \\
\hline & 4.45 & & 6.4 & 9.05 & 8.65 & 8.0 & 5.15 & 4.1 & 3.95 & 3.75 & $a 4.0$ & 4.0 \\
\hline $31 \ldots$ & 4.5 & & 6.25 & & 8.9 & & 5.05 & 4.1 & 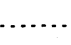 & 3.75 & $\ldots .$. & 3.9 \\
\hline
\end{tabular}

$a$ Gage height estimated.

Station rating table for Kings River near Sanger, Cal., from January 1 to December 31, 1905.

\begin{tabular}{|c|c||c|c||c|c|c|c|}
\hline $\begin{array}{c}\text { Gage } \\
\text { height. }\end{array}$ & Discharge. & $\begin{array}{c}\text { Gage } \\
\text { height. }\end{array}$ & Discharge. & $\begin{array}{c}\text { Gage } \\
\text { height. }\end{array}$ & Discharge. & $\begin{array}{c}\text { Gage } \\
\text { height. }\end{array}$ & Discharge. \\
\cline { 1 - 6 } Feet. & Second-feet. & Feet. & Second-feet. & Feet. & Second-feet. & Feet. & Second-feet. \\
3.70 & 150 & 5.00 & 740 & 6.30 & 1,730 & 8.00 & 3,850 \\
3.80 & 175 & 5.10 & 805 & 6.40 & 1,820 & 8.20 & 4,190 \\
3.90 & 205 & 5.20 & 870 & 6.50 & 1,920 & 8.40 & 4,570 \\
4.00 & 240 & 5.30 & 940 & 6.60 & 2,020 & 8.60 & 4,980 \\
4.10 & 280 & 5.40 & 1,010 & 6.70 & 2,120 & 8.80 & 5,425 \\
4.20 & 325 & 5.50 & 1,085 & 6.80 & 2,230 & 9.00 & 5,890 \\
4.30 & 370 & 5.60 & 1,160 & 6.90 & 2,340 & 9.20 & 6,380 \\
4.40 & 420 & 5.70 & 1,235 & 7.00 & 2,460 & 9.40 & 6,915 \\
4.50 & 470 & 5.80 & 1,310 & 7.20 & 2,705 & 9.60 & 7,495 \\
4.60 & 520 & 5.90 & 1,390 & 7.40 & 2,965 & 9.80 & 8,115 \\
4.70 & 570 & 6.00 & 1,470 & 7.60 & 3,240 & 10.00 & 8,770 \\
4.80 & 625 & 6.10 & 1,550 & 7.80 & 3,535 & 10.20 & 9,450 \\
4.90 & 680 & 6.20 & 1,640 & & & &. \\
& & & & & & & \\
\hline
\end{tabular}

Note.-The above table is based on discharge measurements made during 1895-1905. It is well defined throughout. 
Estimated monthly discharge of Kings River near Sanger, Cal., for 1905.

[Drainage area, 1,742 square miles.]

\begin{tabular}{|c|c|c|c|c|c|c|}
\hline \multirow[b]{2}{*}{ Month. } & \multicolumn{3}{|c|}{ Discharge in second-feet. } & \multirow{2}{*}{$\begin{array}{l}\text { Total in } \\
\text { acre-feet. }\end{array}$} & \multicolumn{2}{|c|}{ Run-off. } \\
\hline & Maximum. & Minimum. & Mean. & & $\begin{array}{l}\text { Second-feet } \\
\text { per square } \\
\text { mile. }\end{array}$ & $\begin{array}{l}\text { Depth } \\
\text { in inches. }\end{array}$ \\
\hline January... & 625 & 325 & 447 & 27,480 & 0.257 & 0.296 \\
\hline February...... & 1,235 & 652 & 899 & 49,930 & .516 & . .537 \\
\hline March...... & 2,900 & 1,085 & 1,844 & 113,400 & 1.06 & 1.22 \\
\hline April.......... & 6,010 & 1,685 & 2,731 & 162,500 & 1.57 & 1.75 \\
\hline May....... & 9,622 & 2,965 & 5,887 & 361,600 & 3.38 & 3.90 \\
\hline June........ & 9,795 & 3,770 & 6,448 & 383,700 & 3.70 & 4.13 \\
\hline July ....... & 3,535 & 772 & 1,859 & 114,300 & 1.07 & 1.23 \\
\hline August..... & 710 & 280 & 448 & 27,550 & .257 & .296 \\
\hline Septemker.. & 280 & 150 & 198 & 11,780 & .114 & .127 \\
\hline October... & 260 & 150 & 174 & 10,700 & .100 & .115 \\
\hline November. & 280 & 175 & 191 & 11,360 & .110 & .123 \\
\hline December. & 302 & 205 & 246 & 15,130 & .141 & .163 \\
\hline The year. & 9,795 & 150 & 1,781 & $1,289,000$ & 1.02 & 13.89 \\
\hline
\end{tabular}

MISCELLANEOUS MEASUREMENTS IN KINGS RIVER DRAINAGE BASIN.

North Fork of Kings River.-This stream enters Kings River from the north about 20 miles above point where the main Kings River leaves the foothills and enters the San Joaquin Valley. A measurement was made 500 feet above its junction with main river and about 50 feet above lower trail crossing on August 22, 1905, by R. S. Hawley.

Width, 16 feet; area, 21 square feet; mean velocity, 1.86 feet per second; discharge, 33 second-feet.

Converse Creek.-This stream enters the Kings River 7 miles below the junction of Middle and South forks. A measurement was made 200 feet above its mouth on August 23, 1905, by R. S. Hawley.

Width, 4 feet; area, 1.6 square feet; mean velocity, 0.77 foot per second; discharge, 1.2 second-feet.

Ten Mile Creek. - This creek enters Kings River one-half mile below junction of Middle and South forks. A measurement was made on August 23, 1905, 100 feet above its mouth by R. S. Hawley.

Width, 2 feet; area, 1.6 square feet; mean velocity, 0.69 foot per second; discharge, 1.1 second-feet.

Boulder Creek.-This stream enters the South Fork of Kings River 5 miles above the junction of the Middle and South forks. A measurement was made on August 25, 1905, 2 miles above its mouth and 100 feet above lower trail crossing by R. S. Hawley.

Width, 6 feet; area, 2.8 square feet; mean velocity, 1.14 feet per second; discharge, 3.2 second-feet.

Lightning Creek.-This stream enters the South Fork of Kings River from the south about 10 miles above the junction of the Middle and South forks. An estimate was made of the discharge about 1 mile above its mouth at the trail crossing on August 25, 1905, by R. S. Hawley.

Discharge, 0.60 second-foot.

Lewis Creek.-This stream enters the South Fork of Kings River from the north in Kings River Canyon 2 miles below old Cedar Grove Hotel. A measurement was made August 26, 1905, 500 feet above its mouth by R. S. Hawley.

Width, 3 feet; area, 2.7 square feet; mean velocity, 1.00 foot per second; discharge, 2.7 second-feet. 
South Fork of Kings River.-This stream is the principal tributary to Kings River. A measurement was made in the lower Kings River Canyon one-fourth mile above the mouth of Lewis Creek on August 26, 1905, by R. S. Hawley.

Width, 52 feet; area, 51 square feet; mean velocity, 2.73 feet per second; discharge, 139 second-feet.

South Fork of Kings River.-A measurement was made 700 feet above the mouth of Bubbs Creek on August 26, 1905, by R. S. Hawley.

Width, 35 feet; area, 36 square feet; mean velocity, 1.36 feet per second; discharge, 49 second-feet.

South Fork of Kings River.-A measurement was made August 26, 1905, one-fourth mile below the mouth of Bubbs Creek by R. S. Hawley.

Width, 65 feet; area, 68 square feet; mean velocity, 1.46 feet per second; discharge, 99 second-feet.

Bubbs Creek.-This is the principal tributary of South Fork of Kings River and joins it at the head of Kings River Canyon. From the measurements made on South Fork of Kings River above and below the junction of Bubbs Creek on August 26, 1905, by R. S. Hawley, the discharge of Bubbs Creek at the mouth was estimated to be 50 second-feet. On account of roughness of stream bed and high velocities in the creek it was impossible to make discharge measurement. (See measurement of South Fork of Kings River above and below mouth of Bubbs Creek.)

Copper Creek.--This stream enters the South Fork of Kings River from the north between North Dome and Buck Peak. A measurement was made on August 26, 1905, one-fourth mile above its mouth by R. S. Hawley.

Width, 4 feet; area, 1.0 square foot; mean velocity, 0.82 foot per second; discharge, 0.82 second-foot.

Roaring River.-This stream enters South Fork of Kings River from the south, 5 miles below the junction of South Fork and Bubbs Creek. A measurement was made August 26, 1905,500 feet above its mouth by R. S. Hawley.

Width, 14 feet; area, 11 square feet; mean velocity, 0.87 foot per second; discharge, 9.6 second-feet.

Sheep Creek.-This stream enters the South Fork of Kings River from the south onefourth mile below Cedar Grove Hotel. An estimate was made of the discharge at the mouth August 26, 1905, by R. S. Hawley.

Discharge, 1.8 second-feet.

Hotel Creek.-This stream enters South Fork of Kings River from the north at Cedar Grove Hotel. An estimate was made of the discharge at its mouth on August 26, 1905, by R. S. Hawley.

Discharge, 0.20 second-foot.

Granite Creek.-This stream enters South Fork of Kings River from the north between North Mountain and North Dome. An estimate was made of the discharge at the mouth August 26, 1905, by R. S. Hawley.

Discharge, 0.50 second-foot.

Horseshoe Creek.-This stream enters Middle Fork of Kings River from the south at Simpson Meadow. An estimate was made of the discharge at its mouth on August 28, 1905, by R. S. Hawley.

Discharge, 2.4 second-feet.

Middle Fork of Kings River.-This is one of the principal tributaries of Kings River and drains the high Sierra Nevadas in the northeastern portion of its watershed. A measurement was made on August 28, 1905, 50 feet above the mouth of Horseshoe Creek, at the lower end of Simpson Meadow, by R. S. Hawley.

Width, 41 feet; area, 65 square feet; mean velocity; 1.83 feet per second; discharge, 119 second-feet.

Goddard Creek.-This stream is the principal tributary of Middle Fork of Kings River, entering it from the north. A measurement was made August 28, 1905, one-fourth mile above its mouth at Simpson Meadow by R. S. Hawley. 
Width, 26 feet; area, 29 square feet; mean velocity, 1.93 feet per second; discharge, 56 second-feet.

Dougherty Creek.- This stream enters Middle Fork of Kings River from the south about 1 mile below Simpson Meadow. A measurement was made August 29, 1905, 300 feet above its mouth by R. S. Hawley.

Width, 4 feet; area 4.4 square feet; mean velocity, 0.86 foot per second; discharge, 3.8 second-feet.

Slide Creek.-This is a small stream entering Middle Fork of Kings River from the south 3 miles below Simpson Meadow. An estimate of its discharge was made 300 feet above its mouth August 29, 1905, by R. S. Hawley.

Discharge, 0.50 second-foot.

Crown Creek.-This stream enters Middle Fork of Kings River in Tehipite Valley one-half mile southwest of Tehipite Dome. An estimate of its discharge was made on August 29, 1905,500 feet above its mouth, above the trail crossing, by R. S. Hawley.

Discharge, 8.0 second-feet.

Blue Canyon Creek.- This stream enters Middle Fork of Kings River 2 miles above Tehipite Dome. An estimate was made of the discharge August 29, 1905, 200 feet above its mouth by R. S. Hawley.

Discharge, 1.4 second-feet.

Rancheria Creek.-This stream enters North Fork of Kings River about 4 miles below Cliff Camp. A measurement was made on August 31, 1905, about 4 miles above its mouth and just below its junction with North Fork of Rancheria Creek by R. S. Hawley.

Width, 8 feet; area, 7.2 square feet; mean velocity, 0.78 foot per second; discharge, 5.6 second-feet.

North Fork of Rancheria Creek.-This stream joins Rancheria Creek about 4 miles above its mouth: A measurement was made August 31, 1905, 100 feet above its junction with Rancheria Creek, by R. S. Hawley.

Width, 2 feet; area, 1.2 square feet; mean velocity, 2.00 feet per second; discharge, 2.4 second-feet.

North Fork of Kings River.-A measurement was made on this stream on August 31, 1905, about one-half mile above Cliff Camp and about 15 miles above its mouth, by R. S. Hawley.

Width, 8.0 feet; area, 12.8 square feet; mean velocity, 0.73 foot per second; discharge, 9.4 second-feet.

Bear Creek.-This stream is tributary to North Fork of Kings River through Dinkey Creek. An estimate was made of the discharge of this stream on August 31, 1905, about 2 miles above its junction with Dinkey Creek at trail crossing, by R. S. Hawley.

Discharge, 0.70 second-foot.

Laurel Creek. -This stream is tributary to North Fork of Kings River through Bear Creek and Dinkey Creek. An estimate was made of the discharge on August 31, 1905, about one-fourth mile above junction with Bear Creek, by R. S. Hawley.

Discharge, 0.40 second-foot.

Dinkey Creek.-This stream is a tributary of North Fork of Kings River from the west. A measurement was made about $1 \frac{1}{2}$ miles above the mouth of Bear Creek at Dinkey Meadows on September 1, 1905, by R. S. Hawley.

Width, 6 feet; area, 4 square feet; mean velocity, 0.70 foot per second; discharge, 2.8 second-feet.

\section{MERCED RIVER DRAINAGE BASIN.}

\section{DESCRIPTION OF BASIN.}

Merced River drains that portion of the western slope of the Sierra Nevada located between the Tuolumne River on the north and the San Joaquin River on the south. Its drainage area is much less than that of the Tuolumne River. It has numerous tributaries, several of which are of considerable size.

The topography of the country in this basin is similar to that of the Tuolumne River, being rough and broken in the upper reaches. In this basin is situated the famous Yosemite 
Valley with its precipitous walls and domes and great waterfalls, which occur on the main stream and its tributaries, which discharge into the valley over precipitous cliffs rising 2,000 to 3,000 feet above the floor of the valley. The formation is of granite which on the upper reaches of the basin above Yosemite Valley is bare, rising in precipitous peaks and domes, and is smoothly marked by glacial action. The middle reaches of the basin are well timbered. The Mariposa grove of big trees is situated in the basin of the South Fork. The timber growth extends well down on the lower elevations to the foothills where the covering is of brush and grass, used extensively for pasturage. Numerous lakes are scattered over the upper portion of the basin. The mean annual precipitation varies from 25 inches in the foothills to 60 inches on the higher elevations, where it falls in the form of snow, which melts in the spring months, except on the extreme higher mountain peaks, where it often remains during the entire year. After leaving the foothills at Merced Falls, where the gaging station is located, canals divert the water for irrigation on lands along the river bottom and in San Joaquin Valley. The surplus water during flood discharge enters San Joaquin River.

\section{MERCED RIVER IN YOSEMITE VALLEY, CALIFORNIA.}

This station was established July 11, 1904, by A. E. Chandler and N. W. Currie. It is located at the wagon bridge, near the Sentinel Hotel.

The channel is straight for a distance of 150 feet above and 50 feet below station. The current is sluggish. The right bank is low and subject to overflow. The left bank is high and above high water. The bed of the stream is composed of coarse gravel and sand, with small bowlders, and is not subject to much change.

Discharge measurements are made from the lower side of the bridge. The initial point for soundings is stream face of abutment on right bank.

The gage is a vertical timber, securely fastened to a masonry abutment on west bank. The bench mark is the heads of two large nails driven into the stream face of the bridgeseat timber on the left abutment; elevation, 14.64 feet above the datum of the gage.

A description of this station, with gage height and discharge data, is contained in WaterSupply Paper No. 134, United States Geological Survey.

Discharge measurements of Merced River in Yosemite Valley, California, in 1905.

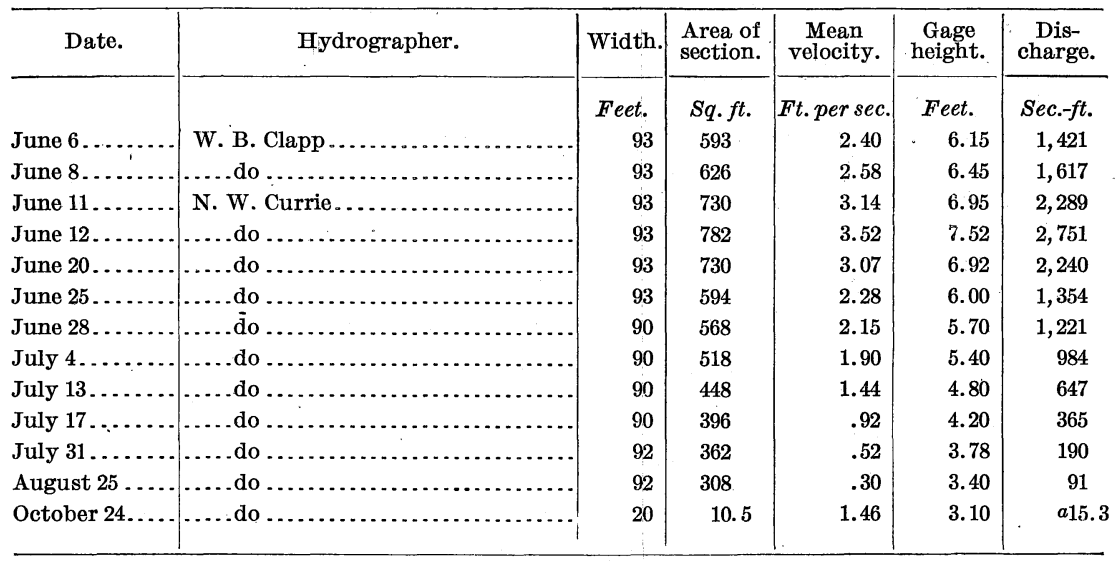

$a$ Wading 400 feet below bridge.

IRR $177-06-14$ 
Daily gage height, in feet, of Merced River in Yosemite Valley, California, for 1905.

\begin{tabular}{|c|c|c|c|c|c|c|c|c|c|c|c|c|c|}
\hline Day. & May. & June. & July. & Aug. & Sept. & Oct. & Day. & May. & June. & July. & Aug. & Sept. & Oet. \\
\hline 1. & & 6.2 & 5.5 & 3.7 & 3. 3 & 3.3 & 17. & $\therefore$ & 7.1 & 4.2 & 3.5 & 3.1 & $a 3.15$ \\
\hline 2. & & 6. 2 & 5.5 & 3.7 & 3.3 & a 3.3 & 18. & & 6.9 & 4.15 & 3.5 & 3.1 & $a 3.15$ \\
\hline 3. & & 6. 1 & 5. 3 & 3. 7 & 3.3 & 3.25 & 19. & & 6.8 & 4.2 & 3.45 & 3.15 & a.3.15 \\
\hline 4. & & 5.95 & 5.4 & 3.7 & 3.25 & a 3.25 & 20. & & 6.9 & 4.2 & 3.45 & 3.2 & a 3.1 \\
\hline 5. & & 5.8 & 5. 35 & 3.65 & 3.2 & $a 3.25$ & 21. & & 7.1 & 4.2 & 3.45 & 3. 2 & 3.1 \\
\hline 6. & & 6.2 & 5. 35 & 3.6 & 3.2 & a 3.25 & 22. & $\ldots$ & 7.0 & 4.2 & 3.45 & 3.2 & $a 3.1$ \\
\hline 7. & & 6.5 & 5.3 & 3.6 & 3.2 & 3.25 & 23. & 6.8 . & 6.5 & 4.2 & 3. 45 & 3.3 & $a 3.1$ \\
\hline 8. & & 6.4 & 5. 25 & 3.6 & 3.2 & a $3.25^{\circ}$ & 24. & 6.8 & 6.5 & 4.2 & 3.4 & 3.3 & 3.1 \\
\hline 9 & & 6.2 & 5.5 & 3.7 & 3.2 & a 3.2 & 25. & 7.1 & 6.0 & 4.1 & 3.4 & 3.3 & 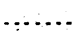 \\
\hline 10. & & 6.55 & 5.5 & 3.7 & 3.2 & 3.2 & 26. & 7.1 . & 6.0 & 4.1 & 3.35 & 3.3 & ........ \\
\hline 11. & & 7.0 & 5.3 & 3.7 & 3.2 & a 3.2 & 27. & 6.3 & 5.8 & 4.1 & 3.3 & 3.3 & - \\
\hline 12. & & 7.5 & 5.1 & 3.6 & 3.15 & 3.2 & 28. & $a 6.0$ & 5. 7 & 4.0 & 3.3 & 3. 4 & $\ldots$ \\
\hline 13. & & 7.45 & 4.8 & 3.6 & 3.1 & a 3.2 & 29. & 5.7 & 5.95 & 3.9 & 3. 3 & 3.4 & - \\
\hline 14. & & 7. 35 & 4.5 & 3. 5 & 3.1 & 3. 15 & 30. & 6.1 & 5.8 & 3.8 & 3.3 & 3.35 & . \\
\hline 15 & & 7.3 & 4.35 & 3.5 & 3.1 & 3.15. & 31. & 6.1 & & 3.8 & 3.3 & & \\
\hline 16. & & 7.4 & 4.3 & 3.5 & 3.1 & a 3.15 & & & & & & & \\
\hline
\end{tabular}

a Gage height estimated.

Station rating table for Merced River in Yosemite Valley, California, from July 11, 1904, to December 31, 1905.

\begin{tabular}{|r|r||r|r|r|r||r|r|}
\hline $\begin{array}{c}\text { Gage } \\
\text { height. }\end{array}$ & Discharge. & $\begin{array}{c}\text { Gage. } \\
\text { height. }\end{array}$ & Discharge. & $\begin{array}{c}\text { Gage } \\
\text { height. }\end{array}$ & Discharge. & $\begin{array}{c}\text { Gage } \\
\text { height. }\end{array}$ & Discharge. \\
\hline Feet. & Second-feet. & Feet. & Second-feet. & Feet. & Second-feet. & Feet. & Second-feet. \\
3.10 & 15 & 4.10 & 326 & 5.10 & 815 & 6.20 & 1,505 \\
3.20 & 38 & 4.20 & 368 & 5.20 & 870 & 6.40 & 1,645 \\
3.30 & 63 & 4.30 & 412 & 5.30 & 925 & 6.60 & 1,795 \\
3.40 & 90 & 4.40 & 457 & 5.40 & .985 & 6.80 & 1,950 \\
3.50 & 118 & 4.50 & 504 & 5.50 & 1,045 & 7.00 & 2,110 \\
3.60 & 147 & 4.60 & 552 & 5.60 & 1,105 & 7.20 & 2,275 \\
3.70 & 178 & 4.70 & 602. & 5.70 & 1,170 & 7.40 & 2,445 \\
3.80 & 212 & 4.80 & 653 & 5.80 & 1,235 & & \\
3.90 & 248 & 4.90 & 706 & 5.90 & 1,300 & & \\
4.00 & 286 & 5.00 & 760 & 6.00 & 1,365 & & \\
& & & & & & & \\
\hline
\end{tabular}

NoTE.-The above table is based on 12 discharge measurements made during 1904-5. It is well defined between gage heights 3.1 feet and 6.5 feet. 
Estimated monthly discharge of Merced River in Yosemite Valley. California, for 1904 and 1905.

\begin{tabular}{|c|c|c|c|c|}
\hline \multirow{2}{*}{ Month. } & \multicolumn{3}{|c|}{ Discharge in second-feet. } & \multirow{2}{*}{$\begin{array}{l}\text { Total in } \\
\text { acre-feet. }\end{array}$} \\
\hline & Maximum. & Minimum. & Mean. & \\
\hline 1904. & & & & \\
\hline July $11-31 \ldots \ldots \ldots \ldots \ldots \ldots \ldots$ & 602 & 286 & 463 & 19,280 \\
\hline August. ................. & 552 & 112 & 248 & 15,250 \\
\hline September $1-24 \ldots \ldots \ldots \ldots \ldots \ldots \ldots \ldots \ldots \ldots \ldots \ldots \ldots$ & 412 & 63 & 131 & 6,236 \\
\hline 1905. & & & & \\
\hline 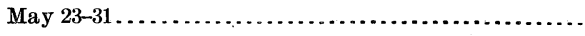 & 2,190 & 1,170 & 1,696 & 30,280 \\
\hline 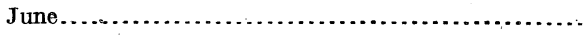 & 2,530 & 1,170 & 1,771 & 105,400 \\
\hline 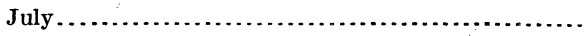 & 1,170 & 212 & 610 & 37,510 \\
\hline August. . . . . . . . . . . . & 178 & 63 & 123 & 7,563 \\
\hline 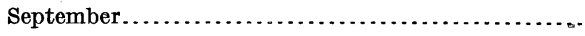 & 90 & 15 & 44.4 & 2,642 \\
\hline 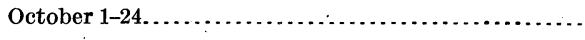 & 63 & 15 & 35.3 & 1,680 \\
\hline The period. & & & & 185,100 \\
\hline
\end{tabular}

MERCED RIVER ABOVE MERCED FALIS, CAL.

The measurement of this stream was undertaken in response to numerous requests from mining and irrigation interests. The midsummer flow of the stream is less than the combined capacity of the irrigation and power canals taking water in the vicinity of Snelling. The station was established April 6, 1901, by H. H. Henderson. It is located 1 mile above Merced Falls.

Both banks are high and rocky and are not subject to overflow. The bed of the stream is composed of gravel and is subject to some change.

Discharge measurements are made from a cable and car.

The gage is a timber bolted to iron stakes driven in the bed of the stream on the left bank. During 1905 the gage was read by Charles Siegfeldt. The bench mark is a three-fourths inch round iron bolt set 10 inches in the slate rock on the right bank of the river; elevation, 69.20 feet above the datum of the gage.

Information in regard to this station is contained in Water-Supply Papers Nos. 81, 85, 100, and 134 of the United States Geological Survey.

Discharge measurements of Merced River above Merced Falls, California, in 1905.

\begin{tabular}{|c|c|c|c|c|c|c|}
\hline Date. & Hydrographer. & Width. & $\begin{array}{l}\text { Area of } \\
\text { section. }\end{array}$ & $\underset{\text { velocity. }}{\text { Mean }}$ & $\begin{array}{c}\text { Gage } \\
\text { height. }\end{array}$ & $\begin{array}{c}\text { Dis- } \\
\text { charge. }\end{array}$ \\
\hline March 16 & F. R. S. Buttemer... & $\begin{array}{r}\text { Feet. } \\
179\end{array}$ & $\begin{array}{r}S q \cdot f t . \\
447\end{array}$ & $\begin{array}{r}\text { Ft.per sec. } \\
2.84\end{array}$ & $\begin{array}{l}\text { Feet. } \\
10.19\end{array}$ & $\begin{array}{r}\text { Sec.-ft. } \\
1,269\end{array}$ \\
\hline April $7 \ldots$ & o. W. Peterson...... & 184 & 597 & 3.79 & 10.75 & 2,263 \\
\hline May $24 \ldots$ & R. S. Hawley ............ & 265 & 862 & 4.94 & 11.91 & 4,261 \\
\hline June 17 . & ....do................... & 272 & 797 & 4.56 & 11.65 & 3,632 \\
\hline July 21 . . & .....do................ & 141 & 282 & 1.74 & 9.04 & 490 \\
\hline September 14 & C. H. Lee.. & 106 & 128 & .43 & 7.82 & 56 \\
\hline October $19 . . .$. & Hawley and Lee...... & 104 & 128 & .37 & 7.80 & 48 \\
\hline October $19 \ldots$ & ....do............. & 44 & 38 & 1.34 & 7.80 & 51 \\
\hline December 7 .. & Hawley and Eaton .......... & 65 & 57 & 1.58 & 8.00 & 90 \\
\hline
\end{tabular}


Daily gage height, in feet, of Merced River above Merced Falls, Cal., for 1905.

\begin{tabular}{|c|c|c|c|c|c|c|c|c|c|c|c|c|}
\hline y. & Jan. & Feb. & Mar. & Apr. & May. & June. & July. & Aug. & Sept. & Oct. & Nov. & Dec. \\
\hline & 9.2 & 9.2 & 9.5 & 10.15 & 11.55 & 11.45 & 10.4 & 8.6 & 8.0 & 7.85 & 7.85 & 8.1 \\
\hline 2. & 8.95 & 13.45 & 9.6 & 10.2 & 11.2 & 11.35 & 10.25 & 8.5 & 7.95 & 7.8 & 7.8 & 8.1 \\
\hline 3. & 8.85 & 10.75 & 9.7 & 10.3 & 10.95 & 11.35 & 10.15 & 8.5 & 7.95 & 7.9 & 7.85 & 8.0 \\
\hline 4. & 8.8 & 10.45 & 9.8 & 10.4 & 10.8 & 11.2 & 10.05 & 8.45 & 8.0 & 7.8 & 7.8 & 7.95 \\
\hline 5.. & 8.7 & 10.8 & 9.8 & 10.5 & 10.7 & 11.05 & 10.05 & 8.4 & 8.05 & 7.9 & 7.8 & 8.0 \\
\hline 6.. & 8.7 & 10.35 & 9.75 & 10.6 & 10.6 & 11.2 & 9.95 & 8.4 & 8.0 & 7.9 & 7.8 & 8.0 \\
\hline 7.. & 8.7 & 9.85 & 9.8 & 10.7 & 10.7 & 11.45 & 9.95 & 8.35 & 7.9 & 7.9 & 7.8 & 8.0 \\
\hline 8.. & 8.7 & 9.65 & 9.85 & 10.7 & 11.05 & 11.45 & 9.9 & 8.35 & 7.9 & 7.9 & 7.8 & 8.0 \\
\hline $9 .$. & 8.7 & 9.5 & 9.85 & 10.7 & 10.85 & 11.35 & 9.95 & 8.4 & 7.9 & 7.9 & 7.8 & 8.0 \\
\hline 10. & 8.7 & 9.4 & 9.85 & 10.75 & 10.65 & 11.35 & 10.0 & 8.3 & 7.9 & 7.9 & 7.85 & 8.0 \\
\hline 11. & 8.7 & 9.3 & 9.85 & 10.6 & 10.6 & 11.6 & 9.9 & 8.35 & .9 & 7.9 & .85 & 8.0 \\
\hline 12. & 8.65 & 9.25 & 9.8 & 10.4 & 10.5 & 11.95 & 9.8 & 8.4 & 7.9 & 7.8 & 7.9 & 7.9 \\
\hline 13. & 8.6 & 9.1 & 10.95 & 10.3 & 10.6 & 11.85 & 9.7 & 8.4 & 7.9 & 7.8 & 7.8 & 7.9 \\
\hline 14.. & 8.6 & 9.1 & 10.6 & 10.25 & 10.85 & 11.75 & 9.55 & 8.35 & 7.85 & 7.85 & 7.8 & 7.9 \\
\hline 15. & 8.6 & 9.1 & 10.25 & 10.4 & 11.35 & 11.7 & 9.35 & 8,3 & 7.8 & 7.85 & 7.8 & 7.95 \\
\hline 16.. & 8.7 & 9.1 & 10.15 & 10.6 & 12.05 & 11.7 & 9.25 & 8.25 & 7.85 & 7.8 & 7.85 & 7.95 \\
\hline 17.. & 8.7 & 9.3 & 11.3 & 10.5 & 12.7 & 11.6 & 9.1 & 8.2 & 7.85 & 7.8 & 7.85 & 8.0 \\
\hline 18.. & 8.6 & 9.3 & 10.7 & 10.55 & 12.5 & 11.45 & 9.1 & 8.2 & 7.85 & 7.8 & 7.85 & 8.1 \\
\hline 19.. & 8.7 & 9.3 & 13.55 & 10.8 & 12.25 & 11.4 & 9.05 & 8.15 & 7.85 & 7.8 & 7.85 & 8.1 \\
\hline 20. & 8.7 & 9.35 & 11.75 & 10.55 & 12.35 & 11.3 & 9.0 & 8.1 & 7.8 & 7.8 & 7.9 & 8.05 \\
\hline 21.. & 8.9 & 9.35 & 10.9 & 10.4 & 12.25 & 11.3 & 9.0 & 8.1 & 7.8 & 7.8 & 7.9 & 8.1 \\
\hline 22. & 9.05 & 9.3 & 10.55 & 10.35 & 11.95 & 11.3 & 9.0 & 8.1 & 7.8 & 7.85 & 7.9 & 8.05 \\
\hline 23. & 8.9 & 9.25 & 10.3 & 10.35 & 11.8 & 11.05 & 9.0 & 8.1 & 7.75 & 7.9 & 7.9 & 8.05 \\
\hline 24.. & 8.85 & 9.25 & 10.3 & 10.55 & 11.9 & 10.85 & 9.0 & 8.1 & 7.8 & 7.85 & 7.9 & 8.0 \\
\hline $25 .$. & 8.85 & 9.3 & 10.25 & 10.8 & 12.05 & 10.65 & 9.0 & 8.1 & 7.7 & 7.85 & 7.9 & 8.0 \\
\hline 26.. & 8.8 & 9.4 & 10.3 & 10.85 & 12.05 & 10.6 & 8.9 & 8.1 & 7.7 & 7.85 & 7.9 & 8.0 \\
\hline 27.. & 8.75 & 9.4 & 10.45 & 11.0 & 11.65 & 10.55 & 8.9 & 8.1 & 7.8 & 7.85 & 8.0 & 8.0 \\
\hline 28.. & 8.7 & 9.4 & 10.2 & 11.4 . & 11.4 & 10.45 & 8.85 & 8.1 & 7.8 & 7.85 & 7.95 & 8.05 \\
\hline 29.. & 8.7 & & 10.4 & 11.75 & 11.15 & 10.5 & 8.8 & 8.0 & 7.8 & 7.85 & 8.25 & 8.2 \\
\hline $30 .$. & 8.7 & . & 10.35 & 11.8 & 11.2 & 10.5 & 8.75 & 8.0 & 7.8 & 7.8 & 8.15 & 8.2 \\
\hline 31... & 8.8 & . & 10.2 & ...... & 11.35 & & 8.65 & 8.0 & $\cdots$ & 7.8 & or & 8.2 \\
\hline
\end{tabular}

Station rating table for Merced River above Merced Falls, Cal., from January 1 to December 31, 1905.

\begin{tabular}{|c|r||r|r||c|c||c|c|}
\hline $\begin{array}{c}\text { Gage } \\
\text { height. }\end{array}$ & Discharge. & $\begin{array}{c}\text { Gage } \\
\text { height. }\end{array}$ & Discharge. & $\begin{array}{c}\text { Gage } \\
\text { height. }\end{array}$ & Discharge. & $\begin{array}{c}\text { Gage } \\
\text { height. }\end{array}$ & Discharge. \\
\hline Feet. & Second-feet. & Feet. & Second-feet. & Feet. & Second-feet. & Feet. & Second-feet. \\
7.70 & 35 & 9.00 & 465 & 10.30 & 1,570 & 11.60 & 3,570 \\
7.80 & 50 & 9.10 & 520 & 10.40 & 1,690 & 11.70 & 3,755 \\
7.90 & 70 & 9.20 & 580 & 10.50 & 1,820 & 11.80 & 3,945 \\
8.00 & 90 & 9.30 & 645 & 10.60 & 1,955 & 11.90 & 4,140 \\
8.10 & 115 & 9.40 & 715 & 10.70 & 2,095 & 12.00 & 4,340 \\
8.20 & 140. & 9.50 & 790 & 10.80 & 2,240 & 12.20 & 4,755 \\
8.30 & 170. & 9.60 & 870 & 10.90 & 2,390 & 12.40 & 5,200 \\
8.40 & 200 & 9.70 & 955 & 11.00 & 2,545 & 12.60 & 5,665 \\
8.50 & 235 & 9.80 & 1,045 & 11.10 & 2,705 & 12.80 & 6,140 \\
8.60 & 275 & 9.90 & 1,140 & 11.20 & 2,870 & 13.00 & 6,630 \\
8.70 & 315 & 10.00 & 1,240 & 11.30 & 3,040 & & \\
8.80 & 360 & 10.10 & 1,345 & 11.40 & 3,215 & & \\
8.90 & 410 & 10.20 & 1,455 & 11.50 & 3,390 & & \\
\hline
\end{tabular}

NoтE.-The above table is based on 9 discharge measurements made during 1905 . It is well defined between gage heights 7.8 feet and 12 feet. 
Estimated monthly discharge of Merced River above Merced Falls, Cal., for 1905.

[Drainage area, 1,090 square miles.]

\begin{tabular}{|c|c|c|c|c|c|c|}
\hline \multirow[b]{2}{*}{ Month. } & \multicolumn{3}{|c|}{ Discharge in second-feet. } & \multirow[b]{2}{*}{$\begin{array}{l}\text { Total in } \\
\text { acre-feet. }\end{array}$} & \multicolumn{2}{|c|}{ Run-off. } \\
\hline & Maximum. & Minimum. & Mean. & & $\begin{array}{l}\text { Second-feet } \\
\text { per square } \\
\text { mile. }\end{array}$ & $\begin{array}{l}\text { Depth } \\
\text { in inches. }\end{array}$ \\
\hline January.... & 580 & 275 & 345 & 21,210 & 0.317 & 0.366 \\
\hline February..................... & 7,760 & 520 & 1,105 & 61,370 & 1.01 & 1.05 \\
\hline March........................ & 8,020 & 790 & 1,774 & 109,100 & 1.63 & 1.88 \\
\hline April........... & 3,945 & 1,400 & 2,050 & 122,000 & 1.88 & 2.10 \\
\hline May............ & 5,900 & 1,820 & 3,316 & 203,900 & 3.04 & 3.50 \\
\hline June............. & 4,240 & 1,755 & 2,980 & 177,300 & 2.73 & 3.05 \\
\hline July............. & 1,690 & 295 & 804 & 49,440 & .738 & .851 \\
\hline August.......... & 275 & 90 & 158 & 9,715 & .145 & .167 \\
\hline September.. & 102 & 35 & 62.8 & 3,737 & .058 & .065 \\
\hline October.... & 70 & 50 & 59.0 & 3,628 & .054 & .062 \\
\hline November....................... & 155 & 50 & 66.4 & 3,951 & .061 & .068 \\
\hline December............ & 140 & 70 & 97.5 & 5,995 & .089 & .103 \\
\hline The year.. & 8,020 & 35 & 1,068 & 771,300 & .979 & 13. 26 \\
\hline
\end{tabular}

YOSEMITE CREEK IN YOSEMITE VALLEY, CALIFORMIA.

This station was established July 9, 1904, by A. E. Chandler and N. W. Currie. It is located at the wagon bridge, about one-half mile from Yosemite, Cal.

The channel is straight for 50 feet above and 100 feet below station. Both banks are above high water. The bed of the stream is composed of small granite fragments and is permanent.

Discharge measurements are made from the lower side of the bridge. The initial point for soundings is the stream face of the abutment on the right bank.

The gage is a vertical timber securely fastened to an alder tree on the right bank 50 feet above bridge. The bench mark is the heads of two nails driven in the alder tree, to which the gage is fastened. Elevation, 9.40 feet above the datum of the gage.

A description of this station, with gage height and discharge data, is contained in WaterSupply Paper No. 134, United States Geological Survey.

Discharge measurements of Yosemite Creek in Yosemite Valley, California, in 1905.

\begin{tabular}{|c|c|c|c|c|c|c|}
\hline Date. & Hydrographer. & Width. & $\begin{array}{l}\text { Area of } \\
\text { section. }\end{array}$ & $\begin{array}{c}\text { Mean } \\
\text { velocity. }\end{array}$ & $\begin{array}{c}\text { Gage } \\
\text { height. }\end{array}$ & $\begin{array}{c}\text { Dis- } \\
\text { charge. }\end{array}$ \\
\hline & & Feet. & Sq. ft. & Ft. per sec. & Feet. & Sec.-ft. \\
\hline June $7 .$. & W. B. Clapp... & 39 & 106 & 2.19 & 6.05 & 232 \\
\hline June 13. . & N. W. Currie.. & 37 & 122 & 2.39 & 6.55 & 290 \\
\hline June 19. & 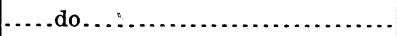 & 40 & 100 & 2.8 & 5.78 & 208 \\
\hline June 24. . & ......do.............. & 35 & 82 & 1.72 & 5.40 & 141 \\
\hline June 27. & .....do... & 35 & 76 & 1.54 & 5.15 & 117 \\
\hline July $4 \ldots$ & .....do... & 33 & 57 & 1.16 & 4.75 & 66 \\
\hline July $14 \ldots$ & .....do. . & 31 & 42 & .71 & 4.30 & 30 \\
\hline July 23... & ....do... & 29 & 35 & .42 & 4.10 & 14.7 \\
\hline August 1. . & ....do............. & 28 & 29 & .08 & 3.90 & 2.3 \\
\hline
\end{tabular}


Daily gage height, in feet, of Yosemite Creek in Yosemite Valley, California, for 1905.

\begin{tabular}{|c|c|c|c|c|c|c|c|c|c|c|c|c|c|}
\hline Day: & ay. & June. & July. & Aug. & Sept. & Oct. & Day. & May. & June & July. & Aug. & Sept. & Oct. \\
\hline & & 6.3 & 5.0 & 3.9 & 3.7 & 3.7 & 17. & $\cdots$ & 6.0 & 4.2 & 3.79 & 3.7 & $a 3.65$ \\
\hline 2. & & 6.4 & 4.9 & 3. 7 & 3.7 & $a 3.7$ & 18. & .... & 5.9 & 4.2 & 3.79 & a. 3.7 & $a 3.65$ \\
\hline 3. & & 6.3 & 4.8 & 3.85 & 3.7 & 3.65 & 19. & & 5.78 & 4.2 & 3.75 & a 3.7 & $a 3.65$ \\
\hline 4. & & 5.95 & 4.75 & 3.85 & 3.7 & a 3.65 & 20. & $\ldots$ & 5.75 & 4.2 & 3.75 & a 3.7 & a 3.65 \\
\hline 5. & & 5.8 & 4.75 & 3.85 & 3. 7 & $a 3.65$ & 21. & & 5.9 & 4.18 & 3.75 & a.3.7 & 3.65 \\
\hline 6. & & 5.9 & 4.75 & 3.8 & 3. 7 & $a 3.65$ & 22. & $\ldots$ & 5.7 & 4.15 & 3.75 & 3.65 & a 3.65 \\
\hline 7. & & 6.2 & 4.7 & 3.8 & 3.7 & 3.65 & 23. & 6.7 & 5.4 & 4.1 & 3. 75 & a 3.65 & a 3.65 \\
\hline 8. & & 6.1 & 4.7 & 3.7 & 3.7 & a 3.65 & 24. & 6.7 & 5.4 & 4.1 & 3.75 & 3.65 & 3.65 \\
\hline 9. & & 6.0 & 4.75 & 3.8 & 3. 7. & a 3.65 & 25. & 6.8 & 5.25 & 4.0 & 3.75 & a 3.65 & 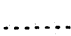 \\
\hline D. & & 6.5 & 4.7 & 3.8 & 3.7 & a 3.65 & 26. & 6.8 & 5.3 & 4.0 & 3.75 & a 3.65 & \\
\hline 1. & & 6.3 & 4.6 & $\begin{array}{l}3.8 \\
\end{array}$ & 3.7 & a 3.65 & 27. & 6.3 & 5.15 & 4.0 & 3. 72 & $a 3.65$. & \\
\hline 12. & & 6.4 & 4.5 & 3.8 & a 3.7 & $a 3.65$ & 28. & 6.2 & 5.0 & 3.95 & 3.7 & 3.7 & \\
\hline 13. & & 6.55 & 4.4 & 3. 78 & 3.7 & a 3.65 & 29. & 6.0 & 5.2 & 3.95 & 3.7 & a 3.7 & ...... \\
\hline 14. & & 6.35 & 4.3 & 3.8 & 3.7 & 3.65 & 30. & 6.3 & 5.0 & 3.9 & 3.7 & 3.7 & .. \\
\hline 15. & & 6.35 & 4.25 & 3.8 & 3.7 & a 3.65 & 31. & 6.3 & $\ldots$ & 3.9 & 3.7 & & $\ldots$ \\
\hline 10 & & 6.35 & 4.25 & 3.79 & 3.7 & a 3.65 & & & & & & & \\
\hline
\end{tabular}

$a$ Estimated.

Station rating table for Yosemite Creek in Yosemite Valley, California, from July 1, 1904, to December 31, 1905.

\begin{tabular}{|r|r||r|r|r|r|r|r|}
\hline $\begin{array}{c}\text { Gage } \\
\text { height. }\end{array}$ & Discharge. & $\begin{array}{c}\text { Gage } \\
\text { height. }\end{array}$ & Discharge. & $\begin{array}{c}\text { Gage } \\
\text { height. }\end{array}$ & Discharge. & $\begin{array}{c}\text { Gage } \\
\text { height. }\end{array}$ & Discharge. \\
\hline Feet. & Second-feet. & Feet. & Second-feet. & Feet. & Second-feet. & Feet. & Second-feet. \\
3.70 & 2 & -4.60 & 50 & 5.50 & 150 & 6.40 & 280 \\
3.80 & 3 & 4.70. & 59 & 5.60 & 163 & 6.50 & 296 \\
3.90 & 5 & 4.80 & 69 & 5.70 & 177 & 6.60 & 312 \\
4.00 & 8 & 4.90 & 79 & 5.80 & 191 & 6.70 & 328 \\
4.10 & 14 & 5.00 & 60 & 5.90 & 205 & 6.80 & 344 \\
4.20 & 20 & 5.10 & 101 & 6.00 & 220 & 6.90 & 360 \\
4.30 & 27 & 5.20 & 113 & 6.10 & 235 & 7.00 & 377 \\
4.40 & 34 & 5.30 & 125 & 6.20 & 250 & & \\
4.50 & 42 & 5.40 & 137 & 6.30 & 265 & & \\
& & & & & & \\
\hline
\end{tabular}

NotE.-The above table is based on 11 discharge measurements made during 1904-5. It is well defined. 
Estimated monthly discharge of Yosemite Creek in Yosemite Valley, California, for 1904 and 1905

\begin{tabular}{|c|c|c|c|c|c|}
\hline \multirow{2}{*}{\multicolumn{2}{|c|}{ Month. }} & \multicolumn{3}{|c|}{ Discharge in second-feet. } & \multirow{2}{*}{$\begin{array}{l}\text { Total in } \\
\text { acre-feet. }\end{array}$} \\
\hline & & Maximum. & Minimum. & Mean. & \\
\hline \multicolumn{6}{|c|}{1904.} \\
\hline & 59 & 8 & 32.2 & 1,341 \\
\hline & 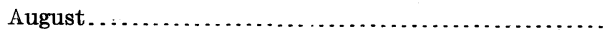 & 59 & 3 & 9.9 & 609 \\
\hline \multirow{2}{*}{\multicolumn{2}{|c|}{$\begin{array}{c}\text { September } 1 \text { to } 24 \ldots \ldots \ldots \ldots \ldots \ldots \ldots \ldots \ldots \ldots \ldots \ldots . . . \\
1905 .\end{array}$}} & 50 & 2.5 & 4.7 & 224 \\
\hline & & & & & \\
\hline \multirow{2}{*}{\multicolumn{2}{|c|}{ 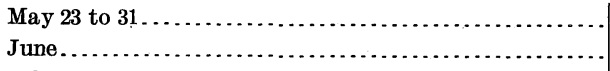 }} & 344 & 220 & 290 & 5,177 \\
\hline & & 304 & 90 & 206 & 12,260 \\
\hline \multicolumn{2}{|c|}{ 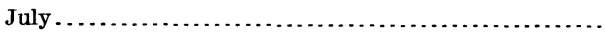 } & 90 & 5 & 34.3 & 2,109 \\
\hline \multicolumn{2}{|c|}{ 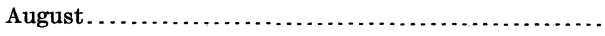 } & 5 & 2 & 2.8 & 172 \\
\hline \multicolumn{2}{|c|}{ September } & 2 & 1.5 & 1.9 & 113 \\
\hline \multirow{2}{*}{$\begin{array}{r}\text { October } 1 \text { to } 24 . \\
\text { The period }\end{array}$} & \multirow{2}{*}{ (n) } & 2 & 1.5 & 1.5 & 71 \\
\hline & & & & & 19,900 \\
\hline
\end{tabular}

TENAYA CREEK IN YOSEMITE VALLEY, CALIFORNIA.

This station was established July 11, 1904, by A. E. Chandler and N. W. Currie. It is located by the wagon bridge, about 2 miles from Yosemite, Cal.

The channel is straight for 200 feet above and below the station. Both banks are above high water. The bed of the stream is composed of small granite fragments and is not subject to change.

Discharge measurements are made from the bridge. The initial point for soundings is the stream face of the abutment on the right bank.

The gage is a vertical timber securely fastened to the bridge stringer on upper side of bridge, 9 feet from left abutment. The bench mark is formed by the heads of two nails in the top of the bridge stringer near the gage; elevation, 12.70 feet above the datum of the gage.

A description of this station, with gage height and discharge data, is contained in WaterSupply Paper No. 134, United States Geological Survey.

Discharge measurements of Tenaya Creek in Yosemite Valley, Calïfornia, in 1905.

\begin{tabular}{|c|c|c|c|c|c|c|}
\hline Date. & Hydrographer. & Width. & $\begin{array}{l}\text { Area of } \\
\text { section. }\end{array}$ & $\begin{array}{c}\text { Mean } \\
\text { velocity. }\end{array}$ & $\begin{array}{c}\text { Gage } \\
\text { height. }\end{array}$ & $\begin{array}{l}\text { Dis- } \\
\text { charge. }\end{array}$ \\
\hline June $7 \ldots . .$. & W. B. Clapp.. & $\begin{array}{r}\text { Feet. } \\
45\end{array}$ & $\underset{119}{S q \cdot f t}$ & $\begin{array}{r}\text { Ft. per sec. } \\
3.36\end{array}$ & $\begin{array}{l}\text { Feet. } \\
\quad 5.95\end{array}$ & $\begin{array}{r}\text { Sec-ft } \\
400\end{array}$ \\
\hline June $14 \ldots . .$. & N. W . durrie $\ldots . . . \ldots \ldots \ldots \ldots$ & 45 & 125 & 3.42 & 6.00 & 428 \\
\hline June $19 \ldots \ldots$. & .....do. & 45 & 110 & 3.03 & 5.65 & 333 \\
\hline June $24 \ldots . . .$. & .....do... & 45 & 90 & 2.46 & 5.18 & 221 \\
\hline June $28 \ldots . . .$. & .....do. . . & 45 & 72 & 2.01 & 4.80 & 145 \\
\hline July 5........ & .....do. & 42 & 51 & -1.59 & 4. 40 & 81 \\
\hline July $12: \ldots \ldots$ & .....do.... & 41 & 37 & 1.19 & 4. 10 & 44 \\
\hline July $24 \ldots \ldots \ldots$ & .....do. & 34 & 16.8 & .84 & 3.60 & 14.1 \\
\hline October $1 a \ldots$ & .....do. & $\cdots$ & - & $\ldots \ldots$ & 3.35 & 3 \\
\hline
\end{tabular}

a Estimated. 
Daily gage height, in feet, of Tenaya Creek in Yosemite Valley, California, for 1905.

\begin{tabular}{|c|c|c|c|c|c|c|c|c|c|c|c|}
\hline Day. & June. & July. & Aug. & Sept. & Oct. & Day. & June. & July. & Aug. & Sept. & Oct. \\
\hline 1. & 5.9 & 4.7 & 3.5 & 3.35 & 3.35 & 17 . & 5.8 & 3.85 & 3. 38 & 3.35 & a 3.35 \\
\hline $2 \ldots$ & 5.9 & 4.6 & 3.5 & 3.35 & u $3: 35$ & 18. & 5.75 & 3.8 & 3.38 & a 3.35 & a 3.35 \\
\hline 3. & 5.9 & 4.5 & 3.5 & 3.35 & a 3.35 & 19. & 5.65 & 3.75 & 3.38 & $a 3.35$ & a 3.35 \\
\hline 4... & 5.7 & 4.4 & 3.45 & 3.35 & $a 3.35$ & 20. & 5.55 & 3.7 & 3.38 & a 3.35 & a 3.35 \\
\hline 5. & 5.6 & 4.4 & 3.45 & 3.35 & $a 3.35$ & 21. & 5.55 & 3.7 & 3.38 & $a 3.35$ & 3.35 \\
\hline 6. & 5.7 & 4.35 & 3.4 & 3.35 & $a \cdot 3.35$ & 22. & 5.5 & 3.65 & 3.38 & a 3.35 & 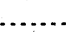 \\
\hline 7.. & 6.0 & 4.3 & 3.4 & 3.35 & 3.35 & 23. & 5.3 & 3.6 & 3.38 & 3.35 & \\
\hline 8.. & 5.8 & 4.25 & 3.4 & 3.35 & a 3.35 & 24. & 5.18 & 3.6 & 3.38 & 3.35 & \\
\hline 9.. & 5.75 & 4.2 & 3.4 & 3.35 & $a 3.35$ & $25 \ldots$ & 5.1 & 3.6 & 3.38 & $a 3.35$ & \\
\hline $10 .$. & 6.0 & 4.1 & 3.4 & 3.35 & 3.35 & $26 \ldots$ & 5.0 & 3.55 & 3.38 & $a 3.35$ & \\
\hline $1 .$. & 6.01 & 4.1 & 3.38 & 3.35 & $a 3.35$ & $27 \ldots$ & 4.9 & 3.55 & 3.38 & $a 3.35$ & \\
\hline $2 .$. & 6.0 & 4.1 & 3.38 & 3.35 & $a 3.35$ & 28. & 4.83 & 3.55 & 3.38 & $a 3.35$ & - \\
\hline $13 .$. & 6.15 & 4.0 & 3.38 & 3.35 & a 3.35 & $29 .$. & 4.8 & 3.5 & 3.35 & $a 3.35$ & \\
\hline $14 .$. & 5.98 & 3.95 & 3.38 & 3.35 & 3.35 & 30.. & 4.7 & 3.5 & 3.35 & 3.35 & \\
\hline $15 \ldots$ & 5.95 & 3.9 & 3.38 & 3.35 & 3.35 & 31. & ..... & 3.5 & 3.35 & & 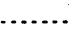 \\
\hline $16 .$. & 5.9 & 3.85 & 3.38 & 3.35 & a 3.35 & & & & & & \\
\hline
\end{tabular}

$a$ Gage heights estimated.

Station rating table for Tenaya Creek in Yosemite Valley, California, from July 1, 1904, to December 31, 1905.

\begin{tabular}{|c|c||c|r||r|r||r|r|}
\hline $\begin{array}{c}\text { Gage } \\
\text { height. }\end{array}$ & Discharge. & $\begin{array}{c}\text { Gage } \\
\text { height. }\end{array}$ & Discharge. & $\begin{array}{c}\text { Gage } \\
\text { height. }\end{array}$ & Discharge. & $\begin{array}{c}\text { Gage } \\
\text { height. }\end{array}$ & \multicolumn{2}{c|}{ Discharge. } \\
\cline { 1 - 5 } Feet. & Second-feet. & Feet. & Second-feet. & Feet. & Second-feet. & Feet. & Second-feet. \\
3.40 & 4.5 & 4.20 & 54 & 5.00 & 184 & 5.80 & 368 \\
3.50 & 8 & 4.30 & 66 & 5.10 & 204 & 5.90 & 395 \\
3.60 & 12 & 4.40 & 80 & 5.20 & 225 & 6.00 & 422 \\
3.70 & 17 & 4.50 & 95 & 5.30 & 247 & 6.10 & 450 \\
3.80 & 22 & 4.60 & 111 & 5.40 & 270 & 6.20 & 480 \\
3.90 & 28 & 4.70 & 128 & 5.50 & 293 & 6.30 & 512 \\
4.00 & 35 & 4.80 & 146 & 5.60 & 317 & & \\
4.10 & 44 & 4.90 & 165 & 5.70 & 342 & & \\
\hline
\end{tabular}

Noте,-The above table is applicable only for open-channel conditions. It is based on 11 discharge measurements made during 1904-5. It is well defined between gage heights 3.3 feet and 6 feet.

Estimated monthly discharge of Tenaya Creek in Yosemite Valley, California, for 1904 and 1905.

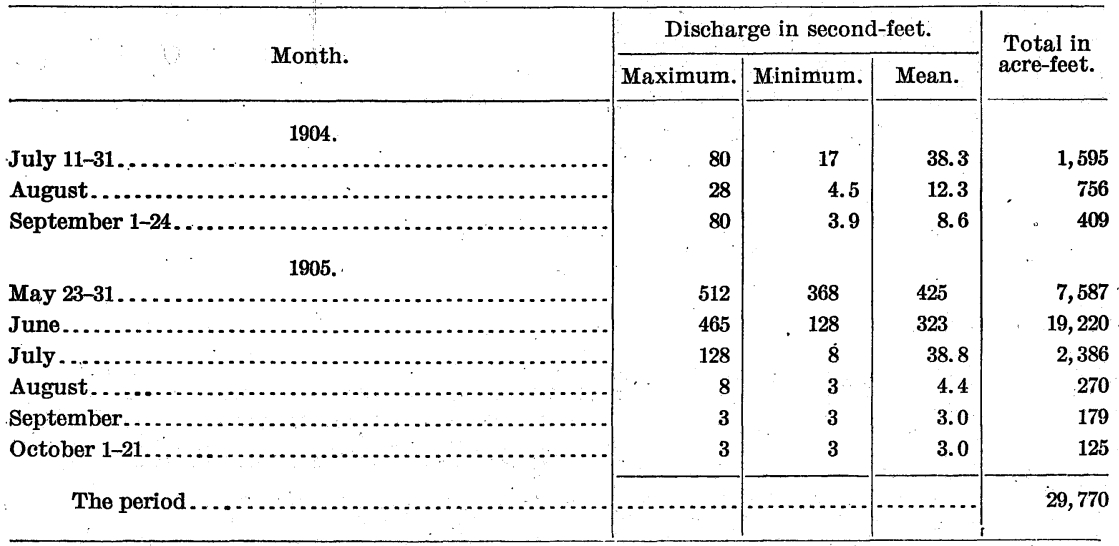




\section{MISCELLANEOUS MEASUREMENTS IN MERCED RIVER DRAINAGE BASIN.}

Bridal Veil Creek, Yosemite Valley, California.-This stream is a tributary of the Merced River. An estimate was made of the discharge at a point 500 feet above its junction with Merced River and about 1,000 feet below Bridal Veil Falls on June 7, 1905, by W. B. Clapp. It was impossible to make a measurement on account of the rough bed and heavy grade of the stream.

Estimated discharge, 100 second-feet.

Cascade Creek, Yosemite Valley, California.-This stream is a tributary of the Merced River. An estimate was made of the discharge at a point 500 feet below Cascade Falls near . mouth of creek on June 6, 1905, by W. B. Clapp.

Estimated discharge, 150 second-feet.

Ribbon Falls Creek, Yosemite Valley, California.-This stream is tributary to Merced River in Yosemite Valley. A measurement was made where the creek crosses the road on the west side of the valley on June 7 by W. B. Clapp.

The stream was flowing in several shallow channels.

Total width, 20.5 feet; area, 9.0 square feet; mean velocity, 1.89 feet per second; discharge, 17.0 second-feet.

\section{TUOLUMNE RIVER DRAINAGE BASIN.}

\section{DESCRIPTION OF BASIN.}

Tuolumne River rises on the western slope of the Sierra Nevada and drains the country located between Stanislaus River on the north and Merced River on the south. It has numerous tributaries, several of which produce a heavy discharge. The country throughout this basin is rough and rugged, especially along the main river, which cuts through solid granite, with high precipitous cliffs on either side. Along this stream is found some of the grandest scenery of the Sierra Nevada. This stream drains the northern portion of the Yosemite National Park, where is located the Grand Canyon of the Tuolumne and the Hetch Hetchy Valley, which is pronounced by many to exceed the famed Yosemite Valley in grandeur and beauty. The formation is of granite, which on the higher elevations is bare and glaciated, often rising thousands of feet in vertical cliffs and domes. Along the middle reaches of this basin there is good soil covering, with a heavy timber growth of pine, fir, cedar, and other kindred trees. On the lower reaches the covering is a heavy growth of brush, which diminishes in the foothills where the stream enters the San Joaquin Valley. This portion of the basin has a light soil covering, with grass growth, which is used for pasturage. There are several glacial lakes throughout the upper reaches of this basin, many of the larger of which offer exceptional opportunities for the construction of storage reservoirs. There are also many reservoir sites on the main river. The stream has a heavy fall, and the opportunities for power development are numerous. Several diversions are made above the gaging station, which is located at Lagrange, where the stream breaks from the foothills. The precipitation on the upper half of this basin falls in the form of snow, a greater portion of which disappears in the spring months, but on the higher elevations remains until late in the summer. The mean annual rainfall varies from about 30 inches on the lower foothills to about 60 inches on the higher elevations.

\section{TUOLUMNE RIVER AT IAGRANGE, CAL.}

This station was established August 29, 1895, by J. B. Lippincott. It is located at the wagon bridge, in the town of Lagrange. It is below the high dam, where the diversions are made by the Turlock and Modesto canals, and also below the head of the canal of the Lagrange Ditch and Hydraulic Mining Company, which diverts water from the left bank of the river above the dam.

The channel is straight for 400 feet above and 600 feet below the station. It is broken by two iron piers and has a width at ordinary stages of 300 feet. During the season of 
low flow all the water is taken out by the Turlock and Modesto canals above the station. The record of flow of these canals is kept. The bed of the stream is composed of gravel and is fairly permanent. The current is swift at high stages and very sluggish during low water. The discharge has gradually increased each year for the same gage heights. Both banks are high and not subject to overflow.

Discharge measurements are made from the downstream side of the bridge. The initial point for soundings is a mark on the railing of the bridge 100 feet north from the center of first pier on right bank of the river.

The gage is a vertical timber fastened to the right abutment of the bridge. During 1905 the gage was read by R. A. Trumbly. Bench marks were established as follows: (1) Top of a rock marked with white paint, situated on the left bank below the bridge and opposite the 573 foot mark of the cross section; elevation, 19.10 feet. (2) The top of rock marked with white paint, situated 75 feet downstream from bench mark No. 1; elevation, 16.89 feet. Elevations refer to the datum of the gage.

Information in regard to this station is contained in Water-Supply Papers Nos. 81, 85, 100, and 134 of the United States Geological Survey.

Discharge measurements of Tuolumne River at Lagrange, Cal., in 1905.

\begin{tabular}{|c|c|c|c|c|c|c|}
\hline Date. & Hydrographer. & Width. & $\begin{array}{l}\text { Area of } \\
\text { section. }\end{array}$ & $\begin{array}{l}\text { Mean } \\
\text { velocity. }\end{array}$ & $\begin{array}{c}\text { Gage } \\
\text { height. }\end{array}$ & $\begin{array}{l}\text { Dis- } \\
\text { charge. }\end{array}$ \\
\hline March 15 & F. R. S. Buttemer.. & $\begin{array}{r}\text { Feet. } \\
325\end{array}$ & $\begin{array}{r}S q \cdot f t . \\
1,426\end{array}$ & $\begin{array}{r}\text { Ft. per sec. } \\
1.79\end{array}$ & $\begin{array}{l}\text { Feet. } \\
6.10\end{array}$ & $\begin{array}{c}\text { Sec.-ft. } \\
2,548\end{array}$ \\
\hline April 6.. & O. W. Patterson... & 332 & 1,591 & 2.40 & 6.60 & 3,817 \\
\hline May 24. & R. S. Hawley...... & 344 & 2,068 & 3.79 & 7.90 & 7,846 \\
\hline June 16. . & .....do. . . . & 344 & 2,068 & 3.66 & 7.90 & 7,574 \\
\hline June 17 & .....do........... & 344 & 1,906 & 3.28 & 7.45 & 6,249 \\
\hline September 13 .. & c. H. Lee.......... & 30 & 20 & .45 & 3. 30 & 9.2 \\
\hline October 18..... & Lee and Hawley.......... & 38 & 36 & 1.47 & 3.68 & 53 \\
\hline
\end{tabular}


Daily gage height, in feet, of Tuolumne River at Lagrange, Cal., for 1905.

\begin{tabular}{|c|c|c|c|c|c|c|c|c|c|c|c|c|}
\hline Day. & Jan. & Feb. & Mar. & Apr. & May. & June. & July. & Aug. & Sept. & Oct. & Nov. & Dec. \\
\hline $1 .$. & 5.7 & 5.75 & 5.7 & 5.9 & 7.3 & 7.0 & 6.05 & 3.2 & 3.1 & 3.7 & 3.65 & 4.05 \\
\hline $2 .$. & 5.1 & 8.1 & 5.85 & 6.05 & 6.7 & 7.0 & 6.0 & 3.2 & 3.1 & 3.85 & 3.65 & 4.05 \\
\hline 3.. & 5.0 & 6.3 & 5.9 & 5.9 & 6.3 & 6.9 & 5.8 & 3.2 & 3.1 & 3.8 & 3.65 & 4.0 \\
\hline $4 .$. & 4.8 & 6.0 & 5.9 & 5.9 & 6.35 & 6.85 & 5.45 & 3.2 & 3.1 & 3.7 & 3.65 & 4.0 \\
\hline 5.. & 4.8 & 6.35 & 5.9 & 5.95 & 6.15 & 6.75 & 5.5 & 3.2 & 3.1 & 3.6 & 3.65 & 4.0 \\
\hline $6 \ldots$ & 4.8 & 6.1 & 5.9 & 6.3 & 8.15 & 6.7 & 5.5 & 3.2 & 3.1 & 3.6 & 3.65 & 4.0 \\
\hline 7.. & 4.75 & 5.6 & 5.9 & 6.65 & 6.35 & 6.85 & 5.35 & 3.2 & 3.1 & 3.6 & 3.65 & 4.0 \\
\hline 8. & 4.75 & 5.5 & 5.9 & 6.6 & 6.5 & 7.1 & 5.4 & 3.2 & 3.1 & 3.6 & 3.65 & 4.0 \\
\hline $9 .$. & 4.75 & 5.4 & 5.85 & 6.45 & 6.2 & 7.6 & 5.15 & 3.2 & 3.1 & 3.5 & 3.65 & 4.0 \\
\hline $10 .$. & 4. 75 & 5.4 & 5.8 & 6.5 & 6.1 & 7.9 & 5.0 & 3.2 & 3.1 & 3.5 & 3.65 & 4.0 \\
\hline 11.. & 4.8 & 5.2 & 5.85 & 6.4 & 6.0 & 8.0 & 4.85 & 3.2 & 3.1 & 3.5 & 3.65 & 3.9 \\
\hline $2 .$. & 4.8 & 5.1 & 5.8 & 6.05 & 6.0 & 8.05 & 4.7 & 3.2 & 3.4 & 3.5 & 3.65 & 3.9 \\
\hline 3... & 4.8 & 5.1 & 6.95 & 5.8 & 5.9 & 8.0 & 4.55 & 3.2 & 3.3 & 3.5 & 3.65 & 4,0 \\
\hline $14 .$. & 4.8 & 5.0 & 6.55 & 5.75 & 6.1 & 7.85 & 4.5 & 3.2 & 3.3 & 3.5 & 3.7 & 3.95 \\
\hline 5.. & 4.8 & 5.0 & 6.1 & 5.9 & 6.8 & 7.85 & 4.75 & 3.2 & 3.2 & 3.5 & 3.7 & 3.95 \\
\hline 6 & 4.8 & 4.9 & 6.1 & 5.95 & 7.7 & 7.8 & 4.7 & 3.2 & 3.2 & 3.5 & 3.7 & 3.95 \\
\hline $17 .$. & 4.8 & $\$ .1$ & 7.3 & 5.9 & 8.7 & 7.6 & 3.65 & 3.2 & 3.2 & 3.5 & 3.7 & 4.0 \\
\hline $18 .$. & 4.8 & 5.1 & 7.0 & 5.95 & 8.25 & 7.45 & 3.4 & 3.1 & 3.2 & 3.6 & 3.7 & 4.0 \\
\hline $19 .$. & 4.8 & 5.1 & 9.3 & 5.9 & 8.0 & 7.3 & 3.3 & 3. 1 & 3.2 & 3.65 & 3.7 & 4.0 \\
\hline $20 .$. & 4.85 & 5.85 & 7.6 & 5.9 & 8.0 & 7.25 & 3.2 & 3.1 & 3.2 & 3.65 & 3.75 & 4.05 \\
\hline $21 .$. & 5.0 & 5.65 & 6.85 & 5.9 & 8.25 & 7.35 & 3.2 & 3.1 & 3.2 & 3.7 & 3.8 & 4.15 \\
\hline 2. & 5.1 & 5.4 & 6.6 & 5.95 & 7. & 6.9 & 3.2 & 3.1 & 3.2 & 3. & 3.75 & 4.1 \\
\hline 23.. & 5.15 & 5.4 & 6.5 & 6.6 & 7. & 6.25 & 3.2 & 3.1 & 3.2 & 3.7 & 3.75 & 4.05 \\
\hline $24 .$. & 5.2 & 5.4 & 6.3 & 7.1 & 7.8 & 6.15 & 3.2 & 3.1 & 3.2 & 3.7 & 3.75 & 4.05 \\
\hline $25 .$. & 5.1 & 5. 55 & 6.1 & 7.1 & 8.0 & 6.15 & 3.2 & 3.1 & 3.2 & 3.7 & 3.8 & 4.05 \\
\hline $26 .$. & 5.0 & 5.8 & 6.1 & 7.2 & 7.85 & 6.1 & 3.2 & 3.1 & 3.2 & 3.7 & 3.8 & 4.0 \\
\hline $27 .$. & 4.85 & 5.7 & 6.2 & 7.2 & 7.65 & 6.05 & 3.2 & 3.1 & 3.3 & 3.7 & 3.85 & 4.0 \\
\hline 28 . & 4.8 & 5.8 & 6.1 & 7.35 & 7.35 & 6.05 & 3.2 & 3.1 & 3. 25 & 3.7 & 4.0 & 4.1 \\
\hline 29. & 4.8 & & .45 & 7.4 & 6.7 & 6.20 & 3.2 & 3.1 & 3.4 & 3.65 & 4. & 4.15 \\
\hline $30 .$. & 4.9 & & 6.25 & 7.7 & 6.85 & 6.05 & 3.2 & 3. 1 & 3.5 & 3.65 & 4.05 & 4.15 \\
\hline 31.. & 4.9 & & 6.1 & & 6.9 & & 3.2 & 3. 1 & & 3.65 & & 4.2 \\
\hline
\end{tabular}


Station rating table for Tuolumne River at Lagrange, Cal., from January 1 to December 31, 1905.

\begin{tabular}{|r|r|r|r|r|r||r|r|}
\hline $\begin{array}{c}\text { Gage } \\
\text { height. }\end{array}$ & Discharge. & $\begin{array}{c}\text { Gage } \\
\text { height. }\end{array}$ & Discharge. & $\begin{array}{c}\text { Gage } \\
\text { height. }\end{array}$ & Discharge. & $\begin{array}{c}\text { Gage } \\
\text { height. }\end{array}$ & Discharge. \\
\cline { 1 - 4 } Feet. & Second-feet. & Feet. & Second-feet. & Feet. & Second-feet. & Feet. & Second-feet. \\
3.10 & 1 & 4.50 & 340 & 5.90 & 2,200 & 7.60 & 6,700 \\
3.20 & 5 & 4.60 & 410 & 6.00 & 2,400 & 7.80 & 7,360 \\
3.30 & 10 & 4.70 & 490 & 6.10 & 2,610 & 8.00 & 8,060 \\
3.40 & 20 & 4.80 & 580 & 6.20 & 2,830 & 8.20 & 8,780 \\
3.50 & 30 & 4.90 & 680 & 6.30 & 3,060 & 8.40 & 9,500 \\
3.60 & 40 & 5.00 & 790 & 6.40 & 3,300 & 8.60 & 10,260 \\
3.70 & 55 & 5.10 & 910 & 6.50 & 3,550 & 8.80 & 11,050 \\
3.80 & 70 & 5.20 & 1,030 & 6.60 & 3,800 & 9.00 & 11,850 \\
3.90 & 90 & 5.30 & 1,170 & 6.70 & 4,060 & 9.20 & 12,660 \\
4.00 & 120 & 5.40 & 1,310 & 6.80 & 4,320 & 9.40 & 13,480 \\
4.10 & 150 & 5.50 & 1,470 & 6.90 & 4,600 & 9.60 & 14,320 \\
4.20 & 190 & 5.60 & 1,630 & 7.00 & 4,880 & 9.80 & 15,160 \\
4.30 & 230 & 5.70 & 1,810 & 7.20 & 5,470 & 10.00 & 16,000 \\
4.40 & 280 & 5.80 & 2,000 & 7.40 & 6,070 & & \\
\hline
\end{tabular}

Estimated monthly discharge of Tuolumne River at Lagrange, Cal., for 1905.

[Drainage area, 1,501 square miles.]

\begin{tabular}{|c|c|c|c|c|c|c|}
\hline \multirow[b]{2}{*}{ Month. } & \multicolumn{3}{|c|}{ Discharge in second-feet. } & \multirow{2}{*}{$\begin{array}{l}\text { Total in } \\
\text { acre-feet. }\end{array}$} & \multicolumn{2}{|c|}{ Run-off. } \\
\hline & Maximum. & Minimum. & Mean. & & $\begin{array}{l}\text { Second-feet } \\
\text { per square } \\
\text { mile. }\end{array}$ & $\begin{array}{l}\text { Depth } \\
\text { in inches. }\end{array}$ \\
\hline January.. & 1,810 & 535 & 745 & 45,810 & 0.496 & 0.572 \\
\hline February.. & 8,420 & 745 & 1,926 & 107,000 & 1.28 & 1.33 \\
\hline March.... & 13,070 & 1,871 & 3,487 & 214,400 & 2.32 & 2.68 \\
\hline April.... & 7,770 & 2,696 & 4,016 & 239,000 & 2.68 & 2.99 \\
\hline May....... & 11,360 & 2,866 & 5,927 & 364,400 & 3.95 & 4.55 \\
\hline June.... & 9,075 & 3,258 & 5,969 & 355,200 & 3.98 & 4.44 \\
\hline July...... & 3,403 & 427 & 1,344 & 82,640 & .895 & 1.03 \\
\hline August.. & 345 & 103 & 212 & 13,040 & .141 & .163 \\
\hline September...... & 131 & $a 8$ & 78.1 & 4,647 & .052 & .058 \\
\hline October.. & 80 & 30 & 45.5 & 2,798 & .030 & .035 \\
\hline November....... & 135 & 48 & 62.2 & 3,701 & .041 & .046 \\
\hline 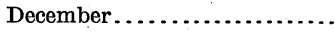 & 190 & 90 & 129 & 7,932 & .086 & .099 \\
\hline The year.. & 13,070 & 8 & 1,995 & $1,441,000$ & 1.33 & 17.99 \\
\hline
\end{tabular}

$a$ The minimum of 8 feet in September was caused by closing head-gate of Turlock Canal and holding water back in reservoir until dam overflowed.

Note.-The above discharge includes that of Turlock and Modesto canals.

\section{MODESTO GANAL AT LAGRANGE, CAL.}

The Modesto canal is the property of the Modesto irrigation district. The water is diverted from the right side of the Tuolumne River at the Lagrange dam. This canal was designed to carry 660 second-feet and to irrigate land in the vicinity of Modesto, Stanislaus County, Cal. The principal part of the construction work was done on this canal prior to 1892, but on account of litigation the canal was not completed until April, 1903.

On April 26, 1903, a gage rod was set in and a rating made of Indian Hill flume, near Lagrange, Cal. From May $10^{\circ}$ to June 3 and from June 10 to June 25, inclusive, boards were placed in the flumes to back the water up and keep the flumes saturated. During this time gage heights were obtained by taking the depth of the water in the canal below Indian Hill flume. 
On July 12, 1904, the station was moved to the flume near the intake. This was done so that more gage readings and explanations of their fluctuations could be obtained by having J. L. Montgomery, the regulator of the gates at the intake, act as gage observer. This flume is 11.85 feet in width.

Information in regard to this station is contained in Water-Supply Papers Nos. 100 and 134 of the United States Geological Survey.

Discharge measurements of Modesto canal at Lagrange, Cal., in 1905.

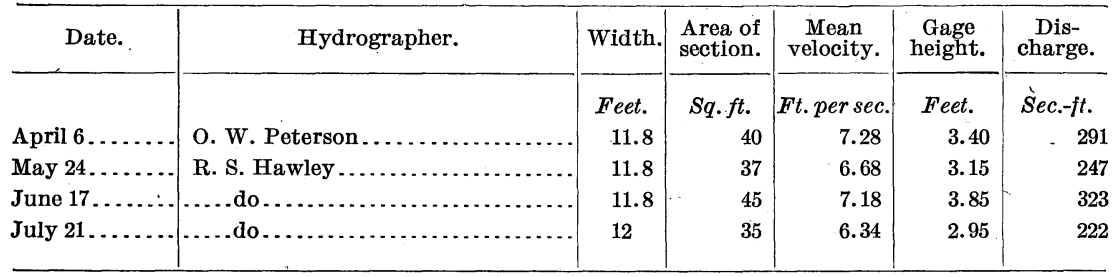

Daily gage height, in feet, of Modesto canal at Lagrange, Cal., for 1905.

\begin{tabular}{|c|c|c|c|c|c|c|c|c|c|}
\hline Day. & Jan & Feb. & Mar. & Apr. & May. & June. & July. & Aug. & Sept. \\
\hline $1 .$. & 0.0 & $\dot{2} .8$ & $a 1.2$ & 2.6 & 3.0 & 3.5 & 4.0 & 1.7 & 1.0 \\
\hline 2. & 0 & $b_{0}$ & 1.2 & 2.9 & 3.0 & 3.5 & 4.0 & 1.8 & .95 \\
\hline 3.. & 0 & a 1.3 & 1.2 & 3.1 & 3.0 & 3.5 & $b_{0}$ & 1.8 & .95 \\
\hline 4.. & 0 & 2.0 & 1.2 & 3.2 & 3.0 & 3.5 & $a 2.5$ & 1.6 & .95 \\
\hline 5. & 0 & 2.1 & 1.2 & 3.3 & 3.0 & 3.5 & 3.5 & 1.65 。 & .95 \\
\hline 6... & 0 & 2.0 & 1.25 & 3.3 & 3.0 & 3.5 & 4.0 & 1.65 & .8 \\
\hline 7. & 0 & 1.95 & 1.25 & 3.3 & 3.0 & 3.6 & 4.0 & 1.4 & .85 \\
\hline 8.. & 0 & 2.45 & 1.25 & 3.2 & 3.0 & 3.6 & 4.0 & 1.55 & .8 \\
\hline 9. & 0 & 2.8 & 1.25 & 3.2 & 3.0 & 3.6 & 4.5 & 1.55 & .8 \\
\hline $10 .$. & 0 & 3.0 & 1.25 & 3.1 & 3.0 & 3.6 & 4.5 & 1.4 & .8 \\
\hline $11 . . .$. & 0 & 3.0 & 1.25 & 3.2 & 3.0 & 2.5 & 4.5 & 1.5 & .4 \\
\hline $12 \ldots .$. & 0 & 3.1 & .85 & 3.2 & 3.0 & 3.5 & 4.0 & 1.4 & \\
\hline $13 . . .$. & 0 & 3.2 & .5 & 3.2 & 3.0 & 2.75 & 4.0 & 1.4 & \\
\hline 14. & 0 & 3.3 & .7 & 3.1 & 3.0 & 3.7 & 4.0 & 1.4 & \\
\hline $15 .$. & 0 & 3.3 & .7 & 3.0 & 3.0 & 3.7 & 4.0 & 1.3 & $\cdots$ \\
\hline 16. . & $a 1.0$ & 3.3 & .7 & 3.0 & 3.0 & 3.7 & 4.0 & 1.3 & \\
\hline 17... & .9 & 3.3 & .8 & 3.0 & 3.0 & 3.7 & 4.0 & 1.3 & \\
\hline 18. . & .9 & 3.3 & .8 & 3.0 & 3.0 & 3.75 & 4.0 & 1.3 & \\
\hline 19. . & .9 & 3.3 & $b_{0}$ & 3.0 & 3.0 & 3.75 & 3.0 & 1.25 & \\
\hline $20 . \ldots \ldots$ & .9 & 3.0 & 0 & 3.0 & 3.2 & 3.75 & 3.0 & 1.0 & \\
\hline $21 \ldots \ldots$ & 1.05 & 2.7 & 0 & 3.0 & 3.2 & 3.8 & 2.9 & 1.1 & \\
\hline 22. & 1.05 & 3.3 & $a 2.0$ & 3.0 & $b_{0}$ & 3.9 & 2.9 & 1.15 & \\
\hline 23. & 1.05 & 3.0 & 2.1 & 3.0 & 0 & 3.9 & 2.5 & 1.15 & \\
\hline 24. & 1.05 & 2.75 & 2.5 & 3.0 & $a 3.15$ & 4.0 & 2.5 & 1.15 & \\
\hline 25. & 1.05 & 2.75 & 2.5 & 3.0 & 3.3 & 1.75 & 2.75 & 1.15 & \\
\hline 26. & 1.6 & $b_{0}$ & 2.8 & 3.0 & 3.35 & 1.55 & 2.7 & 1.15 & \\
\hline 27. & 2.0 & 0 & $b_{0}$ & 3.0 & 3.35 & 4.0 & 2.55 & 1.1 & 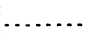 \\
\hline $28 \ldots$ & 2.05 & 0 & 0 & 3.0 & 3.5 & 4.0 & 2.4 & .9 & \\
\hline $29 .$. & 2.05 & & 0 & 3.0 & 3.5 & 4.0 & 2.55 & .9 & \\
\hline $30 \ldots \ldots \ldots$ & 2.35 & & $a 1.5$ & 3.0 & 3.5 & 4.0 & 2.1 & .9 & $\therefore$ \\
\hline 31.. & 2.7 & & 2.0 & $\cdots$ & 3.5 & $\ldots$. & 2.1 & 1.0 & \\
\hline
\end{tabular}

$a$ Water turned in.

$b$ Water turned out.

Note.-Canal dry September 12 to December 31. 
Station rating table for Modesto canal at Lagrange, Cal., from January 1 to December 31, 1905.

\begin{tabular}{|r|r||r|r||r|r|r|r|}
\hline $\begin{array}{c}\text { Gage } \\
\text { height. }\end{array}$ & Discharge. & $\begin{array}{c}\text { Gage } \\
\text { height. }\end{array}$ & Discharge. & $\begin{array}{c}\text { Gage } \\
\text { height. }\end{array}$ & Discharge. & $\begin{array}{c}\text { Gage } \\
\text { height. }\end{array}$ & Discharge. \\
\hline Freet. & Second-feet. & Freet. & Second-feet. & Feet. & Second-feet. & Feet. & Second-feet. \\
0.10 & 2 & 1.10 & 53 & 2.10 & 140 & 3.20 & 252 \\
20 & 4 & 1.20 & 61 & 2.20 & 150 & 3.40 & 274 \\
30 & 7 & 1.30 & 69 & 2.30 & 160 & 3.60 & .296 \\
40 & 11 & 1.40 & 77 & 2.40 & 170 & 3.80 & 318 \\
50 & 16 & 1.50 & 85 & 2.50 & 180 & 4.00 & 340 \\
60 & 21 & 1.60 & 94 & 2.60 & 190 & 4.20 & 364 \\
70 & 26 & 1.70 & 103 & 2.70 & 200 & 4.40 & 388 \\
80 & 32 & 1.80 & 112 & 2.80 & 210 & & \\
90 & 38 & 1.90 & 121 & 2.90 & 220 & & \\
1.00 & 45 & 2.60 & 130 & 3.00 & 230 & & \\
\hline
\end{tabular}

Note.-The above table is based on discharge measurements made during 1904-1905. It is well defined between gage heights 0.5 foot and 4 feet. Monthly estimates are included with those of Tuolumne River, p. 210.

TURIOOK CANAL AT LAGRANGE, CAL.

The Turlock canal, the property of the Turlock irrigation district, takes water from the left bnnk of the Tuolumne River at the Lagrange dam. This canal was designed to carry 1,500 second-feet and to irrigate a large area of fertile.land in the vicinity of Turlock and Ceres, Stanislaus County, Cal.

During 1898 water was first turned into the canal in small quantities and used for puddling the banks. A record of the gage height has been kept since July, 1899. Meter measurements are made when the gaging station on the Tuolumne River at Lagrange is visited, and Morgan flume, or flume No. 2, has been rated. It is 13.75 feet in width.

In the spring of 1905 a station was established in flume No. 1 and used for some time, but it did not give satisfactory results during high stages of the canal. Gage readings were discontinued on this flume and resumed on the Morgan flume. The record since May 22, 1905, is on the Morgan flume. The observer is J. J. R. Johnson, the canal tender.

Information in regard to this station is contained in Water-Supply Papers Nos. 81, 85, 100, and 134 of the United States Geological Survey.

Discharge measurements of Turlock canal at Lagrange, Cal., in 1905.

\begin{tabular}{|c|c|c|c|c|c|c|}
\hline Date. & Hydrographer. & Width. & $\begin{array}{l}\text { Area of } \\
\text { section. }\end{array}$ & $\begin{array}{c}\text { Mean } \\
\text { velocity. }\end{array}$ & $\begin{array}{c}\text { Gage } \\
\text { height. }\end{array}$ & $\begin{array}{c}\text { Dis- } \\
\text { charge. }\end{array}$ \\
\hline & & Feet. & sq. ft. & Ft. per sec. & Feet. & Sec.-ft. \\
\hline April 8 . & O. W. Peterson. & 19.5 & 24 & 1.96 & 1.22 & 47 \\
\hline April 8 . & ..... do ............ & 19.5 & 40 & 3.20 & 2.05 & 128 \\
\hline April 8 & ..... do ... & 19.5 & 55 & 3.82 & 2.80 & 210 \\
\hline April 8. & .....do ......... & 19.5 & 81 & 4.60 & 4.15 & 375 \\
\hline May 24. & R. S. Hawley ...... & 13.8 & 72 & 7.13 & 5.30 & 514 \\
\hline June $17 \ldots$ & $\ldots$. do $\ldots . . . \ldots \ldots$ & 13.8 & 76 & 7.58 & 5.55 & 576 \\
\hline July $21 \ldots$ & .....do . ........... & 13.8 & 66 & 6.55 & 4.65 & 432 \\
\hline September $14 .$. & C. H. Lee.......... & 13.6 & 28 & 3.07 & 2.04 & 86 \\
\hline
\end{tabular}

Note.-Measurements on April 8 were made on little flume near head of canal others made at Morgan flume. 
Daily gage height, in feet, of Turlock canal at Lagrange, Cal., for 1905.

\begin{tabular}{|c|c|c|c|c|c|c|c|}
\hline Day. & Mar. & Apr. & May. & June. & July. & Aug. & Sept. \\
\hline $1 \ldots \ldots \ldots \ldots$ & & 4.15 & 5.3 & 5.4 & 5.55 & 3.1 & 2.0 \\
\hline $2 \ldots \ldots \ldots$ & & 4.1 & 5.3 & 5.4 & 5.6 & 3.2 & 2.0 \\
\hline $3 \ldots \ldots \ldots \ldots$ & & 4.1 & 5.4 & 5.4 & 5.6 & 3.15 & 1.9 \\
\hline $4 \ldots \ldots \ldots \ldots \ldots$ & & 4.15 & 5.2 & 5.4 & 5.6 & 2.9 & 1.9 \\
\hline $5 \ldots \ldots \ldots \ldots$ & & 4.2 & 5.2 & 5.4 & 5.6 & 2.95 & 1.9 \\
\hline $6 \ldots \ldots \ldots$ & & 0 & 5.2 & 5.4 & 5.6 & 2.95 & 1.8 \\
\hline $7 \ldots \ldots \ldots$ & & 0 & 4.1 & 5.4 & 5.6 & 2.65 & 1.8 \\
\hline $8 \ldots \ldots \ldots$ & & 3.2 & 4.6 & 5.4 & 5.6 & 2.65 & 1.65 \\
\hline $9 \ldots \ldots \ldots$ & & 3.0 & 4.7 & 5.4 & 5.6 & 2.65 & 1.65 \\
\hline $10 \ldots \ldots \ldots$ & & 1.5 & 4.7 & 5.45 & 5.6 & 2.65 & 1.65 \\
\hline $11 \ldots \ldots \ldots \ldots$ & & 4.6 & 4.7 & 5.4 & 5.6 & 2.65 & 1.65 \\
\hline $12 \ldots \ldots \ldots$ & & 4.7 & 4.7 & 5.5 & 5.6 & 2.6 & 1.65 \\
\hline $13 \ldots \ldots \ldots \ldots$ & & 4.8 & 4.7 & 5.5 & 5.6 & 2.7 & 1.8 \\
\hline $14 \ldots \ldots \ldots$ & & 5.5 & 4. 75 & 5.5 & 5.6 & 2.4 & 2.5 \\
\hline $15, \ldots \ldots \ldots$ & & 5.5 & 4.85 & 5.5 & 3.6 & 2.4 & $2: 1$ \\
\hline $16 \ldots \ldots \ldots$ & & 5.5 & 4.9 & 5.5 & 5.6 & 2.4 & 2.1 \\
\hline $17 \ldots \ldots \ldots \ldots$ & & 5.5 & 5.0 & 5.5 & $5.2^{\circ}$ & 2.4 & 1.8 \\
\hline $18 \ldots \ldots \ldots$ & & 5.5 & 5.0 & 5.55 & 5.4 & 2.4 & 1.8 \\
\hline $19 \ldots \ldots \ldots$ & $1-$ & 5.2 & 5.0 & 5.5 & 5.0 & 2.4 & 1.7 \\
\hline $20 \ldots \ldots \ldots$ & & 5.2 & 0 & 5.5 & 5.0 & 2.0 & 1.75 \\
\hline $21 \ldots \ldots \ldots \ldots$ & & 5.3 & 0 & 5.5 & 4.8 & 2.15 & 1.75 \\
\hline 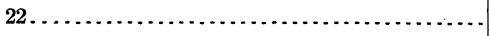 & 1.6 & 5.2 & 5.3 & 5.5 & 4.8 & 2.2 & 1.75 \\
\hline $23 \ldots \ldots \ldots \ldots$ & 2.1 & 5.25 & 5.25 & 5.5 & 4.2 & 2.2 & 1.75 \\
\hline $24 \ldots \ldots \ldots \ldots \ldots$ & 2.1 & . 5.25 & 5.25 & 5.5 & 4.55 & 2.2 & 1.75 \\
\hline $25 \ldots \ldots \ldots \ldots$ & 3.0 & 5.25 & 5.3 & 5.55 & 4.55 & 2.2 & 1.75 \\
\hline $26 \ldots \ldots \ldots \ldots$ & 3.4 & 5.2 & 5.4 & 5.55 & 4.5 & 2.2 & 1.75 \\
\hline $27 \ldots \ldots \ldots \ldots$ & 4. 1 & 5.3 & 5.4 & 5.55 & 4.25 & 2.2 & $\ldots \ldots$ \\
\hline $28 \ldots \ldots \ldots \ldots \ldots$ & 4. 1 & 5.4 & 5.4 & 5.55 & 4.0 & 1.8 & \\
\hline $29 \ldots \ldots \ldots \ldots$ & 2.1 & 5.3 & 5.4 & 5.6 & 4.1 & 1.8 & ......... \\
\hline $30 \ldots$ & 4.1 & 5.3 & 5.4 & 5.55 & 3.6 & 1.8 & $\ldots \ldots$ \\
\hline $31 \ldots \ldots \ldots \ldots \ldots \ldots \ldots \ldots$ & 4.0 & $\ldots$. & 5.4 & $\ldots \ldots$ & 3.6 & 2.0 & ....... \\
\hline
\end{tabular}

Note.-Canal dry January 1 to March 21 and September 26 to December 31.

Station rating table for Turlock canal at Lagrange, Cal., from January 1 to December 31, 1905.

\begin{tabular}{|r|r||r|r|r|r|r|r|}
\hline $\begin{array}{c}\text { Gage } \\
\text { height. }\end{array}$ & Discharge & $\begin{array}{c}\text { Gage } \\
\text { height. }\end{array}$ & Discharge. & $\begin{array}{c}\text { Gage } \\
\text { height. }\end{array}$ & Discharge. & $\begin{array}{c}\text { Gage } \\
\text { height. }\end{array}$ & Discharge. \\
\hline Feet. & Second-feet. & Feet. & Second-feet & Feet. & Second-feet. & Feet. & Second-feet. \\
0.30 & 1 & 1.50 & 40 & 2.70 & 166 & 3.90 & 324 \\
.40 & 2 & 1.60 & 47 & 2.80 & 178 & 4.00 & 338 \\
.50 & 3 & 1.70 & 55 & 2.90 & 190 & 4.20 & 366 \\
.60 & 4 & 1.80 & 64 & 3.00 & 202 & 4.40 & 394 \\
.70 & 5 & 1.90 & 74 & 3.10 & 215 & 4.60 & 422 \\
.80 & 7 & 2.00 & 85 & 3.20 & 228 & 4.80 & 450 \\
.90 & 10 & 2.10 & 96 & 3.30 & 241 & 5.00 & 478 \\
1.00 & 14 & 2.20 & 107 & 3.40 & 254 & 5.20 & 506 \\
1.10 & 18 & 2.30 & 118 & 3.50 & 268 & 5.40 & 535 \\
1.20 & 23 & 2.40 & 130 & 3.60 & 282 & 5.60 & 565 \\
1.30 & 28 & 2.50 & 142 & 3.70 & 296 & & \\
1.40 & 34 & 2.60 & 154 & 3.80 & 310 & & \\
\hline
\end{tabular}

NotE.-The above table is based on discharge measurements made during 1904-5. It is well defined between gage heights 2 feet and 5.5 feet. Monthly estimates are included with those of Tuolumne River, p. 210. 


\section{MISCELLANEOUS MEASUREMENTS IN TUOLUNNE RIVER DRAINAGE BASIN,}

Clavey River.-This stream is tributary to Tuolumne River from the north and enters it about 10 miles above the mouth of the North Fork. A measurement was made on September 15, 1905, just above the bridge for the new trail from Tuolumne to Lake Eleanor, by H. A. Campbell.

Width, 10 feet; area, 14.3 square feet; mean velocity, 0.87 foot per second; discharge, 12.5 second-feet.

Cherry River.- This stream is tributary to the Tuolumne River from the north and enters it about 15 miles below Hetch Hetchy Valley. A measurement was made one-third of a mile above the mouth of Eleanor Creek on September 16, 1905, by H. A. Campbell.

Width, 2 feet; area, 1.4 square feet; mean velocity, 1.00 foot per second; discharge, 1.4 second-feet.

Cherry River at Cherry Valley, Cal.-A measurement was made at the old gaging station in the lower end of Cherry. Valley on main trail from Tuolumne to Lake Eleanor on September 17, 1905, by H. A. Campbell.

Width, 2.2 feet; area, 1.6 square feet; mean velocity, 0.58 foot per second; discharge, 0.92 second-foot.

Eleanor Creek.-A measurement was made on September 18, 1905, 1 mile below outlet from Lake Eleanor and about 3 miles above its junction with Cherry Creek, by H. A. Campbell.

Width, 2.0 feet; area, 0.50 square foot; mean velocity, 0.60 foot per second; discharge, 0.30 second-foot.

Tuolumne River at Hetch Hetchy, Cal.- A measurement was made on September 18, 1905, at the head of Hetch Hetchy Valley, one-half mile above mouth of Rancheria Creek, by H., A. Campbell.

Width, 14 feet; area, 12 square feet; mean velocity, 1.83 feet per second; discharge, 22 second-feet.

Raricheria Creek.-This stream enters Tuolumne River from the north in Hetch Hetchy Valley. A measurement was made on September 19, 1905, 100 yards above trail crossing, about $1 \frac{1}{2}$ miles above its junction with Tuolumne River, by H. A. Campbell.

Width, 4 feet; area, 3.3 square feet; mean velocity, 0.94 foot per second; discharge, 3.1 second-feet.

Tiltill Creek.-This stream enters the Tuolumne River from the north in Hetch Hetchy Valley. A measurement was made on September 19, 1905, at lower end of Tiltill Valley about 2 miles above its junction with Tuolumne River, by $\mathrm{H}$. A. Campbell.

Width, 0.9 foot; area, 0.18 square foot; mean velocity, 0.67 foot per second; discharge, 0.12 second-foot.

Falls Creek.-This stream enters Tuolumne River from the north in Hetch Hetchy Valley. A measurement was made on September 19, 1905, at the outlet of Lake Vernon, by H. A. Campbell.

Width, 4.4 feet; area, 1.6 square feet; mean velocity, 0.75 foot per second; discharge, 1.2 second-feet.

Tuolumne River at Hetch Hetchy Valley, Cal.-A measurement was made on September 21, 1905, at the lower end of Hetch Hetchy Valley about one-half mile below trail to Lake Eleanor, by H. A. Campbell.

Width, 18.5 feet; area, 13.5 square feet; mean velocity, 1.78 feet per second; discharge, 24 second-feet.

Middle Fork of Tuolumne River.-A measurement was made on this stream on September 21,1905 , about 100 yards downstream from the bridge on the road from Sequoia to Hog Ranch by H. A. Campbell.

Width, 2 feet; area, 0.60 square foot; mean velocity. 0.93 foot per second; discharge, 0.56 second-foot. 
South Fork of Tuolumne River.-A measurement was made on this stream on September 22, 1905, about 75 yards upstream from the bridge on the Big Oak Flat and Yosemite Toll Road, near Harden ranch, by H. A. Campbell.

Width, 11.2 feet; area, 10.6 square feet; mean velocity, 0.42 foot per second; discharge, 4.5 second-feet.

Mining ditch near Lagrange, Cal.- This ditch diverts water from the Tuolumne River several miles above the town of Lagrange, Cal. A measurement was made April 6 at a point on the hill above the Lagrange dam.

Width, 6 feet; area, 4.3 square feet; mean velocity, 1.58 feet per second; discharge, 6.8 second-feet.

\section{STANISLAUS RIVER DRAINAGE BASIN.}

\section{DESCRIPTION OF BASIN.}

Stanislaus River drains a portion of the western slope of the Sierra Nevada and heads well back on the crest, at elevations of from 10,000 to 12,000 feet. It drains the country between the basins of the Mokelumne River on the north and the Tuolumne River on the south, and flows in a general southwesterly direction, entering the San Joaquin River 23 miles above Stockton. It has numerous tributaries in the upper reaches of the basin, which have their source in numerous small glacial lakes. The topography is rough and broken with high mountain peaks. The formation is of granite, which is bare and destitute of timber growth above an elevation of 8,000 feet, except where small glacial lakes and moraines occur. In the middle reaches of the basin there is good soil covering and a heavy growth of timber. In this basin is situated the Calaveras grove of big trees (Sequoia gigantea), for which the Sierra Nevada are famous. This is the most northerly grove of these trees, groves of which extend as far south as the Kern River basin. The mean annual rainfall for the basin is about 50 inches. The precipitation falls chiefly in the form of snow on the higher elevations, remaining well into the summer months. Mining operations have been carried on extensively in this basin, and many canals have been taken out of the river, all of which discharge their water into the river again. The canal of the Stanislaus Water Company diverts water 3 miles above Knights Ferry, which is used to irrigate land between Knights Ferry and Stockton. A gaging station is maintained on this canal to determine its discharge.

\section{STANISLAUS RIVER AT KNIGHTS FERRY, CAL.}

A station was first established on this river on May 3, 1895, at the railroad bridge onehalf mile north of the town of Oakdale. On July 30, 1898, a cable was placed 1,000 feet below the railroad bridge. This station was used until February 16, 1901.

The station at Knights Ferry was established May 19, 1903, by W. H. Stearns. It is located 200 feet from the post-office at Knights Ferry.

There is an island 800 feet above the gaging station, and a dam on each channel at the head of the island. The Stanislaus Milling and Power Company's power house is on the right bank of the river below one of these dams and about 1,000 feet above the gaging station. The channel is straight for 500 feet above and below the cable. At ordinary and high stages the stream has a fall of 0.47 foot in the 500 feet above the cable and of 0.68 foot in the 500 feet below. Both banks are composed of cemented gravel and are high. The loft bank is not subject to overflow. In extreme floods the right bank has been known to be overflowed, flooding the yards and houses next to the river. The bed is of gravel and is subject to some change from the addition of material which is washed down from the island above.

Discharge measurements at high and medium stages are made from a car suspended from a cable. Low-water measurements are made by wading 300 feet above the cable. The initial point for soundings is the eyebolt to which the cable is fastened on the right bank.

$$
\text { IRR } 177-06-15
$$


Ordinary and low-water stages were read on an iron pipe driven into the bed of the stream. For high stages the gage was a plank nailed to a post on the right bank of the river. A new gage rod has been placed near the cable on the right bank and gage readings are now made on this rod. It consists of an inclined rod fastened securely to posts set in the ground. This portion of the rod is used for the lower stages. For high water there is a vertical section similar to the above at the north cable support. During 1905 the gage was read once each day by E. J. Coop. Bench marks were established as follows: (1) A spike in a locust tree 50 feet northeast of right cable support; elevation, 19.20 feet. (2). A rock under the spike at the foot of the above locust tree; elevation, 18.74 feet. Elevations refer to the datum of the new gage.

Information in regard to this station is contained in Water-Supply Papers Nos. 81, 100, and 134 of the United States Geological Survey.

Discharge measurements of Stanislaus River at Knights Ferry, Cal., in 1905.

\begin{tabular}{|c|c|c|c|c|c|c|}
\hline Date. & Hydrographer. & Width. & $\begin{array}{l}\text { Area of } \\
\text { section. }\end{array}$ & $\underset{\text { velocity. }}{\text { Mean }}$ & $\begin{array}{l}\text { Gage } \\
\text { height. }\end{array}$ & $\begin{array}{l}\text { Dis- } \\
\text { charge. }\end{array}$ \\
\hline & . & Feet. & $S q . f t$. & Ft. per sec. & Feet. & Sec.-ft. \\
\hline March $14 \ldots .$. & 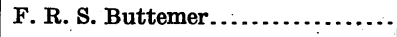 & 197 & 751 & 3.36 & 9.15 & 2,523 \\
\hline April 6. . & O. W. Peterson.. & 198 & 769 & 3.49 & 9.25 & 2,687 \\
\hline April 16. & R. S. Hawley ...... & 194 & 771 & 3.27 & 9.30 & 2,518 \\
\hline May 23. & .....do.... & 217 & 944 & 4.30 & 10.05 . & 4,062 \\
\hline July $20 \ldots$ & .....do........... & 148 & 363 & 1.17 & 7.00 & 425 \\
\hline September 12.. & Lee and Keeler. . & 40 & 51 & 1.68 & 6.19 & 86 \\
\hline September 22.. & R. W. Keeler..... & 40 & 49 & -1.57 & 6.11 & 77 \\
\hline September 29.. & ....do... & 40 & 49 & 1.63 & 6.17 & 80 \\
\hline October 6...... & ....do. . & 40 & 49 & 1.69 & 6.14 & 83 \\
\hline October 13 & $\ldots$. do $\ldots . . . . . . . . .$. & 40 & 48 & 1.77 & 6.19 & 85 \\
\hline October $16 \ldots . .$. & Lee and Hawley ............ & 40 & 47 & . 1.85 & 6.21 & 87 \\
\hline Decemker $21 \ldots$ & R. W. Keeler............... & 120 & 268 & .53 & 6.41 & 142 \\
\hline
\end{tabular}


Daily gage height, in feet, of Stanislaus River at Knights Ferry, Cal., for 1905.

\begin{tabular}{|c|c|c|c|c|c|c|c|c|c|c|c|c|}
\hline Day. & Jan. & Feb. & Mar. & Apr. & May. & June. & July. & Aug. & Sept. & Oct. & Nov. & Dec. \\
\hline 1. & 7.4 & 7.75 & 8.25 & 8.55 & 9.8 & 9.45 & 7.95 & 6.4 & 6.15 & 6.1 & 6.1 & 6.45 \\
\hline 2. & 7.3 & 10.15 & 8.4 & 8.7 & 9.5 & 9.35 & 7.8 & 6.4 & 6.15 & 6.1 & 6.1 & 6.3 \\
\hline 3. & 7.25 & 8.4 & 8.45 & 8.85 & 9.35 & 9,25 & 7.7 & 6.4 & 6.15 & 6.15 & 6.1 & 6.25 \\
\hline 4. & 7.15 & 8.05 & 8.55 & 8.95 & 9.1 & 9.0 & 7.65 & 6.4 & 6.15 & 6.15 & 6.1 & 6.3 \\
\hline 5. & 7.1 & 8.85 & 8.5 & 9.1 & 9.0 & 8.8 & 7.6 & 6.35 & 5.7 & 6.15 & 6.1 & 6.3 \\
\hline 6. & 7.05 & 8.45 & 8.5 & 9.25 & 8.85 & 8.85 & 7.45 & 6.3 & 5.7 & 6.1 & 6.1 & 6.3 \\
\hline 7. & 7.0 & 8.1 & 8.5 & 9.4 & 9.4 & 9.2 & 7.45 & 6.3 & 6.2 & 6.1 & 6.1 & 6.3 \\
\hline $8 .$. & 7.0 & 7.85 & 8.45 & 9.4 & 9.35 & 9.2 & 7.45 & 6.25 & 6.2 & 6.1 & 6.1 & 6.3 \\
\hline $9 .$. & 7.15 & 7.8 & 8.4 & 9.4 & 9.1 & 9.1 & 7.45 & 6.25 & 6.15 & 6.1 & 6.1 & 6.3 \\
\hline 10. & 7.2 & 7.7 & 8. 4 & 9.45 & 8.95 & 9.15 & 7.4 & 6.25 & 6.1 & 6.1 & 6.15 & 6.3 \\
\hline 11.. & 7.1 & 7.65 & 8. 45 & 9.15 & 8.8 & 9.4 & 7.3 & 6.25 & 6.1 & 6.1 & 6.2 & 6.3 \\
\hline 12. & 7.05 & 7.6 & 8.4 & 8.95 & 8.75 & 9.65 & 7.3 & 6.25 & 6.15 & 6.1 & 6.2 & 6.3 \\
\hline 13. & 7.0 & 7.45 & 9.1 & 8.9 & 8. 75 & 9.65 & 7.2 & 6.2 & 6.15 & 6.1 & 6.2 & 6.3 \\
\hline 14. & 7.0 & 7.4 & 9.0 & 8.95 & 9.05 & 9.5 & 7.1 & 6.2 & 6.15 & 6.1 & 6.2 & 6.3 \\
\hline 15. & 7.1 & 7.4 & 8.55 & 9.1 & 9.5 & 9.3 & 7.0 & 6.25 & 6.2 . & 6.15 & 6.2 & 6.3 \\
\hline $16 .$. & 7.4 & 7.4 & 9.1 & 9.25 & 10.15 & 9.4 & 6.9 & 6.2 & 6.2 & 6.2 & 6.2 & 6.3 \\
\hline 17. & 7.3 & 7.7 & 10.35 & 9.05 & 11.2 & 9.25 & 6.85 & 6.2 & 6.2 & 6.15 & 6.2 . & 6.25 \\
\hline 18. & 7.3 & 7.8 & 9.6 & 9.2 & 10.55 & 9.2 & 6.85 & 6.2 & 6.2 & 6.15 & 6.2 & 6.25 \\
\hline 19. & 7.25 & 7.8 & 11. 5 & 9.25 & 10.4 & 9.05 & 6.75 & 6.2 & 6.2 & 6.15 & 6.2 & 6.3 \\
\hline 20. & 7.3 & 8.6 & 10.0 & 8.9 & 10.25 & 8.95 & 6.85 & 6.25 & 6.15 & 6.15 & 6.2 & 6.4 \\
\hline 21. & 7.7 . & 8.45 & 9.35 & 8.8 & 10.35 & 8.95 & 6.75 & 6.25 & 6.15 & 6.15 & 6.25 & 6.4 \\
\hline 22. & 7.5 & 8.2 & 9.25 & 8.75 & 10.05 & 8.9 & 6.7 & 6.25 & 6.1 & 6.15 & 6.3 & 6.3 \\
\hline .23. & 7.7 & 8.1 & 8.85 & 8.9 & 9.9 & 8.7 & 6.75 & 6.25 & 6.15 & 6.15 & 6.3 & 6.25 \\
\hline 24. & 7.65 & 8.1 & 8.8 & 9.05 & 9.85 . & 8. 45 & 6.85 & 6.25 & 6.15 & 6.15 & 6.25 & 6.2 \\
\hline 25. & 7.55 & 8.15 & 8.85 & 9.45 & 9.95 & 8.2 & 6.75 & 6.25 & 6.15 & 6.1 & 6.2 & 6.2 \\
\hline $26 \ldots$ & 7.5 & 8.3 & 8.9 & 9.65 & 10.0 & 8.25 & 6.7 & 6.25 & 6.15 & 6.1 & 6.2 & 6.25 \\
\hline $27 .$. & 7.35 & 8.3 & 9.2 & 10.0 & 9.55 & 8.1 & 6.65 & 6.25 & 6.15 & 6.1 & 6.3 & 6.3 \\
\hline 28. & 7.3 & 8.25 & 8.75 & 10.1 & 9.2 & 8.0 & 6.55 & 6.25 & 6.15 & 6.1 & 6.3 & 6.3 \\
\hline 29. & 7.25 & & 8.95 & 10.35 & 9.1 & 8.0 & 6.55 & 6.15 & 6.15 & 6.1 & 6.4 & 6.4 \\
\hline $30 \ldots$ & 7.05 & 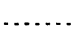 & 8.75 & 10.45 & 9.3 & 8.0 & 6.5 & 6.25 & 6.15 & 6.1 & 6.5 & 6.45 \\
\hline $31 \ldots$ & 6.75 & .... & 8.65 & $\ldots$ & 9.35 & $\ldots$ & 6.45 & 6.2 & 6.15 & 6.1 & $\because$ & 6.4 \\
\hline
\end{tabular}

Station rating table for Stanislaus River at Knights Ferry, Cal., from January 1, 1904, to December 31, 1905.

\begin{tabular}{|c|c||c|c||c|c|c|c|}
\hline $\begin{array}{c}\text { Gage } \\
\text { height. }\end{array}$ & Discharge. & $\begin{array}{c}\text { Gage } \\
\text { height. }\end{array}$ & Discharge. & $\begin{array}{c}\text { Gage } \\
\text { height. }\end{array}$ & Discharge. & $\begin{array}{c}\text { Gage } \\
\text { height. }\end{array}$ & Discharge. \\
\hline Feet. & Second-feet. & Feet. & Second-feet. & Feet. & Second-feet. & Feet. & Second-feet. \\
6.00 & 60 & 7.30 & 630 & 8.60 & 1,840 & 9.90 & 3,550 \\
6.10 & 70 & 7.40 & 710 & 8.70 & 1,960 & 10.00 & 3,700 \\
6.20 & 80 & 7.50 & 800 & 8.80 & 2,080 & 10.20 & 4,040 \\
6.30 & 95 & 7.60 & 890 & 8.90 & 2,200 & 10.40 & 4,400 \\
6.40 & 125 & 7.70 & 980 & 9.00 & 2,320 & 10.60 & 4,800 \\
6.50 & 160 & 7.80 & 1,070 & 9.10 & 2,440 & 10.80 & 5,230 \\
6.60 & 200 & 7.90 & 1,160 & 9.20 & 2,560 & 11.00 & 5,730 \\
6.70 & 245 & 8.00 & 1,250 & 9.30 & 2,680 & 11.20 & 6,330 \\
6.80 & 295 & 8.10 & 1,340 & 9.40 & 2,800 & 11.40 & 6,990 \\
6.90 & 350 & 8.20 & 1,440 & 9.50 & 2,950 & 11.60 & 7,700 \\
7.00 & 410 & 8.30 & 1,540 & 9.60 & 3,100 & & \\
7.10 & 480 & 8.40 & 1,640 & 9.70 & 3,250 & & \\
7.20 & 550 & 8.50 & 1,740 & 9.80 & 3,400 & & \\
\hline
\end{tabular}


Estimated monthly discharge of Stanislaus River at Knights Ferry, Cal., for 1905.

[Drainage area, 935 square miles.]

\begin{tabular}{|c|c|c|c|c|c|c|}
\hline \multirow[b]{2}{*}{ Month. } & \multicolumn{3}{|c|}{ Discharge in second-feet. } & \multirow[b]{2}{*}{$\begin{array}{l}\text { Total in } \\
\text { acre-feet. }\end{array}$} & \multicolumn{2}{|c|}{ Run-off. } \\
\hline & Maximum. & Minimum. & Mean. & & $\begin{array}{l}\text { Second-feet } \\
\text { per square } \\
\text { mile. }\end{array}$ & $\begin{array}{l}\text { Depth } \\
\text { in inches. }\end{array}$ \\
\hline January... & 1,015 & 294 & 642 & 39,480 & 0.687 & 0.792 \\
\hline February.. & 4,002 & 731 & 1,326 & 73,640 & 1.42 & 1.48 \\
\hline March..... & 7,394 & 1,533 & 2,395 & 147,300 & 2.56 & 2.95 \\
\hline April.... & 4,625 & 1,905 & 2,774 & 165,100 & 2.97 & 3.31 \\
\hline May ...... & 6,445 & 2,124 & 3,247 & 99,650 & 3.47 & 4.00 \\
\hline June....... & 3,296 & 1,351 & 2,392 & 142,300 & 2.56 & 2.86 \\
\hline July........... & 1,309 & 225 & 613 & 37,690 & .656 & .756 \\
\hline August..... & 211 & 97 & 150 & 9,223 & 160 & .184 \\
\hline September. & 137 & 90 & 103. & 6,129 & .110 & .123 \\
\hline October.... & 138 & 85 & 97 & 5,964 & .104 & .120 \\
\hline November.... & 189 & 77 & 91 & 5,415 & .097 & .108 \\
\hline December....... & 185 & 96 & 131 & 8,055 & .140 & 1.61 \\
\hline The year. & 7,394 & 77 & 1,163 & 739,900 & 1.24 & 18.29 \\
\hline
\end{tabular}

Note.-The discharge of Stanislaus Water Company's ditch and Schell ditch is included above. Mean daily flow of Schell ditch is estimated at 7 second-feet.

STANISLAUS WATER COMPANY'S DITOH AT KNIGHTS FERRY, CAL.

This station was established June 11, 1904, by S. G. Bennett: The station is located below the point where Schell ditch diverts its water, about 1 mile below the Stanislaus Milling and Power Company's power house and 200 feet below the place where it passes under. Schell ditch flume. The water diverted by this ditch is used for irrigation in the vicinity of Oakdale, Cal.

A meter measurement on Schell ditch 200 feet below its intake gave a discharge of about 7 second-feet, which is said to be its usual constant flow. In computing the estimated monthly discharge of Stanislaus River. a mean daily discharge of 7 second-feet has been used as the capacity of Schell ditch.

The gage is fastened to the upstream side of a small bridge. During 1905 the gage was read once each day by $\mathrm{E}$. J. Coop.

A description of this station, with gage height and discharge data, is contained in WaterSupply Paper No. 134, United States Geological Survey. 
Discharge measurements of Stanislaus Water Company's ditch at Knights Ferry, Cal., in 1905.

\begin{tabular}{|c|c|c|c|c|c|c|}
\hline Date. & Hydrographer. & Width. & $\begin{array}{l}\text { Area of } \\
\text { section. }\end{array}$ & $\begin{array}{c}\text { Mean } \\
\text { velocity. }\end{array}$ & $\begin{array}{c}\text { Gage } \\
\text { height. }\end{array}$ & $\begin{array}{c}\text { Dis- } \\
\text { charge. }\end{array}$ \\
\hline March 14... & F. R. S. Buttemer. & $\begin{array}{r}\text { Feet. } \\
13.2\end{array}$ & $\begin{array}{c}S q . f t . \\
22\end{array}$ & $\begin{array}{r}\text { Ft.per sec. } \\
2.14\end{array}$ & $\begin{array}{r}\text { Feet. } \\
2.58\end{array}$ & $\begin{array}{c}\text { Sec.-ft. } \\
47\end{array}$ \\
\hline May $23 . \ldots . . .$. & R. S. Hawley ........ & 8.7 & 27 & 4.24 & 3.65 & 114 \\
\hline June 16. & .....do.. & 8.5 & 24 & 4.00 & 3.45 & 96 \\
\hline July $20 \ldots$. & .....do.... & 9 & 21 & 3.67 & 3.00 & 77 \\
\hline September $12 .$. & C. H. Lee............ & 11 & 6.1 & .85 & 1.12 & 5.2 \\
\hline September 12 .. & .....do.......... & 8.7 & 5.5 & 1.58 & 1.50 & 8.7 \\
\hline September 12 .. & .....do............ & 9.4 & 13.1 & 2.52 & 2.18 & 33 \\
\hline September 12 .. & ..... do... & 9.4 & 12.6 & 2.38 & 2.08 & 30 \\
\hline October $17 \ldots .$. & R. S. Hawley .................... & 7.5 & 2.1 & .38 & .80 & .8 \\
\hline
\end{tabular}

Daily gage height, in feet, of Stanislaus Water Company's canal at Knights Ferry, Cal., for 1905.

\begin{tabular}{|c|c|c|c|c|c|c|c|c|c|c|c|c|}
\hline Day. & Jan. & Feb. & Mar. & Apr. & May. & June. & July. & Aug. & Sept. & Oct. & Nov. & Dec. \\
\hline 1. & 2.6 & 2.3 & 2.3 & 3.55 & 3.7 & 3.7 & 3.4 & 3.1 & 1.95 & 2.8 & 0.0 & 2.1 \\
\hline $2 \ldots$ & 2.4 & 2.4 & 2.6 & 3.7 & 3.6 & 3.6 & 3.45 & 3.0 & 2.0 & 2.65 & 1.5 & 2.15 \\
\hline 3.. & 2.5 & 2.05 & .0 & 3.5 & 3.5 & 3.6 & 3.35 & 3.05 & 2.1 & 2.4 & 1.8 & 2.2 \\
\hline 4. & 2.5 & 1.9 & .0 & 3.65 & 3.5 & 3.7 & 3.45 & 3.05 & 2.05 & 2.3 & 1.5 & 1.95 \\
\hline 5.. & 2.5 & .0 & .0 & 3.55 & 3.55 & 3.6 & 3.35 & 3.1 & 3.45 & 2.2 & 1.5 & 2.2 \\
\hline $6 \ldots$ & 2.3 & 2.1 & 2.3 & 3.65 & 3.6 & 3.6 & 3.4 & 3.1 & 3.3 & 2.1 & 1.2 & 2.1 \\
\hline $7 \ldots$ & 2.5 & .0 & 2.5 & 3.7 & 3.5 & 2.55 & 3.3 & 3.1 & 2.0 & 2.15 & .0 & 2.0 \\
\hline 8... & 2.5 & .0 & 2.25 & 3.65 & 3.3 & 3.45 & 3.4 & 3.05 & 2.0 & 2.2 & .0 & 2.05 \\
\hline $9 .$. & 2.45 & .0 & 2.55 & 3.6 & 3.2 & 3.6 & 3.4 & 3.0 & 2.1 & 2.05 & .0 & 2.1 \\
\hline $10 .$. & 2.45 & .0 & 2.5 & 3.6 & 3.3 & 3.7 & 3.45 & 2.95 & 2.2 & 1.70 & .0 & 2.1 \\
\hline $11 .$. & 2.25 & .0 & 3.4 & 3.55 & 3.4 & 3.65 & 3.3 & 2.95 & 2.15 & 1.75 & .0 & 1.8 \\
\hline $12 .$. & 2.45 & 1.45 & 3.0 & 3.6 & 3.45 & 3.65 & 3.3 & 2.95 & 2.0 & 1.85 & .0 & 1.95 \\
\hline $13 \ldots$ & 2.05 & .0 & 2.6 & 3.5 & 3.4 & 3.6 & 3.3 & 3.1 & 2.1 & 1.4 & .0 & 2.0 \\
\hline $14 \ldots$ & 2.0 & 1.8 & 2.45 & 3.65 & 3.45 & 3.6 & $3: 25$ & 2.8 & 1.6 & 1.2 & .0 & 1.85 \\
\hline $15 \ldots$ & 2.2 & 1.7 & 2.45 & 3.65 & 3.7 & 3.5 & 3.15 & 2.85 & 1.6 & 1.0 & .0 & 1.65 \\
\hline $16 \ldots$ & 2.15 & 1.9 & 2.6 & 3.7 & 3.7 & 3.5 & 3.35 & 2.9 & 1.5 & 1.4 & .0 & 1.8 \\
\hline $17 .$. & 2.35 & 2.3 & 2.6 & 3.65 & 3.55 & 3.5 & 3.1 & 2.7 & 1.4 & 1.3 & .0 & 2.2 \\
\hline 18. & 2.1 & 2.2 & 2.5 & 3.55 & 3.6 & 3.5 & 3.1 & 2.5 & 1.4 & 1.2 & .0 & 2.1 \\
\hline $19 .$. & 2.1 & 2.4 & 2.45 & 3.6 & 3.7 & 3.5 & 3.0 & 2.45 & 1.1 & 1.3 & .0 & 1.95 \\
\hline $20 \ldots$ & 2.3 & 2.0 & 2.8 & 3.55 & 3.65 & 3.45 & 3.2 & 2.3 & 1.35 & 1.4 & .0 & 2.0 \\
\hline $21 \ldots$ & 2.05 & 2.4 & 2.6 & 3.6 & 3.7 & 3.55 & 3.1 & 2.3 & 1.5 & 1.5 & .0 & 2.0 \\
\hline $22 \ldots$ & 2.2 & 2.3 & 2.8 & 3.6 & 3.65 & 3.45 & 3.0 & 2.05 & 1.6 & 1.55 & .0 & 2.05 \\
\hline $23 .$. & 2.1 & 2.0 & 2.9 & 3.5 & 3.65 & 3.45 & 3.0 & 2.1 & 1.3 & 1.8 & .0 & 1.95 \\
\hline $24 \ldots$ & 2.1 & 2.0 & 3.0 & 3.6 & 3.6 & 3.45 & .0 & 2.0 & 1.7 & 1.4 & .0 & 1.25 \\
\hline $25 \ldots$ & 2.15 & 2.0 & 3.15 & 3.65 & 3.65 & 3.4 & 2.95 & 2.05 & 1.5 & 1.2 & .0 & 1.4 \\
\hline $26 .$. & 2.1 & .0 & 3.2 & 3.7 & 3.65 & 3.35 & 3.0 & 1.9 & 1.65 & 1.3 & .0 & .9 \\
\hline $27 \ldots$ & 2.2 & 2.25 & 3.3 & 3.7 & 3.6 & 3.35 & 3.1 & 1.85 & 1.65 & 1.6 & 1.7 & 2.2 \\
\hline $28 \ldots$ & 2.2 & 2.35 & 3.35 & 3.7 & 3.6 & 3.35 & 3.0 & 1.7 & 1.7 & 1.5 & 1.95 & 2.3 \\
\hline $29 .$. & 2.3 & & 3.4 & 3.7 & 3.6 & 3.4 & 2.95 & 2.15 & 2.2 & 1.45 & 2.0 & 2.2 \\
\hline $30 \ldots$ & 1.65 & & 3.6 & 3.7 & 3.6 & 3.45 & 3.1 & 1.7 & 2.25 & 1.45 & 1.95 & 2.3 \\
\hline $31 \ldots$ & 1.8 & & 3.65 & 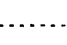 & 3.65 & & 3.05 & 1.5 & $\ldots .$. & 1.4 & 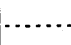 & 2.2 \\
\hline
\end{tabular}


Station rating table for Stanislaus Water Company's ditch near Knights Ferry, Cal., from January 1 to December 31, 1905.

\begin{tabular}{|r|r||r|r||r|r|r|r|}
\hline $\begin{array}{c}\text { Gage } \\
\text { height. }\end{array}$ & Discharge. & $\begin{array}{c}\text { Gage } \\
\text { height. }\end{array}$ & Discharge. & $\begin{array}{c}\text { Gage } \\
\text { height. }\end{array}$ & Discharge. & $\begin{array}{c}\text { Gage } \\
\text { height. }\end{array}$ & Discharge. \\
\hline Feet. & Second-feet. & Feet. & Second-feet. & Feet. & Second-feet. & Feet. & Second-feet. \\
1.00 & 2.5 & 1.70 & 14 & 2.40 & 40 & 3.10 & 79 \\
1.10 & 3.5 & 1.80 & 17 & 2.50 & 45 & 3.20 & 85 \\
1.20 & 5 & 1.90 & 20 & 2.60 & 50 & 3.30 & 91 \\
1.30 & 6.5 & 2.00 & 24 & 2.70 & 55 & 3.40 & 97 \\
1.40 & 8 & 2.10 & 28 & 2.80 & 61 & 3.50 & 104 \\
1.50 & 10 & 2.20 & 32 & 2.90 & 67 & 3.60 & 111 \\
1.60 & 12 & 2.30 & 36 & 3.00 & 73 & 3.70 & 118 \\
& & & & & & \\
\hline
\end{tabular}

Note.-The above table is based on 9 discharge measurements made during $1905^{\circ}$. It is well defined between gage heights 1 foot and 3.7 feet. Monthly estimates are included with those of Stanislaus River, page -

\section{MISCFLIANEOUS MEASUREMENTS IN STANISLAUS RIVER DRATIAGE BASIN.}

Stanislaus River at Parrotts Ferry, Cal.-A measurement was made on this stream frcm the downstream side of the wagon bridge at Parrotts Ferry on September 12, 1905, by H. A. Campbell.

Width, 35 feet; area, 138 square feet; mean velocity, 0.66 foot per second; discharge, 91 second-feet.

South Fork of Stanislaus.-A measurement was made on this stream on September 25, 1905, at trail crossing to Yancey's ranch about 14 miles above its junction with the main river and about 3 miles north of Confidence, Cal. Measurement made by $\mathrm{H}$. A. Campbell.

Width, 5 feet; area, 2.4 square feet; mean velocity, 0.40 foot per second; discharge, 0.96 second-feet.

Middle Fork of Stanislaus.-A measurement was made on this stream on September 26, 1905, at the bridge on the trail to McCormicks, about 5 miles above its junction with the main river. Measurement made by $\mathrm{H}$. A. Campbell.

Width, 21 feet; area, 350 square feet; mean velocity, 0.24 foot per second; discharge, 84 second-feet.

Griswald Creek.-This stream enters Stanislaus River about 2 miles above the mouth of the Middle Fork. A measurement was made on September 26, 1905, at trail crossing, about 5 miles above the mouth, by $\mathrm{H}$. A. Campbell.

Width, 6 feet; area, 5.4 square feet; mean velocity, 0.37 foot; discharge, 2.0 second-feet.

Beaver Creek.-This stream enters Stanislaus River from the east about 5 miles above the mouth of the Middle Fork. A measurement was made on September 26, 1905, at the trail crossing to the Calaveras big trees and about 5 miles above its junction with North Fork of Stanislaus River. Measurement made by H. A. Campbell.

Width, 2.5 feet; area, 2.8 square feet; mean velocity, 1.64 feet per second; discharge, 4.6 second-feet.

North Fork of Stanislaus River at Squaw Hollow, Cal.-A measurement was made about 75 yards upstream from trail bridge at Squaw Hollow on September 27, 1905, by H. A. Campbell.

Width, 29 feet; area, 48 square feet; mean velocity, 1.60 feet per second; discharge, 77 second-feet. 


\section{MOKELUMNE RIVER DRAINAGE BASIN.}

\section{DESCRIPTION OF BASIN.}

Mokelumne River drains an area of 657 square miles of the western slope of the Sierra Nevada. It has numerous tributaries, the North, South, and Middle forks being the most important. This stream heads well back in the main crest of the Sierra Nevada at an elevation of 8,000 feet.

The formation is of granite with good soil covering and heavy timber growth on the middle and higher elevations. On the lower elevations the slopes are less rugged and the soil covering is of brush and scattering oak timber with large areas of cultivated land and pasture. There are numerous small glacial lakes and moraines in the upper reaches of this basin. The precipitation varies from 25 inches on the lower to 50 inches on the higher elevations, where it falls in the form of snow, which melts in the early spring. The greatest discharge usually occurs in April, May, and June. There is some artificial storage in this basin, but not enough to have much effect on the discharge.

Several diversions are made for mining and power purposes within the drainage basin, and this water is returned to the river above the gaging station, which is located at Clement, a few miles above Lodi, Cal.

\section{MOKELUMNE RIVER NEAR CLEMENTS, CAL.}

This station was established October 28, 1904, by O. W. Peterson. It is located at the highway bridge, 1 mile north of Clements.

The channel is straight for 150 feet above and 500 feet below the gaging station. The right bank is high and not subject to overflow. The left bank is subject to overflow when the gage reads above 15 feet. The bed of the stream is composed of gravel and is subject to slight changes.

Discharge measurements are made from the downstream side of the bridge. The initial point is on the end of the bridge near the right bank.

The gage is in two sections. The low-water section is an inclined rod nailed to posts driven in the ground, and the high-water section is a vertical rod nailed to a piie and near the inclined rod. Both are on the right bank. During 1905 the gage was read by Allen Gaskill. The bench mark is the head of a bolt driven in a pile, to which the upper section of the gage is fastened; elevation, 9.60 feet above the datum of the gage.

$\Lambda$ description of this station, with gage height and discharge data, is contained in WaterSupply Paper No. 134, United States Geological Survey.

Discharge measurements of Mokelumne River near Clements, Cal., in 1905.

\begin{tabular}{|c|c|c|c|c|c|c|}
\hline Date. & Hydrographer. & Width. & $\begin{array}{l}\text { Area of } \\
\text { section. }\end{array}$ & $\begin{array}{c}\text { Mean } \\
\text { velocity. }\end{array}$ & $\begin{array}{c}\text { Gage } \\
\text { height. }\end{array}$ & $\begin{array}{l}\text { Dis- } \\
\text { charge. }\end{array}$ \\
\hline January $23 . . .$. & F. R.S. Buttemer. - & $\begin{array}{r}\text { Feet. } \\
132\end{array}$ & $\begin{array}{r}S q \cdot f t \\
293\end{array}$ & $\begin{array}{r}\text { Ft. per sec. } \\
2.69\end{array}$ & $\begin{array}{l}\text { Feet. } \\
\quad 5.15\end{array}$ & $\begin{array}{r}\text { Sec.-ft. } \\
788\end{array}$ \\
\hline January $24 . .$. & . ... .do . . . . . & 128 & 258 & 2.40 & 4.85 & 620 \\
\hline January $24 .$. & .....do..... & 128 & 250 & 2.38 & 4.79 & 596 \\
\hline January $30 \ldots$ & ....do..... & 121 & 191 & 2.01 & 4.28 & 384 \\
\hline February $9 \ldots$ & .....do... & 126 & 259 & 2.39 & 4.87 & 620 \\
\hline February $15 \ldots$ & ....do..... & 122 & 193 & 2.27 & 4.31 & 438 \\
\hline February $20 .$. & ....do....... & 210 & 562. & 2.54 & 6.14 & 1,425 \\
\hline May $18 . . . . .$. & W. B. Clapp... & 248 & 1,238 & 3.06 & 9.00 & 3,788 \\
\hline June $29 . . . . .$. & O. W. Peterson.... & 130 & 237 & 2.36 & 4.64 & 559 \\
\hline July $28 \ldots$ & Peterson and Lee.... & 60 & 80 & 1.56 & 3.14 & 125 \\
\hline September $5 .$. & C. H. Lee. . . . . . . . & 58 & 79 & 1.27 & 3.13 & 100 \\
\hline November 29. & .....do........ & 55 & 66 & .86 & 2.90 & 57 \\
\hline
\end{tabular}


Daily gage height, in feet, of Mokelumne River near Clements, Cal., for 1905.

\begin{tabular}{|c|c|c|c|c|c|c|c|c|c|c|c|c|}
\hline Day. & Jan. & Feb. & Mar. & Apr. & May. & June. & July. & Aug. & Sept. & Oct. & Nov. & Dec. \\
\hline & 5.0 & 4.85 & 5.4 & 6.05 & 7.5 & 7:65 & 4.3 & 3.2 & 3.3 & 3.4 & 3.25 & 3.25 \\
\hline 2. & 55 & 7.55 & 5.45 & 6.2 & 7.0 & 7.45 & 4.3 & 3.3 & 3.3 & 3.25 & 3.2 & 3.1 \\
\hline & 4.5 & 5.8 & 5.5 & 6.3 & 6.9 & 7.15 & 4.1 & 3.3 & 3.25 & 3.3 & 3.25 & 3.1 \\
\hline 4. & 4.4 & 5.35 & 5.65 & 6.45 & 6.65 & 6.9 & 4.0 & 3.3 & 3.3 & 3.25 & 3.1 & 3.0 \\
\hline $5 \therefore$ & 4.15 & 6.55 & 5.6 & 6.7 & 6. & 6.6 & 3.85 & 3.2 & 3.3 & 3.3 & 3.1 & 2.95 \\
\hline 6. & 4.3 & 5.7 & 5.7 . & 6.8 & 6. & 6.8 & 3.85 & 3.25 & 3.3 & 3.3 & 3.1 & 2.95 \\
\hline & 4.1 & 5.3 & 5.5 & 6.95 & & 1 & 8 & 3.15 & 3.3 & 3.45 & 3.1 & 3.1 \\
\hline 8. & 4.1 & 5.0 & 5.6 & 7.05 & 6. & 7.05 & 4.05 & 3.3 & 3.35 & 3.4 & 2.95 & 3.1 \\
\hline 9. & 4.3 & 4.9 & $5: 45$ & 6.85 & 6.55 & 7.0 & 3.65 & 3.25 & 3 & 3.25 & 0 & 3.05 \\
\hline 0. & 4.3 & 4.65 & 5.45 & 7.0 & 6.4 & 7.15 & 3.6 & 3.3 & 3.3 & 3.35 & 2.95 & 3: 05 \\
\hline .. & 4.1 & 4.65 & 5.5 & 6.6 & 6 & 7.55 & 3.7 & 3.2 & 3.2 & 3 & .95 & 3.0 \\
\hline .. & 4.1 & 4.55 & 5.45 & 6.25 & 6.3 & 7.75 & 3.6 & 3.3 & 3.3 & 3.35 & 2.95 & 3.1 \\
\hline & 4.15 & 4.5 & 6.1 & 6.25 & 6.25 & 7.75 & 3.65 & 3.2 & 3.3 & 3.35 & 2.9 & 3.05 \\
\hline .. & 4.15 & 4.55 & 6.15 & 6.45 & & & 3.45 & 2.9 & 3.35 & 3.15 & 2.95 & 3.1 \\
\hline 5. & 4.2 & 4.35 & 5.55 & 6.6 & 7.4 & 6.95 & 3.45 & 3.35 & 3.35 & 3.15 & 2.85 & 3. 05 \\
\hline 6. & 4.7 & 4.4 & 5.6 & 6.75 & 8.25 & 6.85 & 3.35 & 3.3 & 3.3 & 3.25 & .85 & 3. 05 \\
\hline 17. & 4.3 & 4.95 & 6.2 & 6.35 & 10.55 & 6.65 & 3.3 & 3.3 & 3.3 & 3.25 & 2.8 & 3.1 \\
\hline 3. & 4.25 & 4.9 & 6.55 & 6.9 & & & & 3.35 & .35 & 05 & 8 & 3. \\
\hline 9. & 4. 35 & 4.85 & 7.05 & 6.8 & 00 & 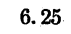 & 3.3 & 3.4 & 3.35 & 3.2 & 2.85 & 3.15 \\
\hline. & 4.4 & 5.8 & 7.3 & 6.35 & & 15 & & 3.25 & 3.35 & 3.1 & .9 & 3.2 \\
\hline 1. & 4.45 & 5.5 & 6.85 & 6.05 & 8.85 & & & 3.25 & 3.35 & 3.05 & 05 & 3.3 \\
\hline 2. & 4.45 & 5.25 & 6.65 & 6. 05 & 8.25 & 5. 75 & 3 & 3. 25 & 3.4 & 3.0 & 2.85 & 3.35 \\
\hline 3.. & 5.0 & 5.15 & 6.3 & 6.25 & & 5.45 & & 3.25 & 3.35 & 2.9 & & 3.3 \\
\hline & 4.85 & 5.1 & 6.55 & 6.7 & 8.65 & 5.35 & 3.3 & 3.25 & 3.4 & 2.95 & 2.95 & 3.2 \\
\hline 5. & 4.6 & 5.25 & 6.5 & 7.25 & & & & 3.3 & 3.3 & 3.1 & .85 & 3.0 \\
\hline & 4.55 & 5.45 & 6.3 & $7: 6$ & 8.95 & 5.1 & 3.25 & 3.35 & 3.35 & 3.0 & 2.85 & 2.95 \\
\hline $7 .$. & 4.45 & 5.55 & 6.9 & 8.3 & 7.35 & & & 3.35 & 3. 35 & 3.2 & 3.2 & 3. 05 \\
\hline 8.. & 4.2 & 5. 35 & 6.45 & 8.3 & 7.1 & 4. 75 & & 3.3 & 3.35 & 3.15 & 3.25 & 3.1 \\
\hline $9 .$. & 4.25 & & 6.85 & 8.4 & 7.45 & 4.65 & 3.15 & 3.25 & 3.35 & 3.0 & 3.3 & 3.1 \\
\hline D... & 4.3 & & 6.4 & 8.15 & 7.45 & 4.4 & 3.15 & 3.3 & 3.45 & 3.05 & 3.15 & 3.1 \\
\hline ... & 4.35 & & 6.2 & 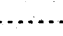 & 7.6 & 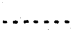 & 3.2 & 3.35 & $\cdots$ & 3.15 & & 3.15 \\
\hline
\end{tabular}

Station rating table for Mokelumne River near Clements, Cal., from January 1 to December 31, 1905.

\begin{tabular}{|r|r||r|r||r|r|r|r|}
\hline $\begin{array}{c}\text { Gage } \\
\text { height: }\end{array}$ & Discharge. & $\begin{array}{c}\text { Gage } \\
\text { height. }\end{array}$ & Discharge. & $\begin{array}{c}\text { Gage } \\
\text { height. }\end{array}$ & Discharge. & $\begin{array}{c}\text { Gage } \\
\text { height. }\end{array}$ & Discharge. \\
\cline { 1 - 5 } Feet. & Second-feet. & Feet. & Second-feet. & Feet. & Second-feet. & Feet. & Second-feet. \\
3.00 & 78 & 4.30 & 391 & 5.60 & 1,059 & 6.90 & 2,000 \\
3.10 & 96 & 4.40 & 427 & 5.70 & 1,124 & 7.00 & 2,080 \\
3.20 & 115 & 4.50 & 465 & 5.80 & 1,191 & 7.20 & 2,240 \\
3.30 & 134 & 4.60 & 506 & 5.90 & 1,260 & 7.40 & 2,400 \\
3.40 & 154 & 4.70 & 550 & 6.00 & 1,330 & 7.60 & 2,560 \\
3.50 & 174 & 4.80 & 597 & 6.10 & 1,400 & 7.80 & 2,730 \\
3.60. & 195 & 4.90 & 647 & 6.20 & $1,47.5$ & 8.00 & 2,900 \\
3.70 & 218 & 5.00 & 700 & 6.30 & 1,550 & 8.20 & 3,070 \\
3.80 & 242 & 5.10 & 755 & 6.40 & 1,625 & 8.40 & 3,250 \\
3.90 & 268 & 5.20 & 812 & 6.50 & 1,700 & 8.60 & 3,430 \\
4.00 & 295 & 5.30 & 871 & 6.60 & 1,775 & 8.80 & 3,610 \\
4.10 & 325 & 5.40 & 932 & 6.70 & 1,850 & 9.00 & 3,800 \\
4.20 & 357 & 5.50 & 995 & 6.80 & 1,925 & & \\
\hline
\end{tabular}

Note.-The above table is based on 12 discharge measurements made during 1905. It is well defined between gage heights 2.9 feet and 6.2 feet. The table has been extended beyond these limits, being based on 1 measurement at 9 feet gage height. 
Estimated monthly discharge of Mokelumne River near Clements, Calif., for 1905.

[Drainage area, 642 square miles.]

\begin{tabular}{|c|c|c|c|c|c|c|}
\hline \multirow[b]{2}{*}{ Month. } & \multicolumn{3}{|c|}{ Discharge in second-feet. } & \multirow{2}{*}{$\begin{array}{l}\text { Total in } \\
\text { acre-feet. }\end{array}$} & \multicolumn{2}{|c|}{ Run-off. } \\
\hline & Maximum. & Minimum. & Mean. & & $\begin{array}{l}\text { Sec.-feet per } \\
\text { sq. mile. }\end{array}$ & $\begin{array}{l}\text { Depth in } \\
\text { inches. }\end{array}$ \\
\hline January .... & 700 & 325 & 428 & 26,320 & .667 & .769 \\
\hline February.. & 2,520 & 409 & 853 & 47,370 & 1.33 & 1. 38 \\
\hline March.. & 2,320 & 932 & 1,410 & 86,700 & 2.20 & 2.54 \\
\hline April ... & 3,250 & 1,365 & 1,959 & 116,600 & 3.05 & 3.40 \\
\hline May ....... & 5,260 & $\dot{1}, 475$ & 2,547 & 156,600 & 3.97 & 4.58 \\
\hline June ..... & 2,688 & 427 & 1,665 & 99,070 & 2.59 & 2.89 \\
\hline July ....... & 391 & 106 & 188 & 11,560 & .293 & .338 \\
\hline August.... & 154 & 60 & 128 & 7,870 & .199 & .229 \\
\hline September. & 164 & 115 & 139 & 8,271 & .217 & .242 \\
\hline October.... & 164 & 60 & 114 & 7,010 & .178 & .205 \\
\hline November. . & 134 & 43 & 794 & 4,725 & .124 & .138 \\
\hline December...... & 144 & 69 & 970 & 5,964 & .151 & .174 \\
\hline The year... & 5,260 & 43 & 801 & $578,101)$ & 1.25 & 16.88 \\
\hline
\end{tabular}

\section{NORTHERN PACIFIC OCEAN DRAINAGE BASIN.}

\section{RUSGIAN RIVER DRAINAGE BASIN.}

\section{MISCELLANEOUS MEASUREMENTS.}

The following is a list of miscellaneous discharge measurements made in Russian River drainage basin during 1905:

Russian River at Calpella, Cal.-A measurement of this stream was made on September 21, 1905, one-fourth mile east of Calpella and about 3 miles above mouth of East Fork of Russian River by R. S. Hawley.

Width, 6 feet; area, 1.6 square feet; mean velocity, 0.75 foot per second; discharge, 1.2 second-feet.

East Fork of Russian River near Ukiah, Cal.-A measurement of this stream was made September 21, 1905, one-fourth mile above its junction with Russian River by R. S. Hawley.

Width, 10 feet; area, 4.6 square feet; mean velocity, 0.48 foot per second; discharge, 2.2 second-feet.

Russian River at Preston, Cal.-A measurement of this stream was made September 22, 1905, 1 mile above Preston, Cal., by R. S. Hawley.

Width, 23 feet; area, 9.8 square feet; mean velocity, 1.05 feet per second; discharge, 10.3 second-feet.

\section{EEL RIVER DRAINAGE BASIN.}

\section{MISCELLANEOUS MEASUREMENTS.}

The following is a list of miscellaneous discharge measurements made in Eel River drainage basin during 1905:

South Eel River at Hearst, Cal.--This stream is tributary to the Eel River from the south. A measurement was made 600 feet below the Hearst bridge on September 19, 1905 , by R. S. Hawley.

Width, 20 feet; area, 7.6 square feet; mean velocity, 1.25 feet per second; discharge, 9.5 second-feet. 
Middle Eel River at Covelo, Cal.-A meàsurement of this stream was made September 19, 1905, at the road crossing between Willets and Covelo about 7 miles above its junction with South Eel by R. S. Hawley.

Width, 34 feet; area, 12.2 square feet; mean velocity, 0.57 foot per second; discharge, 7.0 second-feet.

Middle Eel River at Laytonville, Cal.-A measurement of this stream was made September 20, 1905, 50 feet above its junction with South Eel River by R. S. Hawley.

Width, 18 feet; area, 22 square feet; mean velocity, 0.36 foot per second; discharge, 8.0 second-feet.

Eel River at Laytonville, Cal.-A measurement of this stream was made September 20, 1905, 50 feet below the junction of Middle and South Eel rivers by R. S. Hawley.

Width, 20 feet; area, 16 square feet; mean velocity, 1.69 feet per second; discharge, 27 second-feet.

South Eel River at Laytonville, Cal.-From the difference of the measurement made below the junction of the Middle and South Eel and that made on Middle Eel above on September 20, 1905, the discharge of South Eel at the junction is 19.2 second-feet.

\section{KLAMATH RIVER DRAINAGE BASIN.}

IINK RIVER AT KLAMATH FALLS, OREG, $a$

This station was established May 15, 1904, by J. H. Lewis: It is located at the county bridge at Klamath Falls, Oreg., $1 \frac{1}{2}$ miles below the outlet of Klamath Lake.

The channel is straight for 400 feet above and below the station. The current is swift; the fall in the $1 \frac{1}{4}$ miles between the lake and the gaging station is about 70 feet. The right bank is low and wooded, but not liable to overflow. The left bank is high and rocky. The bed of the stream is composed of gravel, free from vegetation, and subject to some change. There is but one channel at all stages. One irrigation ditch takes water from the river on the left bank about three-fourths mile above the station. Driftwood from a sawmill above the station collects around the bridge piers and interferes materially with the accuracy of measurements. A short distance below the gaging station the river discharges into a lake of considerable size. At flood stages the velocity is checked by water backing up from the lake below, which has a tendency to give decreased velocities with high gage readings. Gage heights may be affected by wind piling up the water of the upper and lower lakes.

Discharge measurements are made from the upstream side of the bridge. The initial point for soundings is the first vertical rod supporting the floor beam at the left end of the bridge, upstream side.

A staff gage is fastened vertically to the plank bracing between steel caissons near the left bank. During 1905 the gage was read once each day by G. H. Woodbury. Bench marks were established as follows: (1) A 30-penny nail driven into the floor beam over the gage; elevation, 12.50 feet. (2) The center of a cross cut in the first sandstone above the ground in the southwest corner of the foundation of the Linkville Hotel; elevation, 15.88 feet. Elevations refer to the datum of the gage.

Gage heights and discharge measurements taken at this station in 1904 are contained in Water-Supply Paper No. 134, United States Geological Survey.

$a$ This station was known as Klamath River at Klamath Falls, Oreg., in report for 1904. 
Discharge measurements of Link River at Klamath Falls, Oreg., in 1905.

\begin{tabular}{|c|c|c|c|c|c|c|}
\hline Date. & Hydrographer. & Width. & $\begin{array}{l}\text { Area of } \\
\text { section. }\end{array}$ & $\underset{\text { velocity. }}{\text { Mean }}$ & $\begin{array}{c}\text { Gage } \\
\text { height. }\end{array}$ & $\begin{array}{l}\text { Dis- } \\
\text { charge. }\end{array}$ \\
\hline January $4 . . .$. & C. T. Darley ......................... & $\begin{array}{r}\text { Feet. } \\
282\end{array}$ & $\begin{array}{r}S q \cdot f t . \\
1,571\end{array}$ & $\begin{array}{r}\text { Ft.per sec. } \\
1.82\end{array}$ & $\begin{array}{l}\text { Feet. } \\
\quad 4.05\end{array}$ & $\begin{array}{r}\text { Sec.-ft. } \\
2,862\end{array}$ \\
\hline January 17 & .....do. & 282 & 1,570 & 1.76 & 4.10 & 2,768 \\
\hline January 17 & .....do......... & 282 & 1,571 & 1.83 & 4. 10 . & 2,872 \\
\hline January 17 . . & ....do... & 282 & 1,571 & 1.67 & 4.10 & 2,627 \\
\hline February $1 \ldots$ & .....do... & 287 & 1,660 & 2.01 & 4.30 & 3,338 \\
\hline February 13. & ......do.. & 288 & 1,683 & $1: 78$ & 4.50 & 2,994 \\
\hline February 17 . & ......do... & 288 & 1,648 & 1.70 & 4.40 & 2,802 \\
\hline March $2 \ldots$ & .....do.... & 288 & 1,642 & 1.82 & 4.40 & 2,984 \\
\hline April $12 \ldots$ & .....do.... & 290 & 1,733 & 2.10 & 4. 49 & 3,645 \\
\hline June $5 . . .$. & .....do.... & 279 & 1,494 & 1.56 & 3.88 & 2,324 \\
\hline June $20 \ldots$ & ......do. & 277 & 1,410 & 1.28 & 3.59 & 1,800 \\
\hline June $28 \ldots$ & .....do... & 283 & 1,354 & 1.12 & 3.40 & 1,517 \\
\hline July $11 \ldots$ & .....do.. & 297 & 1,280 & 1.15 & 3.14 & 1,466 \\
\hline July $18 \ldots$ & .....do.. & 272 & 1,234 & .86 & 2.97 & 1,063 \\
\hline July $28 \ldots$ & .....do.. & 270 & 1,178 & .89 & 2.80 & 1,049 \\
\hline August $7 \ldots$... & .....do... & 267 & 1,162 & .90 & 2.63 & 1,041 \\
\hline August $14 \ldots$ & $\ldots$. do.................. & 267 & 1,146 & .93 & 2.59 & 1,066 \\
\hline August $16 \ldots .$. & W. B. Clapp and C. T. Darley.. & 267 & 1,187 & .93 & 2.60 & 1,104 \\
\hline September $7 .$. & C. T. Darley ............. & 267 & 1,141 & .90 & 2.58 & 1,032 \\
\hline September $16 .$. & .....do... & 268 & 1,155 & .93 & 2.58 & 1,073 \\
\hline November 2 ... & .....do... & 272 & 1,254 & 1.02 & 2.90 & 1,283 \\
\hline
\end{tabular}


Daily gage height, in feet, of Link River at Klamath Falls, Oreg., for 1905.

\begin{tabular}{|c|c|c|c|c|c|c|c|c|c|c|c|c|}
\hline Day. & Jan. & Feb. & Mar. & Apr. & May. & June.: & July. & Aug. & Sept. & Oct. & Nov. & Dec. \\
\hline $1 .$. & 4.1 . & 4.2 & 4.4 & 4.5 & 4.2 & 3.95 & 3. 45 & 2.79 & 2.6 & 2.7 & 2.81 & 3.25 \\
\hline 2. & 4.1 & 4.4 & 4.4 & 4.5 & 4.2 & 3.85 & 3. 45 & 2.75 & 2.6 & 2.65 & 2.8 & 3.3 \\
\hline 3. & 4.1 & 4.4 & 4.4 & 4.5 & 4.2 & 3.85 & 3.3 & 2.7 & 2.58 & 2.7 & 2,81 & 3. 29 \\
\hline 4. & 4.1 & 4.4 & 4.4 & 4.5 & 4.2 & 3.9 & $a 3.3$ & 2.67 & 2.6 & 2.7 & 2.84 & 3.2 \\
\hline 5 & 4.1 & 4.5 & 4.4 & 4.5 & 4.2 & 3.88 & 3.25 & 2.67 & 2.58 & 2.9 & 2.82 & 3.2 \\
\hline 6.. & 4.1 & 4.5 & 4.4 & 4.5 & 4.2 & 3.88 & 3.3 & 2.67 & 2.6 & 2.9 & 2.88 & 3.2 \\
\hline $7 .$. & 4.1 & 4.4 & 4.4 & 4.5 & 4.2 & 3.81 & 3.2 & 2.5 & 2.58 & 2.95 & 2.86 & 3.21 \\
\hline 8.. & 4.1 & 4.4 & 4.4 & 4.5 & 4.2 & 3.88 & 3.2 & 2.63 & 2.58 & 2.7 & 2.99 & 3.23 \\
\hline 9.. & 4.1 & 4.4 & 4.4 & 4.5 & 4.2 & 3.85 & $a 3.25$ & 2.6 & 2.58 & 2.9 & 2.99 & 3.24 \\
\hline $10 .$. & 4.1 & 4.5 & 4.4 & 4.5 & 4.2 & 3.8 & 3.25 & 2.6 & 2.62 & 2.8 & 2.98 & 3.28 \\
\hline 11. & 4.1 & 4.5 & 4.4 & 4.5 & 4.2 & 3.8 & a 3.25 & 2.59 & 2.63 & 2.75 & 2.99 & 3.25 \\
\hline 12. & 4.1 & 4.5 & 4.4 & 4.5 & 4.2 & 3.78 & $a 3.2$ & 2.6 & 2.58 & 2.8 & 2.9 & 3.26 \\
\hline 13.. & 4.1 & 4.5 & 4.4 & 4.5 & 4.2 & 3.75 & 3.2 & 2.6 & 2.58 & 2.8 & 2.95 & 3.27 \\
\hline $14 .$. & 4.1 & 4.5 & 4.4 & 4.5 & 4.2 & 3.7 & 3.1 & 2.59 & 2.6 & 2.8 & 2.95 & 3.24 \\
\hline 15.. & 4.1 & 4.5 & 4.4 & 4.5 & 4.1 & 3.6 & 3.05 & 2.6 & 2.6 & 2.7 & 2.95 & 3. 34 \\
\hline 16.. & 4.1 & :4.5 & 4.4 & 4.5 & 4.1 & 3.7 & $a 3.0$ & 2.6 & 2. 55 . & 2.8 & 2.9 & 3.26 \\
\hline 17. & 4.1 & 4.4 & 4.4 & 4.5 & 4.1 & 3.62 & 2.9 & 2.60 & 2.5 & 2.8 & 2.9 & 3.33 \\
\hline 18. & 4.1 & 4.4 & 4. 4 & 4.45 & 4.05 & a3.61 & 2.9 & 2.63 & 2.55 & 2.8 & 2.9 & 3. 32 \\
\hline 19. & 4.1 & 4.4 & 4.4 & 4.4 & 4.05 & 3.6 & 3.0 & 2.6 & 2.6 & 2.8 & 3.2 & 3: 32 \\
\hline 20. & 4.1 & 4.4 & 4.4 & 4.4 & 4.05 & 3.59 & 2.9 & 2.6 & 2.6 & 2.85 & 3.1 & 3.26 \\
\hline 21.. & 4.1 & 4.4 & 4.4 & 4.35 & 4.0 & $a 3.55$ & 2.9 & 2.6 & 2.6 & 2.85 & 3.3 & 3.27 \\
\hline 22. & 4.1 & 4.4 & 4.4 & 4. 35 & 4.0 & 3.5 & 2.95 & 2.6 & 2.58 & 2.79 & 3.2 & 3.33 \\
\hline 23.. & 4.1 & 4.4 & 4.4 & 4. 35 & 4.0 & 3.5 & 2.85 & 2.6 & 2.6 & 2.8 & 3.15 & 3.3 \\
\hline 24.. & 4.2 & .4 .4 & 4.4 & 4.3 & 4.0 & 3.5 & 2.85 & 2.6 & 2.68 & 2.85 & 3.1 & 3.32 \\
\hline $25 .$. & 4.2 & 4.4 & 4.4 & 4.3 & 4.0 & 3.4 & $a 2.85$ & 2.6 & 2.55 & .2 .81 & 3.2 & 3.29 \\
\hline $26 .$. & 4,2 & 4.4 & 4.4 & 4.3 & 4.0 & 3.4 & $a 2.85$ & 2.7 & 2.58 & 2.8 & 3.25 & 3.35 \\
\hline $27 \ldots$ & 4.2 & 4.4 & 4.4 & 4.3 & 4.0 & 3.4 & $a 2.8$ & 2.8 & 2.6 & 2.89 & 3.15 & 3.36 \\
\hline $28 .$. & 4.1 & 4.4 & 4.5 & 4.25 & 4.0 & 3.4 & $a 2.8$ & 2.6 & 2.62 & 2.88 & 3.15 & 3.33 \\
\hline $29 .$. & 4.2 & & 4.6 & 4.25 & 4.0 & 3.4 & 2.78 & 2.6 & 2.65 & 2.88 & 3.4 & 3.4 \\
\hline $30 \ldots \ldots$ & 4.2 & & 4.5 & 4.25 & 3.95 & 3.35 & 2.79 & 2.65 & 2.65 & 2.81 & 3.2 & 3.53 \\
\hline $31 . . . . . .$. & 4.2 & & 4.5 & & 3.95 & $\ldots$ & 2.78 & 2.55 & ........ & 2.9 & $\ldots$ & 3.53 \\
\hline
\end{tabular}

$a$ Estimated.

Station rating table for Link River at Klamath Falls, Oreg., from May 15, 1904, to December 31, 1905 .

\begin{tabular}{|r|r||r|r|r|r|r|r|}
\hline $\begin{array}{c}\text { Gage } \\
\text { height. }\end{array}$ & Discharge. & $\begin{array}{c}\text { Gage } \\
\text { height. }\end{array}$ & Discharge. & $\begin{array}{c}\text { Gage } \\
\text { height. }\end{array}$ & Discharge. & $\begin{array}{c}\text { Gage } \\
\text { height. }\end{array}$ & Discharge. \\
\hline Fieet. & Second-feet. & Freet. & Second-feet. & Feet. & Second-feet. & Feet. & Second-feet. \\
2.50 & 985 & 3.60 & 1,920 & 4.70 & 3,580 & 5.80 & 5,790 \\
2.60 & 1,035 & 3.70 & 2,045 & 4.80 & 3,770 & 5.90 & 6,020 \\
2.70 & 1,090 & 3.80 & 2,175 & 4.90 & 3,960 & 6.00 & 6,250 \\
2.80 & 1,150 & 3.90 & 2,310 & 5.00 & 4,150 & 6.20 & 6,710 \\
2.90 & 1,220 & 4.00 & 2,450 & 5.10 & 4,340 & 6.40 & 7,180 \\
3.00 & 1,300 & 4.10 & 2,590 & 5.20 & 4,530 & 6.60 & 7,660 \\
3.10 & 1,390 & 4.20 & 2,740 & 5.30 & 4,730 & 6.80 & 8,140 \\
3.20 & 1,485 & 4.30 & 2,900 & 5.40 & 4,930 & 7.00 & 8,640 \\
3.30 & 1,585 & 4.40 & 3,060 & 5.50 & 5,140 & 7.20 & 9,140 \\
3.40 & 1,690 & 4.50 & 3,230 & 5.60 & 5,350 & & \\
3.50 & 1,800 & 4.60 & 3,400 & 5.70 & 5,570 & & \\
& & & & & & & \\
\hline
\end{tabular}

NoTE. - The above table is applicable only for open-channel conditions. It is based on twenty-eight discharge measurements made during 1904-5. It is not very well defined. 
Estimated monthly discharge of Link River at Klamath Falls, Oreg., for 1904 and 1905.

\begin{tabular}{|c|c|c|c|c|}
\hline \multirow{2}{*}{ Month. } & \multicolumn{3}{|c|}{ Discharge in second-feet. } & \multirow{2}{*}{$\begin{array}{l}\text { Total in } \\
\text { acre-feet. }\end{array}$} \\
\hline & Maximum. & Minimum. & Mean. & \\
\hline 1904. & & & & \\
\hline May $15-31 \ldots \ldots \ldots \ldots \ldots$ & 9,015 & 8,140 & 8,640 & 291,300 \\
\hline June.............. & 8,140 & 5,245 & 6,740 & 401,100 \\
\hline July ................ & 5,140 & 3,060 & 4,123 & 253,500 \\
\hline August ............ & 2,980 & 1,860 & 2,336 & 143,600 \\
\hline September ............. & 1,800 & 1,535 & 1,662 & 98,900 \\
\hline October.............. & 1,745 & 1,585 & 1,686 & 103,700 \\
\hline November ............ & 1,982 & 1,745 & 1,837 & 109,300 \\
\hline December........... & 2,520 & 1,860 & 2,185 & 134,400 \\
\hline The period... & $\cdots$ & & & $1,536,000$ \\
\hline 1905. ‘ & & & & \\
\hline January ............ & 2,740 & 2,590 & 2,624 & 161,300 \\
\hline February...$\ldots \ldots \ldots \ldots$ & 3,230 & 2,740 & 3,103 & 172,300 \\
\hline March ............. & 3,400 & 3,060 & 3,087 & 189,800 \\
\hline 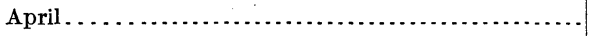 & 3,230 & 2,820 & 3,106 & 184,800 \\
\hline May .................... & 2,740 & 2,380 & 2,597 & 159,700 \\
\hline June................. & 2,380 & 1,638 & 2,004 & 119,200 \\
\hline July .................. & 1,745 & 1,138 & 1,363 & 83,810 \\
\hline August ............... & 1,144 & 985 & 1,051 & 64,620 \\
\hline September............ & 1,062 & 985 & 1,033 & 61,290 \\
\hline October................ & 1,260 & 1,062 & 1,159 & 71,260 \\
\hline November............. & 1,690 & 1,150 & 1,330 & 79,140 \\
\hline December.............. & 1,860 & 1,485 & 1,587 & 100,800 \\
\hline The year... & 3,400 & 985 & 2,004 & $1,448,000$ \\
\hline
\end{tabular}

KLAMATH RIVER AT KENO, OREG.

This station was established August 13, 1904, by T. H. Humphreys. It is located onefourth mile below the county bridge at Keno, Oreg.

The channel is straight for one-fourth mile above and below the station. The current is sluggish at low and moderate at high stages. Both banks are low, clean, and liable to overflow. The bed of the stream is composed of bowlders. An up or down stream wind piles up the water near the gage on account of the low velocities.

Discharge measurements are made by means of a cable and car one-fourth mile downstream from the bridge. The initial point for soundings is at the cable support on the right bank.

A staff gage is fastened vertically to the downstream side of the second bent from the left end of the bridge. During 1905 the gage was read once each day by S. Padgett. Bench marks were established as follows: (1) The top of projecting cap of bridge bent to which the gage is nailed; elevation, 16.80 feet. (2) A 60-penny nail driven into the south gatepost on the west side of the road 58 feet from the south end of the bridge; elevation, 18.85 feet. Elevations refer to the datum of the gage.

Gage heights and discharge measurements taken at this station in 1904 are contained in Water-Supply Paper .No. 134, United States Geological Survey. 
Discharge measurements of Klamath River at Keno, Oreg., in 1905.

\begin{tabular}{|c|c|c|c|c|c|c|}
\hline Date. & Hydrographer. & Width. & $\begin{array}{l}\text { Area of } \\
\text { section. }\end{array}$ & $\begin{array}{c}\text { Mean } \\
\text { velocity. }\end{array}$ & $\begin{array}{c}\text { Gage } \\
\text { height. }\end{array}$ & $\begin{array}{l}\text { Dis- } \\
\text { charge. }\end{array}$ \\
\hline January $10 \ldots$. & C. T. Darley... & $\begin{array}{r}\text { Feet. } \\
407\end{array}$ & $\begin{array}{r}S q . f t . \\
3,800\end{array}$ & $\begin{array}{r}\text { Ft. per sec. } \\
0.68\end{array}$ & $\begin{array}{l}\text { Feet. } \\
13.00\end{array}$ & $\begin{array}{r}\text { Sec.-ft. } \\
2,590\end{array}$ \\
\hline January 23. & .....do. & 420 & 3,794 & .77 & 13.30 & 2,930 \\
\hline Januiary 30 . . & .....do. & 420 & 3,804 & .76 & 13.20 & 2,875 \\
\hline February $9 . .$. & .....do. & 425 & 3,700 & .80 & 13.25 & 2,975 \\
\hline February $18 \ldots$ & .....do.. & 428 & 3,838 & .83 & 13.30 & 3,192 \\
\hline March 3...... & .....do... & 432 & 3,850 & .82 & 13.30 & 3,145 \\
\hline March 31 & .....do... & 425 & 3,828 & .83 & 13. 32 & 3,159 \\
\hline April 13... & .....do... & 425 & 3,760 & .80 & 13.28 & 3,015 \\
\hline May 17. & ...do. & 427 & 3,687 & .73 & 13.03 & 2,676 \\
\hline June $19 \ldots$ & ...do. & 410 & 3,545 & .59 & 12.68 & 2,102 \\
\hline July $14 \ldots$ & ....do.. & 407 & 3,348 & .43 & 12.20 & 1,445 \\
\hline August $14 \ldots$ & .....do.. & 402 & 3,129 & .37 & 11.80 & 1,146 \\
\hline August $31 . .$. . & .....do.. & 404 & 3,221 & .29 & 11.75 & 924 \\
\hline November $3 . .$. & .....do... & 409 & 3,325 & .20 & 12.00 & 680 \\
\hline December 3.... & .....do................. & 410 & 3,390 & .25 & 12.28 & 851 \\
\hline
\end{tabular}

Daily gage height, in feet, of Klamath River at.Keno, Oreg., for 1905.

\begin{tabular}{|c|c|c|c|c|c|c|c|c|c|c|c|c|}
\hline Day. & Jan. & Feb. & Mar. & Apr. & May. & June. & July. & Aug. & Sept. & Oct. & Nov. & Dec. \\
\hline $1 .$. & 12.9 & 13.2 & 13.3 & 13.4 & 13.2 & 13.0 & 12.5 & 11.9 & 11.8 & 11.9 & 12.1 & 12.3 \\
\hline $2 \ldots$ & 12.9 & 13.2 & 13.3 & 13.4 & 13.2 & 13.0 & 12.4 & 11.9 & 11.8 & 11.9 & 12.1 & 12.3 \\
\hline $3 .$. & 13.0 & 13.3 & 13.3 & 13.4 & 13.2 & 13.0 & 12.4 & 11.9 & 11.8 & 11.9 & 12.1 & 12.3 \\
\hline $4 .$. & 13.0 & 13.3 & 13.3 & 13.4 & 13.2 & 13.0 & 12.4 & 11.9 & 11.8 & 11.9 & 12.1 & 12.3 \\
\hline & 13.0 & 13.3 & 13.3 & 13.4 & 13.2 & 13.0 & 12.4 & 11.9 & 11.8 & 11.9 & 12.1 & 12.3 \\
\hline $6 .$. & 13.0 & 13.3 & 13.3 & 13.4 & 13.2 & 12.9 & 12.3 & 11.8 & 11.8 & 11.9 & 12.1 & 12.3 \\
\hline 7.. & 13.0 & 13.3 & 13.3 & 13.4 & 13.2 & 12.9 & 12.3 & 11.8 & 11.8 & 11.9 & 12.1 & 12.3 \\
\hline 8... & 13.1 & 13.3 & 13.3 & 13.4 & 13.1 & 12.9 & 12.2 & 11.8 & 11.8 & 11.9 & 12.1 & 12.4 \\
\hline $9 \ldots$ & 13.1 & 13.3 & 13.3 & 13.4 & 13.1 & 12.9 & 12.2 & 11.8 & 11.8 & 11.9 & 12.1 & 12.4 \\
\hline ... & 13.1 & 13.3 & 13.3 & 13.4 & 13.1 & 12.9 & 12.2 & 11.8 & 11.8 & 11.9 & 12.1 & 12.4 \\
\hline$\cdots$ & 13.1 & 13.3 & 13.3 & 13.4 & 13.1 & 12.9 & 12.2 & 11.8 & 11.8 & 11.9 & 12.1 & 12.4 \\
\hline .. & 13.1 & 13.3 & 13.3 & 13.3 & 13.1 & 12.8 & 12.2 & 11.8 & 11.8 & 11.9 & 12.1 & 12.4 \\
\hline 3.. & 13.1 & 13.3 & 13.2 & 13.3 & 13.1 & 12.8 & 12.2 & 11.8 & 11.8 & 11.9 & 12.1 & 12.4 \\
\hline 4.. & 13.1 & 13.3 & 13.2 & 13.3 & 13.1 & 12.8 & 12.2 & 11: 8 & 11.8 & 11.9 & 2.1 & 12.4 \\
\hline ... & 13.1 & 13.3 & 13.2 & 13.3 & 13.1 & 12.7 & 12.2 & 11.8 & 11.8 & 11.9 & 12.1 & 12.4 \\
\hline $6 .$. & 13.1 & 13.3 & 13.2 & 13.3 & 13.1 & 12.7 & 12.2 & 11.8 & 11.8 & 11.9 & 12.2 & 12.4 \\
\hline $17 .$. & 13.1 & 13.3 & 13.2 & 13.3 & 13.0 & 12.7 & 12.2 & 11.8 & 11.8 & 12.0 & 12.2 & 12.4 \\
\hline $18 .$. & 13.1 & 13.3 & 13.2 & 13.3 & 13.0 & 12.7 & 12.2 & 11.8 & 11.8 & 12.0 & 12.2 & 12.4 \\
\hline $19 .$. & 13.1 & 13.3 & 13.2 & 13.2 & 13.0 & 12.6 & 12.1 & 11.8 & 11.8 & 12.0 & 12.2 & 12.4 \\
\hline 0. & 13.1 & 13.3 & 132 & 132 & 130 & 126 & 121 & 118 & 11.8 & 12.0 & 12.2 & 124 \\
\hline 1. & 131 & 133 & 132 & 132 & 130 & 6 & 121 & 118 & 118 & 12.0 & 12.3 & 12.4 \\
\hline 22. & 13.1 & 13.3 & 13.2 & 13.2 & 13.0 & 12.5 & 12.1 & 11.8 & 11.8 & 12.0 & 12.3 & 12.4 \\
\hline 23. & 13.3 & 13.3 & 13.2 & 13.2 & 13.0 & 12.5 & 12.1 & 11.8 & 11.8 & 12.0 & 12.3 & 12.4 \\
\hline 1. & 13.1 & 13.3 & 13.2 & 13.2 & 13.0 & 12.5 & 12.1 & 11.8 & 11.8 & .12 .0 & 12.3 & 12.4 \\
\hline $25 .$. & 13.1 & 13.3 & 13.3 & 13.2 & 13.0 & 12.5 & 12.0 & 11.8 & 11.8 & 12.0 & 12.3 & 12.4 \\
\hline 26 . & 13.1 & 13.3 & 13.3 & 13.2 & 13.0 & 12.5 & 12.0 & 11.8 & 11.8 & 12.0 & 12.3 & 12.4 \\
\hline 27. & 13.1 & 13.3 & 13.3 & 13.2 & 13.0 & 12.5 & 12.0 & 11.8 & 11.8 & 12.0 & 12.3 & 12.5 \\
\hline $28 .$. & 13.2 & 13.3 & 13.4 & 13.2 & 13.0 & 12.5 & 12.0 & 11.8 & 11.8 & 12.1 & 12.3 & 12.5 \\
\hline 9. & 13.2 & & 13.4 & 13.2 & 13.0 & 12.5 & 12.0 & 11.8 & 11.9 & 12.1 & 12.3 & 12.5 \\
\hline & 13.2 & & 13.4 & 13.2 & 13.0 & 12.5 & 12.0 & 11.8 & 11.9 & 12.1 & 12.3 & 12.5 \\
\hline 31.. & 13.2 & & 13.4 & & 13.0 & & 11.9 & 11.8 & & 12.1 & & 12.5 \\
\hline
\end{tabular}


Station rating table for Klamath River at Keno, Oreg., from May 31, 1904, to December 31, 1905.

\begin{tabular}{|c|c|c|c|c|c||c|c|}
\hline $\begin{array}{c}\text { Gage } \\
\text { height. }\end{array}$ & Discharge. & $\begin{array}{c}\text { Gage } \\
\text { height. }\end{array}$ & Discharge. & $\begin{array}{c}\text { Gage } \\
\text { height. }\end{array}$ & Discharge. & $\begin{array}{c}\text { Gage } \\
\text { height. }\end{array}$ & Discharge. \\
\cline { 1 - 5 } Feet. & Second-ject. & Feet. & Second-feet. & Feet. & Second-feet. & Feet. & Second-fect. \\
11.60 & 900 & 12.60 & 1,960 & 13.60 & 3,730 & 14.60 & 6,590 \\
11.70 & 970 & 12.70 & 2,100 & 13.70 & 3,960 & 14.70 & 6,940 \\
11.80 & 1,050 & 12.80 & 2,250 & 13.80 & 4,200 & 14.80 & 7,300 \\
11.90 & 1,140 & 12.90 & 2,400 & 13.90 & 4,460 & 14.90 & 7,670 \\
12.00 & 1,240 & 13.00 & 2,560 & 14.00 & 4,730 & 15.00 & 8,050 \\
12.10 & 1,350 & 13.10 & 2,730 & 14.10 & 5,010 & 15.10 & 8,440 \\
12.20 & 1,460 & 13.20 & 2,910 & 14.20 & 5,300 & 15.20 & 8,840 \\
12.30 & 1,580 & 13.50 & 3,100 & 14.30 & 5,600 & 15.30 & 9,250 \\
12.40 & 1,700 & 13.40 & 3,300 & 14.40 & 5,920 & & \\
12.50 & 1,830 & 13.50 & 3,510 & 14.50 & 6,250 & & \\
& & & & & & & \\
\hline
\end{tabular}

NotE.-The above table is applicable only for open-channel conditions. It is based on 22 discharge measurements made during 1904-5. It is well defined between gage heigits 12.2 feet and 13.3 feet.

Estimated monthly discharge of Klamath River at Keno, Oreg., for 1904 and 1905.

\begin{tabular}{|c|c|c|c|c|}
\hline \multirow{2}{*}{ Month. } & \multicolumn{3}{|c|}{ Discharge in second-feet. } & \multirow{2}{*}{$\begin{array}{l}\text { Total in } \\
\text { acre-feet. }\end{array}$} \\
\hline & Maximum. & Minimum. & Mean. & \\
\hline 1904. & & & & \\
\hline June................. & 8,440 & 5,300 & 7,076 & $421 ; 100$ \\
\hline July.................. & 5,300 & 3,300 & 4,177 & 256,800 \\
\hline August............... & 3,100 & 2,100 & 2,513 & 154,500 \\
\hline September............... & 2,100 & 1,700 & 1,778 & 105,800 \\
\hline October................ & 1,830 & 1,700 & 1,775 & 109,100 \\
\hline November............. & 2,100 & 1,960 & 2,007 & 119,400 \\
\hline December............. & 2,400 & 2,100 & 2,235 & 137,400 \\
\hline The period... & & & & $1,304,000$ \\
\hline 1905. & & & & \\
\hline January ....... & 3,100 & 2,400 & 2,716 & 167,000 \\
\hline February ......... & 3,100 & 2,910 & 3,086 & 171,400 \\
\hline March.............. & 3,300 & 2,910 & 3,052 & 187,700 \\
\hline April........... & 3,300 & 2,910 & 3,097 & 184,200 \\
\hline May................ & 2,910 & 2,560 & 2,688 & 165,300 \\
\hline June........... & 2,560 & 1,830 & 2,157 & 128,400 \\
\hline July ... ............. & 1,830 & 1,140 & 1,436 & 88,300 \\
\hline August..... . . & 1,140 & 1,050 & 1,065 & 65,480 \\
\hline September............. & 1,140 & 1,050 & 1,056 & 62,840 \\
\hline October.............. & 1,350 & 1,140 & $1 ; 203$ & 73,970 \\
\hline November........... & 1,580 & 1,350 & 1,445 & 85,980 \\
\hline December...................... & 1,830 & 1,580 & 1,694 & 104,200 \\
\hline The year : & 3,300 & 1,050 & 2,058 & $1,485,000$ \\
\hline
\end{tabular}


SYOAN RIVER NEAR SHVERLAKE, OREG.

This station was established May 2, 1905, by Ivan Landes. It is located about 30 miles south of Silverlake, Oreg., in sec. 19, T. 32 S., R. 14 E.

The channel is straight for about 20 feet above and 150 feet below the station. The current is swift. The right bank is high, rocky, and clean and does not overflow. The left bank is low, clean, and subject to overflow at extreme high stages. The bed of the stream is of rocks, gravel, and sand, free from vegetation, and permanent. There is but one channel at low and two channels at high stages.

Discharge measurements are made from a private bridge. The initial point for soundings is at the west end of the bridge.

A staff gage is fastened vertically to a bent of the bridge. During 1905 the gage was read by 0 . F. Griffith and J. S. Wakefield. The bench mark is a marked point of rock on the right bank a short distance upstream from the bridge; elevation, 6.79 feet above the datum of the gage.

Discharge measurements of Sycan River near Silverlake, Oreg., in 1905.

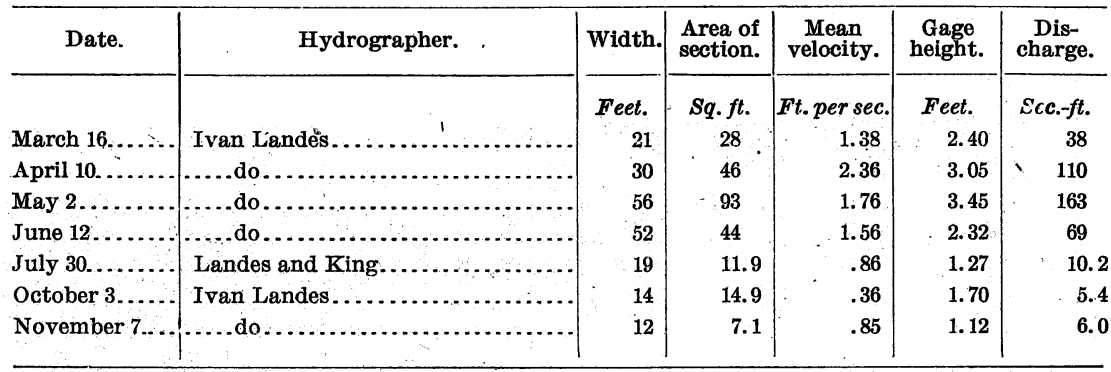

Daily gage height, in feet, of Sycan River, near Silverlake, Oreg., for 1905.

\begin{tabular}{|c|c|c|c|c|c|c|c|c|c|c|c|c|c|}
\hline Day. & May. & June. & July. & Aug. & Sept. & Oct. & Day. & May. & June. & July. & Aug. & Sept. & Oct. \\
\hline 1. & $\cdots$ & 2.6 & 1.8 & 1.25 & 1.4 & & 17. & 3.5 & 2.15 & 1.45 & 1.0 & 1.75 & 1.8 \\
\hline 2. & 3.45 & 2.6 & 1.8 & 1.25 & 1.4 & 1.7 & 18. & 3.4 & 2.05 & 1.45 & 1.05 & 1.8 & 1.8 \\
\hline 3. & 3.35 & 2.6 & 1.75 & 1.1 & 1.5 & 1.85 & 19. & 3.3 & 2.2 & $a 1.3$ & 1.05 & 1.8 & 1.7 \\
\hline 4. & 3.25 & 3.0 & 1.75 & 1.15 & 1.5 & 1.7 & 20. & 3.4 & 2.1 & 1.35 & 1.1 & 1.75 & 1.85 \\
\hline 5. & 3.2 & 3.1 & 1.7 & .8 & 1.55 & 1.85 & 21. & 2.9 & 2.0 & 1.4 & 1.1 & 1.75 & 1.7 \\
\hline $6 \therefore$ & 3.2 & 3.1 & 1.7 & .8 & 1.55 & 1.8 & 22. & 3.0 & 1.8 & 1.4 & 1.15 & 1.75 & 1.7 \\
\hline $7 .$. & 3.25 & 3.2 & 1.7 & $: 8$ & 1.55 & 2.0 & 23. & 3.1 & 1.9 & 1.4 & 1.15 & 1.75 & 1.85 \\
\hline 8. & 3.6 & 3.0 & 1.6 & .8 & 1.55 & 1.9 & 24 & 3.0 & 1.9 & 1.4 & 1.2 & 1.75 & 1.85 \\
\hline 9 & 3.4 & 3.0. & 1.6 & .8 & 1.6 & 1.8 & 25. & 2.9 & 1.9 & 1.3 & 1.25 & 1.75 & 1.85 \\
\hline 10. & 3.35 & $a 2.5$ & 1.5 & .8 & 1.65 & 1.7 & 26. & 3.2 & 1.95 & 1.25 & 1.25 & 1.75 & 1.85 \\
\hline 11. & 3.35 & 2.4 & 1.5 & .8 & 1.7 & 1.7 & 27. & 3.55 & 1.9 & 1.25 & 1.3 & 2.0 & 1.85 \\
\hline 12. & 3.3 & 2.3 & 1.5 & .8 & 1.7 & 1.8 & 28. & 3.1 & 1.9 & 1.25 & 1.3 & 1.9 & 1.7 \\
\hline 13. & 3.4 & 2.4 & 1.5 & .8 & 1.75 & 1.7 & 29. & 2.9 & 1.85 & 1.25 & 1.35 & 1.8 & $\cdots$ \\
\hline 14. & 3.25 & 2.6 & 1.5 & 1.0 & 1.75 & 1.7 & 30. & 2.8 & 1.85 & 1.25 & 1.35 & 1.8 & \\
\hline & 3.3 & 2.4 & 1.5 & 1.0 & 1.75 & 1.65 & $31 \ldots$ & 2.7 & & 1.25 & 1.4 & 1.8 & $\ldots$. \\
\hline $16 \ldots$ & 3.35 & 2.2 & 1.5 & 1.0 & 1.75 & 1.8 & & & & & & & \\
\hline
\end{tabular}

- Water turned into ditch. 


\section{LOST RIVER NEAR CLEAR LAKE, CAL.}

This station was established September 1,1904 , by T. H. Humphreys. It is located about 2 miles downstream from Jessie D. Carr's Clear Lake dam, a short distance below the dam site for Clear Lake reservoir, about 20 miles from Tule Lake post-office, Cal.

The channel is straight for about 150 feet above and below the station. The current is swift. Both banks are high, rocky, and clean. There is a flood plain about 150 feet in width along the left bank. The bed of the stream is composed of rock, gravel, and soil. The channel contains a considerable growth of tules during the spring and summer months.

Discharge measurements are made during flood stages by means of a cable and car, and at low stages by wading. The initial point for soundings is the left-bank end of the cable.

A gage is painted on the vertical rock cliff on the right bank. November 4, 1905, an automatic water-height register was installed for recording gage heights. The bench mark is a mark on the rock to which the left end of the cable is anchored; elevation, 16.62 feet above the datum of the gage.

Gage heights and discharge measurements taken at this station in 1904 are contained in Water-Supply Paper No. 134, United States Geological Survey.

Discharge measurements of Lost River near Clear Lake, Cal., in 1905.

\begin{tabular}{|c|c|c|c|c|c|c|}
\hline Date. & Hydrographer. & Width. & $\begin{array}{l}\text { Area of } \\
\text { section. }\end{array}$ & $\begin{array}{c}\text { Mean } \\
\text { velocity. }\end{array}$ & $\begin{array}{c}\text { Gage } \\
\text { height. }\end{array}$ & $\begin{array}{l}\text { Dis- } \\
\text { charge. }\end{array}$ \\
\hline 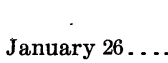 & C. T. Darley.. & $\begin{array}{c}\text { Feet. } \\
102\end{array}$ & $\begin{array}{c}S q . f t . \\
92\end{array}$ & $\begin{array}{r}\text { Ft.per sec. } \\
1.75\end{array}$ & $\begin{array}{l}\text { Feet. } \\
\quad 6.30\end{array}$ & $\begin{array}{c}\text { Sec.-ft. } \\
161\end{array}$ \\
\hline February 3.. & .....do............ & 130 & 196 & 2.31 & 7.20 & 453 \\
\hline February 7 & .....do... & 100 & 104 & 2.08 & 6.39 & 216 \\
\hline February 24 & .....do... & 98 & $70^{\circ}$ & 1.77 & 6.01 & 124 \\
\hline March $7 \ldots$ & .....do... & 102 & 102 & 1.92 & 6.35 & 196 \\
\hline March $13 \ldots$ & .....do... & 110 & 115 & 2.19 & 6.50 & 252 \\
\hline April 3... & .....do... & 130 & 203 & 2.64 & 7.30 & 535 \\
\hline April 8... & .....do... & 92 & 85 & 2.05 & 6.24 & 174 \\
\hline$\Lambda$ pril $28 \ldots$ & .....do.... & 14 & 12.8 & 1.88 & 5.26 & 24 \\
\hline June 2. . & ......do.. & 9.5 . & 8.8 & .90 & 5.00 & 7.9 \\
\hline September 11. & ..... .do... & 10 & 8.8 & .66 & 5.02 & 5.8 \\
\hline October $30 .$. & ......do.. & 16 & 10.1 & .75 & 5.10 & 7.0 \\
\hline
\end{tabular}

IRR $177-06-16$ 
Daily gage height, in feet, of Lost River near Clear Lake, Cal., for 1905.

\begin{tabular}{|c|c|c|c|c|c|c|c|c|c|c|c|c|}
\hline Day. & Jan. & Feb. & Mar. & Apr. & May. & June. & July. & Aug. & Sept. & Oet. & Nov. & Dec. \\
\hline 1... & $5: 4$ & 7.3 & 6.3 & 6.9 & 5. 3 & 5.0 & & & & & 5.1 & 5.15 \\
\hline $2 .$. & 5.4 & 7.8 & 6.2 & 7.5 & 5.3 & 5.0 & & & & & 5.1 & 5.15 \\
\hline 3.. & 5.4 & 7.3 & 6.3 & 7.2 & 5.2 & 5.05 & & & & & 5.1 & 5.2 \\
\hline 4.. & 5.4 & 7.1 & 6.3 & 6.9 & 5.2 & 5. 1 & & & & & 5.1 & 5.2 \\
\hline $5 .$. & 5.4 & 6.8 & 6.4 & 6.7 & 5.1 & 5.15 & & & & & 5.1 & 5.2 \\
\hline 6.. & 5.4 & 6.5 & 6.3 & 6.6 & 5.1 & 5.2 & & & & & 5.1 & 5.2 \\
\hline 7.. & 5.4 & 6.3 & 6.5 & 6.4 & 5.15 & 5. 35 & & & & & 5.1 & 5.2 \\
\hline 8. & 5.4 & 6.1 & 6.4 & 6.2 & 5.2 & 5.6 & & & & & 5.1 & 5.15 \\
\hline $9 .$. & 5.4 & 5.8 & 6.3 & 6.0 & 5.2 & 5.9 & & & & & 5.1 & 5.15 \\
\hline $10 .$. & 5.4 & 5.6 & 6.2 & 5.8 & 5.2 & 5.8 & & & & & 5.1 & 5.15 \\
\hline $11 \ldots$ & 5.4 & 5.2 & 6.2 & 5.7 & 5.3 & 5.7 & & & & & 5. 1 & 5. 15 \\
\hline $12 .$. & 5.4 & 6.05 & 6.3 & 5.8 & 5.4 & 5.6 & & & & & 5.1 & 5.15 \\
\hline $13 .$. & 5.4 & 6.95 & 6.5 & 5.7 & 5.35 & 5.4 & & & & & 5. 1 & 5.15 \\
\hline $14 . \therefore$ & 5.6 & 6.9 & 6.2 & 5.5 & 5.3 & 5.25 & & & & & 5. 1 & 5. 15 \\
\hline 15. & 5.8 & 6.7 & 6.1 & 5.5 & 5.25 & 5.1 & & & & & 5.1 & 5.15 \\
\hline $16 .$. & 6.0 & 6.2 & 6.3 & 5.55 & 5.2 & 5.0 & & & & & 5.1 & 5.2 \\
\hline $17 .$. & 6.25 & 5.6 & 6.9 & 5.6 & 5.15 & 5.0 & & & & & 5.1 . & 5.2 \\
\hline 18.. & 6.3 & 5.5 & 6.7 & 5.6 & 5.1 & 5.0 & & & & & 5.1 & 5.2 \\
\hline $19 .$. & 6.3 & 5.8 & 6.3 & 5.7 & 5.05 & 5.0 & & & & & 5.1 & 5.2 \\
\hline 20. & 6.3 & 6.4 & 6.4 & 5.5 & 5.05 & 5.0 & & & & & 5.2 & 5.2 \\
\hline $21 .$. & 6.35 & 6.3 & 6.6 & 5.5 & 5.0 & 5.0 & & & & & 5.15 & 5.2 \\
\hline $22 .$. & 6.4 & 6.2 & 6.9 & 5.5 & 5.0 & 5.0 & & & & & 5.15 & 5.2 \\
\hline $23 \ldots$ & 6.4 & 6.0 & 6.9 & 5.45 & 5.0 & 5. 0 & & & & & 5.15 & 5.2 \\
\hline $24 \ldots$ & 6.45 & 6.1 & 6.5 & 5.4 & 4.95 & 5.0 & & & & & 5.15 & 5.2 \\
\hline $25 \ldots \ldots$ & 6.5 & 6.2 & 6.3 & 5.14 & 4.9 & 5.0 & & & & & 5.15 & 5.2 \\
\hline $26 \ldots$ & 6.3 & 6.0 & 6.5 & 5.5 & 4.9 & 5.0 & & & & & 5.2 & 5.2 \\
\hline 27... & 6.0 . & 6.1 & .6 .9 & 5.4 & 4.95 & 5.0 & & & & & 5.2 & 5.2 \\
\hline $28 \ldots$ & 6.2 & 6.2 & 6.6 & 5.3 & 5.0 & 5.0 & & & & & 5.2 & 5.2 \\
\hline 29... & 6.4 & ..... & $6: 7$ & 5.3 & 5.05 & 5.0 & & & & 5.1 & 5.15 & 5.2 \\
\hline $30 \ldots$ & $6: 6$ & & 6.0 & 5.3 & 5.1 & 5.0 & & & & 5.1 & 5.15 & 5.2 \\
\hline $31 \ldots \ldots, \ldots$ & 6.8 & & 6.4 & $\therefore \ldots$. & 5.05 & & & & & 5.1 & 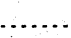 & 5.2 \\
\hline
\end{tabular}

Station rating table for Lost River near Clear Lake, Cal., from September 4, 1904, to December 31, 1905.

\begin{tabular}{|c|c|c|c|c|c|c|c|}
\hline $\begin{array}{c}\text { Gage } \\
\text { height. }\end{array}$ & Discharge. & $\begin{array}{l}\text { Gage } \\
\text { height. }\end{array}$ & Discharge. & $\begin{array}{c}\text { Gage } \\
\text { height. }\end{array}$ & Discharge. & $\begin{array}{c}\text { Gage } \\
\text { height. }\end{array}$ & Discharge. \\
\hline Feet. & Second-feet. & Feet. & Second-feet. & Feet. & Second-feet. & Feet. & Second-feet. \\
\hline $\begin{array}{l}4.90 \\
5.00\end{array}$ & 9 & $\begin{array}{l}0.70 \\
5.80\end{array}$ & $\begin{array}{l}00 \\
81\end{array}$ & $\begin{array}{r}0.50 \\
6.60\end{array}$ & $\begin{array}{r}238 \\
. \quad 268\end{array}$ & $\begin{array}{l}7.30 \\
7.40\end{array}$ & $\begin{array}{l}508 \\
545\end{array}$ \\
\hline 5.10 & 12 & 5.90 & 98 & 6.70 & 300 & 7.50 & 582 \\
\hline 5.20 & 17 & 6.00 & 117 & 6.80 & 332 & 7.60 & 619 \\
\hline 5.30 & 23 & 6.10 & 138 & 6.90 & 365 & 7.70 & 657 \\
\hline 5.40 & 31 & 6.20 & 160 & 7.00 & 400 & $7.80^{\circ}$ & 695 \\
\hline 5.50 . & 41 & 6.30 & 184 & 7.10 & 436 & 7.90 & 733 \\
\hline 5.60 & $\therefore 53$ & 6.40 & 210 & 7.20 & 472 & 8.00 & 772 \\
\hline
\end{tabular}

Note.-The above table is based on 16 discharge measurements made-during 1904-5. It is farly well defined. 
Estimated monthly discharge of Lost River near Clear Lake, Cal., for 1904 and 1905.

\begin{tabular}{|c|c|c|c|c|}
\hline \multirow{2}{*}{ Month. } & \multicolumn{3}{|c|}{ Discharge in second-feet. } & \multirow{2}{*}{$\begin{array}{l}\text { Total in } \\
\text { acre-feet. }\end{array}$} \\
\hline & Maximum. & Minimum. & Mean. & \\
\hline 1904. & & & & \\
\hline September.............. & 20 & 9 & 11.3 & 673 \\
\hline October............... & 23 & 12 & 18.1 & 1,113 \\
\hline November....... & 17 & 12 & 15.5 & 923 \\
\hline December.............. & 27 & 14 & 18.2 & 1,119 \\
\hline 1905. & & 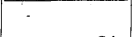 & & \\
\hline January ............... & 332 & 31 & 120 & 7,379 \\
\hline February .............. & 695 & 17 & 217 & 12,050 \\
\hline March........... & 365 & 117 & 224 & 13,770 \\
\hline April........ & 582 & 23 & 127 & 7,557 \\
\hline May................. & 31 & 6 & 14.6 & 898 \\
\hline June.............. & 98 & 9 & 21.6 & 1,286 \\
\hline July . . . . . . . . . . . . & $\cdots$ & $\cdots$ & a 7.0 & 430 \\
\hline August . ............. & $\ldots$ & $\cdots$ & $a_{6.0}$ & 369 \\
\hline September............ & $\ldots$ & $\ldots$ & $a 7.0$ & 417 \\
\hline October............. & - & - & $a_{10.0}$ & 615 \\
\hline November............ & 17 & 12 & 13.0 & 774 \\
\hline December............. & 12 & 14 & 16.0 & 984 \\
\hline The year. . & & & 65.3 & 46,530 \\
\hline
\end{tabular}

$a$ Estimated. 
LOST RIVER NEAR MERRILL; OREG.

This station was established July 26, 1904, by T. H. Humphreys. It is located about $1 \frac{1}{2}$ miles downstream from the Stukel Bridge, 4 miles northwest of Merrill, Oreg.

The channel is straight for 200 feet above and 400 feet below the station. The current is swift at high and medium at low stages. Both banks are high and not liable to overflow. The bed of the stream is composed of clay, rock, and gravel, and is not subject to change. There is but one channel at all stages. During low water the flow over the greater portion of the section is shallow and broken, making it difficult to obtain accurate results with a current meter. Near the right bank the channel is deeper. At low water in the fall of the year a considerable growth of water grass accumulates in the channel. An old rock dam located 200 feet above the gaging station does not materially interfere with the accuracy of discharge measurements.

Discharge measurements are made at flood stages by means of a cable and car and at low water by wading. The initial point for soundings is the stream face of the left-bank cable support.

A staff gage is fastened vertically at the left bank about 40 feet upstream from the cable. During 1905 the gage was read by Mrs. Joseph Stukel. Bench marks were established as follows: (1) A large wire nail driven into post supporting cable at left bank; elevation, 17.92 feet. (2) A nail driven into the sill at the northwest corner of a granary 200 feet downstream from the cable on the right bank; elevation, 20.61 feet. Elevations refer to the datum of the gage.

Gage heights and discharge measurements taken at this station in 1904 are contained in Water-Supply Paper No. 134, United States Geological Survey.

Discharge measurements of Lost River near Merrill, Oreg., in 1905.

\begin{tabular}{|c|c|c|c|c|c|c|}
\hline Date. & Hydrographer. & Width. & $\begin{array}{l}\text { Area of } \\
\text { section. }\end{array}$ & $\underset{\text { Melocity. }}{\text { Mean }}$ & $\begin{array}{c}\text { Gage } \\
\text { height. }\end{array}$ & $\begin{array}{c}\text { Dis- } \\
\text { charge. }\end{array}$ \\
\hline January $18 . .$. & C. T. Darley ............ & $\begin{array}{r}\text { Feet. } \\
87\end{array}$ & $\begin{array}{r}S q \cdot f t . \\
82\end{array}$ & $\begin{array}{r}\text { Ft. per sec. } \\
1.60\end{array}$ & $\begin{array}{l}\text { Feet. } \\
\quad 3.50\end{array}$ & $\begin{array}{r}\text { Sec.-ft. } \\
131\end{array}$ \\
\hline February 1.. & .....do......... & 102 & 208 & 2.72 & 4.80 & 566 \\
\hline February 8. & ....do.. & 108 & 240 & 2.74 & 5.05 & 658 \\
\hline February 27. & .....do... & 92 & 197 & 2.70 & 4.55 & 533 \\
\hline March 5. & ......do... & 96 & 199 & 2.71 & 4.63 & 540 \\
\hline April 5.:. & .....do... & 120 & 425 & 2.67 & 6. 77 & 1,134 \\
\hline April 14... & ....do... & 90 & 140 & 2.03 & 4.00 & 284 \\
\hline April 29... & .....do... & 80 & 95 & 1.53 & 3.50 & 145 \\
\hline May $12 \ldots$ & ....do.... & 89 & 95 & 1.37 & 3.50 & 130 \\
\hline June 14.. & ....do... & 89 & 86 & 1.36 & 3.41 & 117 \\
\hline July $23 \ldots$ & ....do................. & 90 & 86 & 1.36 & 3.45 & 117 \\
\hline August $15 \ldots .$. & C. T. Darley and W. B. Clapp....... & 88 & 82 & 1.26 & 3.41 & 103 \\
\hline August $26 \ldots$. & 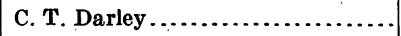 & 87 & 84 & 1.21 & 3.31 & 102 \\
\hline September 15. & .....do........ & 87 & 83 & 1.22 & 3.32 & 101 \\
\hline
\end{tabular}


Daily gage height, in feet, of Lost River near Merrill, Oreg., for 1905.

\begin{tabular}{|c|c|c|c|c|c|c|c|c|c|c|c|c|}
\hline Day. & Jan. & Feb. & Mar. & Apr. & May. & Juñe. & July. & Aug. & Sept. & Oct. & Nov. & Dec. \\
\hline 1. & 3.5 & 4.7 & 4.5 & 4.8 & 3.5 & $3.5^{\circ}$ & 3.4 & 3.4 & 3.3 & 3.3 & 3.3 & 3.3 \\
\hline $2 .$. & 3.5 & 5.7 & 4.5 & 4.8 & 3.5 & 3.5 & 3.4 & 3.4 & 3.3 & 3.3 & 3.3 & 3.3 \\
\hline 3. & 3.5 & 6.3 & 4.5 & 6.0 & 3.5 & 3.5 & 3.4 & 3.4 & 3.3 & 3.3 & 3.3 & 3.3 \\
\hline 4.. & 3.5 & 6.8 & 4.5 & 7.0 & 3.5 & 3.5 & 3.4 & 3.4 & 3.3 & 3.3 & 3.3 & 3.3 \\
\hline $5 .$. & 3.5 & 7.0 & 4.6 & 6.8 & 3.5 & 3.5 & 3.4 & 3.4 & 3.3 & 3.3 & 3.3 & 3.3 \\
\hline $6 \ldots$ & 3.5 & 6.2 & 4.6 & 6.0 & 3.5 & 3.5 & 3.4 & 3.4 & 3.3 & 3.3 & 3.3 & 3.3 \\
\hline $7 .$. & 3.5 & 5.6 & 4.6 & 5.3 & 3.5 & 3.5 & 3.4 & 3.4 & 3.3 & 3.3 & 3.3 & 3.3 \\
\hline $8 .$. & 3.5 & 5.1 & 4.55 & 5.4 & 3.5 & 3.5 & 3.4 & 3.4 & 3.3 & 3.3 & 3.3 & 3.3 \\
\hline $9 .$. & 3.5 & 4.7 & 4.55 & 5.6 & 3.5 & 3.5 & 3.4 & 3.4 & 3.3 & 3.3 & 3.3 & 3.3 \\
\hline $10 \ldots$ & 3.5 & 4.4 & 4.4 & 5.8 & 3.5 & 3.5 & 3.4 & 3.4 & 3.3 & 3.3 & 3.3 & 3.3 \\
\hline $11 \ldots$ & 3.5 & 4.2 & 4.3 & 5.9 & 3.5 & 3.5 . & 3.4 & 3.4 & 3.3 & 3.3 & 3.3 & 3.3 \\
\hline 12. & 3.5 & 4.0 & 4.35 & 4.0 & 3.5 & 3.55 & 3.4 & 3.4 & 3.3 & 3.3 & 3.3 & 3.3 \\
\hline $13 .$. & 3.5 & 3.9 & 4.2 & 4.0 & 3.5 & 3.55 & 3.4 & 3.4 & 3.3 & 3.3 & 3.3 & 3.3 \\
\hline 14. & 3.5 & 3.8 & 4.2 & 4.0 & 3.5 & 3.55 & 3.4 & 3.4 & 3.3 & 3.3 & 3.3 & 3.3 \\
\hline 15. & 3.5 & 3.8 & 4.2 & 4.0 & 3.5 & 3.4 & 3.4 & 3.4 & 3.3 & 3.3 & 3.3 & 3.3 \\
\hline $16 .$. & 3.5 & 3.7 & 4.1 & 3.9 & 3.5 & 3.4 & 3.4 & 3.4 & 3.3 & 3.3 & 3.3 & 3.3 \\
\hline 17. & 3.5 & 3.7 & 4.1 & 3.9 & 3.5 & 3.4 & 3.4 & 3.4 & 3.3 & 3.3 & 3.3 & 3.3 \\
\hline 18. & 3.5 & 3.7 & 4.1 & 3.8 & 3.5 & 3.4 & 3.4 & 3.4 & 3.3 & 3.3 & 3.3 & 3.3 \\
\hline 19. & 3.5 & 3.7 & 4.1 & 3.8 & 3.5 & 3.4 & 3.4 & 3.4 & 3.3 & 3.3 & 3.3 & 3.3 \\
\hline $20 \ldots$ & 3.5 & 3.8 & 4.2 & 3.8 & 3.5 & 3.4 & 3.4 & 3.4 & 3.3 & 3.3 & 3.3 & 3.3 \\
\hline $21 \ldots$ & 3.5 & 3.8 & 4.4 & 3.7 & 3.5 & 3.4 & 3.4 & 3.4 & 33 & 3.3 & 3.3 & 3.3 \\
\hline $22 \ldots$ & 3.5 & 3.9 & 4.5 & 3.7 & 3.5 & 3.4 & 3.4 & 3.4 & 3.3 & 3.3 & 3.3 & 3.3 \\
\hline $23 \ldots$ & 3.5 & 4.4 & 4.6 & 3.7 & 3.5 & 3.4 & 3.4 & 3.4 & 3.3 & 3.3 & 3.3 & 3.3 \\
\hline $24 \ldots$ & 3.5 & 4.4 & 4.7 & 3.6 & 3.5 & 3.4 & 3.4 & 3.4 & 3.3 & 3.3 & 3.3 & 3.3 \\
\hline 25. & 4.5 & 4.5 & 4.9 & 3.6 & 3.5 & 3.4 & 3.4 & 3.4 & 3.3 & 3.3 & 3.3 & 3.3 \\
\hline 26. & 5.4 & 4.5 & 4.6 & 3.5 & 3.5 & 3.4 & 3.4 & 3.4 & 3.3 & 3.3 & 3.3 & 3.3 \\
\hline $27 \ldots$ & 5.3 & 4.55 & 4.6 & 3.5 & 3.5 & 3.4 & 3.4 & 3.4 & 3.3 & 3.3 & 3.3 & 3.3 \\
\hline $28 \ldots$ & 5.0 & 4.55 & 5.0 & 3.5 & 3.5 & 3.4 & 3.4 & 3.4 & 3.3 & 3.3 & 3.3 & 3.3 \\
\hline $29 \ldots$ & 4.7 & . & 4.9 & 3.5 & 3.5 & 3.4 & 3.4 & 3.4 & 3.3 & 3.3 & 3.3 & 3.3 \\
\hline $30 \ldots$ & 4.5 & & 4.8 & 3.5 & 3.5 & 3.4 & 3.4 & 3.4 & 3.3 & 3.3 & 3.3 & 3.3 \\
\hline $31 \ldots \ldots$ & 4. 4 & & 4.8 & & 3.5 & & 3. 4 & 3.4 & & 3. 3 & $\cdots \cdot \cdot$ & 3.3 \\
\hline
\end{tabular}

Station rating 'table for Lost River near Merrill, Oreg., from July 16, 1904, to December 31, 1905.

\begin{tabular}{|c|c|c|c|c|c|c|c|}
\hline $\begin{array}{c}\text { Gage } \\
\text { height. }\end{array}$ & Discharge. & $\begin{array}{c}\text { Gage } \\
\text { height. }\end{array}$ & Discharge. & $\begin{array}{c}\text { Gage } \\
\text { height. }\end{array}$ & Discharge. & $\begin{array}{c}\text { Gage } \\
\text { height. }\end{array}$ & Discharge. \\
\hline Feet. & Second-feet. & Feet. & Second-feet. & Feet. & Second-feet. & Feet. & Second-feet. \\
\hline 3.30 & 102 & 3.60 & 176 & 3.90 & 262 & 4.20 & 362 \\
\hline 3.40 & 125 & 3.70 & 203 & 4.00 & 294 & & \\
\hline 3.50 & 150 & 3.80 & 232 & 4. 10 & 328 & & \\
\hline
\end{tabular}

Note.-The above table is applicable only for open-channel conditions. It is based on 20 discharge measurements made during 1904-5. It is not well defined. Above gage height 4.2 feet the rating curve is a tangent, the difference being 35 per tenth 
Estimated monthly discharge of Lost River near Merrill, Oreg., for 1904 and 1905.

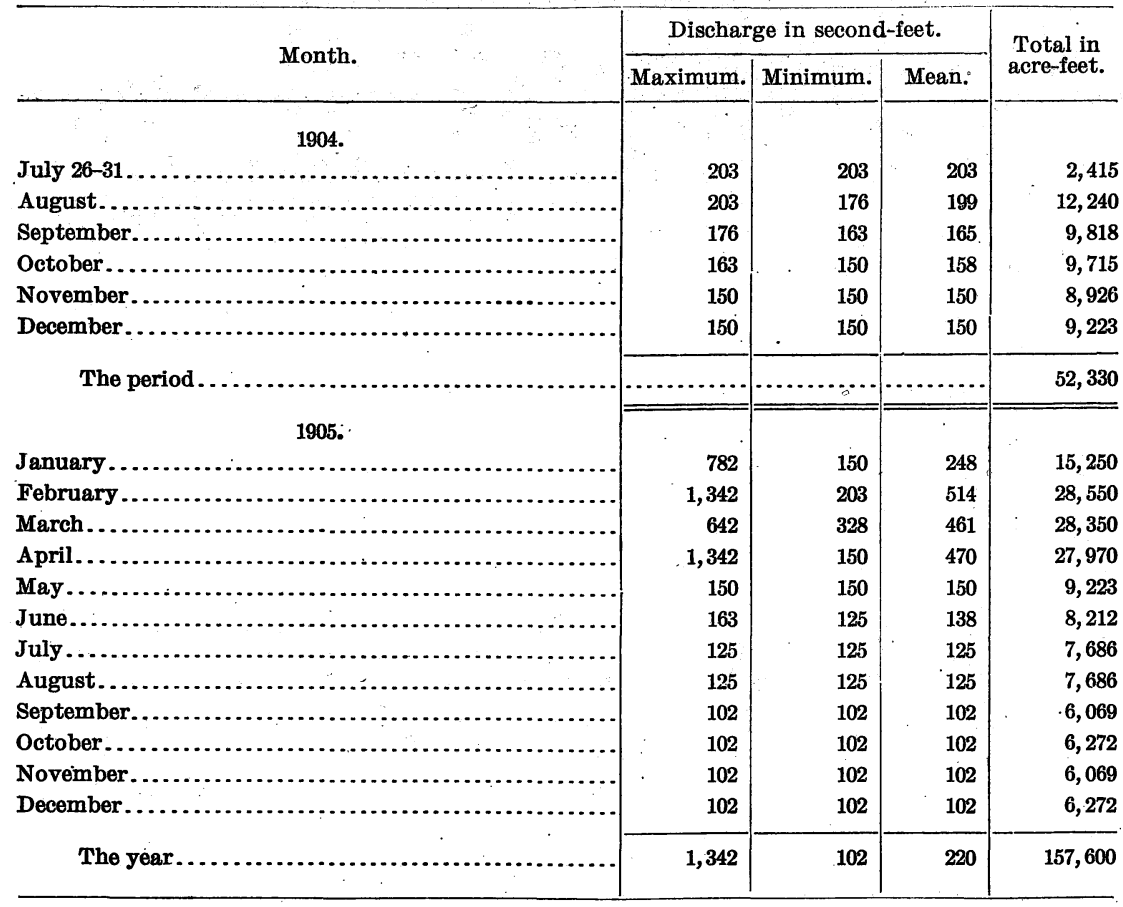

TULE IAKE IFAR MERRIIL, OREG.

This station was established May 17, 1904, by John H. Lewis and Ivan Landes for recording the water level in Tule Lake. It is located on Tule Lake at the mouth of Lost River about 3 miles east of Merrill, Oreg , 25 miles south from Klamath Falls, and near the OregonCalifornia line.

The gage is a vertical timber fastened to posts driven in the lake bed, about 20 feet from the shore of the lake. It is in line with the east lane fence, one-fourth mile south of the residence of J. Frank Adams. The bench mark is a notch cut in large juniper gatepost on the north side of the gate entrance about 60 feet distant from the gage rod; elevation, 13.70 feet above the zero of the gage. 
Daily gage height, in feet, of Tule Lake near Merrill, Oreg., for 1905.

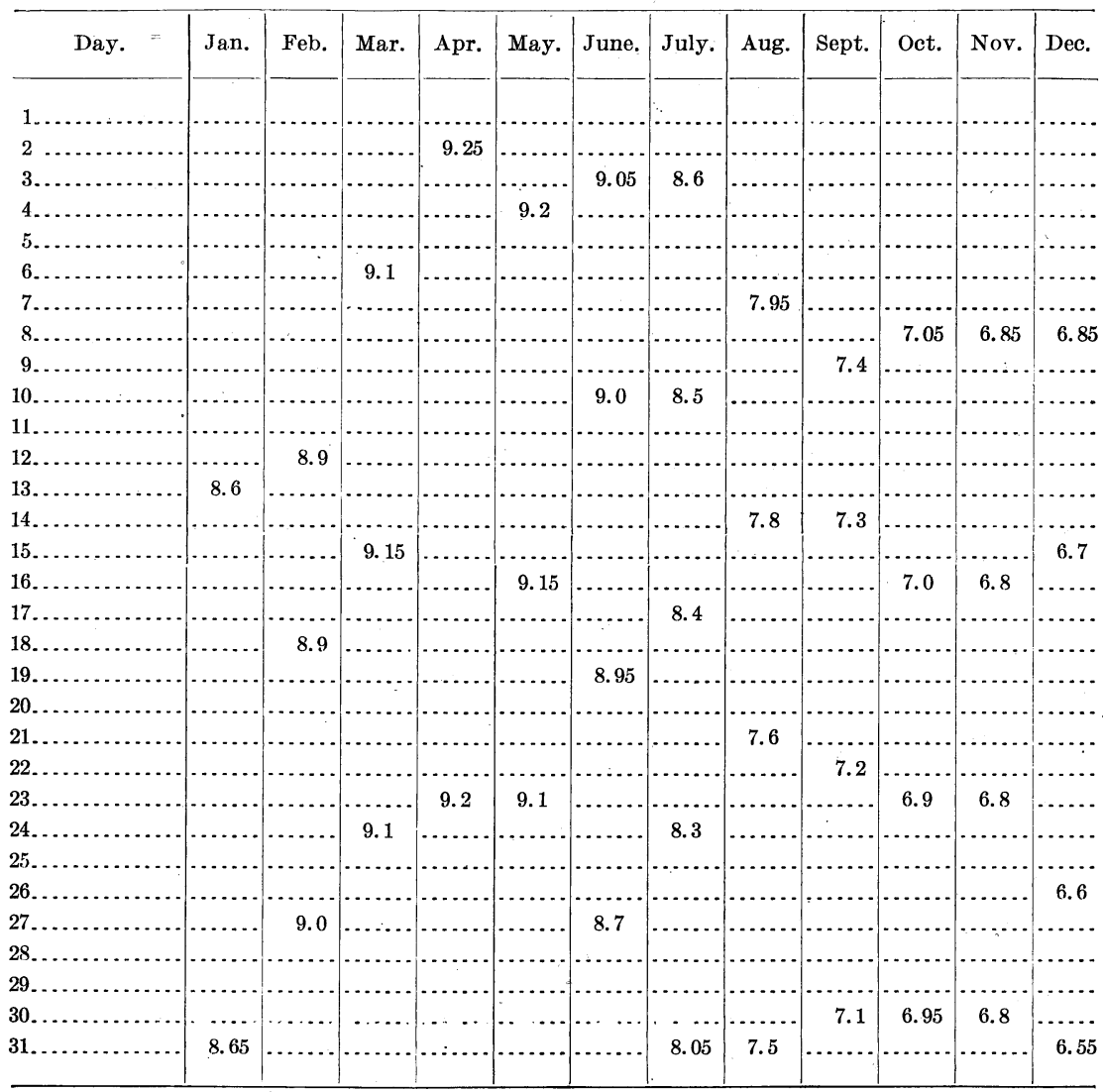

Note.-Ice out of river, Jan. 31.

MILLER CREEK NEAR LORELLA, OREG.

This station was established August 10, 1904, by F. S. Chapman. It is located at Horsefly, 10 miles northeast of Lorella, Oreg.

The channel is straight for 600 feet above and 100 feet below the station. The current is sluggish at low and swift at high stages. Both banks are high, rocky, wooded, and not subject to overflow. The bed of the stream is of rock and gravel, and not liable to change. The channel is obstructed by much vegetation during the season of low stage.

Discharge measurements are made during high stages by means of a cable and car, and at low stages by wading. The initial point for soundings is the stream side of the cable support on the right bank.

A staff gage is fastened vertically at the right bank, 300 feet upstream from the cable, where the water stands in a pool. During 1905 the gage was read by Louis Gerber. On December 9, 1905, an automatic water-height register was installed for recording gage heights. The bench mark is a point on top of a large bowlder 25 feet downstream from the gage; elevation, 12.68 feet above the datum of the gage.

Gage heights and discharge measurements taken at this station during 1904 are contained in Water-Supply Paper No. 134, United States Geological Survey. 
Discharge measurements of Miller Creek near Lorella, Oreg., in 1905.

\begin{tabular}{|c|c|c|c|c|c|c|}
\hline Date. & Hydrographer. & Width. & $\begin{array}{l}\text { Area of } \\
\text { section. }\end{array}$ & $\begin{array}{c}\text { Mean } \\
\text { velocity. }\end{array}$ & $\begin{array}{l}\text { Gage } \\
\text { height. }\end{array}$ & $\begin{array}{c}\text { Dis- } \\
\text { charge. }\end{array}$ \\
\hline & & Feet. & Sq. ft. & Ft. per sec. & Feet. & Sec.-ft. \\
\hline January $28 . .$. & C. T. Darley.. & 110 & 72 & 2.03 & 7.25 & 146 \\
\hline February $4 \ldots$ & ....do............. & 124 & 86 & 1.87 & 7.30 & 161 \\
\hline February $6 \ldots$ & .....do....... & 90 & 58 & 1.50 & 6.99 & 87 \\
\hline February $25 \ldots$ & .....do......... & 118 & 88 & 2.01 & 7.30 & 177 \\
\hline March 8 & …do................. & 91 & 72 & 1.72 & .7 .14 & 124 \\
\hline March 15 & F. S. Chapman... & 90 & 65 & 1.46 & 6.92 & 95 \\
\hline April 2....... & C. T. Darley.......... & 130 & 155 & 2.97 & 8.00 & 460 \\
\hline April 9... & .....do...... & 72 & 47 & 1.32 & 6.88 & 62 \\
\hline April 28. . & .....do....... & 18 & 10.8 & .29 & 6.19 & 3.1 \\
\hline June $1 . . . \ldots .$. & .....do. . . . & 20 & 11.2 & .18 & 6.11 & 2.0 \\
\hline
\end{tabular}

Daily gage height, in feet, of Miller Creek near Lorella, Oreg., for 1905.

\begin{tabular}{|c|c|c|c|c|c|c|c|c|c|c|c|c|}
\hline Day. & Jan. & Feb. & Mar. & Apr. & May. & June. & July. & Aug. & Sept. & Oct. & Nov. & Dec. \\
\hline $1 \ldots$ & 6.3 & $.8 . \overline{0}$ & 7.2 & 7.0 & 6.3 & 6.2 & & & & & & \\
\hline $2 .$. & 6.3 & 8.2 & 7.2 & 8.0 & 6.3 & 6.1 & & & & & & \\
\hline 3.. & 6.3 & 7.7 & 7.3 & 7.8 & 6.3 & 6.1 & & & & & & \\
\hline $4 .$. & 6.3 & 7.3 & 7.3 & 7.6 & 6.3 & 6.2 & & & & & & \\
\hline 5. & 6.3 & 7.7 & 7.3 & 7.4 & 6.3 & 6.2 & & & & & & \\
\hline 6. & 6.3 & 7.0 & 7.2 & 7.2 & 6.3 & 6.3 & & & & & & \\
\hline 7.. & 6.3 & 7.0 & 7.2 & 7.0 & 6.2 & 6.3 & & & & & & \\
\hline 8.. & 6.3 & 7.0 & 7.2 & 6.9 & 6.2 & 6.3 & & & & & & \\
\hline $9 .$. & 6.3 & 7.0 & 7.1 & 6.8 & 6.4 & 6.3 & & & & & & a 6.0 \\
\hline $10 .$. & 6.3 & 6.9 & 7.1 & 6.8 & 6.4 & 6.3 & & & & & & 6.0 \\
\hline 11.. & 6.3 & 6.6 & 7.0 & 6.8 & 6.4 & 6.3 & & & & & & 6.0 \\
\hline 12. & 6.3 & 6.5 & 7.1 & 6.6 & 6.4 & 6.3 & & & & & & 6.0 \\
\hline 13.. & 6.3 & 6.5 & - 7.1 & 6.6 & 6.4 & 6.2 & & & & & & 6.0 \\
\hline 14.. & 6.3 & 6.4 & 7.1 & 6.5 & 6.4 & 6.2 & & & & & & 6.0 \\
\hline $15 \ldots$ & 6.3 & 6.4 & 6.8 & 6.5 & 6.3 & 6.2 & & & & & & 6.0 \\
\hline $16 .$. & 7.5 & 6.4 & 7.3 & 6.6 & 6.3 & 6.2 & & & & & & 6.0 \\
\hline $17 .$. & 7.6 & 6.4 & 7.3 & 6.6 & 6.3 & & & & & & & 6.0 \\
\hline 18. & $7.7^{\circ}$ & 6.4 & 7.3 & 6.6 & 6.3 & & & & & & & 6.0 \\
\hline $19 .$. & 7.5 & 6.8 & 7.4 & 6.5 & 6.3 & & & & & & & 6.0 \\
\hline 20.. & 7.5 & 7.2 & 7.4 & 6.5 & 6.2 & & & & & & & 6.0 \\
\hline 21.. & 7.5 & 7.3 & 7.1 & 6.5 & 6.1 & & & & & & & 6.0 \\
\hline 22. & 7.9 & 7.3 & 7.3 & 6.4 & 6.0 & & & & & & & 6.0 \\
\hline $23 .$. & 8.2 & 7.3 & 7.3 & 6.4 & 6.0 & & & & & & & 6.0 \\
\hline $24 \ldots$ & 7.9 & 7.3 & 7.2 & 6.4 & 6.0 & & & & & & 6.0 & 6.0 \\
\hline $25 \ldots \ldots$ & 7.9 & 7.3 & 7.2 & 6.3 & 5.9 & & & & & & 6.1 & 6.0 \\
\hline $26 \ldots .$. & 7.7 & 7.3 & 7.9 & 6.3 & 6.1 & & & & & & & 6.0 \\
\hline $27 \ldots$ & 7.7 & 7.3 & 7.5 & 6.2 & 6.1 & & & & & & & 6.0 \\
\hline $28 \ldots$ & 7.3 & 7.3 & 7.4 & 6.2 & 6.2 & 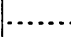 & & & & & & 6.0 \\
\hline $29 \ldots$ & 7.5 & $\cdots .$. & 7.4 & -6.2 & 6.3 & & & & & & & 6.0 \\
\hline $30 \ldots \ldots \ldots \ldots \ldots$ & 8.0 & $\cdots \cdots$ & 7.2 & 6.2 & 6.2 & & & & & & & 6.0 \\
\hline $31, \ldots \ldots \ldots \ldots \ldots$ & 8.3 & $\ldots \ldots$ & 7.2 & $\ldots$. & 6.2 & 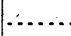 & & & & & & \\
\hline
\end{tabular}

Note.-No flow June 17 to December 31 
Station rating table for Miller Creek near Lorella, Oreg., from August 7, 1904, to December 31,1905 .

\begin{tabular}{|c|c||c|r|r|r|r|r|}
\hline $\begin{array}{c}\text { Gage } \\
\text { height. }\end{array}$ & Discharge. & $\begin{array}{c}\text { Gage } \\
\text { height. }\end{array}$ & Discharge. & $\begin{array}{c}\text { Gage } \\
\text { height. }\end{array}$ & Discharge. & $\begin{array}{c}\text { Gage } \\
\text { height. }\end{array}$ & Discharge. \\
\hline Feet. & Second-feet. & Feet. & Second-feet. & Feet. & Second-feet. & Feet. & Second-feet. \\
6.00 & 0 & 6.80 & 52 & 7.60 & 274 & 8.40 & 665 \\
6.10 & 1.5 & 6.90 & 67 & 7.70 & 317 & 8.50 & 717 \\
6.20 & 4 & 7.00 & 86 & 7.80 & 363 & 8.60 & 770 \\
6.30 & 8 & 7.10 & 109 & 7.90 & 411 & 8.70 & 823 \\
6.40 & 13 & 7.20 & 135 & 8.00 & 460 & 8.80 & 876 \\
6.50 & 20 & 7.30 & 163 & 8.10 & 510 & 8.90 & 929 \\
6.60 & 29 & 7.40 & 196 & 8.20 & 561 & 9.00 & 982 \\
6.70 & 39 & 7.50 & 234 & 8.30 & 613 & & \\
& & & & & & \\
\hline
\end{tabular}

Note.-The above table is based on 13 discharge measurements made during 1904-5. It is well defined between gage heights 6.1 feet and 8 feet.

Estimated monthly discharge of Miller Creek near Lorella, Oreg., for 1904.and 1905.

\begin{tabular}{|c|c|c|c|c|}
\hline \multirow{2}{*}{ Month. } & \multicolumn{3}{|c|}{ Discharge in second-feet. } & \multirow{2}{*}{$\begin{array}{l}\text { Total in } \\
\text { acre-feet. }\end{array}$} \\
\hline & Maximum. & Minimum. & Mean. & \\
\hline 1904. & & & & \\
\hline 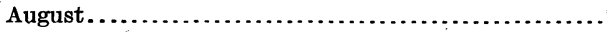 & 1.5 & .75 & 1.3 & 79 \\
\hline September........... & 1.5 & .0 & .2 & 12 \\
\hline October.............. & 8 & 1.5 & 2.4 & 148 \\
\hline November............ & 8 & 1.5 & 3.7 & 221 \\
\hline December.............. & 10.5 & 1.5 & 6.2 & 381 \\
\hline The period... & & $\therefore$. & ..... & 841 \\
\hline 1905. & & & & \\
\hline January .............. & 613 & 8 & 179 & 11,010 \\
\hline February .............. & 561 & 13 & 138 & 7,664 \\
\hline March............. & 411 & 52 & 153 & 9,408 \\
\hline April.................. & 460 & 4 & 71.0 & 4,225 \\
\hline May ........ & 13 & 0 & 6.5 & 400 \\
\hline June................... & 8 & 0 & 3.0 & 179 \\
\hline July . . . . . . . . & 0 & 0 & .0 & 0 \\
\hline August. . . . . . . . . . & 0 & 0 & .0 & 0 \\
\hline 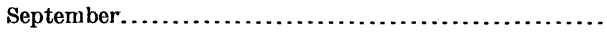 & 0 & 0 & .0 & $\mathbf{0}$ \\
\hline October.............. & 0 & 0 & .0 & 0 \\
\hline$\ldots \ldots \ldots \ldots \ldots \ldots \ldots \ldots \ldots \ldots \ldots \ldots$ & 0 & 0 & .0 & 0 \\
\hline 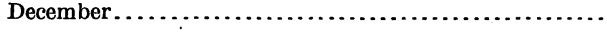 & 0 & 0 & .0 & 0 \\
\hline The year... & 613 & 0 & 46. & 32,890 \\
\hline
\end{tabular}




\section{PRECIPITATION AND EVAPORATION DATA.}

The following table gives the total precipitation in inches, by months, and also the annual totals:

\begin{tabular}{|c|c|c|c|c|c|c|c|c|c|c|c|c|c|}
\hline & Jan. & Feb. & Mar. & Apr. & May. & June. & July. & Aug. & Sept. & Oct. & Nov. & Dec. & Annual. \\
\hline \multicolumn{14}{|l|}{ Keno, Oreg. : } \\
\hline 1904. & & & & & & ..... & $\cdots$ & 0.00 & 1.58 & 2.08 & 1.50 & 3.57 & \\
\hline $1905 \ldots \ldots \ldots$ & 2.08 & 1.91 & 2.30 & 0.66 & 2.29 & 0.15 & 0.00 & 0.00 & 0.75 & 0.72 & 1.30 & 2.23 & 14.39 \\
\hline \multicolumn{14}{|l|}{ Tule Lake, Cal.: } \\
\hline 1904 & & & & & & & & 0.00 & 0.92 & 1.01 & 0.42 & 3.11 & \\
\hline $1905 \ldots \ldots \ldots$ & 1.25 & 1.19 & 1.70 & 0.20 & 2.62 & 0.30 & 0.00 & 0.00 & 0.42 & $\ldots \therefore$ & 1.25 & 0.93 & \\
\hline \multicolumn{14}{|l|}{$\begin{array}{l}\text { Horse Fly, near } \\
\text { Lorella, Oreg.: }\end{array}$} \\
\hline $1904 \ldots$ & $\ldots$ & & & & & $\ldots$ & $\cdots$ & 0.00 & 0.71 & 1.11 & 1.07 & 2.61 & \\
\hline $1905 \ldots \ldots \ldots$ & 1.43 & 1.09 & 2.22 & 0.28 & 2.56 & 1.23 & 0.00 & 0.00 & 0.32 & 0.32 & 0.83 & 2.41 & 12.69 \\
\hline
\end{tabular}

The following table gives the total evaporation in inches, by months, at Keno, Oreg.:

\begin{tabular}{|c|c|c|c|c|c|c|c|c|c|c|c|c|c|}
\hline Year. & Jan. & Feb. & Mar. & Apr. & May. & June. & July. & Aug. & Sept. & Oct. & Nov. & Dec. & Annual. \\
\hline 1904 & & & & & & & & 6.66 & 5.12 & 2.01 & 2.01 & \begin{tabular}{|l|}
2.01 \\
\end{tabular} & \\
\hline $1905 \ldots$ & 1.19 & 0.78 & 0.78 & 2.69 & 4. 12 & 6.20 & 7.57 & 7.03 & 6.15 & 1.98 & 0.46 & & . \\
\hline
\end{tabular}

\section{MISCELLANEOUS MEASUREMENTS IN NORTHERN PACIFIC OCEAN DRAINAGE BASIN.}

Antelope Creek near Mount Hebron, Cal.-This creek rises on the eastern slope of Mount Shasta. It is tributary to Lower Klamath Lake, although its waters sink before reaching the lake. A measurement was made June 17 by C. T. Darley, 12 miles below its source.

Width, 25.5 feet; area, 37 square feet; mean velocity, 3.03 feet per second; discharge, 112 second-feet.

Another measurement was made August 28 by C. T. Darley 15 miles below its source.

Width, 13 feet; area, 9.4 square feet; mean velocity, 1.11 feet per second; discharge, 10.4 second-feet.

Adams ditch near Merrill, Oreg.-This canal diverts water from White Lake, an arm of Lower Klamath Lake. For the first mile this canal is common with the Van Brimmer canal. It is about 16 miles in length and is used for the irrigation of land in the vicinity of Merrill, Oreg. The following measurements were made during 1905 at a point one mile below the head of the canal and 300 feet below the point where the Adams and Van Brimmer canals separate. The gage rod is a 1 by 4 inch timber divided into feet and hundredths. There is no bench mark.

May 14: Width, 36 feet; area, 42 square feet; mean velocity, 1.10 feet per second; gage height, 1.73 feet; discharge, 46 second-feet.

May 21: Width, 37.6 feet; area, 47 square feet; mean velocity, 1.13 feet per second; gage height, 1.90 feet; discharge, 53 second-feet.

June 15: Width, 37.5 feet; area, 40 square feet; mean velocity, 1.28 feet per second; gage height, 2.14 feet; discharge, 51 second-feet.

July 22: Width, 37.5 feet; area, 43 square feet; mean velocity, 1.19 feet per second; gage height, 2.22 feet; discharge, 51 second-feet.

August 24: Width, 37.5 feet; area, 36 square feet; mean velocity, 1.03 feet per second; gage height, 2.13 feet; discharge, 37 second-feet.

August 25: Width, 36.7 feet; area, 32 square feet; mean velocity, 0.91 foot per second; gage height, 2.00 feet; area, 29 second-feet. 
August 25: Width, 37.9 feet; area, 39 square feet: mean velocity, 1.05 feet per second; gage height, 2.26 feet; discharge, 41 second-feet.

Ankeny ditch, station No. 1, near Klamath Falls, Oreg.-Ankeny canal diverts water from Link River at the outlet of Upper Klamath Lake, $1 \frac{1}{2}$ miles above the town of Klamath Falls, Oreg. The water from this canal is used for electric power and irrigation purposes in the vicinity of Klamath Falls. This station is located about 500 feet above point of diversion to electric power plant. Discharges here given represent the amount of water used for both electric power and irrigation purposes. The following measurements were made during 1905 by C. T. Darley.:

June 29: Width,17.4 feet; area,21 square feet; mean velocity,2.71 feet per second; gage height, 1.36 feet; discharge, 57 second-feet.

June 30: Width, 17.4 feet; area, 20 square feet; mean velocity, 2.70 feet per second; gage height, 1.34 feet; discharge, 54 second feet.

May 11: Width, 17.5 feet; area, 22 square feet; mean velocity, 2.86 feet per second; gage height, 1.39 feet; discharge, 63 second-feet.

June 29: Width, 17.4 feet; area, 21 square feet; mean velocity, 2.52 feet per second; gage height, 1.36 feet; discharge, 53 second-feet.

Ankeny canal station No. 2, near Klamath Falls, Oreg.-This station is located about 200 feet below point of diversion by electric power plant. Discharges here given represent the amount of water used for irrigation purposes.

April 23: Width, 13.5 feet; area, 7.0 square feet; mean velocity, 1.24 feet per second; gage height, 1.28 feet; discharge, 8.7 second-feet.

May 2: Width, 13.5 feet; area, 13.1 square feet; mean velocity, 2.21 feet per second; gage height, 1.68 feet; discharge, 29 second-feet.

May 11: Width, 13.5 feet; area, 15 square feet; mean velocity, 2.33 feet per second; gage height, 1.80 feet; discharge, 35 second-feet.

Butte Creek near Mount Hebron, Cal.-Butte Creek rises on the eastern slope of Mount Shasta. It is tributary to Lower Klamath Lake and its waters sink in Butte Valley before reaching the lake. This creek discharges over fissured lava and large quantities of water are lost by seepage. That portion which reaches Butte Valley is used for irrigation. The following measurements were made during 1905 by C. T. Darley, $1 \frac{1}{2}$ miles above Boyce ranch at county road crossing.

May 5: Width, 15.5 feet; area, 51 square feet; mean velocity, 1.33 feet per second; gage height, 3.77 feet; discharge, 68 second-feet.

May 19: Width, 15.9 feet; area, 51 square feet; mean velocity, 1.47 feet per second; gage height, 3.95 feet; discharge, 75 second-feet.

June 16: Width, 16 feet; area, 48 square feet; mean velocity, 1.08 feet per second; gage height, 3.72 feet; discharge, 52 second-feet.

August 29: Width, 14 feet; area, 35 square feet; mean velocity, 0.47 foot per second; gage height, 3.00 feet; discharge, 16.4 second-feet.

November 23: Width, 14 feet; area, 36 square feet; mean velocity, 0.23 foot per second; gage height, 3.30 feet; discharge, 8.1 second-feet.

Bowne's west canal, near Bonanza, Oreg.-This canal takes water from Lost River, the water being raised by means of pumps and used for irrigation in the vicinity of Bonanza, Oreg. A measurement was made on May 31 by C. T. Darley at a point 300 feet below head of flume.

Width, 9.5 feet; area, 5.9 square feet; mean velocity, 1.46 feet per second; discharge, 8.6 second-feet.

Boards ditch, Poe Valley, Oreg.- - This ditch diverts water from Lost River. The water is raised by means of a water wheel and is used for irrigation in the vicinity of Olene, Oreg. A measurement was made on July 19 by C. T. Darley at a point 300 feet below head of canal.

Width, 2 feet; area, 1.56 square feet; mean velocity, 0.22 foot per second; discharge, 0.3 second-foot. 
Coyote Creek, Oreg.-Coyote Creek is tributary to Sycan marsh. During 1905 discharge measurements were made by Ivan Landes as follows:

March 15: Width, 5.5 feet; area, 4.7 square feet; mean velocity, 0.89 foot per second; discharge, 4.2 second-feet.

April 10: Width, 6.0 feet; area, 6.1 square feet; mean velocity, 1.34 feet per second; discharge, 8.2 second-feet.

May 2: Width, 6.0 feet; area, 5.6 square feet; mean velocity, 1.05 feet per second; discharge, 5.9 second-feet.

June 12: Width, 5.0 feet; area, 3.4 square feet; mean velocity, 0.50 foot per second; discharge, 1.7 second-feet.

July 31: Width, 1.4 feet; area, 0.4 square foot; mean velocity, 1.38 feet per second; discharge, 0.6 second-foot.

Crooked Creek near Klamath Agency, Oreg.-This stream is a tributary of Wood River. A measurement was made May 27 by C. T. Darley at the bridge $1 \frac{1}{2}$ miles from Klamath Agency, on road to Fort Klamath.

Width, 24.8 feet; area, 88 square feet; mean velocity, 0.52 foot per second; discharge, 46 second-feet.

Cherry Creek near Crystal, Oreg.-This stream is tributary to Upper Klamath Lake from the west. A measurement was made on May 25 by C. T. Darley at road crossing between Pelican Bay and Fort Klamath.

Width, 27 feet; area, 25 square feet; mean velocity, 1.28 feet per second; discharge, 32 second-feet.

CraneCreek near Fort Klamath,Oreg.-Crane Creek is a tributary to Seven Mile Creek. A measurement was made on May 25 by C. T. Darley at the bridge on wagon road between Fort Klamath and Pelican Bay, Oreg.

Width, 11.5 feet; area, 20 square feet; mean velocity, 0.54 foot per second; discharge, 10.7 second-feet.

Cottonwood Creek near Brownell,Cal.-Cottonwood Creek is tributary to Lower Klamath Lake from the South. The following measurements were made during 1905, 1 mile below its source, at " $F$ " ranch, Brownell:

June 15: Width, 19.5 feet; area, 41 square feet; mean velocity, 0.25 foot per second; discharge, 10.2 second-feet.

August 30: Width, 27.5 feet; area, 24 square feet; mean velocity, 0.53 foot per second; discharge, 12.6 second-feet.

Doris Creek near Picard, Cal.-Doris Creek is tributary to Lower Klamath Lake from the southwest. A measurement was made on June 18 by C. T. Darley at the Doris ranch.

Width, 5.4 feet; area, 4.9 square feet; mean velocity, 1.24 feet per second; discharge, 6.1 second-feet.

Dirty Creek, Oregon.-Dirty Creek is tributary to Sycan marsh. During 1905 discharge measurements were made by Ivan Landes, as follows:

March 15: Width, 3.0 feet; area, 1.4 square feet; mean velocity, 0.98 foot per second; discharge, 1.4 second-feet.

April 10: Width, 3.0 feet; area, 1.5 square feet; mean velocity, 0.99 foot per second; discharge, 1.3 second-feet.

May 2: Width, 3.0 feet; area, 1.4 square feet; mean velocity, 1.0 foot per second; discharge, 1.4 second-feet.

Edson Foulks ditch.-This ditch is in Shasta Valley. A measurement was made at head of ditch September 3, 1905, by L. G. Applegate.

Width, 6.3 feet; area, 6.3 square feet; mean velocity, 1.75 feet per second; discharge, 11 second-feet.

Four MileCreek, Pelican Bay,Oregon.-Four MileCreek is tributary to Upper Klamath Lake from the west. Its source is in Four Mile Lake. A measurement was made May 24 by C. T. Darley at road crossing between Klamath Falls and Pelican Bay. 
Width, 50.5 feet; area, 75 square feet; mean velocity, 0.70 foot per second; discharge, 53 second-feet.

Grass Valley Creek near Lowden, Cal.-Grass Valley Creek is tributary to Trinity River. A measurement was made of this stream August 26, 1905, by W. B. Clapp at lower wagon bridge on Buckhorn road $2 \frac{1}{2}$ miles above junction of creek with Trinity River.

Width, 19 feet; area, 18.7 square feet; mean velocity, 0.98 foot per second; discharge, 18.4 second-feet.

Griffith canal near Olene, Oreg.--This canal diverts water from Lost River. The water is raised by means of a water wheel and used for irrigation of land in the vicinity of Olene: The following measurements were made during 1905 by C. T. Darley at head of canal:

June 2: Width, 2 feet; area, 1.4 square feet; mean velocity, 1.29 feet per second; discharge, 1.8 second-feet.

June 13: Width, 2 feet; area, 1.5 square feet; mean velocity, 1.27 feet per second; discharge, 1.9 second-feet.

June 25: Width, 2 feet; area, 1.16 square feet; mean velocity, 1.08 feet per second; discharge, 1.3 second-feet.

Hot Springs Creek near Klamäth Falls, Oreg.-Hot Springs Creek rises about one-half mile east of Klamath Falls and discharges into Lower Klamath Lake. A measurement was made on August 18 by C. T. Darley near point where creek crosses county road east of Klamath Falls, Oreg.

Width, 1.5 feet; area, 0.36 square foot; mean velocity, 0.94 foot per second; discharge, 4.4 second-feet.

Horton ditch at Poe Valley, Oreg.-This ditch diverts water from Lost River, which is used for irrigation on the south side of the river. A measurement was made on June 23 by C. T. Darley at a point one-fourth of a mile below head of ditch.

Width, 7.5 feet; area, 7.4 square feet; mean velocity, 0.59 foot per second; discharge, 4.4 second-feet.

Little Shasta River at Little Shasta, Cal.-A measurement of this stream was made August 29, 1905, by M. D. Williams.

Width, 9 feet; area, 5.4 square feet; mean velocity், 0.70 foot per second; discharge, 3.8 second-feet.

Little Shasta Springs at Little Shasta, Cal.-A measurement was made August 29, 1905, by M. D. Williams.

Width, 9 feet; area, 5.6 square feet; mean velocity, 3.57 feet per second; discharge, 20 second-feet.

Long Creek, Oreg.--Long Creek is tributary to Sycan Marsh. During 1905 discharge measurements were made by Ivan Landes as follows:

March 15: Width, 22 feet; area, 21.8 square feet; mean velocity, 1.54 feet per second; discharge, 33.6 second-feet.

April 10: Width, 25 feet; area, 36.9 square feet; mean velocity, 1.48 feet per second; discharge, 54.6 second-feet.

May 1: Width, 26 feet; area, 46.8 square feet; mean velocity, 1.45 feet per second; discharge, 68.1 second-feet.

June 12: Width, 25 feet; area, 37.6 square feet; mean velocity, 1.56 feet per second; discharge, 58.6 second-feet.

July 31: Width, 20 feet; area, 10.8 square feet; mean velocity, 1.72 feet per second; discharge, 18.6 second-feet.

Moss Creek near Pelican Bay, Oregon.-Moss Creek is tributary to Upper Klamath Lake from the west. A measurement was made on May 23 by C. T. Darley at road crossing between Pelican Bay and Klamath Falls, Oreg.

Width, 5.5 feet; area, 3.3 square feet; mean velocity, 0.76 foot per second; discharge, 2.5 second-feet.

McCormick mill-race canal near Keno, Oreg.-This canal diverts water from Klamath River about 1 mile below Keno, Oreg. This water is used for power purposes in running a 
sawmill. The following measurements were made during 1905 by C.T. Darley at a point one-fourth of a mile below head of canal:

June 19: Width, 17 feet; area, 43 square feet; mean velocity, 1.51 feet per second; discharge, 64 second-feet.

August 31: Width, 14.5 feet; area, 25 square feet; mean -velocity, 2.12 feet per second; discharge, 53 second-feet.

Moore's power canal near Klamath Falls, Oreg.-This canal diverts water from the head of Link River and is used for power purposes in running a sawmill. The following measurement was made by C. T. Darley at a point one-half mile below head of canal:

March 25: Width, 9.9 feet; area, 34 square feet; mean velocity, 3.03 feet per second; discharge, 103 second-feet.

At a point 200 feet above Penstock the following measurements were made:

March 25: Width, 9 feet; area, 35 square feet; mean velocity, 2.14 feet per second; discharge, 75 second-feet.

June 21: Width, 9.5 feet; area, 34 square feet; mean velocity, 1.74 feet per second; discharge, 59 second-feet.

June 21: Width, 9.5 feet; area, 34 square feet; mean velocity, 1.56 feet per second; discharge, 53 second-feet.

Moore's irrigation canal near Klamath Falls, Oreg.-This canal diverts water from the head of Link River at same point at which water is diverted for Moore's mill-race canal on the west bank. This water is used for power purposes in running sawmill and the irrigation of town lots in the west addition of Klamath Falls, Oreg. The following measurements were made during 1905 by C. T. Darley at a point 300 feet below head of canal. These measurements show total amount of water used for power and irrigation purposes in this canal.

March 25: Width, 10 feet; area, 15.8 square feet; mean velocity, 0.82 foot per second; discharge, 12.9 second-feet.

June 21: Width, 12.4 feet; area, 10.1 square feet; mean velocity, 1.08 feet per second; discharge, 10.9 second-feet.

Phillip's wheel canal near Spring Lake, Oregon.-This canal diverts water from Lost River about 9 miles above Merrill, Oreg. The water is raised from the river by means of a water wheel and is used for irrigation purposes. The following measurements were made during 1905 by C. T. Darley at a point in flume 100 feet below point of diversion.

June 13: Width, 3 feet; area, 1.2 square feet; mean velocity, 1.00 foot per second; discharge, 1.2 second-feet.

July 25: Width, 1.2 feet; area, 0.1 square foot; discharge, 0.2 second-foot.

Rock Creek near Aspin Lake, Oregon.-This portion of Rock Creek is tributary to Upper Klamath Lake and has its source in Aspin Lake. A measurement was made on May 23 by C. T. Darley at bridge on wagon road between Klamath Falls and Pelican Bay.

Width, 23.5 feet; area, 16.9 square feet; mean velocity, 0.57 foot per second; discharge, 9.6 second-feet.

Rock Creek at Crystal, Oreg.-This stream is tributary to Crystal Creek and is a separate stream from the Rock Creek which discharges from Aspin Lake. A measurement was made on May 24 by C. T. Darley at bridge on wagon road between Klamath Falls and Pelican Bay.

Width, 19 feet; area, 8.9 square feet; mean velocity, 0.66 foot per second; discharge, 5.9 second-feet.

Scott River near Fort Jones, Cal.-Scott River is tributary to Klamath River. A measurement of this stream was made August 29, 1905, by W. B. Clapp 500 feet above wagon bridge on main road from Fort Jones to Etna, about 1 mile west from Fort Jones.

Width, 27 feet; area, 23 square feet; mean velocity, 1.17 feet per second; discharge, 27 second-feet.

Seven Mile Creek near Fort Klamath, Oreg.-This creek is tributary to Upper Klamath Lake from the north. A measurement was made on May 25 by C. T. Darley at bridge on wagon road between Fort Klamath and Pelican Bay. 
Width, 33 feet; area, 129 square feet; mean velocity, 0.79 foot per second; discharge, 102 second-feet.

Shasta River near Yreka, Cal.-Shasta River is tributary to Klamath River. A measurement of this stream was made August 29, 1905, by W. B. Clapp 5 miles northeast of Yreka and 500 feet below diversion dam of the Yreka Light and Power Company.

Width, 11.5 feet; area, 8.2 square feet; mean velocity, 1.22 feet per second; discharge, 10 second-feet.

There was on this date 107 second-feet being diverted into canal 500 feet above this point, giving a total discharge of 117 second-feet for this stream.

Sheepy Creek near Brownell, Cal.- This creek is tributary to Lower Klamath Lake from the southwest. The following measurements were made during 1905 by C. T. Darley at a point 2 miles east of Doris ranch, at wagon bridge near rock ford:

May 18: Width, 78 feet; area, 226 square feet; mean velocity, 0.12 foot per second; discharge, 27 second-feet.

June 18: Width, 80 feet; area, 218 square feet; mean velocity, 0.12 foot per second; discharge, 27 second-feet.

Sprague River near Yainax, Oreg.-Sprague River is tributary to Williamson River. A measurement was made on February 22 by C. T. Darley at bridge 5 miles below Yainax Agency. The gage is a 2 by 8 inch plank graduated to feet and tenths and nailed in a vertical position on the downstream side of the second bent from the south end of the bridge. The bench mark is a 2 by 4 inch stake driven flush with the ground on left bank of the stream in line with upstream side of the bridge and distant 74 feet from end of bridge. The zero of the gage is 21.9 feet below the bench mark.

Width, 133.2 feet; area, 1,077 square feet; mean velocity, 0.55 foot per second; discharge, 589 second-feet; gage height, 14.10 feet.

Spring Creek near Fort Klamath, Oreg.-This creek is tributary to Williamson River from the west. A measurement was made on August 13 by J. B. Lippincott and W. B. Clapp at a point 200 feet above its junction with Williamson River.

Width, 110 feet; area, 195 square feet; mean velocity, 1.86 feet per second; discharge, 362 second-feet.

Swingle flume canal near Lorella, Oreg.-This canal diverts water from Miller Creek above Lorella Bridge. The water is used for irrigation purposes in the vicinity of Lorella. A measurement was made on June 2 by C. T. Darley in small flume at road crossing on Langell Valley and Lorella road.

Width, 2 feet; area, 1.0 square foot; mean velocity, 0.20 foot per second; discharge, 0.2 second-foot.

Three Mile Creek near Fort Klamath, Oreg.-This creek is tributary to Upper Klamath Lake from the northwest. A measurement was made on May 25 by C. T. Darley at crossing of wagon road between Fort Klamath and Pelican Bay.

Width, 13.2 feet; area, 6.2 square feet; mean velocity, 1.00 foot per second; discharge, 6.2 second-feet.

Trinity River near.Lowden, Cal.--Trinity River is tributary to Klamath River. A measurement of this stream was made August 26, 1905, by W. B. Clapp from a temporary pontoon bridge 1 mile above Lowden's ranch post-office and 3 miles below Lewiston, Cal. A permanent county highway bridge was being constructed at this point.

Width, 190 feet; area, 468 square feet; mean velocity, 0.50 foot per second; discharge, 233 second-feet.

Varney Creek near Pelican Bay, Oregon.-Varney Creek is tributary to Four Mile Creek. A measurement was made May 24 by C. T. Darley at road crossing between Pelican Bay and Klamath Falls.

Width, 9 feet; area, 13 square feet; mean velocity, 0.70 foot per second; discharge, 9.1 second-feet.

Van Brimmer ditch, south branch, station No. 1, near Merrill, Oreg.-This canal diverts water from White Lake, an arm of Lower Klamath Lake. For the first mile this canal is 
common with the Adams canal. This water is used for irrigation in the vicinity of Merrill, Oreg. The following measurements were made on this branch by C. T. Darley during 1905 at point 1 mile below the head of canal and at a point 300 feet below where Adams and Van Brimmer canals become separate and distinct. The gage rod is a 1 by 4 inch inclined timber graduated to feet and hundredths and nailed to posts set firmly in the bank of the canal. There is no bench mark.

May 14: Width, 20 feet; area, 23 square feet; mean velocity, 1.04 feet per second; gage, 4.01 feet; discharge, 24 second-feet.

May 21: Width, 18 feet; area, 17.2 -square feet; mean velocity, 1.22 feet perr second; gage, 3.78 feet; discharge, 21 second-feet.

June 14: Width, 18.6 feet; area, 16 square feet; mean velocity, 1.28 feet per second; gage, 3.92 feet; discharge, 20 second-feet.

July 22: Width, 18.3 feet; area, 13.9 square feet; mean velocity, 0.83 foot per second; gage, 3.84 feet; discharge, 11.5 second-feet.

August 24: Width, 17.5 feet; area, 10.4 square feet; mean velocity, 0.72 foot per second; gage, 3.72 feet; discharge, 7.5 second-feet.

August 24: Width, 18.2 feet; area, 12.3 square feet; mean velocity, 0.80 foot per second; gage, 3.85 feet; discharge, 9.9 second-feet.

August 25: Width, 19.8 feet; area, 17.9 square feet; mean velocity, 0.91 foot per second; gage, 4.12 feet; discharge, 16.3 second-feet.

August 25: Width, 16.5 feet; area, 5.3 square feet; mean velocity, 0.49 foot per second; gage; 3.40 feet; discharge, 2.6 second-feet.

August 25: Width, 17.0 feet; area, 6.9 square feet; mean velocity, 0.62 foot per second; gage, 3.55 feet; discharge, 4.3 second-feet.

Van Brimmer ditch, north branch, station No. 2, near Merrill, Oreg.-This canal diverts water from. White Lake, an arm of Lower Klamath Lake. For the first mile this canal is common with the Adams canal. The following measurements were made of this branch during 1905 by C. T. Darley at a point 1 mile below heading and 300 feet below where Adams and Van Brimmer canals become separate and distinct. The gage is a 1 by 4 inch timber graduated to tenths and half tenths. It is set firmly to posts driven in the ground on the right bank. There is no bench mark.

May 14: Width, 13.8 feet; area, 8 square feet; mean velocity, 0.75 foot per second; gage, 1.95 feet; discharge, 6 second-feet.

May 21: Width, 14.3 feet; area, 7.5 square feet; mean velocity, 0.83 foot per second; gage, 1.99 feet; discharge, 6.2 second-feet.

June 15: Width, 15.4 feet; area, 7.4 square feet; mean velocity, 1.03 feet per second; gage, 2.21 feet; discharge, 7.6 second-feet.

July 24: Width, 16.5 feet; area, 10.4 square feet; mean velocity, 0.93 foot per second; gage, 2.34 feet; discharge 9.7 second-feet.

July 24: Width, 16.5 feet; area, 10.2 square feet; mean velocity, 0.93 foot per second; gage, 2.34 feet; discharge, 9.5 second-feet.

August 24: Width, 15.5 feet; area, 5.6 square feet; mean velocity, 0.79 foot per second; gage, 2.20 feet; discharge, 4.6 second-feet.

Willow Creek near Brownell, Cal.-Willow Creek is tributary to Lower Klamath Lake from the south. The following measurements were made during 1905 at bridge on Merrill and Brownell road by C. T. Darley:

May 20: Width, 20 feet; area, 17.2 square feet; mean-velocity, 0.72 foot per second; discharge, 12 .s second-feet.

June 15 Width, 35.5 feet; area, 24 square feet; mean velocity, 0.48 foot per second; discharge, 11.5 second-feet.

August 30: Width, 18 feet; area, 13.9 square feet; mean velocity, 0.70 foot per second; discharge, 9.7 second-feet.

November 23: Width, 10 feet; area, 12.6 square feet; mean velocity, 0.87 foot per second; discharge, 11 second-feet. 
Williamson River near Klamath Agency, Oreg.-This stream is tributary to Upper Klamath Lake from the north. The following measurements were made during 1905 at bridge on county road between Klamath Falls and Fort Klamath, Oreg.:

May 27: Width, 175.8 feet; area, 1,532 square feet; mean velocity, 0.89 foot per second; discharge, 1,371 second-feet.

August 22: Width, 176 feet; area, 1,479 square feet; mean velocity, 0.58 foot per second; discharge, 858 second-feet.

At Chillaquin Bridge on road between Klamath Agency and Yainax Agency, November 14: Width, 40 feet; area, 230 square feet; mean velocity, 2.72 feet per second; discharge, 625 second-feet.

Wood River near Fort Klamath, Oreg.-Wood River is tributary to Upper Klamath Lake from the north. The following measurements were made during 1905 by C. T. Darley at bridge on county road, 4 miles below Fort Klamath, Oreg.:

May 27: Width, 52.3 feet; area, 297 square feet; mean velocity, 1.54 feet per second; discharge, 458 second-feet.

At bridge one-fourth mile below Fort Klamath, Oreg.:

August 22: Width, 47.6 feet; area, 166 square feet; mean velocity, 1.60 feet per second; discharge, 266 second-feet.

November 14: Width, 49 feet; area, 162 square feet; mean velocity, 1.59 feet per second; discharge, 259 second-feet.

December 10: Width, 49 feet; area, 162 square feet; mean velocity, 1.69 feet per second; discharge, 274 second-feet.

Yreka'Light and Power Company's canal near Y reka, Cal.-A measurement of this canal was made August 29, 1905, by W. B. Clapp from footbridge at house 500 feet below concrete diversion dam at head of canal and distant about 5 miles northeast from Yreka. This canal diverts water from Shasta River.

Width, 17.5 feet; area, 36 square feet; mean velocity, 2.97 feet per second; discharge, 107 second-feet.

For total flow of Shasta River see measurement made same date below diversion dam.

ROGUE RIVER DRAINAGE BASIN.

ROGUE RIVER AT GOLD RAY, OREG.

This station was established August 30, 1905, by L. R. Allen. It is located at Gold Ray, Oreg., $1 \frac{1}{2}$ miles below Tolo post-office, just below the Condor Water and Power Company's dam and bridge, and a short distance below the mouth of Stewart Creek.

The channel is straight for 400 feet above and 300 feet below the station. The current at the measuring section is uniform. Both banks are high and rocky and are covered with a light growth of brush. The bed of the stream is rocky, somewhat broken in places, free from vegetation, and permanent. There is but one channel at all stages.

Discharge measurements are made by means of a cable, car, tagged wire, and stay line. The initial point is a tag on the tagged wire, 6.2 feet from the cable support on the left bank.

The gage is a vertical staff bolted to concrete pier of bridge about 300 feet above the cable. During 1905 the gage heights were furnished by the Condor Water and Power Company, observations being taken twice each day by C. E. Wertz. The bench mark is the top of the anchor bolt for stay line on the right bank. This bolt is well wedged in solid rock; elevation, 21.34 feet above the datum of the gage.

IRR $177-06-17$ 
Discharge measurements of Rogue River at Gold Ray, Oreg., in 1905.

\begin{tabular}{|c|c|c|c|c|c|c|}
\hline Date. & Hydrográpher. & Width. & $\begin{array}{l}\text { Area of } \\
\text { section. }\end{array}$ & $\begin{array}{c}\text { Mean } \\
\text { velocity. }\end{array}$ & $\begin{array}{c}\text { Gage } \\
\text { height. }\end{array}$ & $\begin{array}{c}\text { Dis- } \\
\text { charge. }\end{array}$ \\
\hline August & L. R. & $\begin{array}{r}\text { Feet. } \\
180\end{array}$ & $\begin{array}{r}S q \cdot f t \\
1,013\end{array}$ & $\begin{array}{r}\text { Ft. per sec. } \\
1.29\end{array}$ & $\begin{array}{r}\text { Feet. } \\
1.10\end{array}$ & $\begin{array}{l}\text { Sec.-ft. } \\
1,307\end{array}$ \\
\hline October $17 .$. & .....do ....... & 180 & 1,023 & 1.18 & $1: 20$ & 1,210 \\
\hline December $26 . .$. & .....do ........................... & 184 & 1,100 & 1.38 & 1.57 & 1,514 \\
\hline
\end{tabular}

Daily gage height, in feet, of Rogue River at Gold Ray, Oreg., for 1905.

\begin{tabular}{|c|c|c|c|c|c|c|c|c|c|}
\hline Day. & Sept. & Oct. & Nov. & Dec. & Day. & Sept. & Oct. & Nov. & Dec. \\
\hline $1 .$. & 1.1 & 1.2 & 1.2 & 1.32 & $17 \ldots$ & 1.2 & 1.25 & 1.22 & 1.32 \\
\hline $2 \ldots$ & 1.1 & 1.17 & 1.2 & 1.3 & $18 \ldots \ldots$ & 1.1 & 1.25 & 1.3 & 1.4 \\
\hline 3... & 1.1 & 1.15 & 1.2 & 1.3 & $19 \ldots \ldots$ & $1: 1$ & 1.25 & 1.3 & 1.45 \\
\hline $4 \ldots$ & 1.15 & 1.15 & 1.2 & 1.27 & $20 \ldots \ldots \ldots$ & 1.05 . & 1.25 & 1.5 & 1.47 \\
\hline 5. & 1.15 & 1.17 . & 1.2 & 1.45 & 21. & 1.05 & 1.25 & 1.3 & 1.4 \\
\hline $6 \ldots$ & 1.12 & 1.3 & 1.2 & 1.32 & $22 \ldots$ & 1.1 & 1.25 & 1.25 & 1.35 \\
\hline 7... & 1.1 & 1.45 & 1.2 & 1.35 & $23 \ldots$ & 1.1 & 1.2 & 1.25 & 1.17 \\
\hline $8 \ldots$ & 1.1 & 1.6 & 1.2 & 1.35 & $24 \ldots$ & 1.1 & 1.2 & 1.22 & 1.25 \\
\hline $9 .$. & 1.1 & 1.35 & 1.2 & 1.3 & $25 \ldots \ldots$ & 1.15 & 1.2 & 1.2 & 1.32 \\
\hline $10 \ldots$ & 1.6 & 1.25 & 1.2 & 1.3 & $26 . \therefore \ldots \ldots$ & 1.15 & 1.37 & 1.2 & 1.6 \\
\hline $11 \ldots$ & 1.1 & 1.2 & 1.2 & 1.2 & $27 \ldots \ldots \ldots$ & 1.17 & 1.35 & 1.3 & 1.82 \\
\hline $12 \ldots$ & 1.15 & 1.2 & 1.2 & 1.2 & $28 \ldots$. & 1.3 & 1.3 & 1.3 & 1.65 \\
\hline $13 .$. & 1.15 & 1.2 & 1.2 & 1.2 & $29 .$. & 1.2 & 1.35 & 1.4 & 1.5 \\
\hline $14 \ldots$ & 1.15 & 1.17 . & 1.2 & 1.3 & $30 \ldots$ & 1.2 & 1.3 & 1.3 & 1.6 \\
\hline $15 \ldots \ldots \ldots$ & 1.1 & 1.2 & 1.2 & 1.3 & $31 .$. & ...... & 1.22 & $\ldots$ & 1.52 \\
\hline $16 \ldots \ldots \ldots$ & 1.1 & 1.22 & 1.2 & 1.32 & & & & & \\
\hline
\end{tabular}

UMPQUUA RIVER DRAINAGE BASIN.

\section{SOUTH FORK OF UMFQUA RIVER NEAR BROCKWAY, OREG.}

This station was established December 6, 1905, by L. R. Allen. It is located just below Winston's highway bridge, 3 miles east of Brockway, Oreg., and 3 miles below the mouth of Lookingglass Creek.

The channel is straight for 700 feet above and below the station. The current is uniform, somewhat broken by riffles at low water. Both banks are high, covered with brush, and not liable to overflow. The bed of the stream is of sand and gravel, free from vegetation, and shifting. There is but one channel at all stages.

Discharge measurements are made by means of a cable, car, tagged wire, and stay wire. The initial point for soundings is a tin tag on the top of the cable support at the right bank:

The gage is a staff in two sections. The lower section is inclined; the upper section is vertical. During 1905 the gage was read once each day by George Brosi. The bench mark is the top of square-headed iron bolt driven into the downstream end of sill of the bridge at the left bank; elevation, 23.31 feet above the datum of the gage.

Discharge measurements of South Fork of Umpqua River near Brockway, Oreg., in 1905.

\begin{tabular}{|c|c|c|c|c|c|c|}
\hline Date. & Hydrographer. & Width. & $\begin{array}{l}\text { Area of } \\
\text { section. }\end{array}$ & $\underset{\text { velocity. }}{\text { Mean }}$ & $\begin{array}{c}\text { Gage } \\
\text { height. }\end{array}$ & $\begin{array}{l}\text { Dis- } \\
\text { charge. }\end{array}$ \\
\hline December $28 . .$. & L. R. Allen... & $\begin{array}{r}\text { Feet. } \\
302\end{array}$ & $\begin{array}{r}S q \cdot f t . \\
1,616\end{array}$ & $\begin{array}{r}\text { Ft.per sec. } \\
1.85\end{array}$ & $\begin{array}{r}\text { Feet. } \\
4.16\end{array}$ & $\begin{array}{r}\text { Sec. }-f t . \\
2,998\end{array}$ \\
\hline
\end{tabular}


Daily gage height, in feet, of South F'ork of Umpqua River near Brockway, Oreg., for 1905.

\begin{tabular}{|c|c|c|c|c|c|c|c|c|c|}
\hline Day. & Dec. & Day. & Dec. & Day. & Dec. & Day. & Dec. & Day. & Dec. \\
\hline & & 8. & 2.3 & 15. & 1.1 & 22 & 2.65 & 29. & 3.4 \\
\hline $2 \ldots$ & 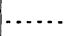 & $9 \ldots$ & 2.1 & $16 \ldots$ & 1.05 & 23. & 2.2 & $30 \ldots$ & 4.35 \\
\hline $3 \ldots$ & & $10 \ldots$ & 1.4 & $17 \ldots$ & 1.05 & $24 \ldots$ & 1.85 & $31 \ldots$ & 6.1 \\
\hline $4 \ldots$ & $\ldots$. & 11. & 1.4 & 18. & 1.1 & $25 \ldots$ & 1.1 & & \\
\hline $5 \ldots$ & ..... & 12. & 1.35 & 19. & 1.45 & 26. & 2.65 & & \\
\hline $6 .$. & 1.9 & 13. & 1.2 & $20 \ldots$ & 3.65 & 27. & 4.4 & & \\
\hline $7 \ldots \ldots$ & 1.9 & $14 \ldots$ & 1.15 & $21 \ldots$. & 3.3 & $28 \ldots \ldots$ & 4.1 & & \\
\hline
\end{tabular}

\section{INORTH FORK OF UMPQUA RIVER NEAR OAKCREEK, OREG.}

This station was established September 6,1905 , by L. R. Allen. It is located 3 miles west of Oakcreek, Oreg., $1 \frac{1}{4}$ miles above J. R. Dixon's farmhouse, about 10 miles below the mouth of East Fork of North Fork of Umpqua River.

The channel is straight for a half mile above and below the station. The current is swift. The right bank is low, covered with .brush, and liable to overflow during floods. The left bank is similar, but somewhat higher, and not as apt to overflow. The bed of the stream is of gravel and rock, somewhat broken, free from vegetation, and shifting during floods. There is but one channel at all stages. Measurements may be affected by uncertainties of soundings due to the irregularities of the bed of the stream.

Discharge measurements are made by means of a cable, car, tagged wire, and stay wire. The initial point for soundings is a tag on the tagged wire, 2 feet from the cable support on the right bank.

Two gage rods were installed-one at the place of measurement and the other about 1 mile below. The lower portion of both rods is inclined, and the upper portion is vertical. During 1905 the gage was read once each day by Ethel Dixon. The bench mark at the lower gage is on top of a $\frac{3}{4}$-inch bolt, driven into sand rock about 50 feet east and 50 feet south of the gage; elevation, 28.47 feet above the datum of the gage.

Discharge measurements of North Fork of Umpqua River near Oakcreek, Oreg., in 1905.

\begin{tabular}{|c|c|c|c|c|c|c|}
\hline Date. & Hydrographer. & Width. & $\begin{array}{l}\text { Area of } \\
\text { section. }\end{array}$ & $\underset{\text { velocity. }}{\text { Mean }}$ & $\begin{array}{c}\text { Gage } \\
\text { height. }\end{array}$ & $\begin{array}{c}\text { Dis- } \\
\text { charge. }\end{array}$ \\
\hline September 6. & L. R. Allen.................. & $\begin{array}{r}\text { Feet. } \\
215\end{array}$ & $\begin{array}{r}S q \cdot f t . \\
457\end{array}$ & $\begin{array}{r}\text { Ft. per sec. } \\
1.94\end{array}$ & $\begin{array}{l}\text { Feet. } \\
1.35\end{array}$ & $\begin{array}{r}\text { Sec.-ft. } \\
886\end{array}$ \\
\hline October $19 \ldots$ & 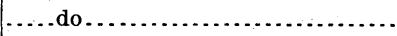 & 222 & 580 & 2.45 & 2.10 & 1,419 \\
\hline December $27 .$. & .....do... & 279 & 1,447 & 4.91 & 5.35 & 7,110 \\
\hline
\end{tabular}


Daily gage height, in feet, of North Fork of Umpqua River near Oakcreek, Oreg., for 1905.

\begin{tabular}{|c|c|c|c|c|c|c|c|c|c|}
\hline Day. & Sept. & Oct. & Nov. & Dec. & Day. & Sept. & Oct. & Nov. & Dec. \\
\hline 1. & 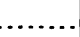 & 1.6 & 1.6 & 2.6 & 17. & 1.3 & 2.0 & 1.45 & 2.6 \\
\hline $2 \ldots$ & $\ldots:$ & 1.5 & 1.6 & 3.3 & 18. & 1.3 & 2.2 & 1.75 & 2.4 \\
\hline $3 \ldots$ & $\ldots$. & 1.4 & 1.55 & 3.35 & 19. & 1.35 & 2.1 & 1.9 & 2.6 \\
\hline $4 \ldots$. & $\ldots$ & 1.4 & 1.5 & 3.7 & $20 \ldots$ & 1.35 & 1.8 & 2.8 & 2.7 \\
\hline 5. & ...... & 1.4 & 1.5 & 3.3 & 21. & 1.35 & 1.65 & 2.2 & 3.1 \\
\hline $6 \ldots$ & 1.35 & 1.8 & 1.45 & 3.1 & $22 \ldots$ & 1.35 & 1.7 & 1.95 & ,2.8 \\
\hline $7 \ldots$ & 1.35 & 2.3 & 1.45 & 3.35 & 23. & 1.35 & 1.65 & 1.8 & 2.5 \\
\hline $8 .$. & 1.35 & 2.85 & 1.45 & 3.9 & $24 .$. & 1.3 & 1.6 & 1.75 & 2.4 \\
\hline $9 .$. & 1.3 & 2.1 & 1.45 & 3.1 & 25. & 1.35 & 1.75 & 1.65 & 2.35 \\
\hline $10 .$. & 1.35 & 1.75 & 1.4 & 3.75 & $26 .$. & 1.4 & 2.75 & 1.8 & 6.25 \\
\hline $11 \ldots$ & 1.3 & 1.65 & 1.4 & 2.6 & $27 \ldots \ldots$ & 1.5 & 2.3 & 2.7 & 5.35 \\
\hline $12 \ldots$ & 1.3 & 1.55 & 1.4 & 2.55 & 28.. & 1.65 & 2.0 & 2.2 & 4.35 \\
\hline $13 .$. & 1.3 & 1.5 & 1.4 & 2.5 & 29. & 1.6 & 1.8 & 2.2 & 3.6 \\
\hline $14 .$. & 1.3 & 1.45 & 1.4 & 2.4 & 30. & 1.5 & 1.7 & 2.7 & 4.5 \\
\hline $15 \ldots$ & 1.3 & 1.65 & 1.4 & 2.35 & 31.. & $\therefore .$. & 1.6 & $\cdots$ & 4.3 \\
\hline $16 .$. & 1.3 & 2.5 & 1.35 & 2.3 & & & & & \\
\hline
\end{tabular}

\section{SILETZ RIVER DRAINAGE BASIN.} SILETZ RIVER AT SILETZ, OREG.

This station was established November 25,1905 , by L. R. Allen. It is located at Siletz, Oreg., about 1 mile above the ferry on the Siletz and Toledo stage road, 6 miles below the mouth of Rock Creek.

The channel is straight for about 1,000 feet above and 400 feet below the station. The current is swift and rough. Both banks are high and not liable to overflow. The right bank is covered with brush; the left bank is timbered. The bed of the stream is of coarse gravel and sand, free from vegetation, and shifting. There is but one channel at all stages.

Discharge measurements are made by means of a cable, car, tagged wire, and stay wire. The initial point for soundings is a tin tag on the tagged wire, about 4 feet from the lower end of the turn-buckle on the right bank.

The gage is in two sections on the right bank. The lower section is inclined; the upper is vertical. During 1905 the gage was read once each day by John Kentta. The bench mark is the top of the head of a bolt driven into a large alder tree on the right bank, about 100 feet above the cable; elevation, 18.48 feet above the datum of the gage.

Discharge measurements of Siletz River at Siletz, Oreg., in 1905.

\begin{tabular}{|c|c|c|c|c|c|c|}
\hline Date. & Hydrographer. & Width. & $\begin{array}{l}\text { Area of } \\
\text { section. }\end{array}$ & $\begin{array}{c}\text { Mean } \\
\text { velocity. }\end{array}$ & $\begin{array}{c}\text { Gage } \\
\text { height. }\end{array}$ & $\begin{array}{l}\text { Dis- } \\
\text { charge. }\end{array}$ \\
\hline November 26 & L. R. Allen... & $\begin{array}{r}\text { Feet. } \\
157\end{array}$ & $\begin{array}{r}S q . f t . \\
394\end{array}$ & $\begin{array}{r}\text { Ft. per sec. } \\
2.95\end{array}$ & $\begin{array}{l}\text { Feet. } \\
\quad 2.15\end{array}$ & $\begin{array}{r}\text { Sec. }-f t . \\
1,167\end{array}$ \\
\hline
\end{tabular}

Daily gage height, in feet, of Siletz River at Siletz, Oreg., for 1905.

\begin{tabular}{|c|c|c|c|c|c|c|c|c|c|c|c|}
\hline Day. & Nov. & Dec. & Day. & Nov. & Dec. & Day. & Nov. & Dec. & Day. & Nov. & Dec. \\
\hline 1. & & 3.75 & 9. & & 2.9 & 17. & & 7.8 & 25. & & 3.2 \\
\hline 2. & & 4.2 & 10. & & 2.6 & $18 \ldots$ & & 6.0 & $26 .$. & 2.1 & 7.5 \\
\hline 3.. & & 3.8 & $11 .$. & 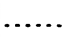 & 2.5 & 19. & & 5.3 & 27. & 2.0 & 5.2 \\
\hline $4 .$. & & 3.3 & 12. & & 2.3 & $20 \ldots$ & & 5.6 & $28 .$. & 2.0 & 4.3 \\
\hline $5 .$. & & 3.0 & $13 \ldots$. & & 2.2 & $21 \ldots$ & & 4.9 & $29 .$. & 2.7 & 3.8 \\
\hline 6. & & 3.7 & $14 \ldots \ldots$ & $\ldots$ & 2.1 & $22 \ldots$ & & 4.0 & $30 .$. & 3.2 & 4.3 \\
\hline $7 \ldots$ & & 3.3 & $15 .$. & & 2.0 & 23. & & 3.9 & $31 .$. & & 5.7 \\
\hline $8 \ldots$ & & 3.1 & $16 . .$. & 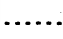 & 2.2 & 24. & & 3.1 & & & \\
\hline
\end{tabular}




\section{N D E X.}

A.

Acknowledgments and cooperation

Acre-foot, definition of .

Adams ditch near-

Merrill, Oreg.:

description

Adin, Cal.

Ash Creek at:

description

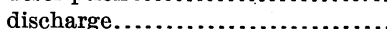

discharge, monthly ..............

gage heights ......................

rating table.

Rush Creek near:

description

discharge..........................

Willow Creek near:

description

discharge........................

Alamitos canal at-

Calexico, Cal.:

discharge....................... 31

discharge, monthly...............

gage keights.

Alamo channel near-

Rockwood, Cal.:

discharge..

gage heights.

Alturas, Cal.

E. Lauer \& Son's ditch near:

description

discharge.

Fitz Hugh Creek near:

description

discharge.

Pine Creek near:

description

discharge

Alvord, Cal.

Sanger canal at:

description

discharge

gage heights.

American River near-

Fairoaks, Cal.:

description

discharge.

discharge monthly.................

gage heights.................... 177

rating table .................. 177, 178

American River, North and Middle forks, near-

Auburn, Cal.:

description

discharge.
12

4

242

242

144

145

147

146

146

152

152

152

152

31

31,32

32

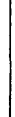

30

151

151

151

151

151 152

70

71

71

176

176

(778

8

American River, Silver and South forks, near-

Slippery Ford, Cal.:

description ....................... $\quad 179$

discharge..................... 179

American River, South Fork, near-

Placerville, Cal.:

description ...................... 178

discharge....................... 178

American River basin:

description ......................... 175-176

miscellaneous measurements ......... 178-179

Ankeny ditch and canal near-

Klamath Falls, Oreg.:

description ....................... 243

discharge......................... 243

Antelope Creek near-

Mount Hebron, Cal.:

description ........................ $\quad 242$

discharge....................... 242

Red Bluff, Cal.

description ...................... 132

discharge........................ 132

Arrowhead Springs, Cal.

East Twin Creek near: description ...................... 103

discharge...................... 103

Waterman Canyon (West Twin Creek) near:

description ...................... 104

discharge........................ 104

Arroyo Seco near-

Pasadena, Cal.:

description ..................... 109

discharge....................... 109

Soledad, Cal.:

description ..................... 123-124

discharge........................ 124

discharge, monthly .............. 126

gage heights..................... 125

rating table ...................... 125

Ash Creek at and near-

Adin, Cal.:

description ..................... 144

discharge......................... 145

discharge, monthly .............. 147

gage heights..................... 146

rating table ..................... 146

Lone Pine, Cal.:

description ..................... 84

discharge....................... 84

Aspin Lake, Oreg.

Rock Creek near:

description ...................... 246

discharge........................ 246 
Auburn, Cal.

American River, North and Middle forks, near:

description .

discharge.

Azusa, Cal.

San Gabriel River and canals nзar:

description

discharge........................ 105, 107

discharge, monthly ............... 107

gage heights...................... 105

rating table

B.

Baird Station, Cal.

Sacramento, River at:

description ......................... 131

discharge......................... 131

Bakersfield, Cal.

Kearn River near:

description ....................... $\quad 187$

discharge......................... 188

discharge, monthly ............... 189

Baldwin ditch near-

El Monte, Cal.:

description ............... 108

discharge........................ 108

Balls Ferry, Cal.

Battle Creek near:

description

Mill ditch near:

description ........................ 132

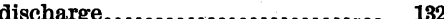

Banta ditch near-

Los Nietos, Cal.:

description

discharge

Battle Creek near-

Balls Ferry, Cal.:

description

Bay Counties Power Company flume near-

Nevada City, Cal.:

description ...................... 164

discharge....................... 164

Bear Creek, California:

description .......................... 200

discharge............................ 200

Bear River above and near-

Wheatland, Cal.:

description

discharge.

discharge, monthly

gage heights......................

rating table

Colfax, Cal.:

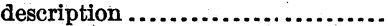

discharge

Bear River basin:

description ...........................

miscellaneous measurements

Beaver Creek, California:

description

discharge.

Beckwith, Cal.

Grizzly Creek near:

description ...................... 159

discharge....................... 159

Bieber, Cal.

Pit River near:

description $. . . \ldots \ldots \ldots \ldots \ldots \ldots \ldots . .136$

discharge........................ 137

discharge, monthly ............... 139

gage heights ..................... 138

rating table ..................... 138

Big Creek, California:

description ......................... 185

discharge.......................... 186

Big Pine, Cal.

Big Pine Creek near:

description $. . . \ldots \ldots \ldots \ldots \ldots \ldots \ldots . \quad 78$

discharge........................ 79

discharge, monthly ............... 80

gage heights..................... $\quad 79$

rating table ...................... 80

Clear Creek near:

description ..................... 84

discharge....................... 84

Fish Springs near:

description ...................... $\quad 85$

discharge........................ 85

Big Pine and Owens River canal near-

Bishop, Cal.:

description ...................... 69

discharge......................... 69

gage heights ..................... 70

Big Pine Creek near-

Big Pine, Cal.:

description $. . . \ldots \ldots \ldots \ldots \ldots \ldots \ldots \ldots . \quad 78$

discharge..................... 79

discharge, monthly .............. $\quad 80$

gage heights...................... 79

rating table ..................... 80

Big Tejunga Creek near-

Sunland, Cal.:

description ........... 109

discharge....................... 109

Birch Creek near-

Bishop, Cal::

description ....................... 84

discharge...................... 84

Tinemaha, Cal.

description ...................... $\quad 80$

discharge........................ 81

gage heights .................... 81

Bishop, Cal.

A. O. Collins canal near:

description ....................... 66

discharge........................ 67

gage heights ................... 67

Big Pine and Owens River canal near: description ....................... 69

discharge......................... $\quad 69$

gage heights ..................... 70

Birch Creek near: description ....................... 84

discharge...................... 84

Bishop Creek near:

description ...................... 62

discharge.......................... 63 
Bishop, Cal.-Continued.

Bishop Creek near-Continued.

discharge, monthly

gage heights.

Page.

rating table.

Bishop Creek canal near

description

discharge

gage heights.

Dell canal near:

description

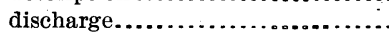

gage heights.

Farmers' canal near:

description

discharge....................

gage heights

George Collins canal near:

description

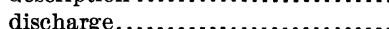

gage heights....................

McGee Creek near:

description

discharge.......................

McNally canal near:

description

discharge......................

gage heights.

North Hillside canal near:

description

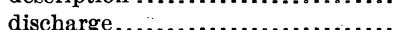

gage heights....................

Owens River canal near:

description

gage heights

Powers canal near:

description

discharge.

Rawson canal near:

description .

discharge. gage heights.................... 65,66

South Hillside canal near:

description

discharge

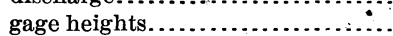

Bishop Creek near-

Bishop, Cal.:

description

discharge.......

discharge, monthly................

gage heights...................6 63,

rating table.

Bishop Creek canal near-

Bishop, Cal.:

description

discharge........................

gage heights......................

Blue Canyon Creek, California:

description

discharge.

Boards ditch, Poe Valley, Oregon:

description

discharge.
Bonanza, Oreg.

Bowne's west canal near:

description ....................... 243

discharge...................... 243

Boulder Creek, California:

description ................ 198

discharge......................... 198

Boundary canal near-

Calexico, Cal.:

discharge....................... 35

discharge, monthly .............. 35

gage heights..................... 35

Bowne's west canal near-

Bonanza, Oreg.:

description ....................... 243

discharge....................... 243

Bridal Veil Creek in-

Yosemite Valley, California:

description ........................ 209

discharge....................... 209

Brockway, Oreg.

Umpqua River, South Fork, near:

description ..................... 250

discharge....................... 250

gage heights .................... 251

Brownell, Cal.

Cottonwood Creek near:

description.

discharge...................... 244

Sheepy Creek near:

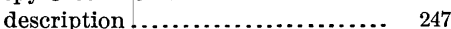

discharge...................... 247

Willow Creek near:

description ..................... 248

discharge........................ 248

Bubbs Creek, California:

description .......................... 199

discharge........................... 199

Butte Creek near-

Mount Hebron, Cal.:

description

discharge.

\section{C.}

Cable Canyon Creek near-

Glen Helen, Cal.:

description ...................... 102

discharge....................... 102

Cable station, figure showing ............. 8

Cache Creek at and near-

Lower Lake, Cal.:

description ...................... 169

discharge........................ 170

discharge, monthly .............. 172

gage heights...................... 171

rating table...................... 171

Yolo, Cal.:

description ...................... 172

discharge......................... 173

discharge, monthly .............. 174

gage heights.................... 173

rating table ...................... 174

Cache Creek basin:

description .......................... 168

miscellaneous measurements ........ 178-179 
Calabasas, Cal.

Malibu Creek near:

description ..................... 111

discharge....................... 111

discharge, monthly ............. 113

gage heights.................... 112

Triunfo Creek near:

description ..................... 113

discharge....................... 114

discharge, monthly ............... $\quad 115$

gage heights..................... 114

Calexico, Cal.

Alamitos canal at:

discharge..............

discharge, monthly ............... 32

gage heights..................... 31, 32

Boundary canal near:

discharge....................... 35

discharge, monthly ................

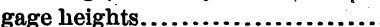

Hemlock canal near:

discharge...... 28

discharge, monthly .............. 29

gage heights.................... 28, 29

Holt canal near:

discharge...................... 26

discharge, monthly .............. 27

gage heights.................... 26, 27

Main canal near:

discharge....................... 33

discharge, monthly ................ 34

gage heights..................... 33,34

New Holt canal near:

discharge, monthly

New River near:

discharge.

Wisteria canal near:

discharge

gage heights.................... 36,37

California, southern:

Miscellaneous measurements...........

California-Mexico Land and Cattle Company's flume near-

Mexicala, Cal.:

description .........................

discharge, monthly ...............

Calpella, Cal.:

Russian River at:

description

discharge

Camulos, Cal.

Camulos ditch near:

description

Santa Clara River near

description

discharge.

Canals below Yuma, Ariz., measi rement of......................... 18-42

Canby, Cal.

Pit River near:

description

discharge....

discharge, monthly

gage heights......................

rating table
Capay ditch at-

Capay, Cal.:

description ...................... 175

discharge...................... 175

Cascade Creek in-

Yosemite Valley, California:

description .................... 209

discharge....................... 209

Cate ditch near-

El Monte, Cal.:

description ....................... 108

discharge....................... 108

Centerville, Cal.

Valley Counties Power Company's canal near:

description ....................... 133

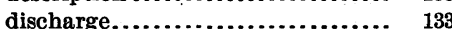

Cherry Creek near-

Crystal, Oreg.:

description ...................... 244

discharge...................... 244

Cherry River, California:

description ......................... 216

discharge............................ 216

Chino Creek near-

Rincon, Cal.:

description ....................... 102

discharge....................... 102

Citrus, Cal.

East Side canal near:

description ..................... 71

discharge....................... 72

gage heights..................... 72

Owens River near:

description ........................ 74

discharge $. . . . \ldots \ldots \ldots \ldots \ldots \ldots \ldots . .6 .75$

discharge, monthly............... $\quad 76$

gage heights...................... 75

rating table....................... 76

Stevens canal near:

description ....................... 73

discharge $. . . \ldots \ldots \ldots \ldots \ldots \ldots \ldots \ldots . .73$

gage heights..................... 74

City Creek near-

Highlands, Cal.:

description ...................... 102

discharge..............................

Clavey River, California:

description .......................... 216

discharge........................... 216

Clear Creek near-

Big Pine, Cal.:

description ...................... 84

discharge....................... 84

Stella, Cal.:

description ....................... 131

discharge........................ 131

Clear Lake at-

Lakeport, Cal.: description ..................... 168

evaporation ..................... 169

Clear Lake, Cal.

Lost River near:

description ...................... 233

discharge......................... 233

discharge, monthly............... 235

gage heights..................... 234

rating table ...................... 234 
Clements, Cal.

Mokelumne River near:

description ........................ 223

discharge....................... 223

discharge, monthly............... 225

gage heights...................... 224

rating table $. . . \ldots \ldots \ldots \ldots \ldots \ldots \ldots . \quad 224$

Clover Creek near-

Upper Lake, Cal.:

description

discharge.

Clover Creek and ditch at-

Millville, Cal.:

description

Cole Creek at-

Kelseyville, Cal.:

description ....................... $\quad 175$

discharge....................... 175

Colfax, Cal.

Bear River near:

description

discharge........................

Green Horn River near:

description

discharge........................

South Yuba Mining Company's ditch near:

description $. . . \ldots \ldots \ldots \ldots \ldots \ldots \ldots . . . . . . .$.

discharge .......................

Collins, A. O., canal near-

Bishop, Cal.:

description

discharge..

grge heights

Collins, George, canal near-

Bishop, Cal.:

description

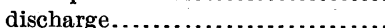

gage heights....................

Colorado River, diversion of, by Imperial

Canal, measurement of..........

Colorado River at and below-

Yuma, Ariz.

description

discharge, monthly............... 16

gage heights.................. 14, 15, 17

Heading No. 3 of Imperial Canal:

description

discharge.........................

gage heights.

Colorado River basin:

miscellaneous measurements in........

- Colorado Valley Pumping and Irrigation

Yuma, Ariz. Company's canal at-

description.

mputation, methods of............... 10-12

rules for

Converse Creek, California:

description .................... 198

discharge......................... 198

Cooperation and acknowledgments ....... 12

Copper City, Cal.

Pit River near:

description

discharge.
Copper City, Cal.-Continued.

Squaw Creek near:

description ...................... 132

discharge....................... 132

Copper Creek, California:

description ........................... 199

discharge......................... 199

Corporation ditch near-

Likely, Cal.:

description ..................... 151

discharge........................ 151

Cottonwood Creek near-

Brownell, Cal.:

description ...................... 244

discharge....................... 244

Jamul, Cal.:

description ...................... 126

discharge....................... 126

gage height....................... 126

Lakeview, Oreg.:

description ..................... 151

discharge...................... 151

Olancha, Cal.:

description $. . . \ldots \ldots \ldots \ldots \ldots \ldots \ldots \ldots . .6 \%$

discharge......................... $\quad 85$

gage heights.................... 85

Cottonwood Creek, North and Middle forks, near-

Gas Point, Cal.:

description ..................... 131, 132

discharge...................... 131, 132

Covelo, Cal.

Middle Eel River at:

description....................... 226

discharge........................ 226

Cow Creek at and near-

Millville, Cal.:

description ..................... 132

discharge...................... 132

Palocedro, Cal.:

description ........................ 132

discharge....................... 132

Coyote Creek, Oregon:

description .......................... 244

discharge.......................... 244

Crane Creek near-

Fort Klamath, Oreg.:

description ..................... 244

discharge........................ 244

Crescent Mills, Cal.

Indian Creek near:

description ................. 159

discharge....................... 159

Crooked Creek near-

Klamath Agency, Oreg.:

description .................... 244

discharge....................... 244

Crown Creek, California:

description....................... 200

discharge.......................... 200

Crystal, Oreg.

Cherry Creek near:

description $\ldots . \ldots \ldots \ldots \ldots \ldots \ldots . .244$

discharge....................... 244

Rock Creek at:

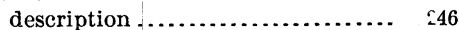

discharge....................... 246

Current meters, classes of ................ 8

methods of using.................... $8-10$ 
Curves for area, discharge, and mean velocity, figure showing........

\section{D.}

\section{Davis ditch near-}

Downieville, Cal.:

description

discharge.

Deer Creek near-

Vina, Cal.:

description

discharge.

Dell canal near-

Bishop, Cal.:

description

discharge...

gage heights.

Devil Canyon near-

Irvington Station, Cal.:

description

Dinkey Creek, California:

description

discharge

Dirty Creek, Oregon:

description

133

133

discharge

Division Creek near-

Independence, Cal.:

description

discharge.

Doris Creek near-

Picard, Cal.:

description

discharge.....

Dougherty Creek, California:

description ........................... 200

discharge............................ 200

Downieville, Cal.

Davis ditch near:

description .

discharge......................... 165

Wheeler flume near:

description ....................... 164

discharge......................... 164

Yuba River, East Fork of North Fork, at: description ...................... 165 discharge........................ 165

Yuba River, Nork Fork, at: description discharge.

Yuba River, North Fork of North Fork at and near:

description

164,165

discharge.

$164,1 \mathrm{C5}$

Drainage basins, list of ................. 2-3

Duke's dutch near-

Likely, Cal.:

description ......................

discharge.

Duty of water in Imperial Valley

151

151

E.

East Highlands, Cal.

Plunge Creek near:

description

discharge
East Side canal neur-

Citrus, Cal.:

description ..................... 71

discharge........................ 72

gage heights..................... 72

East Twin Creek near-

Arrowhead Springs, Cal.:

description ...................... 103

discharge. 103

Edson Foulks ditch, Shasta Valley:

description ......................... 244

discharge............................ 244

Eel River at-

Laytonville, Cal.:

description ...................... 226

discharge...................... 226

Eel River basin:

miscellaneous measurements......... 225-226

Eight Mile Creek near-

Independence, Cal.:

description

discharge $\ldots \ldots \ldots \ldots \ldots \ldots \ldots \ldots \ldots \ldots . \quad 85$

El Dorado ditch below-

Slippery Ford, Cal.:

description........................ $\quad 179$

discharge ........................ 179

El Monte, Cal:

Baldwin ditch near:

description ...................... 108

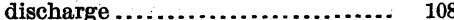

Cate ditch near:

description ....................... 108

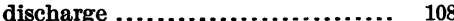

Ranchito or Standerford ditch near:

description ....................... 108

discharge ....................... 108

Rincon ditch near:

description ...................... 108

discharge ....................... 108

Rio Hondo near:

description ................... 108

discharge. ....................... 108

San Gabriel River near:

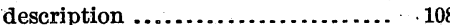

discharge ....................... 108

Sheep Creek ditch near:

description $\ldots \ldots \ldots \ldots \ldots \ldots \ldots \ldots . .108$

discharge...................... 108

Eleanor Creek, California:

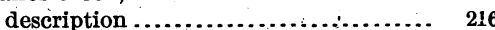

dischärge........................... 216

Equivalents, table of ................... $5-6$

Esperanza, Cal.

Piru Land and Water Company's upper diversion near:

description ..................... 116

discharge....................... 116

F.

Fairoaks, Cal

American River near:

description ..................... 176

discharge........................ 176

discharge, monthly................ 178

gage heights..................... 177

rating table .................... 177, 178 
Falls Creek, California:

description

discharge

Farmers' canal near-

Bishop, Cal.:

description

Santa Paula, Cal.

description ...................... 116

discharge......................... 116

Yuma, Ariz.:

description ...................... 19

discharge........................ 19

Feather River at-

Oroville, Cal.:

description ..................... 155-156

discharge....................... 156

discharge, monthly .............. 158

gage heights................... 157

rating table...................... 158

Prattville, Cal.:

description ...................... 159

discharge....................... 159

Feather River, Middle Fork, at-

Mohawk Valley, Cal.:

description

.......... 159

Feather River basin:

description

miscellaneous measur

Fernando, Cal.

Pacoima Creek near:

description

discharge

Fish Creek, California:

description

discharge.

Fish Springs near-

Big Pine, Cal.:

description

discharge.

Fitz Hugh Creek near-

Alturas, Cal.:

description

Floats, use of in measuring dis..........

Fort Klamath, Oreg.

Crane Creek near:

description

Seven-Mile Creek near:

description

Spring Creek near:

description

Three Mile Creek near:

description

discharge.

Wood River near:

description

discharge

Fort Jones, Cal.

Scott River near:

description

discharge.
Four Mile Creek, Pelican Bay, Oregon:

description......................... 244

discharge.......................... $\quad 245$

Fruto, Cal.

Stony Creek basin:

description ...................... 153

discharge...................... 153

discharge, monthly ............... 155

gage heights..................... 154

rating table ..................... 154

G.

Gaging stations, equipment of .......... $\quad 7-8$

Ganstad's ditch near-

Likely, Cal.:

description ...................... 151

discharge....................... 151

Gas Point, Cal.

Cottonwood Creek, North and Middle forks, near:

description .................... 131, 132

discharge...................... 131, 132

Georges Creek near-

Independence, Cal.description ...................... 85 discharge..................... 85

Glen Helen, Cal.

Cable Canyon Creek near: description ...................... 102

discharge..................... 102

Goddard Creek, California:

description ......................... 199

discharge........................... 200

Gold Ray, Oreg.

Rogue River at:

description ....................... 249

discharge....................... 250

gage heights................... 250

Gooch's ditch near-

Lookout, Cal.:

description ..................... 151

discharge....................... 151

Goodale Creek near-

Tibbetts, Cal.:

description $. . \ldots \ldots \ldots \ldots \ldots \ldots \ldots \ldots . .85$

discharge....................... 85

Goodyears bar, California.

Yuba River, North Fork, near:

description ...................... 164

discharge..................... 164

Granite Creek, California:

description ........................ 199

discharge.......................... 199

Grass Valley Creek near-

Lowden, Cal.:

description...................... 245

discharge......................... 245

Green Horn River near-

Colfax, Cal.:

description ..................... 168

discharge....................... 168

Grees ditch near-

Santa Paula, Cal.:

description ...................... 116

discharge........................ 116 
Gregory, Cal.

McCloud River near:

description ...................... 147

discharge....................... 148

discharge, monthly .............. 150

gage heights...................... 148

rating table....................... 149

Sacramento River at:

description ..................... 131

discharge........................ 131

Griffith canal near-

Olene, Oreg.:

description ................... 245

discharge........................ 245

Griswald Creek, California:

description ...................... 222

discharge............................. 222

Grizzly Creek near-

Beckwith, Cal.:

description

discharge.

Guenoc, Cal.

Puta Creek near:

description

discharge.

discharge, monthly ...............

gage heights

rating table

H.

Heading No. 3 of Imperial Canal.

Colorado River below:

description

discharge.

Hearst, Cal.

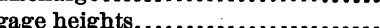

South Eel River at:

description

Hemlock Canal near-

Calexico, Cal.:

discharge.

discharge, monthly

gage height

Herndon, Cal.

San Joaquin River at:

description

gage heights

Hetch Hetchy, Cal.

Tuolumne River at:

description

Highlands, Cal

City Creek near:

description ..................... 102

discharge........................ 102

Holt Canal near-

Calexico, Cal.:

discharge....................... 26

discharge, monthly ................ 27

gage heights...................... 26, 27

Home Camp Creek, California:

description ...................... 185

discharge....................... 185

Horse Fly, near Lorella, Oreg., precipitation at..... 242

Horseshoe Creek, California:

description ......................... 199

discharge.
Horton ditch at-

Poe Valley, Oreg.:

description

Hot Springs Creek near-

Klamath Falls, Oreg.:

description ........................ 245

discharge........................ 245

Hotel Creek, California:

description ......................... 199

discharge........................... 199

Hydrographic surveys, annual appropriations for .................... 1

organization and scope of ............ 1-3

I.

Ice-covered streams, methods of measuring

Imperial, Cal. flow of.........................

McKim flume near:

description ...................... 40

discharge, monthly ............... 41

rainfall records at..................... 41

Tamarack canal near:

discharge...................... 38

discharge, monthly ............... 38

gage heights.................... 38

Imperial canal below-

Quail River cût-off, Mexico:

discharge.

Imperial canal in-

United States and Mexico:

description

discharge....................... 23, 24

discharge, monthly ................ 25

Imperial Valley:

duty of water in.................... $\quad 40$

lands irrigated in .................... 40

soil of, tests of ........................ 40,41

Imperial Valley canals:

description .......................... 25

discharge........ 26, 28, 30, 31, 33, 35, 36, 38,39

discharge, monthly ..... 27-29, 32, 34, 35, 37, 38

gage heights............. 27, 29, 32, 34, 37, 28

Independence, $\mathrm{Cal}$.

Division Creek near:

description

discharge....................... 85

Eight Mile Creek near:

description ...................... 85

discharge...

Georges Creek near:

description ....................... 85

discharge.......................... \&5

Independence Creek near:

description ....................... 81

discharge........................ 82

discharge, monthly ............... 83

gage heights..................... 82

rating table ..................... 83

Moffett Creek near:

description ...................... 86

discharge......................... 86

Oak Creek near:

description ....................... 83

discharge........................ 83

gage heights...................... 
Independence, Cal.-Continued.

Shepherds Creek near:

description

discharge........................

Independence Creek near-

Independence, Cal.

description .....................

discharge.

discharge, monthly. . ............

gage heights....................

rating table.

Indian Creek near-

Crescent Mills, Cal.:

description

discharge.......................

Irrigation in Imperial Valley, amount of .

Irvington Station, Cal.

Devil Canyon near:

description

$$
\text { discharge. }
$$

Ivy, Cal.

Pit River, South Fork, near:

description

discharge

\section{J.}

Jamul, Cal.

Cottonwood Creek near:

description

discharge.

gage heights

K.

Kaweah River below-

Three Rivers, Cal.:

description

discharge

discharge, monthly

gage heights

rating table.

Kaweah River, East Fork, at-

Mount Whitney Power Company's power house, California:

description .......................

discharge........................

Kaweah River, Middle Fork, in-

Sequoia National Park, Cal.:

description

discharge ......................

Kaweah River, North and South forks, near-

Three Rivers, Cal.:

description ....................... 194, 195

discharge...................... 194, 195

Kaweah River basin:

description .......................... 192

miscellaneous measurements ........ 194-195

Kelsey Creek near-

Kelseyville, Cal.:

description ..................... 175

discharge...........................

Kelseyville, Cal.

Cole Creek at:

description ....................... 175

discharge ....................... " 175
Keno, Oreg.

McCormick mill-race canal near:

description ........................ 245

discharge....................... 246

Klamath River at:

description ....................... 229

discharge........................ 230

discharge, monthly ............... 231

gage heights...................... 230

rating table ...................... 231

precipitation......................... 242

evaporation......................... 242

Kern River near-

Bakersfield, Cal.:

.description ...................... 187

discharge....................... 188

discharge, monthly ............... 189

Kern River basin:

description ......................... 187

King Creek, California: ,

description .......................... 186

discharge.......................... 186

Kings River near-

Sanger, Cal.:

description ....................... 196

discharge....................... 196

discharge, monthly ................ 198

gage heights...................... 197

rating table .................... 197

Kings River, Middle, North, and South forks, California:

description .................... 198, 199, 200

discharge...................... 198, 199, 200

Kings River basin: description ......................... 195

miscellaneous measurements ........ 198-200

Klamath Agency, Oreg.

Crooked Creek near:

description ...................... 244

discharge........................ 244

Williamson River near:

description ...................... 249

discharge........................ 249

Klamath Falls, Oreg.

Ankeny ditch and canal near: description ........................ 243

discharge........................ 243

Hot Springs Creek near:

description ....................... 245

discharge......................... 245

Link River at:

description ....................... 226

discharge....................... 227

discharge, monthly ............... $\quad 229$

gage heights......................

rating table .................... 228

Moore's irrigation and power canals near:

description ....................... 246

discharge........................... 246

Klamath River at-

Keno, Oreg.

description ....................... 229

discharge........................ $\quad 230$

discharge, monthly .............. 231

gage heights..................... 230

rating table .................... 231 
Knights Ferry, Cal.

Stanislaus River at:

description

Page.

217-218

discharge........................ 218

discharge, monthly .............. 220

gage heights..................... $\quad 219$

rating table $\ldots \ldots \ldots \ldots \ldots \ldots \ldots \ldots \ldots . \quad 219$

Stanislaus Water Company's ditch at:

description

discharge.

gage heights....

rating table

L.

Lagrange, Cal.

Mining ditch near:

description .

discharge.

Modesto canal at:

description ...................... 212-213

discharge.........................

gage heights...................... 213

rating table ....................... 214

Tuolumne River at:

description ...................... 209-210

discharge.......................... 210

discharge, monthly ............. $\quad 212$

gage heights...................... 211

rating table ....................... 212

Turlock canal at:

description ...................... 214

discharge......................... 214

gage heights..................... 215

rating table ...................... 215

Lakeside, Cal.

San Diego River near:

description

discharge.........................

Lakeview, Oreg.

Cottonwood Creek near:

description

discharge.

Lauer, E., \& Son's ditch near-

Alturas, Cal.:

description ........................

discharge........................... 151

Laurel Creek, California:

description

Laytonville, Cal.

Eel, Middle Eel, and South Eel rivers at:

description .........................

discharge.

Lewis Creek, California:

description

discharge.

Lightning Creek, California:

description

discharge.

Likely, Cal.

Corporation ditch near:

description

Duke's ditch near:

description

discharge.
Likely, Cal.-Continued.

Ganstad's ditch near:

description ....................... 151

discharge....................... 151

Van Loam's ditch near:

description ..................... 152

discharge........................ 152

West Valley Creek near:

description ....................... 140

discharge........................ 141, 143

discharge, monthly ............... 144

gage heights .................... 142

Link River at-

Klamath Falls, Oreg.:

description ...................... 226

discharge......................... 227

discharge, monthly .............. $\quad 229$

gage heights ..................... 228

rating table...................... 228

Little Cow Creek near-

Palocedro, Cal.:

description $. . . \ldots \ldots \ldots \ldots \ldots \ldots \ldots . .132$

discharge........................ 132

Little Shasta River and Springs at-

Little Shasta, Cal.:

description ....................... 245

discharge......................... 245

Little Tejunga Creek near-

Sunland, Cal.:

description ...................... 110

discharge........................ $\quad 110$

Lone Pine, Cal

Ash Creek near:

description ...................... 84

discharge........................ 84

Lone Pine Creek near:

description ..................... 85

discharge........................ 85

Tuttle Creek.near: description ...................... $\quad 86$

discharge......................... 86

Lone Pine Creek near-

Lone Pine, Cal.:

description ...................... 85

discharge........................ 85

Long Creek, Oregon:

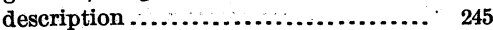

discharge............................. 245

Lookout, Cal.

Gooch's ditch near: description ........................ 151 discharge....................... 151

Lookout; Cal.

Pit River, at:

description ....................... 152

discharge........................ 152

Lorella, Oreg.

Miller Creek near:

description ....................... 239

discharge......................... $\quad 240$

discharge, monthly ............... 241

gage heights ....................... 240

rating table ....................... 241

Swingle flume canal near:

description ....................... 247

discharge........................... 247

precipitation near.................... $\quad 242$ 
Los Angeles River, at-

Los Angeles, Cal.:

description

Page. | McNally canal near-

Bishop, Cal.:

discharge.

Los Angeles River basin:

description

discharge

gage heights.

Page.

description ......................... 109

miscellaneous measurements ........ 109-110

Los Nietos ditch near-

Los Nietos, Cal.:

description........... 108

discharge....................... 108

Lost River near-

Clear Lake, Cal.:

description.

discharge.........

discharge, monthly

gage heights ......................

rating table

Main canal near-

Calexico, Cal.:

discharge......................... 33

discharge, monthly................ 34

gage heights.................... 33, 34

Malibu Creek near-

Calabasas, Cal.:

description ...................... 111

discharge........................ 111

discharge, monthly............... 113

gage heights................... 112

Malibu Creek basin.

description .......................... 111

Merrill, Oreg.:

description................... 236

discharge......................... 236

discharge, monthly .............. $\quad 238$

gage heights .................... 237

rating table $\ldots \ldots \ldots \ldots \ldots \ldots \ldots \ldots \ldots . .237$

Lowden, Cal.

Grass Valley Creek near:

description.................... 245

discharge....................... 245

Trinity River near:

description....................... 247

discharge....................... 247

Lower Lake, Cal.

Cache Creek at:

description ...................... 169

discharge........................ 170

discharge, monthly ............... 172

gage heights ................... 171

rating table ..................... 171

Ludy canal near-

Yuma, Ariz.:

description .................... 19-20

discharge........................ 20

gage heights ..................... 20

Lytle Creek near-

Rialto, Cal.:

description

discharge.

M.

McCloud River near-

Gregory, Cal.:

description ........................

discharge.

discharge, monthly ............... 150

gage heights..................... 148

rating table ...................... $\quad 149$

McCormick mill-race canal near-

Keno, Oreg.

description

ble Fork in-

Sequoia National Park, Cal.:

description .......................

discharge.

Mentone, Cal.

Mill Creek near:

description...................... 103

discharge......................... 103

Morton Canyon Creek near:

description ..................... 103

discharge........................ 103

Redlands tunnel near:

description .................... 103

discharge........................ 103

Santa Ana River near:

description ..................... 94-95

discharge........................ 95, 98

discharge, monthly................ 98

gage heights..................... 96

rating table...................... 96,97

Merced Falls, Cal.

Merced River above:

description...$\ldots \ldots \ldots \ldots \ldots \ldots \ldots .203$

discharge........................ 203

discharge, monthly............... 205

gage heights...................... 204

rating table....................... 204

Merced River in-

Yosemite Valley, California:

description ...................... 201

discharge........................ 201

discharge, monthly................ 203

gage heights...................... 202

rating table...................... 202

Merced River basin.

description ......................... 200

miscellaneous measurements ......... 209

Merrill, Oreg.

Adams ditch near:

description ...................... 242

discharge $. . . \ldots \ldots \ldots \ldots \ldots \ldots \ldots \ldots . .242$

Lost River near:

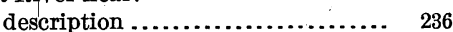

discharge...................... $\quad 236$

discharge, monthly................ 238

gage heights..................... 237

rating table..................... 237

Tule Lake near:

description ....................... $\quad 238$

gage heights.................... 239

1

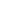

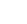

11

3

1

4

94

3

3

03


Merrill, Oreg.-Continued.

Van Brimmer ditch near: description discharge.

Merrillville, Cal.

Willow Creek at:

description

discharge, monthly

gage heights.

rating table

Methods of computing run-off

of measuring stream flow

Mexicala, Cal.

California-Mexico Land and Cattle Company's flume near:

description

discharge, monthly

Middle Creek near-

Upper Lake, Cal.:

description

discharge.

Middle Eel River at-

Covelo, Cal.:

description

discharge.

Laytonville, Cal.:

description

discharge.

Middle Fork, etc. See name of river.

Mill Creek near-

Mentone, Cal.:

description

discharge:

Tehama, Cal.:

description

discharge.

Mill ditch near-

Balls Ferry, Cal.:

description

Miller Creek near-

Lorella, Oreg.:

description

discharge

discharge

gage heights. .........................

rating table

Millrace canal near-

Tehama, Cal.:

description

Millville, Cal.

Clover Creek and ditch at:

description

discharge.

Cow Creek at:

description

discharge.

Miner's inch, definition of

Mining company's flume near-

Sierra City, Cal.:

description

discharge.

Modesto canal at-

Lagrange, Cal.:

description

discharge.

gage heights

rating table
Page.

247,248

248

5

45

46

46

10-11

6-10

41

41

175

175
Moffett Creek near-

Independence, Cal.:

description

discharge.

Mohave River at-

Victorville, Cal.: description ..................... 87

discharge....................... 88, 90

discharge, monthly ............... 90

gage heights .................... 89

Mohave River basin:

description $\ldots . \ldots \ldots \ldots \ldots \ldots \ldots \ldots \ldots, \quad 87$

Mohawk Creek near-

New Mohawk, Cal.:

description ...................... 159

discharge....................... 159

Mohawk Valley, Cal.

Feather River, Middle Fork, at:

description ...................... 159

discharge....................... 159

Mokelumne River near-

Clements, Cal.:

description ..................... 223

discharge......................... 223

discharge, monthly .............. 225

gage heights .................... 224

rating table ..................... 224

Mokelumne River basin:

description .......................... 223

Mono Creek, California:

description ........................ 186

discharge............................. 186

Moore's ditch near-

Woodland, Cal.:

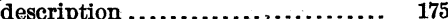

discharge........................ 175

Moore's irrigation and power canals nearKlamath Falls, Oreg.:

description ...................... 246

discharge.......................... 246

Morton Canyon Creek near-

Mentone, Cal.: description ...................... 103

discharge....................... 103

Moss Creek near-

Pelican Bay, Oregon:

description ........................ 245

discharge........................ 245

Mount Hebron, Cal.

Antelope Crcek near: description ...................... 242

discharge........................ $\quad 242$

Butte Greek near:

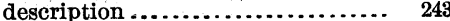
discharge........................ 243

MountWhitney PowerCompany's flumeatSequoia National Park, Cal.:

description ........................ 195

discharge....................... 195

Mount Whitney Power Company's power house, California.

Kaweah River, East Fork, at: description ...................... 195

discharge........................ 195

Mugler Creek, California:

description .......................... 186

discharge........................... 186

Multiple-point method of measuring discharge, description of ..........

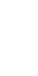

7

9

59

3

4

3

6

75

75

\section{.} . ${ }_{215}^{215}$

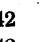

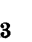
政 西

${ }_{105}^{105}$


N.

Nevada City, Cal.

Bay Counties Power Company flume near:

description

discharge.

Yuba River, Middle Fork, near:

description

discharge.

New Holt canal near-

Calexico, Cal.:

discharge, monthly

New Mohawk, Cal.

Mohawk Creek near:

description

discharge..........................

New River near-

Calexico, Cal.:

discharge.

gage heights.....................

North Fork, etc. See name of river.

North Hillside canal near-

Bishop, Cal.:

description

gage heights. ....................

o.

Oak Creek near-

Independence, Cal.:

description

discharge........................

gage heights......................

Oakcreek, Oreg.

Umpqua River, North Fork, near:

description .......................

discharge.........................

gage heights......................

Olancha, Cal.

Cottonwood Creek near:

description

discharge.

gage heights.

Olene, Oreg.

Griffith Canal near:

description

discharge.

Page.

Owens River basin:

Page.

description ........... 49

miscellaneous measurements in....... 84-86

Owens River Canal near-

Bishop, Cal.:

description $. . . \ldots \ldots \ldots \ldots \ldots \ldots \ldots . .57$

discharge....................... 57

gage heights .................. 57, 58

\section{P.}

Pacific Ocean drainage, northern:

miscellaneous measurements

Pacoima Creek near-

Fernando, Cal.:

description ...................... $\quad 110$

discharge..................... 110

Pala, Cal.

San Luis Rey River near:

description ........................ 91

discharge...................... 92

discharge, monthly ............... 93

gage heights...................... 92

Palocedro, Cal.

Cow Creek near:

description $. . . \ldots \ldots \ldots \ldots \ldots \ldots \ldots . .132$

discharge...................... 132

Little Cow Creek near:

description ....................... 132

discharge........................ 132

Parrotts Ferry, Cal.

Sțanislaus River at:

description ....................... 222

discharge......................... 222

Pasadena, Cal.

Arroyo Seco, Cāl.:

description ....................... 109

discharge...................... 109

Pelican Bay, Oregon.

Moss Creek near:

description ....................... 245

discharge....................... 245

Varney Creek near:

description ...................... 247

discharge.................... 247

Phillip's wheel canal near-

Spring Lake, Oreg.

description ..................... 246

discharge....................... 246

Picard, Cal.

Doris Creek near:

description ...................... 244

discharge.

Pine Creek near-

Alturas, Cal.:

description ...................... 151

discharge...................... 152

Round Valley, Cal.:

description ...................... 55

discharge....................... 55

discharge, monthly .............. 57

gage heights...................... 、 56

rating table....................... 56

Piru City, Cal.

Piru Creek near:

description ...... 116

discharge....................... 116

rating table $\ldots \ldots \ldots \ldots \ldots \ldots \ldots \ldots \ldots \ldots . . . \ldots$

IRR $177-06-18$ 
Piru Land and Water Company's upper diversion near-

Esperanza, Cal.

description ......................

discharge.

Pit River at and near-

Bieber, Cal.;

description .

discharge.........................

discharge, monthly ...............

gage heights......................

rating table..................... 138

Canby, Cal.:

description ...................... 134

discharge........................ 134

discharge, monthly .............. 136

gage heights....................... 135

rating table...................... 135

Copper City, Cal.:

description ...................... 132

discharge....................... 132

Lookout, Cal.:

description ..................... 152

discharge........................ 152

Pit River, South Fork, near-

Ivy, Cal.:

description

discharge.

Pit River basin:

description

miscellaneous measurements.

Pitman Creek, California:

description

discharge.............................

Placerville, Cal.:

American River, South Fork, near-

description. ......................

discharge.

Plunge Creek, near-

East Highlands, Cal.:

description

discharge.

Poe Valley, Oreg.

Horton ditch at:

description

discharge

Portersville, Cal.

Tule River, near:

description

discharge........................

discharge, monthly ................

gage heights.

rating table.......................

Powers canal near-

Bishop, Cal.:

description

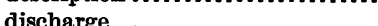

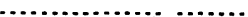

Prattville, Cal.

gage heights..

Feather River at-

description

discharge.

Preston, Cal.

Russian River at-

description.

discharge.
Puta Creek at and near-

Guenoc, Cal.:

description ....................... 180

discharge..................... 180

discharge, monthly .............. 182

gage heights..................... 181

rating table ...................... 182

Winters, Cal.:

description ................... 182-183

discharge....................... 183

gage heights..................... 183

Puta Creek basin:

description

Q.

Quail River cut-off, Mexico.

Imperial canal below:

discharge.

R.

Rainfall, record of, at-

Imperial, Cal....................... 41

Keno, Lorella, and Tule Lake,Oreg .... 242

Rancheria Creek, California:

description ......................... 216

discharge.......................... 216

\section{Red Bluff, Cal.}

Antelope Creek near:

description

discharge........................ 132

Sacramento River near:

description

discharge........................ 129

discharge, monthly ............... 130

gage heights..................... 129

rating table ....................... 130

Redlands, Cal.

Santa Ana River near:

description ..................... 108

discharge....................... 108

Redlands tunnel near-

Mentone, Cal.:

description ...................... 103

discharge........................ 103

Reds Creek, California:

description .......................... 186

discharge........................... 186

Rialto, Cal.

Lytle Creek near:

description ................... 103

discharge........................ 108 
Ribbon Falls Creek in-

Yosemite Valley, California:

description ......................

discharge.

Rincon, Cal.

Chino Creek near:

description

Santa Ana River near:

description .

gage height....

Rincon ditch near-

El Monte, Cal.:

description .......................

discharge.

Rio Hondo near-

El Monte, Cal.:

description

discharge.

Roaring River, California:

description

discharge.

Rock Creek at and near-

Aspin Lake, Oregon:

description

discharge.

Crystal, Oreg.:

description

discharge

Round Valley, Cal.:

description ......................

discharge.

discharge, monthly

gage heights.

Rockwood, Cal.

Alamo channel near:

discharge...

gage heights.

Rogue River, at-

Gold Ray, Oreg.:

description

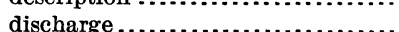

gage heights......................

Round Valley, Cal.

Pine Creek near:

description

discherge, m.......................

charge, monthly...............

gage heights......................

rating table...$\ldots \ldots \ldots \ldots \ldots \ldots \ldots$.

Owens River near:

description

discharge........................

discharge, monthly

gage heights......................

rating table.

Rock Creek near:

description ......................

discharge........................

discharge, monthly..............

gage heights, $. . \ldots \ldots \ldots \ldots \ldots \ldots . . . . .$.

Rules for computation...................

Run-off, office methods of computing, in inches, definition of .......... 10-12
Rush Creek near- $\quad$ Page.

Adin, Cal.:

description ..................... 152

discharge........................ 152

Russian River, East Fork, near-

Ukiah, Cal.:

description ...................... 225

discharge...................... 225

Russian River basin:

miscellaneous measurements

225

S.

Sacramento, Cal.

Sacramento River at:

gage heights

Sacramento River at and near-

Gregory (Baird Station), Cal.:

description ...................... 131

discharge...................... 131

Red Bluff, Cal.:

description ....................... 128

discharge........................ 129

discharge, monthly ............... 130

gage heights .................... 129

rating table ..................... 130

Sacramento, Cal.: gage heights.................... 131

Sacramento River basin:

description ......................... 127

miscellaneous measurements .......... 127

Salinas River basin:

description .......................... 123

Salton sea, discharge into, amount of..... $\quad 39$

San Diego River near-

Lakeside, Cal.:

description ..................... 126

discharge......................... 127

San Francisquito Creek near-

Saugus, Cal.:

description .................... 116

discharge....................... 116

San Gabriel River near-

El Monte, Cal.:

description ....................... 108

discharge....................... 108

San Gabriel River and canals near-

Azusa, Cal.:

description ..................... 104

discharge..................... 105, 107

discharge, monthly ............... 107

gage heights.................... 105

rating table.................... 106

San Gabriel River basin:

description ........................ 104

miscellaneous measurements .......... 108

San Joaquin River:

description ........................ $\quad 184$

miscellaneous measurements ........ 185-187

San Joaquin River at-

Herndon, Cal.:

description ...................... 134

gage heights.................... 185

San Joaquin power house, California:

description ..................... 187

discharge...................... 187 
San Joaquin River, Middle Fork, Cali- $\quad$ Page. fornia:

description

186

discharge...........................

San Joaquin River, North Fork, California: description

discharge............................

San Joaquin River, South Fork, California:

description

discharge.............................

San Luis Rey River near-

Pala, Cal.:

description

discharge

discharge, monthly

gage heights

San Luis Rey River basin:

description

San-Pasqual, Cal.

Santa Ysabel River near

description

discharge......

gage heights.

Sanger, Cal.

Kings River near:

description

discharge...

discharge, monthly

gage heights

rating table

Sanger canal at-

Alṿord, Cal.:

description. ......................

discharge.........................

gage heights.

Santa Ana River, Cal.

Canals from:

discharge

Santa Ana River near-

Mentone, Cal.:

description ........................ 94-95

discharge........................ 95, 98

discharge, monthly $: . . . . . . . . . . .98$

gage heights...................... 96

rating table $\ldots \ldots \ldots \ldots \ldots \ldots \ldots \ldots \ldots .96,97$

Redlands, Cal.:

description.

discharge........................ 103

Rincon, Cal.:

description . ..................... 103

discharge....................... 103

gage heights $: \ldots \ldots \ldots \ldots \ldots \ldots \ldots \ldots$. 103

Santa Ana River basin:

description .......................... 94

miscellaneous measurements ........ 102-104

seepage measurements ................ 99

Santa Anita Creek near-

Sierra Madre, Cal.:

description

discharge..................... 108

Santa Barbara, Cal.

Santa Ynez River near: description ........... 117-118

discharge, monthly ................ 119

gage heights................... 118

rating table.
Santa Clara River near-

Camulos, Cal.:

description ....................... 117

discharge....................... $\quad 117$

Santa Paula, Cal.:

description ...................... 117

discharge........................ 117

Saticoy, Cal.

description ...................... 117

discharge........................ 117

Saugus, Cal.:

description ...................... 117

discharge........................ 117

Santa Clara River basin:

description ........................... 115

miscellaneous measurements ....... 115-117

Santa Clara Water and Irrigation Company's canal near-

Saticoy, Cal.:

description ..................... $\quad 117$

discharge........................ 117

Santa Maria, Cal.

Santa Maria River near:

description ...................... 120

discharge........................ 121

discharge, monthly ............... 123

gage heights ..................... 121

rating table ..................... 122

Santa Maria River basin:

description ...20.0.0.120

Santa Paula, Cal.

Farmers' canal near:

description ........................ 116

discharge....................... 116

Grees ditch near:

description .......................... 116

discharge....................... 116

Santa Clara River near:

description ..................... 117

discharge........................ 117

Santa Paula Creek near:

description ...................... 116

discharge........................ 116

Santa Ynez River near-

Santa Barbara, Cal.:

description ..................... 117, 118

discharge, monthly .............. 119

gage heights..................... 118

rating table ..................... 119

Santa Ynez River basin:

description ........................ 117

Santa Ysabel River near-

San Pasqual, Cal.:

description ..................... 127

discharge....................... 127

gage heights..................... 127

Saticoy, Cal.

Santa Clarả River near:

description ..................... $\quad 117$

discharge 117

Santa Clara Water and Irrigation Company's canal near:

description ..................... 117

discharge....................... 117 
Saugus, Cal.

San Francisquito Creek near:

description ..................... $\quad 116$

discharge....................... 116

Santa Clara River near:

description

Scott River near-

Fort Jones, Cal:

description

discharge

Second-foot, definition of.

Seepage measurements:

-Santa Ana River.

Sequoia National Park, Cal.

Kaweah River, Middle Fork, in:

description ........................

discharge......................... 194

Marble Fork in:

description ...................... 194

discharge......................... 194

Mount Whitney Power Company's flume at:

description

discharge.

Sespe Creek near-

Sespe, Cal.:

description

discharge.

Sespe Land and Water Company's canal near-

Sespe, Cal.:

description .......................

discharge....................... 116

Seven Mile Creek near-

Fort Klamath, Oreg.

description .

Shasta River near-

Yreka, Cal.:

description

Sheep Creek, California:

description .

discharge

Sheep Creek ditch near-

El Monte, Cal.:

description .............................

discharge.

Sheepy Creek near-

Brownell, Cal.:

description

discharge.

Shepherds Creek near-

Independence, Cal.:

description

discharge.

Sierra City, Cal.

Mining Company's flume near:

description.

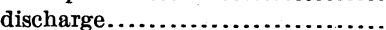

Yuba River, North Fork of North Fork, near:

description

discharge.
195

Page.
116
116
117
Sierra City, Cal.-Continued.

Page.

Yuba River, South Fork of North Fork, near:

description ..................... 165

discharge........................ 165

Sierra Madre, Cal.

Santa Anita Creek, near:

description .................. 108

discharge...................... 108

Siletz River at-

Siletz, Oreg.:

description ...................... 252

discharge....................... 252

gage heights..................... 252

Silver Creek and South Fork, California:

description ......................... 179

discharge............................. 179

Silverlake, Oreg.

Sycan River near:

description .

gage heights .......................

Single-point method of measuring discharge, description of .......... 9

Slide Creek, California:

description ........................ 200

discharge......................... 200

Slippery Ford, Cal.

American River, Silver and South Forks, near:

description .................... 179

discharge...................... 179

El Dorado ditch below:

description ...................... 179

discharge....................... 179

Slope method of measuring discharge, use and value of................... 6

Small Creek, California:

description ......................... $\quad 186$

discharge......................... 186

Smartville, Cal.

Yuba River, near:

description ..................... 160

discharge....................... 161,163

discharge, monthly............... 164

gage heights .................... 162

Soil; classification and test of, in Imperial Valley...................... 40,41

Soledad, Cal.

Arroyo Seco near:

description

123-124

discharge........................ 124

discharge, monthly ................ 126

gage heights...................... 125

rating table ...................... 125

South Eel River at-

Hearst, Cal.;

description ...................... 225

discharge........................ 225

Laytonville, Cal.:

description ....................... 226

discharge......................... 226

South Fork, etc. See name of river.

South Hillside canal near-

Bishop, Cal.:

description....................... $\quad 77$

discharge......................... 77

gage heights ................... 77 
South Yuba Mining Company's ditch near-

Colfax, Cal,: description ....................... 168

discharge....................... 168

Sprague River near-

Yainax, Oreg.:

description $. . . \ldots \ldots \ldots \ldots \ldots \ldots . . . . .$.

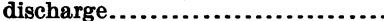

Spring Creek near-

Fort Klamath, Oreg.:

description

discharge...........................

Spring Lake, Oreg.

Phillip's wheel canal near:

description

discharge

Springville, Cal.

Tule River, North and Middle forks, near:

description

191,192

discharge

191,192

Squaw Creek near-

Copper City, Cal.:

description

132

discharge.

Squaw Hollow, Cal.

Stanislaus River at:

description

discharge..........................

Standerford ditch near-

El Monte, Cal.:

description .........................

discharge.

Standish, Cal.

Willow Creek near:

description.

discharge.........................

discharge, monthly ................

gage heights.......................

rating table.......................

Stanislaus River at-

Knights Ferry, Cal.:

description

217-218

discharge........................ 218

discharge, monthly................. 220

gage heights..................... . 219

rating table ...................... 219

Parrotts Ferry, Cal.:

description

discharge.

Stanislaus River, North, Middle, and South forks, Cal.

description

Stanislaus River basin:

description

miscellaneous meas

Stanislaus Water Company's ditch at-

Knights Ferry, Cal.:

description

discharge.........................

gage heights......................

rating table.

Stations, river, location of, map showing .:
Stella, Cal.

Clear Creek near:

description ....................... 131

discharge....................... 131

Stevens canal near-

Citrus, Cal.:

description ....................... 73

discharge......................... 73

gage heights....................... 74

Stevenson Creek, Cal.

description $. . \ldots \ldots \ldots \ldots \ldots \ldots \ldots \ldots \ldots, 186$

discharge.......................... 186

Stony Creek near-

Fruto, Cal.:

description ..................... 153

discharge...

discharge, monthly .............. 155

gage heights..................... 154

rating table .......................

Stony Creek basin:

description ......................... 152

Stream flow, field methods of measuring... $6-10$ Success, Cal.

Tule River, South Fork, near:

description ..................... 191

discharge........................ 191

Sunland, Cal.

Big Tejunga Creek near:

description ........................ 109

discharge......................... 109

Little Tejunga Creek near:

description ...................... 110

discharge........................ 110

Susan River near-

Susanville, Cal.:

description ...................... 42-43

discharge........... 43

discharge, monthly............... . 45

gage heights...................... 44

rating table...................... 44

Susan River basin:

description'

Susanville, Cal.

Susan River near:

description ........................ 42-43

discharge........................ 43

discharge, monthly............... 45

gage heights...................... 44

rating table...................... 44

Sweetwater River near-

Descanso, Cal.:

description $\ldots \ldots \ldots \ldots . .127$

discharge....................... 127

gage heights..................... 127

Swingle flume canal near-

Lorella, Oreg.:

description ....................... 247

discharge......................... 247

Sycan River near-

Silverlake, Oreg.:

description ....................... 232

discharge....................... 232

gage heights ....................... 232 
Page.

Tables, explanation of

Taboose Creek near-

Tibbetts, Cal.:

description

discharge.

Tamarack canal near-

Imperial, Cal.:

discharge......................

discharge, monthly

gage heights..

Tehama, Cal.

Mill Creek near:

description

Millrace canal near:

description

Temecula Creek near-

Temecula, Cal.:

description .......................

discharge.........................

gage heights' ....................

Tenaya Creek in-

Yosemite Valley, California:

description ....................

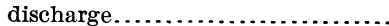

discharge, monthly ..............

gage heights.....................

rating table.

Ten Mile Creek, California:

description

discharge.

Three Mile Creek near-

Fort Klamath, Oreg.:

description

discharge......................

Three Rivers, Cal.

Kaweah River below:

description .....................

discharge........................

discharge, monthly..............

gage heights.....................

rating table.

Kaweah River, North and South forks, near:

description

discharge..................... 194, 195

Tibbetts, Cal.

Goodale Creek near:

description

discharge

Taboose Creek near:

description

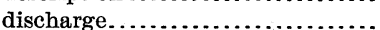

gage heights.....................

Tiltill Creek, California:

description

Tinemaha, Cal.

Tinemaha Creek near:

description

discharge.

Birch Creek near:

description

discharge.

gage heights.
4
Trinity River near-

Lowden, Cal.:

description .

discharge.

Triunfo Creek near-

Calabasas, Cal.:

description ....................... 113

discharge........................ 114

discharge, monthly ............... 115

gage heights..................... $\quad 114$

Tule Lake, California:

precipitation

Tule Lake near-

Merrill, Oreg.:

description ...................... 238

gage heights.................... 239

Tule River near-

Portersville, Cal.:

description ...................... 189

discharge...................... 190

discharge, monthly .............. 191

gage heights..................... 190

rating table..................... 191

Tule River, North and Middle forks, nearSpringville, Cal.:

description ..................... 191, 192

discharge...................... 191, 192

Tule River, South Fork, near-

Success, Cal.:

description ....................... 191

discharge..................... 191

Tule River basin:

description ......................... 189

miscellaneous measurements ........ 191-192

Tuolumne River at-

Hetch Hetchy, Cal.

description...................... 216

discharge:....................... 216

Lagrange, Cal.:

description .................... 209-210

discharge........................ $\quad 210$

discharge, monthly .............. $\quad 212$

gage heights.................... 211

rating table...................... 212

Tuolumne River, Middle and South forks, California:

description..................... 216, 217

discharge....................... 216, 217

Tuolumne River basin:

description ...................... 209

miscellaneous measurements ........ 216-217

Turlock canal at-

Lagrange, Cal.:

description ...................... 214

discharge......................... 214

gage heights.................... 215

rating table...................... 215

Tuttle Creek near-

Lone Pine, Cal.:

description ...................... 86

discharge...................... 86

U.

Ukiah, Cal.

Russian River, East Fork, near:

description ...................... 225

discharge......................... 
Umpqua River, North Fork, near-

Oakcreek, Oreg.:

description

gage heights

Umpqua River, South Fork, near-

Brockwaý, Oreg.:

description ................ 250

discharge $. . . . \ldots \ldots \ldots \ldots \ldots \ldots \ldots . .250$

gage heights...................... 251

Upper Lake, Cal.

Clover Creek near:

description ....................... 175

discharge......................... 175

Middle Creek near:

description ..................... 175

discharge.

V.

Valley Counties Power Company's canal near-

Centerville, Cal.:

description

discharge....

Van Brimmer ditch near-

Merrill, Oreg.:

description $. . \ldots \ldots \ldots \ldots \ldots \ldots \ldots \ldots .247,248$

discharge....................... 248

Van Loam's ditch near-

Likely, Cal.:

description

discharge...

Varney Creek near-

Pelican Bay, Oregon:

description

discharge.

Velocity method of measuring discharge, description of....................

Vertical integration method of measuring discharge, description of....

Vertical velocity-curve method of measuring discharge, description of...

Victorville, Cal.

Mohave River at:

description

discharge....

discharge, monthly

Vina, Cal.

gage hєights

Deer Creek near:

description

discharge.

W.

Waterman Canyon Creek near-

Arrowhead Springs, Cal.:

description

discharge..

Weir method of measuring discharge, requirements of

West Twin Creek near-

Arrowhead Springs, Cal.:

description

discharge.
West Valley Creek near- $\quad$ Page.

Likely, Cal.:

description ...................... 140

discharge.................... 141, 143

discharge, monthly............... 144

gage heights..................... 142

Wheatland, Cal.

Bear River above:

description ....................... 165

discharge......................... 166

discharge, monthly ............... 167

gage heights..................... 166

rating table ...................... 16

Wheeler flume near-

Downieville, Cal.:

description ............... 164

discharge........................ 164

Williamson River near-

Klamath Agency, Oreg.:

description ....................... 249

discharge........................ 249

Willow Creek at and near-

Adin, Cal.:

description ...................... 152

discharge........................ 152

Brownell, Cal.:

description ....................... 248

discharge........................ $\quad 248$

Merrillville, Cal.:

description ..................... 45

discharge, monthly .............. 47

gage heights..................... 46

rating tuble...................... 46

Standish, Cal.:

description ...................... 47

discharge........................ 47

dischargè, monthly ............... 49

gage heights...................... 48

rating table...................... 48

Willow Creek, North Fork, California:

deseription ......................... 187

discharge........................... 187

Winters, Cal.

Puta Creek at:

description ..................... 182-183

discharge........................ 183

gage heights...................... 183

Wisteria canal near-

Calexico, Cal.:

discharge....................... 36

discharge, monthly............... 37

gage heights.................... 36, 37

Wood River near-

Fort Klamath, Oreg.:

description ...................... 249

discharge.......................... 249

Woodland, Cal.

Moore's ditch near:

description

discharge.

Y.

Yainax, Oreg.

Sprague River near:

description

discharge. 
Yolo, Cal.

Cache Creek near:

description $\ldots \ldots \ldots \ldots \ldots \ldots \ldots \ldots \ldots, 172$

discharge........................ $\quad \mathbf{1 7 3}$

discharge, monthly............... 174

gage heights..................... 173

rating table...................... 174

Yosemite Creek in-

Yosemite Valley, California:

description ..................... 205

discharge...................... 205

discharge, monthly ............... 207

gage heights..................... 206

rating table ..................... 206

Yosemite Valley, California.

Bridal Veil Creek in:

description

Cascade Creek in:

description

discharge.

Merced River in

description ......................

discharge.

gage heights

rating table

Ribbon Falls Creek in:

description

discharge.

Tenaya Creek in:

description

discharge

discharge, month

.

gage heights

rating table.

Yosemite Creek in:

description

discharge..........................

discharge, monthly ..............

gage heights......................

rating table $\ldots \ldots \ldots \ldots \ldots \ldots \ldots \ldots .206$

Yreka, Cal.

Shasta River near:

description

discharge....................... 247

Yreka Light and Power Company's canal near:

description ........................ 249

discharge...................... 249

Yuba River, near-

Smartsville, Cal.:

description ..................... 160

discharge.

161,163
Yuba River, near-Continued.

Smartsville, Cal.-Continued.

discharge, monthly.............. 164

gage heights..................... 162

Yuba River, East Fork of North Fork, at-

Downieville, Cal.:

description ....................... 165

discharge....................... 165

Yuba River, Middle Fork, near-

Nevada City, Cal.:

description ....................... 164

discharge....................... 164

Yuba River, North Fork, at and near-

Downieville, Cal.:

description ....................... 165

discharge....................... 165

Goodyears bar, California:

description ...................... 164

discharge...................... 164

Yuba River, North Fork of North Fork, at and near-

Downieville, Cal.:

description ..................... 164,165

discharge..................... 164, 165

Sierra City, Cal.:

description ..................... 165

discharge....................... 165

Yuba River, South Fork of North Fork, near-

Sierra City, Cal.:

description ....................... 165

discharge...................... 165

Yuba River basin:

description ......................... 159-160

miscellaneous measurements......... 164-165

Yuma, Ariz., canals below, measurements of.......................... 18-42

Colorado River at:

description ...................... 13

discharge....................... 14-16

discharge, noonthly ............... 16

gage heights................ 14, 15, 17

Colorado Valley Pumping and Irrigation Company's canal at:

description ....................... 18

discharge....................... 19

Farmers' canal near:

description ...................... 19

discharge....................... $\quad 19$

Ludy canal near:

description ...................... 19-20

discharge........................ 20

gage heights..................... 20 



\section{CLASSIFICATION OF THE PUBLICATIONS OF THE UNITED STATES GEOLOGICAL SURVEY.}

[Water-Supply Paper No. 177.]

The publications of the United States Geological Survey consist of (1) Annual Reports; (2) Monographs; (3) Professional Papers; (4) Bulletins; (5) Mineral Resources; (6) Water-Supply and Irrigation Papers; (7) Topographic Atlas of United States-folios and separate sheets thereof; (8) Geologic Atlas of United States-folios thereof. The classes numbered 2,7 , and 8 are sold at cost of publication; the others are distributed free. A circular giving complete lists can be had on application.

Most of the above publications can be obtained or consulted in the following ways:

1. A limited number are delivered to the Director of the Survey, from whom they can be obtained, free of charge (except classes 2, 7, and 8), on application.

2. A certain number are delivered to Senators and Representatives in Congress, for distribution.

3. Other copies are deposited with the Superintendent of Documents, Washington, D. C., from whom they can be had at practically cost.

4. Copies of all Government publications are furnished to the principal public libraries in the large cities thruout the United States, where they can be consulted by those interested.

The Professional Papers, Bulletins, and Water-Supply Papers treat of a variety of subjects, and the total number issued is large. They have therefore been classified into the following series: A, Economic geology; B, Desscriptive geology; C, Systematic geology and paleontology; D, Petrography and mineralogy; E, Chemistry and physics; F, Geography; G, Miscellaneous; H, Forestry; I, Irrigation; J, Water storage; K, Pumping water; L, Quality of water; M, General hydrographic investigations; N, Water power; O, Underground waters; P, Hydrographic progress reports.

Series P.-The hydrographic progress reports contain the results of stream measurements. A report is issued for every calendar year, containing the results of data collected during that year. These reports were first published as a part.of the Director's annual report or as a bulletin; they are now published as water-supply and irrigation papers. The following is a list, by years, of the publications containing the progress reports of stream measurements. A detailed index of these reports (1888-1903) is published as Water-Supply Paper No. 119.

1888. Tenth Annual Report, Part II.

1889. Eleventh Annual Report, Part II.

1890. Twelfth Annual Report, Part II.

1891. Thirteenth Annual Report, Part III.

1892. Fourteenth Annual Report, Part II.

1893. Bulletin No. 131.

1894. Bulletin No. 131; Sixteenth Annual Report, Part II.

1895. Bulletin No. 140.

1896. Water-Supply Paper.No. 11; Eighteenth Annual Report, Part IV.

1897. Water-Supply Papers Nos. 15 and 16; Nineteenth Annual Report, Part IV.

1898. Water-Supply Papers Nos. 27 and 28; Twentieth Annual Report, Part IV.

1899. Water-Supply Papers Nos. 35, 36, 37, 38, and 39; Twenty-first Annual Report, Part IV.

1900. Water-Supply Papers Nos. 47,48,49, 50,51, and 52; Twenty-second Annual Report, Part IV.

1901. East of Mississippi River, Water-Supply Papers Nos. 65 and 75.

West of Mississippi River, Water-Supply Papers Nos. 66 and 75. 
1902. East of Mississippi River, Water-Supply Papers Nos. 82 and 83.

West of Mississippi River, Water-Supply Papers Nos. 84 and 85.

1903. East of Mississippi River, Water-Supply Papers Nos. 97 and 98.

West of Mississippi River, Water-Supply Papers Nos. 99 and 100.

1904. East of Mississippi River, Water-Supply Papers, Nos. 124, 125, 126, 127, 128, and 129.

West of Mississippi River, Water-Supply Papers Nos. 130, 131, 132, 133, 134, and 135.

1905. East of Mississippi River, Water-Supply Papers Nos. 165, 166, 167, 168, 169, 170, and 171.

West of Mississippi River, Water-Supply Papers Nos. 171, 172, 173, 174, 175, 176, 177, and 178.

The Geological Survey and the Reclamation Service have suboffices in different parts of the United States, from which hydrographic and reclamation work in the respective localities is carried on and where data may be obtained on application. These offices are located as follows:

Boston, Mass., 6 Beacon street; Utica, N. Y., 75 Arcade; Atlanta, Ga., 409 Temple court; Austin, Tex., University of Texas; Chicago, Ill., 876 Federal Building; Belle Fourche, S. Dak.; Cody, Wyo.; Denver, Colo., Chamber of Commerce Building; Salt Lake City, Utah; Los Angeles, Cal., 1108 Braly Building; San Francisco Cal., 432 Merchants' Exchange Building; Phoenix, Ariz.; Carlsbad, N. Mex.; El Paso, Tex.; Billings, Mont.; Great Falls, Mont.; Hazen, Nev.; Boise, Idaho; Spokane, Wash., 424 Peyton Block; Pendleton, Oreg.

Correspondence should be addrest to

The Director,

United States Geological Survey,

ОстовеR, 1906. Washington, D. C. 